\title{
32nd Annual Meeting and Pre-Conference Programs of the Society for Immunotherapy of Cancer (SITC 2017): Part Two
}

National Harbor, MD, USA. 8-12 November 2017

Published: 7 November 2017

\section{About this supplement}

These abstracts have been published as part of Journal for ImmunoTherapy of Cancer Volume 5 Supplement 2, 2017. The full contents of the supplement are available online at https://jitc.biomedcentral.com/articles/supplements/volume-5-supplement-2. Please note that this is part 2 of 2

\section{Poster presentations \\ Clinical Trials (In Progress)}

\section{P218}

KEYNOTE-585: randomized, phase 3 study of chemotherapy + pembrolizumab vs chemotherapy + placebo as neoadjuvant/ adjuvant treatment for patients with gastric or gastroesophageal junction (G/GEJ) cancer

Yung-Jue Bang', Eric Van Cutsem², Charles Fuchs' ${ }^{3}$, Atsushi Ohtsu', Josep Tabernero ${ }^{5}$, David Ilson ${ }^{6}$, Woo Jin Hyung ${ }^{7}$, Vivian Strong ${ }^{6}$, Thorsten Goetze $^{8}$, Takaki Yoshikawa ${ }^{9}$, Laura Tang ${ }^{6}$, Linda Sun ${ }^{10}$, Aisha Hasan ${ }^{10}$, Minori Koshiji ${ }^{11}$, Kohei Shitara ${ }^{4}$

${ }^{1}$ Seoul National University Hospital, Seoul, Republic of Korea; ${ }^{2}$ University Hospitals Gasthuisberg Leuven and KU Leuven, Leuven, Belgium; ${ }^{3}$ Yale Cancer Center, New Haven, CT, USA; ${ }^{4}$ National Cancer Hospital East, Chiba, Japan; ${ }^{5}$ Vall d'Hebron University Hospital and Institute of Oncology (VHIO), Barcelona, Spain; ${ }^{6}$ Memorial Sloan Kettering Cancer Center, New York, NY, USA; ${ }^{7}$ Yonsei Cancer Hospital, Yonsei University Health System, Seoul, Republic of Korea; ${ }^{8}$ Institute of Clinical Cancer Research, UCT University Cancer Center, Frankfurt, Germany; ${ }^{9}$ Kanagawa Cancer Center, Kanagawa, Japan; ${ }^{10}$ Merck \& Co., Inc., Kenilworth, NJ, USA; ${ }^{11}$ Merck \& Co., Inc., New York, NY, USA

Correspondence: Yung-Jue Bang (bangyj@snu.ac.kr)

Journal for ImmunoTherapy of Cancer 2017, 5(Suppl 2):P218

\section{Background}

In KEYNOTE-012 (NCT01848834) and KEYNOTE-059 (NCT02335411), pembrolizumab demonstrated manageable safety and promising antitumor activity alone or in combination with chemotherapy in patients with advanced G/GEJ cancer. Compared with chemotherapy alone, chemotherapy combined with pembrolizumab in the neoadjuvant/adjuvant setting can provide additional benefit to patients with locally advanced, resectable G/GEJ cancer. KEYNOTE-585 is a phase 3, randomized, double-blind study of chemotherapy combined with pembrolizumab versus chemotherapy combined with placebo as neoadjuvant/adjuvant treatment for locally advanced resectable G/GEJ cancer.

Methods

Key eligibility criteria in KEYNOTE-585 (NCT03221426) include age $\geq 18$ years; previously untreated G/GEJ adenocarcinoma (Siewert type 2 or 3 tumors; Siewert type 1 tumor eligibility limited to those for whom planned treatment is perioperative chemotherapy and resection), with no evidence of metastatic disease; planning to undergo surgery after preoperative chemotherapy; Eastern Cooperative Oncology Group performance status
0-1; adequate organ function; no active autoimmune disease. Patients will be randomly assigned 1:1 to receive chemotherapy + pembrolizumab (arm 1) or chemotherapy + placebo (arm 2). Stratification factors are geographic region (Asia vs Non-Asia), primary tumor location (stomach vs GEJ), and tumor stage (II/III vs IVa). All patients will receive neoadjuvant (preoperative) chemotherapy + pembrolizumab every 3 weeks (Q3W) for 3 cycles or chemotherapy + placebo Q3W for 3 cycles followed by surgery and then adjuvant chemotherapy + pembrolizumab Q3W for 3 cycles or chemotherapy + placebo Q3W for 3 cycles followed by monotherapy with pembrolizumab or placebo Q3W for 11 cycles; treatment will continue for up to 17 cycles overall. Chemotherapy consists of cisplatin $80 \mathrm{mg} / \mathrm{m}^{2}$ intravenously + either capecitabine $1000 \mathrm{mg} / \mathrm{m}^{2}$ twice daily orally or 5fluorouracil $800 \mathrm{mg} / \mathrm{m}^{2}$ intravenously (investigator's choice). Pembrolizumab $200 \mathrm{mg}$ was administered intravenously. Adjuvant monotherapy consists of pembrolizumab (arm 1) or placebo (arm 2). Primary end points are overall survival (OS), event-free survival, and rate of pathologic complete response (defined as no invasive disease and histologically negative nodes) per central review. Adverse events (AEs) are graded per National Cancer Institute Common Terminology Criteria for Adverse Events v4.0 and will be monitored for 30 days after treatment end (90 days for serious AEs). Patients are followed up for survival every 12 weeks until death, withdrawal from study, or study termination. Planned enrolment is approximately 800 patients.

Trial Registration

ClinicalTrials.gov, NCT03221426

\section{P219}

Phase 1/1b, first-in-human study of the PI3K-gamma inhibitor IPI549 as monotherapy and combined with nivolumab in patients with advanced solid tumors

Antoni Ribas', David Hong ${ }^{2}$, Anthony Tolcher ${ }^{3}$, Ryan Sullivan ${ }^{4}$, Geoffrey Shapiro ${ }^{5}$, Bartosz Chmielowski ${ }^{1}$, Les Brail ${ }^{6}$, Lucy Lee ${ }^{6}$, Suresh Mahabhashyam ${ }^{6}$, Claudio Dansky Ullmann ${ }^{6}$, Michael Postow ${ }^{7}$, Jedd Wolchok

${ }^{1}$ University of California, Los Angeles, Los Angeles, CA, USA; ${ }^{2} \mathrm{MD}$ Anderson Cancer Center, Houston, TX, USA; ${ }^{3}$ South Texas Accelerated Research Therapeutics (START), San Antonio, TX, USA; ${ }^{4}$ Massachusetts General Hospital, Boston, MA, USA; ${ }^{5}$ Dana-Farber Cancer Institute, Boston, MA, USA; ${ }^{6}$ Infinity Pharmaceuticals, Inc., Cambridge, MA, USA; ${ }^{7}$ Memorial Sloan Kettering Cancer Center, New York, NY, USA

Correspondence: Les Brail (Les.Brail@infi.com)

Journal for ImmunoTherapy of Cancer 2017, 5(Suppl 2):P219 


\section{Background}

IPI-549 is a potentially first-in-class, oral, potent, selective PI3K-gamma inhibitor being developed as an immuno-oncology therapeutic in multiple cancer indications. Preclinical solid tumor research has shown that PI3K-gamma blockade in tumor-associated macrophages by IPI-549 results in transcriptional reprogramming of the pro-tumor macrophage phenotype (M2) to its anti-tumor counterpart (M1). Within this setting, IPI-549 has demonstrated activity as monotherapy and still greater activity when combined with checkpoint inhibitor therapies. Importantly, the latter approach has been found to overcome checkpoint inhibitor resistance in specific resistant models.

\section{Methods}

This study is being conducted to evaluate the safety, tolerability, pharmacokinetics, and pharmacodynamics of IPI-549 to ultimately determine its recommended Phase 2 dose and activity, both as monotherapy and in combination with nivolumab, in patients with advanced solid tumors. As shown in Fig. 1, the design includes four parts: 1) dose escalation (DE) of IPI-549 monotherapy; 2) DE of IPI-549 with fixed-dose nivolumab; 3) monotherapy expansion; and 4) combination expansion in specific tumor types, including non-small cell lung cancer, melanoma, and squamous cell carcinoma of the head and neck, with de novo or acquired resistance to checkpoint inhibitors (Fig. 1).

Results

This trial is currently in progress. No results are available.

Progress update: Monotherapy DE enrollment is complete for oncedaily (QD) IPI-549 doses up to $60 \mathrm{mg}$, while monotherapy expansion enrollment has been initiated at $60 \mathrm{mg} \mathrm{QD}$. Combination DE enrollment is complete for QD doses of $20 \mathrm{mg}$ and $30 \mathrm{mg} \mathrm{IPI-549} \mathrm{com-}$ bined with fixed-dose nivolumab ( $240 \mathrm{mg}$ once every 2 weeks). Conclusions

The collective preclinical data highlight the key role of PI3K-gamma in the immuno-suppressive tumor microenvironment and provide a strong rationale for the ongoing clinical study of IPI-549.

Trial Registration

ClinicalTrials.gov: NCT02637531

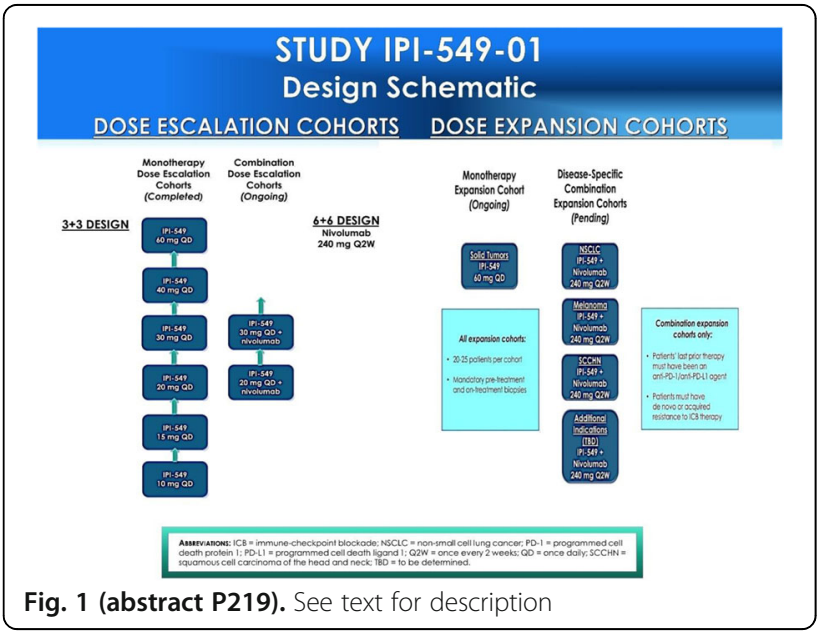

P220

A phase 2, multicenter study to evaluate the efficacy and safety using autologous tumor infiltrating lymphocytes (LN-145) in patients with recurrent, metastatic, or persistent cervical carcinoma Amir Jazaeri ${ }^{1}$, Robert Edwards' ${ }^{2}$, Emese Zsiros ${ }^{3}$, Robert Brown ${ }^{4}$, Igor Gorbatchevsky ${ }^{4}$, Sam Suzuki ${ }^{4}$, Maria Fardis ${ }^{4}$, Robert Wenham ${ }^{5}$ 'University of Texas MD Anderson Cancer Center, Houston, TX, USA; ${ }^{2}$ Magee-Women's Hospital of University of Pittsburgh Medical Center, Pittsburgh, PA, USA; ${ }^{3}$ Roswell Park Cancer Institute, Buffalo, NY, USA; ${ }^{4}$ Iovance Biotherapeutics, San Carlos, CA, USA; ${ }^{5}$ Moffitt Cancer Center, Tampa, FL, USA

Correspondence: Robert Brown (robert.brown@iovance.com) Journal for ImmunoTherapy of Cancer 2017, 5(Suppl 2):P220

\section{Background}

Adoptive cell therapy (ACT) may be effective in treating immunogenic tumors with high mutational load such as melanoma and virallyassociated tumors like cervical cancer with several patients in studies performed by other institutions achieving durable complete response for years. HPV infection increases mutational load, thus providing additional neoantigen targets ideal for the polyclonal nature of ACT. As outcomes for patients with recurrent, metastatic or persistent cervical cancer remain extremely poor, there is an enormous need for novel immunotherapeutic approaches with curative potential such as ACT.

Methods

Clinical trial C-145-04 (NCT03108495) is a prospective, phase 2 multicenter, open-label study evaluating the efficacy of a single autologous tumor infiltrating lymphocyte infusion (LN-145) followed by IL-2 after a non-myeloablative lymphodepletion (NMA-LD) regimen in patients with recurrent, metastatic, or persistent cervical cancer who have failed at least one prior systemic therapy. The clinical trial protocol requires resection of a tumor lesion which is then shipped to a central GMP manufacturing facility for TIL extraction, expansion, and preparation of the final infusion product ( $(\mathrm{N}-145)$. One week prior to LN-145 shipment and infusion, patients undergo NMA-LD consisting of cyclophosphamide $(60 \mathrm{mg} / \mathrm{kg}$ ) daily $\times 2$ days followed by fludarabine $\left(25 \mathrm{mg} / \mathrm{m}^{2}\right)$ daily $\times 5$ days. LN-145 is infused 24 hours after the last dose of fludarabine followed by up to 6 doses of IL-2 $(600,000 \mathrm{lU} / \mathrm{kg})$ every 8-12 hours. Simon's two-stage optimal design with one-sided alpha level $=0.025$ and $80 \%$ power will be used to compare an objective response rate (ORR) of $5 \%$ vs. $20 \%$ in the first stage ( $\mathrm{n}=15$ subjects). If two or more ORR are observed, trial will expand to Stage $2(n=47)$. The primary endpoint is the ORR per RECIST v1.1. Secondary endpoints include complete response, duration of response, disease control rate, progression free- and overall survival; and the safety summarization of treatment-emergent adverse events (AEs) including serious AEs, AEs leading to discontinuation, and clinical laboratory tests. Patients must have been treated with at least 1 systemic chemotherapy or immunotherapy treatment for recurrent, metastatic, or persistent cervical cancer and, in addition to the tumor targeted for excision and TIL manufacture, must have an additional measurable lesion for assessment of response. Other major eligibility criteria include amongst others: adequate bone marrow, liver, pulmonary, cardiac and renal function; ECOG performance status of 0 or 1 . Systemic steroids greater than 10 $\mathrm{mg} /$ day prednisone equivalents are prohibited as are a history of serious immunotherapy-related adverse events.

Trial Registration

ClinicalTrials.gov identifier: NCT03108495

P221

A phase 2 study to evaluate the safety and efficacy using autologous tumor infiltrating lymphocytes (LN-145) in patients with recurrent and/or metastatic squamous cell carcinoma of the head and neck

Rom Leidner ${ }^{1}$, James Ohr ${ }^{2}$, Robert Brown ${ }^{3}$, Sam Suzuki ${ }^{3}$,

Igor Gorbatchevsky ${ }^{3}$, Maris Fardis ${ }^{3}$, Robert Ferris ${ }^{2}$

'Earle A. Chiles Research Institute - Providence Cancer Center, Portland, OR, USA; ${ }^{2}$ Hillman Cancer Center at UPMC, Pittsburgh, PA, USA; ${ }^{3}$ lovance Biotherapeutics, San Carlos, CA, USA

Correspondence: Rom Leidner; Robert Brown

(robert.brown@iovance.com)

Journal for ImmunoTherapy of Cancer 2017, 5(Suppl 2):P221

\section{Background}

Adoptive cell therapy (ACT) may be effective in treating immunogenic tumors with high mutational load such as melanoma and virally-associated tumors like cervical cancer with several patients in studies performed by other institutions achieving durable complete response for years. Despite the heterogeneity of squamous cell carcinomas of the head and neck (HNSCC), most tumors are either virally-associated (e.g., HPV in oropharyngeal) or carry high mutational load (e.g., tobacco-related) providing an increased diversity of potential targets ideal for the polyclonal nature of ACT. Furthermore, outcomes for patients with recurrent and/or metastatic HNSCC 
remain poor. Therefore, a clear rationale exists for the potential application of ACT in patients with HNSCC.

Methods

Clinical trial C-145-03 (NCT03083873) is a prospective phase 2 multicenter, open-label study evaluating the efficacy of a single autologous tumor infiltrating lymphocyte infusion (LN-145) followed by IL-2 after a nonmyeloablative lymphodepletion (NMA-LD) regimen in patients with recurrent and/or metastatic HNSCC. Study-related therapy begins with resection of a tumor lesion that is then shipped to a central GMP manufacturing facility where TIL are extracted, expanded, packaged, and shipped for administration (LN-145). One week prior to LN-145 infusion, patients undergo NMA-LD consisting of cyclophosphamide $(60 \mathrm{mg} / \mathrm{kg})$ daily $\times 2$ days followed by fludarabine $\left(25 \mathrm{mg} / \mathrm{m}^{2}\right)$ daily $\times 5$ days. LN- 145 is infused 24 hours after the last dose of fludarabine followed by up to 6 doses of IL-2 (600,000 IU/kg) every 8-12 hours. Simon's two-stage optimal design with one-sided alpha level $=0.025$ and $80 \%$ power will be used to compare an objective response rate (ORR) of $5 \%$ vs. $20 \%$ in the first stage ( $n=15$ subjects). If two or more ORR are observed, trial will expand to Stage $2(n=47)$. The primary efficacy endpoints are the objective response rate per RECIST v1.1 and the safety summarization of treatmentemergent adverse events (AEs) including serious AEs, AEs leading to discontinuation, and clinical laboratory tests. Secondary efficacy endpoints include CR, DOR, PFS, and OS. Patients must have been treated with at least one systemic chemotherapy or immunotherapy treatment for recurrent and/or metastatic HNSCC and, in addition to the tumor targeted for excision and TIL manufacture, must have an additional measurable lesion for assessment of response. Additional eligibility criteria include amongst others: adequate bone marrow, liver, pulmonary, cardiac, and renal function; ECOG performance status of 0 or 1 . Systemic steroids greater than $10 \mathrm{mg} /$ day prednisone equivalents are prohibited as are a history of serious immunotherapy-related adverse events.

Trial Registration

ClinicalTrials.gov identifier:NCT03083873

\section{P222}

Clinical trial in progress: $\mathrm{A}$ phase $1 \mathrm{~b}$ trial of talimogene

laherparepvec (T-VEC) in combination with dabrafenib and trametinib in advanced melanoma with an activating BRAF mutation

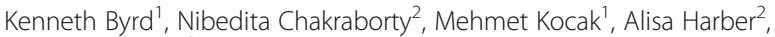
Ari Vanderwalde

${ }^{1}$ University of Tennessee Health Science Center, Memphis, TN, USA, Memphis, TN, USA; ${ }^{2}$ West Cancer Center, Germantown, TN, USA,

Germantown, TN, USA

Correspondence: Kenneth Byrd (kbyrd@westclinic.com)

Journal for ImmunoTherapy of Cancer 2017, 5(Suppl 2):P222

\section{Background}

Patients with BRAF-mutant advanced melanoma have been shown to respond at high rates to combination BRAF plus MEK inhibition. Response rates to single-agent immune therapies tend to be modest, but are often durable, while response rates to BRAF plus MEK inhibition tend to be high but transient. While early attempts at combining targeted and immune therapy resulted in dose-limiting hepatic toxicity, more recent attempts with newer agents have shown significant promise. T-VEC is an oncolytic viral immunotherapy which was designed to selectively replicate in tumors resulting in lytic cell death, antigen release, and production of GM-CSF to enhance systemic immune response. In a prior randomized, phase 3 study of T-VEC versus GM-CSF, durable response rate was statistically improved with T-VEC, with a strong trend towards improved overall survival. Dabrafenib and trametinib, inhibitors of BRAF and MEK, respectively, have been shown in combination to result in response rates of up to $75 \%$ and improvement in progression-free and overall survival compared to single-agent targeted therapy and chemotherapy. Combining T-VEC with dabrafenib and trametinib may further enhance antitumor immune responses in addition to preserving the targeted effect.

Methods

This is a Phase Ib, prospective, single arm study of T-VEC given in combination with standard doses of dabrafenib and trametinib in advanced melanoma with an activating BRAF mutation. The primary endpoint of the study is tolerability as measured by dose-limiting toxicities seen in the first 5 weeks of treatment. Key secondary endpoints include progression-free survival, objective response rate, change in tumor burden, time to response, and duration of response among responders. Tumor-level responses in injected and uninjected tumors, and characterization of immune markers in pre-study and on-study biopsies will be exploratory endpoints. Key eligibility criteria include unresectable stage IIIB-IV BRAF mutant melanoma, presence of measurable and injectable disease, no active cerebral metastases or autoimmune diseases, and any number of prior lines of therapy but no prior receipt of T-VEC. T-VEC ( $10^{6} \mathrm{PFU} / \mathrm{mL}$ first dose, $10^{8} \mathrm{PFU} / \mathrm{mL}$ subsequent doses) will be administered by intralesional injection into cutaneous, subcutaneous, or nodal lesions on week 1 day 1 , week 4 day 1 , and every 2 weeks thereafter until disappearance of injectable lesions, complete response, progressive disease, intolerance of study treatment, or 24 months after starting therapy, whichever occurs first. Dabrafenib and trametinib will be given until progression or intolerance. Twenty subjects are to be enrolled at a single U.S. institution.

Trial Registration

NCT03088176

\section{P223}

KEYNOTE-199: Phase 2 nonrandomized study of pembrolizumab in patients with PD-L1+ and PD-L1- metastatic castration-resistant prostate cancer

Johann de Bono ${ }^{1}$, Josep Piulats ${ }^{2}$, Marine Gross-Goupil ${ }^{3}$, Jeffrey Goh ${ }^{4}$, Kristiina Ojamaa ${ }^{5}$, Christopher Hoimes ${ }^{6}$, Ulka Vaishampayan,

Raanan Berger ${ }^{8}$, Ahmet Sezer ${ }^{9}$, Ronald De Wit ${ }^{10}$, Charles Drake ${ }^{11}$, Haiyan Wu ${ }^{12}$, Christian Poehlein ${ }^{12}$, Emmanuel S. Antonarakis ${ }^{13}$

${ }^{1}$ Royal Marsden Hospital and the Institute of Cancer Research, London, United Kingdom; ${ }^{2}$ Instituto Catalan de Oncologia, Hospital Duran i Reynals, Hospitalet de Llobregat, Barcelona, Spain; ${ }^{3}$ Institut Bergonie, Bordeaux, France; ${ }^{4}$ Royal Brisbane \& Women's Hospital, Brisbane, Australia; ${ }^{5}$ East Tallinn Central Hospital, Tallinn, Estonia; ${ }^{6}$ University Hospitals Seidman Cancer Center, Cleveland, OH, USA; ${ }^{7}$ Karmanos Cancer Institute, Detroit, MI, USA; ${ }^{8}$ Chaim Sheba Medical Center, RamatGan, Israel; ${ }^{9}$ Baskent Üniversitesi Adana Uyg. ve Arast, Adana, Turkey;

${ }^{10}$ Erasmus MC Cancer Institute, Rotterdam, Netherlands; ${ }^{11}$ Columbia University, New York, NY, USA; ${ }^{12}$ Merck \& Co., Inc., Kenilworth, NJ, USA; ${ }^{13}$ Johns Hopkins Kimmel Cancer Center, Baltimore, MD, USA

Correspondence: Johann de Bono (Johann.DeBono@icr.ac.uk) Journal for ImmunoTherapy of Cancer 2017, 5(Suppl 2):P223

\section{Background}

Metastatic castration-resistant prostate cancer (mCRPC) treatment has included suppression of androgen receptor signaling, palliative radiation therapy, and chemotherapy. As expression of the programmed death 1 (PD-1) receptor and its ligand PD-L1 is present in a subset of $\mathrm{mCRPC}$ lesions, targeting this pathway may be an attractive treatment option. KEYNOTE-199 (NCT02787005) is a nonrandomized, multinational, multicohort open-label phase 2 study to evaluate the anti-PD-1 antibody pembrolizumab in patients with $\mathrm{mCRPC}$.

Methods

Patients must be $\geq 18$ years old with histologically or cytologically confirmed prostate adenocarcinoma without small-cell histology, measurable disease (RECIST v1.1) or detectable bone metastases by whole-body bone scintigraphy and no RECIST V1.1 measurable tumors, supplied tumor sample for PD-L1 expression (new or archived), disease progression within 6 months before screening, and ECOG performance status 0 2. As of June 2017, 2 additional cohorts were added called cohorts 4 and 5 . For these cohorts patients had to fail or show signs of failure on current prechemotherapy enzalutamide; patients could fail abiraterone treatment before enzalutamide. For cohort 4, patients will be enrolled with RECIST v1.1-measurable disease $(n=80)$ and for cohort 5 patients will be enrolled with bone metastases only or bone-predominant disease $(n=40)$. For both cohorts patients will receive pembrolizumab 200 $\mathrm{mg}$ every 3 weeks (Q3W) plus current enzalutamide regimen. For the original cohorts (cohorts 1-3), patients must have been treated with $\geq 1$ targeted endocrine therapy (abiraterone or enzalutamide) and $\leq 2$ 
chemotherapy regimens; one must have contained docetaxel. Patients also must be undergoing androgen deprivation with serum testosterone $<50 \mathrm{ng} / \mathrm{dL}$. Patients will be enrolled based on PD-L1 status and RECIST v1.1 measurability and will receive pembrolizumab 200 mg Q3W: PD-L1-positive, RECIST v1.1 measurable disease (cohort 1), PD-L1-negative, RECIST V1.1 measurable disease (cohort 2; cohorts 1 and 2 combined, $n=200$ ), and bone metastases and RECIST v1.1 nonmeasurable disease (cohort 3, $n=50$ ). All patients will continue until documented confirmed disease progression, unacceptable adverse events (AEs), or illness that prevents further treatment. Imaging response will be assessed every 9 weeks for approximately 1 year and every 12 weeks thereafter, per central imaging vendor review (RECIST v1.1) and the Prostate Cancer Clinical Trials Working Group 3 guidelines. AEs will be monitored throughout the study. Primary end points are overall response rate for cohorts 1 and 2 combined and for cohorts 1, 2, and 4. Key secondary end points include safety and tolerability, duration of response, disease control rate, radiographic progression-free survival, and overall survival.

Trial Registration

ClinicalTrials.gov, NCT0278700

\section{P224}

Immunological correlates observed in an Interim analysis of the Phase 3 ADAPT Trial evaluating Rocapuldencel-T (AGS-003), for the treatment of patients with metastatic renal cell carcinoma (mRCC) Mark DeBenedette, Alicia Gamble, Ana Plachco, Marcus Norris, Charles Nicolette

Argos Therapeutics Inc, Durham, NC, USA

Correspondence: Mark DeBenedette

(mdebenedette@argostherapeutics.com)

Journal for ImmunoTherapy of Cancer 2017, 5(Suppl 2):P224

\section{Background}

Rocapuldencel-T is an investigational patient-specific immunotherapeutic comprised of autologous dendritic cells programmed with RNA from the patient's tumor to express tumor-specific antigens and thereby induce a memory $T$ cell response. Rocapuldencel-T is engineered to secrete IL-12, a critical cytokine for memory-T cell formation, to induce an antigen-specific memory $\mathrm{T}$ cell response. We previously reported results from a Phase 2 study showing a correlation between the magnitude of the induced memory-T cell response and overall survival (OS) in advanced RCC patients. We therefore measured the antigen-specific memory $\mathrm{T}$ cell response and the level of secreted IL-12 in patients receiving Rocapuldencel$\mathrm{T}$ to assess the relationship of these parameters to each other and to overall survival (OS).

Methods

The ADAPT trial is designed to evaluate OS in subjects with newly diagnosed mRCC receiving Rocapuldencel-T in combination with standard-of-care (SOC) versus SOC alone. As part of this interim analysis, immune monitoring was performed on patients $(N=146)$ enrolled in the United States and randomized to the combination arm. The change in the number of CD28+/CD45RA- antigen-specific memory $\mathrm{T}$ cells present after in vitro stimulation of PBMCs with Rocapuldencel-T was measured with multi-color flow cytometry. The amount of IL-12 secreted by each patient's immunotherapeutic was measured by a cytokine bead capture method $(N=179)$. The increase in the number of antigen-specific memory $T$ cells after administration of Rocapuldencel-T and the concentration of IL-12 secreted by each patient's immunotherapeutic where correlated with OS.

Results

Data from this interim analysis revealed a statistically significant increase in the number of CD28+/CD45RA- memory T cells after as few as three doses of Rocapuldencel-T administered three weeks apart. The increase above baseline became a statistically significant correlate with OS after seven doses. Furthermore, the amount of IL-12 each patient's immunotherapeutic produced showed a statistically significant correlation with both the magnitude of the memory $\mathrm{T}$ cell response and OS.

\section{Conclusions}

This interim analysis demonstrated that administration of Rocapuldencel$T$ resulted in an increase in antigen-specific memory $T$ cells and that the magnitude of the induced memory $T$ cell response after seven doses correlated with OS. Additionally, the amount of IL-12 secreted by each patient's immunotherapeutic correlated with both the magnitude of the induced memory $\mathrm{T}$ cell response and OS. Therefore, the level of IL-12 secretion may serve as a predictive biomarker for $\mathrm{T}$ cell response to Rocapuldencel-T and favorable clinical outcome, warranting further investigation.

Trial Registration

ClinicalTrials.gov identifier-NCT01582672

\section{P225}

Functional reversal of Foxp3+ $T$ regulatory activity in patients enrolled in the Phase 3 ADAPT Trial evaluating Rocapuldencel-T for the treatment of patients with metastatic renal cell carcinoma (mRCC)

Mark DeBenedette, Joe Horvatinovich, Elizabeth Grogan, Larissa

Benavente, Irina Tcherepanova, Charles Nicolette

Argos Therapeutics Inc, Durham, NC, USA

Correspondence: Mark DeBenedette

(mdebenedette@argostherapeutics.com); Joe Horvatinovich

Journal for ImmunoTherapy of Cancer 2017, 5(Suppl 2):P225

\section{Background}

Rocapuldencel-T is an investigational patient-specific immunotherapeutic comprised of autologous dendritic cells programmed with RNA from the patient's tumor to express tumor-specific antigens and induce a memory-T cell response. The Phase 3 ADAPT trial is designed to evaluate overall survival (OS) in subjects with newly diagnosed $\mathrm{mRCC}$ receiving Rocapuldencel-T in combination with standard-of-care (SOC) versus SOC alone. Current SOC first-line treatment with sunitinib is shown to decrease regulatory $T$ cells (Tregs), thus potentially modulating anti-tumor activity. Therefore, FoxP3+ Treg cell counts in patients receiving Rocapuldencel-T were measured ex vivo in whole blood samples and the relationship to OS was assessed for each arm of the study. Methods

For this interim analysis, whole blood samples collected at intervals during the course of the ADAPT trial were stained immediately ex vivo by multi-color flow cytometry to determine the number of CD4+/CD25+/CD127-/Foxp3+ Treg cells. The correlation between the number of Tregs present and overall survival was determined for the combination arm subjects $(N=177)$ and the SOC arm subjects $(N=80)$ for whom data was available. We further studied the impact of Rocapuldencel-T on Treg phenotype and function after in vitro stimulation of PBMCs from treated subjects.

Results

As reported elsewhere, data from this interim analysis showed a decline in the number of Tregs after the first cycle of sunitinib. Surprisingly, the number of Tregs at baseline or at any time point measured, positively correlated with OS in the combination arm, but negatively correlated with OS in the SOC arm. Furthermore, in vitro stimulation of autologous PBMCs with Rocapuldencel-T resulted in conversion of Tregs to a proinflammatory cell phenotype that proliferates in culture, thus providing a possible explanation for the positive correlation between the number of Tregs and OS in the combination arm.

\section{Conclusions}

In this interim analysis, baseline Tregs positively correlated with survival only in the Rocapudencel-T arm and not in the SOC arm. These data suggest that Rocapudencel-T exerts a biologic effect catalyzed by the presence of Tregs, potentially by conversion to T effector cells, resulting in favorable clinical outcome. This is consistent with the in vitro observation that Rocapudencel-T can convert Tregs to T effector cells. Therefore, the level of Tregs in whole blood samples may serve as a useful baseline biomarker predictive of favorable long-term clinical outcome in patients treated with Rocapudencel-T while also predicting poor outcome in patients receiving conventional SOC.

Trial Registration

ClinicalTrials.gov identifier-NCT01582672. 
P226

FRACTION-RCC: a randomized, open-label, adaptive, phase 2 study of nivolumab in combination with other immuno-oncology agents in patients with advanced RCC

Corina Candiani Taitt', Robert J. Motzer ${ }^{2}$, Toni K. Choueiri ${ }^{3}$, Bernard J. Escudier ${ }^{4}$, Timothy Kuzel ${ }^{5}$, Michael A. Carducci ${ }^{6}$, Suresh Nair ${ }^{7}$, Scott S. Tykodi ${ }^{8}$, Sarah Tannenbaum-Dvir ${ }^{1}$, Megan Wind-Rotolo ${ }^{1}$, Katy L. Simonsen', Paula M. Fracasso'

${ }^{1}$ Bristol-Myers Squibb, Princeton, NJ, USA; ${ }^{2}$ Memorial Sloan Kettering Cancer Center, New York, NY, USA; ${ }^{3}$ Dana-Farber Cancer Institute, Boston, MA, USA; ${ }^{4}$ Gustave Roussy Cancer Centre, Villejuif Cedex, France; ${ }^{5}$ Rush University Medical Center, Chicago, IL, USA; ${ }^{6}$ Sidney Kimmel Comprehensive Cancer Center, Johns Hopkins University, Baltimore, MD, USA; ${ }^{7}$ Lehigh Valley Health Network, Allentown, PA, USA; ${ }^{8}$ University of Washington and Fred Hutchinson Cancer Research Center, Seattle, WA, USA

Correspondence: Corina Candiani Taitt (ea@chrysalismedical.com) Journal for ImmunoTherapy of Cancer 2017, 5(Suppl 2):P226

\section{Background}

Nivolumab, a fully human IgG4 monoclonal antibody (mAb) that targets the PD-1 receptor, is approved for patients with advanced renal cell carcinoma (RCC) after prior antiangiogenic therapy based on superior overall survival vs everolimus (CheckMate 025; Motzer RJ, et al. N Engl J Med. 2015). Nivolumab has also shown promising antitumor activity in combination with ipilimumab (a fully human IgG1 mAb that targets cytotoxic T-lymphocyte antigen 4) in patients with metastatic RCC, supporting the rationale that nivolumab in combination with other immuno-oncology (IO) agents or targeted therapies may improve outcomes in patients with advanced RCC. Given the rapid development of novel 10 agents, traditional study designs may not efficiently evaluate all possible IO-IO and IO-targeted therapy combinations. Fast Real-time Assessment of Combination Therapies in Immuno-ONcology (FRACTION) is an innovative clinical trial program with a rolling, adaptive platform design that allows for the addition of new regimens as well as the withdrawal of ineffective regimens. Here we describe the study concept, key design components, and first IO treatment combinations of FRACTION-RCC, a phase 2, randomized, open-label, adaptive study in advanced RCC (NCT02996110).

Methods

FRACTION-RCC is envisioned to accelerate the development of the next generation of $\mathrm{IO}$ combinations for patients with metastatic RCC. Patients with advanced RCC with a clear-cell component will be enrolled based on prior $\mathrm{IO}$ treatment and randomized to receive nivolumab plus BMS-986016 (a fully human lgG4 mAb that targets lymphocyte activation gene 3 ) or nivolumab plus ipilimumab. Enrollment is continuous and may offer patients consecutive treatment options based on treatment exposure and response. Primary endpoints include objective response rate, duration of response, and progressionfree survival rate at 24 weeks. The secondary endpoint is safety. Biomarker analyses will also be performed. New treatment combinations will be added over time to explore their potential benefits and provide a continuous flow of treatment options for patients whose cancer progresses on existing treatments.

Trial Registration

ClinicalTrials.gov, NCT02996110

\section{P227}

A trial to evaluate the safety, immunogenicity and clinical activity of a helper peptide vaccine plus PD- 1 blockade

Craig Slingluff, Jr, Elizabeth Gaughan, William Grosh, Gina Petroni, Kimberly Bullock

University of Virginia, Charlottesville, VA, USA

Correspondence: Elizabeth Gaughan (egaughan@virginia.edu)

Journal for ImmunoTherapy of Cancer 2017, 5(Suppl 2):P227

\section{Background}

PD-1 blocking antibodies are standard first line therapy for management of advanced melanoma. Monotherapy response rates to these agents are reported between $30-40 \%$; therefore, the majority of patients require additional therapy [1,2]. Strategies to improve the benefit of these agents are currently focused on combination with other systemic agents and adjunctive local treatment measures. We are evaluating the combination of pembrolizumab with a vaccine incorporating 6 peptides that induces CD4+ helper T cell $\left(\mathrm{T}_{\mathrm{H}}\right)$ responses (6MHP) and has proven activity in patients with advanced melanoma [3]. The primary end point is determination of the safety and tolerability of the combination of $6 \mathrm{MHP}$ vaccine plus Pembrolizumab and estimation of the CD4+ T cell response rate to $6 \mathrm{MHP}$ in the blood and sentinel immunized node. Secondary endpoints include evaluation of the induction of epitope-spreading and the T cell infiltration of the tumor microenvironment.

Methods

This is an open-label, phase $1 /$ II study to evaluate the safety, immunogenicity and clinical activity of the 6MHP vaccine and pembrolizumab (MEL64, NCT02515227). Candidates may be resistant or naïve to systemic immunotherapy agents. Patients with prior PD-1 antibody exposure are included if the patient failed to experience a clinical response after 12 weeks or progressed on treatment. All subjects will receive $6 \mathrm{MHP}$ on days $1,8,15,43,64$, and 85 . Pembrolizumab will be administered intravenously (IV) every 3 weeks for up to 2 years. Biopsy specimens will be collected from the tumor (days 1 and 22) and a sentinel immunized node (SIN; day 22). Biopsy and peripheral blood specimens will be used in the immunologic analyses. Overall target sample size is based upon having sufficient information to assess whether combined treatment with $6 \mathrm{MHP}$ vaccine plus pembrolizumab increases the immunogenicity of $6 \mathrm{MHP}$ alone in the entire study population. Maximum target sample size is 40 patients. Trial is currently enrolling patients.

Results

Trial in Progress.

Conclusions

Trial in Progress.

Trial Registration

NCT02515227

\section{References}

1. Robert C, Schachter J, Long GV, et al. Pembrolizumab versus Ipilimumab in Advanced Melanoma. N Engl J Med. 2015;372(26):2521-32.

2. Larkin J, Chiarion-Sileni V, Gonzalez R, et al. Combined Nivolumab and Ipilimumab or Monotherapy in Untreated Melanoma. N Engl J Med. 2015;373(1):23-34

3. Slingluff $C L$ Jr, Petroni GR, Olson W, et al. Helper T cell responses and clinical activity of a melanoma vaccine with multiple peptides from MAGE and melanocytic differentiation antigens. J Clin Oncol. 2008;26(30):4973-80.

P228

Pembrolizumab and decitabine for relapsed and refractory acute myeloid leukemia (PD-AML)

Catherine Lai, Oetjen Karolyn, Katherine Lindblad, Christin Destefano,

Meghali Goswami, Hong Yuen Wong, Therese Intrater, Debbie Draper, Laura Dillon, Christopher Hourigan

National Heart, Lung and Blood Institute, National Institutes of Health, Bethesda, MD, USA

Correspondence: Christopher Hourigan (hourigan@nih.gov)

Journal for ImmunoTherapy of Cancer 2017, 5(Suppl 2):P228

\section{Background}

While a variety of different treatment regimens have been studied for patients with relapsed/refractory acute myeloid leukemia (AML) clinical outcomes unfortunately remain dismal. There appears to be no single superior therapeutic approach and the current standard of care is referral to an appropriate clinical trial. The unique combination of pembrolizumab and decitabine used in this trial was selected for investigation based on several factors: 1) Largely nonoverlapping adverse reaction profiles for these agents. 2) Both agents already FDA approved potentially allowing for rapid translation/adoption. 3) Both agents have previously evaluated dosing schedules that are compatible with one another. 4) Theoretical possibility of synergy given respective mechanisms of action. 
Methods

PD-AML (17-H-0026, NCT02996474) is an investigator sponsored, single-institution, single-arm open-label ten subject pilot study to evaluate the feasibility of a novel combination of pembrolizumab and decitabine in adults with relapsed/refractory AML. Secondary objectives will explore efficacy and determine time to first response, best response and duration of best response. Laboratory objectives include investigation of changes in AML clonal composition and disease burden during therapy, and measurement of changes in immune parameters associated with clinical efficacy and/or toxicity. Up to eight 21 day cycles of pembrolizumab are given with decitabine given on days 8-12 and 15-19 on alternative cycles (ie: cycles 1, 3, 5 and 7) (Fig. 1).

Results

This clinical trial opened in February 2017 and is currently in progress. Currently seven patients have reached response and toxicity assessment time-points. Updated results will be presented at the meeting.

Conclusions

Acute myeloid leukemia is a heterogeneous group of diseases with distinct molecular and phenotypic characteristics. Even within a single patient AML may be polyclonal at any examined time-point, and this clonal composition can change over time with the clone predominant at presentation not necessarily the one responsible for relapse and death. We hypothesize that effective pembrolizumab therapy for refractory/relapsed AML may be associated with changes in the leukemic clonal composition due to differences in immunogenicity between clones. The oligoclonal nature of AML biology, together with a blood and bone marrow distribution highly amenable to repeated sampling of the sites of disease burden, provides a near unique opportunity to investigate fundamental mechanisms underpinning treatment efficacy of this new combination of immunotherapeutic drugs.

Trial Registration

[https://clinicaltrials.gov/ct2/show/NCT02996474]

FDA-IND: 131826

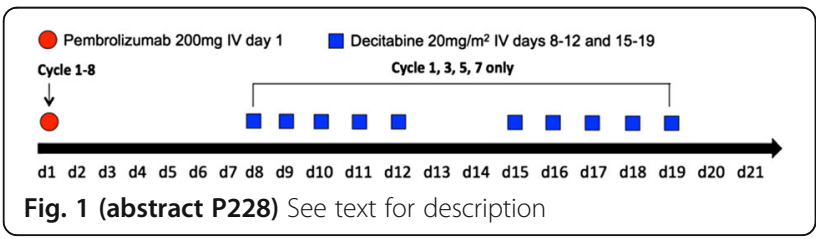

P229

Withdrawn

P230

Phase I/II safety and efficacy study of image guided intratumoral CD40 agonistic monoclonal antibody APX005M in combination with systemic pembrolizumab in metastatic melanoma patients Daniel Johnson H Johnson, Srisuda Lecagoonporn, Chantale Bernatchez, Cara Haymaker, Salah Bentebibel, Marc Uemura, Cassian Yee, Rodabe Amaria, Sapna Patel, Hussein Tawbi, Isabella Glitza, Michael A. Davies, Michael K. Wong, Wen-Jen Hwu, Patrick Hwu, Willem Overwijk, Adi Diab UT-MD Anderson Cancer Center, Houston, TX, USA

Correspondence: Daniel Johnson H Johnson

(dhjohnson@mdanderson.org); Adi Diab

Journal for ImmunoTherapy of Cancer 2017, 5(Suppl 2):P230

\section{Background}

Checkpoint blockade has become a major modality in the treatment of metastatic melanoma (MM). However, long-term survival and durable remission rates remain low and new treatment options are needed. CD40 activation on antigen presenting cells (APCs) initiates their maturation and ability to prime and activate CD8+ T cells through upregulation of co-stimulatory molecules (CD80, CD86, CD70, 4-1BBL, OX40L, and GITR-L) as well as expression of cytokines such as IL-12. Furthermore, CD40 activation cause macrophages to develop a more tumoricidal phenotype and tumor cells to increase MHC I expression.

Direct intratumoral (IT) immune modulation utilizes the tumor as a "vaccine site" to generate a tumor specific immune response. We hypothesize that (IT) injection of a CD40 agonist such as APX005M, will "immunize" patients against melanoma neoantigens through "licensing" of tumor infiltrating APCs for tumor specific T cell priming and activation. In preclinical mouse models, we have shown that IT administration of the recombinant adenovirus encoding the dendritic cell-activating $\mathrm{CD} 40 \mathrm{~L}$ induces $\mathrm{CD} 8^{+} \mathrm{T}$ cell-mediated systemic activity against B16 melanoma. Importantly, IT rAdCD40L also augmented the activity of anti-PD-1.

Methods

This phase I/II trial (NCT02706353) evaluates the safety, efficacy, and immunological impact of IT administration of APX005M (CD40 agonistic $\mathrm{mAb}$ ) in combination with systemic pembrolizumab in pts with MM. An accelerated $3+3$ design was used for the phase 1 dose escalation portion of this study. Pts will receive IT APX005M at escalating doses every 3 weeks for a total of 4 doses. Image guidance will allow for injection of visceral, nodal, and soft tissue metastases. The singlearm phase 2 expansion will evaluate the overall response rate (ORR) of this regimen 12 weeks after initiation of treatment. Some key inclusion criteria: confirmed cutaneous or mucosal melanoma; measurable, unresectable stage-III or stage-IV disease, and at least 2 injectable lesions. Key exclusion criteria include: prior immunotherapy, uveal melanoma, active autoimmune disease, or active immunodeficiency. A sample size of 26 patients will have $75 \%$ power to detect an improvement from a null ORR of $33 \%$ to $55 \%$, using a one group chi-square test and assuming a one-sided a-level of 5\%. Immune analysis will be performed on pre and on-treatment tumor/liquid biopsies including but not limited to quantification of dendritic cells and $T$ cells both in injected and non-injected tumors.

Trial Registration

NCT02706353

P231

KEYNOTE-590: randomized, phase 3 study of chemotherapy + pembrolizumab vs chemotherapy + placebo as first-line therapy for patients with advanced esophageal or esophagogastric junction (E/EGJ) cancer

Ken Kato ${ }^{1}$, Manish Shah'², Peter Enzinger ${ }^{3}$, Jaafar Bennouna ${ }^{4}$, Lin Shen ${ }^{5}$ Antoine Adenis ${ }^{6}$, Ying Zhu' ${ }^{7}$, Pooja Bhagia ${ }^{7}$, Minori Koshijii, Toshihiko Doi

${ }^{1}$ National Cancer Center Hospital, Tokyo, Japan; ${ }^{2}$ Weill Cornell Medical College, New York Presbyterian Hospital, New York, NY, USA; ${ }^{3}$ Dana Farber Cancer Institute, Boston, MA, USA; ${ }^{4} \mathrm{CHU}$ de Nantes, Nantes, France; ${ }^{5}$ Beijing Cancer Hospital, Beijing, China; ${ }^{6}$ nstitut du Cancer de Montpellier, Montpellier, France; ${ }^{7}$ Merck \& Co., Inc., Kenilworth, NJ, USA; ${ }^{8}$ National Cancer Center East, Chiba, Japan

Correspondence: Ken Kato (kenkato@ncc.go.jp)

Journal for ImmunoTherapy of Cancer 2017, 5(Suppl 2):P231

\section{Background}

No chemotherapeutic regimens or targeted agents are approved specifically for esophageal cancer; available options have limited benefit and substantial toxicity. In the phase $1 \mathrm{~b}$ KEYNOTE-028 study (NCT02054806), pembrolizumab monotherapy demonstrated manageable safety and durable antitumor activity in heavily pretreated patients with PD-L1-positive advanced esophageal carcinoma. In KEYNOTE-059 (NCT02335411), combining chemotherapy (cisplatin and 5-fluorouracil) and pembrolizumab as first-line treatment for patients with advanced gastric or gastroesophageal junction cancer resulted in encouraging efficacy and manageable safety. This suggests chemotherapy plus pembrolizumab as a potential therapeutic strategy for esophageal cancer KEYNOTE-590 is a randomized, double-blind, multicenter phase 3 study of cisplatin and 5-fluorouracil plus pembrolizumab versus cisplatin and 5 -fluorouracil plus placebo in patients with advanced E/EGJ carcinoma. Methods

Eligible patients are $\geq 18$ years of age; have locally advanced unresectable or metastatic adenocarcinoma or squamous cell carcinoma of 
esophagus or metastatic Siewert type 1 adenocarcinoma of EGJ, measurable disease per RECIST 1.1, ECOG performance status 0-1, adequate organ function; have had no prior therapy for advanced disease, no autoimmune disease, no active infection; and can provide a newly obtained or archival tissue sample. Patients will be randomly assigned 1:1 to cisplatin $80 \mathrm{mg} / \mathrm{m}^{2}$ IV every 3 weeks (Q3W) for 6 cycles plus 5fluorouracil $800 \mathrm{mg} / \mathrm{m}^{2}$ continuous IV on days 1-5 Q3W IV plus pembrolizumab $200 \mathrm{mg}$ IV Q3W or cisplatin $80 \mathrm{mg} / \mathrm{m}^{2}$ IV Q3W for 6 cycles plus 5-fluorouracil $800 \mathrm{mg} / \mathrm{m}^{2}$ continuous IV on days 1-5 Q3W plus placebo Q3W IV. Treatment will continue up to 2 years. Response will be assessed using CT (preferred) or MRI every 9 weeks by central imaging per RECIST v1.1. Adverse events will be graded per NCI CTCAE v4.0 and up to at least 30 days after the end of treatment. Primary end points are PFS per RECIST V1.1 and OS in all patients and in patients with PDL1 positive or negative tumor expression (combined positive score $\geq 10 \%$ or $<10 \%$ using immunohistochemistry). Secondary end points include ORR per RECIST v 1.1, duration of response, safety, and healthrelated quality of life. PFS and OS will be compared between groups using a stratified log-rank test; hazard ratios will be estimated using a Cox regression model. The Kaplan-Meier method will be used to estimate event rates within groups. Enrollment is planned for approximately 700 patients.

Trial Registration

ClinicalTrials.gov, NCT03189719

\section{P232}

A pilot study to evaluate the clinical and immunological effects of incorporating a CD40-agonistic antibody into the multimodality treatment of resectable esophageal and GE junction cancers

Andrew H. Ko, Lawrence Fong

University of California San Francisco, San Francisco, CA, USA

Correspondence: Andrew H. Ko (andrew.ko@ucsf.edu

Journal for ImmunoTherapy of Cancer 2017, 5(Suppl 2):P232

\section{Background}

Targeting CD40, a member of the TNF receptor superfamily found on antigen presenting cells (APCs), represents a promising cancer immunotherapeutic strategy. Activation of this costimulatory molecule results in improved antigen processing and presentation and cytokine release from activated APCs, enhancing T cell responses. Additionally, CD40 is expressed on many tumor cells that, when activated, results in tumor cell apoptosis and inhibition of tumor growth. APX005M (Apexigen, San Carlos, CA) is a humanized IgG1 anti-CD40 agonistic antibody that binds to CD40 with high affinity. In a FIH phase I clinical trial in patients with advanced solid tumors, APX005M was relatively well tolerated, with cytokine release syndrome (CRS) as the DLT at doses above the RP2D. Importantly, correlative studies show that APX005M produces dose-dependent activation of APCs, T cell activation, and increases in circulating cytokine levels.

Methods

This pilot trial represents the first to evaluate a CD40-agonistic antibody in esophageal/GE junction cancer, a disease in which $I O$ agents (particularly PD-1 mAbs) have demonstrated promising activity. The study is also the first to explore combining $\mathrm{IO}$ with chemoradiation in the neoadjuvant setting, as this multimodality approach represents the standard of care for patients with resectable esophageal/GE junction cancer and is optimally conducive for serial tumor tissue acquisition. A total of 16 patients with resectable (UT1-3N0-1) squamous cell or adenocarcinoma of the esophagus or GE junction will be enrolled. Chemoradiation consists of radiation (5040cGy in 28 daily fractions) and low-dose carboplatin plus paclitaxel weekly x 5, as per standard of care. APX005M $0.3 \mathrm{mg} / \mathrm{kg}(1$ dose level below single-agent MTD) is given every 3 weeks for a total of 4 doses, with the first dose administered two weeks prior to the initiation of concurrent chemoradiation. APX005M administration is offset by 2-3 days from chemotherapy to avoid the steroid premedication administered with paclitaxel. Tumor tissue is acquired via endoscopic biopsy at baseline and following the single-dose "run-in" of APX005M; and then again at esophagectomy, which occurs 1-2 months following completion of chemoradiation. Serial blood collections are also performed at multiple pre-defined timepoints. In addition to assessing the feasibility, safety, and preliminary efficacy (as measured by pathologic complete remission rate) of this novel combination, analyses of tumor tissue and blood will be performed, including Tissue Multiplex Immunohistochemistry and flow cytometry for both APC and T cell activation, as well as $T$ cell receptor sequencing for $\mathrm{T}$ cell repertoire diversity.

P233

A phase I study to evaluate the safety of multi-antigen stimulated tumor specific cellular therapy (MASCT-I) in patients with advanced solid tumors

Ruihua $\mathrm{Xu}^{1}$, Xiaoshuang $\mathrm{Li}^{2}$, Yanjun Kong ${ }^{2}$, Jianchuan Xia' ${ }^{1}$ Desheng Weng' ${ }^{1}$ Xiaoshi Zhang' ${ }^{1}$ Xing Zhang'

'Sun Yat-sen university affiliated oncology hospital, Guangzhou, China;

${ }^{2}$ HRYZ Biotech Company, Shenzhen, China

Correspondence: Ruihua Xu (kyj8@sina.com)

Journal for ImmunoTherapy of Cancer 2017, 5(Suppl 2):P233

\section{Background}

Tumor-specific immune responses are known to be initiated by tumor associated and/or specific antigen-sensitized dendritic cells (DCs), that can effectively process and present tumor antigens to CD4+ and CD8+ T cells. MASCT-I is a sequential immune cell therapy for solid tumor, which included multi-antigen loaded DC vaccines followed by the adoptive transfer of anti-tumor specific T cells. DC vaccines are produced from patients' autologous PBMC-derived DC loaded with multiple tumor associated antigen peptides and are injected into patients to induce active anti-tumor immunity. Antitumor specific $\mathrm{T}$ cells are stimulated from the same patient by cocultured with DC vaccines and Anti-PD1 antibody in vitro, are then infused into the patient to target tumor cells.

Methods

This is a single center, three stages phase I study. Stage 1 and 2 will enroll ( $3+3$ design) patients with advanced (unresectable) or recurrent bladder cancer or soft tissue sarcoma who have failed all standard therapies, Patients will be treated with MASCT-I. If the dose limitation toxicity (DLT) in the first cycle of MASCT-I is $<33.3 \%$, the stage 2 will begin. Patients who have advanced recurrent or metastatic bladder cancer with Gemcitabine and Cisplatin (GP) chemotherapy achieving clinical benefit (group 1) will be treated with MASCT-I as maintenance therapy. Also, patients who have advanced recurrent or metastatic sarcoma with achieving clinical benefit after MAID or CAV/IE (predominant Doxorubicin regimens) (group 2) will be treated with MASCT-I combined with Ifosfamide as maintenance therapy too. During the stage 2 , if, in the first cycle of MASCT-I treatment, the DLT is $<33.3 \%$, stage 2 will extend to stage 3 . Approximately additional 24 patients will be enrolled in group 1 and 2 . The primary objective is safety and tolerability. Secondary objectives include DCR, PFS, TTP, OS. As of 17 May 2017, Three patients were enrolled and completed Stage 1 without any DLT and treatment related SAEs. Five AEs are related with treatment, they are pain on the injection site, fatigue, pruritus and arthralgia, which were all grade 1. Recruitment is ongoing for dose expansion (stage 2). Clinical trial information: NCT030343

\section{P234}

AST-VAC2: An allogeneic dendritic cell cancer immunotherapy entering clinical trials in patients with lung cancer in the advanced and adjuvant setting

Christian Ottensmeier ${ }^{1}$, Hayley Farmer-Hall ${ }^{2}$, Gary Acton ${ }^{2}$, Heike Lentfer ${ }^{3}$, Kevin Nishimoto ${ }^{4}$, Uzma Shoukat-Mumtaz ${ }^{4}$, Erik Whiteley ${ }^{4}$, Rob Allen ${ }^{4}$, Jane Lebkowski ${ }^{4}$

${ }^{1}$ University of Southampton, Southampton, United Kingdom; ${ }^{2}$ Cancer Research UK, London, United Kingdom; ${ }^{3}$ Cancer Research UK, Potters Bar, United Kingdom; ${ }^{4}$ Asterias Biotherapeutics, Fremont, CA, USA Correspondence: Jane Lebkowski (rallen@asteriasbio.com) Journal for ImmunoTherapy of Cancer 2017, 5(Suppl 2):P234 


\section{Background}

Primary lung cancer is the most common malignancy after nonmelanocytic skin cancer with deaths exceeding those from any other type of malignancy worldwide. In advanced (TNM stage IIIA, IIIB and IV) disease, only palliative therapy is available which carries a high risk of toxicity and limited potential to extend life. Intradermal delivery of dendritic cells (DCs) carrying an immunogenic cargo offers a novel approach to address malignancy mediated through upregulated telomerase expression and decreased cell death. AST-VAC2 is a mature, allogeneic $\mathrm{DC}$ vaccine derived by differentiating $\mathrm{H} 1$ human embryonic stem cells (hESCs) into mature DCs, transfected to express the tumor associated antigen human telomerase reverse transcriptase (hTERT) and lysosomal associated membrane protein 1 (LAMP-1) [1,2]. LAMP-1 fusion proteins enable target hTERT peptides to be directed to HLA (Human Leukocyte Antigen) II and HLA I receptors on the surface of endogenous DCs, invoking dual, antigen-specific CD4+ and CD8+ responses [3]. Preclinical investigations have shown that AST-VAC2 phagocytose, process, and present antigen upon maturation. Furthermore they produce immunostimulatory cytokines, migrate in response to cytokines, and activate antigen specific T cell responses.

\section{Methods}

The first-in-human trial will evaluate safety, tolerability, immunogenicity and therapeutic potential in adult NSCLC patients in advanced (metastatic or locally advanced disease) and adjuvant (currently radiologically disease free) settings. Patients who are positive for the HLA-A2 allele (expressed by AST-VAC2) will receive 6 weekly doses of $1 \times 10^{7}$ AST-VAC2 cells and will be monitored for one year as the primary follow-up point and subsequently for up to five years for long-term follow-up. Safety assessments as well as immunological monitoring for the generation and maintenance of hTERT specific T cells will be key endpoints. Results will be used to expand the clinical indications for assessment and support future combination immunotherapy approaches.

\section{References}

1. Tseng SY, Nishimoto KP, Silk KM, Dawes GN, Waldmann H, Fairchild PJ, Lebkowski JS, Reddy A. Generation of immunogenic dendritic cells from human embryonic stem cells without serum or feeder cells. Regen. Med. 2009; 4(4):513-526.

2. Nishimoto KP, Tseng S-Y, Lebkowski JS, Reddy A. Modification of human embryonic stem cell-derived dendritic cells with mRNA for efficient antigen presentation and enhanced potency. Regen. Med. 2011;6(3):303-318.

3. Su Z, Dannull J, Yang B, Dahm P, Coleman D, Yancey D,Sichi S, Niedzwiecki D, Boczkowski D, Gilboa E, Vieweg J. Telomerase mRNA-Transfected Dendritic Cells Stimulate Antigen-Specific CD8+and CD4+T Cell Responses in Patients with Metastatic Prostate Cancer. J Immunol. 2005.174: 3798-3807.

\section{P235}

Nivolumab in patients with advanced or metastatic non-small cell lung cancer (Stage IIIb/IV) who have received at least one prior systemic chemotherapeutic regimens

Sung Yong Lee, Sang Mi Chung, Ju Whan Choi, Young Seok Lee, Jong Hyun Choi, Jee Youn Oh, Kyung Hoon Min, Gyu Young Hur, Jae Jeong Shim, Kyung Ho Kang

Korea University Medical Center, Seoul, Republic of Korea

Correspondence: Sung Yong Lee (syl0801@korea.ac.kr)

Journal for ImmunoTherapy of Cancer 2017, 5(Suppl 2):P235

\section{Background}

Nivolumab, a human programmed death 1 (PD-1) immune checkpoint inhibitor antibody, has been shown to increase overall survival in non-small cell lung cancer (NSCLC) patients. This immune checkpoint blockade has been approved in Korea, United States, the European Union, and other countries for the treatment of advanced NSCLC that has progressed after platinum-based chemotherapy. Nivolumab has demonstrated longer overall survival than docetaxel among the previously treated NSCLC patients. We have assessed the safety of nivolumab in 8 patients with previously treated, locally advanced or metastatic NSCLC who were enrolled in the NSCLC Expanded Access Program (EAP) in Korea University Guro Hospital.

\section{Methods}

This EAP program included subjects with histologically or cytologically documented NSCLC who have relapsed after systemic treatment with a minimum of 1 prior systemic treatment for stage IIIB/stage IV disease. Subjects were treated with $3 \mathrm{mg} / \mathrm{kg}$ of nivolumab IV every 2 weeks for a maximum of 24 months. Each 14-day dosing period constituted a single cycle. Patients included in the analysis had received $\geq 1$ dose of nivolumab and were monitored for adverse events (AEs). Results

As of June 30,2017, eight patients participated. Median age was 63.0 years. All participants were male. Except for 1 (12.5\%) current smoker and $1(12.5 \%)$ never smoker, other $6(75 \%)$ patients were former smokers. The average of smoking period was 27 pack-years. 2 had squamous and 6 had non-squamous histology. At the time of enrollment, 4 had bone, 3 had brain and 3 had brain metastases. Best response rates was $12.5 \%$ and disease control rate was $50 \%$. Median progression free survival was 99.5 days $(95 \% \mathrm{Cl} 50.1-174.7)$. During the nivolumab chemotherapy, 2 patients had pneumonia and 1 had stroke. Other 5 patients had no critical complication during the treatment.

Conclusions

Our study showed that EAP participants had some lower response rate and more prolonged PFS compared to previously reported in Checkmate-017 and in Checkmate-057. And nivolumab EAP showed good safety profiles. In conclusion, treatment with Nivolumab is safe and effective for patients who have previously received heavily chemotherapy.

P236

Innate immunotherapy of neuroblastoma and PD-1 checkpoint blockade

Holger Lode', Maxi Zumpe', Madlen Juettner', Sascha Troschke-Meurer ${ }^{1}$, Evelyne Janzek ${ }^{2}$, Romana Schaefer ${ }^{2}$, Hans Loibner ${ }^{2}$, Nikolai Siebert ${ }^{1}$

${ }^{1}$ University Medicine Greifswald, Greifswald, Germany; ${ }^{2}$ Apeiron Biologics, Vienna, Austria

Correspondence: Holger Lode (lode@uni-greifswald.de) Journal for ImmunoTherapy of Cancer 2017, 5(Suppl 2):P236

\section{Background}

Passive immunotherapy of cancer is established for a variety of malignant diseases. Anti-GD $\mathrm{GD}_{2}$ antibody $(\mathrm{Ab}) \mathrm{ch} 14.18 / \mathrm{CHO}$ (dinutuximab beta) showed activity for the treatment high-risk neuroblastoma (NB) patients and received recently marketing approval in the EU. Here we demonstrate that one important mechanism of action in patients is antigen specific Ab-dependent cellular cytotoxicity (ADCC) and we report that ADCC impacts on PD-1/PD-L1 checkpoint regulation that can be targeted by co-treatment with an inhibitor.

Methods

53 patients received $100 \mathrm{mg} / \mathrm{m}^{2} \mathrm{ch} 14.18 / \mathrm{CHO}$ (d8-17), $6 \times 10^{6} \mathrm{IU} / \mathrm{m}^{2}$ sc IL-2 (d1-5; 8-12 and $160 \mathrm{mg} / \mathrm{m}^{2}$ oral 13-cis-RA (d19-32) in a closed single center program (53 pts). Polymorphisms in Fcy-receptor genes $2 \mathrm{~A}(\mathrm{H} 131 \mathrm{R}),-3 \mathrm{~A}(\mathrm{~V} 158 \mathrm{~F})$ and $-3 \mathrm{~B}$ (NA1/NA2) were determined by realtime PCR. Expression of PD-L1 and PD-1 was analyzed by RT-PCR and flow cytometry. Effect of PD-1/PD-L1 blockade and ch14.18/CHO-mediated anti-NB immune response was evaluated using anti-PD-1 Ab both in vitro (Nivolumab) and in the syngeneic $\mathrm{GD}_{2}^{+} \mathrm{NB}$ NXS2 mouse model (anti-mouse PD-1).

Results

We identified $33 / 53$ patients with low affinity FCGR alleles (FCGR2AH131R/R and/or FCGRA3A-V158 F/F). These patients showed lower PFS rates compared to $20 / 53$ patients with high affinity polymorphisms $(p<0.01)$. ADCC levels on day 15 of cycle 1 in pts with high affinity polymorphisms showed an ADCC increase of $20 \pm 6 \%$ compared to $11 \pm 2 \%$ in the control. The correlation with functional immune parameter ADCC and clinical outcome confirm its role for clinical efficacy.

Interestingly, tumor specific ADCC in the presence of LA-N-1 neuroblastoma cells, leukocytes and sub-therapeutic ch14.18/CHO concentrations $(10 \mathrm{ng} / \mathrm{ml})$ results in a strong increase of the PD-L1 expression and incubation with IL-2 further enhanced this effect. Blockade with Nivolumab reversed the PD-L1-dependent inhibition 
of ADCC. Finally, mice treated with ch $14.18 / \mathrm{CHO}$ in combination with PD-1 blockade showed strongest reduction of tumor growth, longest survival rate as well as the highest level of NB cell lysis mediated by serum and leukocytes of treated mice compared to controls.

\section{Conclusions}

Patient studies clearly reveal ADCC as mechanism of ch14.18/CHO against neuroblastoma, and this upregulates the inhibitory checkpoint PD-1/PD-L1. Combination of ch14.18/CHO with PD-1/PD-L1 blockade results in synergistic treatment effects. Similar upregulation of PD-L1 expression by suboptimal ADCC was also seen with the human GD2+ osteosarcoma cell line MG63. This suggests a broader applicability and to consider combinations of passive immunotherapy of cancer with PD-1/PD-L1 checkpoint inhibition.

\section{P237}

Phase I study of adoptive transfer of iNKT cells for treating patients with relapsed/advanced hepatocellular carcinoma Jun Lu', Xuli Bao', Jia Guo', Wenfeng Sun², Hui Chen², Yanpin Ma', Xiongwei Cui

${ }^{1}$ Beijing YouAn Hospital, Capital Medical University, Beijing, China; ${ }^{2}$ Beijing Gene Key Life Technology Co.Ltd., Beijing, China

Correspondence: Jun Lu (lujun98@ccmu.edu.cn)

Journal for ImmunoTherapy of Cancer 2017, 5(Suppl 2):P237

\section{Background}

Invariant Natural Killer T (iNKT) cells represent a distinctive subset of T lymphocytes characterized by an invariant receptor Va24/Ja18 in human, which play critical roles in regulating anti-tumor immunity by bridging innate and adaptive immune responses. We have previously demonstrated that the accumulation of circulating iNKT cells provide a better prognosis for hepatocellular carcinoma (HCC) patients, the adoptive transfer of ex vivo expanded human iNKT cells in HCC tumor-bearing NOD/SCID mice led to the decrease in tumor size, revealing the iNKT immunotherapy may bring clinical benefits for the HCC patients.

\section{Methods}

The phase I trial enrolls patients who have relapsed/advanced HCC tumor relapsed or metastasized through the body after standard treatment or the patients cannot receive standard treatment under current conditions. The purpose of this study is to find the biggest dose of iNKT cells that is safe and tolerance, to see how long they last in the body, to learn the immuneresponse, the side effects and if the iNKT cells will help people with relapsed/advanced HCC. Key eligibility criteria include age $\geq 18$ years, with HCC (BCLC, stage C) proved by histopathology or proved by $\mathrm{CT}$ or MRI imaging system, relapsed after previous therapy and no effective therapies known at this time and life expectancy of $\geq 12$ weeks. Three different dosing schedules will be evaluated. Three patients will be evaluated on each dosing schedule. The following dose levels will be evaluated: Loading Dose 1: $3 \times 10^{7} / \mathrm{m}^{2}$; Loading Dose 2: $6 \times 10^{7} / \mathrm{m}^{2}$; Loading Dose 3: $9 \times 10^{7} / \mathrm{m}^{2}$. The doses are calculated according to the actual number of iNKT cells. Human Interleukin-2 will be given at a dose of $25,000 \mathrm{IU} / \mathrm{kg} /$ day for 5-14 days. Tegafur will be given at a dose of $40 \sim 60 \mathrm{mg}$ bis in die (BID) 2 weeks. immune responses were measured by Elispot and ELISA; flow cytometry assays were performed to evaluate the effects on immune cell subsets. Tumor biopsies were evaluated for iNKT cells by immunohistochemistry. Incidence of treatment-emergent adverse events were defined as signs/symptoms, laboratory toxicities, and clinical events that are possibly, likely, or definitely related to study treatment adverse events assessed according to $\mathrm{NCl}$ CTCAE v4.0 criteria 2. HCC progression was evaluated by imaging according to the irRC standard.
Results

To date, one patient has been started on therapy and is in week 3 monitoring period, is tolerating treatment well, with no significant toxicities thus far. Data regarding the peripheral blood iNKT cells response will be presented.

Trial Registration

ClinicalTrials.gov identifier NCT03175679.

\section{P238}

Phase I study of adoptive transfer of specific hepatocellular carcinoma antigens $\mathrm{CD}^{+} \mathrm{T}$ cells for treating patients with relapsed/advanced HCC

Jun Lu', Xuli Bao', Yanpin Ma', Hui Chen², Wenfeng Sun², Jia Guo', Xiongwei Cui ${ }^{1}$

${ }^{1}$ Beijing YouAn Hospital, Capital Medical University, Beijing, China;

${ }^{2}$ Beijing Gene Key Life Technology Co.Ltd., Beijing, China

Correspondence: Jun Lu (lujun98@ccmu.edu.cn)

Journal for ImmunoTherapy of Cancer 2017, 5(Suppl 2):P238

\section{Background}

Hepatocellular carcinoma (HCC) is one of the most prevalent cancers for neoplastic deaths and shows a high recurrence rate. Adoptive T cell therapy, involving the ex vivo selection and expansion of antigen-specific $\mathrm{T}$ cell clones by $\mathrm{MHC} /$ peptide tetramer sorting, provides a method of augmenting antigen-specific immunity against HCC. To generate of a high number of cytotoxic $T$ lymphocytes (CTLs), kind of effective $\mathrm{T}$ cells that specific recognizing and killing antigen targeted cells through cloning amplification after receiving antigen information from antigen presented cells, is a promising strategy for adoptive therapy.

Methods

The phase I trial enrolls patients who have HCC tumor relapsed or metastasized through the body after standard treatment or the patients cannot receive standard treatment under current conditions. The purpose of this study is to evaluate the safety and tolerance as well as the potential clinical efficacy of an adoptive transfer of $\mathrm{CD} 8^{+}$ T cells, sorted with human leukocyte antigen (HLA)-peptide multimers and specific for Glypican (GPC)-3 /New York Esophageal Squamous-1 (NY-ESO-1) /alpha-fetoprotein (AFP) antigens and cultured in vitro. Key eligibility criteria include age $\geq 18$ years, with HCC (BCLC, stage C) proved by histopathology or proved by CT or MRI imaging system, relapsed after previous therapy and no effective therapies known at this time and life expectancy of $\geq 12$ weeks. Three different dosing schedules will be evaluated. Three patients will be evaluated on each dosing schedule. The following dose levels will be evaluated: Loading Dose 1: $3 \times 10^{7} / \mathrm{m}^{2}$; Loading Dose 2: $6 \times 10^{7} / \mathrm{m}^{2}$; Loading Dose $3: 9 \times 10^{7} / \mathrm{m}^{2}$. The doses are calculated according to the actual number of GPC3/NY-ESO-1/AFP CTLs. Human Interleukin-2 will be given at a dose of $25,000 \mathrm{IU} / \mathrm{kg} / \mathrm{day}$ for 5-14 days. Tegafur will be given at a dose of 40 60 mg bis in die (BID) for 2 weeks. Immune responses were measured by Elispot and ELISA, flow cytometry assays were performed to evaluate the effects on immune cell subsets. Tumor biopsies were evaluated for CTLs by immunohistochemistry. Incidence of treatment-emergent adverse events were defined as signs/symptoms, laboratory toxicities, and clinical events that are possibly, likely, or definitely related to study treatment adverse events assessed according to NCl-CTCAE v4.0. HCC progression was evaluated by imaging according to the irRC standard.

Results

To date, 5 patients have been enrolled and 2 of them are in week 2 monitoring period, with no significant toxicities thus far. Data regarding the peripheral blood antigen-specific CTL cells response will be presented.

Trial Registration

ClinicalTrials.gov identifier NCT03175705 
P239

Phase 3 study of Pembrolizumab plus Chemoradiation (CRT) vs CRT alone for locally advanced head and neck squamous cell carcinoma (LA-HNSCC): KEYNOTE-412

Jean-Pascal Machiels', Chia-Jui Yen ${ }^{2}$, Lisa Licitra ${ }^{3}$, Danny Rischin ${ }^{4}$, John Waldron ${ }^{5}$, Barbara Burtness ${ }^{6}$, Vincent Gregoire', Sanjiv Agarwala ${ }^{7}$, Yun Gan Tao ${ }^{8}$, Jeffrey Yorio ${ }^{9}$, Sercan Aksoy ${ }^{10}$, Sadakatsu Ikeda ${ }^{11}$, Ruey-Long Hong ${ }^{12}$, Joy Yang Ge ${ }^{13}$, Holly Brown ${ }^{13}$, Behzad Bidadi ${ }^{13}$, Lillian $\mathrm{Siu}^{5}$

${ }^{1}$ Cliniques Universitaires Saint-Luc, Brussels, Belgium; ${ }^{2}$ National Cheng Kung University Hospital, Tainan City, Taiwan; ${ }^{3}$ Fondazione IRCCS Istituto Nazionale dei Tumori, Milan, Italy; ${ }^{4}$ Peter MacCallum Cancer Centre, East Melbourne, Australia; ${ }^{5}$ Princess Margaret Cancer Centre, Toronto, ON, Canada; ${ }^{6}$ Yale University School of Medicine, New Haven, CT, USA; ${ }^{7}$ St. Luke's Cancer Center-Anderson, Easton, PA, USA; ${ }^{8}$ Institut Gustave Roussy, Villejuif, France; ${ }^{9}$ Texas Oncology-Austin Central, Austin, TX, USA ${ }^{10}$ Hacettepe Universitesi Tip Fakultesi, Ankara, Turkey; ${ }^{11}$ Medical Hospital, Tokyo Medical and Dental University, Tokyo, Japan; ${ }^{12}$ National Taiwan University Hospital, Taipei City, Taiwan; ${ }^{13}$ Merck \& Co., Inc., Kenilworth, NJ, USA

Correspondence: Jean-Pascal Machiels (Jean-

pascal.machiels@uclouvain.be)

Journal for ImmunoTherapy of Cancer 2017, 5(Suppl 2):P239

\section{Background}

CRT with cisplatin is the standard of care for patients with LA-HNSCC not treated by surgery. Preclinical data in murine cancer models show improved tumor growth control and survival when RT is combined with a programmed death 1 (PD-1) inhibitor. Pembrolizumab has been found to be effective for treating recurrent/metastatic HNSCC, and initial results from a phase $1 \mathrm{~b}$ study suggest that pembrolizumab plus CRT is tolerable in patients with LA-HNSCC. KEYNOTE-412 (NCT03040999) is a phase 3, randomized, placebocontrolled, double-blind trial to determine the efficacy and safety of pembrolizumab given concomitantly with CRT and as maintenance therapy versus placebo plus CRT in LA-HNSCC.

Methods

Eligibility includes patient age $\geq 18$ years; newly diagnosed, treatment-naive, oropharyngeal p16 positive (any T4 or N3), oropharyngeal p16 negative (any T3-T4 or N2a-N3), or larynx/hypopharynx/ oral cavity (any T3-T4 or N2a-N3) SCC; evaluable tumor burden (RECIST v1.1); ECOG performance status $0-1$; results available from local testing of human papillomavirus status for oropharyngeal cancer; eligible for definitive CRT and not considered for primary surgery per investigator decision; tissue from a core or excisional biopsy for programmed death ligand 1 (PD-L1) biomarker analysis. Patients will be randomly assigned (1:1) to receive pembrolizumab $200 \mathrm{mg}$ every 3 weeks plus CRT, including radiotherapy (RT; accelerated [70 Gy, six 2 Gy fractions/wk] or standard [70 Gy, five 2 Gy fractions/week] fractionation) plus cisplatin $100 \mathrm{mg} / \mathrm{m}^{2}$ Q3W for 3 cycles only, or placebo Q3W plus CRT. Treatment will be stratified by RT regimen (accelerated vs standard), tumor site/p16 status (oropharynx p16 positive vs p16 negative or larynx/hypopharynx/oral cavity), and disease stage (III vs IV). A priming dose of pembrolizumab or placebo will be given 1 week before CRT, followed by 2 doses during CRT, and an additional 14 doses after CRT, for a total of 17 pembrolizumab or placebo infusions. Treatment will be discontinued upon centrally confirmed disease progression, unacceptable toxicity, or patient/ physician decision to withdraw. Disease status will be assessed by computed topography or magnetic resonance imaging 12 weeks after CRT, every 3 months for 3 years, then every 6 months for years 4 and 5. Safety will be monitored throughout the study and for 30 days after treatment. The primary end point is event-free survival. Secondary end points include overall survival, safety, and patient- reported outcomes. Recruitment will continue until $\sim 780$ patients are enrolled.

Trial Registration

ClinicalTrials.gov, NCT03040999.

\section{P240}

Phase $1 \mathrm{~b}$ trial of cabozantinib in combination with atezolizumab in patients with locally advanced or metastatic urothelial carcinoma or renal cell carcinoma

Manuel Caitano Maia', Neeraj Agarwal ${ }^{2}$, Bradley McGregor ${ }^{3}$, Ulka Vaishampayan ${ }^{4}$, Toni K. Choueiri' ${ }^{3}$, Marjorie Green ${ }^{5}$, Colin Hessel ${ }^{6}$, Christian Scheffold ${ }^{6}$, Gisela Schwab ${ }^{6}$, Thomas Powles ${ }^{7}$, Sumanta Pal

${ }^{1}$ City of Hope, Duarte, CA, USA; ${ }^{2}$ Huntsman Cancer Hospital, Salt Lake City, UT, USA; ${ }^{3}$ Dana-Farber Cancer Institute, Boston, MA, USA;

${ }^{4}$ Karmanos Cancer Center, Detroit, MI, USA; ${ }^{5}$ Genentech, South San Francisco, CA, USA; ${ }^{6}$ Exelixis Inc., South San Francisco, CA, USA; ${ }^{7}$ Barts Cancer Institute, London, United Kingdom

Correspondence: Manuel Caitano Maia (hannah.welz@fishawack.com) Journal for ImmunoTherapy of Cancer 2017, 5(Suppl 2):P240

\section{Background}

Cabozantinib is an oral receptor tyrosine kinase inhibitor targeting MET, VEGFR, and TAM family receptors (TYRO3, AXL, and MER). It is approved for use in patients with advanced renal cell carcinoma (RCC) after prior therapy with antiangiogenic/VEGFR-targeted therapy, and has demonstrated clinical activity in urothelial carcinoma (UC). In clinical studies, cabozantinib exposure resulted in an increase in circulating CD8+ T cells and reduction of immune-suppressive monocytes and Tregs. In preclinical tumor models, treatment with cabozantinib resulted in an increase of MHC class 1 expression on tumor cells and a reduction of myeloid-derived suppressor cells. These observations support that cabozantinib may facilitate an immune-permissive tumor environment and may enhance the response to immune checkpoint inhibitors. Atezolizumab, an anti-PDL1 monoclonal antibody, is approved for use in locally advanced or metastatic UC for patients who are either cisplatin-ineligible or have disease progression during or following platinum-containing chemotherapy. It is also approved for use in patients with metastatic nonsmall cell lung cancer who have disease progression during or following platinum-containing chemotherapy. Here, we present the study design of an ongoing phase $1 \mathrm{~b}$ study combining cabozantinib with atezolizumab in patients with locally advanced or metastatic UC or RCC.

\section{Methods}

This multicenter, phase $1 \mathrm{~b}$, open-label study aims to assess safety, tolerability, preliminary efficacy, and pharmacokinetics of cabozantinib in combination with atezolizumab (NCT03170960). The study will enroll patients with advanced UC (including bladder, renal pelvis, ureter, urethra) or RCC. It consists of two stages: a doseescalation stage and an expansion-cohort stage. In the dose-escalation stage ( $3+3$ design), a recommended cabozantinib dose for the combination will be established. In the expansion stage, four tumor-specific cohorts will be enrolled, and the primary objective is to determine the objective response rate in each cohort. The four expansion cohorts are (1) patients with UC who have progressed on or after platinumcontaining chemotherapy; (2) chemotherapy-naïve patients with UC who are ineligible for cisplatin; (3) chemotherapy-naïve patients with UC who are eligible for cisplatin; and (4) previously untreated patients with RCC with clear cell histology. Exploratory objectives include correlation of tumor and plasma biomarkers, and changes in immune cell profiles with clinical outcome. The study has been initiated and enrollment target is up to 120 patients across the 4 cohorts in the expansion-cohort stage. 
Trial Registration

ClinicalTrials.gov: NCT03170960.

\section{P241}

A first-in-human study of ALX148: CD47 blockade to enhance innate and adaptive immunity for advanced solid tumor malignancy and non hodgkin lymphoma

Nehal Lakhani', Patricia LoRusso ${ }^{2}$, Anuradha Krishnamurthy ${ }^{3}$

Timothy O'Rourke', Philip Fanning ${ }^{4}$, Yonggang Zhao ${ }^{5}$, Hong Wan ${ }^{4}$, Jaume Pons ${ }^{4}$, Sophia Randolph" ${ }^{4}$, Wells Messersmith

${ }^{1}$ START Midwest, Grand Rapids, MI, USA; ${ }^{2}$ Yale Cancer Center, New Haven, CT, USA; ${ }^{3}$ University of Colorado Cancer Center, Aurora, CO, USA; ${ }^{4}$ Alexo Therapeutics Inc., South San Francisco, CA, USA; ${ }^{5}$ Skyview Research, Norristown, PA, USA

Correspondence: Sophia Randolph (srandolph@alexotherapeutics.com) Journal for ImmunoTherapy of Cancer 2017, 5(Suppl 2):P241

\section{Background}

CD47, a marker of self, is upregulated by tumors to evade the immune system. Blocking the interaction between CD47 and SIRPa, its receptor on myeloid cells, disrupts a key immune checkpoint and may enhance innate and adaptive immunity against cancer. ALX148 is a high affinity, engineered fusion protein containing the $\mathrm{N}$ terminal D1 domain of SIRPa, which binds and blocks CD47, and is genetically linked to an inactive human Fc domain to minimize toxicity. ALX148 enhanced activity of multiple anti-cancer targeted antibodies and checkpoint inhibitors with minimal effect on normal blood cells in nonclinical models. This phase 1 study evaluates the safety, tolerability, pharmacokinetic (PK) and pharmacodynamic profiles of ALX148 in patients with advanced malignancy.

\section{Methods}

The primary study objective is to characterize the safety profile of ALX148 first as a single agent and then in combination with established anti-cancer antibodies. Cohorts (3-6 pts) with advanced malignancy receive escalating doses of ALX148, intravenously, once weekly or once every other week. Tumor response, PK, and target occupancy (TO) are characterized as secondary objectives. Preliminary single agent data are reported from the data cutoff, July 21, 2017 and will be updated at the time of presentation.

Results

Ten patients received ALX148 ( 4 males/ 6 females; $0.3 \mathrm{mg}$ per kilogram (mpk), 3; $1.0 \mathrm{mpk}, 4 ; 3.0 \mathrm{mpk}, 3$ ) as of data cutoff. Median age was 63 (37-76) yrs and ECOG PS 0/1: 1/9. Four patients experienced treatment related adverse events (AEs) which were predominantly low grade and included 1 each at $0.3 \mathrm{mpk}$ (G1 Headache, Rash, Fatigue); $1.0 \mathrm{mpk}$ (G3 Anemia, G1 Dysgeusia); and at 3.0 mpk (G2 Decreased Appetite, Hypersensitivity). As of the data cut-off no pts have experienced a doselimiting toxicity. One patient achieved SD (0.3 mpk; leiomyosarcoma) for 16 weeks. ALX148 initial PK showed increased exposure with increasing dose and noticeable accumulation with repeated dosing, likely driven by target saturation. Dose dependent TO on CD47 by ALX148 was observed on RBCs and T cells. The magnitude and duration of TO increased with repeat dosing.

\section{Conclusions}

ALX148 is well tolerated in patients with advanced solid tumors with favorable PK/TO characteristics and no significant hematologic toxicity at doses evaluated. Accrual is ongoing. When the maximum tolerated dose/optimal biological dose of single agent ALX148 is established, patients with advanced malignancy will be evaluated with ALX148 in combination with anticancer antibodies.
Trial Registration

ClinicalTrials.gov identifier NCT03013218.

References

1. Weiskopf K. Cancer immunotherapy targeting the CD47/SIRPa axis.Eur J Cancer. 2017;76:100-109.

P242

A phase 1 multicenter, dose escalation study of CBT-501, a novel anti-PD-1 inhibitor in subjects with select advanced or relapsed/ recurrent solid tumors

Purvi Patel, Mamatha Reddy, Melissa Lopez, Neil Sankar, Sarath Kanekal, Mike Li, Sanjeev Redkar, Gavin Choy

CBT Pharmaceuticals, Inc., Pleasanton, CA, USA

Correspondence: Sanjeev Redkar (sanjeev.redkar@cbtpharma.com)

Journal for ImmunoTherapy of Cancer 2017, 5(Suppl 2):P242

\section{Background}

Programmed death-1 (PD-1, CD279) is an inhibitory co-receptor expressed on antigen-activated and exhausted T and B cells. PD-1/ PD-L1 axis inhibition by targeted-antibodies, increases the T cell proliferation and cytotoxicity. This represents a promising mechanism to stimulate the anti-tumor activity of the immune system. CBT-501, genolimzumab (GB226) is a novel humanized lgG4 monoclonal antibody targeting the PD-1 membrane receptor on T lymphocytes and other cells of the immune system. CBT-501 demonstrated highly specific binding to PD-1 of human $(\mathrm{Kd}=505 \mathrm{pM})$ and cynomolgus $(\mathrm{Kd}=7.2 \mathrm{nM})$. CBT-501 efficiently inhibited the binding of PD-L1/L2 to PD-1 for both human and monkey and enhanced human T cell activation in the Mixed Lymphocyte Reaction (MLR) assay. CBT-501 has demonstrated anti-tumor activity in the in vivo animal model and no abnormal drug-related toxicity has been observed in the GLP toxicology studies. Data from all pre-clinical pharmacodynamics and toxicology studies of CBT-501 indicate pharmacological activity at effective doses with a wide margin of safety. Based on these findings, a Phase 1 study has been initiated with CBT-501 in Australia.

Methods

CBT-501-01 is a Phase 1, multicenter, dose escalation study of CBT501 in subjects in select advanced or relapsed/recurrent solid tumors The primary study objective is to identify the overall safety and tolerability, including any dose limiting toxicities (DLT), and determine the recommended Phase 2 dose (RP2D) in subjects with advanced solid tumors. Secondary objectives include assessing efficacy by overall response rate (ORR), best overall response rate (BOR) per RECIST v1.1 and irRECIST, time to response, duration of response (DOR), disease control rate (DCR) by RECIST V1.1 and irRECIST, progression free survival (PFS), and determining the pharmacokinetic (PK) parameters. Exploratory objectives involve the assessment of PD-1 and PD-L1 expression, receptor occupancy and the host immune response (immune modulation) in blood peripheral-blood mononuclear cells (PBMCs) or formalin-fixed paraffin-embedded (FFPE) samples. This is a 2-part study with a dose-escalation segment and dose and disease expansion cohorts of CBT-501. In Part 1, dose escalation ( $3+3$ design) will occur among 3 cohorts to determine the RP2D. The tumor type(s) with the most robust clinical signal relative to response rate and safety/tolerability will be selected for further evaluation in the expansion cohort (Part 2). Approximately 32 subjects will be enrolled in the dose and disease expansion and treated at the RP2D, as determined in Part 1. 
Trial Registration

Clinical Trial Registry Number: NCT03053466.

\section{P243}

Withdrawn

P244

A phase 1, first-in-human, open-label, dose escalation study of MGD013, a bispecific DART ${ }^{\circledR}$ protein binding PD-1 and LAG-3 in patients with unresectable or metastatic neoplasms

Sadhna Shankarr', Manish Patel ${ }^{2}$, George Blumenschein ${ }^{3}$, Erika Hamilton ${ }^{4}$, Jason Luke ${ }^{5}$, Ross La-Motte Mohs' ${ }^{1}$, Kalpana Shah', Lisa Adali-Piston', Syd Johnson', Ezio Bonivini', Paul Moore', Jon Wigginton'1, Jim Vasselli ${ }^{1}$ MacroGenics Inc., Rockville, MD, USA; ${ }^{2}$ Florida Cancer Specialists, Sarah Canon Research Institute, Sarasota, FL, USA; ${ }^{3}$ MD Anderson Cancer Center, Houston, TX, USA; ${ }^{4}$ Sarah Cannon Research Institute, Nashville, TN, USA; ${ }^{5}$ University of Chicago, Chicago, IL, USA

Correspondence: Sadhna Shankar (shankars@macrogenics.com) Journal for ImmunoTherapy of Cancer 2017, 5(Suppl 2):P244

\section{Background}

Lymphocyte-activation gene 3 (LAG-3) is a membrane protein in the immunoglobulin superfamily that binds to major histocompatibility complex class II (MHC-II). LAG-3 engagement negatively regulates $T$ cell proliferation and differentiation. Blockade of PD-1 and LAG-3 in animal tumor models enhanced antitumor immunity via distinct, non-redundant signaling pathways that fostered the accumulation of functionally competent $\mathrm{CD} 8^{+} \mathrm{T}$ cells in mice [1]. Dual targeting of PD-1 and LAG-3 may help reverse effector cell exhaustion and increase the response rates and/or effectiveness of immunotherapy beyond that observed with single agents alone. MGD013 is an Fc-bearing bispecific tetravalent (bivalent for each antigen) DART ${ }^{\oplus}$ protein engineered as a hinge-stabilized immunoglobulin G4 molecule and designed to concomitantly bind PD-1 and LAG-3, thereby contributing to sustain or restore the function of exhausted T cells. MGD013 may enhance T cell activation in a synergistic fashion beyond that observed with the anti-PD-1 and anti-LAG-3 monoclonal antibodies alone or in combination. A bispecific format for target engagement may confer biologic advantages that may translate to clinical advantages over antibody combinations.

Methods

This is an open-label, dose escalation / cohort expansion phase 1 study (NCT03219268) designed to characterize the safety, tolerability, pharmacokinetics, pharmacodynamics, and preliminary antitumor activity of MGD013. Patients with unresectable, locally advanced or metastatic solid tumors of any histology are enrolled in the dose escalation phase. Sequential escalating flat doses ranging from $1 \mathrm{mg}$ to $1600 \mathrm{mg}$ every 2 weeks are evaluated in successive cohorts of 1 to 6 patients each. A single patient dose escalation design is utilized in the lower dose cohorts. The escalation approach transitions to a conventional $3+3$ design after the first three cohorts. Occurrence of a drug-related Grade 2 adverse event in a single patient cohort will lead to enrollment of 3 additional patients at that dose level. Occurrence of a DLT in a single patient cohort will trigger transition to a conventional $3+3$ design. Response is first determined at 8 weeks. Patient management is guided by response assessment according to irRECIST. MGD013 dosing may continue up to 2 years based on response. Cohort expansion phase will start after maximum tolerated dose is determined and will be restricted to 5 tumor types, including solid tumors and hematological malignancies.

Trial Registration

Clinicaltrials.gov- NCT03219268.

\section{References}

1. Grosso JF, Kelleher CC, Harris TJ, Maris CH, Hipkiss EL, De Marzo A, et al.LAG-3 regulates CD8+ T cell accumulation and effector function in murine self- and tumor-tolerance systems. J Clin Invest.

2007; 117(11):3383-92.
P245

CAPRA: A Phase $1 \mathrm{~b}$ study of intratumoral Coxsackievirus A21 (CVA21) and systemic pembrolizumab in advanced melanoma patients

Ann Silk', Howard Kaufman ${ }^{1}$, Nashat Gabrail ${ }^{2}$, Janice Mehnert ${ }^{1}$, Jennifer Bryan', Jacqueline Norrell', Azra Haider', Daniel Medina', Praveen Bommareddy ${ }^{3}$, Darren Shafren ${ }^{4}$, Mark Grose ${ }^{4}$, Andrew Zloza ${ }^{1}$

${ }^{1}$ Rutgers Cancer Institute of New Jersey, New Brunswick, NJ, USA;

${ }^{2}$ Gabrail Cancer Center, Canton, OH, USA; ${ }^{3}$ Rutgers University,

Piccataway, NJ, USA; ${ }^{4}$ Viralytics Limited, Sydney, Australia

Correspondence: Ann Silk (ann.w.silk@rutgers.edu)

Journal for ImmunoTherapy of Cancer 2017, 5(Suppl 2):P245

\section{Background}

Coxsackievirus A21 (CVA21) is a novel bio-selected oncolytic, immunotherapeutic agent. Intratumoral (i.t.) CVA21 injection can induce selective tumor-cell infection, immune-cell infiltration, IFN-g response gene up-regulation, increased PD-L1 expression, tumor cell lysis and systemic anti-tumor immune responses. A clinical trial evaluating combination CVA21 and pembrolizumab in patients with melanoma was initiated and preliminary data on a pre-established futility endpoint are presented here.

Methods

This is a single-arm, multi-institutional open-label phase lb clinical trial of i.t. CVA21 and i.v. pembrolizumab for treated or untreated unresectable Stage IIIB-IVM1c melanoma. Subjects with injectable disease receive up to $3 \times 10^{8} \mathrm{TCID}_{50}$ CVA21 i.t. on Days 1, 3, 5, 8, and then every 3 weeks for up to 19 injections. Subjects also receive pembrolizumab $(2 \mathrm{mg} / \mathrm{kg})$ i.v. every 3 weeks starting on Day 8. The primary endpoint is safety/tolerability by incidence of doselimiting toxicity. Secondary endpoints include best ORR by immune-related response criteria, progression-free survival, overall survival, quality of life.

Results

To date, 22 subjects have started on protocol therapy. Overall, the adverse events have been low-grade constitutional symptoms related to CVA21 and expected pembrolizumab-related side effects. One subject had Grade 3 increased hepatic enzymes that was considered related to pembrolizumab. No DLT's have been reported. Currently, 19 patients are evaluable for investigator response assessment. Among the evaluable subjects $(n=19)$, the ORR was $63 \%(12 / 19)$. The DCR (CR +PR+SD) is currently $84 \%(16 / 19)$. In subjects with stage IVM1c disease, the ORR is $78 \%(7 / 9)$. The study has met its primary statistical futility endpoint of achieving $\geq 2$ confirmed objective responses (CR or PR) in the first 12 patients enrolled. One of the 12 responders displayed early pseudo-progression and later developed a partial response.

Conclusions

Based on these initial results, the sample size has now been expanded to enroll up to 50 patients including subjects refractory to anti-PD1 therapy. Combination therapy of CVA21 and pembrolizumab may represent a new approach for the treatment of patients with injectable advanced melanoma.

Trial Registration

NCT02565992.

Consent

Written informed consent was obtained from all of the patients for participation in the study and use of the data for publication.

\section{P246}

Phase $1 / 2$ study of in situ vaccination with tremelimumab + intravenous (IV) durvalumab + poly-ICLC in patients with select relapsed, advanced cancers with measurable, biopsy-accessible tumors

Craig Slingluff, Jr. ${ }^{1}$, Sunita Hack ${ }^{2}$, Paul Schwarzenberger ${ }^{3}$, Toni Ricciardi ${ }^{3}$, Mary Macri ${ }^{3}$, Aileen Ryann ${ }^{3}$, Ralph Venhaus ${ }^{3}$, Nina Bhardwaj ${ }^{4}$

${ }^{1}$ University of Virginia, Charlottesville, VA, USA; ${ }^{2}$ Ludwig Institute for Cancer Research, New York, NY, USA; ${ }^{3}$ Ludwig Cancer Research, New York, NY, USA; ${ }^{4}$ Icahn School of Medicine at Mt Sinai, New York, NY, USA Correspondence: Craig Slingluff, Jr. (cls8h@virginia.edu) Journal for ImmunoTherapy of Cancer 2017, 5(Suppl 2):P246 


\section{Background}

Immunotherapy has demonstrated promising antitumor activity in various advanced cancers. Combined tumor targeting from multiple drugs with unique mechanisms may provide further improved outcomes. Tremelimumab (TRE) is a CTLA-4 antibody and durvalumab (DUR) blocks PD-L1. Poly-ICLC is a toll-like receptor 3 agonist. Intratumoral (intra-T) injection of poly-ICLC directly alters the tumor microenvironment (TME), and by creating an in situ vaccination, may trigger a clinically effective systemic anti-tumor response when also combined with DUR and TRE.

Methods

This is an ongoing Phase 1/2, open-label, multicenter study (NCT02643303). The study evaluates the use of intra-T administration of TRE and IV DUR + poly-ICLC (intra-T and intramuscular [IM]) to determine the safety, preliminary efficacy and immune activity of this regimen in patients with advanced, measurable, biopsyaccessible tumors: head and neck squamous cell carcinoma, breast cancer, sarcoma, merkel cell carcinoma, cutaneous T cell lymphoma, melanoma, genitourinary cancer, and other solid tumors. Phase 1 determines the recommended combination dosing (RCD) for the regimen with dose de-escalation based on dose limiting toxicities (DLTs) and standard $3+3$ rules. Starting doses are: DUR, $1500 \mathrm{mg}$ IV; TRE, $75 \mathrm{mg}$ IV; TRE, $10 \mathrm{mg}$ intra-T; poly-ICLC, $1 \mathrm{mg}$ intra-T/IM. Phase 1 starts with Cohort 1A (DUR + poly-ICLC). Upon demonstration of tolerability, enrollment proceeds with Cohort 1B (DUR + IV TRE + poly-ICLC) and Cohort $1 C$ (DUR + intra-T TRE + poly-ICLC). The RCD is the highest dose at which $<2 / 6$ patients have DLTs. In Phase 2 , up to 66 evaluable patients are treated using the RCD regimen, with enrollment of 6 patients per tumor type initially, and enrollment of 6 additional patients per 3 tumor types contingent upon at least 1 response among the initial 6 patients. Study endpoints are RCD and safety, objective response rate, progression-free survival, and overall survival. Exploratory endpoints are biological activity, including effects on the TME and immunological responses. Enrollment opened on 28 Dec 2016.

Results

Trial in Progress.

Conclusions

Trial in Progress.

Trial Registration

Clinicaltrials.gov: NCT02643303.

\section{P247}

Study Design: Phase 1 dose escalation, multi-tumor study to assess safety, tolerability and antitumor activity of genetically engineered MAGE-A4 SPEAR T cells in HLA-A2 ${ }^{+}$subjects with MAGE-A4 ${ }^{+}$ tumors

David S Hong ${ }^{1}$, Melissa Johnson ${ }^{2}$, Anthony J Olszanski ${ }^{3}$, Marcus Butler ${ }^{4}$, Connie Erickson-Miller, Malini lyengar ${ }^{5}$, Trupti Trivedi ${ }^{5}$, Karen Chagin $^{5}$, Rafael Amado ${ }^{5}$

${ }^{1}$ The University of Texas MD Anderson Cancer Center, Houston, TX, USA; ${ }^{2}$ Sarah Cannon, Nashville, TN, USA; ${ }^{3}$ Anthony.Olszanski@FCCC.edu, Philadelphia, PA, USA; ${ }^{4}$ Princess Margaret Cancer Centre, Toronto, ON, Canada; ${ }^{5}$ Adaptimmune, Philadelphia, PA, USA

Journal for ImmunoTherapy of Cancer 2017, 5(Suppl 2):P247

\section{Background}

MAGE-A4 is a cancer/testis antigen that has been identified in 13$48 \%$ of non-small cell lung cancer (NSCLC), urothelial, melanoma, head and neck, ovarian, gastric and esophageal tumors. This study (NCT03132922) will evaluate the safety and tolerability of genetically engineered autologous specific peptide enhanced affinity receptor (SPEAR) T cells (MAGE-A4 ${ }^{\mathrm{c} 1032} \mathrm{~T}$ cells) directed towards a MAGE-A4 peptide expressed on tumors in the context of HLA-A *02. Antitumor activity will also be assessed.

Methods

This first-in-human $T$ cell dose escalation study utilizes a modified 3 +3 design to evaluate safety, including dose limiting toxicities (DLT). Secondary objectives include anti-tumor activity (overall response (per RECIST v1.1), duration of response, time to response, progression-free survival, overall survival) and translational research assessments. Patients are screened under a separate protocol (NCT03132922). Those who are HLA-A*02 positive (with the exception of $\left.A^{*} 02: 05\right)$ and have inoperable or metastatic (advanced) NSCLC, urothelial cancer, melanoma, or squamous cell head and neck, ovarian, gastric or esophageal tumors with MAGE-A4 expression and meet all other entry criteria are eligible for treatment. Subjects must have prior treatments as described in the table (Table 1). Patients must have received standard of care therapies and have measurable disease.

Following leukapheresis, the $T$ cells are isolated, transduced with a lentiviral vector containing the MAGE-A4 ${ }^{\text {c1032 }}$ TCR, and expanded with $\mathrm{CD} 3 / \mathrm{CD} 28$ beads. Subjects are given lymphodepleting chemotherapy (fludarabine $30 \mathrm{mg} / \mathrm{m}^{2} /$ day and cyclophosphamide $600 \mathrm{mg} /$ $\mathrm{m}^{2} /$ day, on days $-7,-6$ and -5 ) prior to infusion of transduced cells. Groups 1, 2 and 3 will consist of 3-6 subjects, and the transduced cell doses will be as follows, respectively: $0.1 \times 10^{9}( \pm 20 \%), 1 \times 10^{9}$ $( \pm 20 \%)$, and $5 \times 10^{9}$ (range: $>1.2-6 \times 10^{9}$ ). The DLT observation period will be during the first 30 days following the infusion of MAGE-A4 SPEAR T cells for each patient in all groups. Following the dose escalation, up to 10 patients will be enrolled at the target dose. Disease assessments will be conducted at week 6, 12, 18 and 24, and then every 3 months until confirmation of disease progression or at 2 years post-infusion. On study tumor biopsies and blood samples will be evaluated to compare the pre- and post-T cell infusion immune profile for association with treatment outcome.

Trial Registration

NCT03132922.

Table 1 (abstract P247). Eligibility Criteria

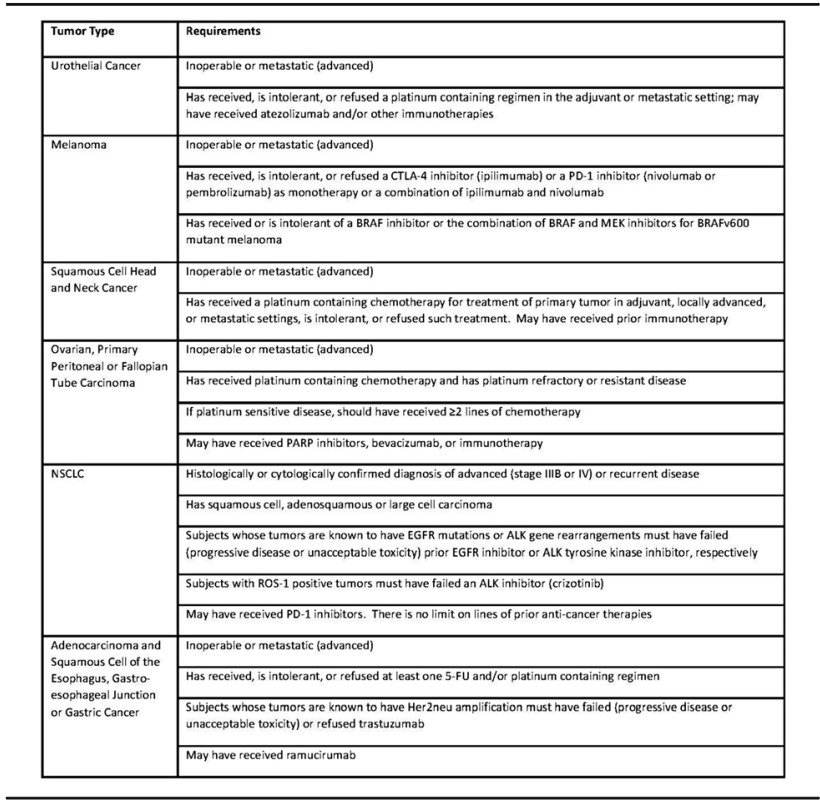

P248

Study Design: An open-label randomized pilot study of NY-ESO-1 SPEAR T cells alone or in combination with pembrolizumab in $\mathrm{HLA}-\mathrm{A2}^{+}$subjects with relapsed and refractory multiple myeloma (NCT03168438)

Aaron P Rapoport ${ }^{1}$, James E Hoffman², Myo Htut ${ }^{3}$, Taiga Nishihori ${ }^{4}$, Karen Chagin ${ }^{5}$, Thomas Faitg ${ }^{5}$, Elliot Norry ${ }^{5}$, Trupti Trivedi ${ }^{5}$, Rafael Amado ${ }^{1}$ University of Maryland School of Medicine, Baltimore, MD, USA; ${ }^{2}$ Sylvester Cancer Center at the University of Miami, Miami, FL, USA; ${ }^{3}$ City of Hope, Duarte, CA, USA; ${ }^{4}$ Moffit Cancer Center, Tampa, FL, USA;

${ }^{5}$ Adaptimmune, Philadelphia, PA, USA

Journal for ImmunoTherapy of Cancer 2017, 5(Suppl 2):P248 


\section{Background}

NY-ESO-1 and LAGE-1a are cancer/testis antigens that are expressed frequently in multiple myeloma (MM) and are often associated with poor prognosis. This two-arm randomized study will evaluate the safety and efficacy of genetically engineered autologous specific peptide enhanced affinity receptor (SPEAR) T cells (NY-ESO- ${ }^{\text {C259 }} \mathrm{T}$ cells) directed towards a NY-ESO-1/LAGE-1a peptide expressed on tumor cells in the context of HLA-A*02, alone and in combination with pembrolizumab. Methods

This open label randomized pilot study will evaluate safety, efficacy (using the International Myeloma Working Group Uniform Response Criteria), and translational research endpoints. Patients must meet these criteria: $\geq 18$ yrs old; HLA-A*02:01, $A^{*} 02: 05$ or $A^{*} 02: 06$ positive; have histologically confirmed diagnosis of MM with either primary refractory or relapsed/refractory disease expressing NY-ESO-1 and/or LAGE-1a; prior therapies including IMiD and a proteasome inhibitor as separate lines or a combined line of therapy; and adequate organ function. Subjects who have relapsed after autologous hematopoietic cell transplantation (HCT) or are unable to receive autologous HCT are eligible. Target enrollment for this study is 20 subjects, with 10 in each arm; patients will be randomly assigned to a treatment arm. Eligible subjects who do not receive the $T$ cell infusion may be replaced.

Following apheresis, the T cells are isolated and expanded with CD3/CD28 beads, transduced with a lentiviral vector containing the NY-ESO-1 259 TCR, and $1-8 \times 10^{9}$ transduced T cells are infused intravenously on day 1 after lymphodepletion with fludarabine $30 \mathrm{mg} / \mathrm{m}^{2} /$ day and cyclophosphamide $600 \mathrm{mg} / \mathrm{m}^{2} /$ day on days -7 to -5 and granulocyte-colony stimulating factor support starting on day -4 . In Arm 1, SPEAR T cell infusion is the only investigational product administered. Subjects in Arm 2 will receive SPEAR T cell infusion followed by an initial $200 \mathrm{mg}$ dose of pembrolizumab on day 22 (week 3). If toxicities preclude week 3 treatment, the first dose may be given at week 6 . Subsequent doses of pembrolizumab will be given every 3 weeks up to week 108 post T cell infusion.

In both arms, safety will be assessed at each clinic visit. Disease response is assessed at weeks 1 and 3, every 3 weeks until week 24, every 6 weeks until week 72, and then every 12 weeks until confirmed progression of disease. On study biopsies and blood samples will be evaluated to compare the pre- and post-T cell infusion immune profile for association with treatment outcome.

Trial Registration

NCT03168438.

\section{P249}

A phase 1 study of the safety, tolerability, and pharmacokinetics (PK) of MGA012 (anti-PD-1 antibody) in patients with advanced solid tumors

Nehal Lakhani ${ }^{1}$, Janice M. Mehnert ${ }^{2}$, Drew Rasco ${ }^{3}$, Michael Gordon ${ }^{4}$, Joanna Lohr ${ }^{5}$, Pepi Pencheva ${ }^{5}$, Sharad Sharma ${ }^{5}$, Hua Li ${ }^{5}$, Ross LaMotte-Mohs ${ }^{5}$, Paul Moore $^{5}$, Jichao Sun ${ }^{5}$, Bradley Sumrow ${ }^{5}$, Jon Wigginton ${ }^{5}$, John Powderly ${ }^{6}$ ${ }^{1}$ START Midwest - South Texas Accelerated Research Therapeutics, LLC, Grand Rapids, MI, USA; ${ }^{2}$ Rutgers Cancer Institute of New Jersey, New Brunswick, NJ, USA; ${ }^{3}$ START - South Texas Accelerated Research

Therapeutics, LLC, San Antonio, TX, USA; ${ }^{4}$ Honor Health Research Institute, Scottsdale, AZ, USA; ${ }^{5}$ MacroGenics, Inc., Rockville, MD, USA;

${ }^{6}$ Carolina BioOncology Institute, Huntersville, NC, USA

Correspondence: Bradley Sumrow (sumrowb@macrogenics.com) Journal for ImmunoTherapy of Cancer 2017, 5(Suppl 2):P249

\section{Background}

MGA012 is a humanized, IgG4K monoclonal antibody (mAb) that recognizes human programmed cell death protein 1 (PD-1). MGA012 binds to PD-1 expressing T cells, inhibits PD-1 and PD-L1/PD-L2 interactions, and disrupts the negative signaling axis to restore $T$ cell function. The biological activity of MGA012 is comparable to replicas of approved anti-PD-1 mAbs when assessed in vitro, including blockade of PD-1 and PD-L1/PD-L2 interactions, inhibition of PD-1 signaling, and enhancement of $\mathrm{T}$ cell effector function.

\section{Methods}

This phase 1, dose escalation study will characterize the safety, tolerability, PK/PD, immunogenicity, and preliminary anti-tumor activity of MGA012 administered IV every two or four weeks in patients with advanced solid tumors. MGA012 has been evaluated in sequential dose escalation cohorts $(1-10 \mathrm{mg} / \mathrm{kg})$ of 3 to 6 patients each, using a $3+3$ design. Four tumor-specific expansion cohorts will be treated at the maximum tolerated dose of MGA012. Selective cohort expansion was allowed during escalation to gather further safety and PK/PD data.

Results

At the data cutoff, 33 patients $(12 \mathrm{M} / 21 \mathrm{~F}$, median age 63 years) with diverse tumor types were treated at doses from $1-10 \mathrm{mg} / \mathrm{kg}$, including 21 patients on treatment at the time of data cutoff. MGA012 has demonstrated acceptable tolerability with no dose-limiting toxicities (DLTs). Treatment-related adverse events (AEs) occurred in 20/33 $(61 \%)$ patients, most commonly fatigue $(n=9)$ and nausea $(n=5)$. Treatment-related Grade $\geq 3$ AEs occurred in 3/33 (9\%) patients and include increased lipase $(n=2)$ and vaginal ulceration/inflammation $(n=1)$. A single treatment-related serious adverse event (SAE) has been reported (aphasia occurring in conjunction with the emergence of new brain metastases). Immune-related AEs (irAEs) were limited to rash $(n=3)$, infusion-related reaction $(n=1)$, and vaginal ulceration/inflammation $(n=1)$. MGA012 has PK features consistent with other lgG4 monoclonal antibodies, as well as full and sustained receptor occupancy at all dosing levels tested, consistent with its known binding characteristics. Twenty-two patients were response evaluable at the data cutoff. Three patients have experienced unconfirmed partial responses (including ovarian, MSI-high colorectal and uterine papillary serous carcinoma), and 4 additional patients experienced stable disease as a best response. Others had radiographic progressive disease or clinical progression.

Conclusions

MGA012 has demonstrated an acceptable safety profile, predictable PK/PD, and early evidence of anti-tumor activity. Subsequent to dose escalation, patients will be enrolled on tumor-specific monotherapy expansion cohorts. Future trials also are planned for combination testing of MGA012 with T cell directed, CD3-based DART ${ }^{\oplus}$ molecules. Trial Registration NCT03059823.

\section{P250}

LTX-315, an oncolytic peptide converts "cold" tumors to "hot" in a majority of patients with advanced cancer: results from an ongoing phase I study

Aurelien Marabelle', Jean-Francois Baurain ${ }^{2}$, Ahmad Awada ${ }^{3}$, Rebecca Kristeleit ${ }^{4}$, Dag-Erik Jøssang ${ }^{5}$, Nina-louise Jebsen ${ }^{5}$, Delphine Loirat ${ }^{6}$,

Andrew Saunders ${ }^{7}$, Wenche Olsen ${ }^{7}$, Berit Nicolaisen ${ }^{7}$, Baldur Sveinbjornsson ${ }^{7}$, Vibeke Sundvold Gjerstad7, Øystein Rekdal7, Pal Brunsvig ${ }^{8}$, Jerome Galon', Fabienne Hermitte ${ }^{10}$, Bjorn-Tore Gjertsen ${ }^{5}$, Anna Armstrong ${ }^{11}$, James Spicer ${ }^{12}$ 'Gustave Roussy, Villejuif, France; ${ }^{2}$ Saint-Luc University Hospital, Brussel, Belgium; ${ }^{3}$ nstitut Jules Bordet, Brussel, Belgium; ${ }^{4}$ Univerity College London Hospital, London, United Kingdom; ${ }^{5}$ Haukeland Univeristy Hospital, Bergen, Norway; ${ }^{6}$ Institut Curie, Paris, France; ${ }^{7}$ Lytix Biopharma, Oslo, Norway; ${ }^{8}$ Oslo University Hospital, Oslo, Norway; ${ }^{9}$ Laboratory of Integrative Cancer Immunology, INSERM, Paris, France; ${ }^{10}$ INSERM, Paris,

France; ${ }^{11}$ Christie Hospital, Manchester UK, Manchester, United Kingdom; ${ }^{12}$ King's College London, London, United Kingdom

Correspondence: Øystein Rekdal

(baldur.sveinbjornsson@lytixbiopharma.com)

Journal for ImmunoTherapy of Cancer 2017, 5(Suppl 2):P250

\section{Background}

LTX-315 disintegrates cytoplasmic organelles (e.g mitochondria) and induces immunogenic cancer cell death in preclinical in vivo models. Intratumoral LTX-315 increases tumor-infiltrating lymphocytes (TILs) and induces complete tumor regression in several rodent models. 
Systemic (abscopal) anti-tumor immune responses can be enhanced upon combination with immune checkpoint inhibitors (ICl). Here we report preliminary results of the phase 1 trial which evaluates intratumoral LTX-315 in monotherapy or in combination with ICl.

Methods

Patients with advanced solid tumors were immunologically primed with weekly intratumoral LTX-315 into a single accessible lesion over 6 weeks. Additional injections could be administered thereafter every 2 weeks. ICl combinations included ipilimumab (melanoma cohort) and pembrolizumab (TNBC cohort). Biopsies of injected lesions were taken at baseline and after LTX-315 treatment. Immunoprofiling was performed using immunohistochemistry and Nanostring analysis. Twenty nine patients have been treated with LTX-315 monotherapy. LTX-315 monotherapy was administered at doses of 2-7mg to a median of 1.8 tumor lesions (range 1-6) for a median of 9 weeks (range 1-33). Results

In 29 patients, all LTX-315-related adverse events were CTC grade 1 or 2, most commonly local erythema, flushing, pruritis and hypotension, usually resolving within minutes of injection. Related grade 3 (3 patients) or 4 (1) allergic/anaphylaxis adverse events occurred and resolved without sequelae. Of 44 injected lesions in 20 evaluable LTX-315 monotherapy patients, 2 lesions regressed completely, $>50 \%$ regression was seen in 5 lesions, and 20 remained stable. Significant increases in CD8+ TILs occurred in 67\% (14 of 21) patients with evaluable biopsies. The HalioDx Immune Gene Signature analysis of LTX-315-treated tumors showed upregulation of genes involved in immune-mediated tumor regression (effector $\mathrm{T}$ cells, TH1 orientation, chemokines and cytokines). Regression of distant non-injected tumors (irRC) criteria has been observed in 11 of 30 tumors in 9 patients. Stable disease (SD) of at least 7 weeks duration in non-injected tumors (median duration 11 weeks) by immune-related RECIST criteria (irRC in evaluable patients) occurred in $50 \%$ of LTX-315 monotherapy patients (4 melanoma, 3 sarcoma, and 1 breast cancer patients).

\section{Conclusions}

This phase 1 study demonstrates that intratumoral LTX-315 is generally safe and tolerable. Intratumoral LTX-315 triggers an increase in TILs as assessed by IHC, and generates a transition from a cold to a hot tumor transcriptome as assessed by Immunosign ${ }^{\circledR}$ gene signature. Moreover, local and systemic clinical benefit (achievement of SD in non-injected sites per irRC criteria) was observed.

Trial Registration

Clinical trial information: NCT01986426.

\section{P251}

A study to evaluate the safety and efficacy of the CD40 agonistic antibody APX005M in combination with nivolumab in subjects with non-small cell lung cancer and subjects with metastatic melanoma

Linda Garland', Ravi Salgia', Melissa Johnson³, Amy Weise ${ }^{4}$, Gerald Linette ${ }^{5}$, Thomas Tremblay ${ }^{6}$, Ovid Trifan ${ }^{6}$, Martin Edelman ${ }^{7}$ ${ }^{1}$ University of Arizona Cancer Center, Tucson, AZ, USA; ${ }^{2}$ City of Hope, Duarte, CA, USA; ${ }^{3}$ Sarah Cannon Research Institute, Tennessee Oncology, Nashville, TN, USA; ${ }^{4}$ Karmanos Cancer Center, Detroit, MI, USA;

${ }^{5}$ Abramson Cancer Center, University of Pennsylvania, Philadelphia, PA, USA; ${ }^{6}$ Apexigen Inc., San Carlos, CA, USA; ${ }^{7}$ Fox Chase Cancer Center, Philadelphia, PA, USA

Correspondence: Martin Edelman (otrifan@apexigen.com) Journal for ImmunoTherapy of Cancer 2017, 5(Suppl 2):P251

\section{Background}

Blocking immune checkpoint PD-1, PD-L1 and CTLA-4 function enhance antitumor immunity, leading to durable clinical responses for a subset of patients with melanoma, lung cancer and other tumor types. However, the majority of patients with melanoma or lung cancer continue to have short or no response to checkpoint blockade therapies and thus require novel approaches to stimulate the antitumor immune response such as immune stimulatory antibodies. Recently, Zippelius and co-authors [1] showed in preclinical models that CD40 engagement with an agonistic mAb leads to a T cell and IFN- $\gamma$ dependent upregulation of PD-L1 on tumor infiltrating monocytes and macrophages, thereby promoting a negative feedback loop, which hampers CD40 induced T-cell responses. This resistance mechanism was successfully circumvented by co-administration of PD-1/PD-L1 blocking antibodies. To this end, Apexigen Inc., is developing APX005M a humanized monoclonal IgG1 CD40 agonistic antibody that stimulates both innate and adaptive immune response. APX005M recognizes a unique epitope that overlaps with the CD40 ligand binding sites and uses FcRpllb to cluster CD40, thus mimicking CD40L engagement. As a result of antigen presenting cell (APC) activation, APX005M enhances Tcell response to tumor antigens. APX005M combined with antibodies against PD-1 or PD-L1 synergistically enhances T-cell responses. In a phase 1 trial, APX005M was administered IV every 21 days to adult subjects up to $1 \mathrm{mg} / \mathrm{kg}$ with an acceptable safety profile and has demonstrated a dose-dependent activation of APCs and T cells and increases in circulating levels of cytokines.

Methods

Study APX005M-002 is a Phase 1b-2 study of APX005M administered in combination with nivolumab every 21 days to adult subjects with platinum pre-treated immunotherapy naïve non-small cell lung cancer (NSCLC) or metastatic melanoma after failure of anti-PD-1/PD-L1 therapy (MM). The Phase $1 \mathrm{~b}$ portion will establish the maximum tolerated dose and the recommended Phase 2 dose of APX005M with nivolumab. The Phase 2 portion will evaluate safety and efficacy of the combination in each of the two distinct tumor types. Inclusion criteria include: age $\geq 18$ years, histologically documented NSCLC or MM, measurable disease by RECIST 1.1, ECOG performance status 01, adequate organ function. Exclusion criteria include: concomitant anti-cancer therapy, history of bone marrow transplantation, active coagulopathy, previous immune mediated disorders, active infections or uncontrolled intercurrent illness. Recruitment is ongoing, with a target enrollment of approximately 100 subjects across 7 centers in the United States.

Trial Registration

Clinical trial information: NCT03123783.

\section{References \\ 1. Zippelius A, Schreiner J, Herzig P, Müller P. Cancer Immunol Res. 2015;3:236-44}

P252

Phase 1 study to evaluate the safety and tolerability of the CD40 agonistic monoclonal antibody APX005M in subjects with solid tumors

Marwan Fakih', David Bajor ${ }^{2}$, Ronac Mamtani ${ }^{3}$, Thomas Tremblay ${ }^{4}$,

Ovid Trifan ${ }^{4}$, Robert Vonderheide ${ }^{3}$

${ }^{1}$ City of Hope, Duarte, CA, USA: ${ }^{3}$ University Hospitals Cleveland Medical

Center, Cleveland, $\mathrm{OH}, \mathrm{USA} ;{ }^{3} \mathrm{Abramson}$ Cancer Center, University of Pennsylvania, Philadelphia, PA, USA; ${ }^{4}$ Apexigen Inc., San Carlos, CA, USA

Correspondence: Ovid Trifan (otrifan@apexigen.com)

Journal for ImmunoTherapy of Cancer 2017, 5(Suppl 2):P252

\section{Background}

APX005M is a humanized monoclonal IgG1 CD40 agonistic antibody developed by Apexigen, which mimics the natural ligand CD154. APX005M binds with high affinity to human CD40 leading to antigen presenting cell (APC) activation and subsequent $T$ cell activation. APX005M enhances T cell proliferation, IFN- $\gamma$ production and T-cell response to tumor antigens. In comparison with other anti-human CD40 agonistic antibodies, such as CP-870,893/RG7876, SGN-40, and ADC-1013/JNJ-64457107 analogs, APX005M is the most potent CD40 agonist and outperforms all others in many measures of immune activation. In a first in human clinical trial APX005M was administered IV every 21 days and has demonstrated a dose-dependent activation of APCs and T cells and increases in circulating levels of IL-12, IFN- $\gamma$, TNFa and IL-6. APX005M was escalated up to $1 \mathrm{mg} / \mathrm{kg}$ with a good safety profile.

Methods

Study APX005M-001 was originally designed as a multicenter Phase 1 dose escalation study of APX005M administered every 3 weeks to 
subjects with solid tumors and was amended to introduce two new dosing schedules for APX005M (every 2 weeks and every 1 week). Currently, primary objectives of the study are to evaluate the safety of APX005M administered intravenously (IV) every 2 weeks and every week and to determine the maximum tolerated dose (MTD) and recommended Phase 2 dose (RP2D) of APX005M for the every 2 weeks and every 1 week schedules. Secondary objectives include determining the pharmacokinetics (PK) of APX005M and preliminary assessment of clinical response. Inclusion criteria include: age $\geq 18$ years, histologically or cytologically documented diagnosis of urothelial carcinoma, melanoma, squamous cell carcinoma of the head and neck, non-small cell lung cancer, or any solid tumor with high microsatellite instability status (MSI-high), measurable disease by RECIST 1.1, ECOG performance status 0-1, adequate organ function. Exclusion criteria include: concomitant anti-cancer therapy, history of bone marrow transplantation, active coagulopathy, previous immune mediated disorders, active infections or uncontrolled intercurrent illness. Recruitment is ongoing, with a target enrollment of approximately 20 subjects across 3 centers in the United States.

\section{Trial Registration}

Clinical trial information: NCT02482168.

\section{P253}

Prioritizing tumor types for treatment with a novel

immunotherapy: LYC-55716 a small-molecule RORY agonist

Xiao Hu', Xikui Liu', Hongxiu Li', Madhumita Bogdan', Yilin Gao', Brian Fox ${ }^{2}, H$. Jeffrey Wilkins ${ }^{3}$, Laura Carter ${ }^{1}$

${ }^{1}$ Lycera Corp., Ann Arbor, MI, USA; ${ }^{2}$ Celgene Corp., Seattle, WA, USA;

${ }^{3}$ Lycera Corp., Plymouth Meeting, PA, USA

Correspondence: $\mathrm{H}$. Jeffrey Wilkins (autumn@ahkcommunications.com) Journal for ImmunoTherapy of Cancer 2017, 5(Suppl 2):P253

\section{Background}

RORY is the master transcription factor for Type 17 effector T cell differentiation and function. RORyt expression is induced by cytokines such as IL- 6 , TGF- $\beta$, IL- $1 \mathrm{~b}$, and IL- 23 and is activated by endogenous ligands derived from the cholesterol biosynthetic pathway. Synthetic RORY agonists augment the activity of this transcriptional regulator by modulating a gene expression program in immune cells, resulting in enhanced effector functions and decreased immunosuppression. LYC-55716 and other RORY agonists have shown promise as monotherapy and combination therapy in syngeneic tumor models. During Phase 1 clinical testing of this compound, preclinical and bioinformatics assessments were undertaken to prioritize tumor types that may respond to RORY agonist therapy, for possible inclusion in a Phase 2a trial.

Methods

An RORy agonist signature was derived from transcriptional profiling of primary murine and human T cells treated with or without RORY agonists. Using a panel of murine syngeneic models and The Cancer Genome Atlas (TCGA) dataset, a series of bioinformatic analyses were conducted to provide information across tumor types on (a) RORY expression, (b) RORy biology, including sterol mobilizing genes and correlations with prognosis, and (c) general immune parameters. Public data sets were also assessed for correlations of RORy signature genes and prognosis. For each assessment, tumor types were prioritized, then compared across categories to determine a final ranking.

Results

Target expression: 15 tumors types were identified, with $>20 \%$ of samples expressing RORyt. However, based on lack of correlation between baseline RORyt expression and efficacy of RORy agonists in preclinical models, these criteria were extended to include additional tumors that express mRNA for factors known to induce RORY expression. Target biology: As a surrogate for endogenous ligand levels, TCGA analysis revealed differentially expressed sterol synthesis and efflux genes across tumor types. RORyt expression correlated significantly with patient survival in 5 tumor types. Immune parameters: Consideration of tumors with immune infiltrates, high mutational burden, and reports of prior immunotherapy success highlighted 8 tumor types. After combining these findings, 7 tumor types met all selection criteria: non-small-cell lung; ovarian; stomach adenocarcinoma; and head and neck squamous cell, renal clear cell, hepatocellular, and esophageal carcinomas.

Conclusions

Preclinical data from syngeneic tumor models and bioinformatic analyses of TCGA database prioritized 7 tumors for LYC-55716 monotherapy. Our findings support the inclusion of these tumor types in the Phase 2a clinical trial.

P254

A phase $1 \mathrm{~b} / 2$ study of CD40 agonistic monoclonal antibody (APX005M) together with gemcitabine and nab-paclitaxel with or without nivolumab in untreated metastatic pancreatic adenocarcinoma patients

Mark O'Hara' ${ }^{1}$, Rosemarie Mick², Jaclyn Lyman ${ }^{3}$, Jingying $\mathrm{Xu}^{3}$,

Maryam Hosseini ${ }^{3}$, Theresa LaVallee ${ }^{3}$, Pier Federico Gherardini ${ }^{3}$,

Barbara Vance ${ }^{1}$, Ovid Trifan $^{4}$, Ute Dugan ${ }^{5}$, Aiman Shalabi ${ }^{6}$,

Ramy Ibrahim ${ }^{3}$, Robert Vonderheide

${ }^{1}$ University of Pennsylvania, Philadelphia, PA, USA; ${ }^{2}$ University of Pennsylvania Perelman, Philadelphia, PA, USA; ${ }^{3}$ Parker Institute for

Cancer Immunotherapy, San Francisco, CA, USA; ${ }^{4}$ Apexigen, San Carlos,

CA, USA; ${ }^{5}$ Bristol-Myers Squibb, New York City, NY, USA; ${ }^{6}$ Cancer

Research Institute, New York, NY, USA

Correspondence: Ramy Ibrahim; Robert Vonderheide

(jxu@parkerici.org)

Journal for ImmunoTherapy of Cancer 2017, 5(Suppl 2):P254

\section{Background}

Pancreatic cancer is one of the most lethal malignancies of the gastrointestinal tract. While check point inhibitors such as aCTLA4 and APD-1 have been effective in melanoma and lung cancer, clinical benefit of immunotherapy in the management in pancreatic cancer subjects has not been yet established. A recent study using a genetically engineered mouse model of pancreatic ductal adenocarcinoma (PDA) demonstrated that despite robust expression of PD-1 and PDL1 in the tumor microenvironment, treatment with aPD-1 with or without aCTLA-4 failed to improve the survival of mice or slow the growth of PDA tumors. However, administration of aCD40, gemcitabine (Gem) and nab-paclitaxel (NP), induces T cell immunity in mice with PDA, controls tumor growth and significantly improves survival in a $\mathrm{CD}^{+} \mathrm{T}$ cell dependent manner. In particular, aCD40/Gem/NP plus aPD-1 nearly double the median overall survival in genetically engineered KPC mice with pre-established spontaneous pancreatic tumors. Moreover, the capability of treated mice to reject second and third subcutaneous tumor challenges in a CD8+ T cell-dependent fashion thereby rendering long-term survival suggests the establishment of antitumor immune memory with curative potential. Methods

This is a multi-center, open label study evaluating the combination of APX005M with Nivolumab and standard chemotherapy (Gem and NP). The Phase $1 \mathrm{~b}$ will define the recommended Phase 2 dose of APX005M when combined with the standard dose of Gem and NP, with or without Nivolumab. The second part of the study is a 3-arm randomized Phase 2 ( 35 subjects per treatment arm) aimed to evaluate the activity (overall survival) of APX005M combined with Gem and NP, with or without Nivolumab with Gem and NP. Main inclusion criteria include: age $\geq 18$ years, documented diagnosis of pancreatic adenocarcinoma with metastatic disease, measurable disease by RECIST 1.1, ECOG performance status $0-1$, normal organ function. Main exclusion criteria include: concomitant anti-cancer therapy, previous exposure to CD40, PD-1, PD-L1, CTLA-4 mAbs or any other immunomodulatory agents, previous immune mediated disorders, active infections or uncontrolled intercurrent illness. The study will be exploring different doses of APX005M in combination with approved doses of Nivolumab and chemotherapy. The main safety endpoints include the frequency of DLT and incidence of AEs. The main efficacy endpoints include overall survival, and 1-year OS rate in each treatment arm.

Results

This trial is open for enrollment.

Conclusions

Clinical trial information: NCT02482168. 


\section{Combination Therapy (10/10, 10/Standard of Care, 10/Other)}

\section{P255}

Depleting blood arginine with AEB1102 (Pegzilarginase) exerts additive anti-tumor and synergistic survival benefits when combined with immunomodulators of the PD-1 pathway

Giulia Agnello, Mark Badeaux, Susan Alters, David Lowe, Scott Rowlinson

Aeglea BioTherapeutics, Inc., Austin, TX, USA

Correspondence: Giulia Agnello (gagnello@aegleabio.com); Scott

Rowlinson

Journal for ImmunoTherapy of Cancer 2017, 5(Suppl 2):P255

\section{Background}

Tumor dependence on specific amino acids for survival and proliferation is well recognized and has been exploited effectively in the clinic through the use of asparaginases for the treatment of acute lymphoblastic leukemia. Sensitivity of tumors to L-Arginine (L-Arg) deprivation results from an impaired ability to synthesize L-Arg, most commonly due to decreased functional expression of argininosuccinate synthase. Native human arginase 1 is not a viable drug candidate due to low activity and low stability in serum. We have developed a novel cobalt substituted, PEGylated human arginase 1 (AEB1102, Pegzilarginase) with enhanced pharmacological properties. We and others have successfully utilized arginase 1 to impart an anti-tumor effect through L-Arg starvation in multiple tumor types in vitro and in vivo (e.g. AEB1102 single agent efficacy in melanoma, SCLC, sarcoma, large cell NSCLC, Merkel cell carcinoma). Given that arginase 1 has been reported to be immune suppressive, immune neutral (PMID: 23717444), or immune promoting (PMID: 27043409) in different experimental settings and by different groups, we have investigated the impact of systemic depletion of L-Arg on the antitumor efficacy of immune checkpoint inhibitors.

Methods

Murine syngeneic models (e.g. CT26, MC38) were dosed with AEB1102 alone and in combination with immunomodulatory anti-PD-L1 monoclonal antibody (mAb).

Results

Combination therapy of AEB1102 with anti-PD-L1 resulted in an additive anti-tumor effect with improved survival benefit (increased life span (ILS) 55-129\%) compared to AEB1 102 (ILS 29-33\%) and anti-PD-L1 (ILS 7-33\%) monotherapies. In addition, in the CT26 model, complete tumor regression (non-palpable tumors) was observed in $37 \%$ of the mice; importantly, complete responses were observed only in the combination therapy group. When the complete responders were rechallenged with fresh CT26 cells, tumors failed to establish, suggesting the development of an immune memory response as a result of the previously administered combination therapy of AEB1102 and anti-PDL1. Administration of AEB1102 as a monotherapy or in combination with anti-PD-L1 in the CT26 model was associated with an increase in tumor-infiltrated CD45+ cells, indicating that AEB1102 promotes T-cells accumulation in the tumor microenvironment.

\section{Conclusions}

Collectively, these results demonstrate that in addition to tumor growth inhibition, L-Arg depletion in the tumor microenvironment enhances the effectiveness of immunotherapy. AEB1102 is currently in Phase 1 (monotherapy) clinical trials. These data open the possibility of clinical combination of AEB1102 with immunomodulators of the PD-1 pathway to further improve outcomes in cancer patients.

\section{P256}

G100 and ZVex ${ }^{\oplus}$-based combination immunotherapy induces near complete regression of established glioma tumors in mice

Tina C. Albershardt, Jordan E. Krull, Reice D. James, Peter Berglund, Jan ter Meulen

Immune Design, Seattle, WA, USA

Correspondence: Tina C. Albershardt

(tina.albershardt@immunedesign.com)

Journal for ImmunoTherapy of Cancer 2017, 5(Suppl 2):P256

\section{Background}

Glioblastoma (GBM) is a malignant brain tumor with an overall survival of $<3.3 \%$ at 5 years. Novel immunotherapies are being explored but face limitations due to low infiltration of activated $T$ cells. ZVex is an integration-deficient lentiviral vector-based platform that targets dendritic cells in vivo to generate tumor-specific T cells. Intratumoral injections of G100, which contains glucopyransosyl lipid A (GLA, a synthetic TLR4 agonist), modulate the tumor microenvironment through induction of proinflammatory responses. We have previously shown in the B16 model that G100 induces T cell homing chemokines CXCL9 and CXCL10, increases number of $T$ cells in the tumor, enhances intratumoral antigen presentation, and results in antigen spreading of the $T$ cell response. Combined systemic and in situ immunization with ZVex/OVA and G100, respectively, completely eradicated established B16/OVA melanomas in mice. Here, we report that, in an orthotopic GBM model, the combined regimen with ZVex/OVA and G100 induced near complete regression of established GL261 syngeneic gliomas in C57BL/6 mice.

Methods

Female C57BL/6 mice were stereotactically cannulated in the left striatum. At study initiation, $2 \times 10^{5} \mathrm{GL} 261$ glioma cells expressing ovalbumin (GL261/OVA) were inoculated through the cannula. Tumor growth was monitored via imaging of luciferase activity, twice weekly. On Day 7 , mice with tumors $\left(6.8 \times 10^{5}\right.$ avg. radiance) were immunized by a single subcutaneous injection of ZVex/OVA. G100 ( $2 \mu \mathrm{g} \mathrm{GLA})$ was administered through the cannula, also on Day 7, and then once weekly for the duration of the study, for a total of three injections. Animals were sacrificed when displaying signs of cachexia and > 20\% weight loss, usually around 25 days after tumor inoculation.

Results

GL261/OVA-bearing mice treated with G100 or ZVex/OVA alone exhibited delayed tumor growth or modest tumor regression, respectively (mean reduction of $17 \%$ and $72 \%$, respectively, ranging from none to $83 \%$ ), while mice treated with ZVex/OVA and G100 combination exhibited significantly greater reduction in tumor size, averaging 98\% (74\%-99.8\%).

Conclusions

This study demonstrates that ZVex/OVA and G100 combination therapy was very successful in controlling growth of GL261 gliomas, presumably in part by chemotactically directing peripheral T cells to the brain, and suggest the potential for treatment of patients with GBM. The precise mechanism of synergy in this GBM model is currently under investigation. Both ZVex-based therapies and G100 are being evaluated in multiple Phase 1 and 2 clinical trials, and their combination is being investigated in a Phase 1 trial in soft tissue sarcoma patients.

\section{P257}

Phase II basket study of olaparib and durvalumab: Biomarker analysis in germline BRCA-mutated (gBRCAm) HER2-negative metastatic breast cancer (MBC) and relapsed small-cell lung cancer (SCLC) patients

Helen Angell', Vidalba Rocher Ros', Nathan Standifer', Zhongwu Lai', Christopher Gresty', Jean-Pierre Delord ${ }^{3}$, Maja De Jonge ${ }^{4}$, Sophie Postel-Vinay ${ }^{5}$, Antoine Italiano ${ }^{6}$, Matthew G Krebs ${ }^{7}$, Bella Kaufman ${ }^{8}$, Yeon Hee Park', Susan Domchek ${ }^{10}$, Pia Herbolsheimer ${ }^{11}$, Darren Hodgson ${ }^{1}$

${ }^{1}$ AstraZeneca, Cambridge, United Kingdom; ${ }^{2}$ Medlmmune, Mountain View, CA, USA; ${ }^{3}$ Institut Claudius Regaud-Oncopole, Toulouse, France; ${ }^{4}$ Erasmus MC Cancer Institute, Rotterdam, Netherlands; ${ }^{5}$ Gustave Roussy Cancer Campus, Villejuif, France; ${ }^{6}$ Institut Bergonié, Bordeaux, France; ${ }^{7}$ The University of Manchester and The Christie NHS Foundation Trust, Manchester, United Kingdom; ${ }^{8}$ Sheba Medical Centre, Ramat Gan, Israel; ${ }^{9}$ Samsung Medical Centre, Sungkyunkwan University School of Medicine, Seoul, Republic of Korea; ${ }^{10}$ Hospital of the University of Pennsylvania, Philadelphia, PA, USA; ${ }^{11}$ AstraZeneca, Gaithersburg, MD, USA

Correspondence: Helen Angell (Helen.Angell@astrazeneca.com) Journal for ImmunoTherapy of Cancer 2017, 5(Suppl 2):P257 


\section{Background}

Poly(ADP-ribose) polymerase inhibitors (PARPi) trap PARP at sites of single-strand DNA breaks, and generate genomic instability and cell death in homologous recombination deficient tumour cells. Both intrinsic and PARPi-induced DNA repair defects may attract tumourinfiltrating lymphocytes (TILs), upregulate programmed cell death ligand-1 (PD-L1) and release tumour neo-antigens upon cell death. The MEDIOLA study (NCT02734004) assessed the effect of the PARPi, olaparib, alone and in combination with durvalumab (anti-PD-L1), on immune cell pharmacodynamics, and the correlation of candidate predictive markers on disease control rate (DCR).

Methods

Patients with HER2-negative, gBRCAm MBC $(n=25)$, or relapsed SCLC $(n=38)$ received olaparib (300 mg PO BID) for 4 weeks, followed by a combination of olaparib and durvalumab (1.5 g IV every 4 weeks) until progression. At 4 weeks after initial olaparib, paired biopsies were conducted for immuno-oncology profiling. Primary endpoints were DCR at 12 weeks, safety and tolerability. Biomarker endpoints included archival (Fig. 1) and paired biopsy analysis of tumour cell (TC) and immune cell (IC) PD-L1 expression (SP263), densities of CD3 and CD8 T-cells (cells $/ \mathrm{mm}^{2}$ ), tumour mutation status and peripheral blood immunophenotyping.

Results

Olaparib caused small reductions in circulating T, B and Natural Killer (NK) cells on day 29 and thereafter in $86-93 \%$ of MBC and $81-91 \%$ of SCLC patients, followed by evidence of post-durvalumab 2 -fold or greater increases in Ki67+CD8+ T-cells in $46 \%$ of MBC and $50 \%$ of SCLC patients on day 57. In MBC ( $n=25)$ 5/5 (100\%) PD-L1 TC $\geq 1 \%$ patients had controlled disease at 12 weeks, compared with 9/14 (64.3\%) PD-L1 TC $<1 \%$ patients (no data for $6 / 25$ patients). In SCLC ( $n=38)$ PD-L1 $\mathrm{TC} \geq 1 \%$ patients had a DCR of $1 / 6(16.7 \%)$ compared with a PD-L1 TC $<1 \%$ DCR of $9 / 27$ (33.3\%) at 12 weeks (no data for $5 / 38$ patients). At the time of analysis, $1 / 2 \mathrm{MBC}$ with paired biopsy data had a partial response (PR) and demonstrated dramatic increases in CD3/CD8 densities and PD-L1 TC positivity 4 weeks post-olaparib monotherapy. 1/3 SCLC paired biopsies had PRs and showed increases in CD3/CD8 densities, but no PD-L1 TC expression, compared with no change in $2 / 3$ patients with stable disease. Updated results will be presented.

Conclusions

Olaparib results in modest reductions in circulating $\mathrm{T}, \mathrm{B}$ and NK cells: however, this effect does not disrupt durvalumab-based increases in circulating, proliferating cytotoxic T-cells. Preliminary data suggest that olaparib treatment may increase levels of TILs, but more data are required. Data collection and analysis is ongoing.

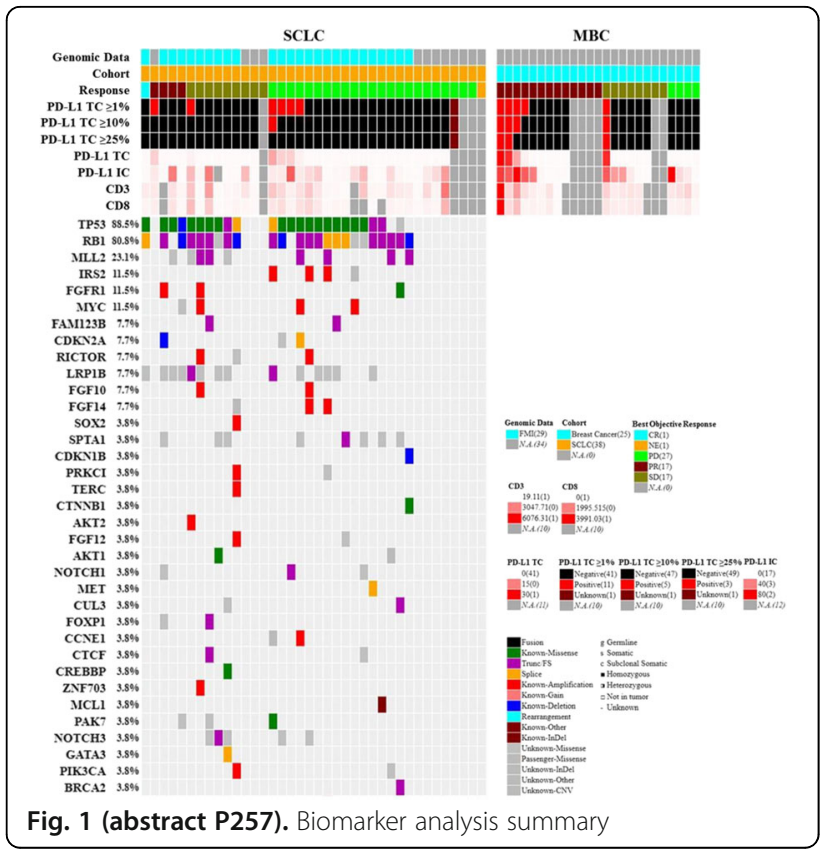

P258

Resource use and cost implications associated with treatment-free interval experienced on immunotherapies

Michael Atkins ${ }^{1}$, Ahmad Tarhini ${ }^{2}$, Apoorva Ambavane ${ }^{3}$, David

McDermott ${ }^{4}$, Komal Gupte-Singh ${ }^{5}$, Josh Weng ${ }^{3}$, Corey Ritchings ${ }^{5}$,

Meredith Regan 7 , Agnes Benedict ${ }^{6}$, Sumati Rao ${ }^{5}$

${ }^{1}$ Georgetown-Lombardi Comprehensive Cancer Center, Washington, DC, USA;

'University of Pittsburgh School of Medicine and Cancer Institute, Pittsburgh,

PA, USA; ${ }^{3}$ Evidera, Inc., Bethesda, MD, USA; ${ }^{4}$ Beth Israel Deaconess Medical

Center, Boston, MA, USA; ${ }^{5}$ Bristol-Myers Squibb, Princeton, NJ, USA; ${ }^{6}$ Evidera,

Inc., Budapest, Hungary; ${ }^{7}$ Dana-Farber Cancer Institute, Boston, MA, USA

Correspondence: Michael Atkins (mba41@georgetown.edu)

Journal for ImmunoTherapy of Cancer 2017, 5(Suppl 2):P258

\section{Background}

Immunotherapies, including ipilimumab, nivolumab, and nivolumab +ipilimumab, are recommended for the treatment of advanced melanoma. Patients treated with immunotherapies often experience clinical benefit beyond treatment discontinuation, resulting in a significant treatment-free interval (TFI) until subsequent treatment is needed. However, resource use during TFI is not well understood. The objective of this study was to estimate the resource use and costs associated with the TFI experienced after first-line immunotherapies.

Methods

Patient-level data $(N=882)$ from the phase 3 CheckMate 067 and phase 2 CheckMate 069 trials were analyzed to estimate the healthcare resource use associated with the management of advanced melanoma. TFI is defined as time from first-line discontinuation to second-line initiation. Concomitant medications, laboratory tests, procedures, consultations, hospitalizations, and surgeries were analyzed. The annual rate of resource use was estimated for ipilimumab, nivolumab, and nivolumab+ipilimumab, and stratified by disease phase (progression-free or progressed defined by RECIST v.1.1). Mean annual total costs and $95 \%$ confidence intervals (Cls) were estimated by applying unit costs from publicly available sources in the United States.

Results

Annual costs per patient during the TFI on various immunotherapies are presented below (Table 1) Although confidence intervals are wide, ipilimumab is associated with higher total cost/year compared to nivolumab+ipilimumab and nivolumab in the progression-free phase (difference in costs $\$ 5,624-\$ 6,481$ ), and compared to nivolumab+ipilimumab and nivolumab in the progressed phase (difference in costs $\$ 354-\$ 3,834)$. Progression increases the costs of melanoma management across all treatments by $\$ 12,486-\$ 16,823$. Key resources utilized across all treatments were hospitalizations (34-62\% of total costs) in the progression-free phase, due to management of adverse events, and hospitalizations and surgeries ( $>94 \%$ of total costs) in the progressed phase, due to disease worsening.

Conclusions

Immunotherapies for advanced melanoma, particularly nivolumab +ipilimumab, may lead to a long TFI before starting second-line therapy. The estimated annual cost of managing advanced melanoma is low in the TFI prior to disease progression, especially with nivolumab and nivolumab+ipilimumab. These results require comparison with real-world evidence to measure the impact of bias due to the controlled clinical trial setting.

Table 1 (abstract P258). See text for description

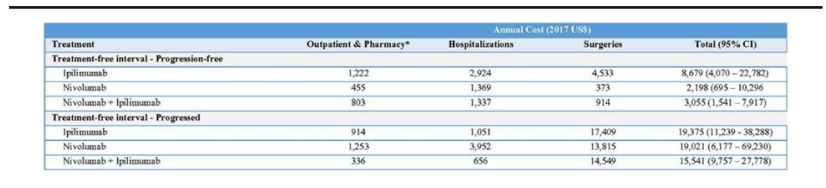

*Includes costs associated with concomitant medications, laboratory tests, procedures, and consultations 
P259

Pushing the accelerator and releasing the brake: testing the soluble LAG-3 protein (IMP321), an antigen presenting cell activator, together with pembrolizumab in unresectable or metastatic melanoma

Victoria Atkinson', Andrew Haydon ${ }^{2}$, Melissa Eastgate ${ }^{3}$, Amitesh Roy ${ }^{4}$ Adnan Khattak ${ }^{5}$, Christian Mueller ${ }^{6}$, Tina Dunkelmann ${ }^{6}$, Chrystelle Brignone ${ }^{7}$, Frederic Triebel ${ }^{7}$

${ }^{1}$ Princess Alexandra Hospital, 4102 Woolloongabba, Australia; ${ }^{2}$ Alfred Hopital, 3004 Melbourne, Australia; ${ }^{3}$ Royal Brisbane and Women's Hospital, 429 Herston, Australia; ${ }^{4}$ Flinders Medical Centre, Bedfork Park, Australia; ${ }^{5}$ Fiona Stanley Hospital, Murdoch, Australia; ${ }^{6}$ Prima BioMed GmbH, Berlin, Germany; ${ }^{7}$ Immutep S.A.S., Châtenay-Malabry, France

Correspondence: Victoria Atkinson

(Victoria.Atkinson@health.qld.gov.au)

Journal for ImmunoTherapy of Cancer 2017, 5(Suppl 2):P259

\section{Background}

IMP321 is a recombinant soluble LAG-3Ig fusion protein binding to $\mathrm{MHC}$ class II molecules and mediating antigen presenting cell (APC) activation followed by CD8 T-cell activation. The activation of the dendritic cell network and the subsequent $T$ cell recruitment at the tumor site with IMP321 may lead to stronger anti-tumor CD8 T cell responses than observed with pembrolizumab alone. This combination of an APC activator with an immune checkpoint inhibitor $(\mathrm{ICl})$ is aiming to increase efficacy without additional toxicity. We report initial results of the first 2 cohorts of a dose escalation phase I trial (TACTI-mel, NCT02676869) with pembrolizumab and IMP321 at different dose levels.

Methods

In this study, melanoma patients treated with pembrolizumab being without a complete response or fast progression after 3 cycles received pembrolizumab per standard dosing plus either $1 \mathrm{mg}(\mathrm{n}=6$; cohort 1$)$ or $6 \mathrm{mg}$ ( $n=6$, cohort 2) IMP321 injections s.c. (every 2 weeks for 6 months) from cycle 5 onwards. Patients without progressive disease (PD) at the end of the 6 months combination therapy continue on pembrolizumab monotherapy.

Results

In total, 12 patients ( 11 male, 1 female) with a median age of 62 years (range 48-85) were enrolled between Apr 2016 and Feb 2017. Four patients completed the 6 months combination treatment. One patient discontinued due to serious adverse event (SAE), unrelated to both study drugs. Two patients withdrew consent and five discontinued prematurely due to PD. No dose limiting toxicities for the combination have been reported. No SAE were found related to IMP321. All patients were evaluable according to irRC and 6 showed a reduction of $\sim 50 \%$ after IMP321 initiation. This includes one patient with a confirmed complete response while having PD on pembrolizumab monotherapy before study start.

\section{Conclusions}

One (1) and $6 \mathrm{mg}$ IMP321 given s.c. every 2 weeks in combination with pembrolizumab are safe and testing of the highest dose (30 $\mathrm{mg}$ ) is underway. The 6 late tumor responses seen after the combination was started may point out to the benefit of adding a systemic APC activator to an ICI.
P260

Glembatumumab vedotin (GV), an anti-gpNMB antibody-drug conjugate (ADC), in combination with varlilumab (V), an anti-CD27 antibody, in advanced melanoma

Omid Hamid ${ }^{1}$, Anna C. Pavlick', C. Lance Cowey ${ }^{3}$, Lowell Hart ${ }^{4}$, Douglas B. Johnson ${ }^{5}$, Jose Lutzky ${ }^{6}$, Aaron Alizadeh', David Spigel ${ }^{8}$, Neal Rothschild ${ }^{9}$, April Salama ${ }^{10}$, Robert Weber ${ }^{11}$, Jason J. Luke ${ }^{12}$, Ying Wang ${ }^{13}$, Michael Yellin ${ }^{13}$, Yi He $^{13}$, Rebecca G. Bagley ${ }^{13}$, Patrick Ott ${ }^{14}$ ${ }^{1}$ The Angeles Clinic and Research Institute, Los Angeles, CA, USA; ${ }^{2} \mathrm{New}$ York University School of Medicine, New York, NY, USA; ${ }^{3}$ Baylor University Medical Center, Dallas, TX, USA; ${ }^{4}$ Florida Cancer Specialists, Fort Myers, FL, USA; ${ }^{5}$ Vanderbilt University Medical Center and Vanderbilt Ingram Cancer Center, Nashville, TN, USA; ${ }^{6}$ Mount Sinai Comprehensive Cancer Center, Miami Beach, FL, USA; ${ }^{7}$ Northside Hospital, Atlanta, GA, USA; ${ }^{8}$ Tennessee Oncology, PLLC, Nashville, TN, USA; ${ }^{9}$ Florida Cancer Specialists, West Palm Beach, FL, USA; ${ }^{10}$ Duke University, Durham, NC, USA; ${ }^{11}$ St. Mary's Medical Center, San Francisco, CA, USA; ${ }^{12}$ University of Chicago, Chicago, IL, USA; ${ }^{13}$ Celldex Therapeutics, Inc., Hampton, NJ, USA; ${ }^{4}$ Dana-Farber Cancer Institute, Boston, MA, USA

Correspondence: Omid Hamid (rbagley@celldex.com)

Journal for ImmunoTherapy of Cancer 2017, 5(Suppl 2):P260

\section{Background}

gpNMB is an internalizable transmembrane glycoprotein expressed in multiple tumor types. The ADC GV delivers the potent cytotoxin MMAE to gpNMB + cells and showed promising activity as monotherapy in advanced melanoma refractory to checkpoint inhibitors (CPI). Preclinical results showed synergistic antitumor activity between ADC with CPI [1, 2], via dendritic cell (DC) activation; ADC-MMAE augmented the immune response induced by $\mathrm{V}$, an agonist monoclonal antibody against the $T$ cell costimulatory molecule CD27. This Phase 2 study was conducted to evaluate the activity and safety of the GV/ $\mathrm{V}$ combination in advanced melanoma.

Methods

Patients with advanced melanoma, progressive after $\leq 1$ chemotherapy, $\geq 1$ checkpoint inhibitor (CPI), and if BRAF mutation $\geq 1$ BRAF or MEK + BRAF inhibitor, were treated with GV $(1.9 \mathrm{mg} / \mathrm{kg}$ q3w until progression/ intolerance) in combination with $V(3.0 \mathrm{mg} / \mathrm{kg})$ on day 1 of weeks 1,3 , $9,15,21$, and 27 . Retrospective analysis on pre-study tumors and skin biopsies include gpNMB expression and infiltrating lymphocytes by immunohistochemistry plus gene expression profiling. Primary objective is to evaluate objective response rate (ORR) (RECIST 1.1).

Results

Thirty-four patients enrolled: median age of 61 years; $59 \%$ male; $18 \%$ $\mathrm{BRAF}^{\mathrm{V} 600}$ mutated; $59 \% \geq 3$ lines prior therapy; $76 \%$ prior anti-CTLA-4; 100\% prior anti-PD-1/PD-L1 inhibitor; $97 \%$ Stage IV; 71\% M1c. Of 30 response evaluable patients, emerging tumor response data shows 2 confirmed partial responses (PR) (ORR $=7 \%, \mathrm{Cl}: 0.8,22.1)$, and 1 single time point PR before patient withdrew consent. $48 \%$ patients had tumor shrinkage. Median PFS $=2.6$ months and median OS $=4.4$ months; 3 patients remain on treatment and 18 patients in survival follow up. Most common treatment related toxicities include alopecia, rash, fatigue, neuropathy, nausea, and vomiting. Tumor cells in $84 \%$ of patients with samples $(n=25)$ tested to date are $100 \% \mathrm{gpNMB}+$.

Conclusions

The GV/N combination was well tolerated without evidence of additive toxicity. There was no apparent enhanced clinical benefit of GVN over GV alone in this patient population, perhaps because immune checkpoint molecules remained unblocked and/or of a dearth of antigen 
presenting cells in tumors. Correlative biomarker analyses are ongoing and will be presented. For further insights into the synergy of ADC and immunotherapy, a cohort evaluating GV with anti-PD-1 in CPI-refractory melanoma is enrolling and a cohort investigating GV with the DC growth factor FLT3L (CDX-301) is planned.

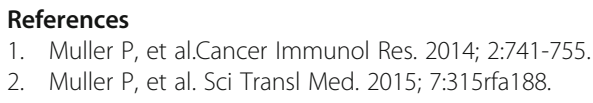

\section{P261}

Molecular signatures of combination immunotherapy of prostate cancer using a Listeria-based PSA vaccine and radiation Emily Bongiorno', Trevor Baybutt', Carla Portocarrero', Adam Snook' Adam Dicker ${ }^{1}$, Sandra Hayes ${ }^{2}$, Ulrich Rodeck

${ }^{1}$ Thomas Jefferson University, Philadelphia, PA, USA; ${ }^{2}$ Advaxis Immunotherapeutics, Inc., Princeton, NJ, USA

Correspondence: Emily Bongiorno (emily.bongiorno@jefferson.edu) Journal for ImmunoTherapy of Cancer 2017, 5(Suppl 2):P261

\section{Background}

Radiotherapy (RT) has the potential to amplify immune responses triggered by tumor vaccines, including ADXS-PSA, a live-attenuated Listeria monocytogenes $(\mathrm{Lm})$-based vector expressing human PSA. Earlier observations suggest that the two treatment modalities cooperatively induce regression of syngeneic mouse prostate cancer cells expressing human PSA (TPSA23), though immune correlates of efficacy and tumor recurrence are poorly understood.

Methods

We compared efficacy of different sequencing regimens of combination RT/vaccine treatments on TPSA23 tumor growth in syngeneic mice. Using the optimal sequencing protocol, tumors were collected post-implantation to assess immune infiltrate and function during initial tumor regression (day 20) and upon resumption of tumor growth (day 38). Correlates of treatment efficacy were determined by transcriptome analysis, phenotypic analyses of infiltrates and TCR sequencing.

Results

We confirmed that combination RT/ADXS-PSA is superior to single modality treatments. Concurrent administration of RT/vaccine was the most effective treatment schedule and was associated with enhanced T cell activation and robust IFNg signatures in the tumor microenvironment. This was reflected in increased intratumoral CD4 and CD8 T cell infiltration in mice receiving RT/vaccine. TCRb chain sequencing revealed elevated and sustained T cell diversity in tumor tissues of RT/vaccine-treated mice, when compared with mice receiving single modality treatments. In these residual tumors resident and/or memory $\mathrm{T}$ cell phenotypic markers were increased. Transcriptome analysis of recurring tumors further revealed induction of PD-L1 as a function of treatment. Targeting of the PD1/PD-L1 axis via a PD1 blocking antibody administered in addition to radiation and ADXS-PSA (triple combination) further amplified tumor growth inhibition in mice receiving dual RT/vaccine therapy.

Conclusions

Combining RT with the ADXS-PSA vaccine leads to effective tumor growth inhibition and induces robust, persistent antitumor immunity within the tumor environment. Transcriptome analysis during treatment revealed increased PD1 expression as a potential resistance mechanism and a PD1 blocking antibody provided further therapeutic benefit. These results support the rationale for combining Listeria-based vaccines with radiation in the clinic.
P262

Phosphatidylserine targeting antibody in combination with tumor radiation and immune checkpoint blockade promotes anti-tumor activity in mouse B16 melanoma

Sadna Budhu', Rachel Giese1, Olivier De Henau', Roberta

Zappasodi ${ }^{1}$, Luis Felipe Campesato ${ }^{1}$, Aditi Gupta', Christopher

Barker ${ }^{1}$

Bruce Freimark², Jedd D. Wolchok', Taha Merghoub ${ }^{1}$

${ }^{1}$ Memorial Sloan Kettering Cancer Center, New York, NY, USA; ${ }^{2}$ Peregrine

Pharmaceuticals, Inc., Tustin, CA, USA

Correspondence: Jedd D. Wolchok (budhus@mskcc.org); Taha

Merghoub

Journal for ImmunoTherapy of Cancer 2017, 5(Suppl 2):P262

\section{Background}

Phosphatidylserine (PS) is a phospholipid that is exposed on surface of apoptotic cells, viable tumor cells, tumor endothelium and activated immune cells. It has been shown to promote immunosuppressive signals in the tumor microenvironment. In a mouse B16 melanoma model, targeting PS in combination with immune checkpoint blockade promoted greater anti-tumor activity than either agent alone. This combination was shown to enhance CD4 + and CD8+ T cell infiltration and activation in the tumors of treated animals. Radiation therapy (RT) is an effective focal treatment of primary solid tumors, but is less effective in treating metastatic solid tumors as a monotherapy. RT induces immunogenic tumor cell death and enhances tumor-specific T cell infiltration in treated tumors. The abscopal effect, a phenomenon in which tumor regression occurs outside the site of RT, has been observed in both preclinical and clinical trials when RT is combined with immunotherapy.

Methods

Mice were injected intradermally on the hind limb with $10^{5}$ B16F10 melanoma cells. 7-10 days after implantation, tumors were treated locally with 15 Gy RT. 1 day after RT, mice were given antibodies to PS (mch1N11) and PD-1 (RMP 1-10) intraperitoneally every 3 days. Tumor surface area and overall survival of mice were used to determine efficacy of the combinations. For FACS analysis, tissues were collected between 1-10 days after RT.

Results

In this study, we show that irradiation of B16 melanoma causes an increase in PS expression on the surface of viable tumor and immune infiltrates. We subsequently examined the effects of combining RT with an antibody that targets PS and anti-PD-1. We found that treatment with mch1N11 synergizes with RT to improve anti-tumor activity and overall survival in tumor bearing mice. In addition, the triple combination of mch1N11, RT and anti-PD-1 treatment displayed even greater anti-tumor and survival benefit. Analysis of the immune response in the tumors of treated animals revealed an increase in M1-like macrophages in the tumors after treatment with RT and mch1N11. In addition, analysis of the systemic immune responses revealed an increase in antigen-specific CD8 T cell infiltration in the tumors as well as increased activation, effector function and differentiation in the triple combination therapy.

Conclusions

This finding highlights the potential of combining these agents to improve outcome in patients with advanced-stage melanoma and may inform the design of future clinical trials with PS targeting in multiple cancers. 
P263

Preclinical development of a Vaccine-Based Immunotherapy Regimen (VBIR) that breaks immunological tolerance and induces high titer and long lived T-cell responses to a tumor-associated self-antigen

Helen Cho ${ }^{1}$, Risini Weeratna ${ }^{2}$, Joe Binder ${ }^{1}$, Bassel Akache ${ }^{2}$, Rajeev Nepal ${ }^{3}$, Paul Cockle ${ }^{4}$, Marianne Martinic ${ }^{1}$, Michael Dermyer ${ }^{1}$, Stanley Dai',

James Merson ${ }^{1}$, Karin Jooss ${ }^{4}$

${ }^{1}$ Pfizer Inc., San Diego, CA, USA; ${ }^{2}$ National Research Council of Canada, Ottawa, ON, Canada; ${ }^{3}$ Pfizer Canada Inc., Kirkland, QC, Canada; ${ }^{4}$ Gritstone Oncology Inc., Emeryville, CA, USA

Correspondence: Helen Cho (Helen.k.cho@pfizer.com)

Journal for ImmunoTherapy of Cancer 2017, 5(Suppl 2):P263

\section{Background}

A successful therapeutic cancer vaccine will activate the immune system to rapidly induce potent, balanced and durable CD8 and CD4 T-cell responses to selected tumor antigens that can reduce tumor growth, lead to tumor regression or prevent/delay the onset of new lesions.

Methods

The magnitude and quality of T-cell responses against self (rhesus Prostate Specific Membrane Antigen; rhPSMA) and non-self (human PSMA; hPSMA) antigens delivered by various vaccine platforms in nonhuman primates (NHPs) given with and without a check point inhibitor, anti-CTLA4 monoclonal antibody (tremelimumab; anti-CTLA4), were evaluated.

Results

Assessment of T-cell responses against self (rhPSMA) and non-self (hPSMA) antigens delivered by various vaccine platforms in NHPs, demonstrated that the AdV vector was the most potent delivery platform for breaking of immune tolerance to a self-antigen.

Many humans have immunity to adenovirus that could blunt the ability of an adenovirus vaccine to effectively prime an immune response. This limitation can be overcome by utilizing an AdV of a different serotype for which humans do not have pre-existing immunity. However, even with a low/no seroprevalent AdV, an AdV boost vaccination following an AdV prime vaccination can only be effective when the neutralizing immunity to the vector wanes in between the vaccinations, making the regimen less suitable for patients with high tumor burden or fast progressive disease. Therefore, plasmid DNA boost vaccinations delivered intramuscularly by electroporation were evaluated for durable expansion of AdV primed T-cell responses. Administration of anti-CTLA4 delivered subcutaneously to achieve high local concentrations in the vaccine draining lymph nodes, given concurrently with the AdV prime and DNA boost vaccinations was shown to induce and expand durable and polyfunctional (IFNy ${ }^{+}, \mathrm{TNFa}^{+}$and/or IL-2 ${ }^{+}$) T-cell responses to a tumor-associated self-antigen in NHPs.

Conclusions

In summary, we have developed VBIR that consists of a heterologous prime-boost vaccine approach given in combination with local delivery of tremelimumab to maximize vaccine potency while minimizing the negative impact of neutralizing antibodies to the AdV vector. These results have encouraged clinical development of this unique immunotherapeutic regimen.

\section{P264}

Immune modulation by low dose sunitinib combined with a cancer vaccine based immunotherapeutic regimen provides therapeutic benefit to tumor bearing mice

Helen Cho ${ }^{1}$, Cindy Li ${ }^{1}$, Antonio Boccia', Steve Burgess', Terri Harder ${ }^{1}$, Paul Cockle ${ }^{2}$, Jim Eyles', Marianne Martinic', Bryan Clay ${ }^{1}$, Kam Chan', Stanley Dai ${ }^{1}$, Michael Dermyer', Joe Binder ${ }^{1}$, Steve Kurzyniec ${ }^{3}$,

Terrina Bayone', Joan Guo ${ }^{4}$, Robert Hollingsworth', James Merson', Karin Jooss ${ }^{2}$

${ }^{1}$ Pfizer Inc., San Diego, CA, USA; ${ }^{2}$ Gritstone Oncology Inc., Emeryville, CA, USA; ${ }^{3}$ Shimadzu Scientific Instruments, San Diego, CA, USA; ${ }^{4}$ Moderna

Therapeutics, Cambridge, MA, USA

Correspondence: Helen Cho (Helen.k.cho@pfizer.com)

Journal for ImmunoTherapy of Cancer 2017, 5(Suppl 2):P264

\section{Background}

Sunitinib is a receptor tyrosine kinase (RTK) inhibitor approved as a monotherapy for the treatment of advanced renal cell carcinoma (RCC), gastrointestinal stromal tumors and advanced pancreatic neuroendocrine tumors. It inhibits angiogenesis and targets RTKs (PDGFR, VEGFR, KIT, FLT3, and RET) involved in tumor cell growth. Sunitinib also has been shown, in preclinical studies and patients, to have immunomodulatory properties that reduce myeloid derived suppressor cells (MDSCs) and restore a Th1 cytokine profile. The approved dosing regimens for RCC have a safety profile that is overall manageable with the most commonly reported sunitinib-related grade 3 adverse events including hypertension, fatigue, diarrhea and hand-foot syndrome. Although the tolerability of sunitinib as monotherapy was acceptable in advanced stage, metastatic cancer patients, the drug's side effect profile could potentially pose challenges for use in patients with earlier stage cancer and/or when combined with other oncology therapies. Thus, lowering the dose of sunitinib might improve the safety of combinations, including combinations with cancer vaccines, thus enabling treatment of cancers of all stages.

Methods

The anti-tumor efficacy of lowered doses of sunitinib as a monotherapy, and in combination with a cancer vaccine, was investigated in immune-competent mouse tumor models. To understand the mechanism of such efficacy, the impact of the treatments on MDSC profiles in the periphery and tumors, T cell IFNy production, and antigen-specific immune responses was evaluated. Additionally, adoptive immune cell transfer experiments were conducted to monitor survival of MDSCs and $T$ cells in mice treated with sunitinib.

Results

At doses below the maximum biologic efficacy dose in mice $(<40 \mathrm{mg} /$ $\mathrm{kg}$ q.d.), sunitinib selectively and significantly reduced the survival and frequency of MDSCs in the periphery and tumors, which resulted in increased IFNg production by T cells. Importantly, when low dose sunitinib was given concurrently with a rat HER2 (rHER2)-based heterologous prime-boost vaccine, it significantly reduced the growth of subcutaneous tumors and prolonged survival of tumor-bearing rHER2 transgenic mice compared to mice receiving only monotherapies. Addition of an anti-CTLA4 monoclonal antibody (anti-CTLA4) significantly enhanced the vaccine-induced immune response and prolonged survival. Conclusions

The combination of the vaccine, sunitinib, and anti-CTLA4 further increased efficacy and survival. These results indicate that low dose sunitinib, alone or together with either a cancer vaccine or a cancer vaccine plus anti-CTLA4, maintains its immune modulatory properties and may be a valuable component of a safe and efficacious vaccinebased immunotherapy regimen.

P265

Optimizing targeted therapy and immune checkpoint blockade therapy in Kras mutant lung cancer

Hyejin Choi', Jiehui Deng ${ }^{2}$, Tarik Silk', Ann Powers ${ }^{1}$, Jonathan Boiarsky',

Taha Merghoub ${ }^{3}$, Kwok-Kin Wong' ${ }^{2}$, Jedd Wolchok'

${ }^{1}$ MSKCC, New York, NY, USA; ${ }^{2}$ New York University Langone Medical

Center, New York, NY, USA; ${ }^{3}$ Medicine, New York, NY, USA

Correspondence: Taha Merghoub (choih3@mskcc.org); Kwok-Kin

Wong; Jedd Wolchok

Journal for ImmunoTherapy of Cancer 2017, 5(Suppl 2):P265

\section{Background}

KRAS is the most commonly identified driver oncogene in lung cancer. However, to date there is no effective therapy available for KRAS mutant lung cancers. To identify the most effective therapy, we studied the impact of Kras signaling targeted therapy (MEK inhibition) on the immune microenvironment, in order to formulate a combinatorial therapy using targeted therapy and immunotherapy, with a goal of optimally enhancing tumor apoptosis and promoting long-term immune response simultaneously. MEK signaling is a downstream of Kras signaling pathway and critical for tumor growth and T cell activation. Conventionally, MEK inhibitor is treated daily/continuously. However, to 
enhance tumor apoptosis and promote $\mathrm{T}$ cell activation on MEK inhibition, we hypothesized intermittent administration of MEK inhibitor will confer $T$ cells temporal release from MEK signaling inhibition allowing $T$ cells activated, while tumor growth is suppressed and immunotherapy can be combined based on T cell activation/inhibitory markers change on this treatment schedule.

Methods

We have treated T cells from Kras lung cancer bearing mice or tumor bearing mice with Selumetinib or Trametinib in a pulsatile/intermittent or continuous way, then analyzed T cell phenotype changes, tumor progression, and survival.

Results

Ex vivo T cell study showed that pulsatile treatment of MEK inhibitor, Selumetinib and Trametinib, showed highly increased CTLA4 expression and mild increase of PD1 in CD8+ T cells and CD4 +Foxp3- T cells, compared to continuously treated group which is a standard regimen. This result was confirmed in intermittently treated KrasG12D/+; p53-/- transplantable mouse model and GEMM as well. Moreover, CD8+ T cells from pulsatile group showed increase of Ki67 and 4-1BB expression, suggesting that CD8+ T cells are more activated in pulsatile group. In vivo tumor study using Kras mutant lung cancer animal model showed intermittent treatment suppressed tumor growth better than continuous treatment. Better response, more activated phenotype of CD8+ T cells including increased CTLA4 expression in intermittent group lead us to test combination of intermittent MEK inhibitor treatment with antiCTLA4 antibody to maximize anti-tumor $\mathrm{T}$ cell activity and it is currently under investigation.

\section{Conclusions}

In summary, we found that pulsatile/intermittent treatment with MEK inhibitor showed better response and more activated phenotype of T cells including increased CTLA4 expression compared to continuous treatment. This study suggests that optimized intermittent schedule of MEK inhibitor treatment is essential to maximize T cell mediated anti-tumor activity in combination with anti-CTLA4 therapy and this will benefit Kras mutant lung cancer patients.

\section{P266}

Significant enhancement of expanded natural killer cells against GD2 pediatric solid tumors (ST) in combination with ALT-803 (IL-15 superagonist) and dinutuximab

Yaya Chu', Nang Kham Su', Jeremy Rosenblum', Hing C. Wong²,

Dean A. Lee ${ }^{3}$, Mitchell S Cairo ${ }^{1}$

${ }^{1}$ New York Medical College, Valhalla, NY, USA; ${ }^{2}$ Altor Bioscience, Miramar, FL, USA: ${ }^{3}$ Nationwide Children's Hospital, Columbus, OH, USA

Correspondence: Yaya Chu (yaya_chu@nymc.edu)

Journal for ImmunoTherapy of Cancer 2017, 5(Suppl 2):P266

\section{Background}

Children with recurrent and/or metastatic osteosarcoma (OS), neuroblastoma (NB) and glioblastoma (GBM) have a dismal even-free survival (EFS) $(<25 \%)$. Dinutuximab is an anti-GD2 monoclonal antibody that has significantly increased EFS in children with $\mathrm{GD}^{+}$neuroblastoma [1]. ALT-803 is a superagonist of an IL-15 variant bound to an IL15RaSu-Fc fusion with enhanced biological activity [2]. Our group has successfully expanded peripheral blood Natural Killer cells (exPBNK) with irradiated feeder cells [3].

In this research, we aim to determine if the combination of ALT-803 and dinutuximab significantly enhances exPBNK cell in vitro cytotoxicity against $\mathrm{GD} 2^{+} \mathrm{OS}, \mathrm{NB}$ and $\mathrm{GBM}$.

Methods

PBMCs were expanded with lethally irradiated K562-mbIL21-41BBL cells [4]. ExPBNK cells were isolated using Miltenyi NK cell isolation kits as we previously described. ${ }^{3}$ ALT-803 was generously provided by Altor BioScience Corporation. NK proliferation, NK receptors expression and cytotoxicity were assessed as we previously described [3]. Dinutuximab (generously provided by United Therapeutics) was used for antibody-dependent cellular cytotoxicity (ADCC) assays. IFN$\gamma$ and perforin levels were evaluated by ELISA assays. GD2 ${ }^{+}$OS, NB and GBM cell lines were used as target cells.
Results

ALT-803 significantly promoted exPBNK in-vitro proliferation by increasing the phosphorylation of Akt, Stat $3 / 5$ and p38 MAPK. ALT-803 increased NK activating receptors expression: NKG2D, NKp30, NKp44, and NKp46.

ALT-803 significantly enhanced exPBNK mediated ADCC with dinutuximab in a E:T dependent manner $(p<0.001)$ against OS, NB and GBM cells (Fig. 1). ALT-803 significantly enhanced IFN- $\gamma(p<0.001)$ and perforin $(p<0.001)$ release from exPBNK when it was combined with dinutuximab against $\mathrm{OS}, \mathrm{NB}$ and GBM cells compared to exPBNK, ALT-803+exPBNK, or dinutuximab+exPBNK.

\section{Conclusions}

ALT-803 significantly enhanced exPBNK ADCC and IFN- $\gamma$ and perforin release with dinutuximab against $\mathrm{GD2}^{+} \mathrm{OS}, \mathrm{NB}$ and $\mathrm{GBM}$ cells. In vivo studies using NOD/SCID human solid tumor xenografts are under investigation.

\section{References}

1. $Y u$ AL, Gilman AL, et al. Anti-GD2 antibody with GM-CSF, interleukin-2, and isotretinoin for neuroblastoma. The New England journal of medicine 2010; 363:1324-34.

2. Xu W, Jones $\mathrm{M}$, et al. Efficacy and mechanism-of-action of a novel superagonist interleukin-15: interleukin-15 receptor alphaSu/Fc fusion complex in syngeneic murine models of multiple myeloma. Cancer Res 2013; 73:3075-86.

3. Chu Y, Hochberg J, et al. Targeting CD20+ Aggressive B-cell Non-Hodgkin Lymphoma by Anti-CD20 CAR mRNA-Modified Expanded Natural Killer Cells In Vitro and in NSG Mice. Cancer Immunol Res 2015; 3:333-44.

4. Denman CJ, Senyukov W, et al. Membrane-bound IL-21 promotes sus tained ex vivo proliferation of human natural killer cells. PLoS One 2012; 7:e3026

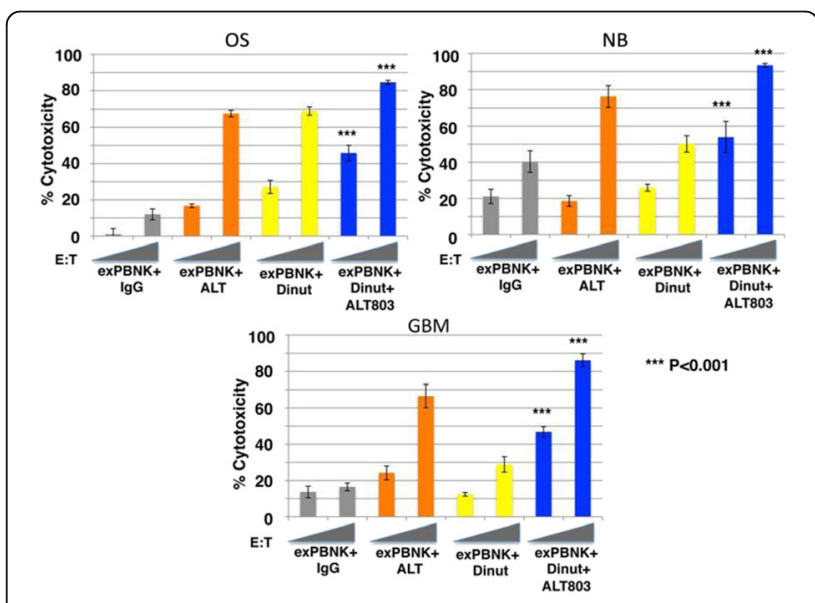

Fig. 1 (abstract P266). See text for description

P267

Withdrawn

P268

Targeting the tumor microenvironment with first-in-class Semaphorin4D MAb for combination immunotherapy

Elizabeth Evans' ${ }^{1}$, Holm Bussler ${ }^{1}$, Crystal Mallow', Christine Reilly',

Sebold Torno', Maria Scrivens', Cathie Foster', Alan Howell', Leslie

Balch', John E. Leonard', Terrence L. Fisher', David Jenkins², Clint Allen³

Paul Clavijo ${ }^{3}$, Siwen Hu-Lieskovann ${ }^{4}$, Antoni Ribas ${ }^{5}$, Ernest Smith',

Maurice Zauderer

${ }^{1}$ Vaccinex, Rochester, NY, USA; ${ }^{2}$ Tesaro, Waltham, MA, USA; ${ }^{3} \mathrm{NIH} / \mathrm{NIDCD}$, Bethesda, MD, USA; ${ }^{4}$ UCLA, Los Angeles, CA, USA; ${ }^{5}$ Vaccinex, Los

Angeles, CA, USA

Correspondence: Elizabeth Evans (eevans@vaccinex.com)

Journal for ImmunoTherapy of Cancer 2017, 5(Suppl 2):P268 


\section{Background}

Mechanistic findings in preclinical studies demonstrate that antibody blockade of Semaphorin 4D (SEMA4D, CD100) reduces expansion of MDSC and shifts the balance of immune cells within the TME to facilitate tumor rejection. Efficacy is further enhanced when combined with various immunotherapies.

Methods

Anti-SEMA4D antibodies were evaluated in combination with other immunotherapies in preclinical models. Anti-tumor activity and immune response was characterized by immunohistochemistry, flow cytometry, functional assays, and cytokine, chemokine and gene expression analysis. These data support clinical combination trials of VX15/2503, a humanized IgG4 antibody targeting SEMA4D, with immune checkpoint inhibition.

Results

SEMA4D restricts migration of monocytes and promotes expansion of suppressive myeloid cells in vitro. Strong expression of SEMA4D at the invasive margins of growing tumors in vivo restricts the infiltration and modulates polarization of leukocytes in the TME. Antibody blockade of SEMA4D in preclinical models facilitated recruitment of activated DCs and T lymphocytes with concurrent reduction in $\mathrm{M} 2$ macrophage and Treg within TME [1]. MDSCs were significantly reduced in tumor and blood following treatment and new data characterizing MDSC function will be described. This significant shift in the immune contexture is associated with durable tumor rejection and immunologic memory in murine colon, breast, HNSCC, and melanoma models. New translational data characterizing expression of SEMA4D and its receptors in human tumors will be shown. Importantly, anti-SEMA4D treatment can further enhance activity of immunotherapies and chemotherapy. For example, combinations with immune checkpoint inhibitor anti-LAG3 or anti-CTLA-4 cause complete tumor regression in $90 \%$ or $100 \%$ of mice, as compared to $\sim 20 \%$ with monotherapy $(p<0.01)$. New preclinical data include synergistic activity of combinations of anti-SEMA4D with anti-LAG3 and additional studies combining with epigenetic modulators, including treatment of established tumors.

\section{Conclusions}

SEMA4D blockade represents a novel mechanism to promote functional immune infiltration into the tumor and enhance immunotherapy. Treatment with VX15/2503 was well tolerated in a Phase I trial in patients with advanced refractory solid tumors [2]. Plans for several clinical trials will be presented, including a Phase $1 \mathrm{~b} / 2$ of combination therapy with avelumab in immunotherapy naïve NSCLC, and combination with anti-PD-1 or Ipilimumab in various indications. A neoadjuvant trial of VX15/2503 with anti-PD-1 in patients with metastatic colorectal and pancreatic cancers will be described, as well as introduction of a Phase 1/2 trial of VX15/2503 in pediatric and osteosarcoma patients.

Trial Registration

NCT01313065.

\section{References \\ 1. Evans, EE et al. Cancer Immunol Res. 2015 Jun;3(6):689-701. \\ 2. Patnaik A et al. Clin Cancer Res. 2016 Feb 15;22(4):827-36.}

\section{P269}

Epigenetic reprogramming of the tumor microenvironment increases tumor sensitivity to multivalent immunotherapy combinations with an IL-15 superagonist plus vaccine or immune checkpoint blockade

Kristin C. Hicks ${ }^{1}$, Karin M. Knudson ${ }^{1}$, Anthony S. Malamas', Frank R. Jones ${ }^{2}$, Peter Ordentlich ${ }^{3}$, Shahrooz Rabizadeh ${ }^{4}$, Hing C. Wong ${ }^{5}$, James W. Hodge', Jeffrey Schlom?', Sofia R. Gameiro

${ }^{1}$ National Cancer Institute, National Institutes of Health, Bethesda, MD, USA; ${ }^{2}$ Etubics Corporation, Seattle, WA, USA; ${ }^{3}$ Syndax Pharmaceuticals, Inc., Waltham, MA, USA; ${ }^{4}$ NantCell, LLC, Culver City, CA, USA; ${ }^{5}$ Altor BioScience Corporation, Miramar, FL, USA

Journal for ImmunoTherapy of Cancer 2017, 5(Suppl 2):P269

\section{Background}

The clinical promise of cancer immunotherapy relies on the immune system recognizing and eliminating tumor cells identified as non-self. However, solid malignancies evade host immune surveillance by multiple mechanisms, including epigenetic deregulation and promoting a tumor microenvironment (TME) that suppresses infiltration and function of immune effector cells. The TME hampers T and NK cell maturation, recruitment, and function, ultimately causing their functional exhaustion via numerous immunosuppressive pathways, including upregulation of immune checkpoints such as PD-L1. Epigenetic silencing of genes involved in antigen processing and tumor immune recognition has been associated with worse prognosis in a wide spectrum of malignancies. Hence, there is an unmet clinical need to develop effective therapeutic strategies that can reprogram the TME to restore tumor immune recognition and reverse immune evasion. We recently demonstrated that entinostat, a class I histone deacetylase (HDAC) inhibitor reverses carcinoma immune escape to $T$ cellmediated lysis.

Methods

We hypothesize that the immune-mediated tumor elimination promoted by the IL-15/IL-15Ra superagonist ALT803 in combination with PD-L1 checkpoint blockade or a therapeutic adenoviral vaccine targeting CEA (Ad-CEA) will be augmented by the epigenetic reprograming of the TME induced by entinostat.

Results

In preclinical studies, ALT803 has been shown to exhibit potent antitumor activity in multiple murine models of cancer through the expansion of NK and CD8+ T cells with high effector function. Here, we demonstrate that entinostat modulates the cell-surface phenotype of murine colon and breast carcinoma cells to become more amenable to immune-mediated elimination. In the MC38-CEA murine model of colon carcinoma, proper scheduling of entinostat administration significantly augmented the antitumor activity promoted by ALT803 plus Ad-CEA, resulting in increased survival. Further, in the 4T1 murine model of triple-negative breast cancer, the combination of entinostat with ALT803 and a monoclonal antibody (mAb) targeting PDL1 significantly reduced primary tumor weight relative to ALT803 plus anti-PD-L1 therapy. Treatment with the triple therapy in the neoadjuvant setting resulted in significant reduction of the number of $4 \mathrm{~T} 1$ tumor-forming cells in the lung, with over $85 \%$ of animals cured. Current studies aim to elucidate the immune mechanisms associated with the antitumor effects observed with these therapeutic interventions.

Conclusions

Overall, these studies examine the rationale for combining entinostat with multivalent immunotherapy combinations, including cytokines, mAbs targeting PD-L1, and therapeutic cancer vaccines.

P270

Simultaneous PD-1 blockade is detrimental to the anti-tumor effects mediated by the agonist $0 \times 40$ antibody

Seema Gupta ${ }^{1}$, Rajeev Shrimali ${ }^{2}$, Shamim Ahmad $^{3}$. Vivek Verma ${ }^{1}$ Peng Zeng', Sudha Ananth', Pankaj Gaur ${ }^{1}$, Rachel Gittelman ${ }^{4}$ Erik Yusko ${ }^{4}$, Catherine Sanders ${ }^{4}$, Harlan Robins ${ }^{4}$, Scott Hammond ${ }^{5}$ John Janik', Mikayel Mkrtichyan ${ }^{3}$, Samir Khleif

${ }^{1}$ Georgia Cancer Center, Augusta University, Augusta, GA, USA; ${ }^{2}$ Georgia

Cancer Center, Augusta University, Dallas, TX, USA; ${ }^{3}$ Georgia Cancer Center, Augusta University, South San Francisco, CA, USA; ${ }^{4}$ Adaptive Biotechnologies, Seattle, WA, USA; ${ }^{5}$ Medlmmune LLC, Gaithersburg, MD, USA

Correspondence: Samir Khleif (segupta@augusta.edu)

Journal for ImmunoTherapy of Cancer 2017, 5(Suppl 2):P270

\section{Background}

The checkpoint inhibitor antibodies (Abs) have improved the antitumor response although in a limited number of patients [1]. The combination of these Abs has enhanced the efficacy but with increased adverse events [1]. Since immune-stimulatory agonist Abs 
like anti-OX40 significantly increase the immune response [2], their combination with the anti-PD-1 Abs is being tested in clinical trials [3] with at least one clinical trial demonstrating lack of benefit [4] and the rest are not yet reported. Moreover, the potential immune outcome of such a combination are currently lacking. Therefore, purpose of this study was to determine the therapeutic and immune outcome of combining anti-PD-1 with anti-OX40 in an immuneprimed environment.

Methods

Effects of adding PD-1 blockade to anti-OX40/vaccine treatment on tumor growth and survival were evaluated in a TC-1 tumor mouse model using different treatment schedules and immune responses were assessed. In vitro mechanistic studies were carried out in pMel$1 \mathrm{CD}^{+} \mathrm{T}$-cells.

Results

Anti-OX40 treatment resulted in significant enhancement of antigenspecific $\mathrm{CD} 8^{+} \mathrm{T}$-cells tumor-infiltration leading to strong anti-tumor response and prolonged survival of mice. Interestingly, we found that simultaneous addition of anti-PD- 1 to anti-OX40 completely abrogated these effects. Despite an increase in IFNY-producing E7-specifc $\mathrm{CD}^{+} \mathrm{T}$-cells in the spleens of mice treated with the combination, these cells underwent significant apoptosis in both the periphery and the tumor. Consistent with increased apoptosis, immunoSequencing analysis of the tumor and spleen T-cell population showed reduction in T-cell fraction and clonality following the addition of antiPD-1. We further showed that delay in anti-PD-1 addition resulted in no significant change in T-cell apoptosis, however, it did not add to the anti-tumor response of the anti-OX40 treatment.

\section{Conclusions}

These results indicate that anti-PD- 1 added at the initiation of therapy exhibits a detrimental effect on the positive outcome of antiOX40. This may provide an important insight into why some of the immune combination clinical trials are not producing the intended outcome, demonstrating the need to rigorously test the combination partners and sequencing before employing them in the clinic.

\section{References}

1. Page DB, et al. Immune modulation in cancer with antibodies. Annu Rev Med. 2014;65:185-202.

2. Linch SN et al: OX40 Agonists and Combination Immunotherapy: Putting the Pedal to the Metal. Front Oncol. 2015;5:34.

3. "ClinicalTrials.gov."April 15, 2017 from https://clinicaltrials.gov/ct2/ results?term $=0 \times 40 \& a m p ; S e a r c h=S e a r c h ">h t t p s: / /$ linicaltrials.gov/ct2/ results?term=0x40\&amp;Search $=$ Search.

4. Infante, et al. A phase $\mathrm{Ib}$ dose escalation study of the OX40 agonist MOXR0916 and the PD-L1 inhibitor atezolizumab in patients with advanced solid tumors. Presentation in ASCO Annual Meeting, 2016.

\section{P271}

Phase $1 \mathrm{~b} / 2$ dose-escalation study of ARRY-382, an oral inhibitor of colony-stimulating factor-1 receptor (CSF1R), in combination with pembrolizumab for treatment of patients with advanced solid tumors

Wael A. Harb¹, Melissa L. Johnson² Jonathan W. Goldman ${ }^{3}$, Amy Weise ${ }^{4}$, Justin A. Call, Arkadiusz Z. Dudek ${ }^{6}$, Rene Gonzalez ${ }^{7}$, C. Lance Cowey ${ }^{8}$, Sybil Zildjian", Kati Maharry ${ }^{9}$, Ashwin Gollerkeri ${ }^{9}$, Justin F. Gainor ${ }^{10}$ ${ }^{1}$ Horizon Oncology Research, Inc., Lafayette, IN, USA; ${ }^{2}$ Sarah Cannon Research Institute, Nashville, TN, USA; ${ }^{3}$ UCLA Medical Center, Santa Monica, CA, USA; ${ }^{4}$ Karmanos Cancer Institute, Detroit, MI, USA; ${ }^{5}$ Utah Cancer Specialists, Salt Lake City, UT, USA; ${ }^{\circ}$ Regions Cancer Care Center, St. Paul, MN, USA; ${ }^{7}$ University of Colorado Cancer Center, Aurora, CO, USA; ${ }^{8}$ Baylor Health Care System, Dallas, TX, USA; ${ }^{9}$ Array BioPharma Inc., Boulder, CO, USA; ${ }^{10}$ Massachusetts General Hospital, Boston, MA, USA

Correspondence: Wael A. Harb (drharb@me.com)

Journal for ImmunoTherapy of Cancer 2017, 5(Suppl 2):P271

\section{Background}

CSF1 regulates tumor-associated macrophages and myeloid-derived suppressor cells, which are critical tumor microenvironment modulators of immune response. Combining a programmed death 1 (PD-1) inhibitor with a CSF1R inhibitor in preclinical models shows enhanced antitumor activity. ARRY-382 is a highly selective oral inhibitor of CSF1R. Studies of ARRY-382 monotherapy identified the maximum tolerated dose (MTD) as $400 \mathrm{mg}$ once daily (QD), with biologic activity at doses $\geq 200 \mathrm{mg}$ QD. This 3-part, phase $1 \mathrm{~b} / 2$ study evaluates ARRY-382 in combination with pembrolizumab, a humanized monoclonal antibody targeting PD-1.

Methods

Phase $1 \mathrm{~b}$ (part A) determined the MTD and recommended phase 2 dose (RP2D) of ARRY-382 plus pembrolizumab in patients with selected advanced solid tumors. Patients were enrolled in 3 successive cohorts of ARRY-382 at doses of 200,400 , or $300 \mathrm{mg}$ QD plus pembrolizumab $2 \mathrm{mg} / \mathrm{kg}$ every 3 weeks (Q3W). The primary endpoint was the incidence of dose-limiting toxicities (DLTs). Secondary endpoints included safety, pharmacokinetics of ARRY-382, and objective response rate.

Results

Twenty patients enrolled in part A; 19 were treated: $200 \mathrm{mg} Q D, n=6$; $400 \mathrm{mg}$ QD, $n=7 ; 300 \mathrm{mg}$ QD, $n=6$. Median age was 59 years; tumor types were pancreatic $(n=6)$, colorectal $(n=5)$, ovarian $(n=3)$, gastric and melanoma ( $n=2$ each), and triple-negative breast $(n=1)$. The mean number of prior therapies was 2.9 (range, 1-5). DLTs of grade 3 increased aspartate aminotransferase (AST)/alanine aminotransferase/bilirubin and grade 3 increased creatine phosphokinase $(n=1$ each, 400-mg dose) and grade 3 pancreatitis ( $n=1,300-\mathrm{mg}$ dose) were observed. The most common ( $>2$ patients across doses) grade $3 / 4$ adverse events (AEs) by dose cohort (200,300, and $400 \mathrm{mg}$ ) were increased AST (1, 1, and 2 patients), increased lipase $(0,3,0)$, and rash $(1,1,1)$, respectively. One patient in the 200-mg cohort discontinued treatment because of an AE of pneumonitis in cycle 5. ARRY382 pharmacokinetics was unaffected by pembrolizumab. Preliminary efficacy data will be presented.

Conclusions

ARRY-382 plus pembrolizumab has a manageable safety profile. The RP2D of ARRY-382 plus pembrolizumab $2 \mathrm{mg} / \mathrm{kg}$ Q3W is $300 \mathrm{mg}$ QD. This combination is currently being evaluated in the study in patients with melanoma and non-small-cell lung cancer.

Trial Registration

ClinicalTrials.gov: NCT02880371.

\section{P272}

The anti-tumor effect of radiation therapy is enhanced with the addition of TTI-621 (SIRPaFc), an immune checkpoint inhibitor blocking the CD47 "do not eat" signal

Lei Cui, Hui Chen, Alison O'Connor, Debbie Jin, Jeffrey Winston,

Robert Uger, Lisa Johnson

Therapeutics Inc., Mississauga, ON, Canada

Correspondence: Lisa Johnson (lisa@trilliumtherapeutics.com) Journal for ImmunoTherapy of Cancer 2017, 5(Suppl 2):P272

\section{Background}

CD47 is an immune checkpoint that binds to signal regulatory protein alpha (SIRPa) and delivers a "do not eat" signal to suppress macrophage phagocytosis. Tumor cells frequently overexpress CD47 to evade macrophage-mediated destruction. TTI-621 (SIRPaFc) is an immune checkpoint inhibitor consisting of the CD47 binding domain of human SIRPa linked to the Fc region of human IgG1 designed to both: 1) block the CD47 "do not eat" signal, and 2) engage macrophage Fcy receptors with IgG1 Fc to enhance phagocytosis and antitumor activity. Radiation therapy (RT), a primary mode of cancer treatment, induces immunogenic cell death, and is associated with release of tumor 
antigens, induction of pro-inflammatory cytokines, and enhanced migration and infiltration of immune cells, including macrophages, to tumor sites. Thus, the combination of RT and TTI-621 may improve the therapeutic effect over either treatment modality alone. Herein, we report the efficacy of the combination of RT and the CD47blocking agent TTI-621 in xenograft tumor models.

Methods

The in vivo efficacy of RT, TTI-621, and RT+TTI-621 was evaluated in B cell lymphoma (SU-DHL-6) and solid tumor xenografts, including the radio-insensitive A549 lung adenocarcinoma. TTI-621 (10 mg/kg) or vehicle were administered intratumorally 30 min prior to RT, 3 times per week for 4 weeks. Tumors were locally irradiated using an imageguided small animal irradiator ( $225 \mathrm{kVp}, 13 \mathrm{~mA}$ ) at a dose of 4-6 Gy for 3 fractions. Tumor volumes were monitored using standard caliper measurement. Systemic toxicity of the treatments was evaluated by body weight change. Tumor-associated macrophages were quantitatively assessed using flow cytometry and immunohistochemistry.

Results

RT+TTI-621 had a more profound effect on tumor control in both lymphoma and solid tumor models than RT alone. In SU-DHL-6, 88\% (7/8) of mice treated with both TTI-621 and radiation were tumorfree at the end of the study whereas none were tumor-free when treated with RT or TTI-621 alone, although there was tumor growth delay with each individual therapy compared to vehicle. RT+TTI-621 led to an increased infiltration of macrophages at the tumor sites in SU-DHL-6. Significant tumor control was also observed in A549 tumor bearing mice treated with RT+TTI-621 compared to either treatment alone. No toxicity was observed for any of the treatments.

Conclusions

The current study demonstrates the combination of TTI-621 and radiation therapy is superior to either treatment alone and provides supportive evidence for dual modality therapy in a variety of tumors.

\section{P273}

Pembrolizumab and afatinib for recurrent or metastatic head and neck squamous cell carcinoma

Hsiang-Fong Kao, Huai-Cheng Huang, Bin-Chi Liao, Ruey-Long Hong National Taiwan University Hospital, Taipei, Taiwan

Correspondence: Hsiang-Fong Kao (hfkao.tw@gmail.com); Ruey-Long Hong

Journal for ImmunoTherapy of Cancer 2017, 5(Suppl 2):P273

\section{Background}

Head and neck squamous cell carcinoma (HNSCC) is an important malignancy in Taiwan. Anti-PD-1, including nivolumab or pembrolizumab, have shown the efficacies against head and neck squamous cell carcinoma. Afatinib, an irreversible EGFR tyrosine kinase inhibitor (TKI), had showed its activity against HNSCC. The role of afatinib for cancer immunotherapy have not been explored. In animal model, afatinib can suppress the carcinogenesis by inhibiting the function of macrophage [1]. EGFR targeted therapy can increase the MHC expression, enhance dendritic cell function, and increase the infiltrating T cell in the tumor [2]. In syngeneic mice cancer models, EGFR TKI can suppress the glycosylation of PD-L1 and sensitize the mice to anti-PD-1 therapy [3]. We hypothesized that adding afatinib with pembrolizumab may improve the treatment efficacy for patients with recurrent or metastatic HNSCC.

\section{Methods}

Patients with locally advanced or metastatic HNSCC were retrospectively reviewed in a university hospital in Taiwan. For patients taking pembrolizumab, the combination with afatinib will be discussed between the physician and the patient. For patients taking pembrolizumab and afatinib $(P+A)$, the medical records were reviewed.

Results

From Nov 2016 to Jul 2017, there were 35 patients taking P+A. The median age was 59 years, and 32/35 pts were men. Oral cavity cancer is the most common type of HNSCC in the cohort (29/35 pts), The treatment were: pembrolizumab 200mg: $30 \mathrm{pts} ; 2 \mathrm{mg} / \mathrm{kg}$ : $5 \mathrm{pts}$; afatinib 40mg QD: 34pts; 30mg QD: 1 pt. The maximal cycles of pembrolizumab per patient is four cycles. Sixteen patients took $P+A$ as first line therapy. Until Jul 30, 2017, 29 pts had evaluable outcomes. The ORR (CR+PR) is $48.2 \%(14 / 29)$, DCR (CR+PR+SD) was $69.0 \%(20 /$ 29), and the median PFS: 184 days (32-332). The common toxicities were: diarrhea $40 \%$; skin rash 37\%; mucositis 29\%; hand-foot-skin reaction 23\%; weight loss 17\%; and elevated AST/ALT 9\%. Grade >= 3 toxicities happened in $8.6 \%$ patients. There was no treatment related pneumonitis in the cohort.

Conclusions

The addition of afatinib with pembrolizumab showed good efficacy (ORR: $48.2 \%$, DCR: $69.0 \%$, PFS: 6.0 months) and tolerable toxicities. Further confirmatory prospective trial is indicated.

References

1. Lanaya, Hanane, et al. EGFR has a tumor-promoting role in liver macrophages during hepatocellular carcinoma formation. Nature cell biology. 2014; 16.10:972

2. Concha-Benavente, Fernando, and Robert L. Ferris. Oncogenic growth factor signaling mediating tumor escape from cellular immunity. Current Opinion in Immunology. 2017:45:52-59.

3. Li, Chia-Wei, et al. Glycosylation and stabilization of programmed death ligand-1 suppresses T-cell activity. Nature Communication. 2016;7:12632.

P274

Combination of NKTR-214 and radiotherapy (RT) to reverse anergy and expand tumor-specific CD8 T Cells

Joshua Walker', Melissa Kasiewicz'ㄹ, Michael McNamara², lan Hilgart-Martiszus², Ute Hock ${ }^{3}$, Deborah Charych ${ }^{3}$, William Redmond ${ }^{2}$

${ }^{1}$ Oregon Health \& Science University, Portland, OR, USA; ${ }^{2}$ Providence

Portland Medical Center, Portland, OR, USA; ${ }^{3}$ Nektar Therapeutics, San Francisco, CA, USA

Correspondence: William Redmond (melissa.kasiewicz@providence.org) Journal for ImmunoTherapy of Cancer 2017, 5(Suppl 2):P274

\section{Background}

We investigated therapeutic and mechanistic synergy between single-dose RT and systemic administration of NKTR-214.The effects of NKTR-214 with and without RT on anergic, tumor-specific CD8 T cells were characterized. NKTR-214 is a CD122-biased cytokine agonist conjugated with releasable chains of polyethylene glycol. NKTR214 provides sustained signaling through the IL2 receptor pathway (IL2RßY) to preferentially activate and expand effector CD8 T and NK cells over regulatory T cells. Preclinical models demonstrated NKTR214 preferentially expands effector CD8 T and NK cells in the tumor resulting in marked tumor growth suppression as a single-agent and in combination with checkpoint inhibitors. RT can induce antigenrelease and epitope spreading, while NKTR-214 activates and expands antigen-specific effector populations. We hypothesized the combination of systemic NKTR-214 and RT would generate better therapeutic responses than either treatment alone. A phase $\mathrm{I} / \mathrm{Il}$ trial is in progress to evaluate NKTR-214 safety and efficacy in an outpatient setting as monotherapy and in combination with nivolumab.

Methods

NKTR-214 was dosed $0.8 \mathrm{mg} / \mathrm{kg}$ alone or together with high-dose RT (20 Gy x 1) in multiple murine models, including an established CD8 T cell anergy model using Nur77-GFP reporter CD8 T cells. Activation markers on CD4, CD8 and NK cells in blood, lymph and tumor were evaluated by flow cytometry and gene expression (mRNA) profiling of the irradiated and non-irradiated tumors. In addition, immunohistochemistry was utilized to visualize immune infiltration.

Results

Consistent with prior observations, NKTR-214 induced activation marker expression by CD4, CD8 T and NK cells in the blood, lymph nodes and tumor. The combination of RT and NKTR-214 resulted in unique effects including increased absolute lymphocyte counts, increased expression of CD8 activation markers (CD25, PD1) in the blood and tumor, increased intratumoral NK cells, and a significant survival benefit over monotherapy. Evaluation of tumor infiltrating lymphocytes (TIL) indicates combination therapy reverses tumorassociated T cell anergy and results in a higher frequency of recently activated CD8 TIL. 


\section{Conclusions}

The combination of NKTR-214 and radiotherapy is synergistic, provides significantly better anti-tumor responses and reverses T cell anergy.

\section{P275}

Harnessing the innate and adaptive immune system to eradicate treated and distant untreted solid tumors

Saul Kivimae, Werner Rubas, Rhoneil Pena, Marlene Hennessy,

Yolanda Kirksey, Wildaliz Nieves, Myong Lee, Clive Law, Kavitha Bhasi,

Phi Quach, Janet Cetz, John L. Langowski, Christie Fanton,

Jode Zandro Aquino, Zhongxu Ren, Haiying Cai, BoLiang Deng,

Wen Zhang, Neel K. Anand, Jennifer Riggs-Sauthier, Steve Doberstein, Jonathan Zalevsky

Nektar Therapeutics, San Francisco, CA, USA

Correspondence: Saul Kivimae (skivimae@nektar.com); Jonathan

Zalevsky

Journal for ImmunoTherapy of Cancer 2017, 5(Suppl 2):P275

\section{Background}

NKTR-262 is a novel therapeutic, which delivers sustained intratumoral engagement of the TLR 7/8 pathway, promoting an immune stimulatory environment and tumor antigen release. When NKTR-262 is administered in combination with NKTR-214, a CD122-biased cytokine agonist currently in clinical trials as a monotherapy and in combination with nivolumab, the combined effect of innate immune stimulation and enhanced antigen presentation with sustained $\mathrm{T}$ cell activation leads to systemic tumor immunity.

Methods

Balb/c mice were implanted s.c. with bilateral CT26 colon carcinoma tumors. Once established, one tumor was treated with a single peritumoral dose of NKTR-262, while NKTR-214 was administered i.v. on q9dx3 schedule. Regression of treated tumors and the abscopal effect in contralateral tumors was assessed by tumor size measurements. Immune cell activation in both tumors was assessed by flow cytometry. Cytokine induction was measured in plasma, blood cells and treated tumors by MSD and qRT-PCR.

Results

Combination treatment with NKTR-262 and NKTR-214 eliminated both tumors in up to $\geq 95 \%$ of mice. Single agent NKTR-262 or NKTR214 treatment led to complete responses in $\leq 30 \%$ of mice. Combination treatment of NKTR-262 plus NKTR-214 induced a two-step immune response in treated and untreated tumors. At early timepoints, accumulation of activated neutrophils correlated with tumor cell death and dendritic cell activation. The innate response was followed by increased CD8+ T cells and a reduction of immunorepressive cells. Single agent treatment showed only a subset of the cellular changes observed in the combination.

\section{Conclusions}

We present a designed combination therapy that mimics a natural immune response by activating a broad immune cell network. Combining NKTR-262 and NKTR-214 engages the entire immune activation cascade required for systemic tumor clearance from local tumor antigen production to a sustained systemic $T$ cell response. Unlike treatments that stimulate downstream components of select immune pathways without eliciting systemic tumor immunity, a comprehensive anti-tumor immune activation by coordinated engagement of innate and adaptive immune cells may increase the success of immune therapy for patients

\section{P276}

Immunoswitch particles target activation of anti-tumor CD8+ T cells to inhibit tumor growth

Alyssa Kosmides, John-William Sidhom, Andrew Fraser, Catherine Bessell, Jonathan Schneck

Johns Hopkins School of Medicine, Baltimore, MD, USA

Correspondence: Alyssa Kosmides (akosmides@gmail.com)

Journal for ImmunoTherapy of Cancer 2017, 5(Suppl 2):P276

\section{Background}

Recent advances in cancer immunotherapy have led to the advent of treatments that boost an anti-tumor response, such as checkpoint blockade and co-stimulatory molecules. However, these approaches are effective for only a subset of patients and target non-specific molecules, thus the large doses required result in off-target toxicities. Here, we have developed a nanoparticle that synergizes checkpoint blockade and $\mathrm{T}$ cell costimulation on a single injectable therapeutic. Nanoparticles are conjugated with antibodies against 4-1BB, expressed by T cells, and anti-PD-L1, expressed by tumor cells and target these pathways while also physically linking the effector and target cell to increase efficacy. These dual targeting particles, termed "immunoswitch particles", localize treatment to the tumor site and are effective at 10-100X lower doses than systemically injected drug and thus have the potential to reduce cost and off-target toxicities.

Methods

Immunoswitch particles were synthesized by conjugating $80 \mathrm{~nm}$ irondextran nanoparticles with agonistic anti-4-1BB and antagonistic antiPD-L1 antibodies. In vitro efficacy was assessed by co-culturing PD-L $1^{\text {hi }}$ tumor cells with 4-1BB/PD-1 ${ }^{\text {hi }}$ CD8+ T cells and measuring cytotoxicity, cytokine secretion, and effector-target cell conjugation. In vivo efficacy was measured by comparing intratumoral immunoswitch and soluble antibody treatment in multiple murine tumor models.

Results

Immunoswitch particles were demonstrated to increase CD8+ T cell cytokine secretion when co-incubated with T cells and cognate B16 tumor cells compared to an equivalent amount of soluble antibody in vitro. Immunoswitch particles also increased effector-target cell conjugation both in vitro (Fig. 1) and in vivo. In vivo, immunoswitch treatment, but not an equivalent amount of soluble antibody, delayed/reversed tumor growth in both a B16-SIY and MC38-OVA tumor treatment model in the absence of adoptively transferred cells (Fig. 2). This was caused by a change in biodistribution, altered CD8+ $T$ cell repertoire, and an increase in cytokine secretion by tumorspecific CD8+ T cells (Fig. 3A). Despite local intra-tumoral treatment, new data shows that MC38-OVA cured mice have systemic immunity against the OVA antigen. These mice have a systemically circulating repertoire of tumor-specific cells, demonstrated by increased in vivo killing of intravenously injected OVA-expressing cells (Fig. 3B) and protection against a re-inoculation of MC38-OVA.

Conclusions

Here, we have demonstrated the efficacy of a new dual-targeting immunoswitch particle for cancer immunotherapy. Immunoswitch particles synergize checkpoint blockade with co-stimulation by confining the antibodies to a rigid nanoparticle surface. This new approach can be extended to different disease models and new checkpoint or co-stimulatory target molecules.

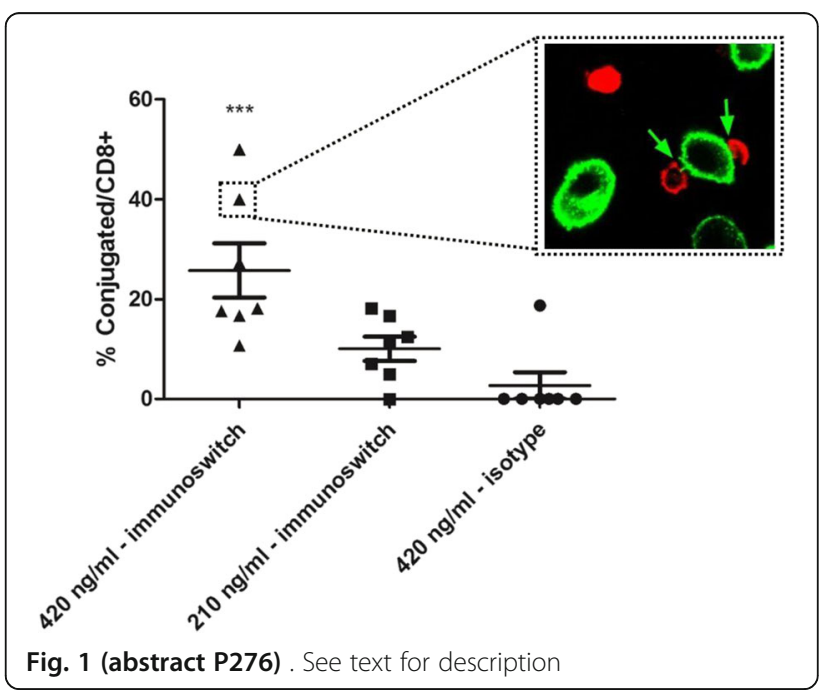




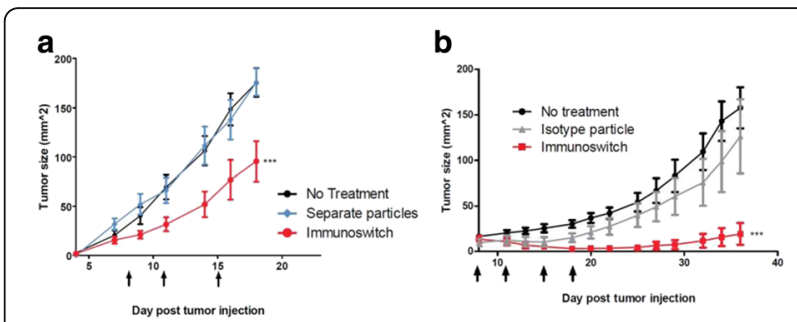

Fig. 2 (abstract P276). See text for description

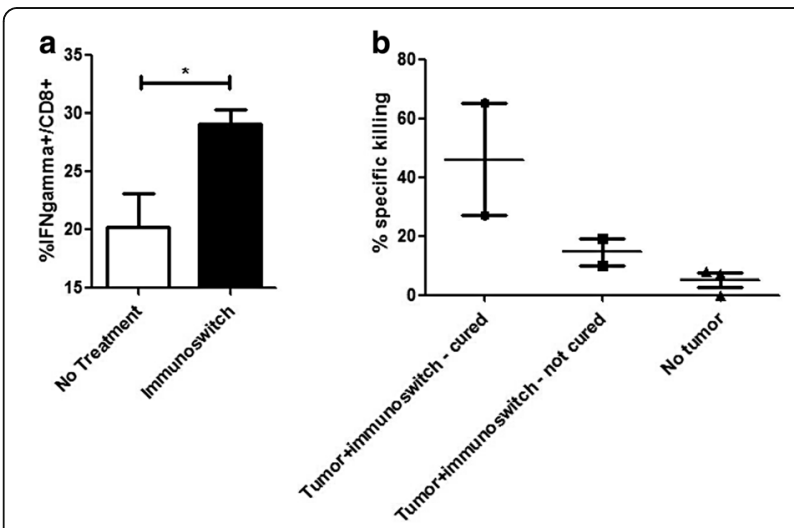

Fig. 3 (abstract P276). See text for description

\section{P277}

STING agonist treatment increases response to chemotherapy and immune checkpoint blockade therapy in a syngeneic murine model of high-grade serous ovarian cancer

Abdi Ghaffari', Nichole Peterson', Kasra Khalaj', Andrew Robinson², Julie-Ann Francis ${ }^{2}$, Madhuri Koti'

${ }^{1}$ Queen's University, Kingston, ON, Canada; ${ }^{2}$ Kingston Health Sciences Center, Kingston, ON, Canada

Correspondence: Madhuri Koti (kotim@queensu.ca)

Journal for ImmunoTherapy of Cancer 2017, 5(Suppl 2):P277

\section{Background}

Background: High Grade Serous Carcinoma of the ovary is mostly diagnosed at late stages and primarily treated with surgery followed by platinum/taxane chemotherapy. Unfortunately, majority of the patients exhibit resistance to chemotherapy and ultimately succumb to the disease. We previously reported that tumours from patients with early recurrence show an immunosuppressed pre-existing tumour immune microenvironment with decreased expression of genes involved in Type I Interferon (IFN1) and T helper type 1 response [1, 2, 3]. We thus hypothesized that response to chemotherapy and overall survival of HGSC patients can be improved by stimulating the IFN1 response in the TME post chemotherapy.

\section{Methods}

In this pre-clinical study we tested the efficacy of a novel "Stimulator of Interferon Genes" agonist in immunocompetent mice implanted with ID8-TRP5 $3^{-/-}$mouse ovarian cancer cells. Post treatment tumour immune cell profiles were measured using a combination of flow cytometry and CyTOF based profiling. Tumour immune transcriptomic alterations were measured using the NanoString mouse pan cancer immune profiling panel. Log-rank test based survival analysis was performed to determine significant differences in overall survival post treatment.
Results

Flow cytometry and NanoString based analysis of tumours collected at endpoint showed higher intra-tumoural PD- $1^{+}$and $\mathrm{CD}^{+} 9^{+} \mathrm{CD} 62 \mathrm{~L}^{-}$, CD8 ${ }^{+} \mathrm{T}$ cells, increased expression of IFN response, antigen presentation and MHCll, genes, respectively, in tumours from STING agonist treated mice compared to those from vehicle treated mice. In addition to significantly decreased ascites accumulation and decreased tumour burden, survival of mice treated with a combination of Carboplatin + STING agonist + anti-PD-1 therapy was significantly longer compared to Carboplatin + STING agonist, Carboplatin only, STING only and vehicle treated mice.

Conclusions

Findings from this study are foundational to future clinical trials aimed at combinatorial immunomodulatory therapies to improve chemotherapy response and overall survival of HGSC patients.

\section{References}

1. Koti $M$, SiuA, Clément I, Bidarimath $M$, Turashvili G, EdwardsA, et al. A distinct pre-existing inflammatory tumour microenvironment is associated with chemotherapy resistance in high-grade serous epithelial ovarian cancer. Br J Cancer [Internet]. 2015;112(7):1215-22.

2. Au KK, Le Page C, Ren R, Meunier L, Clément I, Tryshkin K, et al. STAT1associated intratumoural $T_{H} 1$ immunity predicts chemotherapy resistance in high-grade serous ovarian cancer. J Pathol Clin Res. 2016.

3. Au KK, Peterson N, Truesdell P, Reid-schachter G, Khalaj K, Ren R, et al. Gynecologic Oncology CXCL10 alters the tumour immune microenvironment and disease progression in a syngeneic murine model of highgrade serous ovarian cancer. Gynecol Oncol [Internet]. Elsevier Inc.; 2017;4-13.P278.

P278

A Rational Combination of Standard of Care and Immunotherapy Increases Survival Against Glioblastoma

Erik Ladomersky, Derek Wainwright

Northwestern University, Chicago, IL, USA

Correspondence: Derek Wainwright

(erik.ladomersky@northwestern.edu)

Journal for ImmunoTherapy of Cancer 2017, 5(Suppl 2):P278

\section{Background}

Glioblastoma (GBM) is a fatal primary brain tumor that, even after maximum surgical resection, radiotherapy (RT) and chemotherapy, is associated with a median overall survival (OS) of 14.6 months. Combinatorial treatment approaches that simultaneously address tumor growth, as well as the immunosuppressive microenvironment, may prove to be more effective than current clinical strategies. This work aims to determine the therapeutic efficacy of combining the novel, pharmaceutical-grade IDO1 enzyme inhibitor, BGB-5777, with PD-1 blockade and/or whole brain radiation, in a syngeneic, immunocompetent mouse glioblastoma model. Methods

Mice were intracranially-engrafted with $2 \times 10^{5} \mathrm{GL} 261$ or CT-2A murine GBM cells, then treated, beginning day 14 post-tumor cell implantation, with $500 \mu \mathrm{g}$ loading dose IgG control $\mathrm{mAb}$ followed by three $200 \mu \mathrm{g}$ doses, administered every third day $(n=7)$, or 2 Gy WBRT for 5 days, PD-1 mAb (J43) administered the same as IgG control mAb, and $100 \mathrm{mg} / \mathrm{kg} \mathrm{BGB}-5777$ BID for 4 weeks $(n=9)$.

\section{Results}

Mice engrafted with the GL261 tumors and treated with the combination therapy showed a significant increase in overall survival (53 days) compared to lgG treated mice ( 25 days). This therapy regimen also significantly increased durable survival $(>120$ days $)(P<0.0001)$ in mice ic. unmodified GL261 or CT-2A cells. (Fig. 1A) Paradoxically, mice with GBM cells overexpressing mouse IDO1 CDNA and treated with triple therapy showed a similar proportion of durable survivors (40\%) vs. mice with similar tumors treated with IgG control $(P<0.0001)$. (Fig. 1B) Interestingly, the survival effects seen in engrafted with unmodified GL261 tumors correlated to a significant decrease in GBM-infiltrating Treg levels $(P<0.01)$, however this same effect was not seen in mice with GL261 mIDO1 O/E tumors. (Fig. 1C) These results suggest that effective- 
ness of triple therapy is independent of tumor IDO1 expression level and Treg accumulation. Results from the analysis of WT and IDO1 ${ }^{-1-}$ mice with intracranial tumor indicate that in the presence of nontumor IDO1, IDO1 inhibition is necessary to confer survival benefit $(P<0.01)$. (Fig. 2)

\section{Conclusions}

Ultimately, the data suggest that using radiation to induce potential immunogenicity and/or inflammation in GBM, while co-inhibiting immunosuppression, is a rational and potentially clinically-beneficial pursuit.

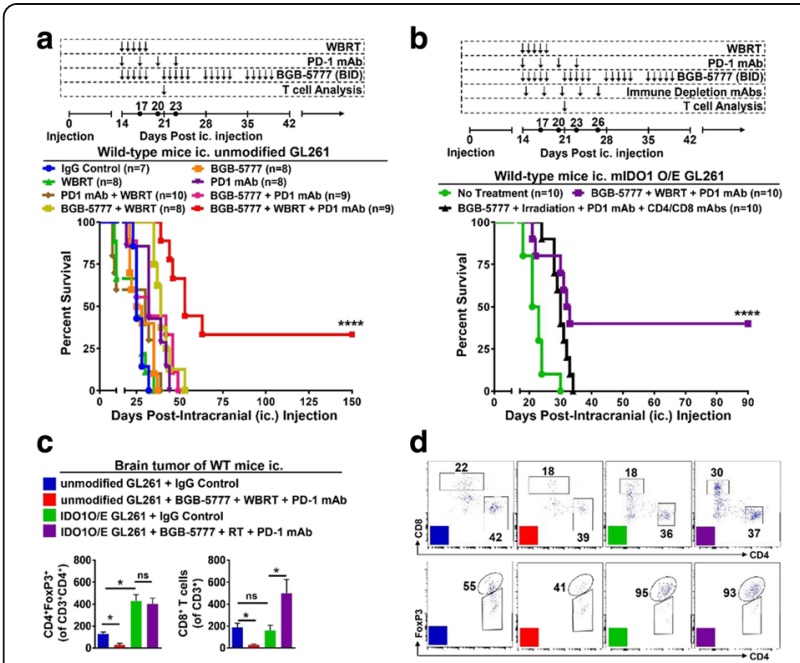

Fig. 1 (abstract P278). See text for description

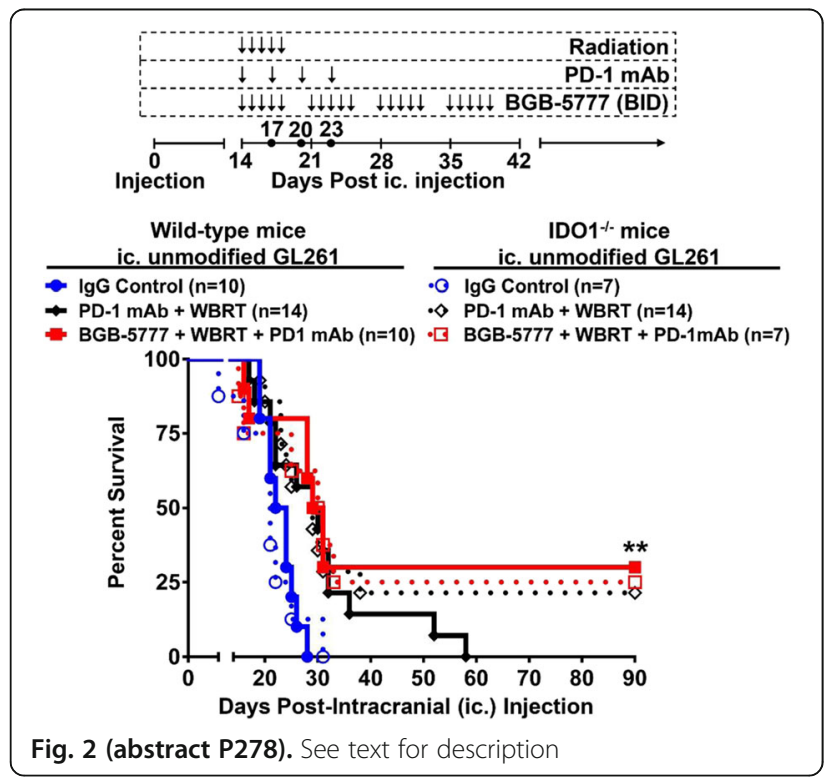

P279

An oral small molecule combination therapy targeting PD-L1,

VISTA and Tim-3 immune inhibitory checkpoints exhibits enhanced anti-tumor efficacy in pre-clinical models of cancer

Adam Lazorchak', Troy Patterson', Yueyun Ding ', Pottayil G. Sasikumar ${ }^{2}$, Naremaddepalli S. Sudarshan², Nagaraj M. Gowda², Raghuveer K.

Ramachandra ${ }^{2}$, Dodheri S. Samiulla ${ }^{2}$, Mohammed Rafi ${ }^{2}$, Nagesh Gowda², Sreenivas Adurthi ${ }^{2}$, Jiju Mani ${ }^{2}$, Rashmi Nair², Murali Ramachandra², David Tuck', Timothy Wyant ${ }^{1}$

${ }^{1}$ Curis, Inc., Lexington, MA, USA; ${ }^{2}$ Aurigene Discovery Technologies Limited, Bengaluru, India

Correspondence:Adam Lazorchak (adam.lazorchak@icloud.com)

Journal for ImmunoTherapy of Cancer 2017, 5(Suppl 2):P279

\section{Background}

Antibodies targeting immune inhibitory checkpoint pathways have transformed cancer therapy. However, the majority of patients fail to respond to therapies targeting single immune checkpoints due, in part, to the compensatory activity of alternative immune suppressive pathways. A therapeutic strategy that targets multiple immune checkpoints may significantly improve the antitumor response rate, however this strategy may also increase the risk of severe treatment related immune mediated side effects. Orally bioavailable, small molecule immune checkpoint antagonists with short in vivo half-lives are ideal candidates for combination cancer immune therapy due to a short drug washout time which may improve safety and flexibility in scheduling which may increase efficacy.

Methods

The anti-tumor efficacy of orally administered CA-170 (a PD-L1/2 and VISTA/PD-1H antagonist), CA-327 (a PD-L1/2 and Tim-3 antagonist) or the combination of CA-170 plus CA-327 was studied in the mouse syngeneic CT26 colon carcinoma tumor model. Compound dosing was initiated within 1-3 days of tumor implantation or in mice bearing established tumors $\left(90-170 \mathrm{~mm}^{3}\right)$. Populations of immune cells were measured by flow cytometric analysis in tumor or blood. Ex vivo functional assays were performed on tumor derived $\mathrm{CD}^{+} \mathrm{T}$ cells to evaluate effector cell function and gene expression profiling was performed on tumors.

Results

Significant anti-tumor efficacy was observed in CT26 tumor bearing mice treated with either CA-170 or CA-327 compared to vehicle treated animals. Flow cytometric analysis of the tumors taken from these mice revealed that both compounds significantly increased the total number of $\mathrm{CD}_{4} 5^{+}$immune cells within the tumor relative to vehicle treated animals. Tumor growth inhibition positively correlated with increased numbers of activated $\mathrm{CD}^{+} \mathrm{T}$ cells and IFN- $\gamma^{+} \mathrm{CD}^{+}$effector $\mathrm{T}$ cells within the tumor. A significant increase in anti-tumor efficacy was observed when CA170 and CA-327 were administered together as an oral combination therapy, when compared to animals treated with either compound alone.

\section{Conclusions}

These non-clinical data demonstrate a proof-of-concept showing that a combination therapy consisting of oral small molecules that antagonize the PD-L1/2, VISTA and Tim-3 pathways significantly enhance anti-tumor efficacy. CA-170 is currently undergoing Phase I clinical testing and CA-327 is in pre-clinical development. The results of our study provide a strong rational for the continued development of combination therapies using small molecule immune checkpoint antagonists for the treatment of advanced cancers. 
P280

Combination lymphoma immunotherapy using intratumoral virus-like particles containing CPG TLR9 agonist combined with checkpoint blockade

Caitlin Lemke-Miltner', Sue Blackwell', Arthur Krieg², George Weiner ${ }^{1}$ 'University of lowa, lowa City, IA, USA; ${ }^{2}$ Checkmate Pharmaceuticals, Cambridge, MA, USA

Correspondence: Caitlin Lemke-Miltner (caitlin-lemke@uiowa.edu) Journal for ImmunoTherapy of Cancer 2017, 5(Suppl 2):P280

\section{Background}

Checkpoint blockade of immune inhibitory pathways is an exciting approach to cancer immunotherapy, however there remains considerable room for improvement through the use of unique immunotherapeutic combinations. We explored the combination of intratumoral injection (in situ immunization) with a virus-like particle (VLP) combined with anti-PD1 antibody therapy. The VLP is designated CMP-001 and is composed of an immunostimulatory CpG oligodeoxynucleotide TLR9 agonist encapsulated in the $\mathrm{Qb}$ bacteriophage protein. CMP-001 was previously evaluated clinically in over 700 subjects in non-cancer trials where it stimulated a strong Th1 cytokine response. The current studies were based on the hypothesis that intratumoral CMP-001 can augment the development of a tumor-specific T cell response that can be maintained by anti-PD-1 therapy.

Methods

The impact of CMP-001 with and without anti-PD1 was assessed in both human (in vitro) and mouse (in vitro and in vivo) systems. In vivo therapy studies in mice included the B16F0 melanoma and A20 $B$ cell lymphoma models. CMP-001, or saline control, was delivered intratumorally starting one week after tumor challenge. Anti-PD-1, or isotype control, was administered systemically starting one week after tumor challenge. Tumor growth and survival was followed. In some experiments, tumor inoculation was done bilaterally while a unilateral tumor was injected with CMP-001 to allow for assessment of response of both the treated and untreated tumor.

Results

In vitro, CMP-001 stimulated IFNa production, as well as other proinflammatory cytokines, from human and mouse mononuclear cells (from the peripheral blood and spleen respectively). This was only seen when anti-Q $\beta$ antibody was present.

Intratumoral CMP-001 enhanced survival, and reduced tumor growth of treated tumors in the B16F0 model, and of both treated and untreated tumors in the A20 lymphoma model. Anti-PD-1 enhanced this effect in the A20 model. Depletion of T cells in mice eliminated the anti-tumor effect in both the treated and untreated tumors. Conclusions

We conclude CMP-001 can induce a robust Th1 response in both murine and human systems in a manner that is dependent on opsonization of the VLP with anti-Qb antibody. The combination of intratumoral CMP-001 and systemic anti-PD-1 leads to development of a systemic anti-tumor T cell response in two murine tumor models and is a promising immunotherapy combination worthy of clinical evaluation. A Phase 1 clinical trial of the combination of anti-PD-1 and CMP-001 is underway in advanced melanoma. These results will be presented separately.

\section{P281}

Treatment with a VEGFR-2 antibody results in intra-tumor immune modulation and enhances antitumor efficacy of PD-L1 blockade in syngeneic murine tumor models

yanxia li', David Schaer', Marguerita O'Mahony ${ }^{1}$, Ivan Inigo ${ }^{1}$, Qi Li',

Nelusha Amaladas', Erik Rasmussen', Thompson Doman', Jason

Manro', Mary Murphy', Macrina Francisco', Gerald Hall', Michael Kalos', Ruslan Novosiadly', Bronislaw Pytowski ${ }^{1}$

${ }^{1}$ Eli Lilly and Company, New York, NY, USA; ${ }^{2}$ Eli Lilly and Company, Indianapolis, IN, USA

Correspondence: David Schaer (yanxia.li@iilly.com)

Journal for ImmunoTherapy of Cancer 2017, 5(Suppl 2):P281

\section{Background}

The activation of VEGFR-2 by its primary cognate ligand VEGF has been principally implicated in tumor angiogenesis. However, emerging data has suggested that the VEGF/VEGFR-2 axis also mediates a suppressive effect on the anti-tumor immune response. Inhibition of this pathway has demonstrated the potential to facilitate $\mathrm{T}$ cell migration into the tumor and to reduce the direct immune inhibitory activity promoted by tumor endothelial cells. Clinical studies that combine anti-angiogenic antibodies to immune checkpoint inhibitors have shown promising results in a number of solid tumors. However, the mechanisms underlying immunomodulatory activity of agents that target the VEGF/VEGFR-2 axis remain incompletely understood. Methods

Ramucirumab is an approved VEGFR-2 targeted antibody currently undergoing clinical trials in combination with PD-1/PD-L1 inhibitory antibodies. The emerging data from these early trials has prompted us to undertake non-clinical studies with potential to uncover mechanisms that mediate the enhancement of anti-tumor immune response by the blockade of VEGFR-2. Using murine EMT6 breast and MC38 colon carcinoma models we investigated the immunomodulatory effects of DC101, a surrogate antibody that blocks mouse VEGFR-2, and its ability to increase the anti-tumor efficacy of checkpoint inhibition. Intra-tumor immune-related changes were evaluated by flow cytometry, immunohistochemistry $(\mathrm{IHC})$ and nCounter gene expression analysis (NanoString).

Results

Anti-VEGFR2 monotherapy resulted in increased T cell infiltration into the tumors. Combination of anti-VEGFR-2 with anti-PD-L1 resulted in greater anti-tumor efficacy compared to anti-PD-L1 monotherapy in both MC38 and EMT6 tumor models. Mice achieving complete tumor regressions after the combination treatment resisted tumor rechallenge demonstrating the development of immunologic memory. Analysis of changes in the tumor microenvironment by flow cytometry during combination therapy showed increased myeloid and $\mathrm{T}$ cell infiltration. nCounter gene expression analysis confirmed that antiVEGFR-2 treatment enhanced inflammation and immune activation gene expression signature in monotherapy, and this effect was much more pronounced after the combination treatment. Pathway analysis highlighted that the combination effect could be attributable to enhanced innate immune response (e.g. dendritic cell maturation, antigen presentation) and $\mathrm{T}$ cell activation. These results were corroborated by flow cytometry, showing increased $\mathrm{MHCl}$ and $\mathrm{MHCll}$ expression on DCs and macrophages, along with increased PD-L1 expression during anti-VEGFR-2 monotherapy.

Conclusions

Taken together, these results highlight the potential of anti-VEGFR2 antibodies to partially ameliorate intra-tumor immune suppression, providing insights into the mechanisms by which combined VEGFR-2/PD-L1 antibody therapy leads to increased anti-tumor efficacy.

\section{P282}

Immunostimulatory CD40L/4-1BBL Gene Therapy Enhances aPD-1 Antibody Therapy in Experimental Models

Jessica Wenthe, Emma Eriksson, Ann-Charlotte Hellström, Angelica Loskog

Uppsala University, Uppsala, Sweden

Correspondence: Angelica Loskog (angelica.loskog@igp.uu.se)

Journal for ImmunoTherapy of Cancer 2017, 5(Suppl 2):P282

\section{Background}

Lung cancer is a deadly disease responding poorly to conventional therapy. Checkpoint blockade antibodies inhibiting PD-1/PD-L1 have shown clinical benefit. Since the success of PD-1 blockade therapy prerequisites the presence of tumor-reactive T-cells, we hypothesized that an activating immunotherapy with high potency to stimulate Tcell responses would be an ideal candidate to combine with anti-PD1 antibody treatment. LOAd703 is a replication-restricted adenovirus serotype $5 / 35$. It is armed with two immunostimulatory transgenes, 4-1BB ligand (4-1BBL) and a trimerized human CD40 ligand (TMZ- 
CD40L). Both molecules are co-stimulatory molecules in the dendritic cell (DC)/T-cell synapse. This study aims to evaluate if immunostimulatory gene therapy, such as LOAd703, is a good enhancer of checkpoint blockade therapy.

Methods

The capacity of LOAd703 was tested using the lung cancer cell lines A549 and H727. Tumor viability was evaluated in vitro using MTS viability assay. Co-culture experiments were analyzed in real time using Incucyte technology. Cells were phenotyped with multicolor flow cytometry and cytokine patterns investigated by MesoScale. A20 cells that can be infected with LOAd703 virus were used in a syngeneic model in BalbC mice.

Results

Tumor cells were killed by oncolysis post infection with high dose virus (100MOI) in vitro and in vivo in multiple xenograft mouse models (lung, ovary, pancreas, colon, bladder). In tumor cell/PBMC co-culture,addition of anti-PD-1 antibody did not increase tumor cell apoptosis. If OKT3/LL2 was added apoptosis was confirmed in the cultures peaking at day 5. Combination of OKT3/IL2 with anti-PD-1 antibody significantly enhanced the effect. LOAd703 was added to tumor cell/PBMC co-cultures at a low $\mathrm{MOI}$ to delay oncolysis. LOAd703 induced tumor cell death, starting already at day 2 and the activity was continuously rising throughout the experiment. Combining LOAd703 with anti-PD-1 antibody significantly increased apoptosis induction of tumor cells while a control virus was less effective. At endpoint, LOAd703 plus anti-PD-1 was twice as effective as OKT3/ IL2 plus anti-PD-1. The T cells were increased in the same groups as tumor cell killing was previously noted. IFNg, TNF, IL2 were all enhanced by LOAd703 and OKT3/LL2. In line with in vitro data, LOAd703 could enhance in vivo tumor control of anti-PD-1 therapy. Conclusions

In conclusion, LOAd703 is a potent stimulator of T cell responses and potentiates the effect of aPD-1 therapy.

\section{P283}

Differential impact of chemotherapy on tumor-associated antigenspecific immunogenicity in cynomolgus macaques

Maria Josic, Aaron Longworth, Joe Binder, Helen Cho, Erick Gamelin, Siradanahalli Guru, Eugenia Kraynov, Steve Burgess, Bryan Clay, Charlie Huang, Jannine Landry, Mark Lesch, Cindy Wei Li, Shangjin Li, Pavinder Kaur, Sophie Muscat-King, Peter Weady, Dan Xu, James Merson, Robert Hollingsworth, Marianne Martinic

Pfizer, San Diego, CA, USA

Correspondence: Marianne Martinic (maria.josic@pfizer.com) Journal for ImmunoTherapy of Cancer 2017, 5(Suppl 2):P283

\section{Background}

Pfizer's vaccine-based immunotherapy regimen (VBIR) is a cancer treatment which combines a tumor-associated antigen (TAA)-specific vaccine, delivered via an AdC68 adenovirus and DNA regimen, with the immunomodulatory agent tremelimumab (anti-CTLA4 antibody) for T cell expansion. Various tumor types that are targets for VBIR, including non-small cell lung, triple negative breast, ovarian, and pancreatic cancer, are treated with chemotherapy as the standard of care (SOC). Accordingly, VBIR clinical administration may take place in patients who are currently undergoing chemotherapy. Synergistic effect has been reported between immune therapies and chemotherapy, either due to immunogenic cell death or a direct effect on immunosuppressive cells. However, limited information is known about the impact of chemotherapy on a vaccine-induced de novo immune response.

Methods

A nonhuman primate model was developed to most precisely mimic clinical therapy conditions, including pharmacokinetics and dynamics, dosing regimens, and supplemental treatments, allowing evaluation of potential chemotherapy impact on the VBIR-induced immune response. Macaques were dosed with a VBIR targeting a selfversion of a human TAA with or without the following SOC treatments: paclitaxel/gemcitabine, paclitaxel/carboplatin, docetaxel/carboplatin, or doxorubicin. Complete blood counts and immunophenotyping were performed to track changes in immune cell profile as a biomarker of response. TAA-specific IFN- $\gamma^{+} \mathrm{CD}^{+}$and $\mathrm{CD}^{+}{ }^{+}$cell responses and humoral responses were measured throughout the study.

Results

In non-tumor bearing macaques, different chemotherapies impacted VBIR-induced immune responses with varying degrees of severity. TAAspecific IFN- $\gamma^{+} \mathrm{CD}^{+} \mathrm{T}$ cell responses ranged from unaffected with doxorubicin, to moderately reduced with carboplatin-based doublet chemotherapies, to almost entirely ablated with paclitaxel/gemcitabine. TAA-specific IFN- $\gamma^{+} \mathrm{CD}^{+} \mathrm{T}$ cell responses, however, were generally unimpaired by chemotherapy: indeed, in many instances chemotherapy boosted $\mathrm{CD}^{+} \mathrm{T}$ cell responses. TAA-specific humoral responses were superior in animals treated with carboplatin-based doublet chemotherapies, while paclitaxel/gemcitabine continued to preclude an effective immune response. Doxorubicin treated animals demonstrated a strongly diminished humoral response, attributed to B cell depletion. The impact of chemotherapy on the VBIR-induced immune response was also dependent on antigenic strength, with responses to a weaker antigen more dramatically reduced than were responses to a more immunogenic antigen.

Conclusions

Careful clinical trial planning is critical for vaccine-based immunotherapy regimen and chemotherapy combinations that will not only permit robust immune response activation and maintenance but also provide the adequate conditions for optimal synergistic anti-tumor effect.

\section{P284}

T cell priming by Toca 511 and 5-FC coupled with T regulatory cell depletion by aCTLA-4 synergistically enhances anti-tumor immune memory in a mouse model of glioma

Leah Mitchell, Kader Yagiz, Anthony Munday, Fernando Lopez,

Daniel Mendoza, Douglas Jolly

Tocagen, San Diego, CA, USA

Correspondence: Leah Mitchell (Imitchell@tocagen.com)

Journal for ImmunoTherapy of Cancer 2017, 5(Suppl 2):P284

\section{Background}

Toca 511 (vocimagene amiretrorepvec) is a gamma retroviral replicating vector that selectively infects cancer cells in vivo and encodes cytosine deaminase. In combination with the prodrug, 5-fluorocytosine (5-FC), Toca 511 produces 5-fluorouracil (5-FU) locally in the tumor microenvironment. Prior work has demonstrated a reduction in immunosuppressive myeloid cells and an increase in CD4 and CD8 T cells in tumors while $T$ regulatory cells remain unchanged with treatment with Toca 511 and 5-FC.

Methods

This work, in a mouse model of glioma, aimed to determine if the addition of a checkpoint inhibitor, aCTLA-4, would provide therapeutic benefit to Toca 511 and 5-FC.

Results

Initially, we noted that Toca 511 and 5-FC was highly efficacious and that it provided little room for further improvement and therefore combination with aCTLA-4 did not show additive benefit against the primary cancer. However tumor associated Regulatory $\mathrm{T}$ cells were significantly reduced with aCTLA-4 treatment and long term memory was significantly improved with the combination as shown in adoptive transfer studies. Adoptive transfer of immune cells from animals that cleared their primary tumor through Toca 511, 5-FC, and aCTLA4 showed $100 \%$ survival benefit to animals bearing orthotopic gliomas, significantly greater than the $\sim 50 \%$ survival seen with transfer from animals that cleared primary tumor through Toca 511 and 5-FC alone. Further, aCTLA-4 treatment during clearance of primary tumors resulted in a marked reduction of memory $T$ regulatory cells in secondary tumors after adoptive transfer. To determine if aCTLA-4 in combination with Toca 511 and 5-FC could reduce primary tumor burden we developed a model using a submaximal infection level of Toca 511. Specifically, restricting Toca 511 infection to only $2 \%$ of 
tumor cells limited the tumor growth arrest activity of 5-FC and the loss of efficacy with $2 \%$ infection was rescued when 5 -FC treatment was combined with aCTLA-4.

\section{Conclusions}

These data suggest that aCTLA-4, and other compounds that target $T$ regulatory $T$ cells, should be evaluated in patients receiving Toca 511 and Toca FC to determine if the combination confers additional clinical benefit.

\section{P285}

Antibody and T cell response profiling of pancreatic cancer patients before and after chemotherapy reveals increased recognition of antigens suitable for immunotherapy Giorgia Mandili', Moitza Principe ${ }^{1}$, Emanuela Mazza' ${ }^{1}$, Sara Bulfamante' Laura Follia', Giulio Ferrero', Andrea Evangelista ${ }^{2}$, Daniele Giordano', Paola Cappello ${ }^{1}$, Francesco Novelli ${ }^{1}$

${ }^{1}$ University of Turin, Torino, Italy; ${ }^{2}$ Hospital Città della Salute e della Scienza di Torino, Torino, Italy

Correspondence: Francesco Novelli (franco.novelli@unito.it)

Journal for ImmunoTherapy of Cancer 2017, 5(Suppl 2):P285

\section{Background}

Pancreatic ductal adenocarcinoma (PDA) is one of the most lethal cancer, both for lack of effective screening method and for resistance to chemotherapy (CTX) and radiotherapy. At present surgical resection is the only potentially curative option. Once diagnosed, CTX, radiation or combination therapy regimens are used to treat patients, but responses remain poor. However, some chemotherapeutic agents have an immune modulating effect and a combination of CTX and immunotherapy could increase therapeutic efficacy. Thus, more immunogenic antigens can be induced by CTX and targeted by passive or active immunotherapy. To discover TAAs that might be selected for immunotherapy, antibody response in PDA patients' sera were analyzed before and after CTX. TAAs selected based on their increased recognition after CTX were used to evaluate whether PDA patient autologous $T$ cells have an increased TAAs specific response after CTX.

Methods

Antibody response in sera of 29 PDA patients, before and after CTX treatments, has been analyzed by Serological Proteome Analysis (SERPA). The production of IFN-g and IL-10 by PBMC stimulated in vitro with recombinant TAAs was evaluated by ELISA.

Results

CTX increases the number of TAAs recognized by PDA patients. Specifically, the set of TAAs we identified are proteins whose mRNA expression have been found up-regulated in PDA patients. We observed a positive correlation between patients survival and the increased antibodies production against alpha-enolase (ENO1), glyceraldehyde-3-phosphate dehydrogenase (G3P), tubulin (TUBB5), and keratin, type II cytoskeletal 8 (K2C8). Of note, 48\% of PDA patients sera after CTX treatment showed an increase of Complement Dependent Cytotoxicity (CDC) against human cell lines. Moreover in about $50 \%$ of the same PDA patient cohort the in vitro specific T cell response to recombinant TAAs that were mostly recognized by antibodies after CTX (ENO1, G3P, K2C8 and Far Upstream Binding Protein 1 (FUBP1) switched from a pro-tumor regulatory (low IFN-g/ IL10 ratio) to anti-tumor effector (high IFN-g/IL10 ratio) phenotype after one or two rounds of CTX.

Conclusions

Data indicated that in PDA patients CTX induces an increase of lgG antibody to ENO1, G3P, TUBB5 and $\mathrm{K} 2 \mathrm{C} 8$ whose expression is upregulated in PDA that may have a prognostic role. CTX also increases the ability of IgG kill PDA cells by CDC. Finally, CTX switches the $T$ cell response to ENO1, G3P, K2C8 or FUBP1 from regulatory to effector and thus renders these TAAs promising targets for the design of new immunotherapeutic approach to improve the efficacy of CTX.
P286

Efficacy of interleukin 2 and interleukin 15 for in situ vaccination in a mouse melanoma model

Alexander Rakhmilevich', Anna Hoefges ${ }^{1}$, Jacob Slowinski ${ }^{1}$, Kayla

Rasmussen', Mackenzie Heck', Michael Meagher ${ }^{2}$, Alan Korman³, Paul Sondel $\left.\right|^{1}$

${ }^{1}$ University of Wisconsin-Madison, Madison, WI, USA; ${ }^{2}$ St. Jude Children's Research Hospital/Children's GMP, LLC, Memphis, TN, USA; ${ }^{3}$ Bristol-Myers Squibb Company, Redwood City, CA, USA

Correspondence: Alexander Rakhmilevich (rakhmil@humonc.wisc.edu) Journal for ImmunoTherapy of Cancer 2017, 5(Suppl 2):P286

\section{Background}

We have previously shown that a direct intratumoral (IT) injection of immunocytokine (IC), an anti-GD2 antibody linked to interleukin 2 (IL-2), can serve as an in situ vaccine and synergize with anti-CTLA-4 antibody to induce T cell-mediated antitumor effects. We have also shown a synergy of this approach with activation of innate immunity with an agonistic anti-CD40 monoclonal antibody (anti-CD40) and CpG (Rakhmilevich et.al. Journal of Immunology 2017). We used these two immunotherapeutic approaches to test whether IT treatment with a mixture of IL-2 and anti-GD2 antibody, hu14.18K $322 \mathrm{~A}$, will be effective against subcutaneous melanoma. In addition, we hypothesized that IL-15, a cytokine having several activities similar to those of IL-2 except induction of $\mathrm{T}$ regulatory cells (Tregs), may be as or more effective than IL2 for in situ vaccination.

\section{Methods}

GD2+ B78 mouse melanoma cells were injected subcutaneously in C57BL/6 mice. Anti-CD40 and anti-CTLA-4 were given intraperitoneally, and CpG, IL-2, IL-15 and K322A were given IT. IL-2 and IL-15 were given at the dose of $7.5 \times 104$ units per injection daily for 5 days. Tumor growth and survival were followed. In some experiments, tumors were analyzed by flow cytometry.

Results

Flow cytometric analyses showed that IL-2 treatment increased Tregs within a tumor whereas IL-15 did not. When the treatments were started on day 6 post tumor cell implantation, IL-2 and IL-15 combined with K322A induced similar reductions of B78 tumor growth, and complete tumor regression with the addition of anti-CTLA-4. When the treatments were started on day 22 post tumor cell implantation, IL-2 and IL-15, combined with K322A, anti-CTLA-4, anti$\mathrm{CD} 40$ and $\mathrm{CpG}$, also induced comparable antitumor effects resulting in survival of $40-60 \%$ of mice.

Conclusions

In two therapeutic approaches, IL-2 and IL-15, combined with other immunotherapeutic agents, induced similar antitumor effects.

\section{P287}

Immunological effects of checkpoint blockade plus galectin-3 inhibition with GR-MD-02 in a first-in-human phase I clinical trial William Redmond1, Yoshinobu Koguchi1, Christopher Fountain1, Peter Traber2, Brendan Curti1

1 Providence Portland Medical Center, Portland, OR, USA; 2Galectin Therapeutics, Norcross, GA, USA

Correspondence: William Redmond (william.redmond@providence.org) Journal for ImmunoTherapy of Cancer 2017, 5(Suppl 2):P287

\section{Background}

Immunosuppression of tumor-infiltrating lymphocytes (TIL) is a major obstacle to creating effective therapies for patients with metastatic cancer. Galectin-3 (Gal3), a lectin family member, is expressed by numerous cancers and immune cell subsets. Serum Gal3 expression is higher in patients with metastatic disease and is associated with reduced survival in patients with metastatic melanoma. Furthermore, Gal3 has been implicated in disease progression via the promotion of angiogenesis and metastasis. Interestingly, extracellular Gal3 
induces immune suppression via inhibiting TIL function, promoting M2 macrophage polarization and mobilizing myeloid cells from the bone marrow to promote a metastatic niche within the tumor. We hypothesized that Gal3 inhibition in conjunction checkpoint blockade immunotherapy would improve TIL function while inhibiting tumor growth and metastasis. Preclinical studies revealed that Gal3 blockade with GR-MD-02 and agonist anti-OX40, anti-CTLA-4 or antiPD-1 mAb therapy enhanced tumor-specific immunity and improved survival in tumor-bearing mice.

Methods

We initiated 2 phase I clinical trials at our Institute to evaluate the safety and immunological effects of GR-MD-02 plus checkpoint blockade immunotherapy. These studies are investigating dose escalations of GR-MD-02 with the standard therapeutic dose of antiCTLA-4 (ipilimumab/ipi) or anti-PD-1 (pembrolizumab/pembro) in patients (pts) with advanced melanoma (ipi; pembro), HNSCC (pembro), or NSCLC (pembro) (NCT02117362, NCT02575404).

Results

The GR-MD-02 + ipi study has completed enrollment. Three patients received GR-MD-02 at $1 \mathrm{mg} / \mathrm{kg}$, 3 at $2 \mathrm{mg} / \mathrm{kg}$ and 2 at $4 \mathrm{mg} / \mathrm{kg}$. There were no DLTs for GR-MD-02; however there was 1 grade $3 \mathrm{AE}$ from ipilimumab (diarrhea) that occurred after dose 4 of ipi. The GRMD-02 + pembro study is ongoing. Six patients were enrolled at the $2 \mathrm{mg} / \mathrm{kg}$ dose level to gain more data from the immunological monitoring assays. Three patients were treated at $4 \mathrm{mg} / \mathrm{kg}$. Eight of the 9 enrolled patients have melanoma and 1 has HNSCC. There have been no DLTs related to GR-MD-02 or pembro, but there one patient experienced transient grade 3 tumor-related pain. Two pts in cohort 1 (2 $\mathrm{mg} / \mathrm{kg}$ GR-MD-02) had objective responses (1 PR, $1 \mathrm{CR}$ ) and two pts in cohort $2(4 \mathrm{mg} / \mathrm{kg})$ have PR at the first response assessment. All responses have been observed in patients with melanoma.

\section{Conclusions}

These data demonstrate that the Gal3 inhibitor GR-MD-02 can be combined safely with checkpoint blockade in patients with metastatic disease and that melanoma regression was observed in multiple patients following combination therapy. Comprehensive immunological monitoring is being conducted to provide insight into potential mechanisms of action.

Trial Registration

NCT02117362, NCT02575404

\section{P288}

KY1044, a novel anti-ICOS antibody, elicits long term in vivo antitumour efficacy as monotherapy or in combination with immune checkpoint inhibitors

Richard C.A. Sainson, Anil Thotakura, Nahida Parveen, Gwenoline Bohris, Robert Rowlands, Miha Kosmac, Jamie Campbell, lan Kirby,

Volker Germaschewski, Matthew McCourt

Kymab Ltd, Cambridge, United Kingdom

Correspondence: Richard C.A. Sainson (richard.sainson@kymab.com);

Matthew McCourt

Journal for ImmunoTherapy of Cancer 2017, 5(Suppl 2):P288

\section{Background}

The inducible co-stimulator molecule (ICOS/CD278) is a member of the CD28/CTLA-4 family that is up-regulated upon T cell activation. In the tumour microenvironment, ICOS expression levels vary in different immune cell subtypes, with expression on highly immunosuppressive regulatory $\mathrm{T}$ cells (Treg: CD4+/FOXP3+) significantly higher than that on effector CD8+ T cells. The high expression levels on Tregs highlights the potential of targeting ICOS to deplete these cells and enhance the anti-tumour immune response when used in combination with immune checkpoint blockers.

Methods

Using the Kymouse ${ }^{\mathrm{TM}}$ platform, we have identified a novel, fully human antibody called KY1044. KY1044 is an anti-ICOS subclass G1 kappa monoclonal antibody that selectively binds to dimeric ICOS (Fc fusion) with an affinity of less than $2 \mathrm{nM}$ as measured by SPR using Fabs of KY1044. This antibody, which binds ICOS from human, cynomolgus monkey, rat and mouse with similar affinity, was used in several in vitro and in vivo assays to refine its mechanism of action and assess the anti-tumour efficacy in pre-clinical models.

Results

Using in vitro reporter and primary cell assays, we have demonstrated that KY1044 can affect the immune context via a dual mechanism of action. On the one hand, KY1044 is extremely potent (low pM EC50) at depleting ICOShigh Tregs and on the other hand KY1044 was shown to stimulate ICOSInt effector T-cells by increasing their IFNY and TNFa production. Since KY1044 is cross reactive to the rodent orthologue of ICOS, a mouse effector enabled version of KY1044 (mlgG2a) was generated and tested in syngeneic tumour models. Using this antibody, we have confirmed strong anti-tumour efficacy as monotherapy or in combination with surrogates of "approved" immune checkpoint blockers. Noteworthy, and confirming the in vitro data, pharmacodynamic studies demonstrated a long-term depletion of Tregs and a significant increase in the effector $\mathrm{T}$ cell to Treg ratio in response to KY1044.

Conclusions

Altogether, the in vitro and in vivo properties of this novel, fully human anti-ICOS antibody support the continued development of KY1044 as a treatment option to activate an anti-tumour immune response.

\section{P289}

Dual cIAP1/XIAP inhibitor ASTX660 synergizes with radiation

therapy and PD-1 blockade to enhance anti-tumor immunity

Roy Xiao ${ }^{1,2}$, Clint Allen ${ }^{1,3}$, Linda Tran', So-Jin Park', Zhong Chen',

Carter Van Waes ${ }^{1}$, Nicole Schmitt ${ }^{1,3}$

${ }^{1} \mathrm{NIDCD}, \mathrm{NIH}$, Bethesda, MD, USA

Correspondence: Nicole Schmitt (nicole.schmitt@nih.gov)

Journal for ImmunoTherapy of Cancer 2017, 5(Suppl 2):P289

\section{Background}

Head and neck squamous cell carcinomas (HNSCCs) frequently harbor genomic mutations in cell death pathways. Nearly $30 \%$ of HNSCC overexpress Fas-Associated Death Domain (FADD), with or without BIRC2/3 genes encoding cellular Inhibitor of Apoptosis Proteins $1 / 2$ (cIAP1/2), critical components of the Tumor Necrosis Factor (TNF) Receptor signaling pathways. ASTX660 is a novel dual cIAP1/XIAP antagonist in clinical trials for advanced solid tumors and lymphomas. Methods

Murine oral cancer 1 (MOC1) cells were used in vitro and in vivo to investigate the anti-tumor activity of ASTX660 alone and in combination with tumor necrosis factor receptor (TNFR) superfamily ligands, radiation, cisplatin chemotherapy, and anti-PD-1 checkpoint blockade. OT-1 T cells were used to investigate the effects of ASTX660 on antigen-specific T cell killing of ovalbumin-expressing MOC1 (MOC1ova) cells.

Results

ASTX660, at nanomolar concentrations, sensitized MOC1 cells to TNFa and stimulated cytotoxic T lymphocyte (CTL) killing of MOC1ova. CTL killing was found to be predominantly mediated by perforin/granzyme B during the earliest stages of killing and release of death ligands TNFa, TRAIL, and FasL as a sustained mechanism of killing. Using MOC1 cells in vivo, ASTX660 synergized with radiation therapy (XRT), cisplatin chemotherapy, and PD-1 blockade to significantly delay or eradicate MOC1 tumors. These combination therapies significantly increased CD8+ T cells and dendritic cells, as well as T cell activity. Depletion of CD8+ T cells and NK cells in vivo revealed both to be important components of the anti-tumor response enhanced by ASTX660+XRT.

\section{Conclusions}

These findings serve to inform future studies of IAP inhibitors and support the potential for future clinical trials investigating ASTX660 with XRT and immunotherapies such as PD-1/PD-L1 blockade in HNSCC. 


\section{P290}

Agonist redirected checkpoint (ARC), TIM3-Fc-OX40L, for cancer immunotherapy

George Fromm, Suresh de Silva, Kellsey Johannes, Arpita Patel, Josiah Hornblower, Taylor Schreiber

Shattuck Labs, Inc., Research Triangle Park, NC, USA

Correspondence: Taylor Schreiber (tschreiber@shattucklabs.com)

Journal for ImmunoTherapy of Cancer 2017, 5(Suppl 2):P290

\section{Background}

Current attempts at combination immunotherapy with bispecific antibodies, linked scFv's or T cell engagers have not demonstrated that both checkpoint blockade and TNF receptor activation (agonism) can be achieved with a single molecule. This is likely due to the fact that these molecules lose target avidity when engineered to bind multiple targets with monovalent antigen binding arms. Fusion proteins incorporating the extracellular domain (ECD) of type I membrane proteins (eg. Enbrel, Orencia) or type II membrane proteins (eg. OX40L-Fc, GITRL-Fc), linked to the hinge- $\mathrm{CH} 2-\mathrm{CH} 3$ domain of antibodies are both functional, despite the fact that the ECDs are in opposite orientation. Here we report the generation of a two-sided fusion protein incorporating the ECD of TIM3 and the ECD of OX4OL, adjoined by a central FC domain.

Methods

Shattuck synthesizes both murine and human versions of ARC proteins, and assesses them using a litany of biochemical assays to determine molecular weight, subunit composition \& binding affinity; molecular assays to characterize in vitro/ex vivo binding, in vitro functional activity; and anti-tumor efficacy in multiple syngeneic tumor model systems. The human TIM3-Fc-OX40L has advanced into cell line development and early manufacturing.

Results

The TIM3 end of the fusion protein binds GAL9 and phosphtidylserine (PS) on the surface of human tumor cells. The OX40L end of the fusion protein binds OX40 on the surface of primary T cells. TIM3-FcOX40L activates NFkB signaling in cells engineered to overexpress OX40 and an NFkB-luciferase reporter. Additionally, the TIM3-FcOX40L ARC added to primary human PBMCs along with the superantigen Staphylococcal enterotoxin $B$, induced robust secretion of the cytokines IL2 and TNFa. In vivo, TIM3-Fc-OX40L stimulates significant expansion of antigen-specific CD4 and CD8 T cells in mice adoptively transferred with OT-I/OT-II cells and vaccinated with ova/alum. Finally, the therapeutic activity of TIM3-Fc-OX40L in established murine MC38 and CT26 tumors was significantly superior to either TIM3 blocking antibody, OX40 agonist antibody or combination antibody therapy. Importantly, a pharmacodynamic biomarker of tumor rejection was identified by coordinated elevations in serum IFNY, IL-2, IL4, IL-5, IL-6 and IL-17A.

\section{Conclusions}

These data demonstrate feasibility and functional activity of a novel chimeric fusion protein platform, providing checkpoint blockade and TNF superfamily costimulation in a single molecule, which is uniquely advantageous because the construct links those two signals in the same microenvironmental context, at the time in which $T$ cells are engaging cognate tumor antigen.

\section{P291}

Agonist redirected checkpoint (ARC), SIRPa-Fc-CD40L, for cancer immunotherapy

Suresh de Silva, George Fromm, Arpita Patel, Kellsey Johannes, Josiah Hornblower, Taylor Schreiber

Shattuck Labs, Inc., Research Triangle Park, NC, USA

Correspondence: Taylor Schreiber (tschreiber@shattucklabs.com)

Journal for ImmunoTherapy of Cancer 2017, 5(Suppl 2):P291

\section{Background}

Current attempts at combination immunotherapy with bispecific antibodies, linked scFv's or T cell engagers have not demonstrated that both checkpoint blockade and TNF receptor activation (agonism) can be achieved with a single molecule. This is likely because these molecules lose target avidity when engineered to bind multiple targets with monovalent antigen binding arms. Fusion proteins incorporating the extracellular domain (ECD) of type I membrane proteins (eg. Enbrel, Orencia) or type II membrane proteins (eg. SIRPa-Fc, GITRL-Fc), linked to the hinge- $\mathrm{CH} 2-\mathrm{CH} 3$ domain of antibodies are both functional, despite the ECDs being in opposite orientation. We report the generation of a two-sided fusion protein incorporating the ECD of SIRPa (CD172a) and the ECD of CD40L, adjoined by a central Fc domain.

Methods

Shattuck synthesizes both murine and human versions of ARC proteins, and assesses them using a litany of biochemical assays to determine MW, subunit composition \& binding affinity; molecular assays to characterize in vitro/ex vivo binding \& functional activity; and antitumor efficacy in syngeneic tumor models. The human SIRPa-Fc-CD40L has completed cell line development and single cell cloning and is in late-stage manufacturing.

Results

The SIRPa end of the ARC binds immobilized CD47 at $3.59 \mathrm{nM}$ affinity and binds CD47 on the surface of human tumor cells both in vitro and in vivo, but does not bind human platelets or RBCs. Importantly, no hemolytic activity has been observed with the human SIRPa-Fc-CD40L ARC; where significant hemolysis has been reported with comparative CD47 mAbs. The CD40L end of the ARC binds immobilized CD40 at $756 \mathrm{pM}$ affinity and binds CD40 on primary macrophages. The SIRPaFc-CD40L ARC stimulates functional activity in NFkB-luciferase reporter cells (CD40 driven activation of NFkB) and when added ex vivo to human PBMCs primed with the super-antigen SEB; increases secretion of IL2 and TNFa. Furthermore, when activated human macrophages were co-cultured with CD47 positive human tumor cells, SIRPa -FcCD40L was shown to enhance phagocytosis of human tumor cells. Finally, the therapeutic activity of SIRPa-Fc-CD40L in established murine MC38 and CT26 tumors was superior to either CD47 blocking antibody, CD40 agonist antibody or combination antibody therapy.

Conclusions

These data demonstrate feasibility and functional activity of a novel chimeric fusion protein platform, providing checkpoint blockade and TNF superfamily costimulation in a single molecule. Signal replacement of CD47 by CD40L may uniquely poise macrophages in the tumor microenvironment for activation and crosspresentation of tumor antigens following enhanced tumor cell phagocytosis.

\section{P292}

Pre-clinical activity of a novel immunotherapy combination of CAVATAK (Coxsackievirus A21), anti-PD1 blockade and an IDO inhibitor in melanoma

Gough Au, Min Yuan, Yvonne Wong, Darren Shafren

Viralytics Limited, Sydney, Australia

Correspondence: Darren Shafren (darren.shafren@viralytics.com)

Journal for ImmunoTherapy of Cancer 2017, 5(Suppl 2):P292

\section{Background}

Coxsackievirus A21 (CAVATAK ${ }^{\mathrm{TM}}$ ) is a bio-selected oncolytic immunotherapy virus. A Phase Ib trial of i.v. CAVATAK (NCT01227551) in advanced cancer patients has displayed viral tumor targeting and initial indications of antitumor activity in some lesions. Intratumoral CAVATAK injection of melanoma lesions can induce selective tumor-cell infection, immune-cell infiltration, IFN-g response gene up-regulation, increased PD-L1 and IDO (indoleamine-pyrrole 2,3-dioxygenase) expression, tumor cell lysis and systemic anti-tumor immune responses. Blockade of programmed death protein-1 (PD1) and/or IDO inhibition in many cancer patients has resulted in substantial tumor responses. We investigated anti-tumor activity in a B16-ICAM-1 melanoma im mune competent mouse model of a novel combination of CAVATAK, an anti-PD1 mAb and an IDO inhibitor.

Methods

Palpable flank tumor of murine melanoma B16-cells expressing human ICAM-1 were propagated to assess the antitumor activity of CAVATAK, an anti-mouse PD1 (mPD1) mAb and an murine IDO inhibitor in an 
immune competent mouse model. CAVATAK was administered i.v, while anti mPD1 mAb was delivered via the i.p route and the IDO inhibitor in drinking water.

Results

Notable single agent antitumor activity against the B16-ICAM-1 tumors was only observed in mice treated with anti-PD1 blockade relative to saline controls. Significant survival benefits were only observed in mice treated with the CVA21-anti-mPD1 doublet or the CVA21, anti-PD1 and IDO inhibitor triplet combinations. While not significant, we observed a positive trend in both reduction of overall tumor burden and survival in mice treated with the CVA21, antimPD1 doublet and the CVA21, anti-mPD1 and IDO inhibitor triplet compared with mice receiving anti-mPD1 blockade and IDOinhibition. All combinations of CVA21, anti-mPD1 and IDO blockade appeared to be generally well tolerated.

Conclusions

The notable anti-tumor activity and survival benefit mediated by the combination of CAVATAK, PD1 blockade and IDO inhibition observed in the presented melanoma model supports potential clinical evaluation of such a novel immunotherapeutic combination treatment regimen.

\section{P293}

Talimogene Laherparepvec combined with anti-PD-1 based immunotherapy for unresectable stage III-IV melanoma: a case series

Lillian Sun ${ }^{1}$, Pauline Funchain ${ }^{2}$, Jung-Min Song ${ }^{2}$, Michael McNamara², Brian Gastman ${ }^{3}$

${ }^{1}$ Cleveland Clinic Lerner College of Medicine of Case Western Reserve University, Cleveland, OH, USA; ${ }^{2}$ Cleveland Clinic Taussig Cancer Institute, Cleveland, OH, USA; ${ }^{3}$ Cleveland Clinic, Cleveland, $\mathrm{OH}$, USA

Correspondence: Brian Gastman (sunl2@ccf.org)

Journal for ImmunoTherapy of Cancer 2017, 5(Suppl 2):P293

\section{Background}

Checkpoint inhibitors have become standard of care for treating advanced melanoma. By blocking key inhibitory pathways, checkpoint inhibitors reinvigorate effector T cells to overcome an immunosuppressed microenvironment. A key preceding step to effective tumor killing is the release and presentation of tumor antigen. Talimogene Laherparepvec (T-VEC) is an oncolytic virus recently approved as an intratumoral therapy for treating unresectable stage IIIB-IV metastatic melanoma. As monotherapy, it confers modest efficacy in controlling disease progression in patients with locoregional lesions. T-VEC is used to directly lyse tumor cells and promote anti-tumor immunity via the release of tumor antigens and virus-encoded GM-CSF. The mechanisms of action for T-VEC and checkpoint inhibitors are highly complementary, raising the possibility of synergistic benefit from combining these two therapies.

Methods

We reviewed 10 consecutive cases of stage IIIC to stage IVM1b melanoma patients that received T-VEC plus either pembrolizumab or ipilimumab/nivolumab, treated between January 2016 and July 2017 at the Cleveland Clinic with a median follow-up of 7 months (range: 4 to 13 months). Responses of injected (on-target) and uninjected (off-target) lesions were evaluated according to RECIST 2.0.

Results

The overall response rate for on-target lesions was $90 \%$, with 5 patients experiencing a complete response of injected lesions. Two patients had off-target lesions, both of whom experienced complete response of their uninjected distant metastases. Overall survival of this cohort was $80 \%$. Of the two patients who died, one died of causes unrelated to melanoma. Checkpoint inhibitor therapy was interrupted for 2 patients due to adverse events, with one patient experiencing Grade 3 nephritis and the other patient experiencing Grade 3 diarrhea. There were 2 patients who experienced progression of disease. Taking this into account with the 2 patients who died, overall progression-free survival for this cohort was $60 \%$.

\section{Conclusions}

There may be potential synergistic benefit in combining checkpoint inhibitors with T-VEC injection in patients with unresectable melanoma. Although this study is limited by the lack of randomization and short follow-up time, the data revealed positive signals in the responses of both on-target and off-target lesions to the combination of T-VEC and checkpoint inhibitor treatment. In addition, the results show a better response rate than previous publications using T-VEC. Ongoing clinical trials will elucidate the true clinical benefit of this combinational therapy.

\section{P294}

Cost of adverse events associated with immunotherapy monotherapy versus targeted therapy in elderly metastatic melanoma patients

Jackson Tang ${ }^{1}$, Zhiyi Li ${ }^{1}$, Syed Mahmood ${ }^{2}$, Sameer Ghate ${ }^{2}$

${ }^{1}$ Asclepius Analytics, New York, NY, USA ; ${ }^{2}$ Novartis Pharmaceuticals

Corporation, East Hanover, NJ, USA

Correspondence: Jackson Tang (jtang@asclepiusanalytics.com)

Journal for ImmunoTherapy of Cancer 2017, 5(Suppl 2):P294

\section{Background}

The National Comprehensive Cancer Network guidelines recommend the use of immunotherapy (IO) or targeted therapy (TT) (if BRAFmutated) for the first-line treatment of metastatic melanoma (MM). Both $I O$ and $\Pi$ can lead to treatment-related adverse events (AEs). The objective of the study is to estimate and compare the cost of treatment-related AEs between elderly MM patients receiving 10 monotherapy and those receiving $\Pi$.

Methods

A retrospective cohort study was conducted using Medicare research identifiable files from 2006 to 2014 . Eligible patients had $\geq 1 \mathrm{MM}$ diagnosis and $\geq 1$ prescription for an 10 (ipilimumab or pembrolizumab) or $\Pi$ (dabrafenib, trametinib, vemurafenib, or dabrafenib+trametinib) that was FDA-approved during the study period. Patients were assigned to the $\mathrm{IO}$ or $\Pi \mathrm{T}$ cohort based on the most recent therapy used and had a minimum of 3 months follow-up within the study period. 10 categories of AEs were identified based on a review of $\mathrm{IO}$ or $\Pi \pi$ package inserts, as well as AEs that were common among MM patients. Cost per $\mathrm{AE}$ was calculated as the average per-episode cost (inpatient + outpatient) incurred in the 30 days after the first occurrence of the $A E$. Costs were inflated to 2017 USD and compared between the cohorts using the Wilcoxon rank-sum test, with a significance level of 0.05 .

Results

The study included 26610 patients and $159 \pi$ patients. The $\Pi$ cohort was younger $(72.8 \pm 14.3$ vs. $73.7 \pm 12.6$ years; $\mathrm{p}=0.04)$ and less likely to be male $(57.2 \%$ vs. $63.2 \%$; $p=0.01)$ compared with the IO cohort. The 30-day AE costs for each cohort are summarized in Table 1. The average costs associated with gastrointestinal, respiratory, and pain-related AEs were significantly higher in the 10 cohort than the $\Pi$ cohort (difference: $\$ 9270, \$ 2654$, and $\$ 2155$, respectively; $p<0.01$ ). In contrast, the average costs associated with pyrexia and/or chills, and other AEs (including decreased appetite/anorexia, fatigue, or infections) were significantly lower in the 10 cohort (difference: $-\$ 5761$ $-\$ 598$ respectively; $p<0.05$ ). There was no significant difference in the costs associated with hematologic/lymphatic, central nervous system/psychiatric, metabolic/nutritional, skin/subcutaneous tissue, or cardiovascular AEs.

Conclusions

The costs associated with treatment-related AEs among elderly MM patients were substantial. Patients treated with 10 incurred higher costs after gastrointestinal, respiratory, and pain-related AEs, while patients treated with $\Pi$ incurred higher costs after pyrexia/chills, decreased appetite/anorexia, fatigue, or infections.

\section{P295}

Economic burden of adverse events associated with immunotherapy and targeted therapy for metastatic melanoma in the US elderly population

Jackson Tang ${ }^{1}$, Zhiyi Li', Syed Mahmood ${ }^{2}$, Sameer Ghate ${ }^{2}$

${ }^{1}$ Asclepius Analytics, New York, NY, USA; ${ }^{2}$ Novartis Pharmaceuticals

Corporation, East Hanover, NJ, USA

Correspondence: Jackson Tang (jtang@asclepiusanalytics.com)

Journal for ImmunoTherapy of Cancer 2017, 5(Suppl 2):P295 


\section{Background}

The National Comprehensive Cancer Network guidelines for the firstline treatment of metastatic melanoma (MM) recommend the use of immunotherapy $(\mathrm{IO})$ or targeted therapy (TT) (if BRAF-mutated), both of which are associated with treatment-related adverse events (AEs). The occurrence of AEs is associated with increased healthcare resource use and costs. The objective of this study is to estimate the incremental costs of experiencing treatment-related AEs among elderly $\mathrm{MM}$ patients receiving $\mathrm{IO}$ monotherapy or $\mathrm{T}$.

Methods

A retrospective cohort study was conducted using Medicare research identifiable files from 2006 to 2014 . Eligible patients had $\geq 1 \mathrm{MM}$ diagnosis, $\geq 1$ prescription for an IO (ipilimumab or pembrolizumab) or $\Pi$ (dabrafenib, trametinib, vemurafenib, or dabrafenib+trametinib) that was FDA-approved during the study period, and a minimum of 3 months of follow-up. 10 categories of AEs were identified based on a review of $I O$ or $T$ package inserts, as well as AEs that were common among MM. The incremental cost for each AE category was determined by comparing the 30-day expenditures (inpatient + outpatient) between patients with the AE versus patients without the AE using a generalized linear model with a log-link function and gamma distribution, adjusting for baseline covariates. All costs were inflated to 2017 USD.

Results

A total of 425 patients were included. The mean age was 73.4 years ( $\mathrm{SD} \pm 13.8$ ), 61\% were male, 94\% were white, and the mean baseline Charlson comorbidity index was 8.5 ( $\mathrm{SD} \pm 2.3)$. Table 1 summarizes the adjusted 30-day incremental cost of each AE. The adjusted 30day incremental cost was highest for respiratory AEs $(\$ 24,150 ; 95 \%$ confidence interval $[\mathrm{Cl}] \$ 17,630-30,671)$, followed by central nervous system/psychiatric disorders (\$21,932; 95\% Cl \$16,011-27,854), metabolic/nutritional disorders $(\$ 19,776 ; 95 \%$ Cl $\$ 14,239-25,314)$, skin/ subcutaneous tissue AEs $(\$ 19,183 ; 95 \% \mathrm{Cl} \$ 14,195-24,170)$, fever (pyrexia) and/or chills $(\$ 18,976 ; 95 \%$ Cl $\$ 13,663-24,289)$, pain (\$18,406; 95\% Cl \$13,805-23,008), cardiovascular AEs $(\$ 16,393 ; 95 \%$ $\mathrm{Cl} \$ 12,131-20,655)$, hematologic/lymphatic AEs $(\$ 15,850 ; 95 \% \mathrm{Cl}$ $\$ 11,888-19,813)$, gastrointestinal AEs $(\$ 13,699 ; 95 \%$ Cl $\$ 10,138-$ 17,261 ), and other AEs (e.g. decreased appetite/anorexia fatigue, infections (including folliculitis)) $(\$ 9,754 ; 95 \% \mathrm{Cl} \$ 7,315-12,192)$.

\section{Conclusions}

Among elderly MM patients in the United States, the incremental costs of treatment-related AEs are substantial. These findings may help to inform comparisons between treatments, and thereby aid in clinical and budgetary decision-making in this population.

\section{P296}

Exosomes shuttle TREX1-sensitive IFN-stimulatory dsDNA from irradiated cancer cells to dendritic cells

Claire Vanpouille-Box', Julie Diamond ${ }^{1}$, Nils Rudqvist ${ }^{1}$, Karsten Pilones ${ }^{1}$, Yasmeen Sarfraz', Silvia Formenti ${ }^{2}$, Sandra Demaria'

${ }^{1}$ Weill Cornell Medicine, New York, NY, USA; ${ }^{2}$ Weill Cornell, New York, NY, USA

Correspondence: Sandra Demaria (claire.vanpouille.box@gmail.com) Journal for ImmunoTherapy of Cancer 2017, 5(Suppl 2):P296

\section{Background}

Radiotherapy (RT) used at immunogenic doses leads to accumulation of cytosolic dsDNA in the cancer cells, which activates interferon type I (IFN-I) via the CGAS/STING pathway. Cancer cell-derived IFN-I is required to recruit BATF3-dependent dendritic cells (DCs) to poorly immunogenic tumors and trigger anti-tumor immune responses in combination with immunotherapies. Importantly, we have recently demonstrated that TREX1 regulates radiation immunogenicity by degrading cytosolic dsDNA (Vanpouille-Box et al., 2017 Nat Commun). Tumor-derived dsDNA has also been shown to be critical for cGAS/ STING-mediated IFN-I by tumor-infiltrated DCs (Woo et al., 2014 Immunity). Here we hypothesized that activation of DCs by tumorderived dsDNA is modulated by RT and regulated by TREX1.
Exosomes are secreted by cancer cells and can carry dsDNA. Thus, we also tested whether tumor-derived exosomes (TEX) can deliver dsDNA to DCs.

Methods

TEX were purified from supernatants of mouse carcinoma TSA or TSA knock-in for Trex1 (TSA ${ }^{\mathrm{KI}}{ }^{\text {Trex1}}$ ) cells that were mock-treated (UT-TEX) or irradiated with 3 doses of 8Gy (RT-TEX). Protein composition and content of dsDNA was analyzed. TEX were incubated in vitro with primary DCs, or used to vaccinate BALB/c mice $(n=6)$ by 3 s.c. injections followed by challenge with TSA cells to evaluate the development of protective anti-tumor immunity. Tumor-specific $\mathrm{CD}^{+} \mathrm{T}$ cells were identified using $\mathrm{H} 2-\mathrm{L}^{\mathrm{d}} / \mathrm{AH} 1$ peptide pentamers.

Results

Double-stranded DNA content of RT-TEX was significantly higher than that of UT-TEX. In vitro, RT-TEX but not UT-TEX induced the upregulation CD40, CD80 and CD86 on DCs, and the production of IFNb, which was dependent on STING expression by DCs. When TREX1 is upregulated in TSA ${ }^{\mathrm{KI} T r e x 1}$ cells, dsDNA amount of RT-TEX was markedly reduced, indicating that it is largely derived from cytosolic dsDNA present in the irradiated parent cells. Most importantly, enforced TREX1 expression abrogated the ability to RT-TEX to induce IFN-I in recipient DCs. In vivo, vaccination with UT-TEX led to $100 \%$ tumor outgrowth, while 2/6 mice vaccinated with RT-TEX were protected from tumor development. Remaining tumors grew significantly slower compared to UT-TEX treated animals. Tumor-specific $\mathrm{CD}^{+} \mathrm{T}$ cells were significantly increased in the tumors and spleen of RT-TEX vaccinated mice.

Conclusions

Overall these results identify RT-TEX as a mechanism whereby IFNstimulatory dsDNA is transferred from cancer cells to DCs. Importantly, they also demonstrate that TREX 1 in the irradiated cancer cells regulate the production of IFN-I by DCs. Findings further support the use of RT doses that do not induce TREX1 to achieve in situ vaccination by RT.

P297

Predicting the efficacy of combination immunotherapy in animal models using tumor microenvironment immune cell profiling

Ava Vila-Leahey, Genevieve Weir, Alecia MacKay, Valarmathy Kaliaperumal, Cynthia Tram, Marianne Stanford

Immunovaccine, Halifax, NS, Canada

Correspondence: Ava Vila-Leahey (avilaleahey@imvaccine.com) Journal for ImmunoTherapy of Cancer 2017, 5(Suppl 2):P297

\section{Background}

Due to the complex nature of the tumor microenvironment, combinations of immune modulating compounds are likely required for maximal clinical benefit in advanced cancer patients. In our previous work, we have demonstrated that a therapeutic cancer vaccine combined with metronomic cyclophosphamide (mCPA) can enhance T cell infiltration of the tumor. This treatment can be added to treatments such as anti-PD-1 to provide enhanced immune infiltration and better tumor control in murine models. A part of this increased efficacy was due to the increased levels of these molecules in the tumors of treated animals. In this study, we have conducted a systematic flow cytometry survey of checkpoint molecules expressed on $T$ cells within the tumor microenvironment after immune therapy, and utilized this to predict the potential of combining vaccine therapy with other clinical stage antibodies targeting the checkpoint system.

Methods

HPV16 E7-transformed C3 cells were implanted subcutaneously into C57BL/6 mice, and then treated with mCPA (20mg/kg/day PO), followed by vaccination with HPV16 E7 $7_{49-57}$ peptide in a DepoVax ${ }^{\mathrm{TM}}$ platform (DPX-R9F). Additional mice were also treated with anti-PD-1 (200 mg) or anti-OX40 (100 mg). Tumors were collected for analysis 10 days post-vaccine treatment, and digested for staining for flow cytometry to observe differences in infiltrating immune cells and markers on tumor infiltrating immune cells. 
Results

We found that expression of the inhibitory checkpoint markers such as PD-1 and TIM-3 were increased, but activating checkpoint molecules GITR and OX40 were not increased, on T cells after treatment with $\mathrm{DPX} / \mathrm{mCPA}$, and no checkpoint molecules are altered further with either anti-PD-1 or OX40 antibody. We performed a tumor challenge study with the C3 model and antibodies targeting either PD-1, GITR or OX40 to determine whether targeting a molecule upregulated by the vaccine would have greater effect than targeting a molecule that is not altered. Antibodies targeting PD-1, GITR or OX40 had significant synergistic activity with DPX-R9F and MCPA treatment, but anti-PD-1 antibody was most potent in tumor growth inhibition. (Fig. 1)

Conclusions

In conclusion, we have found that an extensive tumor immune profile of treatment regimes in animal models can accurately predict the efficacy of combination immunotherapy, and activating checkpoint molecules do not necessarily enhance the efficacy of treatment in our tested models. Current studies are exploring additional checkpoint molecules in this model that were shown to be elevated in tumor infiltrating $\mathrm{CD}^{+} \mathrm{T}$ cells with vaccine treatment to maximize combination immunotherapy potential.

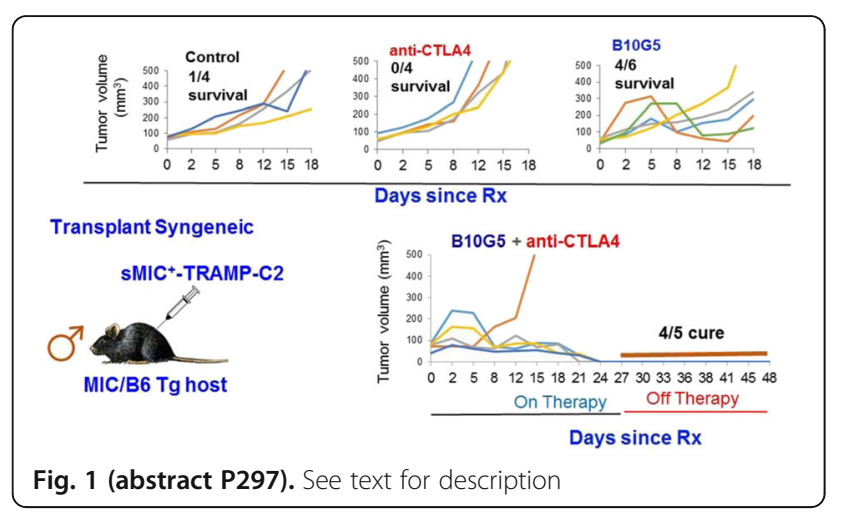

\section{Emerging Models and Imaging}

\section{P298}

Utilization of murine breast cancer models in preclinical immunooncology pharmacology

Dylan Daniel, Sumithra Urs, Kevin Guley, David Draper, Alden Wong, Dan Saims, Scott Wise, Maryland Rosenfeld Franklin

Ml Bioresearch, Ann Arbor, MI, USA

Correspondence: Dylan Daniel (ddaniel@molecularimaging.com)

Journal for ImmunoTherapy of Cancer 2017, 5(Suppl 2):P298

\section{Background}

The field of immuno-oncology is persistently challenged by the need for more syngeneic mouse models in any given tissue type. The most prevalently used syngeneic model for breast cancer is the 4T1 mammary cancer cell line. The 4T1 cell line has useful traits for immunooncology research including a highly metastatic phenotype that leads to extensive lymph node and lung metastasis. The tumors have a highly immunosuppressed microenvironment with regulatory $\mathrm{T}$ cells (Tregs) and large numbers of granulocytic myeloid derived suppressor cells (G-MDSCs). Radiation can induce changes in an immunosuppressive microenvironment, and focal beam radiotherapy remains an important therapeutic strategy for the treatment of breast cancer. For this reason, we established a radiation dose response on established 4T1 tumors for the purpose of guiding future immunotherapy combinations.
Nonetheless, there are some important challenges with using the 4T1 model, in particular with studying the activity of T cell checkpoint inhibitors and costimulatory antibodies. Mice bearing 4T1 tumors develop a fatal pulmonary hypersensitivity upon repeated treatment with rat antibodies to PD-1, PD-L1 or OX40. 4T1 tumors are completely resistant to PD-1 pathway blockade and the hypersensitivity reaction makes it challenging to perform combination studies with novel test agents.

Methods

We have characterized two mammary cancer alternatives to $4 \mathrm{~T} 1$, EMT-6 and E0771. The in vivo growth characteristics and baseline immune cell profiling have been completed for these models with some notable immune profile differences. Further, we have completed an efficacy study of E0771 using an array of immunotherapies. Results

All three models have a similar proportion of CD4+ T cells and Tregs. In contrast, 4T1 has a much larger proportion of G-MDSCs while E0771 is almost entirely lacking in G-MDSCs with EMT-6 in between the two. The content of monocytic MDCSs (M-MDSCs) is nearly reciprocal with E0771 having a proportionately high population and 4T1 having a minimal number. These immune profile characteristics can be factored into model selection decisions.

An efficacy study in the E0771 model showed that it is highly sensitive to several checkpoint inhibitors, including anti-PD-1, anti-PD-L1, and anti-CTLA-4. E0771 shows a more modest response to anti-LAG3. Costimulatory agonist antibodies to OX40, CD137 (4-1BB) and glucocorticoid induced TNFR-related protein (GITR) were highly active. The indoleamine 2,3-dioxygenase 1 (IDO1) inhibitor epacadostat had no impact on the growth of E0771.

Conclusions

These data enable rational combination strategies and provide alternatives to breast cancer studies in $4 \mathrm{~T} 1$.

\section{P299}

Cell therapy - TRAcking, Circulation, \& Safety (CT-TRACS): The Health and Environmental Sciences Institute (HESI)'s new collaborative effort to address the challenges of cell therapies translation

Brooke Helfer', Charles O'Hanlon'1, Patricia Murray², Keiji Yamamoto ${ }^{3}$, Gregory Mullen ${ }^{4}$, Carla Herberts ${ }^{5}$, William Shingleton ${ }^{6}$, Lucilia Mouriès ${ }^{7}$ ${ }^{1}$ Celsense, Inc, Pittsburgh, PA, USA; ${ }^{2}$ University of Liverpool, Liverpool L69 3BX, United Kingdom; ${ }^{3}$ Takeda Pharmaceutical Company Limited, Fujisawa, Kanagawa, Japan; ${ }^{4}$ King's College London, London, United Kingdom; ${ }^{5}$ Medicines Evaluation Board, Netherlands, 3531 AH Utrecht, Netherlands; ${ }^{6} \mathrm{GE}$ Healthcare, Amersham, United Kingdom; ${ }^{7} \mathrm{ILSI}$ Health and Environmental Sciences Institute, Washington, DC, USA

Correspondence: Brooke Helfer (brooke@celsense.com)

Journal for ImmunoTherapy of Cancer 2017, 5(Suppl 2):P299

\section{Background}

Cell therapies show great therapeutic promise in the field of immunotherapy. To realize their full clinical potential there is a need for greater understanding of their mode of action, migration after administration, delivery, persistence at sites of action, and whether their localization or distribution may cause safety issues. There are several existing and emerging tools available to develop pharmacokinetics data on cell-derived therapies to improve our understanding but adoption by investigators has been limited. Furthermore, the regulatory landscape is not clearly defined for these emerging therapeutics. Methods

The Health and Environmental Sciences Institute's Emerging Issues Committee recently launched a multi-sector collaborative subcommittee to identify key needs for assessing the safety of cell therapies and identify opportunities to meet these needs. This program, the Cell Therapy - TRAcking, Circulation, \& Safety (CT-TRACS) subcommittee, provides a platform for developers, researchers, regulators, imaging specialists and other stakeholders to interact, discuss challenges and identify best practices to ensure that therapies are 
safe and effective. The sub-committee aims to bring awareness on how existing cell tracking technologies, methods, and best practices can benefit the clinical translation of these new therapies. Results

Since its inception in December 2015, CT-TRACS gathered more than 60 members from 25 organizations across the United States, Europe and Japan. The sub-committee has convened monthly and the focus of the group has been narrowed to Cell Fate, i.e., distribution, survival/engraftment and phenotype, post-administration, in vivo, as well as evaluating the tumorigenic potential of cell-based therapies. Our initial goals have been to: 1 . evaluate current cell-based therapies safety assessment practice and tools; 2 . develop best practices for application of available tools for safety assessment of cell therapies and/or identify gaps in safety assessment; 3 . organize a workshop to present findings of the sub-teams and develop recommendations for next steps; and 4. initiate a manuscript describing the needs and gaps identified, to build confidence in safety assessment approaches for clinical applications. To date, we have held our first scientific session at an international cell therapy meeting and are compiling the results of a survey of therapeutic stakeholders' needs and wishes in the imaging of cellular therapeutics, survey shared herein.

Conclusions

The CT-TRACS project is open to all current HESI members as well as new participants with relevant technical expertise. The program encourages inquiries by those with interest in providing support for these innovative efforts.

\section{P300}

PSMA-associated PET imaging of CAR T cells

IL Minn ${ }^{1}$, David Huss², Hye-Hyun Ahn', Tamara Chinn², Andrew Park',

Jon Jones ${ }^{2}$, Mary Brummet ${ }^{1}$, Steven Rowe', Polina Sysa-Shah', Yong Du', Hyam Levitsky², Martin Pomper ${ }^{3}$

${ }^{1}$ Johns Hopkins Medical Institutions, Baltimore, MD, USA; ${ }^{2}$ Juno

Therapeutics, Seattle, WA, USA

Correspondence: Hyam Levitsky (david.huss@junotherapeutics.com); Martin Pomper

Journal for ImmunoTherapy of Cancer 2017, 5(Suppl 2):P300

\section{Background}

Chimeric antigen receptor (CAR) T cells have demonstrated clinical benefit in numerous hematological malignancies and hold promise for application in solid tumors. As a "living drug", CAR T cells traffic throughout the body with dynamic expansion and contraction kinetics. This dynamic nature limits the utility of standard blood pharmacokinetic analyses where the temporal and spatial distribution of CAR T cells is only partially captured. Therefore, a non-invasive imaging technique that allows tracking of CAR T cells may enhance the understanding of in vivo CAR-T behavior and inform associations with safety and efficacy. Previous attempts to track gene modified T cell therapies with PET imaging have been limited by immunogenicity of PET reporters, poor tissue penetration of large molecular weight PET ligands and/or limited sensitivity.

Methods

To improve the ability to track CAR T cells, we engineered CD19directed CAR T cells to express a truncated form of human prostatespecific membrane antigen (tPSMA) as a reporter. We then used the PSMA-directed small molecule PET ligand $\left[{ }^{18} \mathrm{~F}\right] \mathrm{DCFPyL}$ for in vitro and in vivo CAR T visualization.

Results

We demonstrate high level surface co-expression of tPSMA and the CD19-directed CAR. The addition of tPSMA to the CAR T cells did not impact in vitro or in vivo anti-tumor functionality. Phantom imaging studies demonstrated that PET with $\left[{ }^{18} \mathrm{~F}\right] \mathrm{DCFPyL}$ could reliably detect as few as 4000 CD19-tPSMA CAR T cells in a 50uL volume. We developed a spontaneous metastatic acute lymphoblastic leukemia model by subcutaneously injecting CD19+ Nalm6ffLuc-eGFP tumor cells into immunodeficient NSG mice. Metastatic lesions were observed in liver, spleen and bone marrow, providing an opportunity to evaluate CAR $\mathrm{T}$ infiltration into primary subcutaneous and secondary disseminated tumor sites. CD19tPSMA CAR T cell treatment eradicated Nalm6 tumors from mice up to 90 days post-treatment. PET/CT with $\left[{ }^{18} \mathrm{~F}\right]$ DCFPyL visualized infiltration of CAR T cells at primary and metastatic tumor sites, which was confirmed by immunohistochemical analyses.

Conclusions

These preclinical results establish a new technology for whole body, non-invasive tracking of CAR T cells and support translation into the clinic.

\section{P301}

A dual in vivo and in silico system to model tertiary lymphoid structure formation and anti-tumor immune response in the murine tumor microenvironment

Rana Falahat, Yohsuke Yagawa, Mark Robertson-Tessi, Susan L. Zhou,

Alexander Anderson, James J. Mulé, Adam Mailloux

H. Lee Moffitt Cancer Center, Tampa, FL, USA

Correspondence: Adam Mailloux (adam.mailloux@moffitt.org)

Journal for ImmunoTherapy of Cancer 2017, 5(Suppl 2):P301

\section{Background}

Tertiary Lymphoid Structures (TLS) are highly organized foci of lymphocytes and antigen-presenting cells that predict increased survival across multiple solid tumors, and are associated with a previously identified gene expression signature composed of twelve chemokines (12-CK-GES) [1]. These chemokines likely result from chronic lymphotoxin-beta receptor activation on resident stromal cells or by paracrine production involving subsequent infiltrates. The exact nature and timing of this induction is unclear due in part to insufficient models of TLS formation. Here, we build upon insights gained from the 12-CK-GES using an implantable three-dimensional bioscaffold to study the interaction of developing murine tumor, endogenous infiltrate, and implanted stroma. Concurrently, we use this system to parameterize an integrated mathematical model of the microenvironment, which can then re-inform our three-dimensional bioscaffold model.

\section{Methods}

Injectable bioscaffolds were prepared using primary lymph node reticular fibroblast cells implanted in either Matrigel or chitosan hydrogels loaded with recombinant Lymphotoxin-a1 $\beta 2$ (LT) or individual chemokines from the 12-CK-GES, with or without murine MC38 colon carcinoma cells. In some experiments, recombinant factors were loaded in lipid-coated silica microparticles to delay release. Preparations were then injected subcutaneously in C57BL/6 mice. Implants were resected at later time points and dissociated for flow cytometry or cryosectioned for histology and immunofluorescent staining. Resulting data, along with those from in vitro chemotaxis assays, were used to parameterize an in silico model of TLS formation simulated in two dimensions using fixed stromal cells, three types of immune cells, and discrete chemokine fields.

Results

LT, when implanted with stromal cells, can induce organized aggregates of endogenous lymphocytes, significantly increase infiltration of $\mathrm{T}$ cells, B cells, and dendritic cells (all $p<0.05$ ), and prevent the growth of MC-38 tumors in resected implants $(p<0.001)$. In silico model runs predict polarized activation of stromal cells and subsequent production of CCL19, CCL21, and CXCL13 is sufficient to compartmentalize lymphoid aggregates into discrete $\mathrm{B}$ and $\mathrm{T}$ cell zones and promote anti-tumor activity.

Conclusions

This dual-model system has identified an important role for LT activation of stromal cells in the induction of TLSs and suggests components of the 12-CK-GES are vital for TLS organization.

\section{References}

1. Messina JL, Fenstermacher DA, Eschrich S, et al. 12-Chemokine gene signature identifies lymph node-like structures in melanoma: potential for patient selection for immunotherapy? Scientific Reports 2012; 2:765-770 
P302

Characterizing immunotherapy-induced lymphocyte infiltration at the single patient level using CANscript ${ }^{\mathrm{TM}}$, an ex-vivo human tumor model

Munisha Smalley, Basavaraja Shanthappa, Hans Gertje, Mark Lawson, Baraneedharan Ulaganathan, Allen Thayakumar, Laura Maciejko,

Padhma Radhakrishnan, Aaron Goldman

Mitra Biotech, Woburn, MA, USA

Correspondence: Aaron Goldman (msmalley@mitrabiotech.com)

Journal for ImmunoTherapy of Cancer 2017, 5(Suppl 2):P302

\section{Background}

The presence and activity of lymphocytes within the tumor is critical for clinical response to cancer immunotherapy, such as immune checkpoint blockade. Poor lymphocyte infiltration into the tumor, known as a 'cold' phenotype, is associated with modest clinical response. High baseline infiltration of effector lymphocytes is considered 'hot', and patients are predicted to respond more favorably to treatment. Despite these fundamental predictive biomarkers, patient-to-patient response and durability remains highly variable. There is an urgent gap in available methods to study lymphocyte infiltration, trafficking and spatial heterogeneity induced by different cancer immunotherapies in individual patients.

Methods

Here, we used CANscript ${ }^{\mathrm{TM}}$, an ex-vivo human tumor model that recapitulates and preserves the native, patient-autologous tumor microenvironment, including peripheral blood mononucleated cells (PBNC). Utilizing tissue from breast cancer patients classified as either 'cold' $(N=5)$ or 'hot' $(N=5)$, we studied lymphocyte infiltration under pressure of immune checkpoint blockade for $72 \mathrm{~h}$ using pembrolizumab (a-PD-1) and avelumab (a-PDL-1). Using flow cytometric analysis, we characterized infiltrating lymphocytes, and coupled these data with multiplex immunohistochemistry $\left(\mathrm{CD}^{+}, \mathrm{CD}^{+}, \mathrm{CD}^{+}, \mathrm{CD}^{+} 6^{+}\right.$, $\mathrm{CD}_{25^{+}}$) to map proximity of tumor cells to lymphocytes before and after treatment, ex-vivo.

Results

We determined that immune checkpoint blockade induced unique patterns of migration and infiltration of effector T-cells $\left(T_{\text {eff }}\right), T$-regulatory $\left(T_{\text {reg }}\right)$ cells and natural killer (NK)-cells in 'hot' vs 'cold' tumors. Furthermore, we determined that, in some instances, 'cold' tumors can be driven towards a 'hot' phenotype characterized by trafficking of active immune lymphocytes following treatment, which corresponded to differential ratio of $T_{\text {eff }}$ to $\mathrm{T}_{\text {reg }}$ compared to baseline.

\section{Conclusions}

Taken together, these data demonstrate the utility of CANscript ${ }^{\mathrm{TM}}$ as a platform to characterize response to immunotherapy in a spatial context. Such an advance in our preclinical methods to study immuno-modulators at the individual patient level can help guide treatment decisions for clinicians while simultaneously functioning as a platform to study and discover mechanisms of clinical efficacy for emerging drugs.

\section{References}

1. Brijwani N, Jain M, Dhandapani M. Rationally co-targeting divergent pathways in KRAS wild-type colorectal cancers by CANscript technology reveals tumor dependence on Notch and Erbb2, Sci. Rep. 2017;7:1502-1513.

2. Majumder B, Baraneedharan U, Thiyagarajan S. Predicting clinical response to anticancer drugs using an ex vivo platform that captures tumour heterogeneity, Nat. Comm. 2015;6:6169-6183.

\section{P303}

Immune checkpoint inhibitor responses in humanized mouse melanoma models using patient-derived xenografts

Rajasekharan Somasundaram, Marilda Bequiri, Ling Li, Meenhard Herlyn, Meaghan Kiernan, Kar Muthumani, Hyeree Choi

The Wistar Institute, Philadelphia, PA, USA

Correspondence: Rajasekharan Somasundaram (shyam@wistar.org) Journal for ImmunoTherapy of Cancer 2017, 5(Suppl 2):P303

\section{Background}

Melanoma patients develop resistance to both chemo- and targeted-therapy drugs. Promising pre-clinical and clinical results with immune checkpoint inhibitors using antibodies directed against CTLA-4 and PD-1 have re-energized the field of immunebased therapies in melanoma. However, only a third of melanoma patients respond to immune checkpoint blockade. Currently available mouse xenograft and transgenic mouse melanoma models have several short comings and are unable to address the basis of drug resistance and immune non-responsiveness that are frequently observed in melanoma patients. Thus, there is an urgent need to establish an in vivo model with a human immune microenvironment that can address issues of therapy resistance.

Methods

For this, our laboratory has developed a humanized mouse melanoma model using patient-derived xenografts (PDX). Immunodeficient NSG mice are reconstituted with human CD34+ cells and after 7-9 weeks, mature human CD45+ cells are observed in circulating blood. Humanized mice were then challenged with HLA-matched melanoma PDX and the functional ability of human immune cells to restrict tumor growth is monitored.

Results

Delayed tumor growth was observed in humanized mice indicating in-vivo sensitization of human immune cells to melanoma. This was confirmed by in-vitro demonstration of human lymphocytes from tumor-bearing mice showing enhanced cytokine expression after stimulation with melanoma antigen peptides. Further, cytotoxic Tcells derived from melanoma peptide stimulation could functionally lyse tumor cells in vitro. In preliminary therapy studies, most tumorbearing humanized mice treated with anti-PD-1 antibody showed restricted tumor growth. Anti-PD-1 antibody therapy resulted in enhanced infiltration of CD4+ and CD8+ T-cells that correlated with tumor response.

Conclusions

Our results suggest that humanized mouse melanoma model can be explored further to understand the causes of therapy resistance and immune non-responsiveness.

\section{P304}

Predicting pre-clinical tumour response to anti-PD-1 immunotherapy with computational modelling

Damijan Valentinuzzi ${ }^{1,2}$, Urban Simončic ${ }^{1}{ }^{1,2}$, Katja Uršič ${ }^{3}$, Martina Vrankar ${ }^{3}$, Maruša Turk', Robert Jeraj, ${ }^{1,2,4}$

${ }^{1}$ Jožef Stefan Institute, Ljubljana, Slovenia; ${ }^{2}$ Faculty of Mathematics and

Physics - University of Ljubljana, Ljubljana, Slovenia; Institute of

Oncology Ljubljana, Ljubljana, Slovenia, Ljubljana, Slovenia; ${ }^{4}$ University of Wisconsin - Madison (WI)

Correspondence: Damijan Valentinuzzi (damijan.valentinuzzi@ijs.si)

Journal for ImmunoTherapy of Cancer 2017, 5(Suppl 2):P304

\section{Background}

Majority of patients fail to respond to anti-programmed death-1 (anti-PD-1) immunotherapy and the reasons for this remain largely unknown. The aim of this study was to develop a computational model, able to predict antitumour response to anti-PD-1 on the basis of key biological properties that might serve as predictive biomarkers of response.

Methods

Interplay between tumour cells and tumour infiltrating lymphocytes (TILs) is described with deterministic population model. It incorporates intrinsic tumour parameters (growth rate (k)), as well as other biological parameters, such as major histocompatibility complex (MHC) class I and PD-1 ligand (PD-L1) appearance on tumour cells, PD-1 appearance on TILs, anti-PD-1 pharmacodynamics, dosing and scheduling regimen, etc. Most of the parameters were taken from literature. The remaining free parameters ( $k$, TILs infiltration rate, tumour cells-TILs interaction rate) were fitted to experimental data, where B16-F10 melanoma was treated with anti-PD-1 [1]. Predictive ability of the model was tested on independent experiment from literature, namely B16-OVA treated with 
anti-PD-1 [2]. Simulated tumour growth curves were compared to experimental data and sensitivity study of key parameters was performed.

Results

Simulated tumour growth curves are in good agreement with experimental data. Maximum deviation of simulated control tumour volume (solid line - blue) to experimental data (blue squares) is $-25 \%$ (day 13 ). On the other hand, the model slightly underestimates the effect of treatment with anti-PD-1. Maximum deviation of simulated anti-PD-1-treated tumour volume (solid line - red) to experimental data (red squares) is $+40 \%$ (day 17 ). Sensitivity study reveals that dosing and scheduling regimen does not importantly affect treatment outcome (data not shown). The most sensitive parameter of the model is MHC class I appearance. By modulating MHC class I appearance from $1 \%$ to $100 \%$, while keeping other parameters fixed, we are able to simulate responders as well as nonresponders to anti-PD-1.

\section{Conclusions}

Model predictions of antitumour response to anti-PD-1 are within expectations. The predictions might be even more reliable if model parameters were, due to their inter-patient variability and presumably dynamic nature, actually measured for every specific experiment/patient. The emphasis should be on MHC class I appearance as it might be one of the predictive biomarkers of response to anti-PD-1.

\section{References}

1. Kleffel, Sonja, et al. Melanoma cell-intrinsic PD-1 receptor functions promote tumor growth. Cell. 2015; 162.6:1242-1256.

2. Sánchez-Paulete, Alfonso R., et al. Cancer immunotherapy with immunomodulatory anti-CD137 and anti-PD-1 monoclonal antibodies requires BATF3-dependent dendritic cells. Cancer discovery. 2016; 6.1:71-79.

\section{P305}

Unravelling the immune contexture of pre-invasive lesions of the lung by multispectral imaging

Angela Vasaturo', Celine Mascaux², Mihaela Angelova', Benedict Buttard', Jean-Paul Sculier ${ }^{3}$, Jerome Galon ${ }^{1}$

${ }^{1}$ Centre de Recherche des Cordeliers, Université Pierre et Marie Curie,

Sorbonne Universités, Paris, France; ${ }^{2}$ Aix Marseille University, APHM,

Marseille, France; ${ }^{3}$ Institut Jules Bordet, Centre des Tumeurs de I'Université

Libre de Bruxelles, B-1000 Brussels, Belgium, Bruxelles, Belgium

Correspondence: Jerome Galon (angelavasaturo@gmail.com)

Journal for ImmunoTherapy of Cancer 2017, 5(Suppl 2):P305

\section{Background}

Lung cancer is the leading cause of cancer deaths world- wide and despite advances in therapy, the overall survival rate for lung cancer patients remains only $15 \%$.

As most of sporadic cancers, lung cancer emerges from pre-neoplastic lesions characterized by morphological and molecular changes. If morphological changes of pre-invasive bronchial lesions are well characterized, the cause and effect relationship between those changes and the immune response is still un- known. Though, we identified gene expression alterations that suggest a role of the innate and adaptive immunity in the transformation towards carcinoma.

Methods

In order to characterize the evolution of the immune response in pre-invasive bronchial lesions, we have optimized different multispectral 7 colors immunofluorescence panels, by using the Tyramide Signal Amplification (TSA) technology.

Results

We have performed multiplex staining on FFPE human bronchial biopsies ( $N=114)$ at 8 successive morphological stages of lung squamous carcinogenesis, from normal, to low grades dysplasia, high grades, to carcinoma. Images of each biopsy have been acquired multispectrally and digitally analyzed to identify and quantify the density and the tissue distribution of different immune cell types. We aimed to characterize the immune infiltrates at different stages of carcinogenesis and elucidate the role of different cell subtypes in tumor development and progression and the possible causal relationship between the immune phenotypes in pre-neoplastic lesions and tumor progression and patient prognosis.

Conclusions

Evaluation of the immune contexture and prognostic assessment of precancerous lesions of the lung may identify promising new biomarkers for early detection and targets of novel therapeutic strategies for lung cancer.

Immune Modulation, Cytokines, and Antibodies

P306

A protein extract from fermented wheat germ promotes NK cell-mediated lymphoma eradication in mouse xenografts Gustavo Barisone', Yunpeng Ma ${ }^{1}$, Mastewal Abuhay', Robert O'Donnell ${ }^{1}$ Kathleen Lundeberg', Sonia Gowda', William Murphy', Joseph Tuscano ${ }^{1}$ ${ }^{1}$ University of California Davis, Sacramento, CA, USA; ${ }^{2}$ University of California, Sacramento, CA, USA

Correspondence: Joseph Tuscano (gabarisone@ucdavis.edu) Journal for ImmunoTherapy of Cancer 2017, 5(Suppl 2):P306

\section{Background}

Proteomic and genomic data has allowed for the development of promising targeted agents for NHL [1]. Most have acute and chronic toxicities that limit efficacy. The use of complementary and alternative medicines has increased during the last decade. However, scientific evidence of their efficacy is scarce. Fermented wheat germ extract (FWGE) has been claimed to have anti-cancer properties in many tumor types. FWGE therapeutic activity has been attributed to its content of benzoquinones [2].

Methods

A protein fraction (FWGP) was isolated by FPLC and proteins identified by mass spectrometry. Direct cytotoxic was studied in vitro using $\mathrm{NHL}$ cell lines. Immunomodulatory properties were evaluated ex vivo by measure immune cell activation in human PBMCs and isolated NK cells. In vivo experiments used $\mathrm{nu} / \mathrm{nu} \mathrm{NHL}$ xenografts with or without NK cell depletion; endpoints were tumor volume and toxicity. In vivo immunomodulatory effects were evaluated by treating tumor-free BALB/c mice with FWGP and measuring NK cell killing activity and degranulation.

Results

FWGP was cytotoxic in 17 cancer cell lines (IC50 $=20-171 \mu \mathrm{g} / \mathrm{ml}$ in $\mathrm{NHL}, 12-27 \mu \mathrm{g} / \mathrm{ml}$ in colon and $70-144 \mu \mathrm{g} / \mathrm{ml}$ in lung) and induced apoptosis by increasing levels of caspase-3, PARP, BAK, BAD and p53, while reducing levels of AKT. FWGP increased $\%$ NK cells, production of $\mathrm{IFg}$ and $\mathrm{GrB}$, and NK-mediated killing. In vivo efficacy was confirmed, with no toxicity, in pre-emptive and established models. In vivo treatment with FWGP+rituximab was as effective as R-CHOP, with $90 \%$ complete remission. NK depletion resulted in no response to FWGP. These results support the hypotheses that FWGP augments NK-mediated tumor killing. Proteomic profiling identified 844 proteins. An active fraction consisted of 169 proteins.

Conclusions

FWGP represents a promising immunomodulatory agent with antitumor activity, minimal toxicity and low cost. Our results suggest FWGP has direct lymphomacidal activity by inducing apoptosis and indirect anti-tumor efficacy by enhancing NK-mediated tumor eradication. Further experimental validation will allow translation of al "alternative" product into mainstream medicine.

References

1. Abuhay, M., et al. The HB22.7-vcMMAE antibody-drug conjugate has efficacy against non-Hodgkin lymphoma mouse xenografts with minimal systemic toxicity. Cancer Immunol Immunother. 2016; 65(10):1169-75.

2. Hidvegi, M., et al. MSC, a new benzoquinone-containing natural product with antimetastatic effect. Cancer Biother Radiopharm. 1999. 14(4):277-89. 
P307

Expression and function of PD-1 and TIM-3 in non-small cell lung cancer (NSCLC)

Jonathan Travers, Krtisten McEachern, Srimoyee Ghosh, Sridhar

Ramaswamy, David Jenkins

${ }^{1}$ TESARO Inc., Waltham, MA, USA

Correspondence: David Jenkins (dianna.bartel@ashfieldhealthcare.com)

Journal for ImmunoTherapy of Cancer 2017, 5(Suppl 2):P307

\section{Background}

The use of anti-programmed cell death protein 1 (PD-1) and PD-Ligand 1 agents in the treatment of non-small cell lung cancer (NSCLC) has been well established but many patients are either intrinsically resistant or become refractory during therapy. One potential resistance mechanism is the upregulated expression of additional checkpoint receptors such as $\mathrm{T}$ cell immunoglobulin and mucin domain 3 (TIM-3), a transmembrane receptor that binds multiple putative ligands, and that has been shown to negatively regulate the function of $T$ cells that coexpress PD-1 [1].

\section{Methods}

We examined the immunophenotype and checkpoint receptor expression of over $100 \mathrm{NSCLC}$ samples from primary surgical resections. From a subset of these samples, we evaluated T cell functional status by gene expression analysis on sorted PD-1+ and TIM-3+ CD8+ T cells as well as ex vivo stimulation assays to evaluate cytokine production. Furthermore, we used ex vivo and in vivo studies to assess the effect of blockade of PD-1 and TIM-3 alone and in combination on $\mathrm{T}$ cell activation and anti-tumor activity.

Results

We showed that primary NSCLC samples display heterogeneity in both their baseline immune infiltrate and also PD-1 and TIM-3 checkpoint receptor expression. We examined mRNA expression of multiple immune genes on sorted PD-1+ and TIM-3+ CD8+ T cells, and found that PD-1/TIM-3 double positive cells express reduced interleukin-2 (IL-2) and tumor necrosis factor alpha (TNFa), but similar mRNA levels of interferon gamma (IFNY) when compared to double negative cells. This phenotype is recapitulated in CD8+ T cells derived from patient samples stimulated with PMA and ionomycin, where we found PD-1 and TIM-3 double positive cells to be significantly deficient in IL-2, but not IFNY production. Importantly, in addition to their expression being associated with T-cell dysfunction, we also found that blockade of PD-1 and TIM-3 was associated with increased T cell activation and anti-tumor activity in ex vivo and in vivo models, suggesting a potential functional role for the inhibition of TIM-3, in addition to PD-1, in the enhancement of anti-tumor immunity.

\section{Conclusions}

Taken together, these data provide further evidence that TIM-3 may play a role in intrinsic resistance to single agent anti-PD1 therapy in NSCLC and support evaluating the combination of anti-PD-1 and anti-TIM-3 agents in the clinic.

\section{References}

1. Koyama, et al. Adaptive resistance to therapeutic PD-1 blockade is associated with upregulation of alternative immune checkpoints. Nat Commun. 2016;7:10501.

\section{P308}

A PD-1 x CTLA-4 bispecific DART ${ }^{\circledR}$ protein with optimal dual checkpoint blockade and favorable tolerability in non-human primates

Alexey Berezhnoy ${ }^{1}$, Kurt Stahl ${ }^{1}$, Kalpana Shah', Tim Gaynutdinov ${ }^{1}$, Gurunadh Chichili ${ }^{1}$, Daorong Liu', Rebecca Johnson ${ }^{1}$, Ross La Motte-Mohs ${ }^{1}$, Jessica Hill', Jonathan Li $i^{2}$, Sergey Gorlatov', Valentina Ciccarone', Ralf Alderson', Hua Li', James Tamura', Jennifer Brown ${ }^{1}$, Jon Wigginton ${ }^{1}$, Ezio Bonvini ${ }^{1}$, Paul Moore ${ }^{1}$, Syd Johnson ${ }^{1}$

${ }^{1}$ Macrogenics, INC, Rockville, MD, USA; ${ }^{2}$ Macrogenics, INC, South San Fancisco, CA, USA

Correspondence: Alexey Berezhnoy (berezhnoya@macrogenics.com) Journal for ImmunoTherapy of Cancer 2017, 5(Suppl 2):P308

\section{Background}

Immunotherapy with the combination of monoclonal antibodies that block PD-1 and CTLA-4 has shown clinical benefit beyond that observed with either mAb alone. A PD-1xCTLA-4 bispecific DART protein was designed to induce antitumor immunity through simultaneous targeting of both checkpoint pathways via administration of a single molecule. The DART protein increases checkpoint blocking activity on PD-1/CTLA-4 dually expressing cells, while displaying distinct immunological effects of CTLA-4 blockade in vivo absent evidence of toxicity. Methods

A PD-1xCTLA-4 DART protein was engineered as a tetravalent bispecific molecule from humanized anti-PD- 1 and anti-CTLA-4 mAb sequences in a human hinge-stabilized IgG4 backbone. PK, PD and toxicology studies were performed in cynomolgus monkeys.

Results

The PD-1xCTLA-4 DART molecule demonstrated binding to immobilized PD-1 protein and PD-1-expressing cells lines, inhibition of PD-1 interaction with PD-L1 or PD-L2, as well as reversal of PD-1-mediated T-cell signal inhibition in gene-reporter assays comparable to that supported by a replica of nivolumab. Similarly, binding, ligand blocking and rescue of CTLA-4-mediated T-cell suppression was comparable to that supported by a replica of ipilimumab. The DART molecule demonstrated activation properties comparable to the combination of ipilimumab and nivolumab replicas in a variety of human primary T-cell assays and showed enhanced B7-ligand binding blockade over that mediated by the ipilimumab replica on PD-1/CTLA4 double-positive cells. In the cynomolgus monkey, the PD-1xCTLA-4 DART molecule exhibited a PK profile consistent with that of an IgG4 and was well tolerated, with no mortality or significant adverse findings up to $75 \mathrm{mg} / \mathrm{kg} \mathrm{QWx3}$, the highest dose tested. T-cell expansion in the peripheral blood and lymphoid organs was observed, which was attributable to the CTLA-4 blocking arm, since no such finding was observed with similar or higher doses of the anti-PD-1 constituent of the bispecific molecule.

Conclusions

- PD-1xCTLA-4 DART protein binds and blocks its targets, with increased activity on dual PD-1/CTLA-4-expressing cells.

- The DART molecule enhances T-cell responses in vitro to the level achieved by a combination of nivolumab and ipilimumab replicas.

- PD-1xCTLA-4 DART protein was well tolerated in cynomolgus monkeys, with a safety profile similar to that observed with PD-1 blockade alone, while demonstrating biological effects of CTLA-4 antagonism.

The favorable safety and tolerability profile of the PD-1xCTLA-4 DART molecule combined with its enhanced activity on PD-1/CTLA-4 doublepositive cells suggest a potential for an improved therapeutic window for PD-1/CTLA-4 co-blockade strategies, with the administration of a single molecule providing dosing convenience and ease of incorporation into additional therapeutic regimens.

\section{P309}

Treatment with heterodimeric IL-15 promotes effector T cell infiltration into tumors

Cristina Bergamaschi', Konstantinos Dimas', Dimitrios Stellas ${ }^{1}$, Bethany Nagy ${ }^{1}$,

Shawn Jensen², Bernard Fox², Barbara Felber', George Pavlakis

${ }^{1}$ National Cancer Institute at Frederick, Frederick, MD, USA; ${ }^{2}$ Earle A Chiles Research Institute, Portland Providence Cancer Center, Portland, OR, USA

Correspondence: Cristina Bergamaschi (cristina.bergamaschi@nih.gov) Journal for ImmunoTherapy of Cancer 2017, 5(Suppl 2):P309

\section{Background}

The presence of tumor-infiltrating effector $T$ cells is considered the most predictive biomarker for clinical benefit in response to immunotherapies. IL-15 is a cytokine important for the proliferation, activation and mobilization of lymphocytes, including natural killer and 
CD8 ${ }^{+} \mathrm{T}$ cells. We have previously shown that bioactive IL-15 in vivo comprises a complex of the IL-15 chain with the IL-15 receptor alpha chain that are together termed heterodimeric IL-15 (hetIL-15). Several preclinical models have indicated the ability of IL-15 to enhance the response of the immune system against cancer, and based on these results hetIL-15 has advanced to clinical trials.

Methods

We have produced hetIL-15 and tested its anti-tumor activity in several murine cancer models. Analysis of lymphocytes in lymphoid organs and in tumors was performed by flow cytometry and multicolor immunohistochemistry. Chemokine and cytokine levels were determined using electrochemiluminescence (MSD) and ELISA assays. Results

Repeated injections of hetIL-15 in mice were effective in delaying tumor growth in the MC38 colon carcinoma, TC-1 cervical carcinoma and B16 melanoma models. The combination of hetIL-15 and adoptive cell transfer of melanoma specific Pmel-1 cells showed antitumor efficacy in B16-bearing mice in absence of lymphodepletion. The E0771 orthotopic breast cancer model showed delay in tumor progression and significantly reduced lung metastasis upon hetlL-15 treatment. A significantly reduced onset of lung metastasis was also observed in 4T1 breast cancer-bearing mice. Flow cytometry and multi-color immunohistochemistry assays showed increased trafficking and persistence of $\mathrm{CD}^{+} \mathrm{T}$ cells, including tumor specific T cells, into the tumors and an increased $\mathrm{CD}^{+} /$Treg ratio, upon hetIL- 15 administration. Importantly, hetlL-15 treatment led to preferential enrichment of adoptively transferred tumor-specific $C D 8^{+} T$ cells in the B16 tumor in an antigen-dependent manner. Tumor infiltration by CD8 ${ }^{+} \mathrm{T}$ cells was accompanied by increased plasma levels of CXCL10. Tumor-resident $\mathrm{CD}^{+}{ }^{+} \mathrm{T}$ cells showed features of activated effector cells with enhanced proliferation $\left(\mathrm{Ki}_{6} \mathrm{7}^{+}\right)$and high cytotoxic potential $\left(\right.$ Granzyme $B^{+}$). Upon ex-vivo stimulation, an increased frequency of both $\mathrm{CD}^{+}$and $\mathrm{CD}^{+} \mathrm{T}$ cells producing IFNg was observed in the tumors of mice treated with hetIL-15.

\section{Conclusions}

Our results show that hetlL-15 administration may be a general method to enhance T cell entry in non-inflamed tumors, increasing the success rate of immunotherapy interventions. Preclinical cancer studies support the use of hetlL-15 in tumor immunotherapy approaches to promote the development of anti-tumor responses by favoring effector over regulatory cells. The effect of hetlL-15 on metastasis establishment in orthotopic models may provide synergies against metastatic disease.

\section{P310}

CSF1/CSF1R signaling blockade triggers release of matrix-degrading proteases in mouse models

Stefan Bissinger ${ }^{1}$, Martina Schmittnaegel ${ }^{2}$, loanna Keklikoglou',

Michele De Palma², Sabine Hoves' ${ }^{1}$, Carola Ries ${ }^{1}$

${ }^{1}$ Roche Innovation Center Munich, 82377 Penzberg, Germany; ${ }^{2}$ The

Swiss Institute for Experimental Cancer Research (ISREC), School of Life Sciences Ecole Polytechnique Fédérale de Lausanne (EPFL), Lausanne,

Switzerland

Correspondence: Stefan Bissinger (stefan.bissinger@roche.com) Journal for ImmunoTherapy of Cancer 2017, 5(Suppl 2):P310

\section{Background}

The heterotypic interplay between cancer cells and their microenvironment provides an opportunity for therapeutic targeting. The abundant tumor-associated macrophage (TAM) infiltrate can be substantially reduced in mouse tumor models and cancer patients by colonystimulating factor 1 receptor (CSF1R) signaling blockade [1]. Notably, TAM depletion provides marked clinical benefits in diffuse-type tenosynovial giant cell tumors $[2,3]$. However, facial edema is reported as the most common adverse event of TAM elimination in patients [2,4]. We here sought to gain insight into the molecular mechanisms mediating edema formation. To this aim, we characterized antibody exposure, CSF1 levels and an array of extracellular matrix-degrading and restructuring metalloproteinases (MMPs) in tumor-bearing and tumor-free mice treated with an anti-CSF1R antibody.

\section{Methods}

Western Blotting and multiplex assays of tumors and sera of anti-mouse CSF1R mAb (clone 2G2)-treated mice showed an association of TAM elimination with an early intratumoral and systemic release of a specific set of MMPs, including MMP-2, -3 and -8 , in multiple transplant (MC38, E0771, KPL-4 and PyMT) and de novo (MMTV-PyMT) tumor models.

Results

In addition, we found that the early increase of MMPs in the face of CSF1/CSF1R pathway blockade was independent of tumor burden and was accompanied by a significant increase of body weight in tumor-free mice following long-term exposure to the antibody. The body weight gain may therefore indicate the enhanced retention of body fluids. Discontinuation of the CSF1R antibody reinstated unaltered body weight and MMP levels. We excluded platelets and neutrophils as sources of MMP release, even if they accumulated in tumor-bearing mice during CSF1R inhibition. We also examined the effects of blocking the CSF1R ligand CSF1, which is one of the two known ligands of the CSF1R [2,4]. CSF1 antibodies had no impact on the binding of IL-34 (the second CSF1R ligand) to CSF1R. Similar to CSF1R blockade, an anti-mouse CSF1 antibody (clone 5A1) provoked an early systemic surge of a subset of MMPs.

Conclusions

Collectively, our data suggest that CSF1 and CSF1R blocking antibodies induce the release of a distinct set of matrix-degrading proteases (MMP-2,-3 and -8), but not metastasis-promoting proteases (MMP-9 and -12), which may be potentially causative of edema formation. Further studies may inform optimized dosing schedules of CSF1/CSF1R targeting regimens for cancer patients.

\section{References \\ Ries CH, et al. Cancer Cell 2014;25(6):846-59. \\ 2. Cassier PA, et al. Lancet Oncol 2015;16(8):949-56. \\ Tap WD, et al. N Engl J Med 2015;373(5):428-37. \\ 4. Papadopoulos KP, et al. Clin Cancer Res. 2017.}

P311

Clinical outcomes of PD-1 inhibition by PD-L1 expression level across malignancies in 204 consecutive patients in a real world oncology setting

Kenneth Byrd', Tristan Hayes ${ }^{1}$, Mike Martin², Lee Schwartzberg ${ }^{2}$,

Ari Vanderwalde

'University of Tennessee Health Sciences Center, Memphis, TN, USA;

${ }^{2}$ West Cancer Center, Germantown, TN, USA

Correspondence: Kenneth Byrd (kbyrd@westclinic.com)

Journal for ImmunoTherapy of Cancer 2017, 5(Suppl 2):P311

\section{Background}

The utility of the PD-L1 biomarker in predicting response to anti-PD1 agents has been inconsistent across malignancies. In this study, we describe outcomes of patients treated with anti-PD-1 agents by PDL1 expression level.

Methods

Molecular profiling of tumors in patients with advanced cancer was performed at West Cancer Center using Caris Molecular Intelligence Profile testing, which includes PD-L1 percentage assessed by immunohistochemical staining. Patients were included in this retrospective analysis if they were treated with a PD-1 inhibitor and had available PD-L1 results between November 2014 and May 2017. Patients were assessed by PD-L1 expression level, defined as negative ( $0 \%$ expression) or positive ( $>1 \%$ expression), regardless of staining intensity. PD-L1 positive samples were further subclassified into PDL1 low (1-4\%), intermediate (5-49\%) and high ( $\geq 50 \%)$. Best overall response using RECIST 1.1 criteria was retrospectively assessed using 2-physician review of radiologic data. Progression free survival (PFS) and overall survival (OS) were assessed using the Kaplan-Meier method.

Results

204 patients with quantifiable PD-L1 expression were treated with PD-1 inhibitors. Primary tumors included 125 non-small cell lung cancers, 31 melanomas, 12 renal cell carcinomas, and 36 others. 110 
(54\%) tumors were PD-L1 negative and $94(46 \%)$ were PD-L1 positive (22 [11\%] low, 37 [18\%] intermediate, and 35 [17\%] high). ORR was $39 \%$ for PD-L1 positive versus $17 \%$ for PD-L1 negative $(p=<0.001)$. Best response for each PD-L1 level is shown in Table 1. The estimated median PFS was 6.4 months for PD-L1 positive versus 3.0 months for PD-L1 negative (HR 0.59; $p=0.001 ; 95 \% \mathrm{Cl}, 0.43$ to 0.81 ). The estimated median OS was 17.3 months for PD-L1 positive versus 6.9 months for PD-L1 negative (Fig. 1; HR 0.64; $p=0.021,95 \% \mathrm{Cl}, 0.44$ to 0.94). Increasing PD-L1 expression was associated with a statistically significant improvement in PFS and OS. There was a statistically significant difference among PFS and OS with increasing PD-L1 levels (PFS $p=0.002$; OS $p=0.026$ ). Multivariate analysis did not identify tumor type as a predictor of response.

Conclusions

PD-L1 staining of any level predicted for improvements in ORR, PFS and OS with PD-1 inhibitors across multiple malignancies. The higher the PD-L1 staining, the greater the likelihood of benefit. These data provide important real world confirmation for the potential utility of global PD-L1 testing in clinical practice, regardless of malignancy.

\section{P312}

AGEN2034, a novel anti-PD-1 antibody that combines effectively with CTLA-4 pathway blockade to enhance $T$ cell activity

Dhan Chand ', David Savitsky', Ana Gonzalez', Christopher Clarke', Andrea Schuster², Elise E. Drouin', Jeremy D. Waight', Cornelia Mundt ${ }^{2}$ Gerd Ritter ${ }^{1}$, Taha Merghoub ${ }^{4}$, David Schaer ${ }^{4}$, Rikke B Homlgaard ${ }^{4}$, Roberta Zappasodi', Marc van Dijk ${ }^{5}$, Jennifer S. Buell ${ }^{1}$, Jean-Marie Cuillerot ${ }^{1}$, Robert Stein , Nicholas S Wilson

${ }^{1}$ Agenus Inc., Lexington, MA, USA; ${ }^{2}$ Former employee of Agenus Switzerland Inc., Basel, Switzerland; ${ }^{4}$ Memorial Sloan Kettering Cancer Center, New York, NY, USA; ${ }^{5}$ Agenus Switzerland Inc., Basel, Switzerland Correspondence: Dhan Chand (dhan.chand@agenusbio.com) Journal for ImmunoTherapy of Cancer 2017, 5(Suppl 2):P312

\section{Background}

PD-1 (or CD279) is a co-inhibitory receptor that suppresses T cell function upon binding to its ligands, PD-L1 or PD-L2. PD-1 signaling functions cooperatively with CTLA-4 to limit T cell activation during priming by antigen presenting cells, leading to reduced proliferation, cytokine and chemokine production and cell survival. Anti-PD-1 antibody therapies that block the interaction between PD-1 and its ligands have shown durable clinical benefit both as single agents, but particularly in combination with antibodies that antagonize CTLA-4.

\section{Methods}

AGEN2034 (anti-PD-1; lgG4) was discovered using a proprietary mammalian display technology, Retrocyte Display ${ }^{\mathrm{TM}}$. Binding kinetics and affinity to PD-1 were characterized by surface plasmon resonance. Cell-based potency was determined in a Jurkat PD- $1^{+}$NFAT reporterbased assay where luciferase activity was measured as an endpoint of PD-1/PD-L1 antagonism. The pharmacological effects of AGEN2034 alone or in combination with CTLA-4 pathway blockade using a novel high affinity anti-CTLA-4 IgG1 antibody, AGEN1884, was assessed in vitro using peripheral blood mononuclear cells (PBMC) from healthy donors. Prior to human clinical trials, pharmacokinetic and pharmacodynamic profiling of AGEN2034 were performed in cynomolgus monkeys both alone and in combination with CTLA-4 blockade.

Results

AGEN2034 selectively binds to human and cynomolgus PD-1 with high affinity $(\mathrm{Kd}<1 \mathrm{nM})$ and sub-nanomolar $\mathrm{EC}_{50}$. The $\mathrm{Fc}$ interactions of AGEN2034 are minimized via selection of a human IgG4 Fc region. In primary human immune cell assays, AGEN2034 showed a dose-dependent increase in $T$ cell cytokine and proliferative responses. Notably, AGEN2034 combined effectively with AGEN1884 in a dose-dependent manner to further enhance $T$ cell responsiveness. AGEN2034 was well tolerated, and no-observed-adverse-effect level (NOAEL) could be established up to $40 \mathrm{mg} / \mathrm{kg}$ in non-human primates. Furthermore, the combination of AGEN2034 and antiCTLA-4 blockade promoted a dynamic pharmacodynamic response in cynomolgus monkeys, including a transient increase in proliferation and ICOS (inducible co-stimulator molecule) expression in a subset of central memory and effector memory $\mathrm{T}$ cells.

Conclusions

The functional attributes of AGEN2034 combined with the favorable pharmacokinetic and pharmacodynamic profile in cynomolgus monkeys are ideally suited for clinical development. Moreover, AGEN2034 combined effectively with CTLA-4 blockade in a range of preclinical assays to enhance antigen-specific $T$ cell responsiveness and produced a dynamic pharmacodynamic response in non-human primates. AGEN2034 is currently under evaluation in a Phase $1 / 2$ study in subjects with advanced tumors and cervical cancer (NCT03104699) and clinical studies to evaluate AGEN2034 in combination with AGEN1884 are planned.

P313

In vivo effect of albumin binding domains attached to immune modulators

Haomin Huang, Keneshia Haenssen, Anil Bhate, Supriya Sanglikar, John Baradei, Shan Liu, Senthil Kumar, Zihao Cui, Richard Hampton, Robert Kramer, John Cini

Sonnet BioTherapeutics, Cranbury, NJ, USA

Correspondence: Haomin Huang (johncini@sonnetbio.com); Keneshia Haenssen; Anil Bhate; Supriya Sanglikar; John Baradei; Shan Liu; Senthil Kumar; Zihao Cui; Richard Hampton

Journal for ImmunoTherapy of Cancer 2017, 5(Suppl 2):P313

\section{Background}

Recombinant therapeutic proteins $<50 \mathrm{Kd}$ (eg., receptor ligands, cytokines) exhibit short circulation half-lives (mins/hour vs. days for lgGs) which limit their therapeutic utility. A specific way to increase the pharmacokinetic half-life of these agents is via conjugation to circulating albumin. Here we describe the creation of an albumin binding single chain fragment antibody (ScFv-ABD) that binds albumin in circulation, is recycled by binding to the FcRn (similar to lgG's) and then recycled after cellular uptake resulting in increased half-life of the appended therapeutic protein. A second advantage to linking a therapeutic protein to an ScFv-ABD is improved tumor target delivery as numerous studies have shown that albumin accumulates in tumors and inflamed tissues.

Methods

Sonnet BioTherapeutics, using a XOMA phage library, has developed scFv ABD fusion constructs with several different small therapeutic proteins (recombinant interleukin proteins and scFvs targeting relevant immune-oncology receptors). These various $A B D$ constructs have high binding affinity to mouse, human \& cyno circulating serum albumin thereby preventing renal clearance and retaining benefits of $F c R n$ mediated recycling of albumin for extended PK. Early studies to investigate improved tumor accumulation translates into anti-tumor efficacy in vivo and have shown that ScFv-ABD enhances tumor targeting. Results

Our characterized scFV-ABD constructs have demonstrated that;

1. biologic activity is retained when the therapeutic protein is attached via the $\mathrm{N}$ - or C-terminus suggesting utility for delivering more than one therapeutic protein/scFv;

2. half-life in mouse serum in vivo was extended from minutes to hours/days for three different recombinant proteins and scFv with MWs of $10-80 \mathrm{Kd}$;

3. in an established B6F10 melanoma model these ABD constructs have demonstrated markedly superior reductions in tumor growth and improved overall survival compared to the same constructs without the ABD. Superior efficacy was observed with lower doses and with a single dose of the ScFv-ABD constructs vs free recombinant protein

\section{Conclusions}

We will describe several examples of improved half-life, tumor accumulation and efficacy using our albumin linkage approach that is leading to the selection of drug candidates for clinical development. 
P314

Glioblastoma stem-like cell targeting antibodies identified using yeast display biopanning

Paul Clark, Michael Zorniak, Benjamin Umlauf, Yongku Cho, Eric Shusta,

John Kuo

University of Wisconsin - Madison, Madison, WI, USA

Correspondence: John Kuo (clark@neurosurgery.wisc.edu)

Journal for ImmunoTherapy of Cancer 2017, 5(Suppl 2):P314

\section{Background}

BACKGROUND: Glioblastoma stem-like cells (GSCs) are hypothesized to evade current therapies and cause tumor recurrence, contributing to poor patient survival. Existing cell surface markers for GSCs are developed from embryonic or neural stem cell systems; however, currently available GSC markers are suboptimal in sensitivity and specificity. We hypothesized that the GSC surface proteome could be mined with a yeast display antibody library to reveal novel immunophenotypes.

Methods

MATERIALS AND METHODS: A naïve yeast expression library of single-chain human antibodies ( $\mathrm{scFv}$ ) was mined using biopanning against patient-derived GSCs. Discovered unique clones were characterized for qualitative binding affinity against 5 patient-derived GSC lines, 5 matched non-GSC/GBM lines, and 2 normal cell lines. Presumptive GSC-specific antibodies were purified from yeast, and GSC specificity and affinity determined by confocal microscopy and flow cytometry. GSC targeting in vitro was evaluated using flow cytometry, and in vivo after intravenous administration of near infrared fluorescent tagged scFv to immunodeficient mice harboring orthotopic GBM xenografts.

Results

RESULTS: Nine rounds of positive selection against patient-derived GSCs enriched for GSC-binding SCFv, with selected pools also negatively screened against normal human astrocytes, neural stem cells, and serum-cultured GBM (i.e. non-GSC). We identified 62 unique scFv clones from $\sim 600$ candidates by differential PCR and restriction analysis. Clone 9.7 (heavy chain only, termed VH-9.7) demonstrated specificity against 5 patient-derived GSC lines, with minimal binding to non-GSC and normal controls. Purified VH-9.7, produced predominantly in a monomeric form, had a GSC binding affinity $(\mathrm{Kd})$ of $74.3 \pm 9.85 \mathrm{nM}$. Flow cytometry using $5 \mathrm{GSC}$ lines verified VH-9.7 specific GSC labeling: 17-115-fold higher compared to normal astrocytes and 10-65-fold higher compared to non-GSC/ GBM lines. After intravenous injection, $\mathrm{VH}-9.7$ significantly localized to GSC-derived GBM xenografts in mice [92 \pm 11 relative fluorescent units (RFI), $n=3, p<0.05]$, compared to control non-targeting sCFv $(11 \pm 7.8 \mathrm{RFI})$.

\section{Conclusions}

CONCLUSIONS: Rapid screening via yeast antibody library biopanning identified human-specific antibodies that demonstrated GSC specificity compared to both non-GSC (i.e. bulk of GBM) and normal neural cells. Identified antibodies could potentially be developed into immunotargeted diagnostics and therapeutics in brain cancer.

\section{P315}

A bispecific fusion protein, ACDClx, can selectively activate T cells against glioblastoma cells in vitro

Rebecca Cook', Sierra Bichler ${ }^{1}$, Andrew Diamos ${ }^{1}$, Braeden Schaefer ${ }^{1}$, Andrew Niemann', Hugh Mason', Tsafrir Mor', Rachael Sirianni², Joseph Blattman ${ }^{1}$

${ }^{1}$ Arizona State University, Tempe, AZ, USA; ${ }^{2}$ Barrow Neurological Institute, Phoenix, AZ, USA

Correspondence: Rebecca Cook (Rebecca.McCall@asu.edu) Journal for ImmunoTherapy of Cancer 2017, 5(Suppl 2):P315

\section{Background}

Glioblastoma (GBM) is a highly invasive and fatal form of brain tumor with a median survival of approximately 15 months. Treatment for GBM is hampered by the blood-brain barrier (BBB) and small populations of cells that resist conventional therapies. There is an urgent need for innovative new therapies to target all GBM cells within a patient. Chlorotoxin is a small peptide derived from the venom of the deathstalker scorpion that has been shown to be highly selective for all GBM cells, does not bind healthy tissue, is non-toxic to humans, and has been demonstrated to cross the BBB. To this end, we have designed a $T$ cell engaging molecule, anti-CD3/chlorotoxin (ACDClx), composed of the variable heavy $\left(V_{H}\right)$ and light $\left(V_{L}\right)$ fragments of an anti-CD3 antibody (2C11) tethered to chlorotoxin. Here, we show that $T$ cells can be selectively activated against GBM cells, only in the presence of $A C D C l x$.

Methods

The gene sequence encoding for His-tagged ACDClx was cloned into a deconstructed and improved geminiviral vector and introduced into agrobacteria for needleless infiltration into leaf tissue of Nicotiana benthamiana. ACDClx was extracted from tissue 4 days postinfiltration and purified via nickel affinity chromatography. T cell activation was evaluated via calcium flux assay (Fluo-4) using ionomycin as a positive control and CD69 expression using full length anti-CD3 antibody as a positive control. Activity was measured using freshly isolated mouse splenocytes and mouse GBM cells (GL261-LucNeo). For flow cytometry, lymphocytes were gated from the splenocyte population and further gated based on CD4 and CD8 markers.

Results

Expression and purification from $N$. benthamiana resulted in a yield of 614 ug ACDClx per gram of leaf tissue with greater than $99 \%$ purity (Figure 1A). Expression of CD69 was observed when splenocytes and GBM cells were incubated with ACDClx or anti-CD3 antibody, but not with media alone (Figure 1B). T cell activation as measured by an increase in calcium flux over baseline was observed when ACDClx was added to a mixture of splenocytes and GBM cells (Figure 1C). Flux was not observed when mock isolated protein from mock-infiltrated plants was added to splenocytes and GBM, nor with splenocytes and ACDClx alone.

Conclusions

Our results indicate that $A C D C l x$ can be expressed to high levels in plant tissue and has the capacity to selectively activate $T$ cells in vitro. These results provide further motivation for our current studies of $A C D C l x$ in vitro and in an immunocompetent model of GBM in vivo.

\section{P316}

Impact of anti- PD1 on TIL phenotype and function

Caitlin Creasy ${ }^{1}$, Cara Haymaker ${ }^{2}$, Marie-Andrée Forget ${ }^{2}$, Gopal Singh²,

Coya Tapia², Jeane Marie Painter ${ }^{2}$, Funda Meric-Bernstam²,

Chantale Bernatchez ${ }^{2}$, Aung Naing ${ }^{2}$

${ }^{1} \mathrm{MD}$ Anderson Cancer Center University of Texas Health Graduate

School of Biomedical Sciences, Houston, TX, USA; ${ }^{2}$ University of Texas

MD Anderson Cancer Center, Houston, TX, USA

Correspondence: Chantale Bernatchez (cacreasy@mdanderson.org);

Aung Naing

Journal for ImmunoTherapy of Cancer 2017, 5(Suppl 2):P316

\section{Background}

Therapeutic antibodies targeting PD-1 have demonstrated efficacy in several solid tumor types with durable responses in a subset of patients. However most patients will either not respond to therapy or progress after an initial response. It is not fully understood how this treatment may alter the phenotype or function of Tumor-infiltrating Lymphocytes (TIL). In this study, methods have been optimized to derive TIL from a single core biopsy and applied to growing TIL from pre-treatment and on-treatment biopsies in patients receiving antiPD-1 to investigate drug-induced changes in TIL phenotype and function.

Methods

Tumor samples are obtained from an ongoing Phase II clinical trial of anti-PD-1 in cohorts of patients with rare solid tumor types (NCT02721732). Mandatory core biopsies are taken at baseline and on day 15-21 after the first cycle of anti-PD1 (Pembrolizumab, $200 \mathrm{mg}$ ). TIL are propagated ex vivo utilizing IL-2 and an agonistic anti-4-1BB antibody (Urelumab, BMS), with or without anti-CD3 (clone OKT3). TIL phenotype and function are evaluated after 2 or 3 weeks of culture. 
Results

TIL cultures were first initiated by mincing one core biopsy into 3-6 tumor fragments and cultured in media containing IL-2 and the anti4-1BB mAb. Observed TIL growth was poor with only $11 \%$ of all samples yielding over 2 million TIL per fragment after 3 weeks (2 /17 samples). The addition of anti-CD3 to the regimen dramatically improved TIL outgrowth, with $78 \%$ and $80 \%$ of the baseline biopsy and on-treatment biopsy samples, respectively, growing over 108 million TIL ( $n=38 / 49$ baseline; $24 / 30$ treatment, range 10 million to 357.7 million). Phenotypic analysis of the expanded TIL showed an effector memory differentiation status at both time points. However TIL grown from samples obtained after anti-PD-1 dosing showed enhanced proportion of $\mathrm{CD}^{+} \mathrm{TIL}^{2}$ and enriched CTLA-4 expression in both CD4 and CD8 TIL subsets. PD-1 expression on expanded $\mathrm{CD}^{+}$and $\mathrm{CD} 4^{+}$TIL was maintained or elevated after therapy. Analysis of 4 patients' paired baseline and on-treatment TIL show that $\mathrm{CD}^{+}$and $\mathrm{CD} 8^{+} \mathrm{TIL}$ grown post anti-PD-1 treatment secrete significantly more of the effector cytokines IFN- $\gamma$, IL-13, and TNF- $a$ following anti-CD3 re-stimulation. Conclusions

Our study highlights phenotypical and functional differences of TIL after a single dose of anti-PD-1. Additionally we demonstrate that it is possible to grow TIL in numbers that would be sufficient to proceed with rapid expansion and adoptive cell transfer from one core biopsy. Trial Registration NCT02721732

\section{P317}

In vivo efficacy and mechanism of action of anti-TIGIT monoclonal antibody CASC-674

Peter de Vries, Robert Rosler, Janelle Taylor, Scott Peterson

Cascadian Therapeutics, Inc., Seattle, WA, USA

Correspondence: Peter de Vries (pdevries@cascadianrx.com)

Journal for ImmunoTherapy of Cancer 2017, 5(Suppl 2):P317

\section{Background}

TIGIT is a coinhibitory immune checkpoint receptor expressed on regulatory T cells (Tregs), cytotoxic T cells and NK cells. TIGIT ligands include CD155 and CD112, which are expressed on antigen presenting cells and a variety of tumors. These ligands also bind the activating receptor CD226, often co-expressed with TIGIT, creating a network that modulates adaptive and innate immune response in a manner analogous to the CD28-CTLA4-CD80-CD86 network. We previously reported on the characterization of a panel of fully human antagonistic monoclonal antibodies that bind with subnanomolar affinity to mouse, NHP and human TIGIT and block ligand-receptor interactions and signaling in T cells (AACR, 2017). Here we report on the in vivo mechanism of action and efficacy of CASC-674 in a number of syngeneic tumor models.

Methods

A lead candidate antibody (CASC-674) was selected and produced as mouse lgG2a and tested in syngeneic in vivo mouse tumor models. To explore the mechanism of action in vivo, CT26 tumor bearing mice were treated with CASC-674 alone or in combination with antiPD1/anti-PD-L1 and their tumors and spleens isolated and evaluated by flow cytometry for various lymphoid and myeloid cell subsets. Results

CASC-674 as single agent has significant anti-tumor activity in the anti-PD1/anti-PD-L1 insensitive CT26 colorectal tumor model, resulting in $8 / 10$ complete regressions (CR). Combination treatment with anti-PD-1/PDL-1 did not enhance the anti-tumor effect compared to CASC-674 alone. Tumor free mice from this study were re-challenged 8 weeks after the last treatment with CT26 cells in the opposite flank of the original inoculum and no CT26 tumor growth was observed in any of the mice after 30 days, demonstrating that previous treatments resulted in an immune memory response. In addition to the CT-26 model, CASC-674 demonstrated significant anti-tumor effects in EMT6, H22 and MBT-2 syngeneic mouse tumor models.
In CT26 tumors, treatment with CASC-674 but not an IgG1 version of the same variable domains, shifted the immunosuppressive phenotype to an inflammatory anti-tumor phenotype. CASC-674 alone and in combination with anti-PD1 significantly reduced the \% of Tregs and exhausted (PD1+ Ki67+) CD8+ cells and increased the $\%$ of cytotoxic CD8+ cells (PD-1- Ki67+) and anti-tumor (CD155+) M1 macrophages.

Conclusions

These results combined with the antitumor effect observed suggest Fc functionality is critical for the effect of CASC-674. The observed single agent anti-tumor effect in several different tumor models of CASC-674 and its unique human, non-human primate, and murine cross-reactivity supports consideration of CASC-674 as a therapeutic development candidate.

P318

Anti-tumor efficacy and enhancement of $T$ cell effector functions by EOS084448, an antagonist anti-TIGIT antibody

Gregory Driessens', Julia Cuende', Sofie Denies', Chaterine Hoofd' ${ }^{1}$

Florence Lambolez', Shruthi Prassad', Virginie Rabolli', Anthony Cooper ${ }^{2}$, Christophe Quéva'

${ }^{1}$ iTeos Therapeutics, Gosselies, Belgium; ${ }^{2}$ Adimab, Lebanon, NH, USA

Correspondence: Gregory Driessens

(gregory.driessens@iteostherapeutics.com)

Journal for ImmunoTherapy of Cancer 2017, 5(Suppl 2):P318

\section{Background}

T cell Immunoreceptor with Ig and ITIM domains (TIGIT) is an ITIM domain- containing co-inhibitory receptor preferentially expressed by NK, $\mathrm{CD}^{+}$and $\mathrm{CD} 4^{+} \mathrm{T}$ cells as well as by regulatory $\mathrm{T}$ cells (Treg). Several ligands are described to bind to TIGIT with PVR (CD155) showing the highest affinity. CD226 (DNAM-1), a co-stimulatory receptor also expressed on NK and T cells compete with TIGIT for PVR binding but with a lower affinity. Co-expression of TIGIT and CD226 receptors on T and NK effector cells suggests a role in the fine control of their activation

Methods

Antagonistic anti-TIGIT antibodies were selected by Adimab, LLC. using a synthetic library of human antibodies presented on the surface of yeast. Anti-TIGIT mAbs were characterized for affinity to recombinant human TIGIT (Biacore), for binding to human primary $T$ cells, for competition to CD155 binding as well as for functional activity on cells engineered to express TIGIT or on human primary T cells. An anti-TIGIT mAb cross-reactive to mouse TIGIT was used to evaluate the anti-tumor efficacy of anti-TIGIT in the CT26 syngeneic model.

Results

Anti-TIGIT mAb EOS084448 affinity for human TIGIT was $0.25 \mathrm{nM}$, translating into potent binding to human primary $T$ cells with an average $\mathrm{EC}_{50}$ of $0.09 \mathrm{nM}$. EOS084448 inhibited CD155 binding to TIGIT at the surface of TIGIT-expressing cells with an $E_{50}$ of $0.16 \mathrm{nM}$. EOS084448 antagonistic activity was evaluated in a TIGIT:CD155 reporter bioassay that resulted in activation of the IL-2 promoter and by measuring IFNg secretion by minimally stimulated human primary CD8 ${ }^{+} \mathrm{T}$ cells; EOS084448 potency in these 2 assays was $8 \mathrm{nM}$ and $0.4 \mathrm{nM}$. A surrogate anti-TIGIT mAb with a $26,7 \mathrm{nM} \mathrm{Kd}$ for mouse TIGIT was evaluated in established CT26 mouse tumors. Anti-TIGIT monotherapy delayed CT26 tumor growth and achieved complete response in few mice. Complete tumor regression occurred in most of the animals receiving the anti-TiGIT mAb combined with anti-PD-1 mAb. Anti-tumor efficacy was associated with an increased $\mathrm{CD}^{+} \mathrm{T}$ cell : Treg ratio, an increase $\mathrm{TH} 1 / \mathrm{TH} 2$ cytokine ratio and a transcriptional signature indicating an increased cytolytic $T$ cell activity.

Conclusions

In vitro and in vivo data demonstrate the potential for EOS084448 to promote antitumor immunity and efficacy and supports the rationale for its clinical evaluation. 


\section{P319}

Long-term disease-free survival (DFS) of metastatic melanoma $(\mathrm{mM})$ and renal cell cancer ( $\mathrm{mRCC}$ ) patients following high-dose interleukin-2 (HD IL2)

Joseph Clark', Brendan Curti', Elizabeth Davis ${ }^{3}$, Howard Kaufmann ${ }^{4}$ Asim amin ${ }^{5}$, Ajjai Alva ${ }^{6}$, Theodore Logan?, Ralph Hauke ${ }^{8}$, Gerald Miletello ${ }^{9}$, Ulka Vaishampayan ${ }^{10}$, Douglas Johnson ${ }^{3}$, Richard White ${ }^{5}$, Peter Wiernik ${ }^{11}$, Janice Dutcher ${ }^{12}$

${ }^{1}$ Loyola University, Maywood, IL, USA; ${ }^{2}$ Providence Cancer Center, Portland, OR, USA; ${ }^{3}$ Vanderbilt University, Nashville, TN, USA; ${ }^{4}$ Rutgers University, New Brunswick, NJ, USA; ${ }^{5}$ Carolinas Health Care System, Charlotte, NC, USA; ${ }^{6}$ University of Michigan, Ann Arbor, MI, USA; ${ }^{7}$ University of Indiana, Indianapolis, IN, USA; ${ }^{8}$ Midwest Cancer Center, Omaha, NE, USA; ${ }^{\circ}$ Hematology/Oncology Clinic, LLP, Baton Rouge, LA, USA; ${ }^{10}$ Wayne State University, Detroit, MI, USA; ${ }^{11}$ Cancer Research Foundation of New York, Chappaqua, NY, USA; ${ }^{12}$ Cancer Research Foundation of New York, Bronx, NY, USA

Correspondence: Janice Dutcher (jpd4401@aol.com)

Journal for ImmunoTherapy of Cancer 2017, 5(Suppl 2):P319

\section{Background}

HD IL2 treatment produces durable complete responses (CRs) and surgical CRs. Patients achieving partial response (PR) and stable disease (SD) demonstrate improved survival compared with patients who progress.

Methods

Eleven HD IL2 treatment centers identified patients with survival $>5$ years after HD IL2. DFS was from end of IL2 to June 2017. Treatment courses generally consisted of 2 1-week cycles of HD IL2, 600,000-720,000 U/kg IV every 8 hours. We collected data on patients treated with HD IL2 alone, or HD IL2 plus local therapy (surgery or radiation (SRS) leading to $\mathrm{CR}$ ) with survival $>5$ years after HD IL2.

Results

Ninety-nine patients are reported: $46 \mathrm{mRCC}$ (male 32, female 10, unknown 4) and $53 \mathrm{mM}$ (male 31, female 22). Median age at HD IL2 treatment of mRCC patients is 54 years (range, 39-73 years) and of $\mathrm{mM}$ patients is 53 years (range, 24-76 years). Sites of metastatic disease for $\mathrm{mM}$ patients were lymph nodes (LN), lungs, bone, liver, brain, and other organs, and for MRCC patients were lung, LN, adrenal, bone, and other organs. The majority of patients received 2-3 courses of IL2 (63 of 99 patients) and 18 received 1 course, with the overall number of IL2 courses ranging from 0.5 to 4 courses. Among the $46 \mathrm{mRCC}$ patients, there are $38 \mathrm{CRs}$, 5 surgical CRs and 3 PRs with no further treatment. Among $53 \mathrm{mM}$ patients, there are 42 CRs, 2 near CRs, and 9 Surgical/ SRS CRs without further treatment. DFS in these patients after HD IL2 ranges from $5+$ years to $30+$ years, median $10+$ years. $27 \mathrm{mRCC}$ and 31 $\mathrm{mM}$ are alive $>10$ years after IL2. Long-term toxicity among these 99 patients includes hypothyroidism-5 patients, arthralgias/arthritis-6 patients, vitiligo- 3 patients, and 1 patient each: neuropathy, PVCs, and normal pressure hydrocephalus. Additional patients may be added as records become available.

\section{Conclusions}

We document long-term DFS ( $>5$ years) after CR or PR from HD IL2 alone. Surgical or SRS conversion of PR to CR can produce durable CRs. Long-term DFS was observed in patients with visceral and bone metastases, not only those with LN or lung sites of metastases. Neither age, sex, nor number of courses of IL2 predicted long-term DFS. Chronic toxicity due to IL2 is uncommon in long-term survivors.

\section{P320}

Targeting CD38 beyond haematological malignancies: a panel of anti-CD38 antibodies with unique functional properties

Nina Eissler', Pascal Merchiers', Simone Filosto', Rahul Chaitanya Khanolkar', Beatriz Goyenechea', Dominic Smethurst ${ }^{1}$, Kevin Moulder' ${ }^{1}$, Sergio A. Quezada², Anne Goubier

${ }^{1}$ Tusk Therapeutics, Stevenage, United Kingdom; ${ }^{2}$ UCL Cancer Institute, London, United Kingdom

Correspondence: Anne Goubier (nina.eissler@tusktherapeutics.com) Journal for ImmunoTherapy of Cancer 2017, 5(Suppl 2):P320

\section{Background}

Because of its high level of expression on haematological cancers, depleting antibodies targeting $\mathrm{CD} 38$, an ectoenzyme with hydrolase and cyclase activity, have been generated and showed clinical benefits in particular against multiple myeloma. Interestingly, CD38 is not restricted to haematological cancer cells but also expressed on many different immune subsets including NK and effector T cells, exhausted PD-1 ${ }^{+} \mathrm{T}$ cells, suppressive myeloid cells, and regulatory T and B cells. Anti-CD38 antibodies will therefore not only impact CD38-expressing tumour cells but also both effector and suppressive immune cells, as illustrated by the increased interest for CD38 as a target in Immuno-Oncology.

Methods

An antibody production and screening campaign has been initiated resulting in a panel of fully human CD38-binding antibodies. All antibodies have been screened for their potential to induce ADCC, apoptosis, ADCP, and CDC. The potential to deplete CD38-expressing cells has been verified in vivo in lymphoma engrafted mice. Additionally, all antibodies have been evaluated for their ability to influence effector T cell and NK cell activation.

Results

We have produced a portfolio of antibodies targeting distinct epitopes of CD38. These antibodies exert ADCC when directed against CD38-overexpressing targets, while showing differential capacity to induce $A D C P$ and $C D C$. This translated into differential inhibition of in vivo tumour growth of human lymphoma tumours in SCID mice. Most interestingly, some of these antibodies augment TCR-induced proliferation and activation of human T cells in vitro, with an activity ranging from strong $T$ cell activators that increase proinflammatory cytokine release, to medium or weak activators resulting in low or no cytokine release. In addition, in tumour coculture models, anti-CD38 antibodies increased NK cell activation and proliferation. Regarding CD38 enzymatic function, i.e. hydrolase and cyclase activity, our antibodies display differential profiles in terms of blockade or augmentation of both activities. Effects of anti-CD38 antibodies on immune effector cells, suppressive immune cells as well as tumour cells will be further explored in patient-derived ex vivo tumour models.

Conclusions

We present a portfolio of CD38-targeting antibodies with distinct activity profile. The broad expression and multiple functions described for CD38 underline the importance of being able to choose from a range of antibodies that can address the different functionalities depending on the most prominent role of CD38 in each disease setting. The new class of anti-CD38 antibodies presented here will be further explored for their potential to improve response rates, especially in solid tumours.

\section{P321}

Ibrutinib in combination with agonist aOX40 mAb and CTLA-4

blockade induces Eomes ${ }^{\text {hi }}$ CD8 $\mathrm{T}$ cells and promotes tumor regression

Dana Emerson ${ }^{1}$, Michael Mcnamara², lan Hilgart-Martiszus ${ }^{2}$, Mohammad Farhad $^{2}$, William Redmond ${ }^{2}$

${ }^{1}$ Oregon Health \& Science University, Portland, OR, USA; ${ }^{2}$ Earle A. Chiles

Research Institute, Portland, OR, USA

Correspondence: William Redmond (emersoda@ohsu.edu)

Journal for ImmunoTherapy of Cancer 2017, 5(Suppl 2):P321

\section{Background}

Antagonist monoclonal antibodies (mAb) targeting T cell checkpoints such as CTLA-4 or PD-1 have shown efficacy in treating a subset of patients with metastatic disease. In preclinical models, CTLA-4 blockade synergized with an agonist anti-OX40 mAb to enhance the expansion and effector function of tumor-specific $T$ cells. Previous studies demonstrated that combined aOX40/aCTLA-4 therapy also induced the generation of CD8 T cells expressing high levels of Eomesodermin (Eomes), a transcription factor known to regulate CD8 T cell differentiation and memory. Eomes expression is negatively regulated in T cells by the T cell signaling kinase ITK (interleukin- 2 inducible T cell kinase). Importantly, the FDA-approved Bruton's tyrosine kinase inhibitor ibrutinib also blocks ITK, thus providing a potential means of modulating Eomes expression. We sought to characterize 
these Eomes ${ }^{\text {hi }} \mathrm{CD} 8 \mathrm{~T}$ cells and investigate the mechanisms regulating the generation of this novel subset.

Methods

Wild-type C57BL/ 6 mice were challenged by $1 \times 10^{6}$ TRAMP-C1 tumor cells in the right flank. Wild-type BALB/C mice were challenged by $5 \times 10^{4} 4 \mathrm{~T} 1$ tumor cells orthotopically in the mammary fat pad. Mice were treated starting day 7 with 200ug aCTLA-4 (clone 9D9), 200ug aOX40 (Clone OX86), or 150ug lbrutinib. All treatments were injected i.p. Tumor growth (area) was assessed with microcallipers every 2 to 3 days. Mice were killed when tumors exceeded $175 \mathrm{~mm}^{2}$ for experiments tracking tumor growth and survival. Lymph nodes and tumors were harvested on day 14 to assess and characterize T cell responses by flow cytometry.

Results

Our data revealed that these Eomes ${ }^{\text {hi }}$ CD8 $T$ cells expressed significantly less PD-1 on their surface compared to Eomes ${ }^{\text {lo }}$ CD8 T cells ( $97 \%$ vs. $57 \%$, respectively), while maintaining high levels of IFN-g production within the tumor. Additionally, we confirmed that clinical concentrations of ibrutinib do not inhibit T cell receptor signaling in CD8 T cells. Next, demonstrated that the combination of ibrutinib/ aOX40/aCTLA-4 therapy enhanced the frequency of Eomes ${ }^{\text {hi }}$ CD8 T cells in $4 \mathrm{~T} 1$ tumor-bearing mice, a model of triple negative breast cancer. Additionally, this triple therapy significantly enhanced IFN-g and TNF-a expression by CD4 and CD8 T cells in the tumor and draining lymph nodes, which was associated with tumor regression and enhanced survival in TRAMP-C1 and Myc-CaP models of prostate adenocarcinoma.

Conclusions

Taken together, these data demonstrate that combined ibrutinib/ aOX40/aCTLA-4 therapy induced a robust population of Eomes ${ }^{\text {hi }}$ CD8 $T$ cells with enhanced effector function capable of mediating tumor regression in multiple pre-clinical tumor models.

\section{P322}

Antitumor effects of radiation combined with intratumoral antiTem8 $\mathrm{mAb}$ and IL2

Amy K. Erbe', Kathryn Komro', Arika Feils ${ }^{1}$, Mackenzie Heck', Sabrina VandenHeuvel', Manasi Mohan', Peter M. Carlson', Jacquelyn A. Hank' Alexander Rakhmilevich', Zachary S. Morris ${ }^{1}$, Amit Chaudhary²,

Brad St. Croix ${ }^{3}$, Paul M. Sondel'

${ }^{1}$ University of Wisconsin-Madison, Madison, WI, USA; ${ }^{2}$ Meso Scale

Diagnostics, Rockville, MD, USA; ${ }^{3}$ National Cancer Institute, Frederick, MD, USA

Correspondence: Paul M. Sondel (aerbe@wisc.edu)

Journal for ImmunoTherapy of Cancer 2017, 5(Suppl 2):P322

\section{Background}

Tumor Endothelial Marker 8 (Tem8) is overexpressed on endothelial cells that line the tumor vasculature. Chaudhary et al. [Cancer Cell, 2012] showed in several tumor models that the anti-Tem8 monoclonal antibody, L2mAb, augmented the activity of anti-cancer agents leading to slowed tumor growth. Tem8 is also overexpressed on tumor cells themselves in several tumor types, including melanoma. We have recently shown that radiation (RT) given in combination with intratumoral injection (IT) of a tumor-specific antibody and interleukin-2 (IL2) can elicit an in situ vaccine effect for mice bearing a B78 syngeneic melanoma. Here, we show that RT+L2mAb+IL2 treatment resulted in improved outcome for mice bearing a Tem8+ B78 melanoma.

Methods

B78 melanoma cells were transduced to overexpress Tem8 and injected into C57BL/6 mice. Mice bearing a $\sim 200 \mathrm{~mm}^{3}$ tumor received external beam RT (12Gy), followed by IT injection of L2mAb $(2 \mathrm{mg} / \mathrm{kg})$ and/or IL2 (150,000U) on days 5-9 post-RT. Mice were monitored for tumor growth and overall survival. Statistical differences in tumor volume were determined by Two-Way ANOVA followed by Tukey's test for multiple comparisons, and Log-rank/Mantel-Cox test was used for overall survival. Chi-Square test was used to compare response rates. Results

Treatment with $\mathrm{RT}+\mathrm{L} 2 \mathrm{mAb}$ resulted in slower tumor growth as compared to RT alone $(p<0.001)$. By including IL2 to RT+L2 (i.e. RT
$+\mathrm{L} 2 \mathrm{mAb}+\mathrm{IL} 2)$, tumor growth was significantly reduced as compared to mice treated with either RT alone $(p<0.001)$ or with $\mathrm{RT}+\mathrm{L} 2$ $(p<0.001)$. In our preliminary study, only the combination of RT $+\mathrm{L} 2 \mathrm{mAb}+\mathrm{IL} 2$ yielded any mice that were tumor free (2 tumor-free mice out of 5); treatment with $\mathrm{RT}+\mathrm{L} 2 \mathrm{mAb}$, or RT+IL2 did not generate any tumor-free mice.

Conclusions

Tem8-targeted therapy given in conjunction with other anti-cancer agents has shown promising effects. Prior to this study, anti-Tem8 antibody given in combination with RT or IL2 had not been investigated. Here, we show that RT followed by Tem8-specific antibody significantly improved outcome, and that addition of IL2 further improved outcome. Inhibition of tumor-angiogenesis via anti-Tem8 antibodies may block the function of the Tem8 that is expressed on tumor endothelial cells, slowing tumor growth. However, since RT+L2mAb treatment resulted in improved outcome when given in combination with IL2, antibodydependent-cell-mediated cytotoxicity may also contribute to the mechanism of action of this therapy.

\section{P323}

Novel treatment of cutaneous T cell lymphoma: Targeting TNFR2, an oncogene and marker of potent Tregs, with anti-TNFR2 antibodies

Heather Torrey ${ }^{1}$, Audrey Defusco ${ }^{1}$, Danielle Baum', Ziba Rahbar², Michael Khodadoust ${ }^{2}$, Youn H. Kim², Denise Faustman ${ }^{1}$

'Massachusetts General Hospital/Harvard Medical School, Charlestown, MA, USA; ${ }^{2}$ Stanford University School of Medicine/Cancer Institute, Stanford, CA, USA

Correspondence: Denise Faustman (faustman@helix.mgh.harvard.edu) Journal for ImmunoTherapy of Cancer 2017, 5(Suppl 2):P323

\section{Background}

Tumor necrosis factor receptor 2 (TNFR2) is a lymphoid marker of the most potent regulatory $T$ cell (Treg) subtype, which is enriched in the tumor microenvironment, and a commonly expressed oncogene in human tumors. Anti-TNFR2 antibodies can inhibit both Tregs and tumors with specificity for the tumor microenvironment [1], and recent data shows that TNFR2 is a candidate oncogene in cutaneous T cell lymphoma (CTCL) with recurrent point mutations and gain of function alteration of TNFR2, resulting in abnormal expression of TNFR2 on CD4+CD26- tumor cells [2].

Methods

We screened novel TNFR2-directed antagonistic antibodies for their ability to kill leukemic cells in Stage IV CTCL (Sézary syndrome) subjects with failure on diverse drug regimens, as well as their ability to induce killing of tumor-associated Tregs and unleash effector T cell (Teffector) proliferation. Studies were performed in vitro on sorted CD4+CD26Sézary cells or V-beta specific populations when a tumor was typed. Results

At baseline, CTCL blood samples showed significant burdens of tumor cells within the CD26- subset of CD4 cells (averaging 45-95\% of this subfraction), vs control blood ( $18 \%$ CD26- cells on average). In CTCL subjects, Treg numbers (CD4+CD25hiFoxp3) were elevated at baseline ( $11 \%$ vs $7 \%$ control, $p<0.05)$, Teffectors were depressed $(3 \%$ vs $8 \%$ control, $p<0.05)$, and Treg/Teffector ratios were abnormally elevated ( $8 \%$ vs $1 \%$ control, $p<0.05$ ).

Regardless of underlying therapy used in vivo, TNFR2 antagonism showed dose responsive killing of tumor cells within the CD26fraction of peripheral CD4 T cells. TNFR2 antagonism also had specificity for tumor cells vs CD26- cells from paired controls. In vivo treatment of $C T C L$ subjects with anti-proliferative agents such as methotrexate hindered TNFR2 antagonism driven killing, demonstrating the specificity of TNFR2 antagonism for rapidly proliferating cells.

Dose response experiments in vitro showed TNFR2 antagonism also had the desired dual effect of Treg killing combined with unleashing of Teffector proliferation at 48-72 hours.

Conclusions

TNFR2 is a recurrent genomic gain alteration in CTCL that can potentially be targeted to directly stop tumor cell growth by antibody- 
induced cell death, eliminate Tregs of the tumor microenvironment and unleash Teffector proliferation.

\section{References}

1. Torrey H, Butterworth J, Mera T, et al. Targeting TNFR2 with antagonistic antibodies inhibits proliferation of ovarian cancer cells and tumor-associated Tregs. Sci Signal. 2017;10(462):pii: eaaf8608.

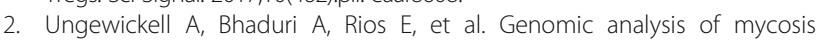
fungoides and Sézary syndrome identifies recurrent alterations in TNFR2. Nat Genet. 2015;47(9):1056-60.

\section{P324}

Co-targeting of mesothelin and CD47 with bispecific antibodies for efficient elimination of solid tumors

Krzysztof Masternak, Limin Shang, Vanessa Buatois, Stefano Majocchi, Eric Hatterer, Xavier Chauchet, Valéry Moine, Lucile Broyer,

Marie H. Kosco-Vilbois, Nicolas Fischer, Walter G. Ferlin

Novimmune S.A., Geneva, Switzerland

Correspondence: Walter G. Ferlin (wferlin@novimmune.com)

Journal for ImmunoTherapy of Cancer 2017, 5(Suppl 2):P324

\section{Background}

Mesothelin (MSLN) is a cell surface glycoprotein overexpressed in several human cancers, including mesothelioma, pancreatic-, ovarian, lung- and gastric cancer. MSLN overexpression is associated with poor prognosis, with serum levels of soluble MSLN a biomarker of disease severity in mesothelioma patients. While a promising target in cancer, monoclonal antibodies (mAbs) targeting MSLN have demonstrated limited efficacy in clinical trials. Current MSLN-targeted approaches in development have thus incorporated novel modalities to enhance tumor-killing potential (e.g., Antibody Drug Conjugates and CAR-T cells). CD47, an immune check-point, interacts with SIRPa providing a 'don't eat me' signal that allows healthy cells to limit elimination by immune cells, in particular macrophages. CD47 upregulation in solid cancers is correlated with poor clinical prognosis, almost certainly by allowing tumor cells to escape immune surveillance by phagocytes. Clinical development of mAbs to CD47 is hindered by the ubiquitous expression of CD47 leading to rapid drug elimination and significant hematological toxicity including anemia. To address these concerns, we have employed a bispecific antibody (biAb) approach that pairs a high affinity anti-MSLN targeting arm to an anti-CD47 arm of an optimized affinity that drives the efficacious binding only on MSLN-positive cells. This MSLN/CD47 biAb approach, therefore, is designed to target the CD47-SIRPa pathway in the tumor microenvironment to more efficiently harness the immune system for tumor eradication.

Methods

Fully human biAbs targeting both MSLN and CD47 were generated. An array of biAbs coupling MSLN-targeting arms binding to different epitopes on MSLN with a common CD47-targeting arm have been tested in antibody dependent cellular phagocytosis (ADCP) and antibody dependent cellular cytotoxicity (ADCC) assays in vitro plus in mouse xenograft experiments. The following human cell lines were used: $\mathrm{NCl}-$ N87 (gastric), HPAC (pancreatic), OVCAR3 (ovarian), Caov-3 (ovarian), $\mathrm{NCl}-\mathrm{H} 226$ (mesothelioma) and MSLN-transfected HepG2 (hepatic).

Results

The MSLN/CD47 biAbs significantly enhanced macrophage-mediated ADCP of NCl-N87, HPAC, OVCAR3 and Caov-3 as compared to the antiMSLN mAb, Amatuximab. In addition, the biAbs also demonstrated superior ADCC of NCl-N87 cells and NCl-H226. When tested in a xenograft tumor model using MSLN-transfected HepG2 cells, therapeutic treatment with MSLN/CD47 biAbs significantly prevented tumor development, while Amatuximab and $\mathrm{B} 6 \mathrm{H} 12$, an anti-CD47 mAb, only slightly delayed tumor growth.

\section{Conclusions}

Using a novel biAb approach that focuses the blockade of the innate immune checkpoint receptor CD47 to MSLN-expressing tumors substantially enhances their elimination in vitro and in vivo. Thus, MSLN/ CD47 targeting biAbs are a potentially superior strategy in managing MSLN-positive solid tumors.
P325

Characterization of the anti-CTLA-4 antibody AGEN1884, including toxicology and pharmacology assessments in non-human primates Randi Gombos', Ana Gonzalez', Mariana Manrique', Dhan Chand',

David Savitsky', Benjamin Morin', Ekaterina Breous-Nystrom², Christopher Dupont ${ }^{1}$, Rebecca Ward', Cornelia Mundt ${ }^{3}$, Benjamin Duckless ${ }^{4}$, Hao Tang ${ }^{4}$, Mark Findeis ${ }^{1}$, Andrea Schuster ${ }^{3}$, Jeremy Waight', Dennis Underwood', Christopher Clarke', Gerd Ritter ${ }^{5}$, Taha Merghoub ${ }^{6}$, David Schaer ${ }^{6}$, Marc van Dijk ${ }^{2}$, Jennifer Buell ${ }^{1}$, Jean-Marie Cuillerot', Robert Stein ${ }^{1}$, Elise Drouin', Nicholas WIIson'

${ }^{1}$ Agenus Inc., Lexington, MA, USA; ${ }^{2}$ Agenus Switzerland Inc., Basel, Switzerland; ${ }^{3}$ Former employee of Agenus Switzerland, Inc., Basel, Switzerland; ${ }^{4}$ Former employee of Agenus Inc., Lexington, MA, USA; ${ }^{5}$ Ludwig Institute for Cancer Research, New York, NY, USA; ${ }^{6}$ Memorial Sloan Kettering Cancer Center, New York, NY, USA

Correspondence: Randi Gombos (randi.gombos@agenusbio.com); Nicholas Willson

Journal for ImmunoTherapy of Cancer 2017, 5(Suppl 2):P325

\section{Background}

Cytotoxic T lymphocyte antigen-4 (CTLA-4) is an important negative regulator of T cell function. Together with CD28, these receptors exemplify a co-inhibitory and co-stimulatory signaling axis that dynamically sculpts the interaction of antigen-specific $T$ cells with antigen presenting cells (APCs). Preclinical studies have demonstrated that anti-CTLA-4 antibodies can enhance tumor-specific immunity through a variety of mechanisms including: i) blockade of CD80 or CD86 binding to CTLA-4; ii) preventing CTLA-4-expressing regulatory $T$ cells from physically removing CD80 and CD86 from the surface of APCs; and iii) selective elimination of CTLA-4-expressing intratumoral regulatory $\mathrm{T}$ cells by an Fcg receptor-dependent mechanism.

Methods

Here we describe the pharmacological and toxicological characterization of a novel human IgG1 anti-CTLA-4 antagonist antibody, AGEN1884. Binding, blocking, T cell activation as well as Fcgamma receptor-mediated activity of AGEN1884 were evaluated in vitro. The activity and tolerability of AGEN1884 was further assessed in vivo using a non-human primate model. Our in vitro and in vivo assessments extended to a direct comparison of AGEN1884 with an IgG2 Fc variant, AGEN2041.

Results

AGEN1884 potently enhanced T cell responsiveness in vitro, and combined effectively with other immunomodulatory antibodies targeting co-inhibitory and co-stimulatory receptors on T cells. AGEN1884 was well-tolerated in non-human primates and was confirmed to modulate cellular and humoral immune responses to co-administered reporter vaccines. In addition to the activity of AGEN1884 as a monotherapy, a memory $T$ cell proliferative response was observed in peripheral blood of animals when co-administered with an anti-PD-1 antibody. Finally, we provide a comparison of the in vitro and in vivo functional properties of an lgG2 variant of AGEN1884, revealing important antibody isotype differences that may have an impact on the design of optimal dosing regimens in patients.

Conclusions

Taken together, the pharmacologic properties of AGEN1884 support its clinical investigation as both a single therapeutic agent and in combination therapies.

\section{P326}

Isoform specific TGF- $\beta$ inhibition in combination with radiation therapy as a novel immune therapeutic approach to cancer therapy

Aditi Gupta', Sadna Budhu', Rachel Giese', Jacques van Snick², Catherine Uyttenhove ${ }^{2}$, Gerd Ritter ${ }^{3}$, Jedd Wolchok', Taha Merghoub ${ }^{1}$ Memorial Sloan Kettering, New York, NY, USA; ${ }^{2}$ Ludwig Institute for Cancer Research Ltd Brussels Branch, Brussels, Belgium; ${ }^{3}$ Ludwig Institute for Cancer Research, Ltd, New York, NY, USA

Correspondence: Taha Merghoub (guptaa@mskcc.org); Jedd Wolchok Journal for ImmunoTherapy of Cancer 2017, 5(Suppl 2):P326 


\section{Background}

TGF- $\beta$ is a pleotropic cytokine, which has emerged as a potential target in cancer treatment due to its dual role in tumorigenesis and homeostasis. There are three isoforms of TGF- $\beta$ (TGF- $\beta 1$, TGF- $\beta 2$ and TGF- $\beta 3$ ), which are secreted by immune and non-immune cells as a latent complex. Depending on the local context, TGF- $\beta$ adopts opposing roles in carcinogensis and in modulating the immune system. These dueling roles of TGF- $\beta$ are dependent on its secretion and activation. Local radiation therapy (RT) can activate TGF- $\beta$ via reactive oxygen species. Such TGF- $\beta$ expression is linked to radioresistance and dose-limiting toxicities, reducing the effectiveness of RT. In these studies, we aim to characterize the effect of RT on the temporal and cell-specific expression patterns of TGF- $\beta$ isoforms in mouse tumor models. This will inform treatment regimens combining isoform specific anti-TGF- $\beta$ therapy with RT.

Methods

Fluorescence-activated cell sorting (FACS): C57BL/6 mice were implanted on the hind limb with B16-F10 melanoma cells. On day 10, tumors were irradiated locally with 15 Gy. Expression of TGF- $\beta$ isoforms was measured at 1, 3 and 5 days post-RT by FACS.

In-vivo: C57BL/6 mice were implanted with tumors and irradiated as described. Mice were treated (10/group) with anti-TGF- $\beta 1$, anti-TGF$\beta 3$ or a pan-TGF- $\beta$ antibody beginning 1 day after RT given intraperitoneally (200 ug/mouse) every other day for 8 doses. Tumor growth and overall survival were monitored. A similar experiment was conducted in the 4T1 breast cancer model, in which mice were treated 1 day prior to radiation.

Results

FACS data indicated that TGF- $\beta 1$ and TGF- $\beta 3$ expression increases on most immune cells in the tumor 1 day after RT, decreases 3 days after RT and reaches a peak 5 days after RT. Preliminary in-vivo studies demonstrate that both aTGF- $\beta 1$ and aTGF- $\beta 3$ as monotherapies have activity against B16 melanoma. In combination with RT, aTGF$\beta 3$ trends towards inhibiting tumor growth. Similar observations were obtained in a 4T1 breast model; however, aTGF- $\beta 3$ alone and in combination with RT as well as aTGF- $\beta 1+$ RT showed a significant delay against tumor growth. No significant differences in survival were seen in either tumor model.

\section{Conclusions}

TGF- $\beta 1$ and TGF- $\beta 3$ are expressed on numerous lymphoid and myeloid cells in B16 tumors and spleens. TGF- $\beta$ isoform expression peaks 5 days post-RT. Anti-TGF- $\beta$ therapy is effective in delaying tumor growth and may synergize with RT in certain cancers. This demonstrates rationale for the use of anti-TGF- $\beta$ therapy to enhance the effectiveness of RT in cancer.

\section{P327}

Intratumor injection of tumor-specific antibody and IL2 triggers in situ vaccination following local radiation therapy

Emily I. Guy', Sara Busche', Peter Carlson', Clinton Heinze', Ciara N. Schwarz ${ }^{1}$ Raghava N. Srirameneni', Jasdeep Kler ${ }^{1}$, Michael M. Meagher' ${ }^{2}$, Amy K. Erbe', Jacquelyn A. Hank', Alexander L. Rakhmilevich', Paul M. Sondel',

Zachary S. Morris'

'University of Wisconsin School of Medicine and Public Health, Madison, WI, USA; ${ }^{2}$ St. Jude Children's Research Hospital/Children's GMP, LLC, Memphis, TN, Memphis, TN, USA

Correspondence: Emily I. Guy (eguy@wisc.edu)

Journal for ImmunoTherapy of Cancer 2017, 5(Suppl 2):P327

\section{Background}

In murine models of $\mathrm{GD}^{+}$melanoma, $\mathrm{GD}^{+}$neuroblastoma, and $\mathrm{EGFR}^{+}$head and neck cancer, we have reported a cooperative interaction between radiation and intratumor (IT) injection of tumorspecific antibody (anti-GD2 hu14.18K322A or anti-EGFR cetuximab). Consistent with a process mediated by antibody-dependent cellmediated cytotoxicity, this interaction required the Fc portion of the antibody, host FcY receptor, NK cells, and tumor expression of the antibody-targeted antigen. In this GD2+ melanoma model, combined treatment with RT + IT-hu14.18-IL2 immunocytokine (a fusion protein of hu14.18 antibody and IL2) markedly enhanced response compared to radiation or IT-IC alone, radiation + IT-hu14.18 antibody, or radiation + intravenous-IC. In those studies, radiation + IT-IC induced an in situ vaccine effect, resulting in a tumor-specific memory $\mathrm{T}$ cell response. Here we test whether IT administration of non-fused tumorspecific antibody and IL2 may elicit an in situ vaccination response following local radiation.

Methods

C57BL/6 mice were flank engrafted with syngeneic GD2+ B78 melanoma and 5-week tumors $\left(\sim 200 \mathrm{~mm}^{3}\right)$ were treated with single fraction 12 Gy radiation, IT-IL2 (150,000U), and/or IT-hu14.18K322A $(50 \mu \mathrm{g})$. IT injections were given daily on days 6-10 after radiation. Outcomes included tumor response and rates of complete regression, overall survival, tumor-specific memory (tested in disease-free mice by contralateral flank injection with B78 melanoma $>90$ days after radiation), and immunohistochemistry on tumors resected at day 12 after radiation.

Results

The combination of local radiation + IT-hu14.18K322A + IT-IL2 resulted in greater tumor regression compared to radiation + IT-hu14.18K322A or radiation + IT-IL2 [50\% (19/38) aggregate complete tumor regression vs $0 \%(0 / 11)$ and $25 \%(3 / 12)$, respectively, $p<0.001]$. No mice were rendered disease-free with these IT-treatments in the absence of radiation. Kaplan-Meier analysis demonstrated improved survival with radiation + IT-hu14.18K322A + IT-IL2 compared to radiation + IT-hu14.18K322A and radiation + IT-IL2 (log-rank $p<0.0001 ; 80 \%$ alive at day 65 vs $0 \%$ and $50 \%$, respectively). Thus far, $100 \%(6 / 6)$ of mice rendered diseasefree by combined radiation + IT-hu14.18K322A + IT-IL2 have rejected subcutaneous B78 re-implantation at $>90$ days after radiation, compared to $0 / 10$ naive control mice. Preliminary immunohistochemistry analyses suggest that IT-IL2 may increase tumor infiltrating CD8+ T cells in this preclinical model.

Conclusions

We present evidence of a cooperative anti-tumor effect with the combination of local radiation and IT injection of both tumor-specific antibody and IL2. Given the widespread availability of tumor-specific antibodies, this may offer a viable approach to pursuing in situ tumor vaccination in many diverse types of cancer using off-the-shelf reagents.

\section{P328}

Therapeutic application of radiation-induced anti-tumor antibody

Tsuguhide Takeshima, Raquibul Hannan

UT Southwestern Medical Center, Dallas, TX, USA

Correspondence: Raquibul Hannan

(Raquibul.Hannan@utsouthwestern.edu)

Journal for ImmunoTherapy of Cancer 2017, 5(Suppl 2):P328

\section{Background}

Radiation therapy (RT) has significant immune modulatory properties that, when strategically integrated with immunotherapy, may improve cancer treatment outcomes. It has been previously reported that RT induces tumor-specific antibodies [1]; however, this response needs to be better characterized and its therapeutic potential evaluated. Antibodies binding to the Fc receptor of dendritic cells (DC) have been shown to direct DC to the tumor site [2]. We hypothesize that radiation-induced anti-tumor antibodies (RT-Ab) increase dendritic cell (DC) trafficking to the irradiated tumor to increase tumor antigen presentation. Here, we explore the therapeutic application of RT-Ab.

\section{Methods}

Multiple syngeneic murine cell lines were used (EG7, B16, LLC, 4T1), along with their respective murine strains. Fluorescence tagged antimouse antibodies (eBiosciences) and anti-CD11c antibodies (eBiosciences) were used for flow cytometry.

Results

Multiple syngeneic murine tumor grafts (B16, LLC, 4T1) were grown in the hind legs of respective murine strains and focally irradiated with a single 15Gy RT dose, with post-RT sera (pRTs) obtained at different time intervals. Flow cytometric analysis using anti-mouse antibodies of the pRTs incubated with the respective tumor cell showed a significant increase in RT-Ab that peaked between days 713. This increase was more pronounced for a single fraction of 15Gy 
when compared to $4.5 \mathrm{~Gy}$ on 5 consecutive days while the tumor growth delay was equal between the two groups. Next, flow cytometry was performed on bone marrow-derived dendritic cells (BMDC) from the respective murine strains co-cultured with pRTs, which showed an increase in the binding of RT-Ab to DCs when compared to normal or non-irradiated tumor bearing mice sera. When an Fc receptor blocking agent was used, this binding was significantly inhibited, which suggested Fc receptor-mediated binding of RT-Ab to the BMDC. Lastly, we demonstrated that RT-Ab-bound-BMDC (RT-DC), when administered as an autologous DC therapy in conjunction with focal RT, increased the therapeutic effect of focal tumor RT significantly compared to multiple control conditions.

\section{Conclusions}

RT induces a tumor-specific antibody response that increases DC trafficking to the tumor via Fc receptor-mediated targeting. This likely contributes to RT-mediated tumor antigen presentation and adaptive anti-tumor immunity. Amplification of the RT-Ab response may have therapeutic applications.

\section{References}

1. Nesslinger NJ et. al. Standard treatments induce antigen-specific immune responses in prostate cancer. Clin Cancer Res. 2007 Mar 1;13(5):1493-502. PubMed PMID: 17332294.

2. Franki SN et. al. Dendritic cells loaded with apoptotic antibody-coated tumor cells provide protective immunity against B-cell lymphoma in vivo. Blood. 2008 Feb 1;111(3):1504-11

\section{P329}

Dual blockade of PD1 and CTLA4 with bispecific antibody XmAb20717 promotes human T cell activation and proliferation Michael Hedvat ${ }^{1}$, Matthew Bernett ${ }^{1}$, Gregory Moore ${ }^{1}$, Christine Bonzon ${ }^{1}$ Rumana Rashid', Seung Chu², Kendra Avery' , Alex Nisthal', Umesh Muchhal ${ }^{1}$, John Desjarlais ${ }^{1}$

${ }^{1}$ Xencor, Inc., Monrovia, CA, USA; ${ }^{2}$ schu@xencor.com, Monrovia, CA, USA Correspondence: John Desjarlais (mhedvat@xencor.com) Journal for ImmunoTherapy of Cancer 2017, 5(Suppl 2):P329

\section{Background}

Treatment of melanoma patients with nivolumab plus ipilimumab increases progression-free-survival compared to each monotherapy. The increase in efficacy of the combination regimen is accompanied by an increase in adverse events. Since PD1 ${ }^{+} \mathrm{CTLA4} 4^{+}$tumorinfiltrating-lymphocytes are dysfunctional in the tumor microenvironment, we developed XmAb20717 to selectively target PD $1{ }^{+} \mathrm{CTLA} 4^{+}$ double-positive $T$ cells in an effort to recapitulate efficacy of the combination regimen while reducing toxicity.

\section{Methods}

XmAb20717 component antibodies binding to PD1 and CTLA4 with favorable stability and functionality were assembled in a bispecific antibody platform with substitutions in the Fc domain to suppress effector function. XmAb20717 was evaluated in vitro by measuring antibody binding and de-repression of super-antigen stimulated peripheral blood lymphocytes (PBMCs) and in vivo by monitoring the engraftment of human PBMCs in NSG mice (huPBMC-NSG) by flow cytometry. To evaluate anti-tumor efficacy we monitored the growth of established cancer cells in huPBMC-NSG following treatment.

Results

Optimized candidate single-chain Fvs were confirmed to bind PD1 and functionally block PDL1 and PDL2 binding to PD1. We also generated optimized anti-CTLA4 Fabs. Anti-PD1 and anti-CTLA4 targeting component antibodies were assembled into XmAb20717, which displayed favorable biophysical and manufacturing properties. XmAb20717 enhanced IL2 secretion in vitro 4.1-fold relative to a negative control antibody ( $p<0.01, n=15$ donors) in response to antigenic challenge of previously stimulated $T$ cells, with 1.6-fold superior activity compared to an anti-PD1 bivalent antibody ( $p<0.01, \mathrm{n}=15$ donors). XmAb20717 enhanced T cell engraftment (9.6-fold) and IFNy secretion (2.9-fold) compared to vehicle controls in huPBMC-NSG mice $(p<0.001, n=10 /$ group), with equivalent activity to a combination regimen of anti-PD1 and anti-CTLA4 bivalent antibodies. Blockade of PD1 and CTLA4 with an equimolar mixture of monovalent component antibodies that compose XmAb20717 lead to inferior activity compared to XmAb20717, suggesting that binding selectivity of the bispecific antibody contributes to function. XmAb20717 also exhibited anti-tumor activity in preliminary anti-tumor studies in mice.

Conclusions

Dual blockade of PD1 and CTLA4 with XmAb20717 resulted in T cell activation that is comparable to a combination of bivalent antibodies targeting PD1 and CTLA4. Specific targeting of human lymphocytes positive for both PD1 and CTLA4 with XmAb20717 may promote similar efficacy compared to a combination of bivalent antibodies while reducing adverse events. These data suggest that clinical development of $\mathrm{XmAb20717}$ is warranted for the treatment of human malignancies.

\section{P330}

T cell immunotherapies trigger innate immunity and aseptic inflammation leading to potent anti-tumor and off-targets effects Daniel Hirschhorn', Jacob Ricca', Bilel Gasmi ${ }^{1}$, Levi Mark Mangarin ${ }^{1}$ Olivier De Henau', Sadna Budhu', Yanyun Li', Czrina Cortez', Yang Xia', Hong Zhong ${ }^{1}$, Cailian Liu', Roberta Zappasodi', Travis Hollmann' Mario Lacouture', Jean Albrengues ${ }^{2}$, Mikala Egeblad ${ }^{2}$, Wolchok Jedd ${ }^{1}$, Taha Merghoub

${ }^{1}$ Memorial Sloan Kettering Cancer Center, New York, NY, USA; ${ }^{2}$ Cold Spring Harbor Laboratory, Cold Spring Harbor, NY, USA

Correspondence: Wolchok Jedd (hirschhd@mskcc.org); Taha Merghoub Journal for ImmunoTherapy of Cancer 2017, 5(Suppl 2):P330

\section{Background}

Mobilizing the immune system to treat advanced cancers is now a clinical reality. Successful immune-based therapies that treat tumors are often accompanied by immune-related adverse events (irAE) including toxicities that can occasionally present with severe and lethal symptoms. The primary immunotherapies currently in clinic include agents that activate $T$ cell responses such as checkpoint blockade of inhibitory pathways and infusion of ex-vivo tumor-derived or $\mathrm{T}$ cell receptor (TCR)-transgenic or chimeric antigen receptor (CAR)-modified T cells, While the beneficial and toxic effects of T cell-based immunotherapies in the clinic are being extensively explored, the precise mechanisms of tumor elimination and irAE remain the subject of intense investigation. Methods

In the present study, we treated established tumors with melanomaspecific adoptive CD4+ T cell transfer and costimulation via OX40 or checkpoint blockade with anti-CTLA-4.

Results

We found that, in spite of co-opting the adaptive immune response to treat cancer, acute local inflammation, resembling delayed-type hypersensitivity, plays a fundamental role in tumor elimination and related toxicities in a model of irAE. While OX40 or CTLA-4 antibodies stimulated T cells are necessary for initiating a therapeutic response, activation of endogenous neutrophils constitutes an important and necessary effector mechanism of tumor destruction and irAE. Upon closer examination, we found extensive neutrophil extracellular traps (NETs) in ear pinnae of treated mice and in melanoma patients suffering from immunotherapy-induced irAE.

Conclusions

Our results illustrate the involvement of innate immunity in promoting tumor elimination and subsequent side effects with immunotherapies that engage $T$ cells.

P331

Involvement of estrogen and progesterone in the modulation of indoleamine 2,3 dioxygenase - IDO - expression in cultured mammary carcinoma cells of female dog

Jose Roberto Kfoury Jr, Pedro Bianchi, Flavia Ponce, Rafael Leandro, Tulio Yoshinaga

School of Veterinary Medicine and Animal Sciences, University of Sao

Paulo, Sao Paulo, Brazil

Correspondence: Jose Roberto Kfoury Jr (jrobertok@usp.br)

Journal for ImmunoTherapy of Cancer 2017, 5(Suppl 2):P331 


\section{Background}

Indoleamine 2,3 dioxygenase - IDO is an enzyme that prevents the establishment of an immune response in the microenvironment in which it is expressed by catabolizing the amino acid tryptophan. The deprivation of tryptophan and the generation of its metabolites, mainly kynurenine, impairs effector $\mathrm{T}$ - cells proliferation leading them to apoptosis [1] and, by its interaction with the aryl-hydrocarbon receptor (AhR) in CD4+ T-cells, favors the expansion of T-regulatory cells [2]. It is known that several cancer cells and leucocytes in the tumor microenvironment express IDO and are sensitive to hormones. Steroidal hormones, such as estrogen and progesterone, are capable of altering immune functions in cells and may influence IDO expression; nonetheless, the mechanisms involved are still poorly understood. Our group previous data have shown that the progesterone was directly involved in IDO expression modulation via its receptor in dendritic and CD4+ T cells from the maternal-fetal interface of Wistar rats [3]. Therefore, this study aims to investigate whether this mechanism is present in the female dog mammary carcinoma microenvironment and if it occurs in a similar way with estrogen.

Methods

Cells of mammary carcinoma from bitches were treated with exogenous progesterone and estrogen and their respective receptor antagonists tamoxifen and mifepristone. IDO expression was analyzed by immunohistochemistry, flow cytometry and the mRNA by real-time PCR. IDO quantification was obtained by western blot technique. Results

IDO quantification exhibited the same pattern as mRNA expression. There was an increase of the enzyme expression and mRNA in the estrogen treated group, in contrast to the decrease observed in the progesterone group. When the cells were subjected to the hormonal inhibitors, an evident decrease of IDO expression percentage and the respective mRNA was verified following the supplementation of tamoxifen and a restoration of IDO expression values and the mRNA after the addition of the progesterone inhibitor, mifepristone.

\section{Conclusions}

These findings strongly suggest that progesterone and estrogen participate indirectly in the modulation of IDO through their membrane receptors.

\section{References}

1. Munn DH., Mellor AL: IDO in the Tumor Microenvironment Inflammation, Counter-Regulation, and Tolerance. Trends in Immunology. 2016; 37(3):193-207.

2. Harden JL, Egilmez NK: Indoleamine 2,3-Dioxygenase and Dendritic Cell Tolerogenicity. Immunol Invest. 2012; 41:738-764.

3. Bianchi PKF, Leandro RM, Poscai AN, Yoshinaga T, Gonçalez PO, Kfoury Junior JR: Progesterone Decreases in vitro Indoleamine 2, 3-dioxygenase Expression in Dendritic and CD4+ Cells from Maternal-Fetal Interface of Rats. Immunol Invest. 2017; 46(5):1-13.

\section{P332}

Pre-clinical efficacy and tolerability of NKTR-255, a polymer-conjugated IL-15 for immuno-oncology

Peter Kirk, Peiwen Kuo, Murali Addepalli, Takahiro Miyazaki, Jane

Gunther, Laurie VanderVeen, Ping Zhang, Palakshi Obalapur, Mekhala

Maiti, Amol Murkar, Arunasree Lanka, Werner Rubas,

Jonathan Zalevsky

Nektar Therapeutics, San Francisco, CA, USA

Correspondence: Peter Kirk (pkirk@nektar.com)

Journal for ImmunoTherapy of Cancer 2017, 5(Suppl 2):P332

\section{Background}

IL-15 is a cytokine that activates T-cells and NK cells and has long been recognized for its potential as an immunotherapeutic agent for the treatment of cancer. Exploiting this potential has been challenging due to unfavorable pharmacokinetic properties. NKTR-255 is a polymer-conjugated IL-15 that shows improved plasma exposure while retaining potency and high affinity for IL-15Ra. Here we investigate the pharmacodynamics, pre-clinical efficacy and tolerability of NKTR-255.

\section{Methods}

To assess the pharmacodynamic effects of NKTR-255 in mice and non-human primates NKTR-255 was delivered intravenously and whole blood was collected at the indicated timepoints; flow cytometry was used to measure signaling activity (STAT5 phosphorylation), proliferative status (Ki-67 expression) and absolute frequency of various lymphocyte subpopulations. Cytotoxicity assays were performed by incubating NK cells purified from spleens of NKTR 255-treated mice with dye-labelled YAC-1 target cells and assessing extent of cell-killing by flow cytometry. Efficacy was studied using the CT-26 lung metastasis model; briefly, $10^{5}$ CT-26 cells were injected into the tail-vein of mice, NKTR-255 treatment was initiated the following day, lungs were harvested on day 13 and metastases were counted.

Results

NKTR-255 induces rapid and sustained signaling in lymphocytes following intravenous administration in mice and non-human primates. This sustained signal results in proliferation of CD8 T-cells and a preferential expansion of the CD8 central memory population. NK cells increase in number and in Granzyme B expression, concomitant with an increase in cytotoxic potential. The robust induction of CD8 and NK cell proliferation is maintained upon repeat dosing. In a mouse model of tumor metastasis to the lungs, NKTR-255 treatment results in an $85 \%$ reduction in the number of metastases. This efficacy is dependent on NK cells but not CD8 T-cells, as demonstrated in celldepletion studies. Toxicology assessments demonstrate that NKTR255 is well tolerated at efficacious dose levels.

Conclusions

NKTR-255 provides a sustained IL-15 signal, resulting in profound and sustained immune activation and anti-tumor activity. NKTR-255 is well tolerated and its pharmacokinetic properties and pharmacodynamic effects translate well from rodents to non-human primates, supporting further development.

\section{P333}

MPL-5821, an ESM ${ }^{\mathrm{TM}}$-p38 MAPK inhibitor, enhances tumor immune response and $M 1$ macrophage polarization in a 3D Ex Vivo system utilizing fresh tumor microspheroids of lung cancer patients

Melanie Mediavilla-Varela', Melba Marie Page ${ }^{1}$, Jenny Kreahling1,

Martin Perry ${ }^{2}$, David Moffat ${ }^{2}$, Scott Antonia', Soner Altiok

${ }^{1}$ Nilogen Oncosystems, Tampa, FL, USA; ${ }^{2}$ Macrophage Pharma Limited,

Windsor, United Kingdom

Correspondence: Soner Altiok (jenny@nilogen.com)

Journal for ImmunoTherapy of Cancer 2017, 5(Suppl 2):P333

\section{Background}

Lung cancer is one of the most common causes of death worldwide. Immunotherapy has demonstrated durable responses and tolerability in subsets of lung cancer patients. Macrophages are a significant component of the tumor microenvironment and the predominant phenotype (M2-like) is frequently associated with one supportive of tumor immune evasion. However, the plasticity of macrophages of fers the opportunity for therapeutic intervention to repolarize the phenotype to one that is non-immunosuppressive and supportive of an anti-tumor immune response (M1-like). MPL-5821 is a p38 MAPK inhibitor employing Esterase Sensitive Motif $\left(\mathrm{ESM}^{\mathrm{TM}}\right)$ technology [1] to principally target myelomonocytic cells. This study evaluates the immu nomodulatory effect of MPL-5821 in a 3D ex vivo assay of lung cancer.

Methods

Fresh tumor tissues obtained from consented patients with nonsmall cell lung cancer at the time of surgical resection were utilized in a proprietary 3D ex vivo tumor microsphere assay with intact tumor immune microenvironment. Tumor microspheres were treated with MPL-5821 for 36 hours. At the end of the treatment, flow cytometry analysis was performed to assess M1/M2 plasticity and TIL proliferation (CD3+/Ki-67+). Additionally, multiplex human cytokine assay was used to simultaneously analyze the differential release of cytokines in culture media and gene expression analysis was performed using the NanoString PanCancer Immune Profiling panel which contains probes to quantitate 770 immune function genes. 
Results

MPL-5821 at 10nM and 50nM effectively inhibited the p38 signaling pathway in the tumor samples and led to profound inhibition of the immunosuppressive cytokine IL-10 and pro-inflammatory cytokines TNFa and IL-6. Evidence of enhanced T-cell proliferation and activation was observed in several of the tumor samples. The most statistical significant up-regulated genes were associated with antigen processing/presentation and MHC class II protein binding and included CD4, CR1, CD74 and multiple HLA genes. CD40 and ICOSLG genes were up-regulated in several tumors as were CXCL9 and 10 . Chemokine genes $\mathrm{CXCL3}, 5,7$ and 8 , associated with a proangiogenic macrophage phenotype, were the most statistically down regulated genes along with the M2 phenotype associated genes CCL13, 23 and 24. Down-regulation of PD-L1 and IDO gene expression was also seen in several tumors.

\section{Conclusions}

This lung patient derived ex vivo approach indicates that MPL-5821 may alleviate myelomonocytic cell induced immunosuppression and enhance antigen responsiveness suggesting potential clinical implications in the treatment of lung cancer.

\section{References}

1. Needham LA, et al, J Pharmacol Exp Ther. (2011), 339 :132-42.

\section{P334}

Inhibition of IDO activity by epacadostat (INCB024360) activates tumor infiltrating lymphocytes in a patient-derived 3D ex vivo system of lung cancer and alleviates stromal immunosuppression

Melanie Mediavilla-Varela', Melba Marie Page', Jenny Kreahling ${ }^{1}$,

Peggy Scherle², Scott Antonia', Soner Altiok

${ }^{1}$ Nilogen Oncosystems, Tampa, FL, USA; ${ }^{2}$ Incyte Corporation, Wilmington, DE, USA

Correspondence: Soner Altiok (jenny@nilogen.com)

Journal for ImmunoTherapy of Cancer 2017, 5(Suppl 2):P334

\section{Background}

Immune evasion is one of the major hallmarks of cancer and identifying mechanisms by which cancer cells evade the immune system have become a major strategy against cancer. IDO (indoleamine 2,3-dioxygenase) is a tryptophan catabolizing enzyme expressed constitutively by tumor cells and different components of immune cells present within the tumor microenvironment. It has been shown that high expression of IDO increases the number of Tregs and blocks the proliferation of effector T cells. Thus, inhibiting the IDO pathway is a promising strategy to restore immune system responses to more easily identify and destroy cancer cells. This study evaluates the immunomodulatory effect of an IDO inhibitor epacadostat (INCB024360) on the immunosuppressive effect of cancer-associated fibroblasts and activation of tumor infiltrating lymphocytes in a 3D ex vivo assay utilizing fresh patient tumor samples

Methods

$3 \mathrm{D}$ ex vivo studies were performed with fresh tumor tissue obtained from consented NSCLC patients. Tumor samples were treated with IDO inhibitor at $1 \mu \mathrm{M}$ for 48 hours. HPLC analysis on kynurenine and tryptophan was performed to verify target inhibition in the ex vivo model. A multiplex human cytokine assay was used to simultaneously analyze the differential release of cytokines in culture media. Additionally, NanoString PanCancer Immune Profiling platform containing probes to quantitate 770 immune function genes was used to determine positive and negative associations between expression of immune function genes and TIL activation by ex vivo treatment. Furthermore, autologous patient-derived cell lines (CAF and TILs) were utilized in an in vitro assay to determine the role of IDO inhibition on CAF-mediated immunosuppression.

Results

$3 \mathrm{D}$ ex vivo studies showed a significant decrease in kynurenine demonstrating that epacadostat effectively inhibited the enzymatic activity of IDO in the tumor microenvironment accompanied by increased release of pro-inflammatory cytokines such as IFNY. Treatment with epacadostat demonstrated decreased expression of genes involved in tumor growth (CCL25) and increased expression of antitumor immune response genes (CXCL14, CCL19 and CCL21). These studies showed epacadostat at an effective concentration of $1 \mu \mathrm{M}$ induced specific changes in the microenvironment and increased immune response. Furthermore, the autologous patient derived cell line in vitro assay determined that epacadostat overcame CAF induced inhibition of TIL activity.

Conclusions

This patient-derived 3D ex vivo approach demonstrated the immunomodulatory activity of epacadostat in NSCLC and indicates that inhibition of IDO activity may overcome stroma-induced immunosuppression in lung cancer. Studies on the effects of epacadostat in combination with anti-PD1 in the same culture systems are currently ongoing.

P335

Characterization of a novel differentiated anti-CTLA-4 antibody

(ADU-1604) in vitro and in vivo

Maaike Hendriks', Joost Kreijtz', Paul Vink', David Lutje Hulsik', Imke

Lodewijks ${ }^{1}$, Astrid Bertens', Jos van de Crommert', Maurice Habraken',

Wout Janssen', Judith Stammen-Vogelzang ${ }^{1}$, Weiwen Deng ${ }^{2}$,

Laura Hix Glickman², Meredith Leong ${ }^{2}$, Sarah McWhirter²,

Thomas Dubensky ${ }^{2}$, Hans van Eenennaam ${ }^{1}$, Andrea van Elsas

${ }^{1}$ Aduro Biotech Europe, Oss, Netherlands; ${ }^{2}$ Aduro Biotech, Inc., Berkeley, CA, USA

Correspondence: Joost Kreijtz (jkreijtz@aduro.com)

Journal for ImmunoTherapy of Cancer 2017, 5(Suppl 2):P335

\section{Background}

Targeting the CTLA-4 immune checkpoint with antibodies, either alone or in combination with PD-1/PD-L1 inhibitors shows clinical activity and durable responses in advanced cancer. The use of anti-CTLA-4 may augment other immunotherapies. Indeed, in syngeneic mouse tumor models anti-CTLA-4 strongly enhanced anti-tumor efficacy of live attenuated double-deleted Listeria monocytogenes (LADD) immunotherapy and of the stimulator of interferon genes (STING) pathway activator ADU-S100, indicating combination potential. Activity of antiCTLA-4 is enhanced by Fc receptor engagement and tumor-selective depletion of regulatory T cells in mice, but this is unproven as of yet for cancer patients. Here we present a novel, humanized anti-CTLA-4 antibody originating from Aduro's proprietary B-Select platform.

Methods

Among a panel of mouse anti-human CTLA-4 antibodies, 27A was humanized and functionally characterized on an IgG1 and IgG4 backbone. Both variants, named hCTLA4.27H6L1.i1 and .i4 were characterized in vitro for binding to human and cynomolgus CTLA-4, blocking of CD80/CD86 and functional activity. Both antibodies are compared for their capacity to induce tumor rejection in patient-derived tumor graft models in humanized mice.

Results

The IgG1 and IgG4 variants bound recombinant human CTLA-4 with a $\mathrm{Kd}$ of $2.7 \pm 2.9 \mathrm{nM}$ and $1.3 \pm 0.5 \mathrm{nM}$ as measured by Bio-Layer Interferometry, and cell-expressed CTLA-4 with an EC50 of $0.042 \pm$ $0.000 \mathrm{nM}$ and $0.048 \pm 0.002 \mathrm{nM}$, respectively. Binding to endogenous CTLA-4 was demonstrated by flow cytometry using primary activated human and non-human primate lymphocytes. Both IgG1 and lgG4 variants potently blocked the interaction of CD80 and CD86 with human CTLA-4 (IC50 ranged from 1-2 nM). Using swap mutants of mouse and human CTLA-4 and cross-competition assays by Bio-Layer Interferometry, these antibodies showed a unique binding profile indicating a previously undiscovered epitope on CTLA-4. Functional characterization demonstrated that both antibodies enhanced activation and IL-2 production by human primary $\mathrm{T}$ cells or peripheral blood mononuclear cells (PBMCs) co-stimulated by Raji cells or Staphylococcal enterotoxin B, in a dose-dependent manner. The lgG1 variant (in contrast to IgG4) bound to low and high-affinity Fcy receptors inducing antibody-dependent cell-mediated cytotoxicity (ADCC) mediated by natural killer (NK) cells and CD16+ monocytes towards CTLA-4+ cells. Similarly, complement-dependent cytotoxicity was differentially induced in vitro. 


\section{Conclusions}

Overall, both hCTLA4.27 derivatives compared favorably to benchmarks in their biophysical and functional characteristics. The IgG1 variant, designated ADU-1604, is progressing through preclinical development.

\section{P336}

Preclinical characterization of MGA012, a novel clinical-stage PD-1 monoclonal antibody

Ross La Motte-Mohs', Kalpana Shah', Jennifer G. Brown', Ralph F. Alderson Doug Smith ${ }^{2}$, Sergey Gorlatov', Valentina Ciccarone', James K. Tamura', Hua Li', Leilei He' ${ }^{1}$, Farha Vasanwala', Jill R. Rillema², Monica Licea², Jessica Hill', Christina Wolff', Massimiliano Pascuccio ${ }^{1}$, Francine Z. Chen², Yan Chen², Anushka De Costa², Ann Easton', Alexey Berezhnoy', Jonathan $\mathrm{Li}^{2}$, Jeffrey Nordstrom ${ }^{1}$, Scott Koenig', Ezio Bonvini', Syd Johnson', Paul A. Moore

${ }^{1}$ MacroGenics, Inc., Rockville, MD, USA; ${ }^{2}$ MacroGenics, Inc., South San Francisco, CA, USA

Correspondence: Ross La Motte-Mohs

(lamottemohsr@macrogenics.com)

Journal for ImmunoTherapy of Cancer 2017, 5(Suppl 2):P336

\section{Background}

Monoclonal antibodies (mAbs) that target immune checkpoint pathways, such as the cytotoxic $T$ lymphocyte-associated antigen- 4 and the programmed cell death protein 1 (PD-1) pathways, have demonstrated broad clinical efficacy against a variety of malignancies as monotherapy or in a combination. MGA012 is a novel anti-PD-1 mAb developed to disrupt the PD-1 interaction with PD-L1/PD-L2 to restore or improve T-cell function as stand-alone therapy or in combination with other immune approaches.

Methods

Murine PD-1 mAbs were generated and benchmarked against replicas of the approved mAbs, nivolumab and pembrolizumab. Several mAbs with favorable characteristics were further chimerized or humanized. MGA012, a humanized, hinge-stabilized IgG4K mAb, was selected based on binding and biophysical properties as well as a functional characterization inclusive of enhanced T-cell activation following superantigen re-stimulation.

Results

MGA012 bound human PD-1 with an affinity equal to or exceeding those of replicas of nivolumab or pembrolizumab. MGA012 bound PD-1-expressing cell lines and chronically-activated T cells, blocked PD-1 interactions with PD-L1/PD-L2, resulting in inhibition of PD-1 signaling and enhanced antigen-driven cytokine secretion to levels comparable to those observed with nivolumab or pembrolizumab replicas. Furthermore, characterization of MGA012 in ex-vivo tumor microenvironment immune models showed activation profiles recapitulating the benchmark PD-1 mAbs. MGA012 showed combinatorial activity in vitro when added to anti-CTLA-4 or anti-LAG-3 mAbs and enhanced the activity of a T-cell redirecting molecule in a mouse tumor model. MGA012 showed no unexpected cross-reactivity in human tissues, with staining observed primarily in lymphocytes and lymphoid organs. In a repeat-dose (10-150 mg/kg QWx4) study in cynomolgus monkeys, PK was linear with a beta half-life of 11.2 days $( \pm 4.6$ SD) and full PD-1 occupancy on circulating T cells at all doses tested. Occupancy of $\geq 80 \%$, persisting for $4-7$ weeks, was also observed in monkeys receiving a single $10 \mathrm{mg} / \mathrm{kg}$ dose. MGA012 was well tolerated in cynomolgus monkeys and demonstrated a favorable safety profile with a no-adverse effect level (NOAEL) of $150 \mathrm{mg} / \mathrm{kg}$.

\section{Conclusions}

MGA012 is a novel anti-PD-1 mAb with favorable preclinical characteristics, including PD-1 binding and biophysical properties, PD-1 pathway blockade, the ability to enhance T-cell responses in vitro and in vivo, and a favorable PK and safety profile in cynomolgus monkeys. Clinical trials are ongoing [NCT03059823] or in planning stage to ascertain the safety and preliminary activity of MGA012 alone or in combination with other immune oncology agents, including T-cell redirecting bispecific DART ${ }^{\circledR}$ molecules.
Trial Registration

ClinicalTrials.gov NCT03059823.

\section{P337}

Preclinical characterization of MGD013, a PD-1 x LAG-3 bispecific DART ${ }^{\circledR}$ molecule

Ross La Motte-Mohs', Kalpana Shah', Jennifer G. Brown', Doug Smith², Sergey Gorlatov', Valentina Ciccarone ${ }^{3}$, James K. Tamura', Hua Li ${ }^{1}$,

Leilei He', Farha Vasanwala', Christine Shoemaker', Jonathan $\mathrm{Li}^{2}$,

Shereen Saini ${ }^{2}$, Jill R Rillema², Monica Licea², Jessica Hill', Arin Whiddon?',

Massimiliano Pascuccio ${ }^{1}$, Francine Z. Chen ${ }^{2}$, Anushka De Costa ${ }^{2}$, Ann

Easton², Peter Lung ${ }^{2}$, Kurt Stahl', Jeffrey Nordstrom', Scott Koenig' ${ }^{1}$ Ezio

Bonvini', Syd Johnson', Paul A. Moore ${ }^{1}$

${ }^{1}$ MacroGenics, Inc., Rockville, MD, USA; ${ }^{2}$ MacroGenics, Inc., South San

Francisco, CA, USA; ${ }^{3}$ MacroGenics, Inc., Rockville, CA, USA

Correspondence: Ross La Motte-Mohs

(lamottemohsr@macrogenics.com)

Journal for ImmunoTherapy of Cancer 2017, 5(Suppl 2):P337

\section{Background}

Monoclonal antibodies (mAbs) that target the immune checkpoints, cytotoxic $T$ lymphocyte-associated antigen-4 (CTLA-4) and programmed cell death protein-1 (PD-1), have shown enhanced clinical anti-tumor activity when given in combination, triggering interest in determining whether additional checkpoint inhibitor combinations may afford enhanced clinical benefit. Lymphocyte activation gene-3 (LAG-3) is another immune checkpoint expressed on activated $T$ cells and tumor infiltrating lymphocytes. Recognizing the therapeutic potential of dual checkpoint blockade, we have engineered MGD013, a IgG4K bispecific DART molecule to bind PD-1 and LAG-3 concomitantly or independently and disrupt these non-redundant inhibitory pathways to further restore exhausted T-cell function.

\section{Methods}

Proprietary PD-1 and LAG-3 mAbs were generated and selected based on binding characteristics, biophysical properties, the ability to block their respective receptor/ligand axes and to synergize in T-cell stimulation assays. Humanized sequences were incorporated into a tetravalent bispecific DART format and benchmarked against combinations of replicas of the approved PD-1 mAb, nivolumab, and BMS-986016 anti-LAG-3 mAb, which is currently under clinical evaluation. MGD013 biological activity was evaluated in various primary cell-based immune assays. Safety was assessed in cynomolgus monkey toxicology studies performed at MPI (Mattawan, MI) under Institutional Animal Care and Use Committee-approved protocols.

Results

MGD013 bound with high affinity to human and cynomolgus monkey PD-1- and LAG-3-expressing cells and blocked PD-1/PD-L1, PD-1/ PD-L2 and LAG-3/HLA (MHC-II) interactions, with resultant signaling blockade. Functional characterization revealed enhanced cytokine secretion in response to antigen stimulation that was greater than that of the combination of individual equimolar amounts of PD-1 and LAG-3 mAbs. MGD013 was well-tolerated in a repeated-dose (Q1Wx4) cynomolgus monkey toxicology study. Except for the occurrence of watery feces in a few animals, no MGD013-related adverse findings were noted, including hematological or clinical chemistry changes, serum cytokine levels or gross and microscopic histological findings, establishing a no-observed adverse-effect level (NOAEL) of $100 \mathrm{mg} / \mathrm{kg}$.

\section{Conclusions}

MGD013 is a bispecific DART molecule capable of simultaneously blocking the PD-1 and LAG-3 pathways, resulting in enhanced Tcell activation compared to single or combination mAb blockade. MGD013 has demonstrated a favorable pre-clinical safety and toxicological profile and is currently initiating clinical testing [NCT03219268].

Trial Registration

ClinicalTrials.gov NCT03219268 
P338

Combined IL- 6 and CTLA4 blockade enhances CD8+ T cell infiltration via CXCR3 and limits growth of pancreatic cancer in murine models

Christopher McQuinn', Matthew Farren², Thomas Mace', Jacob Bowers ${ }^{3}$, Reena Shakya', A. Brad Ferris², Gregory Young', William Carson, III', Chrystal Paulos ${ }^{3}$, Bassel El-Rayes ${ }^{2}$, Gregory Lesinski ${ }^{2}$

${ }^{1}$ The Ohio State University, Columbus, OH, USA; ${ }^{2}$ Winship Cancer Institute of Emory University, Atlanta, GA, USA; ${ }^{3}$ Medical University of

South Carolina, Charleston, SC, USA

Correspondence: Gregory Lesinski (gregory.b.lesinski@emory.edu)

Journal for ImmunoTherapy of Cancer 2017, 5(Suppl 2):P338

\section{Background}

Immune checkpoint blockade has not shown efficacy in pancreatic cancer. We hypothesized the cytokine interleukin-6 (IL-6) enhances immune suppression in this disease, and represents a logical target to augment immunotherapy. Prior publications from our group and others demonstrate IL- 6 is derived from pancreatic stroma, is inversely associated with overall survival in metastatic patients and enhances efficacy of anti-PD-L1 antibodies in pre-clinical models. This report addresses the efficacy of combined IL- 6 and CTLA4 blockade, and identifies a mechanism by which this combination enhances effector T cell infiltration into tumors.

Methods

In vivo efficacy of Ab targeting IL- 6 and CTLA4 was tested in mice bearing subcutaneous MT-5 murine pancreatic tumor cells with $\mathrm{Kras}^{G 12 D}$ and TP53 ${ }^{R 172 H}$ mutations. CXCR3 blocking antibodies were used for in vivo mechanistic studies, while immune biomarkers were analyzed using flow cytometry or immunohistochemistry.

Results

Combined blockade of IL- 6 and CTLA4 inhibited tumor growth rate as compared to controls ( $p$ 's $<0.05$ ). IHC analysis revealed increased T cells in tumors from combination treated mice ( $p=0.035$ vs. anti-IL-6; $p=0.038$ vs. anti-CTLA4; $p<0.0001$ vs. iso control). Analysis of systemic immune biomarkers was conducted via flow cytometric analysis of splenocytes. Despite the role for IL-6 in expanding MDSC, no change in monocytic $\left(\mathrm{CD} 11 \mathrm{~b}^{+}\right.$Ly6G Ly6C $\mathrm{C}^{+}$) or granulocytic (CD11b $\mathrm{b}^{+}$Ly6G ${ }^{+}$Ly6 $\mathrm{C}^{\mathrm{low}}$ ) cells were observed. However, analysis of T cell subsets revealed both anti-CTLA4 alone or the combination increased cells with Th1 or Th17, but not Th2 phenotypes $(p<0.05)$. Cells with a CD4 ${ }^{+} \mathrm{CD} 25^{+} \mathrm{FoxP}^{+}$phenotype were increased in combination treated mice $(p<0.05)$. We are studying if these cells are suppressive or a byproduct of enhanced activation marker expression. Blockade of IL-6, CTLA4 or the combination altered circulating cells with phenotypic properties of naïve, effector and central memory $T$ cells (based on CD44/CD62L). Notably, circulating CD4 ${ }^{+} \mathrm{CD} 44^{+} \mathrm{CD} 62 \mathrm{~L}^{+} \mathrm{T}$ cells were significantly higher in the combination $(p<0.05)$. Consistent with $\mathrm{T}$ cell infiltration data, sequencing of TCR $\beta$ within $n=3$ representative tumors from each group of mice revealed a trend toward increased number of both productive templates and rearrangements. Finally, in vivo administration of $A b$ targeting CXCR3 limited the efficacy of the treatment combination, and reduced $\mathrm{CD} 8^{+} \mathrm{T}$ cell infiltration into tumors.

Conclusions

IL-6 blockade enhances efficacy of antibodies targeting CTLA4 in preclinical models of pancreatic cancer. Mechanistically, this involves CXCR3mediated intratumoral infiltration of $\mathrm{CD}^{+} \mathrm{T}$ cells. These data suggest that clinically-available IL-6/L-6R targeted agents could be repurposed to enhance immune checkpoint blockade as a novel combination therapy.

\section{P339}

Critical role of hematopoietic progenitor kinase 1 in anti-tumor immune responses

Dan You', Stephen Hillerman', Jesse Swanson'1, Ching-Ping Ho', Anwar Murtaza', Rukiye Eraslan', Miguel Sanjuan', John Hunt',

Luisa Salter-Cid ${ }^{1}$, Joshua Curtin ${ }^{2}$

'Bristol-Myers Squibb, Lawrence Township, NJ, USA; ${ }^{2}$ Johnson \& Johnson Pharmaceutical, Spring House, PA, USA

Correspondence: Jinqi Liu (jinqi.liu1@gmail.com)

Journal for ImmunoTherapy of Cancer 2017, 5(Suppl 2):P339

\section{Background}

Hematopoietic progenitor kinase 1 (HPK1) was previously reported as a negative regulator of $\mathrm{T}$ cell responses due to its ability to attenuate T cell receptor (TCR) signaling. T cells from HPK1 knockout (HPK1-/-) mice demonstrated elevated proliferation and cytokine production in response to TCR engagement and are resistant to prostaglandin E2 (PGE2)-mediated suppression. Additionally, HPK1-/- bone marrow derived dendritic cells (BMDCs) eliminate established Lewis Lung Carcinoma more effectively compared with HPK1 +/+ BMDCs, suggesting HPK1 is a critical negative regulatory component in key immune cell types involved in anti-tumor immunity. To further investigate the potential value of targeting HPK1 pharmacologically in cancer immunotherapy, we generated HPK1 kinase dead mice (HPK1 KD). Extensive characterization of these mice was conducted via employing syngeneic tumor models and ex vivo studies. We report here our findings on the blockade of HPK1 kinase activity in modulating anti-tumor immune responses.

Methods

HPK1 KD mice were generated and characterized, including immune cell phenotyping in peripheral blood and secondary lymphoid organs. These mice were then subjected to in vivo stimulation with agonistic anti-CD3 followed by measurement of plasma cytokines. HPK1 WT and $\mathrm{KD}$ mice were further interrogated in syngeneic tumor models in combination with a cyclooxygenase inhibitor (celecoxib) or anti-PD1. Antitumor efficacy was monitored, immune phenotyping was conducted for draining lymph nodes and tumor tissues, and ex vivo studies were performed to evaluate the enhancement of immune responses.

\section{Results}

HPK1 KD mice were grossly normal and immune phenotyping revealed no difference compared with WT mice. HPK1 KD mice demonstrated superior anti-tumor efficacy in a sarcoma model. Significantly better anti-tumor efficacy with $50-70 \%$ tumor free was observed in HPK1 KD mice compared to WT mice treated with either celecoxib or anti-PD1, in sarcoma and colorectal tumor models, respectively. Further immune phenotyping and ex vivo studies indicated enhanced $\mathrm{CD}^{+}$and $\mathrm{CD}^{+}{ }^{+}$cell populations as well as IFNY and TNFa secretion along with reduced ratios of CD8/Tregs. Cytolytic activities against syngeneic tumor cells were also augmented with HPK1 KD CD8 ${ }^{+} \mathrm{T}$ cells compared with WT CD8 ${ }^{+}$cells.

Conclusions

Genetic blockade of HPK1 kinase activity revealed a significant enhancement of immune responses and resulted in improved anti-tumor efficacy in combination with inhibition of PGE2 or PD-1 pathways.

P340

In vitro effects and mechanism of hu14.18-IL2 immunocytokine against $\mathrm{GD}_{2}$ positive pediatric malignancies

Holger Lode', Maxi Zumpe ${ }^{1}$, Madlen Juettner', Sascha Troschke-Meurer ${ }^{1}$, Evelyne Janzek ${ }^{2}$, Romana Schaefer ${ }^{2}$, Bettina Neuchrist ${ }^{2}$, Nikolai Siebert ${ }^{1}$,

Hans Loibner ${ }^{2}$

${ }^{1}$ University Medicine Greifswald, Greifswald, Germany; ${ }^{2}$ Apeiron Biologics, Vienna, Austria

Correspondence: Holger Lode (lode@uni-greifswald.de)

Journal for ImmunoTherapy of Cancer 2017, 5(Suppl 2):P340

\section{Background}

Passive immunotherapy with anti-GD $\mathrm{C}_{2}$ antibody $(\mathrm{Ab}) \mathrm{ch} 14.18 / \mathrm{CHO}$ (dinutuximab beta) showed activity for the treatment high-risk neuroblastoma (NB) patients and received recently marketing approval in the EU. Immunocytokines are antibody-cytokine fusion proteins that combine the targeting properties of monoclonal antibodies with the immune stimulatory function of cytokines. Here we demonstrate activity and mechanism of hu14.18-IL2 against a variety of $\mathrm{GD}_{2}$ positive pediatric tumor cell lines (neuroblastoma, osteosarcoma, Ewing's sarcoma, retinoblastoma) in preclinical models.

Methods

Expression of the target antigen $\mathrm{GD}_{2}$ on LAN-1 (neuroblastoma), MG63 (osteosarcoma), TC-71 (Ewing's sarcoma) and Y79 (retinoblastoma), and PD-1/PD-L1 checkpoint on LAN-1 was analyzed by flow cytometry. 
Hu14.18-IL2 mediated ADCC, CDC and whole blood cytotoxicity (WBT) was determined by ${ }^{51} \mathrm{Cr}$ release assays.

Results

We found expression of antigen $\mathrm{GD}_{2}$ on all cell lines derived of major neuro-ectodermal malignancies in childhood. The highest level was found in neuroblastoma but all other cell lines were clearly $\mathrm{GD}_{2}$ antigen positive. Importantly, hu14.18-IL2 was effective in mediating ADCC, CDC and WBT against all cell lines in vitro, and potency was found higher than that of ch14.18/CHO in the osteosarcoma and retinoblastoma models. The effects were antigen specific as addition of an anti-idiotypic antibody abrogated the cytolytic activity.

Interestingly, tumor specific ADCC in the presence of LA-N-1 neuroblastoma cells, leukocytes and sub-therapeutic hu14.18-IL2 concentrations $(10-100 \mathrm{ng} / \mathrm{ml})$ resulted in a strong increase of PD-L1 expression both on effector and target cells. This effect required cell-cell contact, since separation of effector cells from target cells using a membrane abrogated the activity. This provides a rationale to explore combinatorial approaches with agents that inhibit the PD-1/PD-L1 checkpoint. Conclusions

Immunocytokine hu14.18-IL2 is effective against $\mathrm{GD}_{2}$ positive pediatric malignancies derived of neuroectodermal origin. Engaged immune effector functions by hu14.18-IL2 result in a concomitant upregulation of immune checkpoints that suggests a combinatorial approach with checkpoint inhibitors early during clinical development.

\section{P341}

Peripheral immunomodulatory changes in metastatic renal cell carcinoma (mRCC) patients treated with nivolumab

Lorena Almeida, Manuel Maia, Haejung Won, Dayson Moreira, Paulo Bergerot, Marcin Kortylewski, Sumanta Pal

City of Hope Comprehensive Cancer Center, Duarte, CA, USA

Correspondence: Manuel Maia (mcaitano@coh.org)

Journal for ImmunoTherapy of Cancer 2017, 5(Suppl 2):P341

\section{Background}

CheckMate-009 was a biomarker-based study in which serial biopsies were conducted for patients receiving nivolumab [1]. Lesser data is available for fluxes in circulating biomarkers during the course of treatment. We hypothesized that immunomodulatory changes in blood would mirror previous observations in tissue.

Methods

Patients with $\mathrm{mRCC}$ receiving nivolumab as standard of care were prospectively enrolled. Blood samples were drawn at baseline, week 4 and week 12 during treatment. Analysis of samples for relevant white blood subsets was conducted by immersing peripheral blood mononuclear cells in a mixture of PBS, $2 \%$ FCS and $0.1 \%$ sodium azide with Fclll/IIR-specific antibody to block nonspecific binding and stain the cells with different combinations of fluorochrome-coupled antibodies. We further collected fluorescence data on Fortessa (Beckton Dickinson) and analyzed them using FlowJo software (Tree Star) [2]. Plasmas were analyzed for human cytokines using Luminex. Changes in cells and cytokines overtime in the overall population were interrogated. We also compared baseline levels of cytokines in responders ( $\mathrm{R}$; including partial/complete response and stable disease) and nonresponders ( $P$; primary progression).

Results

A total of 10 patients were included, with a total of 29 samples collected. During treatment, we observed a significant decrease in the proportion of $C D 3+C D 4+T$ cells and an increase in the CD3+CD8+ T cells in the overall population $(p<0.05)$. In addition, the expression of PD-1 was significantly decreased in both CD4+ and CD8+ T cells overtime, while serum level of IL2R and CXCL9 significantly increased $(p<0.05)$. Compared to $P, R$ group showed a trend towards higher baseline levels of IFN-alpha and IFN-gamma ( $p=0.05$ and 0.11 , respectively) as well as VISTA expression on CD4+ T cells $(p=0.15)$

\section{Conclusions}

Our results emphasize the immunomodulatory changes in the peripheral blood occurring during treatment with nivolumab in mRCC pts. Some dynamics contrast with previous observations in tissue [3], reflecting temporal and spatial heterogeneity during immune system modulation by immunotherapies. Differential baseline levels of cytokines and immune checkpoint expression in the $\mathrm{R}$ group may be used as a predictive biomarker for nivolumab response if confirmed in larger series.

\section{References}

1. Choueiri, T. K., et al. Immunomodulatory Activity of Nivolumab in Metastatic Renal Cell Carcinoma. Clinical Cancer Research.2016;22(22): 546.

2. Chalmin F, et al. Membrane-associated Hsp72 from tumor-derived exosomes mediates STAT3-dependent immunosuppressive function of mouse and human MDSC. J Clin Invest. 2010;120:457-71.

3. Gao, J., et al. VISTA is an inhibitory immune checkpoint that is increased after ipilimumab therapy in patients with prostate cancer. Nat Med.2017;23(5): 551-555.

P342

Blood in circulation, with intact cascade systems, as a tool to predict first-infusion reactions and mechanism-of-action of immunotherapeutics

Erika Fletcher ${ }^{1}$, Mohamed Elthahir ${ }^{1}$, Frida Lindqvist ${ }^{2}$, Jonas Rieth?', Gunilla Törnqvist ${ }^{2}$, Justyna Leja-Jarblad ${ }^{1,2}$, Sara Mangsbo ${ }^{1,2}$

${ }^{1}$ Uppsala University, Uppsala, Sweden; ${ }^{2}$ Immuneed AB, Uppsala, Sweden

Correspondence: Sara Mangsbo (sara.mangsbo@farmbio.uu.se)

Journal for ImmunoTherapy of Cancer 2017, 5(Suppl 2):P342

\section{Background}

Since the TGN1412 disaster in London, where an anti-CD28 superagonistic antibody induced a life-threatening cytokine storm in six healthy men during a clinical trial [1], risks of first-infusion reactions are important to study prior clinical trial initiation. These assays can constructed in different ways and depending on the setup, the sensitivity of the assay depends on the antibody tested. It is therefore of great need to validate a cytokine release assay with the potential to detect first-infusion reactions regardless of the nature of the antibody tested.

Methods

As the assays used today to predict cytokine release syndrome (CRS) are devoid of one or several blood components we setout to investigate if a modified chandler loop model could be used to predict CRS. The assay makes use of extracorporeal fresh whole blood in circulation, thus it contains blood immune cells, immunoglobulins along with intact cascade systems [2]. The challenge with such systems is to avoid clotting during assay performance, but correctly performed, will allow for both monitoring of cytokine release along with studies of ADCC and CDC.

Results

Uniquely the assessed agonistic antibodies were scored to induce CRS in blood from all tested donors $(n=22)$ after only 4 hours of incubation in the loop assay, whereas non-agonistic antibodies associated with no or low infusion reactions in the clinic neither induce cytokine release nor cause false positive responses. Additionally, the value of an intact complement system in the assay was highlighted by the possibility to dissect out the mechanism-of-action of alemtuzumab and rituximab.

Conclusions

A modified chandler loop system can complement lymph node-like assays or be used as an excellent stand-alone test to investigate drug/blood interactions during preclinical development, or for individual safety screening prior a first-in-man clinical trial.

\section{References}

1. Suntharalingam G, Perry MR, Ward S, Brett SJ, Castello-Cortes A, Brunner $\mathrm{MD}$, Panoskaltsis N. Cytokine storm in a phase 1 trial of the anti-CD28 monoclonal antibody TGN1412. N Engl J Med. 2006;355(10):1018-28.

2. Mangsbo SM, Sanchez J, Anger K, Lambris JD, Ekdahl KN, Loskog AS, Nilsson B, Totterman TH. Complement activation by $\mathrm{CpG}$ in a human whole blood loop system: mechanisms and immunomodulatory effects. J Immunol. 2009:183(10):6724-32. 


\section{P343}

Novel immuno-oncology biologics derived via directed evolution of IgSF domains

Mark Maurer, Ryan Swanson, Michael Kornacker, Steve Levin, Chris Navas, Chelsea Gudgeon, Lawrence Evans, Martin Wolfson, Dan Ardourel, Daniel DeMonte, Joseph Kuijper, Sean MacNeil, Janhavi Bhandari, Mark Rixon, Stanford Peng

Alpine Immune Sciences, Inc., Seattle, WA, USA

Correspondence: Mark Maurer

(mark.maurer@alpineimmunesciences.com)

Journal for ImmunoTherapy of Cancer 2017, 5(Suppl 2):P343

\section{Background}

Our variant lg domain (vlgD $\left.{ }^{\mathrm{TM}}\right)$ platform creates novel, therapeuticallyapplicable protein domains with tailored specificity and affinity. These vlgDs are created through directed evolution of immunoglobulin superfamily (IgSF) proteins and have unique biochemical properties including small size, single domain structure, and the capacity to interact with multiple counter-structures. Because many IgSF family members and their counter-structures are widely expressed on immune cells and tumors, the vlgD platform is well positioned for the development of immuno-oncology biologics with potential first-in-class mechanisms of action. Here, multiple therapeutic formats for vlgDs were developed and characterized.

Methods

Novel vlgDs were created with tailored affinities and modulatory activities against PD-1, TIGIT, PD-L1, CTLA-4, CD28, and/or ICOS. These domains were successfully developed into multiple therapeutic formats, including single and multiple domain Fc fusion proteins and vlgD-monoclonal antibody ( $\mathrm{V}$-mAb) fusion proteins. In addition to ligand binding and specificity assays, in vitro functional activity was characterized in several T cell-based assays including cell-based reporter systems for pathway agonism or antagonism, primary human mixed lymphocyte reactions (MLRs), and costimulation assays utilizing artificial APCs (assessed by proliferation and IFNg production).

Results

Several functionally active therapeutic vlgD-based molecules were created successfully. (1) Single-domain vlgD-Fc fusion proteins with tailored binding to CD28, CTLA-4, and PD-L1 demonstrated differential activity in T cell activation assays and, depending on their ligand binding profile, resulted in greater or reduced IFNY production and T cell proliferation in human $T$ cell activation assays. (2) Multi-domain vlgD-Fc fusion proteins demonstrated promising targeting of immunomodulatory pathways in cell-based reporter assays and MLRs as assessed by IL-2 signaling and IFNy production. Efficacy was comparable to monoclonal antibodies against the individual vlgD targets. (3) $\mathrm{V}$-mAbs demonstrated target-specific T cell proliferation and IFNY production in vitro, using both recombinant target proteins or targetspecific cell lines.

\section{Conclusions}

The vlgD platform has successfully generated multiple immuno-oncology therapeutic candidates, in various formats including single- and multipledomain Fc fusion proteins as well as V-mAbs. These varied formats confer, from a single molecule, multiple advantages including the multitarget modulation capability of evolved IgSFs, and, where applicable, tumor localizing capability of partner molecules or domains. This platform may contribute to the next generation of immunotherapeutic proteins in an oncology setting and efforts are ongoing to develop these candidates for human therapeutic use.

\section{P344}

Development and functional characterization of COM902, a novel therapeutic antibody targeting the immune checkpoint TIGIT

Sandeep Kumar', Radhika Desai', Hsin-Yuan Cheng', Kyle Hansen', Andy Drake ${ }^{1}$, Patrick Wall ${ }^{1}$, Kathryn Logronio ${ }^{1}$, Gady Cojocaru ${ }^{2}$,

John Hunter ${ }^{1}$, Mark White ${ }^{1}$, Spencer Liang ${ }^{1}$, Maya Kotturi ${ }^{1}$

${ }^{1}$ Compugen USA, Inc., South San Francisco, CA, USA; ${ }^{2}$ Compugen Ltd.,

Holon, Israel

Correspondence: Maya Kotturi (mayak@cgen.com)

Journal for ImmunoTherapy of Cancer 2017, 5(Suppl 2):P344

\section{Background}

TIGIT is a coinhibitory receptor that is expressed on lymphocytes, including effector and regulatory $\mathrm{CD}^{+}{ }^{+}$cells (Tregs), effector $\mathrm{CD} 8^{+}$ $T$ cells, and NK cells, that infiltrate different types of tumors. Engagement of TIGIT with its reported ligands, poliovirus receptor (PVR) and PVR-like proteins (PVRL2 and PVRL3) directly suppresses lymphocyte activation. PVR is also broadly expressed in tumors, suggesting that the TIGIT-PVR signaling axis may be a dominant immune escape mechanism for cancer. We report here the biophysical and functional characterization of COM902, a therapeutic antibody targeting TIGIT. We demonstrate that co-blockade of TIGIT and a new checkpoint inhibitor, PVRIG, augments T cell responses.

Methods

Human phage display and mouse hybridoma antibody discovery campaigns were conducted to generate therapeutic anti-TIGIT antibodies. The resulting antibodies were evaluated for their ability to bind to recombinant and cell surface-expressed human TIGIT with high affinity. Cross-reactivity of the antibodies to cynomolgus and mouse TIGIT was also examined. A subset of antibodies that bound with high affinity to human TIGIT, and cross-reactive to cynomolgus TIGIT were further characterized for their ability to block the interaction between TIGIT and PVR. Blocking antibodies were screened for their ability to enhance antigen-specific T cell activation in vitro either alone, or in combination with an anti-PVRIG antibody, COM701.

Results

We identified a lead antibody, COM902, that binds to human TIGIT with high femtomolar affinity. This antibody bound to TIGIT endogenously expressed on human $\mathrm{CD}^{+} \mathrm{T}$ cells with higher affinity than tested benchmark antibodies, and was also crossreactive to both cynomolgus and mouse TIGIT. When tested for in vitro activity, COM902 augmented cytokine secretion and tumor cell killing by CMV-specific $\mathrm{CD}^{+}{ }^{+} \mathrm{T}$ cells with superior or equivalent potency to the tested benchmark antibodies. Combination of COM902 with an anti-PD1 antibody or COM701 resulted in enhanced CMV-specific CD8 ${ }^{+} \mathrm{T}$ cell activity. Furthermore, we demonstrated that TIGIT is predominantly expressed on Tregs and effector $\mathrm{CD}^{+} \mathrm{T}$ cells from solid tumors compared to peripheral blood, suggesting that these populations will likely be preferentially targeted by COM902.

Conclusions

We describe the development of a high affinity antagonistic TIGIT antibody, COM902, that is currently in preclinical development. We postulate that the femtomolar affinity of COM902 could result in lower and less frequent dosing in patients. COM902 can enhance human T cell activation either alone or in combination with other checkpoint antibodies. Thus, our data demonstrates the utility of targeting TIGIT, PD1, and PVRIG for the treatment of cancer.

\section{P345}

Tumor protective effect of anti-MUC1.IgE in pancreatic cancer Kamiya Mehla', James A Grunkemeyer ${ }^{1}$, Ragupathy Madiyalakan ${ }^{2}$ Christopher F Nicodemus ${ }^{3}$, Michael A Hollingsworth ${ }^{1}$

${ }^{1}$ University of Nebraska Medical Centre, Omaha, NE, USA; ${ }^{2}$ Oncoquest, Edmonton, AB, Canada; ${ }^{3}$ AIT Strategies, Franconia, NH, USA

Correspondence: Michael A Hollingswort (kamiya.mehla@unmc.edu) Journal for ImmunoTherapy of Cancer 2017, 5(Suppl 2):P345

\section{Background}

Pancreatic adenocarcinoma remains a highly lethal disease, that has thus far proven to be highly refractory to immunotherapeutic strategies. Hence, new approaches are needed. There is epidemiological evidence that individuals with allergies have a lower incidence of pancreatic cancer [1]. This suggests that distinct immune surveillance (Th2 based) might underlie protection against pancreatic cancer observed in allergic individuals. We hypothesized that therapeutic targeting with IgE to enhance FcERI signaling would trigger an effective anti-tumor immune response against pancreatic cancer. We posited that tumor antigen directed $\mathrm{IgE} / \mathrm{FcERI}$ cross-linking on mast cell and controlled release of histamine near intra-tumor blood vessels could be exploited to improve drug delivery in pancreatic cancer. 


\section{Methods}

We therefore explored the efficacy of human tumor antigen targeted IgE antibody (humanized anti-MUC1.IgE) in combination of anti-PD-L1 (for relieving T cell exhaustion [2]) and PolyICLC (for effective dendritic cell maturation [2]) in a pre-clinical model of pancreatic cancer using mice transgenic for human MUC1 and FceRI (hMUC1/hFceRl).

Results

We observed that a combination of anti-MUC1.IgE+anti-PD-L1 + PolylCLC induced antigen specific rejection of two different MUC1 expressing pancreatic tumors cell lines (Panc02.MUC1, KPC.MUC1) and prolonged the overall survival of mice challenged with subcutaneous and orthotopic tumors as compared to mice treated with an isotype control antibody (anti-PSA.lgE). Furthermore, anti-MUC1.lgE tanti-PD-L1+PolylCLC combination induced MUC1 specific memory responses as evidenced by antigen specific rejection/delays of tumors in mice re-challenged with Panc02.MUC1 tumors. Importantly, we observed that NK and CD8 T cells were required for the cell mediated anti-tumor responses, as in vivo depletion of these subtypes but not CD4 abrogated the tumor protective in mice bearing orthotopic tumors. Additionally, our study demonstrated a time dependent increase in intra-tumor vascular permeability (increased dextran-647 influx) post anti-MUC1.IgE treatment (iv) in subcutaneous tumor (Panc02.MUC1) bearing dTg mice.

\section{Conclusions}

Taken together, this is the first study to show that cellular immune responses induced by antigen specific stimulation of the lgE/FcERI axis in combination with PolyICLC and anti-PD-L1provided tumor protective benefits against pancreatic cancer. Our study provides evidence for the clinical applicability and rapid translation of anti-MUC1.IgE based combination therapy against pancreatic tumors.

\section{References}

1. Gandini $S$, Lowenfel AB, Jaffee EM, Armstrong TD. Allergies and risk of pancreatic cancer: a meta-analysis with review of epidemiology and biological mechanisms. Cancer Epidemio Biomarker Prev. 2005;14(8):1908-16.

2. Nagato T, Celis E. A novel combinatorial cancer immunotherapy:poly-IC and blockade of the PD-1/PD-L1 pathway. Oncoimmunology. 2014;15:3:e28440.

\section{P346}

Inhibition of MMP9 yields improved effector T cell responses in a PD1-Axis refractory model

Amanda Mikels-Vigdal, Vladi Juric, Chris O'Sullivan, Erin Stefanutti, Andrew Greenstein, Maria Kovalenko, Jeremiah Degenhardt, Peng Yue, Victoria Smith

Gilead Sciences, Inc, Foster City, CA, USA

Correspondence: Amanda Mikels-Vigdal (amanda.mikelsvigdal@gilead.com)

Journal for ImmunoTherapy of Cancer 2017, 5(Suppl 2):P346

\section{Background}

Matrix metalloproteinase 9 (MMP9) acts via diverse mechanisms to promote tumor growth and metastasis, and is a key component of the immune-suppressive myeloid inflammatory milieu. We developed a monoclonal antibody (AB0046) that inhibits murine MMP9 and assessed its mechanism of action in immunocompetent mice as a single agent or in combination with a murine anti-PDL1 antibody.

\section{Methods}

An orthotopic, syngeneic tumor model of Her2-driven breast cancer was utilized for both efficacy and pharmacodynamic studies involving RNA and T cell receptor (TCR) sequencing, flow cytometry, and protein analysis. Enzymatic analyses were performed on $\mathrm{T}$ cell chemoattractant CXCR3 ligands (CXCL9, CXCL10, and CXCL11) which were subsequently evaluated in chemotaxis assays.

Results

Anti-MMP9 treatment alone or in combination with an anti-PDL1 antibody decreased primary tumor growth as compared to IgG controltreated animals (56\% vs $335 \%$ tumor growth increase, $p=0.0005$ ) or anti-PDL1 alone. Profiling of tumors by RNA sequencing revealed that inhibition of MMP9 resulted in elevated expression of genes associated with immune cell activation pathways (Hallmark Interferon Gamma
Response, FDR $p<0.001)$. Treatment with anti-MMP9 and anti-PDL1 antibodies decreased TCR clonality, with evidence of a more diverse TCR repertoire $(p=0.005)$. Immunophenotyping of tumor-associated T cells by flow cytometry showed that anti-MMP9 and anti-PDL1 co-treatment promoted a 2.8-fold increase in CD3+ cells in tumors $(p=0.01)$, which was associated with an increase in CD4+ $T$ cells (3.2-fold increase; $p=0.006$ ) and CD8+ T cells (2.8-fold increase; $\mathrm{p}=0.013$ ). In contrast, antiMMP9 and combination treatment resulted in a decrease in tumorassociated regulatory $T$ cells (CD25+ FoxP3+ cells, $p=0.04)$. MMP9 cleavage of T cell chemoattractant ligands in vitro rendered them functionally inactive for recruitment of activated primary human effector $T$ cells. Luminex analysis of tumor protein lysates showed elevated levels of key immune cytokines IL-12p70, IL-18, and CXCL10.

Conclusions

Inhibition of MMP9 reduces tumor burden and promotes cytotoxic T cell infiltration in a PD1-axis refractory mouse model. The combination of nivolumab and GS-5745, a humanized anti-MMP9 inhibitory antibody, is being evaluated in gastric cancer (NCT02864381).

Trial Registration

ClinicalTrials.gov: NCT02864381.

\section{P347}

Anti-PD1 $x$ anti-ICOS bispecific antibody XmAb23104 brings together PD1 blockade and ICOS costimulation to promote human $\mathrm{T}$ cell activation and proliferation

Gregory Moore, Michael Hedvat, Matthew Bernett, Christine Bonzon,

Rajat Varma, Suzanne Schubbert, Sung-Hyung Lee, Kendra Avery,

Rumana Rashid, Alex Nisthal, Liz Bogaert, Irene Leung, Seung Chu,

Umesh Muchhal, John Desjarlais

Xencor, Inc., Monrovia, CA, USA

Correspondence: John Desjarlais (gmoore@xencor.com)

Journal for ImmunoTherapy of Cancer 2017, 5(Suppl 2):P347

\section{Background}

Tumor infiltrating lymphocytes (TILs) often express multiple immune checkpoints and costimulatory receptors. Checkpoint blockade has demonstrated increased clinical response rates relative to other treatment options; however, many patients fail to achieve a response to checkpoint blockade. We sought to identify an additional therapeutic modality to stack with checkpoint blockade that could increase patient response rate. We hypothesized that engagement of $\mathrm{T}$ cell costimulatory receptors in concert with checkpoint blockade could further increase $T$ cell activation and proliferation. The combination of checkpoint blockade with costimulation could be accomplished using a bispecific antibody format, with the potential benefits of reduced cost and more selective targeting of TILs to improve safety.

Methods

XmAb23104, which binds to immune checkpoint PD1 and T cell costimulatory receptor ICOS, was assembled in a bispecific antibody platform with substitutions in the Fc domain to suppress effector function. XmAb23104 was evaluated in vitro by measuring antibody binding to and cytokine release from Staphylococcal enterotoxin B (SEB) stimulated PBMCs. In vivo activity was evaluated using a mouse model in which human PBMCs are engrafted into NSG mice (huPBMC-NSG) and the extent of T cell engraftment monitored by flow cytometry.

Results

We produced XmAb23104, a PD1 x ICOS bispecific antibody that binds both PD1 and ICOS monovalently. XmAb23104 selectively targeted $\mathrm{PD}^{+}{ }^{+} \mathrm{COS}^{+} \mathrm{T}$ cells more than monovalent controls, indicating that a single bispecific molecule was capable of avid co-engagement of both PD1 and ICOS. Surprisingly, despite monovalent engagement of ICOS, XmAb23104 promoted strong T cell activation above that expected of PD1 blockade alone, both in vitro and in vivo. In vitro, XmAb23104 enhanced IL2 production in an SEB stimulation assay ( $n=16$ donors) relative to control, anti-PD1 alone, and anti-ICOS alone $(p<0.001$ for all comparisons), indicating productive combination of PD1 blockade plus ICOS costimulation. Analysis of XmAb23104-treated cells revealed hallmarks of ICOS signaling, including AKT phosphorylation and a multigene expression signature consistent with ICOS costimulation. In vivo, 
treatment of huPBMC-NSG mice with XmAb23104 promoted superior T cell engraftment to that found for anti-PD1 treatment alone. In a representative study, XmAb23104 induced a 14-fold increase in human CD45 + cells, while anti-PD1 treatment alone only promoted a 2 -fold increase. Conclusions

XmAb23104, a PD1 x ICOS bispecific antibody, brings together PD1 blockade and ICOS costimulation and promotes strong T cell activation in vitro and in vivo. Compelling activity suggests clinical development is warranted for the treatment of human malignancies.

\section{P348}

Dual blockade of PD-L1 and LAG-3 with FS118, a unique bispecific antibody, induces T-cell activation with the potential to drive potent anti-tumour immune responses

Matthew Kraman, Natalie Fosh, Katarzyna Kmiecik, Katy Everett, Carlo Zimarino, Mustapha Faroudi, Mateusz Wydro, Alexander Koers, Lesley Young, Michelle Morrow, Jacqueline Doody, Mihriban Tuna, Neil Brewis F-star, Cambridge, United Kingdom

Correspondence: Michelle Morrow (michelle.morrow@f-star.com) Journal for ImmunoTherapy of Cancer 2017, 5(Suppl 2):P348

\section{Background}

Despite advances with therapies targeting the PD-1/PD-L1 pathway, many patients are refractory or relapse following treatment. LAG-3 expression on exhausted T cells and T-regulatory cells (Tregs) in the tumour may be responsible for this resistance and provides a rationale for co-treatment with antibodies targeting LAG-3 and PD-L1. An alternative approach is the development of a bispecific antibody encompassing binding sites for two antigens. FS118 is a bispecific antibody targeting LAG-3 and PD-L1 that provides dual pathway blockade with the potential to drive unique biology via co-binding of PD-L1 and LAG-3.

Methods

A LAG-3/PD-L1 mAb ${ }^{2}$ bispecific antibody, termed FS118, was engineered by introducing a distinct LAG-3 binding capability into the constant region of a human IgG1 molecule and assembled into a bispecific format with anti-PD-L1 Fabs. Additional mutations introduced into the Fc region suppress effector function. FS118 was evaluated in vitro for antigen binding and de-repression of LAG-3 and PD-L1 function in both a D011.10 T-cell activation system and a superantigen stimulated peripheral blood mononuclear cells (PBMC) assay. Anti-tumour activity of a murine-specific molecule, mLAG-3/PD-L1 $\mathrm{mAb}{ }^{2}$, was assessed in vivo in the MC38 mouse tumour model and associated immunophenotypic changes were evaluated using flow cytometry.

Results

FS118 is bivalent for both LAG-3 and PD-L1. It is capable of binding to both targets simultaneously and can de-repress the inhibitory function of human PD-L1 and human LAG-3 in an engineered murine T-cell system. FS118 is a potent activator of immune cell function generating sub-nanomolar $\mathrm{EC}_{50}$ values in a human $\mathrm{PBMC}$ assay as measured by cytokine with at least equivalent activity to a combination of anti-LAG-3 and anti-PD-L1.

In murine in vitro assay systems, $\mathrm{mLAG3/PD-L1} \mathrm{mAb}^{2}$ recapitulates the function of FS118 in human systems. MC38 tumour growth studies indicated that mLAG-3/PD-L1 could result in significant antitumour activity equivalent to a combination of antibodies targeting LAG-3 and PD-L1. Pharmacodynamic assessment demonstrated changes in the immunophenotype of tumour-infiltrating lymphocytes in the tumour of $\mathrm{mLAG} 3 / \mathrm{PD}-\mathrm{L} 1 \mathrm{mAb} \mathrm{b}^{2}$ treated mice.

\section{Conclusions}

Dual blockade of LAG-3 and PD-L1 with a bispecific antibody results in T-cell activation at least comparable to a combination of antibodies targeting LAG-3 and PD-L1 in primary T-cell assays and murine tumour models. These data provide evidence to support the rationale for clinical development of FS118, a LAG-3/PD-L1 mAb², for the treatment of human cancer.
P349

Molecular-targeted radiotherapy with an alkyl-phosphocholine analog leads toimmunomodulation in a syngeneic murine melanoma model

Suresh Veerankutty, Peter Carlson, Dana Baiu, Clinton Heinze, Alexander Boruch, Amy Erbe, Joseph Grudzinski, Reinier Hernandez, Bryan Bednarz, Jamey Weichert, Paul Sondel, Zachary Morris, Mario Otto

University of Wisconsin, Madison, WI, USA

Correspondence: Mario Otto (motto@pediatrics.wisc.edu); Zachary Morris

Journal for ImmunoTherapy of Cancer 2017, 5(Suppl 2):P349

\section{Background}

lonizing radiation can influence the immune response to tumors, and the combination of external beam radiotherapy with immunotherapy has increasingly garnered attention in clinical trials. Little is known about the immunomodulatory effects of molecular-targeted radiopharmaceuticals. 18-(p-iodophenyl) octadecyl phosphocholine (NM404) is a phospholipid ether analog with selective sequestration in cancer cells [1]. Here, we report on the immunomodulatory properties of the radioactive isostere, ${ }^{131}$ I-NM404 in a syngeneic murine melanoma model.

Methods

In vitro cell uptake of NM404 was evaluated by flow cytometry using a fluorescent-labeled NM404 analog. Mice, bearing syngeneic B78 melanoma tumors, were injected with a subtherapeutic dose of 2.2 $\mathrm{MBq}^{131} \mathrm{I}$-NM404 via lateral tail vein. Mice were euthanized and tumor tissue was harvested at 4 consecutive 1-week intervals post injection; the half-life of iodine-131 is $~ 1$ week. To evaluate markers of immunomodulation over time, quantitative PCR and immunohistochemistry $(\mathrm{IHC})$ were performed on the serially collected tumor tissue samples.

Results

In vitro, murine B78 melanoma cells sequestered 3-4 times more NM404 than normal murine splenocytes, and 6-10 times more than primary human fibroblasts over an 18 hour incubation period. The single, sub-therapeutic dose of ${ }^{131}$ I-NM404 did not significantly reduce the growth rate of B78 tumors in the treated mice compared to a control cohort injected with equivalent mass dose of nonradioactive NM404 (excipient). Gene expression analysis revealed a marked modulation of various immune and tumor-associated markers during the course of radiotherapy, such as IL-1 $\beta$, IFN $\gamma$, CXCL1, IL-18, TGF- $\beta 2$, B7-H3, PD-L1 and PD-L2. Our preliminary data suggest time-dependent patterns. IHC demonstrated that the number of tumor-infiltrating CD4 and CD8 T cells did not change significantly over time.

Conclusions

Our preliminary results suggest time-dependent immunomodulation induced by ${ }^{131}$ I-NM404 in B78 flank tumors. Further research should address the influence of variables such as radioactive dose, choice of radionuclide, tumor type and location on immunomodulation. This information has the potential to provide guidance in the design of more effective therapeutic strategies combining molecular-targeted radiotherapy and immunotherapy for cancer patients.

\section{References}

1. Pinchuk AN et al. Synthesis and structure-activity relationship effects on the tumor avidity of radioiodinated phospholipid ether analogues. J Med Chem. 2006;49(7):2155-65.

\section{P350}

Breast tumor suppression mediated by therapeutic expression of chemerin, an innate leukocyte chemoattractant

Russell Pachynski ${ }^{1}$, Ping Wang ${ }^{1}$, Brian Zabel $^{2}$, Eugene Butcher ${ }^{2}$

${ }^{1}$ Washington University School of Medicine, St Louis, MO, USA; ${ }^{2}$ Stanford University, Palo Alto, CA, USA

Correspondence: Russell Pachynski (rkpachynski@wustl.edu) Journal for ImmunoTherapy of Cancer 2017, 5(Suppl 2):P350 


\section{Background}

The infiltration of immune cells into the tumor microenvironment can regulate growth and survival of neoplastic cells. Several studies have shown a correlation between increases in the number of effector immune cells present in a tumor and clinical outcomes in many human tumors, including breast. Current strategies employing checkpoint inhibitors aim to stimulate effector immune cells, however lack of adequate effector cell numbers within the tumor microenvironment can result in suboptimal responses to these agents. Here, we describe a novel strategy employing therapeutic overexpression of chemerin to recruit immune cells into the tumor microenvironment (TME) and suppress tumor growth.

Methods

Publically available whole genome expression datasets were analyzed for RARRES2 expression, comparing normal breast tissue to invasive breast cancers. Both the JC and EMT6 tumor lines were used in fully immune competent BALB/c mice. Mammary fat pads were used for inoculation, and chemerin-expressing tumors were compared to control tumors. Tumors were measured using standard caliper measurements, and infiltrating leukocytes measured by flow cytometry. Leukocyte depletion studies were performed using specific depleting antibodies. Results

Our analyses of both TCGA and other public whole genome expression datasets show that RARRES2 (the gene for chemerin), a widely expressed endogenous chemoattractant protein for innate immune cells, is downregulated in several studies of human breast cancer. Significant downregulation of RARRES2 is seen in both invasive ductal and lobular breast cancers, compared to normal breast tissue. In mouse models using both the JC and EMT6 tumor lines, we have found that forced overexpression of chemerin within the TME significantly suppressed tumor growth with increased numbers of infiltrating leukocytes compared to controls. Systemic depletion of NK cells using anti-GM1 antibody treatment resulted in significant abrogation of chemerin's anti-tumor effect, suggesting - at least in part - a reliance on the innate response.

\section{Conclusions}

We have shown, for the first time, the use of therapeutic overexpression of chemerin is effective at suppressing breast tumor growth in in vivo mouse models. This approach has been used successfully in our melanoma models, and may be a broadly applicable approach to increase the number of immune effector cells within the TME. Ongoing studies are looking at the combination of therapeutic chemerin modulation in combination with available checkpoint inhibitors for determination of additive or synergistic effects.

\section{P351}

A novel, individualized xenograft model of cancer immunotherapy and tumor growth inhibition by ALKS 4230

Lukas Pfannenstiel', Jared Lopes², Heather Losey², Juan Alvarez², Brian Gastman

${ }^{1}$ Cleveland Clinic, Cleveland, OH, USA; ${ }^{2}$ Alkermes, Inc., Waltham, MA, USA Correspondence: Lukas Pfannenstiel (pfannel@ccf.org)

Journal for ImmunoTherapy of Cancer 2017, 5(Suppl 2):P351

\section{Background}

Recent successes in tumor immunotherapy highlight the curative potential of modulating patient anti-tumor immune responses. However, preclinical in vivo modeling of immune/tumor interactions often depend on a limited number of well-established cell lines. Of available treatment strategies, the use of cytokine therapy offers the advantage of using the patient's own immune cells as anti-tumor effectors. ALKS 4230 is a fusion protein of circularly permuted IL-2 and IL-2 receptor a that is selective for the intermediate-affinity IL-2 receptor expressed on NK cells and subsets of memory and effector T cells. ALKS 4230 is currently in a phase 1 trial to evaluate safety and tolerability in the treatment of patients with refractory solid tumors.

Methods

In order to evaluate the ability of ALKS 4230 to promote and enhance anti-human tumor immune responses preclinically, individual xenograft tumor models were established in NOD-scid IL2Rgamma ${ }^{\text {null }}$ mice (NSG) using tumor cells derived from metastatic melanoma patients following surgical resection. Upon tumor implantation and palpable growth, mice received an adoptive transfer of autologous, unexpanded PBMC from the same patient and treatment with ALKS 4230.

Results

We found that autologous T cells successfully engrafted NSG recipient mice after ALKS 4230 treatment similar to IL-2 and that both treatments induced cellular expansion over vehicle controls. Following treatment with ALKS 4230 and adoptive transfer of autologous PBMCs, PDX tumor-bearing mice consistently displayed increased numbers of both CD8 and CD4 T cells migrating into tumor tissue, preferential expansion of non-regulatory $\mathrm{T}$ cell subsets, and significant delays in tumor growth as compared to vehicle-treated controls. Conclusions

Together these data support the rationale for ALKS 4230 as a novel immunotherapeutic for the treatment of melanoma and potentially other solid cancers, as well as the strategy of screening individual, patient-specific xenograft models to assess potential treatment efficacy.

\section{P352}

A novel CD73 blocking antibody restores $T$ cell function and augments efficacy of Adenosine 2A Receptor (A2AR) inhibitor CPI-444

Emily Piccione, Jenny Rudnick, Barbara Daine-Matsuoka, Glen Mikesell, Richard Miller, lan McCaffery

Corvus Pharmaceuticals, Burlingame, CA, USA

Correspondence: Emily Piccione (epiccione@corvuspharma.com)

Journal for ImmunoTherapy of Cancer 2017, 5(Suppl 2):P352

\section{Background}

Adenosine accumulates in the tumor microenvironment (TME) through degradation of extracellular ATP by ectonucleotidases CD39 and CD73 and suppresses T cell activity through activation of A2AR. CD73 levels are elevated and are prognostic in certain tumors suggesting CD73 is an important immune-suppressive mechanism. Multiple CD73 antibodies are in clinical development that are allosteric CD73 inhibitors that stimulate internalization (type 2 mechanism) leading to incomplete inhibition of cell surface CD73 activity. We describe CPI-006, a novel CD73 antibody that directly inhibits CD73 enzymatic activity (type 1 mechanism).

Methods

CD73 activity was assayed by Malachite Green. Internalization was measured by flow cytometry. T cell proliferation was assayed by flow cytometry and cytokine levels by ELISA. Tumor CD73 was assessed by immunohistochemistry. Gene expression was determined by Nanostring.

Results

CPI-006 (nM affinity) was compared to CPX-016 (pM affinity), a potent type 2 CD73 antibody. CPI-006 completely inhibited CD73 catalytic activity across a broad range of CD73 expression. In contrast, CPX016 incompletely inhibited CD73 activity ( 60\%) and was not effective towards cell lines with high CD73 expression.

CPI-006 was significantly more effective than CPX-016 at blocking suppression of $\mathrm{T}$ cell proliferation and cytokine secretion by adenosine $(80 \%$ restoration compared to $45 \%)$. CPI-006 was effective across a broad range of CD73 expression while CPX-016 efficacy was restricted to samples with lower CD73 levels.

CD73 expression was evaluated by IHC in renal cell, melanoma, nonsmall cell lung and breast cancers ( $N=70$ each). $C D 73$ expression was heterogeneous in the tumor and stromal compartments; however, when expressed, CD73 levels were high, exceeding expression in the high expressing CD73 cell lines that were incompletely inhibited by CPX-016. These data suggest that type 2 antibodies will have limited efficacy in CD73 high expressing tumors.

Tumors with high CD73 expression are more responsive to A2AR inhibition [1] therefore we investigated the combined effect of CPI-006 with A2AR inhibitor, CPI-444. The combination significantly restored 
T cell proliferation and interferon-gamma production and suppressed adenosine responsive gene expression to levels greater than either agent alone.

\section{Conclusions}

$\mathrm{CPI}-006$ is a novel CD73 blocking antibody that directly and completely inhibits CD73 activity, in contrast to type 2 antibodies in development. CPI-006 blocks CD73 activity and improves T cell function independent of CD73 expression levels and augments the effect of CPI-444.

\section{References}

1. Beavis, P.A., et al., Blockade of A2A receptors potently suppresses the metastasis of CD73+ tumors. PNAS. 2013;110(36):14711-6.

\section{P353}

Discovery and pre-clinical development of an orally available small molecule antagonist targeting the CD47/SIRPa pathway for cancer immunotherapy

Pottayil Sasikumar, Chennakrishna Gundala, Wesley Balasubramanian, Sudarshan Naremaddepalli, Archana Bhumireddy, Sandeep Patil, Amit

Dhudashiya, Vijaysai Rayavarapu, Anirudha Lakshminarasimhan, Samiulla Dodheri, Kiran Aithal, Girish Daginakatte, Murali Ramachandra

Aurigene Discovery Technologies Limited, Bangalore, India

Correspondence: Murali Ramachandra (sasikumar_g@aurigene.com) Journal for ImmunoTherapy of Cancer 2017, 5(Suppl 2):P353

\section{Background}

The CD47/signal regulatory protein alpha (SIRPa) axis is a critical regulator of myeloid cell activation and serves as an immune checkpoint for macrophage mediated phagocytosis. Because of its frequent upregualtion in several cancers, CD47 contributes to immune evasion and cancer progression. Most of the current approaches in the immunotherapy focus on T-cell axis. Macrophages and other myeloid immune cells offer much promise as effectors of cancer immunotherapy, hence efforts to modulate them for therapeutic benefit are gaining momentum. In this regard disruption of CD47-SIRPa interaction is now being evaluated as a therapeutic strategy for cancer to stimulating macrophage mediated anti-tumor immune response. In view of the requirement for the intravenous dosing for all reported CD47 targeting agents, we sought to discover and develop an orally available small molecule CD47 antagonist. An oral CD47 agent potentially offers the convenience, flexibility to adjust the dose and schedule to address any emergent adverse events and ease of combination therapy.

Methods

Series of peptide disrupting CD47-SIRPa interactions were identified by rational design strategy based on CD47/SIRPa interacting interface. An FITC probe based cellular binding assay was established to identify high peptide fragments. The elements of this pharmacophore were incorporated into non-peptidic small molecule scaffolds resulting in lead compounds disrupting CD47/SIRPa interaction. The shortlisted compounds were further screened in a phagocytosis assay. In vivo efficacy was evaluated in A20 B Cell lymphoma syngeneic tumor model.

Results

Initial hits identified in FITC probe based cellular binding assay was further optimised for their activity in binding assay. Lead CD47 antagonists induced phagocytototic activity of human macrophages to a similar extent as commercially available anti-CD47 antibodies. Further optimization of these leads resulted in compounds with desirable physico-chemical properties and good oral bioavailability. An advanced lead CD47 antagonist inhibited primary tumor growth $(\sim 90 \% \mathrm{TGl})$ in a mouse syngeneic model of B-cell lymphoma upon twice a day oral dosing. Biomarker characterization and efficacy studies in additional tumor models are ongoing.

\section{Conclusions}

Rational design based on CD47/SIRPa interacting interface led to the identification of a novel and selective CD-47 antagonist with potent activity in cellular binding assay and phagocytosis assay. The lead compound exhibited desirable metabolic stability, solubility and oral bioavailability. In in-vivo studies, lead compound demonstrated significant anti-tumor efficacy in A20 B-cell lymphoma tumor model upon oral dosing. The above findings support further development of these orally bioavailable agents for use in the clinic.

P354

An orally bioavailable small molecule antagonist of VISTA and VSIG8 signaling pathways shows potent anti-tumor activity

Pottayil Sasikumar, Sudarshan Naremaddepalli, Raghuveer Ramachandra, Nagesh Gowda, Govardhan Alluri, Manikyalarao Yerramsetti,

Sreenivas Adurthi, Jiju Mani, Rashmi Nair, Amit Dhudashiya,

Samiulla- Dodheri, Nagaraj Gowda, Murali Ramachandra

Aurigene Discovery Technologies Limited, Bangalore, India

Correspondence: Murali Ramachandra (sasikumar_g@aurigene.com)

Journal for ImmunoTherapy of Cancer 2017, 5(Suppl 2):P354

\section{Background}

Antibody-mediated blockade of immune checkpoint pathways have transformed the outlook for cancer therapy. While PD-1/PD-L1 antibodies primarily focus on T-cells to achieve anti-tumor efficacy, other cells in the tumor microenvironment such as myeloid cells including MDSCs also play a role in immune evasion. To overcome the immune resistance induced by MDSCs, V-domain Ig suppressor of T-cell activation (VISTA) expressed predominantly on myeloid cells and tumorinfiltrating lymphocytes is considered as an ideal target. Recent findings also support the role of VISTA pathway in clearance of apoptotic bodies and prevention of autoimmunity. VISTA is reported to mediate immune suppression through homophilic interaction as well as interaction with V-Set and immunoglobulin domain containing 8 (VSIG8). We sought to discover and develop an orally available small molecule VISTA antagonist targeting both VISTA and VSIG8 pathways. Unlike antibodies an oral gent potentially offers the convenience, flexibility to adjust dose and schedule to address any emergent adverse events and ease of combination therapy.

Methods

VISTA belongs to B7 family and shares sequence homology with the B7 family ligands PD-L1, a focused library of compounds mimicking the interaction of checkpoint proteins of B7 family was designed and synthesized. These compounds were tested in VISTA and VSIG8 specific functional assays. In-vivo efficacy was evaluated in mouse syngeneic B16F10 melanoma and CT26 colon carcinoma models. Impact of test agents on various immune populations was measured by flow cytometric analysis in tumor-bearing animals upon repeated dosing.

Results

Screening and analysis of the focused library of compounds led to the identification of hits capable of functional disruption of the checkpoint protein(s) signaling. Further optimization resulted in lead compounds targeting both VISTA and VSIG8 signaling pathways with desirable drug-like properties. Potent functional activity comparable to that obtained with an anti-VISTA or anti-VSIG8 antibody in rescuing effector functions was observed with the lead compound along with selectivity against other immune checkpoint proteins. An advanced lead compound exhibited sustained immune PD in tumorbearing animals including desirable impact on myeloid and T-cells in both circulation and tumor. The advanced lead compound also exhibited significant efficacy in syngeneic pre-clinical tumor models of melanoma and colon cancers upon once a day oral dosing with excellent tolerability.

Conclusions

Our efforts have resulted in the identification of novel oral antagonists of VISTA and VSIG8 signaling. Desirable drug-like properties and anti-tumor efficacy at well-tolerated doses by the advanced lead compound in pre-clinical models supports its further development towards advancing to the clinic. 
P355

Epigenetic modulation promotes tumor suppression and improves survival when combined with checkpoint inhibition in murine models of breast cancer

Evanthia Roussos Torres, Hayley Ma, Christine Rafie, Brian Christmas, Todd Armstrong, Elizabeth Jaffee

Johns Hopkins University, Baltimore, MD, USA

Correspondence: Evanthia Roussos Torres (ertorres@jhu.edu); Elizabeth Jaffee

Journal for ImmunoTherapy of Cancer 2017, 5(Suppl 2):P355

\section{Background}

Checkpoint inhibition is a very successful treatment strategy in cancers that are naturally immunogenic by attracting $T$ cells into the tumor microenvironment (TME) and promoting cytotoxic signaling pathways. Most breast cancers are not highly immunogenic likely due to an immunosuppressive microenvironment. One strategy to transform this TME is to use epigenetic modulation to affect activation and trafficking of myeloid derived suppressor cells (MDSCs), known to alter the immunogenicity of the TME and sensitize tumors to checkpoint modulation. We hypothesize that combinatorial therapy primes the TME by altering infiltration and function of MDSCs leading to a more robust $\mathrm{T}$ cell response.

Methods

We use the HER-2/neu transgenic mouse model with challenge of syngeneic cell lines. This model enables us to study the efficacy of different combinations of an anti-HER2 antibody (a-Her2), an epigenetic agent, the histone deacetylase inhibitor entinostat, and checkpoint inhibitors, anti -programmed cell death protein (PD-1) and anti-cytotoxic Tlymphocyte-associated protein 4 (CTLA-4) antibodies, on tumor growth and to help identify co-stimulatory and inhibitory factors regulating $T$ cell and MDSC responses. Characterization of tumor infiltrating lymphocytes (TIL) and their functional capabilities are being investigated in primary tumors using fluorescence-activated cell sorting, nanostring gene expression profiling, and immunohistochemistry.

Results

We found significant improvement in survival and delay in tumor growth in tumor bearing mice- treated with entinostat, anti-PD1, and anti-CTLA-4 and with a-Her2, entinostat and anti-PD1 or anti-CTLA4. We show that addition of entinostat to checkpoint inhibition leads to significantly increased infiltration of granulocytic-MDSCs and $C D 8^{+} \mathrm{T}$ effector cells into the TME. Flow cytometric evaluation suggests increased T cell activation, exhaustion, and altered MDSC function. Functional assays of isolated MDSCs from mice treated with combination therapy demonstrates reduced suppressive ability of these cells. Gene expression profiling of isolated MDSCs and TIL is underway to help determine significant changes in immune related pathways that have lead to our observed outcomes.

\section{Conclusions}

Addition of entinostat and checkpoint inhibition to a-Her2 therapy significantly increases infiltration of innate and adaptive immune cells into the highly tolerant breast tumors and leads to improved survival and decreased tumor burden. Results suggest that this combinatorial treatment alters the function of the infiltrating cell types that lead to the observed phenotype. We aim to delineate genetic alterations responsible for these observations. It is our hope that our novel findings will provide further rationale for combination therapy and improve the response rate to immunotherapy in patients with breast cancer.

\section{P356}

Efficacy and Mechanism of Action of CXCR4 Inhibition in B16-OVA Melanoma Model

Ruchi Saxena', Yan Wang ${ }^{2}$, James Mier ${ }^{1}$

${ }^{1}$ Beth Israel Deaconess Medical Center, Boston, MA, USA; ${ }^{2} \times 4$

Pharmaceuticals, Cambridge, MA, USA

Correspondence: James Mier (rsaxena@bidmc.harvard.edu)

Journal for ImmunoTherapy of Cancer 2017, 5(Suppl 2):P356

\section{Background}

In our previous studies, we demonstrated that combination of a CXCR4 antagonist with an anti-angiogenesis agent axitinib relieved myeloidderived suppressor cells (MDSCs) mediated immunosuppression and suppressed HIF-2a expression, which resulted in synergistic antitumor effects in 786-0 and A498 RCC xenografts. To further study the CXCR4 mechanism of action in an immune-proficient background, we investigated the activity of a CXCR4 antagonist in a syngeneic mouse tumor model. As the current murine models for RCC do not share the same genetic alterations with the human disease, the B16-OVA model was selected for this study. The CXCR4 antagonist was tested in combination with axitinib or with checkpoint inhibitors which are part of the SOC in managing melanoma in clinic.

Methods

B16-OVA cells were implanted into C57BL/6 mice. Seven-days post implantation, mice were treated with axitinib, X4-136 (CXCR4 inhibitor) or anti-PD-L1/anti-CTLA4, or the combinations for 16 days. At sacrifice, tumors were excised and flash frozen in liquid nitrogen for Western Blot analysis or treated with collagenase for the analysis of subsets of T-cells by FACS.

Results

Axitinib alone modestly inhibited growth of B16-OVA tumors. X4-136, the CXCR4 inhibitor, alone demonstrated more robust activity than the combination treatment of anti-PD-L1 and anti-CTLA4. The antitumor activity of the anti-PD-L1 and anti-CTLA4 regimen was further enhanced in combination with X4-136.

Preliminary analysis of infiltrating immune cells by flow cytometry showed that axitinib treatment led to an increase in immunosuppressive Treg cell population in tumors, while treatment with X4-136 alone or the anti-PD-L1/anti-CTLA4 combination led to a decrease in Tregs. The decrease was more pronounced when X4-136 was used in combination with axitinib. Additional analysis of other immune cell subsets will also be presented.

This combination also led to a decrease in the expression of HIF-2a and cyclin D1 in comparison to vehicle treated mice as demonstrated by western blot analysis of tumors, suggesting an anti-proliferative effect. PARP cleavage was apparent when X4-136 was used in combination with axitinib indicating the role of apoptosis in anti-tumor effect. Additional analysis of tumors after treatment in earlier time points will also be presented.

Conclusions

X4-136 alone exhibited potent anti-tumor activity in the B16-OVA murine melanoma model. Added benefit was observed when X4-136 was added to either axitinib or to anti-PD-L1/anti-CTLA-4 treatments. Treatment benefits were associated with the reduction of Treg population in the tumor microenvironment. Induction of apoptosis was observed in combination treatment of axitinib and X4-136.

P357

The Tt-cell growth factor cocktail IL-2/IL-15/IL-21 enhances expansion and effector function of tumor-infiltrating $T$ cells in a novel process developed by iovance

lan Frank, Michelle Simpson-Abelson, Michael Lotze, Krit Ritthipichai, Christopher Mosychuk

lovance Biotherapeutics, Tampa Bay, FL, USA

Correspondence: Michael Lotze (michelle.abelson@iovance.com) Journal for ImmunoTherapy of Cancer 2017, 5(Suppl 2):P357

\section{Background}

Adoptive T cell therapy with autologous tumor infiltrating lymphocytes (TIL) has demonstrated clinical efficacy in patients with metastatic melanoma and cervical carcinoma. In some studies, clinical outcomes have positively correlated with the total number of cells infused and/or percentage of CD8+ T cells. Most current production regimens solely utilize IL-2 to promote TIL growth, although enhanced lymphocyte expansion has been reported using IL-15 and IL-21-containing regimens. This study describes the positive effects of adding IL-15 and IL-21 to the standard IL-2-alone TIL generation protocol. 


\section{Methods}

The process for generating TIL includes a pre-Rapid Expansion Protocol (pre-REP), in which TIL emigrate out of tumor fragments of 1-3 $\mathrm{mm}^{3}$ in media containing IL-2. To further stimulate TIL growth, TIL are expanded using a secondary culture period termed the Rapid Expansion Protocol (REP) that includes irradiated PBMC feeders, IL-2 and anti-CD3. In this study, a shortened pre-REP and REP expansion protocol was developed to expand TIL while maintaining the phenotypic and functional attributes of the final TIL product. This shortened TIL-generation protocol was used to assess the impact of IL-2 alone versus a combination of IL-2/IL-15/IL-21. These two culture regimens were compared for the generation of TIL grown from colorectal, melanoma, cervical, triple negative breast, lung and renal tumors. At the completion of the pre-REP, cultured TIL were assessed for expansion, phenotype, function (CD107a+ expression and IFNg release) and TCR $\mathrm{V} \beta$ repertoire.

Results

Enhanced TIL expansion ( $>20 \%$ ), in both CD4+ and CD8+ cells in the IL-2/IL-15/IL-21 culture cocktail was observed across multiple tumor histologies. Preliminary analysis demonstrated a shift towards a predominantly CD8+ TIL population with a skewed TCR V $\beta$ repertoire in TIL cultured with the IL-2/L-15/IL-21, versus IL-2 alone. IFNg release and CD107a expression were also elevated in TIL cultured in the presence of IL-2/IL-15/IL-21 versus those cultured using IL-2 alone.

\section{Conclusions}

Rapidly expanding TIL ex vivo for adoptive cell therapy is essential in treating patients with cancer. We report an increased TIL-product yield, in addition to potentially beneficial phenotypic and functional differences, when TIL are cultured with IL-2/L15/LL-21 as compared to IL-2 alone. We suggest a more robust process encompassing the use of IL-2/IL-15/IL-21 in TIL culture may provide a means to promote TIL expansion particularly in tumors with poor $\mathrm{T}$ cell infiltration.

\section{P358}

Development of CDX-1140, an agonist CD40 antibody for cancer immunotherapy

Lawrence Thomas ${ }^{1}$, Laura Vitale², Thomas O'Neill², Jenifer Widger², Andrea Crocker², Li-Zhen He'2, Jeffrey Weidlick², Karuna Sundarapandiyan², James Storey ${ }^{1}$, Eric Forsberg ', Catherine Pilsmaker', Lauren Gergel ${ }^{1}$, Elizabeth Do', Joel Goldstein ${ }^{2}$, Henry Marsh', ${ }^{1}$ Tibor Keler ${ }^{2}$

${ }^{1}$ Celldex Therapeutics, Needham, MA, USA; ${ }^{2}$ Celldex Therapeutics, Hampton, NJ, USA

Correspondence: Lawrence Thomas (Lthomas@celldex.com) Journal for ImmunoTherapy of Cancer 2017, 5(Suppl 2):P358

\section{Background}

Current limitations for immunotherapy approaches include poorly functioning events early in the immune response cycle, such as efficient antigen presentation and T cell priming. CD40 signaling in dendritic cells (DCs) leads to upregulation of cell surface costimulatory and MHC molecules and the generation of cytokines, which promotes effective priming of CD8+ effector T cells while minimizing T cell anergy and the generation of regulatory $T$ cells. This naturally occurs through interaction with CD40 ligand (CD40L) expressed on CD4+ T-helper cells. CD40 signaling can also be achieved using specific monoclonal antibodies (mAbs), leading to the development of several agonist CD40 antibodies that have initiated clinical development.

\section{Methods}

Our approach for the development of a CD40 agonist antibody was to define a balanced profile between sufficiently strong immune stimulation and the untoward effects of systemic immune activation. Results

CDX-1140 is a human IgG2 antibody derived from human Ig transgenic mice, that activates DCs as demonstrated by upregulation of costimulatory molecules and increased production of cytokines. This activity is Fc independent as it is maintained using an $\mathrm{F}\left(\mathrm{ab} \mathrm{b}^{\prime}\right)_{2}$ fragment of the antibody. CDX-1140 does not block the binding of CD40L and the addition of soluble CD40L greatly enhances DC activation by CDX-1140, suggesting that CDX-1140 may act synergistically with naturally expressed
CD40L. CDX-1140 also activates B cells, and has direct anti-lymphoma activity in xenograft models. However, the antibody does not promote cytokine production in whole blood assays. Importantly, CDX-1140 has shown good pharmacodynamic and safety profiles in a cynomolgus macaque study investigating doses from 0.01 to $10 \mathrm{mg} / \mathrm{kg}$. This study showed a clear dose response with respect to changes in hematologic and circulating cytokine values (IL-12p40) that were expected to result from CD40 activation.

Conclusions

These data support the potential of CDX-1140 as part of a cancer therapy regimen, and the phase 1 dose-escalation clinical study is anticipated to begin this year.

P359

Effect of targeted anti-GD2/-CD16 Bispecific NK cell Engager (BiKE) with or without IL-15 super agonist ALT803 against GD2+

Neuroblastoma (NB) and Ewing Sarcoma (ES)

Aradhana Tiwari ${ }^{1}$, Dina Edani ${ }^{1}$, Janet Ayello ${ }^{1}$, Hing C. Wong ${ }^{2}$,

Mitchell S Cairo

${ }^{1}$ New York Medical College, Valhalla, NY, USA; ${ }^{2}$ Altor

BioscienceWebsiteDirections, Miramar, FL, USA

Correspondence: Aradhana Tiwari (aradhana_tiwari@nymc.edu)

Journal for ImmunoTherapy of Cancer 2017, 5(Suppl 2):P359

\section{Background}

GD2 is a surface disialoganglioside that is a well-characterized immunotherapeutic target in NB and Sarcomas [1]. The efficacy of antiGD2 antibodies depends on engaging functional NK cells to kill GD2positive targets through antibody dependent cellular cytotoxicity (ADCC). However, NK cell number and function are decreased in most cancer patients at diagnosis, are further reduced by radiation chemotherapy, antigen loss and immunosuppressive tumor microenvironment (TME) which contribute to treatment failure. One of the new approaches to overcome TME resistance and improve NK cell mediated ADCC against tumor cells is using a BiKE [2]. ALT-803 is a superagonist of an IL-15 variant bound to an IL-15RaSu-Fc fusion with enhanced IL-15 biological activity.

To investigate the in-vitro activity of hu-anti-GD2/-CD16 BiKE with or without ALT803 against GD2 expressing NB/ES

Methods

Anti-GD2/-CD16 BiKE was constructed in pBudCE4.1 mammalian expression vector and transfected in to HEK293-EBNA Cells. Stable clone were selected by Zeocin for the secretion and purification of anti-GD2/-CD16 plasmid by ProBond ${ }^{\mathrm{TM}} \mathrm{Ni}$ column and validated by Western blot. Cytotoxicity was examined against NB/ES cells with or without ALT803 (generously supplied by Altor Biosciences) with K562-mbIL21-41BBL expanded NK cells by DELFIA cytotoxicity assay at 10:1 E:T ratio.

Results

GD2 expressing NB (54.4 $\pm 10.61 \%)$ and ES $(71.93 \pm 8.33 \%)$ cells were used for functional assays. Anti-GD2/-CD16 BiKE was purified from the transfected culture supernatant and validated by western blot (Fig. 1A). BiKE+NK compared to Medium+NK significantly increased NK mediated cytotoxicity against NB: SKNF1 $(64.5 \pm 5.9 \%$ vs. $20.1 \pm 1.3 \%, p=0.002)$, SKNBE2 $(67.4 \pm 4.02 \%$ vs. $15.1 \pm 0.9 \%, p=0.004)$, SHS5Y5 $(68.9 \pm 0.9 \%$ vs. $30.2 \pm 0.85 \%, p=0.005)$ and ES: EWS502 $(68.05 \pm 3.06 \%$ vs. $28.1 \pm 4.05 \%$, $p=0.004), A 673(66.02 \pm 4.05 \%$ vs. $20.3 \pm 0.8 \%, p=0.005)$ respectively. Further, BiKE+NK+ALT803 compared to BiKE+NK+rhulL15 improved NK mediated cytotoxicity against NB: SKNF1 $(73.9 \pm 9.3 \%$ vs. $56.5 \pm 8.5 \%$, $p=0.007)$ and SKNBE2 (63.9 $\pm 0.6 \%$ vs. $40.5 \pm 0.49 \%, p=0.002)$ respectively, against NB/ES cell lines (Fig. 1B).

Conclusions

Our preliminary results demonstrated that the BiKE with ALT803 significantly enhanced NK cytotoxicity against NB and ES. Future studies will investigate the efficacy of this BiKE with ALT803 against GD2 expressing solid tumor in humanized NSG xenografted mouse model.

\section{References}

1. Perkins S M, Shinohara ET, DeWees T, Frangoul H. Outcome for children 
with metastatic solid tumors over the last four decades. PLoS One. 2014;9.

2. Gleason MK, et al. Bispecific and trispecific killer cell engagers directly activate human NK cells through CD16 signaling and induce cytotoxicity and cytokine production. Mol Cancer Ther. 2012;11:2674-2684.

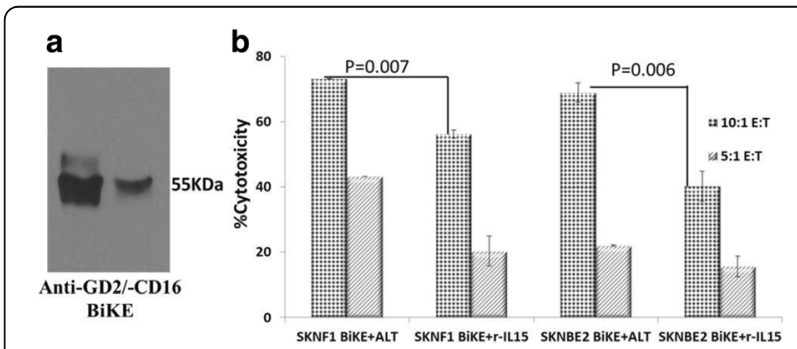

Fig. 1 (abstract P359). See text for description

\section{P360}

APX005M is a potent CD40 agonistic antibody capable of stimulating both innate and adaptive immune responses against cancer

Pia Björck, Erin Filbert, Ovid Trifan, Xiaodong Yang

Apexigen Inc., San Carlos, CA, USA

Correspondence: Xiaodong Yang (otrifan@apexigen.com)

Journal for ImmunoTherapy of Cancer 2017, 5(Suppl 2):P360

\section{Background}

CD40 plays an important role in activation and regulation of both innate and adaptive immunity. Using the proprietary APXiMAB $^{T M}$ discovery platform, Apexigen discovered APX005M, a potent immune stimulatory CD40 agonistic antibody currently being developed for cancer immunotherapy. APX005M is a high-affinity humanized IgG1 antibody targeting human CD40. APX005M was engineered to carry an S267E mutation in its Fc region to enhance CD40 agonistic activity via cross-linking to the FcRyllb.

Methods

PBMCs were obtained from healthy human subjects. Isolated cell subsets were cultured in various conditions with APX005M. The potential synergistic effect of APX005M with immune checkpoint inhibitors (ICl) was assessed by in vitro culture of human dendritic cells (DC) and allogeneic T cells with APX005M and anti-PD-1 or anti-PDL1 antibodies. The duration of APC activation following exposure to APX005M was determined by culturing PBMCs with APX005M for 24 hours. Cell activation was measured at various times post washout. Results

APX005M binds with high-affinity to human CD40 (Kd=0.12 nM) and recognizes a unique epitope that overlaps with the CD40 ligand (CD40L) binding domain thus mimicking CD40L-induced activation. APX005M is capable of activating human $B$ cells $(E C 50=12 \mathrm{pM})$ and $D C(E C 50=0.49$ $\mathrm{nM})$ and also promotes proliferative responses of tumor-infiltrating $T$ cells. APX005M's CD40 agonistic activity depends on cross-linking of Fcgamma receptors. Receptor occupancy studies revealed that $10 \%$ of CD40 receptor occupancy is sufficient to produce maximum APC and T cell activation. Short-term exposure (24 hours) of PBMC to APX005M induced long-lasting activation of $B$ cells, monocytes and $T$ cells that remained activated 2 weeks after removal of APX005M. In vitro coculture of human DC and allogeneic T cells showed that APX005M induces a dose-dependent increase of CD4 and CD8 T cell proliferation and IFN- $\gamma$ secretion, and the T-cell responses were further enhanced by anti-PD-1 or anti-PD-L1 antibodies suggesting that APX005M combined with $\mathrm{ICl}$ can synergistically stimulate $\mathrm{T}$-cell responses.

\section{Conclusions}

The immune stimulatory activity of APX005M results from its unique epitope and is dependent on cross-linking by Fc-gamma receptors. The properties of APX005M make it an optimal CD40 agonist for stimulating anti-tumor immune responses while maintaining a good safety profile. Combination of APX005M with ICl enhances T-cell responses providing a rationale for the ongoing clinical studies combining APX005M with anti-PD-1 antibodies in NSCLC and melanoma patients (trials NCT03123783 and NCT02706353).

\section{P361}

A novel T-cell engaging bispecific antibody platform: Efficient In vivo tumor clearance with minimal cytokine release

Nathan Trinklein, Andrew Boudreau, Ben Buelow, Starlynn Clarke, Kevin Dang, Laura Davison, Shelley Force Aldred, Katherine Harris, Suhasini lyer, Brett Jorgensen, Heather Ogana, Duy Pham, Payal Pratap, Udaya Rangaswamy, Ute Schellenberger, Harshad Ugamraj, Omid Vafa,

Wim van Schooten

Teneobio, Menlo Park, CA, USA

Correspondence: Nathan Trinklein (ntrinklein@teneobio.com)

Journal for ImmunoTherapy of Cancer 2017, 5(Suppl 2):P361

\section{Background}

Using transgenic rats and a unique sequence-based discovery approach, we have created a large collection of fully human anti-CD3 antibodies with diverse T-cell agonist activities. We have used these anti-CD3 antibodies to create a unique multi-specific platform for Tcell redirected tumor cell killing.

Methods

Our novel discovery platform combines antibody repertoire deep sequencing, high-throughput gene assembly, and recombinant expression and generates a much larger diversity of antibodies than traditional approaches. The CD3 antibodies identified by our platform show diverse in vitro T-cell activation profiles measured by CD69 upregulation, IL2, and IFNg production. Using our discovery platform, we have also generated human domain antibodies targeting tumor antigens that may be combined with our unique CD3 antibodies to create multi-specific antibodies.

Results

As one example, we have created a CD3XBCMA bispecific antibody (TNB-383B) for the treatment of multiple myeloma. TNB-383B kills multiple myeloma cells in vitro and in vivo in a BCMA-dependent manner, and kills primary patient myeloma cells ex vivo. The EC50 for cytotoxicity was in the single-digit nanomolar range for TNB-383B against MM cell lines in vitro. TNB-383B showed much reduced (ie IFN- $\gamma$ ) or absent (ie IL-2) cytokine release compared to other anti-CD3 antibodies. In vitro results were consistent across 10 healthy huPBMC donors. Ex vivo, TNB$383 \mathrm{~B}$ efficiently lysed primary MM cells in the presence and absence of supplementary T-cells. In vivo, TNB-383B mediated clearance of MM tumors from NSG mice at doses as low as 10ng of bispecific antibody.

Conclusions

In summary, we have created a T-cell engaging bispecific antibody platform with tunable T-cell agonism that can be used to optimize the therapeutic index for a variety of tumor antigens.

\section{P362}

A novel assay using RNA aptamers to quantitate the fraction of IL2Ra (CD25) receptors occupied by IL2

Suresh Veeramani, Sue Blackwell, William Thiel, Paloma Giangrande, George Weiner

University of lowa, lowa City, IA, USA

Correspondence: George Weiner (suresh-veeramani@uiowa.edu) Journal for ImmunoTherapy of Cancer 2017, 5(Suppl 2):P362

\section{Background}

RNA aptamers bind to antigens in a manner analogous to antibodies and their binding can be quantitated using simple RT-PCR. Using IL2IL2Ra as a model, we report a novel assay using IL2-IL2Ra-binding RNA aptamers that allows for quantitation of receptor occupancy by its ligand.

Methods

A modification of whole cell Systematic Evolution of Ligands by EXponential enrichment (SELEX) was used to select T regulatory (Treg) cell-specific RNA aptamers. Aptamers specific for common $\mathrm{T}$ cell 
antigens were precleared using normal donor $\mathrm{CD}^{+}{ }^{+} \mathrm{L} 2 \mathrm{Ra}^{-} \mathrm{T}$ cells, followed by enrichment for aptamers specific for $\mathrm{CD} 4^{+} \mathrm{IL} 2 \mathrm{Ra}^{+}$Tregs obtained from the same donor. The process was repeated for eight rounds with each round using T cells from a different normal donor. High-throughput sequencing and bioinformatics analysis was used to select top Treg-binding aptamers. Binding of aptamers to unoccupied IL2Ra and IL2Ra occupied by IL2 was determined by RT-qPCR. Some aptamers bound preferentially to unoccupied IL2Ra while others bound preferentially to IL2Ra occupied by IL2. To determine the fraction of IL2Ra occupied by IL2, aptamers that bound preferentially to unoccupied receptor or to IL2-occupied receptor were mixed in equimolar quantities. Aptamer mix was added to IL2Ra-coated Dynabeads that were pre-incubated with various concentrations of IL2 to create various IL2-occupied receptor fractions. Aptamer binding was quantified by RT-qPCR using a set of primers specific for both aptamers. Fluorescent probes specific for the variable region for each aptamer were used to quantify each of the aptamers.

Results

Five of the top 12 Treg-binding aptamers recognized IL2Ra. Some IL2Ra-specific aptamers (e.g. Tr-8) bound preferentially to unoccupied IL2Ra and others (e.g. Tr-7) to the IL2-IL2Ra complex (Fig. 1). Standard curves were developed which allowed for determination of the fraction of IL2Ra occupied by IL2 (Fig. 2).

\section{Conclusions}

A Treg cell-based SELEX was used to select Treg-binding aptamers specific for IL2Ra. Aptamer pairs showing differential binding preferences towards unoccupied IL2Ra versus the IL2-IL2Ra complex were identified. Because the selected aptamer pairs share the same primers, RT-qPCR amplification using a single set of primers allowed for quantitation of the ratio of bound aptamers. The resulting ratio reflects the occupancy of the IL2Ra receptor by its ligand IL2. This technique could be valuable for studying the role of IL2-IL2Ra ligandreceptor complex in anti-tumor immunity. Importantly, modifications of this assay could be used to quantitate other receptor-ligand complexes relevant in the field of cancer immunotherapy.
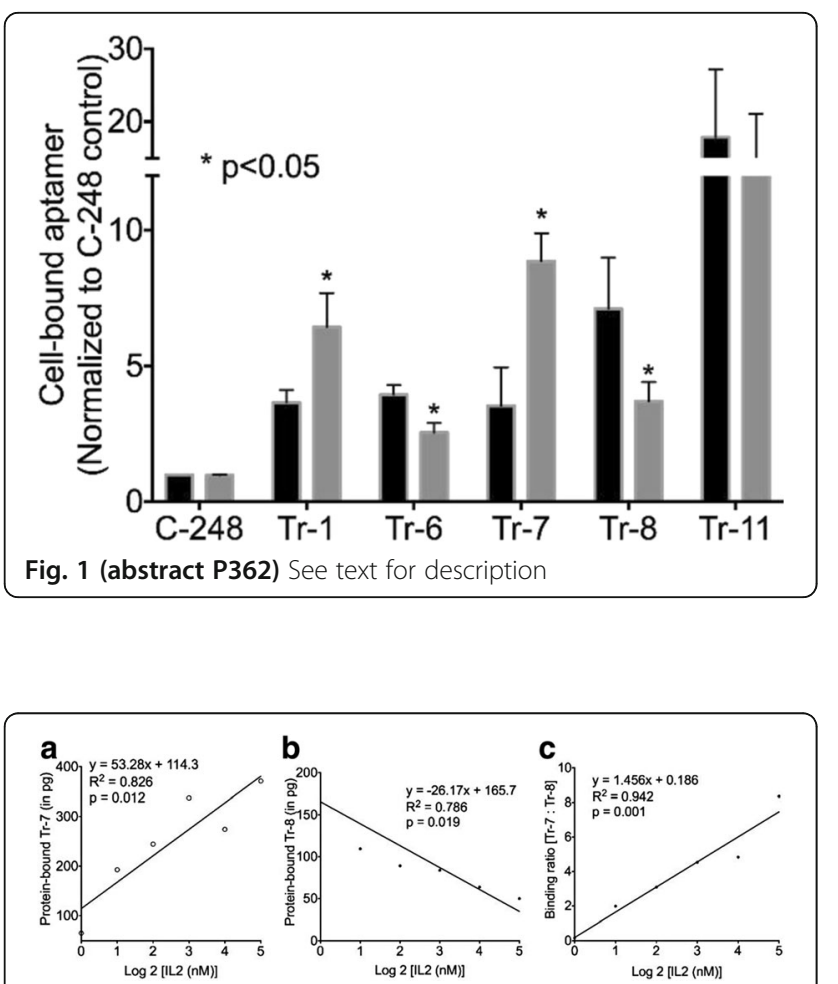

Fig. 2 (abstract P362) See text for description
P363

The OX40-CTLA-4 bispecific antibody, ATOR-1015, induces immune activation and anti-tumor effect

Niina Veitonmäki, Karin Hägerbrand, Mia Thagesson, Doreen Werchau,

Karin Enell-Smith, Anna Rosén, Sara Frizell, Anne Månsson-Kvarhammar,

Per Norlén, Furebring Tina, Peter Ellmark

Alligator Bioscience, Lund, Sweden

Correspondence: Niina Veitonmäki (niv@alligatorbioscience.com)

Journal for ImmunoTherapy of Cancer 2017, 5(Suppl 2):P363

\section{Background}

ATOR-1015 is an OX40-CTLA-4 bispecific immune activating antibody developed for tumor-directed immunotherapy. The compound was generated by fusing a high affinity CTLA-4 binder, derived by FIND ${ }^{\circledR}$ optimization of CD86, to an agonistic OX40 antibody derived from the human antibody library ALLIGATOR-GOLD ${ }^{\odot}$.

ATOR-1015 binds both targets simultaneously resulting in cell-cell interactions expected to enhance the immuno-stimulating effect of the compound. The mode of action of ATOR-1015 is thought to be a combination of effector $\mathrm{T}$ cell activation and regulatory $\mathrm{T}$ cell (Treg) depletion.

Methods

Human primary cells used in in vitro assays were isolated from leukocyte concentrates from healthy donors. The in vitro effects were measured by using either standard cytokine release assays or Promega reporter assays.

Human OX40 transgenic (knock-in) mice were generated by genOway SA and female mice were used for the anti-tumor effect studies using syngeneic mouse models. Tumors and spleens from treated mice were analyzed for Treg and $\mathrm{T}$ cell populations by flow cytometry.

Results

ATOR-1015 dependent T cell activation and Treg depletion is supported by cell-based in vitro studies and in vivo syngeneic tumor models.

The ability to induce ADCC of human Treg was investigated using a FcyR expressing reporter assay demonstrating superior effect of ATOR-1015 compared to the monospecific antibodies. Further, ATOR1015 has been shown to induce activation of $T$ cells in the presence of CTLA-4 expressing cells.

Treatment with ATOR-1015 reduces tumor growth and prolongs survival in syngeneic tumor models in vivo using human OX40 transgenic mice. Further, ATOR-1015 treatment demonstrates an increase in the intratumoral $\mathrm{CD}^{+} \mathrm{T}$ cell/Treg ratio which is superior compared to the monospecific counterparts, without affecting systemic T cells.

Conclusions

ATOR-1015 suppresses/depletes Tregs and activates CD8 ${ }^{+}$T cells in vivo and is currently in the second phase of production, and process development. ATOR-1015 is planned to enter clinical trials in 2018.

P364

Discovery and pharmacological characterization of JNJ-63723283, an anti-programmed cell death protein-1 (PD-1) antibody that blocks PD-1 function

Catherine Ferrante ${ }^{1}$, Nikki DeAngelis ${ }^{1}$, Gordon Powers ${ }^{1}$, Jocelyn Sendecki ${ }^{1}$, Hillary Millar ${ }^{2}$, Bethany Mattson' ${ }^{1}$, Darlene Pizutti ${ }^{1}$, Christina Lourdes Mayer ${ }^{1}$, Weirong Wang ${ }^{1}$, Raymond Brittingham?', John Wheeler', Linda Barone'

Rupesh Nanjunda', Eilyn R. Lacy', Sheng-Jiun Wu', Jinquan Luo ${ }^{3}$, Enrique Zudaire', Kathryn Packman', Brian Geist', Kevin Trouba', Matthew V. Lorenzi', Raluca I. Verona

${ }^{1}$ Janssen Research \& Development, Spring House, PA, USA; ${ }^{2}$ Janssen Research \& Development, Beerse, Belgium; ${ }^{3}$ Janssen Resaerch \&

Development, Spring House, PA, USA

Correspondence: Raluca I. Verona (RVerona@its.jnj.com)

Journal for ImmunoTherapy of Cancer 2017, 5(Suppl 2):P364

\section{Background}

Programmed cell death protein-1 (PD-1), a negative immune checkpoint receptor, suppresses $T$ cell function when bound to its ligands 
PD-L1 and PD-L2. Tumor cells exploit this pathway to evade immune surveillance; therefore, inhibiting the PD-1 pathway can restore T cell function, stimulating their anti-tumor response. Here we report the characterization of JNJ-63723283 (JNJ-283) to support new combination approaches in the future.

\section{Methods}

JNJ-283 was generated by phage panning against human and cynomolgus monkey (cyno) PD-1 extracellular domain followed by affinity maturation. In vitro activity was evaluated using cytomegalovirus (CMV) recall, mixed lymphocyte reaction (MLR), and Jurkat-PD-1 nuclear factor of activated T cells (NFAT) reporter assays. In vivo activity was assessed using human PD-1 knock-in mice implanted with MC38 tumors and a lung patient-derived xenograft (PDX) model (LG1306) using CD34 ${ }^{+}$cord blood humanized NSG mice. Pharmacodynamic, toxicokinetic, and safety assessments were performed in cyno following single $(0.1,1,10$ $\mathrm{mg} / \mathrm{kg})$ and/or repeat $(10,30,100 \mathrm{mg} / \mathrm{kg} / \mathrm{wk}$ up to 5 weeks) intravenous (IV) dosing.

Results

JNJ-283 displayed high affinity for human (1.72 $\pm 0.99 \mathrm{nM})$ and cyno $(0.90 \pm 0.08 \mathrm{nM})$ PD-1, blocked PD-1 binding to PD-L1 (IC ${ }_{50}=111.7 \pm$ $22.0 \mathrm{ng} / \mathrm{mL})$ and PD-L2 ( $\left(\mathrm{C}_{50}=138.6 \pm 12.4 \mathrm{ng} / \mathrm{mL}\right)$, and crosscompeted with nivolumab and pembrolizumab analogs.

JNJ-283 dose-dependently increased T cell-mediated cytokine production in CMV recall and MLR assays and enhanced NFAT activity in a Jurkat-PD-1 reporter assay, indicating functional disruption of the PD-1/PD-L1 interaction. JNJ-283 in vitro activity was comparable to that of competitor molecule analogs.

Following intraperitoneal injection with $10 \mathrm{mg} / \mathrm{kg} \mathrm{JNJ}-283$ or nivolumab analog (2x weekly; day 7 after tumor implantation), mean MC38 tumor volume in PD-1 knock-in mice was significantly lower at days $17(p<0.001)$ and $21(p<0.0001)$ compared to control (Fig. 1). In the lung PDX model, $10 \mathrm{mg} / \mathrm{kg} \mathrm{JNJ-283}$ or pembrolizumab (q5d x 6) reduced $(p<0.01)$ mean tumor volume compared to control.

JNJ-283 was tolerated in cyno following single and/or repeat dosing; mean drug exposures increased in a dose dependent manner. Primary findings attributed to JNJ-283 included proliferation of T-lymphocyte subsets, stimulation-related cytokine expression (in vitro), increased lgM and IgG titer secondary to antigen challenge, and decreased cellularity (lymphocytes) in the thymus.

\section{Conclusions}

JNJ-283, a high affinity anti-PD-1 antibody, was well-tolerated in toxicology studies and demonstrated robust activity in vitro as well as displayed anti-tumor efficacy in vivo comparable to that observed with other agents targeting PD-1. These data support the ongoing clinical study of JNJ-283 and combination potential with other modalities.

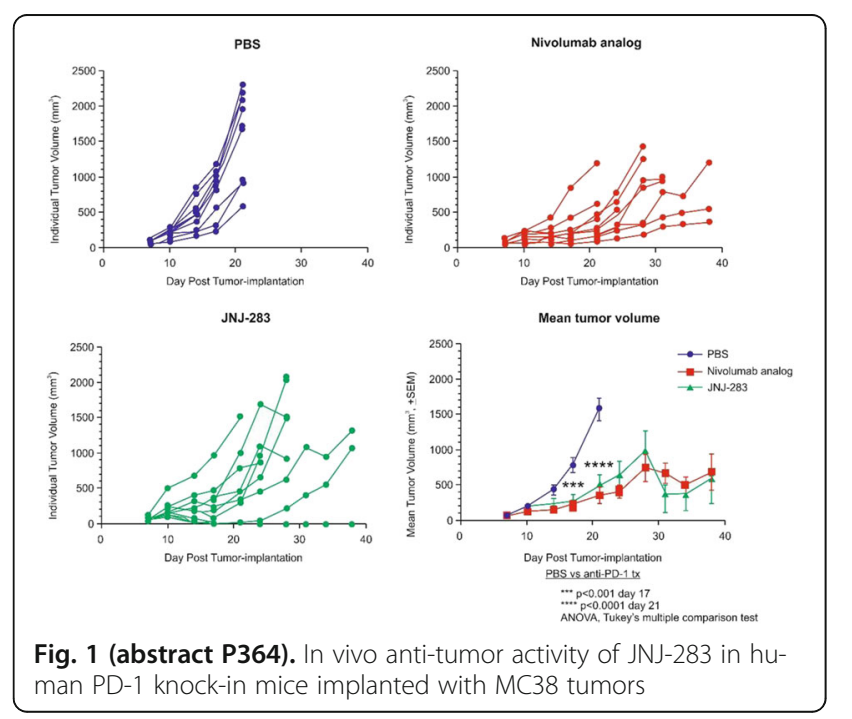

P365

Development of JNJ-64158120, an anti-TIM-3 antibody to overcome innate and acquired mechanisms of resistance to PD-1 therapy Nikki DeAngelis ${ }^{1}$, Gordon Powers' ${ }^{1}$, Keri Dorn ${ }^{1}$, Matthew Chomo Douglas Dolfi', Karla Wiehagen', Douglas Marvel', Bethany Mattson', Darlene Pizutti ${ }^{1}$, Liam Campion' ${ }^{1}$, Caitlin Morgan', Brian Tomkowicz', Nina Sabins', Sandra Santulli-Marotto ${ }^{2}$, Jocelyn Sendecki , John Wheeler', Brian Whitaker', Annmarie Winkis', Linda Barone', Deborah Kwok', Eilyn R. Lacy', Edmund Moon ${ }^{3}$, Steve Albelda ${ }^{3}$, Kathryn Packman', Enrique Zudaire', Matthew V. Lorenzi', Raluca I. Verona'

${ }^{1}$ Janssen Research \& Development, Spring House, PA, USA; ${ }^{2}$ Alios

BioPharma/Janssen Research \& Development, South San Francisco, PA, USA; ${ }^{3}$ University of Pennsylvania, Philadelphia, PA, USA

Correspondence: Raluca I. Verona (RVerona@its.jnj.com) Journal for ImmunoTherapy of Cancer 2017, 5(Suppl 2):P365

\section{Background}

Programmed cell death protein-1 (PD-1) pathway blockade has revolutionized the treatment of a variety of malignancies but has only demonstrated clinical benefit in a subset of patients. Emerging preclinical and clinical data have proposed $\mathrm{T}$ cell immunoglobulin and mucin domain-3 (TIM-3) as an inhibitory mechanism that is co-regulated with PD-1 to restrict T cell-mediated immune responses. Dynamic upregulation of TIM-3 has been observed in patients in response to anti-PD-1/ PD-L1 therapy, implicating TIM-3 as a potential mechanism of immune resistance. These observations suggest that dual blockade of the TIM-3 and PD-1 pathways can broaden the effectiveness of anti-PD-1/PD-L1 therapy as a new immunotherapy for PD-1 responsive malignancies. Here we describe the characterization and functional activity of JNJ64158120 (JNJ-120), an antibody targeting human TIM-3.

Methods

JNJ-120 in vitro activity was assessed by CMV, MART-1, and NK cell activation assays; in vivo activity was evaluated using a NY-ESO TCR transgenic model of T cell exhaustion and a human TIM-3 knock-in mouse model bearing MB49 murine bladder carcinoma tumors.

Results

JNJ-120 is a fully human, phage-derived, IgG2 $\sigma$ monoclonal antibody that binds to human $\left(K_{d}=2.5 \mathrm{nM}\right)$ and cynomolgus monkey $\left(\mathrm{K}_{\mathrm{d}}=0.75\right.$ nM) TIM-3. JNJ-120 inhibited the binding of TIM-3 to two putative TIM3 ligands, phosphatidyl serine and galectin-9 and enhanced T cell function in primary antigen-specific T cell assays (CMV and MART-1). In CMV stimulated PBMCs, JNJ-120 triggered expression of IFN- $\gamma$ and TNF-a and enhanced $\mathrm{CD} 137$ expression on $\mathrm{CD}^{+}$and $\mathrm{CD} 4^{+} \mathrm{T}$ cells. Furthermore, in MART-1 assays, JNJ-120 enhanced IFN- - , TNF- $a$, IL-2, and IL-8 production by $\mathrm{CD}^{+}{ }^{+}$cells. JNJ-120 also enhanced $\mathrm{T}$ cell function in combination with anti-PD-1 antibodies in peripheral blood samples derived from melanoma patients. JNJ-120 led to higher NK cell activation, as measured by CD69 expression and cytokine secretion, in IL-2stimulated PBMCs derived from normal donors and cancer patients. In vivo, JNJ-120 treatment enhanced the anti-tumor activity of PD-1 blockade in tumor-bearing TIM-3 knock-in mice and in a NY-ESO TCR transgenic model of T cell exhaustion. In the NY-ESO TCR model, JNJ120 increased T cell infiltration in NY-ESO-expressing A549 tumors and, in combination with anti-PD-1 antibodies, enhanced the ability of infiltrating $T$ cells to kill tumor cells ex-vivo.

\section{Conclusions}

These data demonstrated that targeting TIM-3 in combination with PD-1 leads to increased tumor T cell infiltration and superior antitumor efficacy compared to PD-1 blockade alone. These data support the clinical testing of PD-1 and TIM-3 in tumor types not broadly responsive to current immunotherapy regimens.

P366

Anti-PD-L1 mAb treatment combined with cisplatin modulates intratumoral immune responses and promotes antitumor effects Daiko Wakita, Toshiki Iwai, Masamichi Sugimoto, Suguru Harada, Miho Suzuki, Kaname Yamamoto

Chugai pharmaceutical CO., LTD., Kamakura, Japan

Correspondence: Kaname Yamamoto (wakita.daiko59@chugai-pharm.co.jp) Journal for ImmunoTherapy of Cancer 2017, 5(Suppl 2):P366 


\section{Background}

Immune checkpoint inhibitors such as anti-PD-L1/PD-1 agents have shown marked antitumor effect in a range of cancer types, and to further expand their effects, combination treatments with chemotherapeutic drugs have been actively investigated. Although cisplatin is widely used as a standard chemotherapy, the antitumor activity of cisplatin combined with immune checkpoint inhibitors remains largely unknown. Here, we investigated if anti-PD-L1 plus cisplatin combination can augment antitumor immunity in a syngeneic mouse tumor model.

Methods

E.G7-OVA cells, expressing ovalbumin (OVA) gene as a model tumor antigen, were subcutaneously inoculated into syngeneic C57BL/6 mouse. After tumor mass was established, anti-mouse PD-L1 mAb (anti-PD-L1; $10 \mathrm{mg} / \mathrm{kg}$, three times a week) and cisplatin $(1 \mathrm{mg} / \mathrm{kg}$, once) were administered intraperitoneally on the day of treatment initiation (Day1). The frequency and activation status of immune cells in tumor tissues were evaluated by flow cytometry. For detection of IFNg-producing cells, the immune cells were stimulated with anti$\mathrm{CD} 3 /$ anti-CD28 mAbs in vitro before staining.

Results

We found that anti-PD-L1 plus cisplatin combination resulted in profound effect leading to tumor shrinkage vs anti-PD-L1 alone or cisplatin alone even though each monotherapy showed significant inhibition of the tumor growth than the control group at Day17. In parallel with the clinical effect, the combination therapy significantly increased tumor-infiltrating CD8+ T cells vs each monotherapy at Day7 even though more tumor-infiltrating CD8+ T cells were observed in mice treated with anti-PD-L1 or cisplatin alone than control. Further, anti-PD-L1 plus cisplatin combination activated tumor-infiltrating CD8+ T cells, characterized by higher frequency of granzyme B-expressing cells than each monotherapy. We also observed increased OVA-specific CD8+ T cells with combination vs monotherapies. Additional analysis of CD4+ T cells showed that antiPD-L1 plus cisplatin combination or anti-PD-L1 alone, but not cisplatin alone, induced IFNg-producing CD4+ $\mathrm{T}$ cells in the tumor tissues.

\section{Conclusions}

Anti-PD-L1 plus cisplatin combination therapy demonstrated marked antitumor effect in this mouse tumor model. This robust therapeutic effects may at least partly be due to the increased tumor-specific CD8+ T cells in tumor tissues. Cisplatin or anti-PD-L1 alone increased tumorinfiltrating CD8+ T cells, however anti-PD-L1 but not cisplatin induced IFNg+ CD4+ T cells at tumor sites. These differences in immune status in response to anti-PD-L1 or cisplatin may lead to the combined therapeutic effect and provide a rationale for the combination.

\section{P367}

X4P-001, an orally bioavailable CXCR4 antagonist, increases T cell infiltration in human metastatic melanoma

Robert Andtbacka', Melinda Yushak ${ }^{2}$, Merrick Ross ${ }^{3}$, Kenneth Grossman', Eleni Tsiroyanni ${ }^{4}$, Sarah Blanchette ${ }^{4}$, Lu Gan $^{4}$, Yan Wang ${ }^{4}$,

Mohammed Milhem

${ }^{1}$ Huntsman Cancer Institute, Salt Lake City, UT, USA; ${ }^{2}$ Winship Cancer Institute, Atlanta, GA, USA; ${ }^{3}$ MD Anderson Cancer Center, Houston, TX, USA; ${ }^{4}$ X4 Pharmaceuticals, Cambridge, MA, USA; ${ }^{5}$ University of lowa, lowa City, IA, USA

Correspondence: Robert Andtbacka (jennifer@theyatesnetwork.com) Journal for ImmunoTherapy of Cancer 2017, 5(Suppl 2):P367

\section{Background}

The CXCR4/CXCL12 axis plays a central role in the trafficking of key immune cells in the tumor microenvironment. Enhanced survival is reported in multiple syngeneic mouse models when a CXCR4 antagonist is combined with a check-point inhibitor. X4P-001 is an oral, selective, allosteric inhibitor of CXCR4, and X4P-001 alone demonstrated robust inhibition of murine B16-OVA melanoma growth. We hypothesize that disruption of CXCR4/CXCL12 signaling will result in modulation of the immune cell profile within the tumor microenvironment and ultimately lead to increased CD8+ T-cell infiltration, favoring an improved response to checkpoint inhibitors in metastatic melanoma.

\section{Methods}

The primary objectives for this ongoing biomarker-driven Phase lb trial of X4P-001 alone and with pembrolizumab are to evaluate the safety and tolerability in patients (pts) with metastatic melanoma, and to characterize the effects of X4P-001 alone and with pembrolizumab on tumor immune cell infiltrates. Serial biopsies of cutaneous or subcutaneous metastatic melanoma lesions and peripheral blood mononuclear cells (PBMCs) are collected at pre-dose, after 3 weeks of X4P-001 treatment and after 6 weeks of combination treatment. Biopsies are assessed by immunohistochemistry (IHC) for multiple markers including CD3, CD8, FoxP3 and CXCL12, and PBMCs are analyzed by flow cytometry for both lymphoid and myeloid-cells.

Results

As of June 30, 2017, 13 pts have been enrolled and 4 of the 13 pts have completed the study. The median age was 74 years (range $53-91$ ). Most pts $(12 / 13)$ had not received prior systemic therapy. X4P-001 was well tolerated. Treatment related AEs (related to either X4P-001 or pembrolizumab; $>5 \%)$ of any grade were: diarrhea (15\%), chill, fatigue, headache, ocular hyperemia, photophobia, pruritus, rash, and vomiting (7.7\%). Two treatment related G3 SAEs were reported: acute diarrhea and immune-mediated drug-reaction. Three of the 4 pts who completed the study had IHC evaluable tumor samples. All showed an increase in T-cell infiltration in the central region of the tumors following both single agent and combination treatment. The percentage of Ki67 positive CD8+ T cells in PBMC were increased post treatment in all 4 pts. These data along with additional biomarker readouts in all enrolled patients will be presented.

Conclusions

Treatment with X4P-001 as a single agent, and in combination with pembrolizumab, is well tolerated with preliminary evidence of enhanced immune cell infiltration and activation. The enrollment of the study is near completion and further biomarker analysis is on-going.

\section{P368}

eFT508, a potent and highly selective inhibitor of MNK1 and

MNK2, is an activator of anti-tumor immune response

Kevin Webster', Rajesh Sharma', Vikas Goel', Craig Stumpf', Jocelyn

Stauton', Peggy Thompson', Gary Chiang', Yichen $\mathrm{Xu}^{2}$, Hyun Yong Jin²,

Davide Ruggero ${ }^{2}$

${ }^{1}$ eFFECTOR Therapeutics, San Diego, CA, USA; ${ }^{2}$ Helen Diller Family

Comprehensive Cancer Center, San Francisco, CA, USA

Correspondence: Kevin Webster (kwebster@effector.com)

Journal for ImmunoTherapy of Cancer 2017, 5(Suppl 2):P368

\section{Background}

eFT508 is a potent and highly selective inhibitor of MNK1 and MNK2, kinases that function to mediate tumor immune evasion downstream of MEK and MAPK signaling. eFT508 treatment establishes a regulatory program that promotes multiple steps in the cancer immunity cycle including antigen presentation and $T$ cell priming, expansion of memory $\mathrm{T}$ cells, and prevention of $\mathrm{T}$ cell exhaustion.

Methods

The immunological effects of eFT508 have been evaluated in the context of normal human immune cells in vitro and in immunocompetent syngeneic and genetically engineered mouse models in vivo.

Results

eFT508 treatment of normal donor T cells has no deleterious effect on aCD3/aCD28 stimulated $\mathrm{T}$ cell proliferation or $\mathrm{T}$ cell viability in contrast to inhibitors acting upstream of MAPK signaling. eFT508 selectively down regulates key immune checkpoint proteins and the production of a subset of proinflammatory and immunosuppressive cytokines. In vitro mechanism of action studies have demonstrated that MNK selectively regulates gene expression at the level of mRNA translation via specific sequence elements in the $5^{\prime}$ and 3 '-untranslated regions. In addition, eFT508 activated antigen presenting cells leading to more effective T cell priming. eFT508 also affected $T$ cell memory formation, both in the context of 
specific peptide antigen stimulation and in a mixed lymphocyte reaction, shifting the distribution of $\mathrm{T}$ cells towards a $\mathrm{CD} 62 \mathrm{~L}^{+} \mathrm{CD} 44^{+}$ central memory T cell population. eFT508 also enhanced the cytotoxic function of $T$ cells from OT-I mice stimulated with SIINFEKL peptide demonstrating a dose-dependent increase of cell killing. Consistent with the mechanisms elaborated upon in vitro, eFT508 showed significant anti-tumor activity mediated through tumor infiltrating lymphocytes in the CT26 syngeneic tumor model as well as genetically engineered mouse models of NSCLC and HCC. Conclusions

eFT508 treatment establishes a regulatory program that promotes anti-tumor immunity. eFT508 is currently under evaluation as a single agent in two phase $1 / 2$ clinical trials for patients with advanced solid tumors and patients with advanced lymphoma. A biomarker driven proof of concept study, including mandatory pre- and on-treatment biopsies, to evaluate the immunological mechanism of action of the drug is planned to be initiated later this year. In addition, a phase 2 study evaluating eFT508, alone or in combination with avelumab, a PD-L1 immune checkpoint inhibitor, in microsatellite stable relapsed or refractory CRC patients is planned.

\section{P369}

Analysis of the TIGIT/PVRIG axis in human cancers to support indication selection and biomarkers for COM701 and COM902 Sarah Whelan, Ling Leung, David Bernardos, John Hunter, Mark White, Spencer Liang

Compugen USA, Inc., South San Francisco, CA, USA

Correspondence: Sarah Whelan (sarahw@cgen.com)

Journal for ImmunoTherapy of Cancer 2017, 5(Suppl 2):P369

\section{Background}

PVRIG and TIGIT were identified by Compugen's Predictive Discovery Platform as immune inhibitory receptors and have been reported to inhibit anti-tumor activity. We are pursuing clinical development of antagonistic antibodies to PVRIG (COM701) and to TIGIT (COM902). Here, we analyzed primary human cancer tissues and immune cells to characterize expression in the TIGIT/PVRIG axis to support indication selection and combination strategies for COM701 and COM902.

\section{Methods}

COM701 and COM902 were identified based on ability to block the interaction of PVRIG and TIGIT with their cognate ligands (PVRL2 and PVR respectively) and were screened for their ability to enhance antigen-specific CD8 T cell activation in a co-culture with tumor cell lines. Immunohistochemistry and Flow cytometry were performed to assess receptor/ligand expression in dissociated bladder, breast, colorectal, head and neck, lung, kidney, ovarian, prostate, and stomach tumors.

Results

Among the cancers examined, PVRIG and PVRL2 expression was highest in endometrial, lung, kidney, ovarian, and head and neck cancers compared to normal adjacent tissue. From dissociated tumors, PVRIG expression was detected on T and NK TILs whereas PVRL2 expression was detected on $\mathrm{CD}^{-} 5^{-}$cells and myeloid cells. A co-expression analysis of PVRIG, TIGIT, and PD1 demonstrated that PVRIG was co-expressed with both TIGIT and PD1 and that PVRIG ${ }^{+} \mathrm{TIGIT}^{+} \mathrm{PD} 1^{+}$cells comprised a major proportion of CD8 TILs. In comparison to PD-L1, PVRL2 expression was more prevalent across several cancer types and expression of PVRL2 was detected in PD-L1 negative samples. In vitro, combination of COM701 with PD1 inhibitors or COM902 enhanced CD8 cytokine production and cytotoxic activity, with the triple combination of COM701, COM902, and PD-1 antibody yielding the greatest increase in functional activity. Several immune receptors were induced in response of PVRIG blockade by COM701 on CD8 T cells. Taken together, these data support indication selection and combination strategies for COM701 and COM902 and potential biomarkers that could be indicators of response.

Conclusions

In summary, we demonstrate that PVRIG and PVRL2 are induced in the tumor microenvironment of human cancers, and the potential of
COM701 as a cancer therapeutic, either as a monotherapy or as a dual- or triple-combination therapy with antibodies targeting TIGIT, and PD-1. These data highlight the potential of this combination approach to expand the immune checkpoint inhibitor responsive cancer patient population, including those who are non-responsive to PD-1 inhibitors.

P370

Enhancement of tumor specific immunity by activation of CD40 through a bispecific molecule targeting CD40 and a tumor surface antigen

Shiming Ye', Diane Yu ${ }^{1}$, Nicole Belmar', Donghee Choi ${ }^{1}$, Debra Chao',

Mien Sho', Han Kim', Jean Cabel', Danying Song ${ }^{2}$, Kelley Hiser²,

John Harlan², Catherine Zhang ${ }^{1}$, Yuni Fang ${ }^{1}$, Steve Keller ${ }^{1}$, Alan Wah ${ }^{3}$,

Patricia Culp ${ }^{4}$, Diane Hollenbaugh

${ }^{1}$ AbbVie Biotherapeutics Inc., Redwood City, CA, USA; ${ }^{2}$ AbbVie Inc., North Chicago, IL, USA; ${ }^{3}$ Former AbbVie Employee, Del Mar, CA, USA; ${ }^{4}$ Former

AbbVie Employee (currently working at Alector Inc.), South San

Francisco, CA, USA

Correspondence: Shiming Ye (shiming.ye@abbvie.com)

Journal for ImmunoTherapy of Cancer 2017, 5(Suppl 2):P370

\section{Background}

CD40 activation can bridge the innate and adaptive immune systems during immune activation. Agonistic anti-CD40 monoclonal antibodies (mAbs) have demonstrated anti-tumor activity in clinical studies. Due to the dose-limiting toxicity observed, an alternative approach to developing an anti-CD40 therapy, and potentially reducing systemic toxicity, is to achieve immune activation via a bispecific molecule that is maximally active in the presence of a tumor antigen (TA), but with limited activity in its absence.

Methods

Different bispecific formats targeting both CD40 and a TA were designed and constructed. Bispecific molecules with desired properties were screened and assayed for activation of primary $B$ cells or monocyte-derived dendritic cells (moDC) co-cultured with TA positive or negative cells. Selected bispecific molecules were tested for anti-tumor activity using PC3 tumor cells with or without TA expression mixed with autologous moDC and T cells, cultured in vitro or inoculated in NSG mice. Syngeneic mouse models of TA positive tumors were used to test bispecific molecules for immune activation, anti-tumor potency, and tolerability.

Results

ABBV-428 is a bispecific molecule with single chain Fv (scFv) domains targeting human CD40 and a TA. ABBV-428 was less potent than a CD40 mAb in stimulating $B$ cells and moDCs when cultured alone or in the presence of cells that do not express TA. However, ABBV-428 exhibited enhanced B cell and moDC activation when cultured with cells expressing TA. T cells were also activated by ABBV-428 when mixed with moDC in the presence of cell-surface $T A$, as evidenced by a reduction in growth of PC3 cells, both in vitro and in NSG mice. Although expression of the cell surface TA is necessary for immune-mediated anti-tumor activity, ABBV-428 inhibited the growth of both TA-positive and TA-negative PC3 cells as long as the TA was expressed within the tumor environment. This phenomenon was confirmed in a mouse model carrying syngeneic $4 \mathrm{~T} 1$ tumors expressing the cell surface TA. A surrogate molecule of ABBV-428 elicited T cell responses against both TAexpressing and non-expressing $4 \mathrm{~T} 1$ cells, where the anti-tumor activity was similar to anti-CD40 mAb, but did not elicit increases in serum cytokines and liver enzyme levels observed in anti-CD40 mAb treated mice.

Conclusions

ABBV-428 exhibits enhanced CD40 activation upon binding to TAexpressing cells, and provides tumor-specific immune stimulation with systemic administration. The enhanced tumor-specific immune activation is hypothesized to maximize anti-tumor potency while limiting systemic toxicity in clinical studies. 
Impact of Diet, Exercise, and/or Stress on Antitumor Immunity

\section{P371}

Featuring adipocytes secretion as pharmacological target for adjuvant immunotherapy against breast cancer

Luis Henrique Correa, LÍVIA PIMENTEL SANT'ANA DOURADO, Kelly Grace Magalhaes

University of Brasilia, Brasilia, Brazil

Correspondence: Kelly Grace Magalhaes (kellymagalhaes@gmail.com) Journal for ImmunoTherapy of Cancer 2017, 5(Suppl 2):P371

\section{Background}

Obesity and adipose tissue have been shown to be associated with low grade inflammation resulting in cellular and humoral inflammatory factors that contribute to carcinogenesis ${ }^{1}$. It has been known that inflammation exerts an important role in carcinogenesis and tumor progression. Inflammatory molecules can potentially be used as adjuvant in immunotherapy against cancer. However, little is known about the differential role of brown and white adipocytes against breast cancer. In the present work, we aimed to characterize the differential function of brown and white adipocytes in breast cancer cell death in vitro and investigate the role of NLRP3 and caspase- $1 / 11$ in this process.

\section{Methods}

Brown and white adipose tissue were isolated from wild type and caspase-1/11 and NLRP3 knockout mice. Conditioned medium from these brown and white adipocytes were used to stimulate breast cancer cells $4 \mathrm{~T} 1$. Breast cancer cells (I) viability was analyzed by MTT assay; (II) cell death was investigated by annexin V/PI staining and flow cytometry analysis (III) membrane pore formation was observed by PI staining and spectrophotometry analysis and (IV) lipid droplet biogenesis was analyzed by Bodipy staining and flow cytometry analysis.

Results

Our data showed that brown adipocytes conditioned medium triggered significant higher levels of breast cancer cell death and pore membrane formation and lower levels of lipid droplet biogenesis and cell viability compared to white adipocytes conditioned medium. Conclusions

The absence of caspase-1/11 in brown adipocytes, but not NLRP3, enhanced these cell death and carcinogenic parameters in breast cancer cells. Identification of molecules from these adipocytes secretion is ongoing and can be potentially used as adjuvant immunotherapy agains breast cancer.

\section{References}

1. Alderton G. The supersized tumour microenvironment. Nature Reviews Cancer. 2015;15:575.

\section{Mechanisms of Efficacy or Toxicity}

\section{P372}

A syngeneic mouse model of CAR-T mediated toxicity and neuroinflammation

Eric Chadwick, Alyssa Noll, Yue Jiang, Ronald Hause, Rafael Ponce, Hyam Levitsky, Ruth Salmon

Juno Therapeutics, Seattle, WA, USA

Correspondence: Ruth Salmon (eric.chadwick@junotherapeutics.com) Journal for ImmunoTherapy of Cancer 2017, 5(Suppl 2):P372

\section{Background}

Interrogating the spectrum of systemic effects of CAR-T cell therapy, especially in the context of adverse events (i.e. systemic cytokine release syndrome (sCRS) and neurotoxicity) that have occurred in clinical trials, may provide important insights into factors contributing to the cause and/or progression of such events and potential interventions. Preclinical CAR-T cell efficacy models in immune-compromised mice do not capture the potential role of the host immune system in SCRS and central nervous system (CNS) pathologies that, in combination with other patient factors, may lead to severe neurotoxicity. We have developed a novel model system where conditioning immune-competent mice with cyclophosphamide (CPA) followed by transfer of murine CD19directed CAR-T cells induces acute symptoms including systemic cytokine release in concordance with what is observed in patients that develop CRS and/or neurotoxicity. This systemic toxicity is accompanied by the alteration of gene expression levels in the mouse brain indicating a possible neuro-inflammatory response.

Methods

We examined the effects of murine CD19-directed CAR-T cell therapy in a syngeneic BALB/C model. Following dose optimization for both CPA conditioning and the intravenous transfer of CAR-T cells, mice were evaluated for acute toxicity and systemic and brain-related pathologies including serum cytokine levels, brain and peripheral organ histopathology by microscopy of hematoxylin and eosinstained sections, evaluation of blood brain barrier (BBB) integrity by extravasation of fluorescently-labeled low-molecular weight dextrans, wet-dry brain weights indicative of cerebral edema, and flow cytometry and gene expression analysis of transcardially-perfused brain tissues.

Results

Conditioning with CPA, followed by administration of murine CD19-directed CAR T cells, but not control CAR-T cells, induced rapid weight loss, peripheral organ pathologies and elevated serum cytokine levels (including a spectrum of cytokines similar to those observed in clinical (RS). In the brain, we observed significant changes in gene expression indicative of neuroinflammation including genes associated with interferon response pathways, vascular endothelial activation and oxidative stress, accompanied by CAR-T cell infiltrate into the brain. No evidence for overt brain histopathology was observed, nor increased BBB permeability or cerebral edema.

Conclusions

These findings describe a new animal model and highlight its potential use to elucidate the mechanisms underlying CAR-T-cell mediated toxicities and test proposed interventions to reduce neuroinflammation that may arise from CD19-directed CAR-T cell therapies. Ongoing work seeks to identify and evaluate various pharmacological interventions with the potential to ameliorate systemic and neuro-inflammation in this model, with the goal to translate these learnings to the clinic.

P373

Optimizing anti-OX40 mediated immunotherapy: preclinical exploration of the relationship between antitumor activity and isotype choice, ligand blocking capacity, dose and schedule

Chan Gao ${ }^{1}$, John Engelhardt' ${ }^{2}$ Gennaro Dito', Susan Glick',

Megan Raymond', Marie-Claude Gaudreau', Alan Korman², Michael Quigley

${ }^{1}$ Bristol-Myers Squibb, Princeton, NJ, USA; ${ }^{2}$ Bristol-Myers Squibb, Redwood City, CA, USA

Correspondence: Michael Quigley (gyrase@gmail.com); Alan Korman Journal for ImmunoTherapy of Cancer 2017, 5(Suppl 2):P373

\section{Background}

Following the clinical success of checkpoint blockade, the field of cancer immunotherapy is rapidly expanding. Extensive preclinical data have demonstrated that treatment with an agonist OX40 antibody can result in anti-tumor immune responses both alone and in combination with other immune-targeting agents including CTLA-4 and PD-1 blockers. The reported mechanism of action for OX40 includes costimulation of effector $\mathrm{T}$ cells as well as reduction in regulatory $\mathrm{T}$ cell $\left(\mathrm{T}_{\text {reg }}\right)$ suppression either through depletion or receptor engagement. However, the vast majority of published work utilizes a single, ligand non-blocking antibody to define the role of anti-OX40 in enhancing tumor immunity in vivo. We generated an alternative agonistic, ligand-blocking mouse OX40 antibody as well as series of isotype variants in order to better define the role of isotype, ligand blocking vs non-blocking epitopes, dose and schedule on the anti- 
tumor activity of anti-OX40 alone and in combination with PD-1 blockade in vivo.

Methods

The activity of OX40 agonist antibodies, OX86 (ligand non-blocker) and OX40.23 (ligand blocker), were tested across a dose range from 0.03 $\mathrm{mg} / \mathrm{kg}$ to $10 \mathrm{mg} / \mathrm{kg}$ in the CT26 tumor model. Anti-PD-1 (10 mg/kg) was dosed either concurrently or 5 days after initial OX40 treatment. Ontreatment immune monitoring of peripheral and tumor-infiltrating lymphocytes was analyzed by flow cytometry.

Results

Fc-competent agonist OX86 antibodies display potent in vivo antitumor activity at $10 \mathrm{mg} / \mathrm{kg}$, whereas an Fc inert antibody at the same dose had no effect on CT26 tumor growth. Relative anti-tumor activity was related to their ability to preferentially bind activating FcyR and deplete intratumoral $\mathrm{T}_{\text {reg }} \mathrm{s}$. Maximal activity of $\mathrm{OX} 40.23$ was achieved at $3 \mathrm{mg} / \mathrm{kg}$ and $0.3 \mathrm{mg} / \mathrm{kg}$ as monotherapy and in combination with anti-PD-1, respectively. Interestingly, administration of OX40.23 at $10 \mathrm{mg} / \mathrm{kg}$ in both monotherapy and combination displayed diminished activity, accompanied by a reduction in peripheral T cell activation. Evaluation of OX40 RO demonstrated that peripheral and intratumoral RO was similar as was RO between monotherapy and combination treatment. Maximal anti-tumor activity of the combination was achieved well below $100 \%$ OX40 RO. Furthermore, for combination treatment, concurrent dosing resulted in greater antitumor activity than a staggered regimen.

\section{Conclusions}

Our results demonstrate the importance of isotype choice, ligand blockade capacity, dose and schedule on the in vivo antitumor activity of mouse OX40 agonist antibodies alone and in combination with PD-1 blockade. These data provide valuable insight relevant in biomarker, dose and schedule selection for agonist OX40 antibody-containing regimens currently in clinical development.

\section{P374}

Activation of 4-1BB on liver myeloid cells triggers hepatitis via an interleukin-27 dependent pathway

Ashvin R. Jaiswal', Todd Bartkowiak', Casey R. Ager', Renee Chin ${ }^{1}$ Chao-Hsien Chen', Pratha Budhani', Midan Ai ${ }^{1}$, Matthew J. Reilley',

Manu M. Sebastian², David S Hong ${ }^{1}$, Michael A Curran'

'The University of Texas MD Anderson Cancer Center, Houston, TX, USA;

${ }^{2}$ The University of Texas MD Anderson Cancer Center, Smithville, TX, USA

Correspondence: Ashvin R. Jaiswal (arjaiswal@mdanderson.org);

Michael A Curran

Journal for ImmunoTherapy of Cancer 2017, 5(Suppl 2):P374

\section{Background}

Agonist antibodies targeting the T cell co-stimulatory receptor 4-1BB (CD137) are among the most effective immunotherapeutic agents across multiple pre-clinical models of cancer. In the clinic, however, development of these agents has been stymied by dose-limiting liver toxicity. Lack of knowledge of the mechanisms underlying this toxicity has limited the potential to separate 4-1BB agonist driven anti-tumor immunity from hepatotoxicity.

Methods

The capacity of 4-1BB agonist antibodies to induce liver toxicity was investigated in immune competent mice, with or without coadministration of checkpoint blockade, via measurement of serum transaminase levels, through imaging of liver immune infiltrates, and via qualitative and quantitative assessment of liver myeloid and $\mathrm{T}$ cells via flow cytometry. Knockout mice were used to clarify the contribution of specific cell subsets, cytokines and chemokines.

Results

We find that activation of 4-1BB on liver myeloid cells is essential to initiate hepatitis. Once activated, these cells produce interleukin-27 which is required for liver toxicity. CD8 T cells infiltrate the liver in response to this myeloid activation and mediate tissue damage triggering transaminase elevation. FoxP3+ regulatory $\mathrm{T}$ cells limit liver damage and their removal dramatically exacerbates 4-1BB agonist hepatitis. Co-administration of CTLA-4 blockade ameliorates transaminase elevation, whereas PD-1 blockade exacerbates it. Loss of the chemokine receptor CCR2 blocks 4-1BB agonist hepatitis without diminishing tumor-specific immunity against B16 melanoma.

Conclusions

4-1BB agonist antibodies trigger hepatitis via activation of myeloid cells to produce Interleukin-27. Co-administration of CTLA-4 and/or CCR2 blockade may minimizing hepatitis but yield equal or greater antitumor immunity.

\section{P375}

Evaluation of surrogate endpoints for overall survival in patients treated with immunotherapies

Howard Kaufman ${ }^{1}$, Lawrence Schwartz ${ }^{2}$, William William ${ }^{3}$, Mario Sznol ${ }^{4}$, Michael del Aguila ${ }^{5}$, Craig Whittington ${ }^{5}$, Kyle Fahrbach ${ }^{6}$, Yingxin $\mathrm{Xu}^{6}$, Eric Masson ${ }^{7}$, Andrea Vergara-Silva ${ }^{8}$

${ }^{1}$ Rutgers Cancer Institute of New Jersey, New Brunswick, NJ, USA;

${ }^{2}$ Columbia University Medical Center, New York, NY, USA; ${ }^{3}$ MD Anderson Cancer Center, Houston, TX, USA; ${ }^{4}$ Yale School of Medicine, New Haven, CT, USA; ${ }^{5}$ Doctor Evidence, Santa Monica, CA, USA; ${ }^{6}$ Evidera, Bethesda, MD, USA; ${ }^{7}$ AstraZeneca, Waltham, MA, USA; ${ }^{8}$ AstraZeneca, Gaithersburg, $M D, U S A$

Correspondence: Howard Kaufman (hk553@cinj.rutgers.edu) Journal for ImmunoTherapy of Cancer 2017, 5(Suppl 2):P375

\section{Background}

Surrogate endpoints have not been clearly determined in oncology immunotherapy (IT). Using both arm- and comparison-level data, this study explored the relationship between overall survival (OS) and clinical endpoints (objective response rate [ORR], disease control rate [DCR], and progression-free survival [PFS]) in patients treated with IT. The aim was to assess whether any of these endpoints could function as surrogates for OS.

Methods

A systematic review was conducted in MEDLINE ${ }^{\mathrm{TM}}$ and Embase (January 2005-March 2017), and supplemented with conference proceedings (2014-2017). Eligible studies were randomized controlled trials (RCTs) that investigated blocking antibodies targeting programmed cell death-1 (PD-1)/programmed cell death ligand-1 (PD-L1) or cytotoxic T-lymphocyte-associated antigen-4 (CTLA-4). Studies were included in the arm-level analyses if the treatment arm's absolute effects could be obtained for ORR, DCR, 6- and 9-month PFS, median PFS, median OS, or OS at 12 or 18 months. They were included in comparison-level analyses if the treatment's relative effects (odds ratios [ORs] on ORR and DCR or hazard ratios [HR] on PFS and OS) were reported/could be derived.

Weighted linear regression models were fitted and adjusted $R^{2}$ values estimated, with analyses stratified by treatment regimen (IT mono or IT plus chemotherapy), by type of IT (PD-1/PD-L1 or CTLA-4), and by indication, as data permitted.

Results

Twenty-nine RCTs involving 66 treatment arms (11,797 patients) were included. In the arm-level analyses, higher PFS rates at 6 months predicted better OS rates at 12 and 18 months, with similar trends across subgroups (Fig. 1A-D); results by type of IT revealed stronger correlations for PD-1/PD-L1 than CTLA-4 as potential surrogates for OS (Fig. 1A, Fig. 1B). Similarly, the comparison-level analyses only found the PFS HR to be moderately correlated with the OS HR $\left(R^{2}=0.372\right.$; $P=0.002$; Fig. 1e); this finding remained consistent when restricted to studies assessing IT monotherapy only $\left(R^{2}=0.460\right.$; $P=0.002$; Fig. $1 \mathrm{~F}$ ) and in subgroups stratified by treatment type or indication.

Conclusions

Among anti-PD-1/PD-L1 studies, PFS was an imperfect surrogate (low to moderate correlation) for OS, whereas other surrogate clinical endpoints were not correlated with OS. Thus, a new surrogate, such as a biomarker, is needed to better predict the OS benefit for IT. 


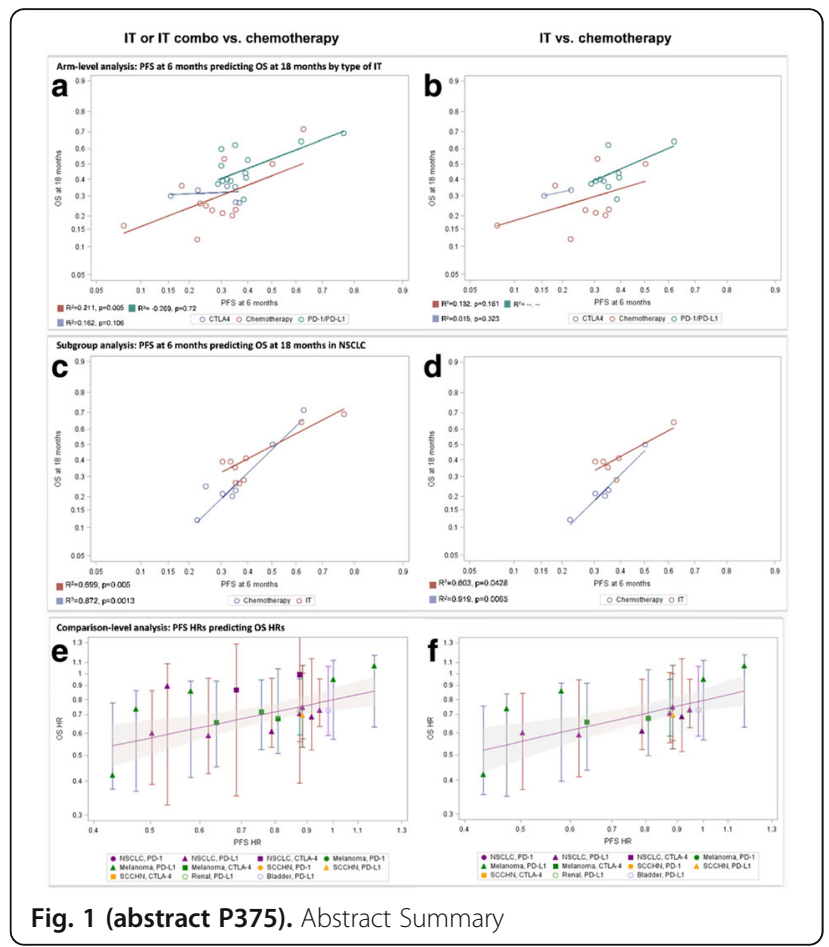

P376

Evaluating CD38 as a therapeutic target in non-small cell lung cancer (NSCLC)

Michelle Kinder, Mark Mendonca, Khaja Syed, Melissa Parker, Patrick Wilkinson, Edward Thompson, Gerald Chu, Christopher Chiu, Natalie Hutnick

Janssen Research and Development, LLC, Spring House, PA, USA

Correspondence: Michelle Kinder (mkinder@its.jnj.com)

Journal for ImmunoTherapy of Cancer 2017, 5(Suppl 2):P376

\section{Background}

Daratumumab (DARA), a human CD38-targeted monoclonal antibody, is approved as monotherapy and in combination with standard of care regimens for patients with relapsed or refractory multiple myeloma. DARA demonstrates direct on-tumor and immunomodulatory mechanisms of action. In multiple myeloma patients treated with DARA, CD38 ${ }^{+}$immunosuppressive cells including regulatory T-cells $\left(T_{\text {reg }}\right)$, myeloid derived suppressor cells (MDSC) and regulatory B-cells $\left(\mathrm{B}_{\text {reg }}\right)$ are depleted. DARA also results in increased $\mathrm{CD} 8^{+} \mathrm{T}$-cells and T-cell clonality. We hypothesized that DARA could be effective in solid tumors that have elevated levels of CD38 expression on the surface of tumor and/ or immunosuppressive cells. Here, we investigated CD38 expression on lung cancer and intra-tumoral immune cells to determine whether NSCLC may be susceptible to the immunomodulatory effects of DARA. Methods

CD38 RNA expression in lung cancer cell lines and tumor sections from The Cancer Genome Atlas (TCGA) database was investigated using RNA-sequencing. Protein expression on lung cancer cell lines, tumorinfiltrating immune cells, and peripheral blood (PB) immune cells was analyzed by flow cytometry. Primary tumor samples were evaluated by immunohistochemistry. The effect of DARA on lung cancer cell viability was measured in an in vitro assay. DARA-mediated depletion of immune cells in the PB of lung cancer patients was assessed ex vivo. Results

CD38 RNA was highly expressed in lung tumor specimens from the TCGA database. Of the lung cancer cell lines investigated, 3/12 (A549, $\mathrm{NCl}-\mathrm{H} 2023$, and $\mathrm{NCl}-\mathrm{H} 2073$ ) showed detectable CD38 receptor. These cell lines demonstrated moderate receptor density $(8,830,20,900$, and 58,100 average receptors/cell, respectively) compared to a multiple myeloma cell line (MM.1R; 75,590 average receptors/cell). Detectable CD38 receptor expression was observed on tumor cells from 5/21 primary NSCLC tumor samples and was highly expressed on intratumoral immunosuppressive cells including: monocytic MDSC, tumor-associated macrophages and $\mathrm{T}_{\text {reg. }}$. In the PB of lung cancer patients, CD38 receptor was expressed on $>50 \%$ of NK cells, B-cells, monocytic MDSC, immature MDSC, and monocytes. Significantly, DARA induced cell-mediated killing of $\mathrm{CD}_{3} 8^{+}$lung cancer cell lines in vitro, and depleted monocytic MDSC from the PB of lung cancer patients ex vivo.

Conclusions

Tumor cell CD38 expression is observed in a subset of lung cancers and is highly expressed on patient tumor-infiltrating immune cells and immunosuppressive cells in PB samples. Because DARA has demonstrated in vitro activity in depleting these cells, DARA may represent a novel therapeutic approach to targeting the immune microenvironment in solid tumors. Clinical trials in NSCLC are underway (NCT03023423) and/or planned.

\section{P377}

Cisplatin induces immunogenic cell death in preclinical models of head and neck squamous cell carcinoma

So-Jin Park', Linda Tran ${ }^{1}$, Roy Xiao ${ }^{1,2}$, Carter Van Waes ${ }^{1}$, Nicole Schmitt ${ }^{1,3}$ ${ }^{1} \mathrm{NIDCD}, \mathrm{NIH}$, Bethesda, MD, USA; ${ }^{2}$ Cleveland Clinic Lerner College of Medicine, Bethesda, MD, USA; ${ }^{3}$ Johns Hopkins University, Bethesda, MD, USA

Correspondence: Nicole Schmitt (nicole.schmitt@nih.gov)

Journal for ImmunoTherapy of Cancer 2017, 5(Suppl 2):P377

\section{Background}

Immunogenic cell death (ICD) is the process by which stressed cells exhibit translocation of markers including calreticulin and heat shock proteins (HSPs) to the cell surface along with release of HMGB1 and ATP. This activates toll-like receptors and adaptive immunity. Studies in several cancer types suggest that oxaliplatin induces superior ICD compared to cisplatin. This has never been studied in head and neck squamous cell carcinoma (HNSCC).

Methods

Three HNSCC cell lines were treated with sublethal doses of the platinum chemotherapy drugs cisplatin, oxaliplatin, and carboplatin for 48 hours. Cell surface levels of HSP70 and calreticulin were then assayed by flowcytometry. Intracellular HMGB1, an indirect measurement of HMGB1 release, was also quantified. Release of HMGB1 was measured in the cell culture supernatants. A syngeneic mouse model was then used to compare the effects of cisplatin vs. oxaliplatin, alone or in combination with anti-PD-1 immunotherapy, on tumor growth and survival. A subset of tumors were analyzed for immune cell infiltrates by flow cytometry.

Results

All three platinum drugs induced translocation of HSP70 and calreticulin to the cell surface, as well as release of HMGB1 in multiple cell lines. Cisplatin was the superior ICD inducer in these cell lines. Cisplatin and oxaliplatin induced similar tumor growth delay when combined with anti-PD-1 immunotherapy in tumor-bearing, immunocompetent mice.

\section{Conclusions}

Treatment of HNSCC cells with platinum chemotherapy drugs appears to induce ICD, which may enhance anti-tumor immunity. Cisplatin, which is the standard chemotherapy drug for treatment of HNSCC, appears to be at least as effective as oxaliplatin as an ICD inducer in these preclinical models.

\section{P378}

Improving the efficacy of cancer immunotherapy: An intimate play of CD8 T and NK cells

Roman Uzhachenko', J Shawn Goodwin', William Hofmeister' Anil Shanker ${ }^{1,3}$

${ }^{1}$ Meharry Medical College School of Medicine, Nashville, TN, USA;

${ }^{2}$ University of Tennessee Space Institute, Tullahoma, TN, USA;

${ }^{3}$ Vanderbilt-Ingram Cancer Center, Nashville, TN, USA

Correspondence: Anil Shanker (ashanker@mmc.edu)

Journal for ImmunoTherapy of Cancer 2017, 5(Suppl 2):P378 


\section{Background}

The interaction between the innate and adaptive immune components is fundamental for an effective antitumor immunity. Our studies in murine models of mastocytoma, kidney, breast and lung solid tumors showed that productive antitumor effector response relies on functional crosstalk between innate immune effectors-natural killer (NK) cells, and adaptive immune effectors-cytolytic CD8 ${ }^{+} \mathrm{T}$ lymphocytes. We found that this lymphocyte cooperativity between $C D 8^{+} T$ and NK cells can prevent the development of antigen-escape tumor variants.

Methods

Using a nanofiber matrix engineered to provide lymphocytes a controlled 3D interaction, we found that activated $C D 8^{+} T$ cells $\left(C D 69^{\text {high }} C D 25^{\text {high }}\right)$ formed multiple intercellular contacts with several naïve NK cells, while naïve $\mathrm{CD}^{+}{ }^{+} \mathrm{T}$ cells made single or no contact with NK cells.

Results

In lymphocyte coculture (physical contact possible), activated $C D 8^{+} T$ and NK cells cross-regulated each other's phenotype wherein NK cells polarized activated $\mathrm{CD} 8^{+} \mathrm{T}$ cells towards $\mathrm{T}$ central memory phenotype and activated $\mathrm{CD} 8^{+} \mathrm{T}$ lymphocytes induced acquisition of effector/ regulatory phenotype by naïve NK cells. This cross-regulation of lymphocytes disappeared in a trans-well system (no physical contact) indicating the necessity of cell-to-cell physical interaction during CD8 ${ }^{+}$T-NK crosstalk. Notably, intercellular physical interaction led to cross-regulation of mitoCa ${ }^{2+}$ oscillations in both activated $\mathrm{CD}^{+} \mathrm{T}$ and NK cells. Inhibition of mitochondrial $\mathrm{Ca}^{2+}$ uptake or $\mathrm{Na}^{+} / \mathrm{Ca}^{2+}$ exchanger with Ru360 and CGP37157, respectively, mimicked observed alterations in both lymphocytes. Further, NK cells displayed increased oxidative signaling, Tyk2, Jak 1 and 3, Stat2 and Stat6 phosphorylation while inhibiting TCR- and various cytokine receptor-mediated signaling. In turn, NK cells selectively restrained IL-2 signaling in CD8 ${ }^{+} \mathrm{T}$ cells by dampening activation-induced up-regulation of CD25, Stat5 phosphorylation, IL-2 synthesis and elevation in IL-2 uptake.

\section{Conclusions}

These data suggest a model, where mitochondrial $\mathrm{Ca}^{2+}$ flux acts as a key biological controller to guide cellular crosstalk allowing acquisition of NK cell effector/regulatory and T cell central-memory phenotypes upon their interaction. Further understanding of the characteristics and regulatory factors involved in this $\mathrm{NK}-\mathrm{CD} 8^{+} \mathrm{T}$ cell physical play in the tumor microenvironment will provide new insights on controlling immune escape variants of tumor. This could lead to novel strategies for effective cancer immunotherapies, with a potential of relapse-free survival in cancer patients.

\section{P379}

Distinct cellular mechanisms underlie anti-CTLA-4 and anti-PD-1 checkpoint blockade

Spencer Wei', Jacob Levine ${ }^{2}$, Alexandria Cogdill', Yang Zhao' Nana-Ama Anang ${ }^{1}$, Miles Andrews', Padmanee Sharma', Jing Wang', Jennifer Wargo ${ }^{1}$, Dana Pe'er ${ }^{2}$, James Allison ${ }^{1}$

${ }^{1}$ The University of Texas MD Anderson Cancer Center, Houston, TX, USA; ${ }^{2}$ Sloan Kettering Institute, New York, NY, USA

Correspondence: Spencer Wei (scwei@mdanderson.org); James Allison Journal for ImmunoTherapy of Cancer 2017, 5(Suppl 2):P379

\section{Background}

Immune checkpoint blockade is able to achieve durable responses in a subset of patients, however despite such significant clinical progress we still lack a fundamental understanding of the mechanisms of antiCTLA-4 and anti-PD-1 induced tumor immune rejection. CTLA-4 and PD-1 regulate $T$ cell activation through different molecular and cellular mechanisms and act at different stages of T cell activation. As such, we hypothesized that anti-CTLA-4 and anti-PD-1 checkpoint blockade induce tumor rejection through distinct cellular mechanisms. Methods

To address this hypothesis, we utilized mass cytometry which enables characterization of more than 40 parameters at single cell resolution and unsupervised cellular classification. Using this approach, we comprehensively profiled the immune infiltrates of MC38 and B16BL6 murine tumors from mice treated with anti-CTLA-4, anti-PD-1, or control antibodies. We also performed similar analyses of surgically resected melanomas from patients being treated with checkpoint blockade therapy.

Results

In both tumor models, more than 13 distinct tumor infiltrating $\mathrm{T}$ cell populations were identified. Both anti-CTLA-4 and anti-PD-1 checkpoint blockade modulated the frequencies of only a subset of these tumor infiltrating $T$ cell populations. Furthermore, of multiple exhausted-like CD8 T cell populations identified, the frequencies of only two subsets correlate with outcome, suggestive of functional heterogeneity within phenotypically exhausted T cells. Most notably, we find that anti-CTLA4, but not anti-PD-1, modulates the CD4 effector compartment by inducing the expansion of Th1-like CD4 effector T cells. Observations from mass cytometry analyses of surgically resected melanoma tumors from patients being treated with anti-CTLA-4 or anti-PD-1 therapy were consistent with these preclinical findings.

Next we utilized similar methodologies to investigate the cellular mechanism of combination anti-CTLA-4 and anti-PD-1 therapy. Although combination therapy largely enhanced effects observed in monotherapies, combination therapy differentially modulated specific exhausted-like CD8 T cell populations. These data suggest that combination therapy modulates $\mathrm{T}$ cell function and mediates tumor rejection through mechanisms that are in part distinct from either monotherapy.

Conclusions

Our findings indicate that anti-CTLA-4 and anti-PD-1 modulate specific tumor infiltrating $T$ cell populations and utilize distinct cellular mechanisms to induce tumor rejection. Anti-CTLA-4, but not anti-PD1 , induces expansion of CD4 effector T cells. Furthermore, these data suggest that combination therapy modulates $T$ cell function differently than monotherapies. These findings have implications for the rational design of combinatorial therapeutic approaches and expand our understanding of the mechanisms that regulate T cell activity.

\section{P380}

The role of radiotherapy in driving anti-tumor CD8 T cell responses

Lauren Zebertavage ${ }^{1}$, Alejandro Alice², Marka Crittenden²,

Michael Gough²

${ }^{1}$ Oregon Health and Science University, Portland, OR, USA; ${ }^{2}$ Earle A

Chiles Research Institute, Portland, OR, USA

Correspondence: Michael Gough (uhde@ohsu.edu)

Journal for ImmunoTherapy of Cancer 2017, 5(Suppl 2):P380

\section{Background}

Radiotherapy (RT) is one of the three main arms of traditional cancer therapy, and has been show to synergize well with immunotherapies in preclinical models. Consistent with the findings of other investigators, in immunogenic murine models of radiation therapy and immunotherapy we have observed CD8+ T cell-dependent clearance of tumors given single, high-dose radiation (20Gy) and anti-PD1 checkpoint blockade. In this setting, RT has been proposed to generate tumor antigen-specific T cells following the release of antigens by dying cancer cells, and PD1 blockade allows $T$ cell control of residual cancer cells in the suppressive tumor environment. We hypothesized that the role of radiation is to boost the number of existing tumor antigen-specific CD8 T cells and drive an influx of tumor-antigen reactive cytotoxic $T$ cells into the tumor. If this hypothesis is true, then providing $T$ cells through other means should be sufficient to replace radiation therapy in this therapeutic combination.

Methods

In order to deliver targeted beams of radiation, we use the Xstrahl Small Animal Radiation Research Platform (SARRP). Tumor-reactive CD8 $T$ cell numbers were boosted using live-attenuated Listeria monocytogenes vaccines against potent tumor-specific CD8 model antigens. Nur77-GFP transgenic mice were used to measure antigen recognition by $T$ cells in vitro and in vivo.

Results

We demonstrate that RT generates marginal increases in tumor-antigen specific $T$ cells in the peripheral circulation. In order to determine whether higher numbers of tumor-responsive CD8s were driving RT plus checkpoint blockade efficacy, we vaccinated mice with attenuated 
Listeria expressing tumor-specific antigens. Using immunohistology and flow cytometry we demonstrate that these antigen-specific cells were present at high levels in the tumor. We demonstrate that antigenspecific $T$ cells generated by vaccination kill antigen-pulsed targets in in vivo cytotoxicity assays, recognize cancer cells ex vivo, and respond to tumor-associated antigen within the tumor. However, mice treated with Listeria vaccination and PD1 blockade showed no tumor growth control advantage when compared to controls, despite the high level of antigen-specific T cells in the tumor.

\section{Conclusions}

These data demonstrate that while radiotherapy generates marginal increases in the number of antigen-specific T cells, which are log-fold fewer than generated by Listeria vaccination, $\mathrm{RT}$ is a superior partner for combination with checkpoint blockade. We find that generating large numbers of tumor antigen-specific T cells cannot substitute for the efficacy of radiotherapy in combination with anti-PD1 checkpoint blockade, and question whether the main driver of radiotherapeutic efficacy is augmenting CD8 T cell numbers.

\section{Mechanisms of Resistance to Immunotherapy}

\section{P381}

The BET bromodomain inhibitor ZEN-3694 modulates the expression of checkpoint receptors and immune suppressive factors in the blood of $\mathrm{MCRPC}$ patients

Eric Campeau', Henrik Hansen', Sanjay Lakhotia², Karen Norek', Laura Tsujikawa', Sarah Attwell ${ }^{1}$

${ }^{1}$ Zenith Epigenetics, Calgary, AB, Canada; ${ }^{2}$ Zenith Epigenetics, San Francisco, CA, USA

Correspondence: Eric Campeau (sattwell@zenithepigenetics.com) Journal for ImmunoTherapy of Cancer 2017, 5(Suppl 2):P381

\section{Background}

Epigenetic regulation of the immune system plays a significant role in the response to immunotherapies. The potential for epigenetic modulators to prime the immune system and increase the duration and frequency of response to checkpoint inhibitors has been supported by both pre-clinical and clinical evidence. ZEN-3694 is an orally available inhibitor of the bromodomain and extra-terminal (BET) domain family of proteins currently in phase I clinical trials in metastatic castration-resistant prostate cancer (mCRPC) (NCT02705469 and NCT02711956). Previously, we have shown that ZEN-3694 modulates multiple checkpoint receptors, immune suppressive factors and cytokines in vitro, and acts synergistically with a PD-1 mAb to inhibit tumor growth in a MC-38 syngeneic mouse model. To follow up with these findings, we examined the ability of ZEN-3694 to modulate the expression of Immuno-Oncology target genes in mCRPC patients at multiple doses in our current phase 1 clinical trial.

Methods

The Nanostring $n$ Counter ${ }^{\oplus}$ PanCancer Immune Profiling Panel was used to measure immune marker expression in patient whole blood RNA taken at 0, 4 and $24 \mathrm{~h}$ post dosing with ZEN-3694.

Results

ZEN-3694 modulates multiple checkpoints, suppressive factors and cytokines in peripheral immune cells 4 hours after a single dose. Most of these changes return to baseline at 24 hours, following clearance of the drug. Significant effects were detected at all doses tested, including at welltolerated doses below the maximum tolerated dose. Multiple checkpoint receptors, including TIM3 and PD-L1, chemokines CCL2/CCR2 and IL-8, and the suppressive factors IDO1 and ARG1, were significantly inhibited 4 $\mathrm{h}$ post-dose across 16 patients. These results were also confirmed by realtime PCR for several of these markers. Furthermore, mRNA levels of multiple co-stimulatory markers, including ICOSLG and CD28, were maintained or induced. Several of these markers show a strong dose/exposure response, and Ingenuity ${ }^{\oplus}$ Pathway Analysis suggests that lower doses may be superior to higher doses for cancer immune response modulation.

\section{Conclusions}

Taken together, these data suggest that ZEN-3694 has the potential to modulate multiple factors involved in adaptive resistance to therapeutic PD-1 blockade, and therefore may improve response rate and duration in combination with a checkpoint inhibitor. This is the first presented clinical evidence that a BET bromodomain inhibitor can modulate PD-L1 and other relevant immuno-oncology targets in patients at therapeutically well-tolerated doses. Follow up studies to correlate these changes with protein expression, immune cell activation, and tumor-specific targets in patients are underway.

P382

ATR inhibition sequenced with radiation therapy abrogates immune exhaustion

David Clump', Christopher Bakkenist', Frank Vindetti', Greg Delgoffe², Robert Ferris

${ }^{1}$ UPMC Hillman Cancer Center, Pittsburgh, PA, USA; ${ }^{2}$ University of

Pittsburgh School of Medicine, Pittsburgh, PA, USA

Correspondence: David Clump (clumpda2@upmc.edu)

Journal for ImmunoTherapy of Cancer 2017, 5(Suppl 2):P382

\section{Background}

Immunotherapy that targets the immunosuppressive interaction between the T cell activation-induced programmed cell death receptor 1 (PD-1) and its ligand PD-L1 and radiation therapy are used in the management of a majority of metastatic non-small cell lung cancer (NSCLC) patients. ATR is a DNA damage signaling kinase activated at damaged replication forks and ATR kinase inhibitors potentiate the cytotoxicity of DNA damaging chemotherapies and radiation Methods

Here we show that ATR kinase inhibitors potentiate radiation-induced tumor immune responses in genetically engineered and syngeneic mouse models of Kras-mutant lung adenocarcinoma and colorectal carcinoma, respectively.

Results

ATR kinase inhibitors attenuate radiation-induced PD-L1 upregulation on tumor cells and dramatically decrease the number of tumor infiltrating Tregs. ATR kinase inhibitors further attenuate radiation-induced T cell exhaustion and increase $T$ cell activity in the tumor microenvironment.

Conclusions

Our work raises the exciting possibility that a single pharmacologic agent may enhance the cytotoxic effects of DNA damaging chemotherapy and radiation while concurrently potentiating radiation-induced tumor immunity.

P383

Molecular and immune characterization of melanoma metastases with heterogeneous PTEN expression

Mariana Petaccia de Macedo ${ }^{1}$, Feng Wang ${ }^{2}$, Diego Marzese ${ }^{3}$

Courtney Hudgens ${ }^{2}$, Meredith McKean ${ }^{2}$, Khalida Wani ${ }^{2}$, Lauren Haydu ${ }^{2}$,

Patrick Danaher ${ }^{4}$, Christopher Merrittt, Giang Ong ${ }^{4}$, Sarah Warren ${ }^{4}$,

Joseph Beechem ${ }^{4}$, Dave Hoon ${ }^{3}$, Weiyi Peng ${ }^{2}$, Lawrence Kwong ${ }^{2}$,

Michael Tetzlaff', Alexander Lazar ${ }^{2}$, Michael Davies ${ }^{2}$

${ }^{1}$ AC Camargo Cancer Center, São Paulo, Brazil; ${ }^{2} \mathrm{MD}$ Anderson Cancer Center, Houston, TX, USA; ${ }^{3}$ John Wayne Cancer Institute, Santa Monica,

CA, USA; ${ }^{4}$ NanoString Technologies, Seattle, WA, USA

Correspondence: Michael Davies (mdavies@mdanderson.org)

Journal for ImmunoTherapy of Cancer 2017, 5(Suppl 2):P383

\section{Background}

There is growing evidence that oncogenic pathways in tumor cells can impact the anti-tumor immune response and the efficacy of immunotherapy. Previously we showed that loss of PTEN inhibits immune infiltration and promotes resistance to immune-mediated tumor killing in melanoma [1]. Although PTEN expression is generally uniform in melanoma, we identified a cohort of melanoma metastases with distinct areas with (+) and without (-) PTEN protein expression. Comparative molecular and immune analyses were performed on the PTEN (+) and $(-)$ regions of these tumors to improve our understanding of the mechanisms, pathogenesis, and significance of PTEN loss.

Methods

Core biopsies were performed on formalin-fixed paraffin-embedded FFPE blocks to isolate analytes from tumor-matched regions with $(+)$ and (-) PTEN expression by immunohistochemistry (IHC). DNA was analyzed for somatic mutations and copy number variations (CNVs) 
in 200 cancer-related genes by next generation sequencing (NGS), and globally for DNA methylation patterns by Illumina Infinium Human Methylation450 arrays. Expression of proteins involved in signaling pathways $(n=42)$ and immune populations/regulators $(n=40)$ in distinct PTEN (+) and (-) regions within the tumors was quantified by Nanostring Digital Spatial Profiling.

Results

NGS showed that PTEN (+) and (-) regions did not differ in overall mutation patterns. PTEN (-) regions had increased rates of chromosomal loss affecting PTEN, EMB and ZNF567; gain of LETM1, WDR17 and SPATA4 was detected in PTEN (+) regions. Hierarchical clustering of DNA methylation patterns demonstrated overall clustering of samples by tumor. Loss of PTEN expression did not correlate with increased methylation of the Pten locus, but differential methylation of several potential regulatory transcription factors (Pax3, Gata2, Ikzf1, Pax5, and Gata5) was detected. Proteomic analysis identified multiple signaling and immune related proteins with significantly different expression between PTEN (+) and (-) regions. Loss of PTEN correlated with significantly increased P-AKT and PP70S6K, consistent with increased activation of PI3K-AKT-mTOR signaling, and significantly decreased VISTA, CD8A, and CD3. PD-L1 expression did not correspond with PTEN status.

\section{Conclusions}

Intratumoral loss of PTEN in melanoma metastases corresponded with genetic alterations in the Pten gene but not its methylation status. Proteomic analysis supports that PTEN status corresponds locally with PI3K-AKT pathway activation and with heterogeneity of components of the anti-tumor immune response. Together the findings further support the biological significance of PTEN loss in melanoma.

Consent to publish

All patients provided informed consent.

\section{References}

1.Peng

W, et al. Loss of PTEN promotes resistance to T-cell mediated immunotherapy. Cancer Discovery. 2016; 6 (2):201-216.

\section{P384}

Mechanisms of efficacy during TGFbR1 inhibition/cytotoxic

combination therapy for rectal cancer

Andrew Gunderson, Kayla McCarty, Michaela Phillips, Emily Hodel, Michael Gough, Marka Crittenden, Kristina Young

Earle A. Chiles Cancer Research Institute, Portland, OR, USA

Correspondence: Andrew Gunderson (agun1107@gmail.com); Kristina

Young

Journal for ImmunoTherapy of Cancer 2017, 5(Suppl 2):P384

\section{Background}

Neoadjuvant chemoradiation is the standard of care for patients with locally advanced rectal cancer with the intention of downstaging and sphincter preservation prior to surgical intervention. Responses may be limited, however, in part due to TGFb1 regulated resistance to these cytotoxic modalities. We have shown previously that blocking ALK5 signaling through use of a small molecule inhibitor sensitizes CT26 tumor-bearing mice to the effects of radiation in part by boosting $\mathrm{CD} 8^{+} \mathrm{T}$ cell mediated cytotoxicity. The mechanism for this efficacy is currently unknown. Methods

By using LY2157299 prior to 5-FU and hypofractionated radiation, tumorbearing animals elicit suboptimal $\mathrm{T}$ cell responses capable of controlling tumor growth in a significant percentage of animals but fail to cure. Results

Early results from a clinical trial of neoadjuvant TGFbR1 inhibition with chemoradiation in 3 rectal cancer patients demonstrate objective clinical responses and markers of enhanced anti-tumor immunity but indicators of compensatory immunosuppression are also observed in surgically excised tissue. One visually prominent aspect in a proportion of these patients is the infiltration of plasma cells into tumor parenchyma. Typically these fully differentiated B cells would be a hallmark of immune mediated rejection through secretion of high affinity immunoglobulin, but previous reports suggest they may also function to suppress cytotoxic $T$ cells through TGFb1, IL-10 and PDL1. Correspondingly, we also observe increases in B regulatory populations and IL-10 expression in CT26 tumor draining lymph nodes from treated animals.

Conclusions

As B cell subsets express apoptotic cellular receptors similar to macrophages, we propose $B$ cells may be one mechanism of resistance to this combination therapy and the direct blockade of their activity may optimize T cell effectiveness for patients with rectal cancer.

\section{P385}

A HSP-TLR-Wnt5a paracrine signaling axis drives CXCR2 ligand recruitment of myeloid-derived suppressor cells and represents a novel adaptive resistance mechanism to anti-PD-1 antibody therapy Bala Theivanthiran', Nicholas C. DeVito', Kathy Evans', Fei Zhao',

Christine Xiao', Benjamin S. Goldschmidt', Rob Edgar², Alisha H. Holtzhausen ${ }^{3}$ April KS. Salama', John Lewicki', John H. Strickler', John A. Viator ${ }^{2}$, Brent A. Hanks 'Duke University, Durham, NC, USA; ${ }^{2}$ Duquesne University, Pittsburgh, PA, USA; ${ }^{3}$ University of North Carolina, Chapel Hill, NC, USA; ${ }^{4}$ OncoMed Pharmaceuticals, Inc., Redwood City, CA, USA; ${ }^{5}$ Duke University, Durham, NC, USA

Correspondence: Brent A. Hanks (hanks004@mc.duke.edu)

Journal for ImmunoTherapy of Cancer 2017, 5(Suppl 2):P385

\section{Background}

Despite the impressive impact generated by the anti-PD-1/PD-L1 antibody (ab) therapies in oncology, a significant percentage of patients exhibit either primary or secondary resistance to these treatment modalities. The evolution of these resistance mechanisms to cancer immunotherapy remains poorly understood. Myeloid-derived suppressor cells (MDSCs) have been demonstrated to suppress anti-tumor immunity and previous work has shown the granulocytic subset of MDSCs (GrMDSDCs) to migrate in response to CXCR2 chemokine gradients.

Methods

We utilized RNAseq differential gene expression analysis, serial tissue biopsy qrt-PCR, and SILAC-AHA metabolic labeling mass spectrometry-based differential secretome analysis to identify those genes and secreted proteins upregulated in melanomas escaping anti-PD-1 ab therapy in an autochthonous $\mathrm{BRAF}^{\mathrm{V} 600 \mathrm{E}} \mathrm{PTEN}^{-1-}$ melanoma model. Multi-parameter flow cytometry and immunohistochemistry were utilized to investigate MDSC recruitment to tumor tissues. Lentiviral shRNA gene silencing and in vivo $\mathrm{CD} 8^{+} \mathrm{T}$ cell ablation assays were conducted to define the underlying signaling mechanisms driving MDSC recruitment to anti-PD-1 ab-treated melanomas. Circulating melanoma cells (CMCs) were derived from patients undergoing active anti-PD-1/PD-L1 ab therapy using a photoacoustic cytometry technique for single cell qrt-PCR validation of pre-clinical findings. Results

Autochthonous $\mathrm{BRAF}^{\mathrm{V} 600 \mathrm{E}} \mathrm{PTEN}^{-/-}$melanomas upregulate the expression of several CXCR2 ligands, including CXCL5, following escape from anti-PD-1 ab therapy (Fig. 1A-B). This is associated with the rapid recruitment of Gr-MDSCs and an inhibition of local $C D 8^{+} \mathrm{T}$ cell responses (Fig. 1C-D). We determined that melanoma CXCL5 expression is induced by a Wnt5a-YAP pathway and that Wnt5a is, in turn, regulated by local $\mathrm{CD}^{+} \mathrm{T}$ cell killing and paracrine heat shock protein (HSP)-TLR2 signaling (Fig. 2). The individual genetic silencing of Wnt5a and CXCL5 reverses Gr-MDSC recruitment in response to antiPD-1 ab therapy and sensitizes these BRAF ${ }^{\mathrm{V} 600 \mathrm{E}} \mathrm{PTEN}^{--}$melanomas to checkpoint inhibition (Fig. 3). Indeed, blockade of Wnt-mediated signaling with a OMP-54F28 'Wnt trap' diminishes Gr-MDSC recruitment and augments the efficacy of anti-PD-1 ab therapy in this autochthonous melanoma model (Fig. 4). Plasma levels of CXCL5 correlate with progression and anti-PD- 1 ab escape in the transgenic melanoma model while patient-derived CMCs also demonstrate CXCR2 ligand upregulation following progression on anti-PD-1/PD-L1 ab therapy (Fig. 5).

Conclusions

A HSP-TLR-Wnt5a paracrine signaling axis mediates the recruitment of Gr-MDSCs in response to anti-PD- 1 ab therapy-mediated $\mathrm{CD}^{+} \mathrm{T}$ cell killing and represents a novel adaptive resistance mechanism to cancer immunotherapy. Additional studies are ongoing to exploit this pathway as a strategy for augmenting the therapeutic efficacy of anti-PD-1 ab therapy and for monitoring the development of immunotherapy resistance in advanced melanoma patients. 


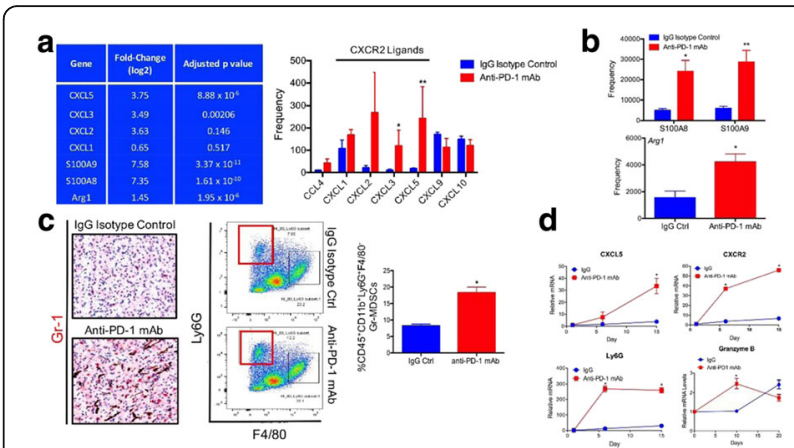

Fig. 1 (abstract P385). Escape from anti-PD-1 Ab Immunotherapy in a Transgenic Melanoma Model Involves the Recruitment of Gr-MDSCs. A. RNAseq differential gene expression analysis showing an upregulation of several CXCR2 ligands following anti-PD-1 ab escape in a transgenic BRAF(V600)-PTEN-/- melanoma model. B. RNAseq differential gene expression analysis demonstrating increased MDSC markers in BRAF(V600E)PTEN-/- melanomas following escape from anti-PD-1 ab therapy. C. Immunohistochemistry and flow cytometry analysis demonstrating increased levels of Gr-MDSCs in anti-PD-1 ab-treated versus lgG isotype controltreated BRAF(V600E)-PTEN-/- melanomas. Representative IHC images and flow cytometry dot plots are provided. D. Serial tissue biopsy and qrt-PCR analysis of anti-PD-1 ab-treated versus lgG isotype control-treated BRAF(V600E)-PTEN-/- melanomas. All data is mean +/- SEM. *P $<0.05$

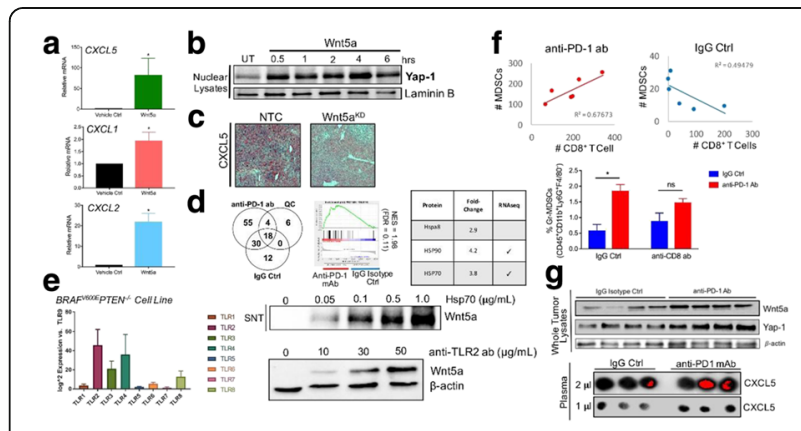

Fig. 2 (abstract P385). Induction of CXCL5 in Melanomas Undergoing Treatment with Anti-PD-1 Ab Therapy is Dependent Upon a Paracrine HSP-TLR-Wnt5a Signaling Pathway. A. Qrt-PCR analysis shows that Wnt5a stimulates the expression of several CXCR2 ligands in a BRAF(V600E)PTEN-/- melanoma cell line. B. Western blot analysis of nuclear YAP1 levels in response to Wnt5a stimulation in a BRAF(V600E)-PTEN-/- melanoma cell line. C. Immunohistochemistry studies show that CXCL5 expression is significantly suppressed in Wnt5a-silenced BRAF(V600E)-PTEN-/melanomas. D. SILAC-AHA metabolic labeling mass spectrometry-based differential secretome analysis and RNAseq differential gene expression analysis of BRAF(V600E)-PTEN-/- melanomas following treatment with anti-PD-1 ab vs lgG isotype control ab. Several cellular stress pathways identified as being upregulated in those melanomas undergoing antiPD-1 ab therapy. Several heat shock proteins including HSP70 noted to be induced in anti-PD-1 ab-treated melanomas. E. BRAF(V600E)-PTEN-/melanomas express elevated levels of TLR2 and TLR4 based on grt-PCR. Western blot demonstrates that HSP70 and agonistic anti-TLR2 ab stimulates Wnt5a expression in the BRAF(V600E)-PTEN-/- melanoma cell line. F. top, CD8+ T cell levels and Gr-MDSC levels correlate significantly in BRAF(V600E)-PTEN-/- melanomas only when treated with anti-PD-1 ab therapy $(p=0.044)$. bottom, Ablation of CD8+ T cells diminishes the recruitment of Gr-MDSCs in response to anti-PD-1 ab therapy. G. top, Whole tissue Western blot analysis shows that anti-PD-1 ab therapy induces the upregulation of Wnt5a and YAP1 in BRAF(V600E)-PTEN-/melanomas. bottom, Dot blot analysis shows that CXCL5 expression is upregulated in the serum of transgenic BRAF(V600E)-PTEN-/- mice undergoing treatment with anti-PD-1 ab therapy (red, blot density analysis). Representative blots shown. All data is mean $+/$ - SEM. ${ }^{*} P<0.05$

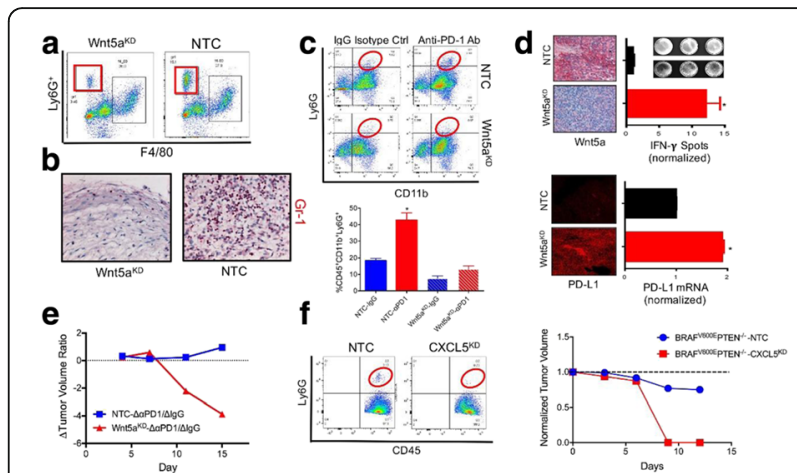

Fig. 3 (abstract P385). The Wnt5a-CXCL5 Pathway Drives Gr-MDSC Recruitment in Response to anti-PD-1 Ab Therapy and Promotes the Development of anti-PD-1 Ab Adaptive Resistance. A. Flow cytometry analysis of Gr-MDSCs in Wnt5a-silenced BRAF(V600E)PTEN-/- melanomas. B. Immunohistochemistry of Gr-MDSCS in Wnt5a-silenced BRAF(V600E)-PTEN-/- melanomas. C. Flow cytometry analysis demonstrates that Gr-MDSC recruitment in response to anti-PD-1 ab therapy is ablated in Wnt5a-silenced BRAF(V600E)PTEN-/- melanomas. Representative flow cytometry dot plots provided. D. IFNg ELISPOT and tissue immunofluorescence show that melanoma Wnt5a silencing enhances the activation of TRP2specific CD8+ T cells and promotes the expression of PD-L1. Representative images provided on the left. E. Wnt5a silencing sensitizes BRAF(V600E)-PTEN-/- melanomas to anti-PD-1 ab therapy. F. CXCL5 silencing suppresses Gr-MDSC recruitment and sensitizes BRAF(V600E)-PTEN-/- melanomas to anti-PD-1 ab therapy. Representative flow cytometry dot plots shown. All data is mean + /- SEM. ${ }^{*} P<0.05$

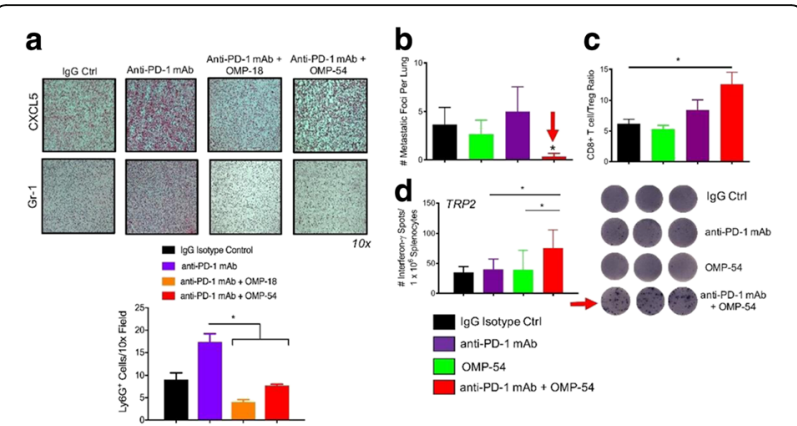

Fig. 4 (abstract P385). Inhibition of Wnt Signaling Suppresses Gr-MDSC Recruitment in Response to anti-PD-1 Ab Therapy and Augments the Efficacy of this Checkpoint Inhibitor in a Transgenic BRAF(V600E)-PTEN-/- Melanoma Model. A. Immunohistochemistry of CXCL5 and Gr-1 in BRAF(V600E)-PTEN-/- melanoma tissue undergoing treatment with anti-PD-1 ab therapy in the absence and presence of OMP-18R5 (vantictumab, anti-Fzd7 ab) or OMP-54F28 (ipafricept, Fzd8-Fc). B. Histologic analysis of resected lung tissues to enumerate lung metastases in the transgenic BRAF(V600E)-PTEN-/- melanoma model following the indicated treatment regimens. C. Flow cytometry analysis of BRAF(V600E)PTEN-/- melanoma tissue following the indicated treatment regimens. $\mathrm{CD} 45+\mathrm{CD} 3+\mathrm{CD} 8+\mathrm{T}$ cells and $\mathrm{CD} 45+\mathrm{CD} 4+\mathrm{FoxP3}+$ regulatory $T$ cell (Treg) populations quantitated and expressed as a CD8+ T cell : Treg ratio. D. TRP2 IFNg ELISPOT analysis performed using harvested splenocytes from transgenic BRAF(V600E)-PTEN-/mice after undergoing the indicated therapy. Representative wells shown on the right. All data is mean $+/$ - SEM. ${ }^{*} P<0.05$ 


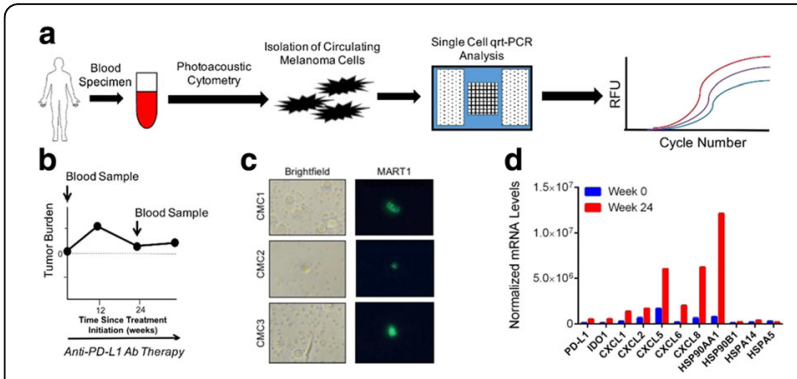

Fig. 5 (abstract P385). Circulating Melanoma Cell (CMC) Qrt-PCR Analysis Demonstrates Enhanced CXCR2 Ligand Expression in Progressing Melanoma Patients Following anti-PD-1/anti-PD-L1 Ab Therapy. A. Schematic showing clinical specimen acquisition and analysis work flow. Samples are harvested from patients before and during checkpoint inhibitor immunotherapy. CMCs are enriched through centrifugation, analyzed by the photoacoustic flow cytometer, and subjected to integrated fluidic chip single cell qrt-PCR analysis of select target genes. Samples positive for CD45 or negative for both MART1 and S100 are excluded from analysis. Target gene expression normalized to Ubb expression levels. 6 CMCs analyzed per patient time point. B. Spider plot of target lesion tumor volume versus time for an advanced melanoma patient undergoing anti-PD-L1 ab therapy. Blood samples taken at week 0 prior to therapy and week 24. C. MART1 immunofluoresence analysis of captured CMCs. Brightfield images shown on left. D. CMC single-cell grt-PCR analysis and quantitation of CXCR2 ligands and other target genes of interest in the same patient described in B. Data has been normalized to week 0 expression levels of respective target genes

\section{P386}

Tracking the dynamic response to vaccine prime/oncolytic boost immunotherapy identifies key mechanisms of immune resistance in metastatic ovarian cancer

AJ Robert McGray', Raya Huang', Sebastiano Battaglia', Cheryl Eppolito', Anthony Miliotto', Mukund Seshadri', Kyle Stephenson'², Brian Lichty ${ }^{2}$, Kunle Odunsi

${ }^{1}$ Roswell Park Cancer Institute, Buffalo, NY, USA; ${ }^{2}$ McMaster University/ Turnstone Biologics, Hamilton \& Ottawa, Canada

Correspondence: Kunle Odunsi (bobmcgray@gmail.com)

Journal for ImmunoTherapy of Cancer 2017, 5(Suppl 2):P386

\section{Background}

While spectacular responses to cancer immunotherapy have been observed, most responses are short-lived with ultimate tumor relapse. Using a vaccine prime/oncolytic boost immunotherapy, we sought to define robust biomarkers indicative of therapeutic response, response durability, as well as changes leading to tumor relapse in a pre-clinical mouse model of intraperitoneal metastatic ovarian cancer.

\section{Methods}

We utilized a heterologous prime/boost vaccine to treat ovarian cancer, combining an adjuvant-based vaccine with a novel oncolytic Maraba viral vector to generate robust anti-tumor $\mathrm{T}$ cell responses. To investigate treatment impact on both anti-tumor immunity and local events within the tumor, we combined analyses of immune cells within the tumor microenvironment with transcriptional profiling of solid tumors, and pre-clinical MRI to directly monitor changes in intraperitoneal tumors in response to therapy. This approach led to the identification/testing of candidate combination treatments with the potential to improve prime/boost vaccination.

Results

Heterologous prime/boost vaccination elicited robust tumor-specific CD8+ $T$ cell responses, with high numbers of therapy-induced CD8+ T cells effectively trafficking to the tumor microenvironment. This approach greatly improved tumor control and long-term survival compared to vaccine priming alone, however the combination therapy was not curative and cellular analysis suggested that $T$ cells within the tumor microenvironment were functionally suppressed. Tumor transcriptional profiling revealed that prime/boost vaccination led to induction of numerous inflammatory processes, with gene signatures consistent with CD8+ $\mathrm{T}$ cell infiltration, upregulation of multiple costimulatory/ immune checkpoint receptors, and induction of chemokine networks associated with lymphoid and myeloid cell trafficking. Based on these signatures, we tested candidate combination therapies to further enhance the impact of prime/boost vaccination, of which checkpoint blockade using aPD-1 produced the greatest benefit. Analysis of the response to prime/boost vaccination + aPD-1 by MRI revealed significant intratumoral inflammation after Maraba boosting followed by dramatic tumor regression, consistent with pseudo-progression. Tumors were effectively controlled, but ultimately relapsed. Using MRI guided assessment, treated tumors were subsequently isolated during all phases of this response and subjected to RNAseq analysis. The findings from these studies will be presented.

Conclusions

Our data suggest that interrogating the tumor microenvironment during the course of vaccine prime/oncolytic boost immunotherapy can identify candidate therapeutic targets, as well as key biomarkers of therapeutic response and/or intratumoral changes leading to tumor progression.

\section{P387}

IFN $\gamma$ signaling in $\mathrm{T}_{\text {regs }}$ acts as a barrier to immunotherapeutic response Abigail Overacre-Delgoffe', Maria Chikina', Rebecca Dadey',

Hiroshi Yano', Erin Brunazzi', Gulidanna Shayan², William Horne',

Jessica Moskovitz ${ }^{2}$, Jay Kolls ${ }^{1}$, Cindy Sander ${ }^{2}$, Yongli Shuai',

Daniel Normolle', John Kirkwood ${ }^{2}$, Robert Ferris², Greg Delgoffe²,

Tullia Bruno ${ }^{2}$, Creg Workman', Dario Vignali'

${ }^{1}$ University of Pittsburgh, Pittsburgh, PA, USA; ${ }^{2}$ University of Pittsburgh

Cancer Institute, Pittsburgh, PA, USA

Correspondence: Abigail Overacre-Delgoffe (overacre@pitt.edu); Dario Vignali Journal for ImmunoTherapy of Cancer 2017, 5(Suppl 2):P387

\section{Background}

Checkpoint blockade (anti-PD1/PDL1) has displayed striking, durable responses in cancer patients; however, many still succumb to disease. The underlying reasons for this lack of response remain obscure. Therefore, identifying resistance mechanisms to current immunotherapies is critical. While regulatory $T$ cells $\left(T_{\text {regs }}\right)$ are required to maintain immune homeostasis, they are also a major barrier to the anti-tumor immune response, and as such, are an attractive therapeutic target. We have previously shown that intratumoral $T_{\text {reg }}$ stability is reliant on the Neuropilin-1 (Nrp1):Semaphorin-4a axis. In its absence, $T_{\text {regs }}$ become fragile and secrete IFNy while maintaining Foxp3 expression [1,2]. However, 1) whether $T_{\text {reg }}$ fragility is required for response to immunotherapy, and 2)

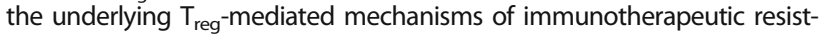
ance in mice and patients remain unclear.

Methods

We utilized syngeneic tumor models, including MC38 (anti-PD-1 sensitive) and B16.F10 (anti-PD-1 resistant) in both Foxp3 $3^{\text {Cre-YFP }}$ and Ifngr $1^{\mathrm{L} / \mathrm{L}}$ Foxp $3^{\text {Cre-YFP }}$ mice. Mice were treated with various immunotherapies to assess the role of IFN $\gamma$ signaling by $T_{\text {regs }}$ in mediating resistance. In addition, we are assessing the direct effect of IFNY in vivo through the use of novel delivery mechanisms. Lastly, we are determining the impact of $\mathrm{T}_{\text {reg }}$ fragility on patient response rate after PD-1 blockade in metastatic melanoma and head and neck squamous cell carcinoma. Results

Using Foxp $3^{\mathrm{Cre}-Y F P}$ and Ifngr $1^{\mathrm{L} / \mathrm{L}}$ Foxp $3^{\mathrm{Cre}-\mathrm{YFP}}$ mice, we found that response to PD-1 blockade required IFNY-induced $T_{\text {reg }}$ fragility, as Ifngr $^{1 / L}$ Foxp $3^{\text {Cre-YFP }}$ mice treated with anti-PD1 were completely resistant to therapy. In addition, PD-1 blockade led to increased IFNy secretion by $\mathrm{T}_{\text {regs, }}$ an effect that was absent in Ifngr $1^{\mathrm{L} / \mathrm{L}} \mathrm{Foxp}^{\mathrm{Cre}-\mathrm{YFP}}$ mice. Furthermore, pre-treatment of tumor-derived $T_{\text {regs }}$ with IFNY led to significantly reduced suppressive function in both mouse and human $\mathrm{T}_{\text {regs. }}$. We are currently extending our studies to patients treated with anti-PD1 in order to determine whether $\mathrm{T}_{\text {reg }}$ fragility is associated with immunotherapeutic response. 


\section{Conclusions}

Overall, we have shown that IFNץ-induced $\mathrm{T}_{\text {reg }}$ fragility is required for anti-PD1 response and that IFNY leads to reduced $\mathrm{T}_{\text {reg }}$ suppressive capacity while maintaining Foxp3 expression. These studies uncover a novel potential resistance mechanism to immunotherapy, and provides an avenue to target $\mathrm{T}_{\text {regs }}$ selectively in the tumor microenvironment to bolster current immunotherapies.

\section{References}

1. Delgoffe $G M$, et al. Stability and function of regulatory $T$ cells is maintained by a neuropilin-1-semaphorin-4a axis. Nature. 2013; 501: 252-256.

2. Overacre-Delgoffe $A E$, et al. Interferon- $\gamma$ Drives Treg Fragility to Promote Anti-tumor Immunity. Cell. 2017. 169. 1130-1141.

\section{P388}

Neutrophil-rich inflammation and PD1/PD-L1 inhibitor resistance in advanced non-small cell lung cancer patients

Wungki Park', Sandra Algaze², Vaia Florou', Diana Saravia',

Deukwoo Kwon', Gilberto Lopes

${ }^{1}$ University of Miami, Miller School of Medicine, Sylvester Comprehensive Cancer Center, Miami, FL, USA; ${ }^{2}$ University of Miami, Miller School of

Medicine, Miami, FL, USA

Correspondence: Gilberto Lopes (wungkipark@gmail.com)

Journal for ImmunoTherapy of Cancer 2017, 5(Suppl 2):P388

\section{Background}

The pro-tumoral role of neutrophils in chronic inflammation in carcinogenesis and metastasis has been well-reported in the animal models in the literature.[1] However, the role of neutrophil-tolymphocyte ratio (NLR) and the acquired resistance to PD1/PD-L1 inhibitors (PD1/PD-L1i) in advanced non-small cell lung cancer patients (NSCLC) has not been described.

\section{Methods}

We retrospectively evaluated 115 advanced NSCLC patients who received PD-1 and PD-L1 inhibitors as their second-line therapy after platinum-based chemotherapy. Two groups were defined: Progressors $(\mathrm{P})$ as patients who had radiographic and clinical progression after at least 2 months of treatment and Clinical Durable Responders (CDR) as patients with at least treatment duration of 6 months and ongoing response. Patients with baseline NLR $>10$ were excluded. We performed paired-samples $T$ test to analyze the difference of each patient's NLR at baseline and at progression in P group. In CDR group, the difference of each patient's NLR at baseline and at censored time while receiving ongoing PD-1/PD-L1i above 6 months were compared. Mann-Whitney test was performed to compare individual patient's NLR at baseline and at progression with the presence of bone metastasis.

Results

There were 72 patients in $\mathrm{P}$ group and 43 patients in the CDR group. Total 82 patients received nivolumab, 25 patients received pembrolizumab, and 8 patients received atezolizumab. The duration of clinical response in $\mathrm{P}$ ranged from 2 to 17.4 months and 6.5 to 23.8 months in CDR group. The mean NLR at baseline in P group was 4.89 and 6.59 at progression. Patients in $\mathrm{P}$ group had statistically significant increases of NLR at progression with mean increase of 1.69 . The $95 \%$ confidence interval was 3.04 to 0.35 ( $p$-value $=0.01$ ). However, there was no statistical difference between the NLR at baseline and censored time while receiving ongoing treatment in CDR group (95\% C.I.: $-1.90-0.822, p$-value $=0.428)$. Bone metastasis did not correlate with the degree of NLR. $(p=0.56)$ Patients who received corticosteroid at the time of progression were not included in the study.

\section{Conclusions}

Persistent increase of NLR may represent unfavorable pro-tumoral inflammation and can represent unfavorable peripheral balance of PD1 and PD-L1 interplay in myeloid-effector T cell immune synapse. This may serve as acquired resistance mechanism of PD-1/PD-L1 $i$ and warrants further investigation.

\section{References}

1. Fridlender ZG, Sun J, Kim S, et al. Polarization of tumor-associated neutrophil phenotype by TGF-beta: "N1" versus "N2" TAN. Cancer cell. 2009;16:183-194.

\section{P389}

Aurora kinase inhibition enhances the efficacy of immunotherapy

Simone Punt ${ }^{1}$, Shruti Malu², Rina Mbofung ${ }^{1}$, Jodi McKenzie ${ }^{1}$, Zhe Wang ${ }^{3}$, Leila Williams' ${ }^{1}$, Jie Qing Chen ${ }^{1}$, Sourindra Maiti ${ }^{1}$, Trang Tieu', Weiyi Peng ${ }^{1}$, Chengwen Liu' ${ }^{1}$, Chunyu Xu' ${ }^{1}$, Marie-Andrée Forget ${ }^{1}$

Cara Haymaker ${ }^{1}$, Jahan Khalili ${ }^{4}$, Nikunj Satani ${ }^{1}$, Florian Muller ${ }^{1}$

Laurence Cooper ${ }^{5}$, Jason Roszik ${ }^{1}$, Rodabe Amaria', Chantale Bernatchez ${ }^{1}$, Timothy Heffernan', Patrick Hwu'

${ }^{1}$ The University of Texas MD Anderson Cancer Center, Houston, TX, USA;

${ }^{2}$ The University of Texas MD Anderson Cancer Center (current: Eli Lilly and Company), Indianapolis, IN, USA; ${ }^{3}$ The University of Texas MD Anderson Cancer Center (current: The Journal of Experimental Medicine), New York, NY, USA; ${ }^{4}$ The University of MD Anderson Cancer Center (current: Personal Peptides LLC), Seattle, WA, USA; ${ }^{5}$ The University of Texas MD Anderson Cancer Center (current: Ziopharm Oncology, Inc.), Houston, TX, USA

Correspondence: Simone Punt (s.vd.garde@outlook.com) Journal for ImmunoTherapy of Cancer 2017, 5(Suppl 2):P389

\section{Background}

Immunotherapy has improved clinical outcomes for a subpopulation of melanoma patients, but over half of treated patients develop primary or secondary resistance. Current efforts are thus focused on developing combinatorial strategies involving immunotherapy and other treatment modalities, such as targeted therapy, to improve clinical outcomes. We aimed to identify factors that regulate the tumor cell response to $\mathrm{T}$ cell mediated cytotoxicity, with the goal to develop more efficacious treatment combinations.

Methods

A high-throughput screen of 850 bioactive compounds was used to identify proteins/pathways that confer resistance to $\mathrm{T}$ cell mediated cytotoxicity (Fig. 1A). A comboscore was calculated for apoptosis (cleaved caspase-3) induced by combined compound and $\mathrm{T}$ cell treatment compared to either treatment alone (Fig. 1B). A library of 576 ORF was subsequently used to identify proteins that induce resistance to T cell mediated cytotoxicity. The genes with the lowest comboscore in the ORF screen were analyzed using Nanostring on 23 samples from adoptive cell therapy (ACT) treated melanoma patients. One of the hits was further validated using in vitro and in vivo assays.

Results

After challenging melanoma cell lines with the compounds and autologous TILs, Aurora kinase inhibitors (AURKi) obtained a high comboscore. Two Aurora kinase inhibitors were validated to synergize with $\mathrm{T}$ cell mediated cytotoxicity in multiple melanoma cell lines (example of enhanced cytotoxicity in one cell line shown in Fig. 1C). Complementary to this finding, Aurora kinase A (AURKA) and AURKB overexpression induced resistance to $T$ cell cytotoxicity. Additionally, patients who did not respond to ACT expressed higher levels of AURKA compared to responding patients $(p=0.04)$, further demonstrating the relevance of Aurora kinase in immune suppression. The combination of AURKi AZD1152 and anti-CTLA4 significantly reduced tumor growth $(p<0.05)$ and enhanced survival $(p<0.01)$ as compared to either therapy alone in an MC38/gp100 in vivo model. Ongoing studies are now aimed at identifying the molecular mechanisms by which AURKi enhance T cell mediated cytotoxicity.

Conclusions

Aurora kinase inhibition may not be cytotoxic per se, but we showed that AURKi treatment sensitizes tumor cells to $T$ cell mediated cytotoxicity and enhances the efficacy of immunotherapy. The combination of AURKi treatment and immunotherapy may thus induce an effective and durable response in melanoma patients, making Aurora kinase inhibitors a promising combination 
strategy with immunotherapy, especially for patients who do not respond to immunotherapy alone.

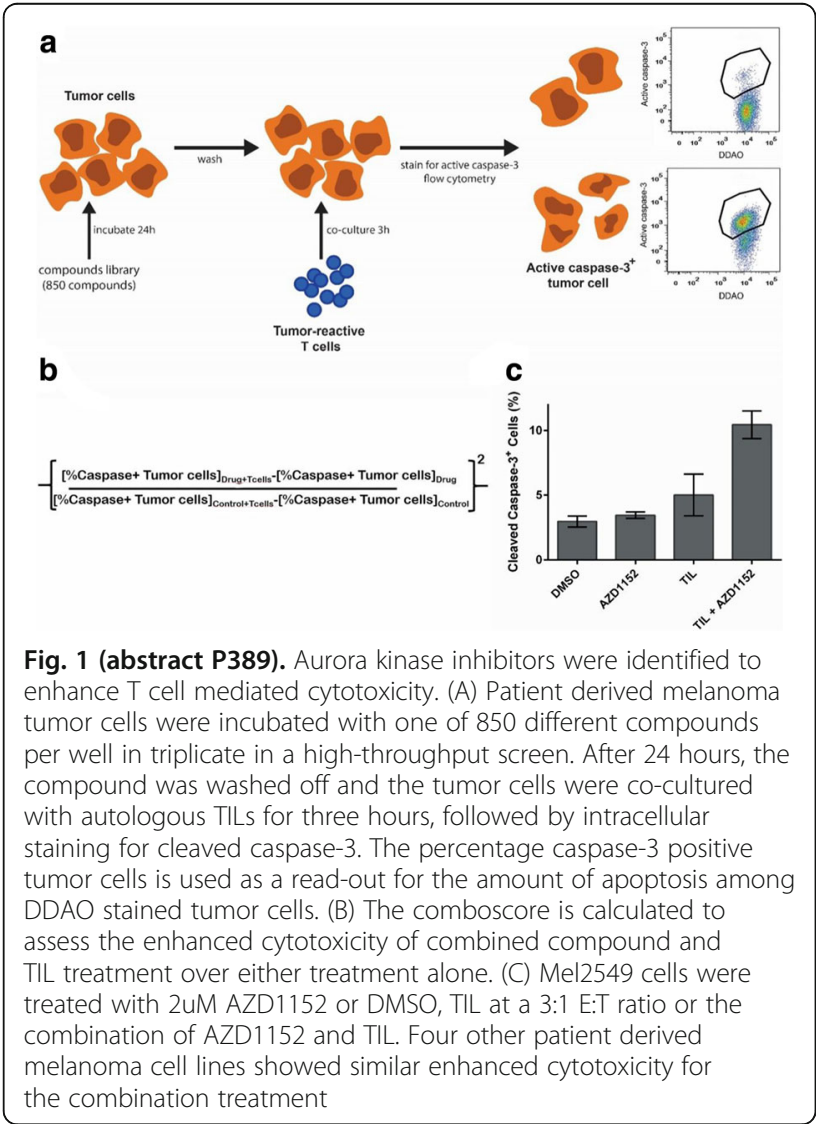

P390

Combination radiotherapy and aOX40/aCTLA-4 immunotherapy reverses anergy and prevents development of functional exhaustion within tumor-specific CD8 T cells

Joshua Walker', Melissa Kasiewicz², William Redmond ${ }^{2}$

${ }^{1}$ Oregon Health \& Science University, Portland, OR, USA; ${ }^{2}$ Providence

Cancer Center, Portland, OR, USA

Correspondence: William Redmond (walkejos@gmail.com)

Journal for ImmunoTherapy of Cancer 2017, 5(Suppl 2):P390

\section{Background}

The immunosuppressive tumor microenvironment and chronic $\mathrm{T}$ cell stimulation that occurs in the presence of cancer results in CD8 T cell dysfunction that has been difficult to reverse with immunotherapy (IT) alone. This dysfunction can be separated into two categories of anergy and exhaustion. Anergy being a poor proliferative response to stimulation after initial priming with inadequate costimulation or suboptimal T cell receptor ligation, and functional exhaustion being the inability of T cells to kill or produce effector cytokines in response to stimulation. We hypothesized the addition of ionizing radiotherapy (RT) to IT with agonist aOX40 \& blocking aCTLA-4 antibodies would reverse tumor associated CD8 T cell anergy and exhaustion.

Methods

We utilized a model of CD8 T cell anergy in which transgenic, OVAspecific, OT-I CD8 T cells are adoptively transferred into centrally tolerant POET-1 mice bearing OVA expressing tumors. After development of anergy/exhaustion, 21 days after transfer, animals were treated with a single 20 Gy fraction of RT, aOX40/aCTLA-4, or combined IT/RT. To assess anergy/exhaustion in endogenous CD8 T cell responses, CT26 colon carcinoma bearing $\mathrm{BALB} / \mathrm{c}$ mice were treated with $\mathrm{IT}$, RT, or combined IT/RT either 10 or 17 days after tumor implantation. The frequency/function of tumor-specific (OT-I or AH1) CD8 T cells was monitored in blood, lymph node (LN), and tumor infiltrating lymphocytes (TIL). Reporter Nur77-GFP-OT-I CD8 T cells expressing green fluorescent protein in response to TCR ligation were used to measure TCR signaling following IT/RT.

Results

We demonstrate OT-I cells become anergic/exhausted and produce few effector cytokines 21 days after adoptive transfer. Combination therapy with IT/RT resulted in increased TCR stimulation (measured by GFP expression) and reversed anergy (measured by proliferation) within OT-I T cells in blood, LN, and TIL compared to IT or RT alone. Combination therapy was able to reverse functional exhaustion (measured by cytokine production) within LN but not TIL in this model. In CT26 tumor bearing mice combination IT/RT significantly increased (up to 4-fold) the frequency of tumor-specific endogenous AH1 CD8 TIL and prevented development of functional exhaustion within TIL compared to IT or RT alone.

Conclusions

Combined ablative RT and IT with aOX40/aCTLA-4 results in increased CD8 TCR signaling, reversal of T cell anergy, and can prevent or reverse functional exhaustion in some cases. These novel results suggest the addition of RT to IT can effectively reverse tumor-associated T cell dysfunction where IT alone is insufficient and provide rationale for early phase clinical trial design.

P391

Non-conventional inhibitory $\mathrm{CD}^{+}{ }^{+}$Foxp3 $3^{-} \mathrm{PD}-1^{\text {hi }} \mathrm{T}$ cells as a

biomarker of immune checkpoint blockade activity

Roberta Zappasodi, Sadna Budhu, Matthew Hellmann, Michael Postow, Yasin Senbabaoglu, Yanyun Li, Cailian Liu, Hong Zhong, Billel Gasmi,

Daniel Hirschhorn-Cymerman, Katherine Panageas, Taha Merghoub, Jedd Wolchok

MSKCC, New York, NY, USA

Correspondence: Jedd Wolchok (zappasor@mskcc.org)

Journal for ImmunoTherapy of Cancer 2017, 5(Suppl 2):P391

\section{Background}

A significant proportion of cancer patients do not respond to immune checkpoint blockade therapy. To deepen our understanding of the mechanisms of action of this strategy, we explored the role of a subset of CD4+Foxp3-negative T cells expressing PD-1 (4PD1hi), which we found to be up-regulated after CTLA-4 blockade in association with limited response to treatment.

Methods

4PD1hi frequency was monitored by flow cytometry. Their function was tested in standard in vitro suppression assays and 3D collagenfibrin gel killing assays. RNAseq gene expression analyses were performed on a Proton sequencing system at the MSK Genomics Core Facility.

Results

We observed that $4 \mathrm{PD} 1 \mathrm{hi}$ accumulate intratumorally as a function of tumor burden in untreated tumor-bearing hosts. In addition, mouse and human circulating and intra-tumor 4PD1 hi inhibit Tcell functions in a PD-1/PD-L1 dependent fashion. Interestingly, CTLA-4 blockade promotes intratumoral and peripheral 4PD1 hi increases in a dose-dependent manner, while combination with PD-1 blockade mitigates this effect and significantly improves anti-tumor activity. In keeping with these observations, we found that patients have a significantly higher risk of death if high 4PD1 hi levels persist after PD-1 blockade. Mechanistically, we provide evidence that mouse and human $4 \mathrm{PD} 1 \mathrm{hi}$ resemble follicularhelper-T-cell(TFH)-like cells and that CTLA-4 blockade increases Bcell co-stimulatory potential in vivo and in vitro, pointing to $4 \mathrm{PD} 1 \mathrm{hi}$ as a possible downstream effect of enhanced T-cell priming during CTLA-4 blockade.

Conclusions

These findings broaden our understanding of the incremental activity of combination checkpoint blockade. They also provide a new 
pharmacodynamic and prognostic biomarker that may inform the design of optimal combination schedules and checkpoint blockade dosage.

\section{Microbiome}

\section{P392}

Microbiome analyses in patients with previously treated, deficient DNA mismatch repair/microsatellite instability-high metastatic colorectal cancer treated with nivolumab \pm ipilimumab: CheckMate 142

Scott Kopetz ${ }^{1}$, Vittorina Zagonel ${ }^{2}$, Michael J. Overman ${ }^{1}$, Ray McDermott ${ }^{3}$, Michael A. Morse ${ }^{4}$, Franklin L. Chen ${ }^{5}$, James Lee ${ }^{6}$, Rebecca A. Moss ${ }^{7}$, Lilan Ling ${ }^{8}$, Alexander Greenfield ${ }^{7}$, Danielle Greenawalt ${ }^{7}$, Z. Alexander $\mathrm{Cao}^{7}$ ${ }^{1} \mathrm{MD}$ Anderson Cancer Center, Houston, TX, USA; ${ }^{2}$ Istituto Oncologico Veneto IOV-IRCSS, Padova, Italy; ${ }^{3}$ St. Vincent's University, Dublin, Ireland; ${ }^{4}$ Duke University Medical Center, Durham, NC, USA; ${ }^{5}$ Novant Health Oncology Specialists, Winston-Salem, NC, USA; ${ }^{6}$ University of Pittsburgh School of Medicine UPMC, Pittsburgh, PA, USA; ${ }^{7}$ Bristol-Myers Squibb, Princeton, NJ, USA; ${ }^{8}$ Memorial Sloan-Kettering Cancer Center, New York, NY, USA

Correspondence: Scott Kopetz (chris.geraci@chrysalismedical.com) Journal for ImmunoTherapy of Cancer 2017, 5(Suppl 2):P392

\section{Background}

Microbiota may have a role in the carcinogenesis of colorectal cancer (CRC) through chronic low-grade inflammation and the influence of microbiota-derived metabolites on host metabolism and immune function. $[1,2]$ Microbiome diversity was significantly different between patients with melanoma who responded to anti-PD-1 therapy and those who did not respond.[3] Here, we explore the relationship between microbiota and response to nivolumab \pm ipilimumab in patients with deficient DNA mismatch repair/microsatellite instabilityhigh (dMMR/MSI-H) CRC enrolled in CheckMate 142 (NCT02060188). Methods

Patients received nivolumab $3 \mathrm{mg} / \mathrm{kg}$ Q2W or nivolumab $3 \mathrm{mg} / \mathrm{kg}+$ ipilimumab $1 \mathrm{mg} / \mathrm{kg}$ Q3W $\times 4$ doses followed by nivolumab $3 \mathrm{mg} / \mathrm{kg}$ Q2W until discontinuation. 16S ribosomal RNA sequencing was performed on baseline stool samples. Alpha diversity and differential measures of operational taxonomic units were evaluated at each taxa level. Microbiota differences were compared between patients with an investigator-assessed best overall response (BOR) of partial response (PR) vs those with a BOR of progressive disease (PD). Due to small patient numbers, those treated with nivolumab and nivolumab + ipilimumab were combined for the analysis.

Results

Baseline stool samples were collected from 72 patients. Among these patients, BORs of PR were achieved in 25, stable disease in 31, and PD in 14; 2 were not evaluable. Alpha diversity was not statistically different between patients with a BOR of PR vs those with PD. The abundance of Actinobacteria Micrococcaceae was 2.7-fold lower in patients with a BOR of PR compared with patients with a BOR of PD ( $P=0.0009$; Fig. 1); significant differences were also observed in the genus Rothia (2.7-fold lower in patients with a $P R ; P=0.0007)$. These results remained significantly different $(P=0.04)$ after corrections for false discovery rate. Analyses on additional microbiota will be presented.

\section{Conclusions}

Patients with dMMR/MSI-H CRC who achieved a BOR of PR with nivolumab \pm ipilimumab treatment were found to have a lower abundance of Actinobacteria Micrococcaceae, and in particular, the genus Rothia, compared with those with a BOR of PD. These results suggest that there may be differences in the gut microbiota of patients with $\mathrm{dMMR} / \mathrm{MSI}-\mathrm{H}$ CRC who respond to treatment with checkpoint inhibitors vs those who progress. Additional analyses investigating correlations with tumor immune phenotype and microenvironment, and other clinical parameters, are warranted.

\section{References}

1. Oke S, Martin A. Therap Adv Gastroenterol. 2017;10:417-428.

2. Gao R et al. Eur J Clin Microbiol Infect Dis. 2017; $36: 757-769$.

3. Sivan A et al. Science. 2015; 350:1084-1089.
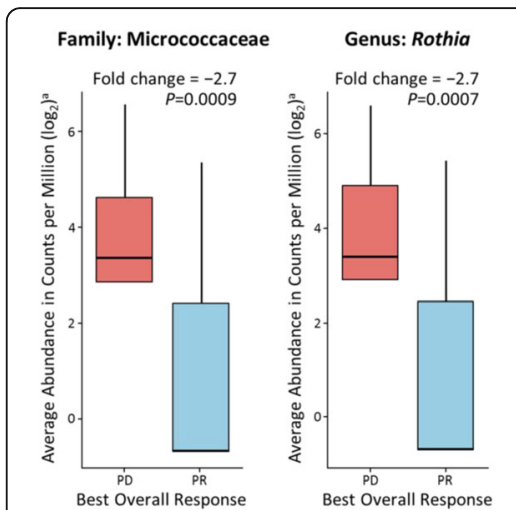

Species: Rothia mucilaginosa

The bold lines and boxes in each plot and the vertical lines denote the range.

Fig. 1 (abstract P392). See text for description

\section{P393}

Association of microbiome to nivolumab response in metastatic renal cell carcinoma ( $\mathrm{mRCC}$ )

Manuel Maia', Valeriy Poroyko ${ }^{1}$, Hae Won², Paulo Bergerot ${ }^{1}$,

Lorena Almeida', Nazli Dizman', JoAnn Hsu', Jeremy Jones²,

Ravi Salgia', Sumanta Pal ${ }^{1}$

${ }^{1}$ City of Hope Comprehensive Cancer Center, Duarte, CA, USA;

${ }^{2}$ Beckman Research Institute, City of Hope Comprehensive Cancer

Center, Duarte, CA, USA

Correspondence: Manuel Maia (mcaitano@coh.org)

Journal for ImmunoTherapy of Cancer 2017, 5(Suppl 2):P393

\section{Background}

Checkpoint inhibitors (CPI) have improved clinical outcomes in various cancer types, including mRCC [1]. Predictive factors of benefit from CPIs are not well defined. Relationship of the microbiome to benefit from CPIs has been suggested in preclinical studies [2]. Here we provide the first evidence of association between microbiome composition and response to nivolumab in mRCC patients (pts).

Methods

Stool samples were prospectively collected from pts with $\mathrm{mRCC}$ at 3 time points relative to nivolumab treatment (baseline, week 4 and week 12) and used to assess gut microbiota composition in two different groups: responders ( $R$; including complete/partial response and stable disease) and non-responders ( $P$; primary progression). In short, microbial DNA was extracted, 16s rRNA gene tags (v4) were generated by PCR amplification and sequenced using MiSeq (Illumina). Sequence reads were processed by Mothur software, assembled in operational taxonomic units (OTUs), taxonomically annotated to the level of genus and used to construct BrayCurtis dissimilarity matrices. The similarity of samples was visualized by principal coordinates analysis (PCoA) and further confirmed by k-means clustering $(k=2)$ and ANOSIM tests. Differentially abundant taxa were determined by METASTATS and compared between $R$ and $P$.

Results

Of 11 pts, 9 were evaluable for response. In this study, 25,304 OTUs were attributed to 165 genera from 8 phyla. PCoA analysis reveals that the first two principal coordinates can explain $49.2 \%$ of data set variation. Subsequent k-means clustering shows an almost complete separation of microbiota in $\mathrm{R}$ and $\mathrm{P}$ groups. The produced clusters almost perfectly aligned with $R$ and $P$ groups, although ANOSIM of this separation was not significant $(p=0.07)$. The analysis of microbiota membership in $\mathrm{P}$ and $\mathrm{R}$ groups revealed 4 differentially abundant taxonomic units at the genus level (Fig. 1), with 2 present above $1 \%$ abundance. Namely, Roseburia spp and Faecalibacterium spp were significantly elevated in $\mathrm{R}$ as compared to $\mathrm{P}(p<0.05)$. Notably, no significant differences were noted in Bifidobacterium spp or Bacteriodes spp in $\mathrm{R}$ and $\mathrm{P}$ ( $P=0.15$ for both). 


\section{Conclusions}

Our results are the first to associate specific microbial genera to nivolumab response in $\mathrm{mRCC}$ and warrant confirmation in larger series. Furthermore, manipulation of the stool microbiome as a means of modulating nivolumab response should be investigated.

\section{References}

1. Motzer RJ, et al. Nivolumab versus Everolimus in Advanced Renal-Cell Carcinoma. N Engl J Med.2015: 1803-1813.

2. Vetizou M,et al. Anticancer immunotherapy by CTLA-4 blockade relies on the gut microbiota. Science. 2015; 350: 1079-84.

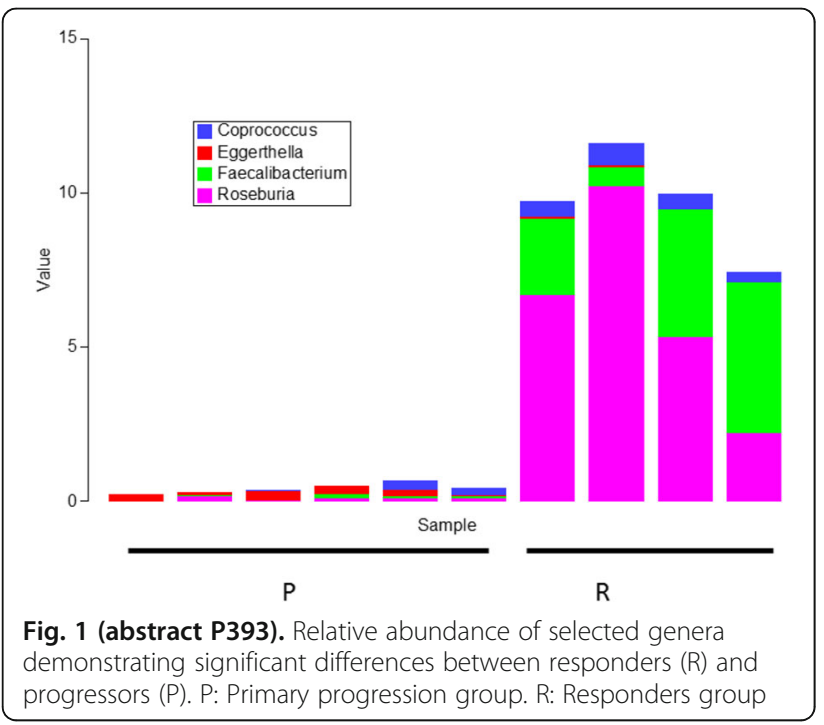

P394

Commensal microbiota associated with anti-PD-1 efficacy in metastatic melanoma patients

Vyara Matson, Jessica Fessler, Riyue Bao, Tara Chongsuwat, Yuanyuan Zha, Maria-Luisa Alegre, Jason Luke, Thomas Gajewski

University of Chicago, Chicago, IL, USA

Correspondence: Thomas Gajewski (vmatson@uchicago.edu)

Journal for ImmunoTherapy of Cancer 2017, 5(Suppl 2):P394

\section{Background}

Anti-PD-1-based immunotherapy is having a major impact on cancer outcomes, but only a subset of patients respond favorably. To predict response and ultimately improve therapeutic efficacy, it is of interest to identify variables that cause this inter-patient heterogeneity. Preclinically, commensal bacterial species have been demonstrated to modulate spontaneous anti-tumor immunity and the outcome of immunotherapy and chemotherapy in mice (1-3). Here, we examine the association between gut microbiota composition and response to anti-PD-1 immunotherapy in patients with melanoma.

\section{Methods}

Stool samples were collected from 42 metastatic melanoma patients prior to immunotherapy and microbial composition was analyzed through an integration of $16 \mathrm{~S}$ rRNA gene sequencing, metagenomic shotgun sequencing, and quantitative PCR for selected bacteria. Clinical response was determined using Response Evaluation Criteria In Solid Tumors (RECIST) version 1.1. Germ-free mice were colonized with stool from responding and non-responding patients, and tumor growth kinetics and anti-tumor immune responses were evaluated. Results

There were 16 responders and 26 non-responders. There was a significant association between the commensal microbiota composition and clinical response. Sixty-two operational taxonomic units (OTUs) assigned from the $16 \mathrm{~S}$ rRNA gene sequencing dataset were differentially abundant in responders relative to non-responders. Forty-three of these OTUs were assigned potential species-level identities, based on metagenomics sequencing of the same stool samples. Selected responder-associated species, including Bifidobacterium longum, Akkermansia muciniphila, Collinsella aerofaciens, and Enterococcus faecium were also confirmed by quantitative PCR. Reconstitution of germ-free mice with fecal material from a responding patient led to improved tumor control, augmented $\mathrm{T}$ cell responses, and a distinct microbiota compared to a non-responding patient.

Conclusions

Our results indicate a correlation between the abundance of certain gut bacteria and clinical response to anti-PD-1 immunotherapy in cancer patients, and suggest a mechanistic impact of the commensal microbiota on anti-tumor immunity

References

1.

Sivan A, Corrales L, Hubert N, et al. Commensal Bifidobacterium promotes antitumor immunity and facilitates anti-PD-L1 efficacy. Science. 2015; 350: 1084-1089.

2. Daillere $R$, Vétizou $M$, Waldschmitt $N$, et al. Enterococcus hirae and Barnesiella intestinihominis Facilitate Cyclophosphamide-Induced Therapeutic Immunomodulatory Effects. Immunity. 2016; 45: 931-943.

3. lida N, Dzutsev A, Stewart CA, et al. Commensal bacteria control cance response to therapy by modulating the tumor microenvironment. Science. 2013; $342:$ 967-970.

P395

DNA repair genetic aberrations and tumor mutation burden in biliary tract cancers

Reham Abdel-Wahab ${ }^{1,2}$, Rachna Shroff ${ }^{1}$, Jean Nicolas Vauthey ${ }^{1}$, Eugene Jon Koay ${ }^{1}$, Siraj $\mathrm{Ali}^{3}$, Lawrence Kwong ${ }^{1}$, Mingxin Zuo ${ }^{1}$,

Asif Rashid', Milind Javle

${ }^{1}$ MD Anderson Cancer Center, Houston, TX, USA; ${ }^{2}$ Assiut University

Hospital, Assiut Faculty of Medicine, Assiut, Egypt; ${ }^{3}$ Foundation Medicine, Cambridge, MA, USA

Correspondence: Milind Javle (raali@mdanderson.org)

Journal for ImmunoTherapy of Cancer 2017, 5(Suppl 2):P395

\section{Background}

Immune checkpoint inhibitors (ICPI) has been approved for several cancers. The association between tumor mutational burden (TMB) and ICPI has been previously studied and high TMB is associated with prolonged progression free survival (PFS) after ICPI. DNA repair gene aberrations (GAs) including those leading to microsatellite instability (MSI) is associated with response to ICPI. Data regarding this in biliary tract cancers (BTC) are limited.[1]

Methods

A comprehensive genomic profiling of 422 formalin-fixed, paraffinembedded tumor tissue from BTC patients, including 92 gall bladder cancer and 330 cholangiocarcinoma, was performed using next generation sequencing. We included 20 DNA repair genes that were previously described.[1] These included direct DNA repair genes (ATM, ATR BRCA1, BRCA2, FANCA, FANCD2, MLH1, MSH2, MSH6, PALB2, POLD1, POLE, $P R K D C, R A D 50, S L X 4)$ and caretaker genes that induce genomic instability (BAP1, CDK12, MLL3, TP53, BLM). TMB was calculated in 205 samples by counting the number of mutations across a $1.25 \mathrm{Mb}$ region and classified into three groups; low ( $<6$ mutation/Mb), intermediate (6 - 19 mutation/Mb), and high ( $\geq 20$ mutation/Mb). We assessed MSI status by a computational algorithm examining 114 intronic homopolymer loci.

Results

Direct DNA repair genes were identified in 61 BTC tumors (14.5\%) including GAs in ATM (5.5\%) or BRCA2 (2.1\%). Majority of the tumors had GAs in caretaker genes (42.9\%), predominately in TP53 (33.4\%), and BAP1 (9.5\%). However, FANCD2, POLD1, POLE, PRKDC, RAD50, $S L X 4, B L M$ mutations were not identified in our cohort. TMB was evaluated in 205 patients. Among patients with direct DNA repair GAs, 20 out of $35(57.2 \%)$ had intermediate or high TMB as compared with 47 out of $170(27.7 \%)$ patients without direct DNA repair GAs ( $P<$ .0001). Patients with caretaker GAs also had significantly higher TMB 
[intermediate and high (48.4\%)] than patients without these mutations $(P<.0001)$. MSI was determined in 88 patients and classified as stable, ambiguous, and high. All patients with MSI high had direct DNA repair GAs $(P<.0001)$.

\section{Conclusions}

DNA repair GAs occur at a relatively high frequency in BTC and are associated with higher TMB and MSI. Future clinical trials targeting this subgroup with ICPI are warranted.

\section{References}

1. Chae YK, Anker JF, Carneiro BA, Chandra S, Kaplan J, Kalyan A, SantaMaria CA, Platanias LC, Giles FJ. Genomic landscape of DNA repair genes in cancer. Oncotarget. 2016;7(17):23312-21.

\section{P396}

Evolving immune response in lung squamous carcinogenesis Celine Mascaux ${ }^{1}$, Mihaela Angelova², Angela Vasaturo ${ }^{2}$, Jennifer Beane ${ }^{3}$, Kahkeshan Hijazi ${ }^{4}$, Geraldine Anthoine ${ }^{5}$, Karen Willard-Gallo ${ }^{5}$ Annick Haller ${ }^{6}$, Vincent Ninane ${ }^{7}$, Arsène Burny ${ }^{5}$, Jean-Paul Sculier ${ }^{8}$, Avrum Spira9 ${ }^{9}$, Jérôme Galon²

${ }^{1}$ Marseille Early Phases Cancer Trials Center CLIP, Marseille, France; ${ }^{2}$ Laboratory of Integrative Cancer Immunology, Paris, France; ${ }^{3}$ Boston Univ. School of Medicine, Boston, MA, USA; ${ }^{4}$ Research Center for Modeling and Simulation, Islamabad, Pakistan; ${ }^{5}$ Institut Jules Bordet, Université Libre de Bruxelles, Brussels, Belgium; ' ${ }^{6}$ niversity Chidren's Hospital Queen Fabiola, Brussels, Belgium; ${ }^{7} \mathrm{CHU}$ Saint-Pierre - Service de Pneumologie, Brussels, Belgium; ${ }^{8}$ Institut Jules Bordet, Centre des Tumeurs de I'Université Libre de Bruxelles, Brussels, Belgium; ${ }^{9}$ Boston University Medical Center, Boston, MA, USA

Correspondence: Jérôme Galon (mihaela.angelova@crc.jussieu.fr) Journal for ImmunoTherapy of Cancer 2017, 5(Suppl 2):P396

\section{Background}

Molecular characterization of pre-invasive and invasive bronchial lesions can provide insights into the mechanisms behind genesis and development of squamous cell carcinoma, revealing potentially promising new biomarkers for early detection and treatment of lung cancer. Methods

At 8 different morphological stages of lung squamous carcinogenesis, from normal, to low grades dysplasia, high grades dysplasia, to carcinoma, fresh frozen human bronchial biopsies $(n=122)$ were subjected to gene expression profiling (Agilent microarrays) from 77 patients. A linear mixed-effects model was applied to infer modules of gene co-expression, adjusted for smoking status, gender, and cancer history as fixed effects, and patient as a random effect. Immune cell-specific gene signatures were used to characterize the immune response through the different development stages.

Results

The gene expression alterations could distinguish four groups of successive stages of development. There were eight modules of co-expressed genes with different expression patterns across the stages: up- or downregulation among the early or late steps, linear increase or decrease in expression, and a biphasic pattern. Transitioning to early grade, there was a depletion of immune gene expression. However, at the transition to high-grade dysplasia, there was a significant up-regulation of immune/inflammatory response genes, representing $\mathrm{T}$ helper 1 , myeloid cells, as well as co-inhibitory and co-stimulatory molecules.

\section{Conclusions}

At a critical stage of carcinogenesis there is a significant modification of the immune response. The up-regulation of immune genes in high-grade suggests a major role of the surrounding microenvironment thorough adaptive and innate immune response, but also a tumor versus host immunosuppressive response before tumor invasion across the basal membrane. This analysis may identify promising new immune signatures of carcinogenesis, and may provide novel therapeutic targets for lung cancer.

\section{P397}

Withdrawn
P398

Tumor mutation load assessment of FFPE samples using an NGS based assay

Ruchi Chaudhary', Asha Kamat ${ }^{1}$, Warren Tom¹, Iris Casuga', Dinesh Cyanam²

Dumitru Brinza', Janice Au-Young ${ }^{1}$, Fiona Hyland ${ }^{1}$

${ }^{1}$ Thermofisher Scientific, South San Francisco, CA, USA; ${ }^{2}$ Thermofisher Scientific, Ann Arbor, MI, USA

Correspondence: Asha Kamat (asha.kamat@thermofisher.com); Ruchi Chaudhary

Journal for ImmunoTherapy of Cancer 2017, 5(Suppl 2):P398

\section{Background}

Background - Tumors with high mutation load respond to therapy with immune checkpoint inhibitors. Hence high mutation load of a tumor can act as a predictive biomarker to immuno-therapy. We developed an lon S5 Sequencing System assay to determine the mutation status of solid tumors.

Methods

Methods: Our assay uses an FFPE sample, utilizing an Ampliseq based target panel that covers $1.7 \mathrm{Mb}$ of genomic DNA including 409 genes. The assay does not require matched normal sample to estimate the mutation status of a tumor.

Results

Results: Our panel exhibits $>90 \%$ sensitivity and $>95 \%$ specificity in distinguishing between high and low mutation burden samples. Using a variety of public annotation sources, we have successfully eliminated approximately $98 \%$ of germline variants. Matched tumor-normal analyses on 9 lung and colorectal samples suggested that our single sample analysis on tumor samples detects mutation load with strong correlation ( $r=0.9358$ ) with tumor-normal analysis. On engineered control from Acrometix, mutation load count consistently comprise true mutations with positive predictive value of $89 \%$. To assess reproducibility, we compared the mutation load in library replicates for a cohort of 10 samples (FFPE and fresh frozen tumors, Acrometrix control, and NIST cell-lines) and observed high correlation ( $r=0.9948)$.

Conclusions

We have developed a single tumor-only assay for accurate estimation of tumor mutation burden, useful for cancer immuno-therapy research and development.

P399

Rationally improving $\mathrm{T}$ cell-mediated cancer immunotherapy using Sleeping Beauty mutagenesis to identify novel drug targets

Laura Rogers, Adam Dupuy, George Weiner

University of lowa, lowa City, IA, USA

Correspondence: Laura Rogers (laura-rogers@uiowa.edu)

Journal for ImmunoTherapy of Cancer 2017, 5(Suppl 2):P399

\section{Background}

T cells have amazing potential to eliminate tumor cells throughout the body, evidenced by the clinical success of immunotherapies designed to enhance $T$ cell function like immune checkpoint blockade and chimeric antigen receptor $T$ cell (CAR-T) therapies. Expanding success to a broader number of patients is a top priority in the field. T cell infiltration into tumors appears to be an important prerequisite for the success of both immune checkpoint blockade and CAR-T therapies. Thus, increasing $\mathrm{T}$ cell infiltration into tumors could have beneficial therapeutic impact.

Methods

We have designed a forward genetic screen to identify $T$ cell genes that contribute to intratumoral T cell accumulation using Sleeping Beauty (SB) transposon insertional mutagenesis in T cells. Our systematic screen approach allows us to evaluate both loss- and gain-of-function mutations across the entire genome in immunocompetent melanoma and lymphoma models that preserve the complexity of the tumor microenvironment. Importantly, the vast majority of genes we identified were not previously known to be involved in tumor-associated T cell biology, and likely would not have been considered as drug targets to enhance immunotherapy. These genes have the potential to modify 
important $\mathrm{T}$ cell functions including trafficking to the tumor, clonal expansion, and sustained viability once inside the tumor.

Results

We identified 312 tumor-enriched genes that were mutated in tumors, but not the spleen, of individual mice. Twenty of these were detected in more than one mouse, representing strong gene candidates for validation. We demonstrate that one gene candidate is functionally associated with $\mathrm{T}$ cell response to activation signals. Specifically, CRISPR-mediated knockout of this gene in a murine T cell line resulted in cytokine production defects after $\mathrm{T}$ cell receptor stimulation. Additional screening has begun using other tumor models and anti-PD-1 therapy. Preliminary data from these indicate that few genes are conserved across tumor models and treatment groups.

\section{Conclusions}

We are currently investigating the role of candidate genes in intratumoral $\mathrm{T}$ cell accumulation and immunotherapy enhancement using an in vivo approach. Together, these experiments have the potential to expand our understanding of $\mathrm{T}$ cell infiltration into tumors and may provide previously unexplored strategies to rationally enhance immunotherapy efficacy.

\section{P400}

Evaluation of tumor genetics and microenvironment through next-generation sequencing (NGS)

Alex So, Shannon Kaplan, Joyee Yao, Shile Zhang, Aaron Wise, Kristina Kruglyak, Naomi O'Grady, Jeremiah Mcdole, Marina Bibikova Illumina, San Diego, CA, USA

Correspondence: Alex So (alexsoas@gmail.com)

Journal for ImmunoTherapy of Cancer 2017, 5(Suppl 2):P400

\section{Background}

The effectiveness of cancer immunotherapy is determined by the genetic basis of the patient's tumor as well as the state of the tumor microenvironment. For instance, tumor mutation burden (TMB) and microsatellite instability (MSI) status correlate with patient response to checkpoint inhibitors [1]. Also, the presence of immune cells in the tumor microenvironment correlates with immunotherapy responsiveness. Thus, this project aims to develop a next-generation sequencing (NGS) workflow that reports various biomarkers that can be used to guide cancer immunotherapy selection. Here we present our data on using whole exome sequencing (WES) and whole transcriptome sequencing (WTS) to report human leukocyte antigen (HLA) sequence, TMB load, prioritized neoantigens, and presence of immune cells. Methods

WES and WTS libraries were generated using Illumina's TruSeq ${ }^{\circledR}$ Exome and RNA Access library preparation kits, respectively. The samples were paired-end sequenced (2x75bp) using $\mathrm{HiSeq}^{\oplus} 2000$ and 2500 instruments.

Results

Here we demonstrate a workflow using tumor/normal WES and tumoronly WTS to determine MSI status and expressed TMB load. We also show data that WES can be used to haplotype HLA Class I genes with 97\% accuracy. Using the HLA haplotype sequence, we computationally predicted putative neoantigens and experimentally validated a subset, as true binders to their respective major histocompatibility complex. In addition, we applied FRICTION, a novel algorithm for cell deconvolution, to WTS data extracted from bulk tumor to quantify the fraction of immune cells present in the tumor microenvironment. Our titration experiments demonstrated our method's linearity in quantifying $\mathrm{CD} 4^{+} \mathrm{T}$ cells, $\mathrm{CD} 8^{+} \mathrm{T}$ cells, and $\mathrm{CD} 19^{+} \mathrm{B}$ cells in a variety of tissue backgrounds (median $R^{2}>0.97$ ). Finally, we applied FRICTION to WTS data from melanoma samples extracted from different subjects and showed that our workflow could also quantify the fraction of immune cells in these samples when compared to quantitation by flow cytometry.

\section{Conclusions}

In summary, we demonstrate the utility of a NGS workflow that uses WES and WTS to report biomarkers relevant to immunotherapy and characterize the inflammation status of the tumor microenvironment.
References

1. Rizvi, NA, et al. Mutational landscape determines sensitivity to PD-1 blockade in non-small cell lung cancer. Science. 2016;348:1-13.

\section{Oncolytic Viruses and Intratumoral Therapies}

P401

Intratumoral expression of IL12 using the ZVex ${ }^{\circledast}$ dendritic celltargeting lentiviral vector exerts potent anti-tumor effects via induction of multiple immune effectors, including CD8 T cell responses

Tina C. Albershardt, Jardin Leleux, Rebecca S. Reeves, Jan ter Meulen, Peter Berglund

Immune Design, Seattle, WA, USA

Correspondence: Tina C. Albershardt

(tina.albershardt@immunedesign.com)

Journal for ImmunoTherapy of Cancer 2017, 5(Suppl 2):P401

\section{Background}

Interleukin 12 (IL12), produced by antigen-presenting cells, plays a pivotal role in the interplay between the innate and adaptive arms of the immune system. IL12 treatment has been shown to augment cytotoxic $T$ lymphocyte and $T_{H} 1$ responses and anti-tumor effects. However, its use as a systemic therapeutic agent is limited due to toxicity. Intratumoral administration of IL12 is therefore being explored as an alternative route. We have previously reported on the strong anti-tumor effects of IL12 delivered intratumorally using Immune Design's dendritic cell-targeting lentiviral platform ZVex in various murine tumor models. Here, we evaluated the mechanism of action mediating the local and systemic immune responses induced by intratumoral ZVex/IL12 in different animal models.

Methods

Tumor cell lines (B16 melanoma, CT26 colon carcinoma, 4T1 breast cancer, A20 lymphoma, P815 mastocytoma, GL261 glioma) were implanted subcutaneously into mice. ZVex was engineered to express murine IL12 and administered as a single intratumoral injection into palpable tumors $\left(50-100 \mathrm{~mm}^{3}\right)$, typically at 7 days post-tumor inoculation. Mice were monitored twice weekly for tumor growth and survival. In lymphocyte depletion studies, anti-CD8, anti-CD4 or antiNK1.1 antibodies were administered twice weekly, and their effect on immediate and long-term anti-tumor response was studied.

Results

A single intratumoral injection of ZVex/mLL12 induced complete tumor regression in B16, CT26, A20, GL261, and P815 models, delayed tumor growth in the $4 \mathrm{~T} 1$ model. In mice depleted of either CD8 T, CD4 T, or NK cells, complete tumor regression was still observed in $13-40 \%$ of mice. However, only depletion of CD8 T cells together with CD4 T and/or NK cells completely abrogated the anti-tumor response, suggesting that CD8 $\mathrm{T}$ cells are required but not sufficient effectors in ZVex/mlL12-mediated anti-tumor control. Furthermore, depletion of CD4 T cells resulted in very pronounced vitiligo, demonstrating that intratumoral ZVex/mlL12 profoundly breaks self-tolerance, likely in the absence of regulatory $T$ cells. Conclusions

Taken together, the results demonstrate that intratumoral administration of IL12 induces complex protective cellular immune responses, requiring CD8 effector T cells for maximum efficacy.

\section{P402}

Chemotherapy enhances oncolytic recombinant poliovirus efficacy Jeffrey Bryant ${ }^{1}$, Michael Brown ${ }^{2}$, Matthias Gromeier ${ }^{2}$

${ }^{1}$ Duke University, Durham, NC, USA; ' ${ }^{2}$ Duke University Medical Center, Durham, NC, USA

Correspondence: Jeffrey Bryant (jeffrey.bryant@duke.edu); Matthias Gromeier

Journal for ImmunoTherapy of Cancer 2017, 5(Suppl 2):P402

\section{Background}

Oncolytic viruses are capable of direct tumor lysis along with the ability to activated pro-inflammatory programs that may contribute to the recruitment of adaptive immune effector responses. We use 
a recombinant poliovirus:rhinovirus hybrid, PVSRIPO, which has tropism for neoplastic cells, due to the ectopic expression of the poliovirus receptor, CD155, and a reliance on oncogenic signaling to initiate viral protein translation. PVSRIPO is currently in Phase-I/II clinical trials against recurrent glioblastoma multiforme (GBM). The first patients treated with PVSRIPO are radiographically tumor-free and clinically normal $>52$ months after single intratumoral infusion of PVSRIPO. On May $10^{\text {th }}, 2016$, the FDA/CBER granted Breakthrough Therapy Designation to PVSRIPO. A co-incidental finding emerging from our ongoing trial is remarkable durable, complete responses in patients upon treatment with chemotherapy following tumor progression after PVSRIPO administration.

Methods

This study used syngeneic mouse tumor models (TRAMPC2 and CT2A) to test the same chemotherapy combination used in the clinic. Both the clinical trial and mouse study use chemotherapy doses that induce severe lymphotoxic side effects which have been suggested to augment antitumor immunity when combined with an immunotherapy regimen. To mirror what is done in the clinic the established mouse tumors receive a single injection of PVSRIPO followed a week later by a single round of the alkylating chemotherapeutic drug Temozolomide (TMZ). Tumor growth and end point immune cells populations were monitored.

Results

This study demonstrates that this PVSRIPO TMZ combination can be tested in syngeneic mouse models resulting in both an increase in survival and a delay in tumor growth over both PVSRIPO and TMZ treatment alone. Our data also suggests that this combination induces an increase in memory and infiltrating Treg cell populations.

Conclusions

Ongoing work is being done to further validate these finding in multiple mouse tumor models including intra-cerebral brain tumors models and to determine the mechanisms contributing to the synergy seen resulting from the PVSRIPO and chemotherapy combination. These intriguing results suggest that the memory $T$ cells seen after PVSRIPO chemotherapy combination may be responsible for the new anti-tumor immune response. However, chemotherapy also induced an increased tumor infiltrating inhibitory Treg cells. Future work will look at ways to enhance this positive memory $T$ cell population while preventing intratumoral Treg cell accumulation.

\section{P403}

Intratumoral administration of a multigene construct by electroporation can effectively modulate anti-tumor response in a murine B16.F10 model

Shawna Shirley ${ }^{1}$, Anandaroop Mukhopadhyay ${ }^{1}$, Christoph Burkart ${ }^{1}$,

Jocelyn Wright ${ }^{1}$, Robert Pierce ${ }^{2}$, Jean Campbell ${ }^{2}$, David Canton'

${ }^{1}$ Oncosec Medical Incorporated, San Diego, CA, USA; ${ }^{2}$ Fred Hutchinson Cancer Research Center, Seattle, WA, USA

Correspondence: David Canton (dcanton@oncosec.com)

Journal for ImmunoTherapy of Cancer 2017, 5(Suppl 2):P403

\section{Background}

Immunomodulatory cytokines, such as interleukin-12 (IL-12) are attractive candidates for cancer immunotherapy. IL-12 is a pro-inflammatory cytokine with potent anti-tumor effects; however, systemic administration of IL-12 shows limited clinical efficacy and dose-associated toxicity. In preclinical and clinical studies, intratumoral (IT) delivery of IL-12 plasmid DNA by electroporation (EP) can provide a safe and effective alternative for efficacious dosing. To augment the effects of IL-12, we developed a DNA plasmid platform that allows for delivery of agents that modulate multiple immune pathways as well as tumor- or patientspecific neoantigens. Polycistronic IL-12 Immune Modulator plasmid (PIIM) is a single plasmid encoding IL-12, and a fusion of Flt3L to an antigen. Flt3L is a ligand that stimulates dendritic cell (DC) maturation and enhances antigen processing and presentation. The encoded antigen can be a viral or shared antigen, or a patient-specific neoantigen, which enables customization to patient populations, as well as providing an aid to monitoring antigen-specific immune response(s) that can be correlated to patient outcomes. Here we demonstrate the first functional characterization of PIIM

Methods

Two PIIM constructs were created for functional characterization: PIIMOVA (IL12 Flt3L-OVA) for mouse experiments, and PIIM-NYESO1 (IL12 Flt3L-NYESO1) for testing in human cells. The expression and functional activity of PIIM components were determined. Treated tumors and spleens were assessed for transcriptional changes by NanoString ${ }^{\circledR}$ and phenotypic changes by flow cytometry. Systemic effects of PIIM were assessed using a syngeneic two-tumor model of B16.F10 in which only one tumor received IT-PIIM-EP while the other contralateral lesion remained untreated.

Results

PIIM-OVA and PIIM-NYESO1 secrete functional IL-12p70, FIt3L-OVA and Flt3L-NYESO1 fusion proteins as assessed by ELISA, flow and cell-based assays. PIIM promotes DC maturation and antigen-specific T cell proliferation both ex vivo and in vivo. Hydrodynamic-based gene delivery of PIIM-OVA lead to splenomegaly and significantly increased splenic CD11c+ DCs. Furthermore, IT-PIIM-EP lead to generation of splenic OVA-specific CD8s and increased APM gene expression. When introduced intratumorally in a mouse two-tumor model, IT-PIIM-EP delays B16.F10 tumor growth in both treated and contralateral tumors compared to untreated controls resulting in increased overall survival.

Conclusions

PIIM represents a novel approach to cancer immunotherapy. A combination of functional immune modulators can be expressed locally in the tumor microenvironment that increase inflammatory infiltrate, enhances antigen presentation and produces a systemic $T$ cell response specific to the antigen encoded on the plasmid. This customizable approach has the potential to improve therapeutic outcome by enhancing adaptive-immunity and addressing patientspecific neoantigens needs.

P404

Targeting T-cells to human cancer associated fibroblasts using an oncolytic virus expressing a FAP-specific T-cell engager

Brian Champion', Joshua Freedman', Rebecca Ashfield ${ }^{2}$, Margaret Duffy ${ }^{2}$ Alice Brown 1 , Leonard Seymour ${ }^{2}$, Kerry Fisher ${ }^{2}$

${ }^{1}$ PsiOxus Therapeutics Ltd, Abingdon, United Kingdom; ${ }^{2}$ Oxford

University, Oxford, United Kingdom

Correspondence: Brian Champion (brian.champion@psioxus.com)

Journal for ImmunoTherapy of Cancer 2017, 5(Suppl 2):P404

\section{Background}

Enadenotucirev (EnAd) is a chimeric oncolytic group B adenovirus with potent and selective anti-tumor activity against a range of epithelial cancer cells, with a blood stability profile that enables systemic dosing and has been administered intravenously to over 100 cancer patients. As an approach to immunogene therapy targeting stromal rich tumors, we have created transgene-modified variants of EnAd expressing a bispecific T-cell engager (BiTE) molecule recognizing human fibroblast activating protein (FAP) on cancer associated fibroblasts (CAFs) and CD3 on T-cells. CAFs play a pivotal role in the development of solid carcinomas by facilitating invasion, coordinating angiogenesis and establishing and maintaining an immune suppressive microenvironment in the tumor stromal tissue. We hypothesize that production of BiTE proteins by virus infected tumor cells could be an effective way of modifying the stromal microenvironment to drive effective anti-tumor immunity and would bypass delivery and safety issues related to systemic dosing of BiTE proteins.

Methods

Bi-specific T-cell engager constructs comprising linked ScFv antibodies specific for human FAP and CD3 were designed and used to generate EnAd viruses expressing the BiTE such that transgene expression was under the control of either a CMV promoter or the virus major late promoter to allow broad or tumor-selective expression, respectively. Tumor and fibroblast cell lines, together with control BiTE proteins and BiTE-encoding viruses, were used to evaluate initial activities of the FAP-BiTE viruses prior to testing with freshly isolated malignant peritoneal ascites from patients with advanced cancer. 
Results

We have shown that the viruses selectively express and secrete functional FAP-specific BiTEs following infection of tumor cell lines. In co-culture with FAP+ fibroblasts and PBMC-derived T cells this leads to $T$ cell-activation and cytotoxicity towards the fibroblasts. Similarly, infection of primary malignant cancer ascites with FAP-BiTE-encoding viruses led to polyclonal activation of endogenous T-cells and depletion of FAP+ cells from the cultures. Unlike activation of T-cells with anti-CD3/CD28, FAP-BiTE activation was also shown to be effective even in the presence of immunosuppressive (cell-free) ascitic fluid samples added to the cultures.

\section{Conclusions}

In this study, we have shown that CAFs can be effectively targeted for T-cell mediated destruction by a FAP-BiTE transgene-bearing oncolytic virus, with an associated strong activation of endogenous T-cells to kill endogenous CAFs even in the presence of an immunosuppressive microenvironment. Systemic dosing of such a virus to patients with stromal rich tumors may provide an effective approach for driving effective anti-tumor immunity.

\section{P405}

Harnessing pre-existing antiviral immunity to treat solid tumors Nicolas Cuburu', Rina Kim', Sergio Pontejo ${ }^{2}$, Cynthia Thompson" Douglas Lowy', John Schiller,

${ }^{1}$ National Cancer Institute, $\mathrm{NIH}$, Bethesda, MD, USA; ${ }^{2}$ National Institute of Allergy and Infectious Diseases, $\mathrm{NIH}$, Bethesda, MD, USA; ${ }^{3} \mathrm{NCI} / \mathrm{NIH}$, Bethesda, MD, USA

Correspondence: Nicolas Cuburu (cuburun@mail.nih.gov); John Schiller Journal for ImmunoTherapy of Cancer 2017, 5(Suppl 2):P405

\section{Background}

Cancer immunotherapy rely on adaptive immune responses against tumor-associated antigens. The diversity of tumor antigens makes the development of cancer vaccines a highly personalized endeavor. Epitope spreading results from the induction of de novo responses against tumor antigens not initially included in vaccines [[1]. Intratumoral therapies also require to induce de novo responses against tumor antigen for abscopal therapeutic effect.

Human cytomegalovirus is highly prevalent in humans with polyfunctional $T$ cell responses expanding with age [2]. We questioned whether redirecting pre-existing anti-cytomegalovirus $\mathrm{T}$ cells into solid tumor could arrest tumor growth, induce epitope spreading, and confer long-term anti-tumor immunity?

\section{Methods}

Persistently infected mice with mouse cytomegalovirus (MCMV) were challenged with TC-1 tumor cells expressing human papillomavirus (HPV) E6 and E7 oncogenes. We generated HPV pseudovirons expressing MCMV antigens or peptides derived from minimal MCMV epitopes. These reagents were injected intratumorally together with the poly $(I: C)$, as adjuvant. We monitored tumor growth, survival and measured antitumor $\mathrm{CD}^{+} \mathrm{T}$ cell responses against an E7 immunodominant epitope using MHC-I tetramer and intracellular cytokine stainings. In rechallenge experiments, we evaluated the induction of long-term anti-tumor immunity. Using PanCancerlmmune panel (Nanostring), we analyzed the tumor immune microenvironment after treatment.

Results

Transduction of TC-1 tumors with HPV pseudovirions expressing MCMV antigens or peptidic MCMV epitopes caused the expansion of MCMVspecific $\mathrm{CD}^{+}$and $\mathrm{CD}^{+} \mathrm{T}$ cells. These treatments also caused broad modifications of the tumor immune environement. Intratumoral injection of MCMV CD8 epitopes provoked the arrest of tumor growth. Intratumoral injection of MCMV CD4 epitopes with poly(l:C) promoted induction of E7-specific CD8 ${ }^{+} \mathrm{T}$ cells. Sequential administration of CD4 and CD8 MCMV epitopes together with poly $(1: C)$ was the best protocol to eradicate pre-existing tumor of 5 to $10 \mathrm{~mm}^{3}$, and rechallenge experiments showed anti-tumor immunity up to 4 months after the last treatment.

\section{Conclusions}

Our results provide a proof of concept to design "antigen-agnostic" intratumoral therapies based on pre-existing antiviral T cells. Such approach change the tumor immune microenvironment, induce epitope spreading, and confer long term anti-tumor immunity. These findings prompt further evaluation in other spontaneous tumor models and provide a model to decipher the mechanisms of epitope spreading and the role of CD4 help.

\section{References}

1. Gulley $J \mathrm{~L}$, et al. Immune impact induced by PROSTVAC (PSA-TRICOM), a therapeutic vaccine for prostate cancer. Cancer Immunol Res. 2014;2(2):133-41.

2. O'Hara GA, et al. Memory T cell inflation: understanding cause and effect. Trends Immunol. 2012;33(2):84-90.

P406

Dissecting the mechanisms underlying antitumor effects of live oncolytic vaccinia and heat-inactivated vaccinia

Weiyi Wang, Peihong Dai, Ning Yang, Stewart Shuman, Taha Merghoub, Jedd Wolchok, Liang Deng

Memorial Sloan Kettering Cancer Center, New York, NY, USA

Correspondence: Liang Deng (dengl@mskcc.org)

Journal for ImmunoTherapy of Cancer 2017, 5(Suppl 2):P406

\section{Background}

Preclinical and clinical studies have shown that viral-based immunotherapy has the potential to overcome resistance to immune checkpoint blockade and to fill the unmet needs of many cancer patients. Oncolytic viruses are defined as engineered or naturally occurring viruses that selectively replicate in and kill cancer cells. Oncolytic viruses also induce antitumor immunity. We recently showed that intratumoral (IT) delivery of inactivated modified vaccinia virus Ankara (iMVA) induces antitumor systemic immunity via the STINGmediated cytosolic DNA-sensing pathway and Batf3-dependent CD103 ${ }^{+} / \mathrm{CD} 8 \mathrm{a}^{+}$dendritic cells (DCs). The combination of IT iMVA and systemic delivery of immune checkpoint blockade is highly effective in treating large established tumors and distant metastasis [1]. MVA is non-replicative in tumor cells, and therefore whether or not viral replication is necessary for antitumor effects of IT oncolytic DNA virus is unknown.

Methods

In the study, we engineered a replication-competent, attenuated vaccinia virus (VC) by removing the Z-DNA-binding domain of vaccinia virulence gene $E 3$ and inserting murine GM-CSF gene into the thymidine kinase (TK) locus (E3L $\Delta 83 \mathrm{~N}-\mathrm{TK}^{-}-\mathrm{mGM}-\mathrm{CSF}$ ). We compared antitumor effects of live VC and VVC in murine tumor models.

Results

We found that IT Heat-inactivated VC (iVC; by heating the live virus at $55^{\circ} \mathrm{C}$ for $1 \mathrm{~h}$ ) is more effective in generating systemic antitumor immunity than live VC murine tumor models. The antitumor effects of live VC also requires Batf3-dependent DCs. IT HeatiVC induces higher numbers of infiltrating activated $\mathrm{CD}^{+}$and $\mathrm{CD}^{+} \mathrm{T}$ cells in the non-injected tumors than live $\mathrm{VC}$ and is more potent in depleting tumor-associated macrophages than live VC in both injected and non-injected distant tumors. The surviving mice treated with Heat-iVC are more effective in rejecting tumor rechallenge than those treated with live-VC, indicating that IT Heat-iVC generates stronger antitumor memory $T$ cell responses than live VC.

Conclusions

In conclusion, our results support the use the IT delivery of inactivated vaccinia virus as a safe and effective cancer immunotherapy either alone or in combination with immune checkpoint blockade antibodies.

\section{References}

1. Dai P, Wang W, Serna-Tamayo C, Ricca JM, Zamarin D, Shuman S. Merghoub T, Wolchok JD, and Deng L. Intratumoral delivery of inactivated modified vaccinia virus Ankara (iMVA) induces systemic antitumor immunity via STING and Batf3-dependent DC. Science Immunology. 2017;2: eaal1713. 
P407

In situ vaccination for cancer immunotherapy: treat locally, respond systemically

Nicole Steinmetz ${ }^{1}$, P. Jack Hoopes², Mee Ree Sheen², Chenkai Mao²,

Scott Sieg ${ }^{1}$, Steven Fiering ${ }^{2}$, Patrick :Lizotte ${ }^{2}$, Amy Wen $^{3}$

${ }^{1}$ Case Western Reserve University, Cleveland, NH, USA; ${ }^{2}$ Geisel School of

Medicine at Dartmouth, Lebanon, NH, USA; ${ }^{3}$ Case Western Reserve

University, Cleveland, OH, USA

Correspondence: Steven Fiering (fiering@dartmouth.edu)

Journal for ImmunoTherapy of Cancer 2017, 5(Suppl 2):P407

\section{Background}

Immunotherapy for cancer is making impressive impacts in the clinic One strategy dating back to William Coley in 1900 is in situ vaccination. This approach puts immunostimulatory reagents into an identified tumor to break the local immunosuppression, stimulate a local anti-tumor response and most importantly stimulate systemic antitumor immune responses to eliminate metastatic disease. This is essentially an antitumor therapeutic vaccination, because the tumor provides the antigens and the adjuvants are the immunostimulatory reagents, thus "in situ vaccination". The approach has been part of standard of care for superficial bladder cancer using BCG bacteria. There are many immunostimulatory reagents that can be used and each has different capabilities. We have done previous studies with attenuated microorganisms including Toxoplasma gondii, and Listeria monocytogenes.

Methods

Our recent focus has been on a plant virus that cannot infect animals, compea mosaic virus (CPMV). Complete CPMV or CPMV lacking nucleic acids (eCPMV) are grown in plants and biochemically purified.

Results

CPMV nanoparticles were used in mouse cancer models and community dogs with oral melanoma and other tumors. These particles are composed of assembled viral capsid proteins. eCPMV has no nucleic acids and no recognized immunostimulatory reagents. However, eCPMV is strongly immunostimulatory through unknown pathways and causes changes in the tumor microenvironment that lead to primary tumor reduction or elimination and potent resistance to metastatic tumors (1). The treatment is immune-mediated but response in the lungs requires different immune components than response in flank tumors of the same B16F10 melanoma cell line. Tumor reduction or elimination occurs in many anatomic locations with multiple tumor types and in multiple strains of inbred mice. Treatment of primary tumors by direct intratumoral injection mediates robust rejection of a rechallenge with the same tumor. Response in community dogs was highly effective at both eliminating the local tumor and resisting metastatic disease. The mechanisms and pathways of immunostimulation are under investigation.

\section{Conclusions}

In situ vaccination has considerable potential for clinical use. In addition to the inherent immunostimulatory adjuvant properties of CPMV, they are a versatile platform to which other reagents for immune modulation can be attached. This demonstration of the value of select viral-like nanoparticles for treatment of cancer opens a new avenue of cancer immunotherapy.

\section{References}

1. Lizotte PH, Wen AM, Sheen MR, Fields J, Rojanasopondist P, Steinmetz NF, Fiering S. In situ vaccination with cowpea mosaic virus nanoparticles suppresses metastatic cancer. Nat Nanotechnol. 2016;11:295-303.

\section{P408}

Tumor cell-intrinsic STING signaling and regulation of IFN- $\beta$ gene expression

Blake Flood ${ }^{1}$, Leticia Corrales ${ }^{2}$, Thomas Gajewski

${ }^{1}$ University of Chicago, Chicago, IL, USA; ${ }^{2}$ Aduro Biotech, Chicago, IL, USA

Correspondence: Blake Flood (blakeflood@uchicago.edu)

Journal for ImmunoTherapy of Cancer 2017, 5(Suppl 2):P408

\section{Background}

Our laboratory has previously shown that immunogenic tumors spontaneously activate the innate immune system through the STING pathway. The STING pathway senses cytosolic DNA, which activates a signal transduction pathway culminating in phosphorylated IRF3 that translocates to the nucleus where it acts as a transcription factor to induce several genes including IFN- $\beta$. STING signaling and IFN- $\beta$ receptor signaling in tumor-infiltrating immune cells, in turn, are required for optimal priming of CD8+ T cells against tumor antigens. Based on this notion, STING agonists were developed and tested as a pharmacologic approach to activate the pathway.

Methods

We stimulated various cell populations present in the tumor microenvironment as well as several tumor cell lines with STING agonists to test their ability produce IFN- $\beta$ and analyze each step in STING pathway signaling.

Results

We observe that tumor cells themselves frequently fail to produce IFN- $\beta$ in response to STING agonists or cytoplasmic DNA, arguing that loss of activation of this pathway might occur regularly as a component of oncogenesis. Surprisingly, we find that tumor cells retain expression of each gene in the STING pathway and STING signal transduction is intact up to and including nuclear translocation of IRF3. ChIP assays demonstrate IRF3 is unable to bind the IFN- $\beta$ promoter but can still bind the promoters of other genes.

Conclusions

These data indicate tumor cells fail to express IFN- $\beta$ following STING pathway activation due to a defect in transcription factor binding to the IFN locus. Based on ChIP experiments the defect in IRF3 DNA binding may be specific to the IFN- $\beta$ locus. Sequencing of the locus shows no mutations in or around the binding site so we are currently investigating epigenetic mechanisms regulating IFN- $\beta$ gene expression.

\section{P409}

Efficacious anti-melanoma immunity induced by OX40 ligandexpressing oncolytic adenovirus Delta-24-RGDOX

Hong Jiang, Andrew Dong, Caroline Carrillo, Verlene Henry, Xuejun Fan, Yisel Rivera-Molina, Francisco Puerta-Martinez, Teresa Nguyen, Karen Clise-Dwyer, Frederick Lang, Candelaria Gomez-Manzano, Juan Fueyo The University of Texas MD Anderson Cancer Center, Houston, TX, USA Correspondence: Hong Jiang (hjiang@mdanderson.org) Journal for ImmunoTherapy of Cancer 2017, 5(Suppl 2):P409

\section{Background}

Oncolytic adenoviruses are highly immunogenic and cancer-selective but show limited efficacy as a single agent. Immune checkpoint modulation has shown efficacy in a variety of cancers but is associated with nonspecific T-cell activation, and has had a limited effect in tumors with a nonimmunogenic microenvironment.Thus, combining these two strategies likely resulted in both efficacious and specific cancer therapy. To this end, we constructed the oncolytic adenovirus Delta-24-RGDOX which expressed the immune co-stimulator OX40L. This new virus induces a superior immunotherapeutic effect in immunocompetent mouse glioma models than its predecessor Delta-24-RGDOX.

Methods

In a s.c./s.c. melanoma mouse model with B16F10-Red-FLuc cells, Delta-24-RGDOX and/or anti-CD47 antibody were injected into the first implanted tumor. The tumor growth of the first and second implanted tumors was monitored with bioluninescence. The survival curves were plotted according to the Kaplan-Meier method. Survival rates in the different treatment groups were compared using the log-rank test. The immune cells in the tumor were isolated and analyzed with flow cytometry.

Results

Delta-24-RGDOX expressed OX40L effectively in mouse melanoma cells. Compared to treatment with PBS, four doses of intratumoral injection of the virus significantly inhibited the growth of both the injected tumors and the untreated distant tumors, resulting in prolonged survival of the mice with $40 \%$ long-term survival $(P=0.01)$. The surviving mice is resistant to rechallenging with the same tumor cells but is 
susceptible to lung cancer cells, suggesting the development of immune memory specific to the virus-injected tumor type. Through flow cytometry analysis of the tumor-infiltrating lymphocytes, we found the virus injection increased the presence of $C D 3+T$ lymphocytes, $C D 3$ $+C D 4+$ helper $T$ cells and CD3+CD8+ cytotoxic $T$ cells in the tumor, causing a decreased ratio of $C D 4+/ C D 8+$ cells. To further increase the efficacy of Delta-24-RGDOX, we combined the virus with intratumoral injection of an antibody against CD47, a "don't eat me" signal overexpressed on tumor cell surface to protect them from phagocytosis. We found high expression levels of CD47 in cultured B16 cells and the tumor cells from the xenografts. Intratumoral injection of Delta-24RGDOX caused much more phagocytes presented at the tumor site than PBS treatment. Combination of anti-CD47 antibody with Delta-24RGDOX mediated longer survival time than the virus alone (median survival: 56 days vs. 26 days)

\section{Conclusions}

Delta-24-RGDOX induced efficacious local and systemic anti-melanoma immunity in B16-C57BL/6 mouse model and combination of the virus with anti-CD47 antibody further increased the therapeutic efficacy.

\section{P410}

Generation of therapeutic RNAs to induce immunogenic cell death and interferon expression in cancer cells

Jaewoo Lee' ${ }^{1}$ Youngju Lee ${ }^{2}$

${ }^{1}$ Duke University Medical Center, Durham, NC, USA; ${ }^{2}$ Duke University, Durham, NC, USA

Correspondence: Jaewoo Lee (jaewoo.lee@duke.edu)

Journal for ImmunoTherapy of Cancer 2017, 5(Suppl 2):P410

\section{Background}

Pattern-recognition receptors (PRRs) are immunological sensors that initiate the host defense response against infections. They are located at the cell surface, within endosomal compartments and in the cytoplasm, where they are poised to recognize different molecular signatures associated with invading pathogens. In addition to anti-infectious immunity, the activation of RNA-sensing PRRs can mediate programmed cell death of infected cells, which allows the host to efficiently block viral replication by sacrificing infected cells. Transfection with certain types of RNA ligands that stimulate RIG-I-like receptor (RLR) family can induce type I interferon (IFN) and immunogenic cell death (ICD) in cancer cells, which together orchestrate anti-tumor immune responses that can prevent tumor recurrence and metastasis. Currently RNA-sensing PRR agonists have demonstrated little or no overall benefit to patients with cancers because of, mostly but not exclusively, toxicity driven by nonspecific induction of immune reactions. In this study, we generate and screen multiple nuclease-resistant RNA molecules that can differentially induce immunogenic cancer cell death with or without concomitant expression of IFN- $\beta$, pro-inflammatory cytokines and pro-coagulation mediators.

\section{Methods}

Therapeutic RNAs are designed using mFold RNA structure prediction program and generated by in vitro transcription. All RNAs contain 5 'triphosphate and 2'fluoro pyrimidine that are recognized by RLRs and resistant to nucleases. Immunogenic cell death in human melanoma, pancreatic cancer and prostate cancer cells and PBMCs was analyzed by western blot, ELISA and Flow cytometry. In vivo anti-tumor effects of the RNAs were induced by intratumoral transfection with RNAs into immunocompromised mice bearing human melanoma. Results

We generated two novel and distinct ssRNA molecules (Immunogenic Cell- killing RNA (ICR)2 and ICR4). ICR2 and ICR4 differentially stimulated cell death and PRR signaling pathways and induced different patterns of cytokine expression in cancer and innate immune cells. Interestingly, damage-associated molecular patterns (DAMPs) released from ICR2- and ICR4-treated cancer cells had distinct patterns of stimulation of innate immune receptors and coagulation. Finally, ICR2 and ICR4 inhibited in vivo tumor growth as effectively as polyl:C. ICR2 and ICR4 are potential therapeutic agents that differentially induce cell death, immune stimulation and coagulation when introduced into tumors.

\section{Conclusions}

ICR2 and ICR4 are potent PRR-stimulating cytotoxic agents against human cancers. ICR2 and ICR4 have distinctive immune stimulatory and coagulating activities. Combination of dual checkpoint inhibitors and ICR4 and/or ICR2 would be a potent and effective anti-cancer therapies against advanced cancers.

P411

Immunotherapy with BO-112, a novel double-stranded RNA-based agent, promotes tumor cell death and boosts $\mathrm{T}$ cell immunity in preclinical mouse models

Lourdes Planelles', Angela Aznar², Majosé Gómez-Sánchez'

Mercedes Pérez-Olivares ${ }^{1}$, Saray Garasa², Mercedes Pozuelo ${ }^{1}$,

Ivan Márquez-Rodas ${ }^{3}$, Marisol Quintero', Ignacio Melero ${ }^{2}$

${ }^{1}$ Bloncotech Therapeutics, Valencia, Spain; ${ }^{2}$ Center for Applied Medical

Research (CIMA) \& University of Navarra, Pamplona, Spain; ${ }^{3}$ Hospital

General Universitario Gregorio Marañón, Madrid, Spain

Correspondence: Lourdes Planelles (Iplanelles@bioncotech.com)

Journal for ImmunoTherapy of Cancer 2017, 5(Suppl 2):P411

\section{Background}

Unresponsive or refractory tumors are the main research objective in cancer immunotherapy. Strategies that promote immunogenic tumor cell death in the context of type I interferon (IFN) signaling can be combined effectively with existing therapies that target checkpoint inhibitors to boost anti-tumor immunity. BO-112 is a double-stranded synthetic RNA currently undergoing phase I clinical trials for intratumor injection. To enhance local antitumor immunity, BO-112 bears poly I:C chains that trigger endosomal Toll-like receptor 3 and cytosolic helicases.

Methods

B16F10 and B16F10-OVA melanoma, MC38 colon carcinoma or 4T1 breast carcinoma cells $\left(5 \times 10^{5}\right)$ were implanted subcutaneously in the right flank of $\mathrm{C} 57 \mathrm{BL} / 6 \mathrm{~J}$ and $\mathrm{BALB} / \mathrm{C}$ mice; a second tumor was engrafted in the left flank to evaluate distant antitumor effects. Beginning 7-10 days post-implant, mice were treated intratumorally with BO-112 only in the primary lesion. Therapeutic efficacy was evaluated by monitoring tumor growth and survival, and cellular responses were analyzed by multiparametric flow cytometry. Antigen-specific CD8+ T cell in vivo priming was addressed by $\mathrm{H}-2 \mathrm{~K}^{\mathrm{b}}$ tetramer analysis in B16F10-OVA tumors. The role of CD4+ or CD8+ T cells was studied by depletion and depletion/rechallenge experiments. Combined BO-112 and anti-PDL1 (programmed death-ligand 1) antibody therapy was evaluated in established B16F10 melanomas.

Results

In vitro and in vivo studies showed that BO-112 triggered cell death in tumor cells of distinct tissue origins (melanoma, colon or breast carcinoma). The cytotoxic effect was also detected in engineered B16 cells that do not respond to type I IFN. Intratumor BO-112 administration in all three mouse tumor models showed therapeutic effects, with complete tumor regression in up to $30 \%$ of mice. FACS analyses of tumor-derived cell suspensions showed that BO-112 increased the tumor immune infiltrate and CD8+ T cell frequency. Moreover, the receptors PD1 and CD137, which denote activation, were upregulated on CD8+ T cells. BO-112 treatment enhanced intratumor OVAspecific CD8+ $T$ cells, and depletion and rechallenge experiments confirmed CD8+ T cell involvement in its immunotherapeutic effects. A combination of BO-112 and anti-PD-L1 antibody significantly improved the poor response to PD-L1 blockade in B16F10 and B16F10OVA tumor-bearing mice. Responses were observed in treated and in untreated distant tumors, which provides a rationale for clinical testing of this combination.

\section{Conclusions}

These data identify local BO-112 release as a new immunotherapy agent beneficial in the treatment of refractory and recurrent tumors, especially when combined with checkpoint inhibitors. A phase Ib clinical trial is being designed to address this question.

Trial Registration

http://ClinicalTrials.gov identifier, NCT02828098. 


\section{P412}

Antitumor immunity in patients with locally advanced soft tissue sarcoma treated with hafnium oxide nanoparticles and radiation therapy

Jérôme Galon ${ }^{1}$, Marick Laé ${ }^{2}$, Juliette Thariat ${ }^{3}$, Sébastien Carrere ${ }^{4}$ Zsuzsanna Papai ${ }^{5}$, Martine Delannes ${ }^{6}$, Philippe Rochaix ${ }^{6}$, László Mangel $^{7}$, Fabienne Hermitte ${ }^{8}$, Zoltán Sapi ${ }^{9}$, Tamas Tornoczky ${ }^{7}$, Vincent Servois ${ }^{2}$, Isabelle Birtwisle Peyrottes ${ }^{3}$, Raphaël Tetreau ${ }^{4}$, Marie-Christine Château ${ }^{4}$ Sébastien Paris ${ }^{10}$, Hervé Brisse ${ }^{2}$, Sylvie Bonvalot ${ }^{2}$

${ }^{1}$ INSERM, Paris, France; ${ }^{2}$ Institut Curie, Paris, France; ${ }^{3}$ Centre Antoine Lacassagne, Nice, France; ${ }^{4}$ Centre Régional De Lutte Contre Le Cancer

Paul Lamarque, Montpellier, France; ${ }^{5}$ Medical Centre, Hungarian Defence Forces, Budapest, Hungary; ${ }^{6}$ Institut Universitaire du Cancer Toulouse Oncopole, Toulouse, France; ${ }^{7}$ Pecs University, Pecs, Hungary; ${ }^{8}$ HalioDX, Marseille, France; ${ }^{9}$ Semmelweis University, Budapest, Hungary;

${ }^{10}$ Nanobiotix, Paris, France

Correspondence: Sébastien Paris (agnes.pottier@nanobiotix.com) Journal for ImmunoTherapy of Cancer 2017, 5(Suppl 2):P412

\section{Background}

Soft tissue sarcoma (STS) is a large and heterogeneous group of malignant mesenchymal neoplasms characterized by a strong tendency toward local recurrence and metastatic spreading. Consistently, the immune microenvironment in sarcomas is highly variable. A new class of material with high electron density, hafnium oxide, was designed at the nanoscale to efficiently absorb ionizing radiation from within the tumor cells and augment the dose deposited to a tumor. These nanoparticles ( $\mathrm{HfO}_{2}-\mathrm{NP}$ ) administered in a single intratumor injection and activated by fractionated radiotherapy are evaluated in a phase II/III trial in patients with locally advanced STS as neoadjuvant treatment. Besides, beyond the broadly cytotoxic effect of radiation therapy (RT), RT may promote the release of tumor neoantigens during cancer cell death and stimulate local immunological effects.

Here, we explore the effects of nanosized hafnium oxide exposed to RT in terms of tumor immune profile changes in patients with STS when compared to RT alone.

Methods

Tumor tissues pre- (biopsy) and post-treatment (resection) are collected from patients with locally advanced STS (NCT02379845), who received either $\mathrm{HfO}_{2}$-NP activated by RT or RT alone. Immunohistochemistry and Digital Pathology for immune biomarkers and Pan-Immune gene expression profiling are analyzed.

Results

A significant increase of CD8+ T cells and a marked increase of CD3+ and PD-1 T cells and CD103+ immune cell infiltration post- vs pretreatment are observed for $\mathrm{HfO}_{2}-\mathrm{NP}+\mathrm{RT}$ while no differences are seen for RT alone (more than 10 patients analyzed in each arm). Functional analysis of genes expression up-regulated in $\mathrm{HfO}_{2}-\mathrm{NP}+\mathrm{RT}$ post- vs pretreatment shows an enrichment of cytokine activity (IL7, IFNA, IL11, IFNG), adaptive immunity (RAG1, TAP1, TAP2, TBX21, IFNG, LTK, CD37, CD22) and T cell receptor signaling pathway (CD28, CTLA4, CD274, BTLA, TIGIT, CD5, ZAP70) when compared to RT.

Conclusions

Promising results are observed in patients who received $\mathrm{HfO}_{2}-\mathrm{NP}+$ $\mathrm{RT}$ in terms of immune cells infiltration post- vs pre-treatment when compared to RT. Moreover, $\mathrm{HfO}_{2}-\mathrm{NP}+\mathrm{RT}$ induces a specific adaptive immune pattern. So far, nanosized hafnium oxide exposed to RT bring substantial changes to the tumor immune profile in patients with STS when compared to RT. As such, it may convert immunologically "cold" tumor into "hot" tumor and be effectively combined with immunotherapeutic agents across oncology. More tissue samples are under evaluation to reinforce these findings.

\section{P413}

Transforming immunologically "cold" tumor into "hot" tumor with hafnium oxide nanoparticles and radiation therapy Sébastien Paris, Audrey Darmon, Ping Zhang, Maxime Bergère, Laurent Levy Nanobiotix, Paris, France

Correspondence: Sébastien Paris (agnes.pottier@nanobiotix.com) Journal for ImmunoTherapy of Cancer 2017, 5(Suppl 2):P413

\section{Background}

Hafnium oxide, an electron-dense material, was designed at the nanoscale to increase the radiation dose deposited from within the cancer cells: "Hot spot" of energy deposit where the nanoparticles are when exposed to radiation therapy (RT). Preclinical studies have demonstrated increase of cancer cells killing in vitro and marked antitumor efficacy in vivo with presence of these nanoparticles $\left(\mathrm{HfO}_{2}-\mathrm{NP}\right)$ exposed to RT, when compared to RT alone. $\mathrm{HfO}_{2}-\mathrm{NP}$ is intended for a single intratumor injection and is currently evaluated in clinical trials including soft tissue sarcoma, head and neck, prostate, liver and rectum cancers.

Here, we explore the ability of nanosized hafnium oxide exposed to RT to bring substantial immune cells infiltrations in the tumors and convert immunologically "cold" tumor into "hot" tumor.

\section{Methods}

CT26 (murine colorectal cancer cells) were subcutaneously injected in the flank of BALB/c mice. Once the mean tumors volume reached 115 $\pm 30 \mathrm{~mm}^{3}$, tumors were intratumor injected with $\mathrm{HfO}_{2}-\mathrm{NP}$ and irradiated with $2 \mathrm{~Gy} \times 3$ or $4 \mathrm{Gyx} 3$, or irradiated only. Tumors were collected 5 days after the last RT fraction and analyzed for immune cell infiltrates by immunohistochemistry (2Gyx3 and 4Gyx3) and cytokines content by flow cytometry (2Gyx3).

A second study evaluated $\mathrm{HfO}_{2}-\mathrm{NP}$ exposed to RT vs RT alone using the 4T1 murine breast cancer model. Cells treated or not with $\mathrm{HfO}_{2}$-NP were exposed to irradiation (40Gy). Irradiated cells $\left(1.10^{6}\right)$ (or phosphate-buffered saline as control) were inoculated subcutaneously into the flank of BALB/C mice (vaccination phase). Seven days after, mice were challenged with untreated $4 \mathrm{~T} 1$ cells $\left(1.10^{6}\right)$ (challenge phase). Grown tumors (challenge site) were collected 19 days after the challenge phase and analyzed for immune cell infiltrates by immunohistochemistry.

Results

In mice bearing CT26 tumors, a marked increase of cytokines content and immune cell infiltrates was observed with $\mathrm{HfO}_{2}-\mathrm{NP}+2 \mathrm{Gyx} 3$ when compared to RT alone. The tumor immune cell infiltrates were further enhanced with $\mathrm{HfO}_{2}-\mathrm{NP}+4 \mathrm{Gyx} 3$.

In mice inoculated with $4 \mathrm{~T} 1$ cells treated with $\mathrm{HfO}_{2}-\mathrm{NP}+40 \mathrm{~Gy}$, a marked increase of immune cell infiltrate (CD8+) was observed in tumors when compared to tumors in mice inoculated with 4T1 cells treated with 40Gy and control.

\section{Conclusions}

These in vivo data generated from $\mathrm{CT} 26$ and $4 \mathrm{~T} 1$ tumor models suggest that $\mathrm{HfO}_{2}-\mathrm{NP}+\mathrm{RT}$ triggers immunogenic conversion of the tumor microenvironement when compared to $\mathrm{RT}$ alone. $\mathrm{HfO}_{2}-\mathrm{NP}$ treatment may represent a therapeutical approach for broad applications since it does not rely on any molecular characteristics of the tumor.

\section{P414}

PVSRIPO is an interferon-resistant, immunotherapeutic oncolytic

virus

Ross Walton, Michael Brown, Eda Holl, David Boczkowski, Vidya

Chandramohan, Smita Nair, Matthias Gromeier

Duke University, Durham, NC, USA

Correspondence: Ross Walton (rww7@duke.edu)

Journal for ImmunoTherapy of Cancer 2017, 5(Suppl 2):P414

\section{Background}

Oncolytic viruses are attractive cancer therapies because they selectively lyse cancer cells, but show attenuation in normal cells. Many oncolytic virus are attenuated through susceptibility to innate antiviral immune responses, like Type I Interferon (IFN) responses. Until recently it was believed that cancer cells lack these responses. However, our lab and others have shown cancer cells can have intact IFN responses which inhibit IFN-susceptible viruses, but may activate potent anti-cancer immunity. Innate immunity in cancer is thus an area of extreme interest.

Our lab developed an oncolytic human rhinovirus:poliovirus chimera, PVSRIPO, which is in clinical trial against glioblastoma multiforme, an aggressive form for brain cancer. Using PVSRIPO, and the related picornavirus encephalomyocarditis virus (EMCV), we investigated MDA5 
and IFNs in infection of cancer cells. MDA5 is a pattern recognition receptor, targeted to viral double-stranded RNA produced by picornaviruses. We also investigated PVSRIPO's role in anti-tumor immune responses after treatment of cancer cells.

Methods

DM440, a human melanoma cell line, was engineered with stable MDA5 knock-down by lentivirus transduction containing MDA5targeting small-hairpin RNA. PVSRIPO or EMCV was added at a multiplicity of infection (MOI) of 0.1 or 0.01 and samples collected for immunoblot, ELISA, or viral titer. Cells were also incubated with IFN-a2 for 24 hours, infected, and assayed as above. Human dendritic cells (DCs) were infected at 1, 10,50, or $100 \mathrm{MOI}$ and collected as above. DCs were also treated with oncolysate from PVSRIPO-infected SUM149 human breast cancer cells, non-infectious lysate, or virusfiltered lysate. DCs were analyzed by flow cytometry or used for a cytotoxic HLA-matched T-cell assay using europium-labeled target cells, including cancer cells, tumor antigen transfected DCs, and control antigen expressing DCs.

Results

DM440 cells produce and respond to IFNs in an MDA5-dependent manner after PVSRIPO and EMCV infection. MDA5 activation and IFN treatment inhibits EMCV replication significantly more than PVSRIPO replication. Human DCs can be productively infected and activated with PVSRIPO without toxicity. Oncolysate activates human DCs in a virus dependent manner, and DCs activated in this way promote cytotoxic T-cell activation against tumor-specific antigen.

Conclusions

PVSRIPO is an IFN-resistant oncolytic virus that provokes MDA5dependent IFN responses in cancer cells, without significant viral inhibition. Further, PVSRIPO infects DCs, activating them without significant cytotoxicity, which can lead to tumor-specific cytotoxic T-cell responses, and may lead to antigen spreading. It is possible this potent DC activation phenotype is due to PVSRIPO's resistance to IFNs.

\section{P415}

Upregulation of PD-L1 in tumor microenvironment is a resistance mechanism for oncolytic virus immunotherapy

Dmitriy Zamarin, Jacob Ricca, Anton Oseledchyk, Mathieu Gigoux,

Taha Merghoub, Jedd Wolchok

Memorial Sloan Kettering Cancer Center, New York, NY, USA

Correspondence: Dmitriy Zamarin (zamarind@mskcc.org)

Journal for ImmunoTherapy of Cancer 2017, 5(Suppl 2):P415

\section{Background}

Intralesional therapy with oncolytic viruses (OV) leads to activation of multiple immune pathways such as type I IFN. Both OV and OVactivated type I IFN pathway can exert a variety of pleiotropic effects on both immune and non-immune cells, activating resistance to the immune system on both local and abscopal level. Identification of such pathways could provide insights into the mechanisms of OV-mediated T cell activation as well as generate rationale for combinatorial strategies. Methods

Using Newcastle Disease Virus (NDV) as a model OV, we explored the effect of intratumoral OV therapy on the microenvironment of the treated and distant tumors in syngeneic bilateral flank tumor models, and explored combination therapies using intratumoral NDV with systemic immune checkpoint blockade.

Results

Intratumoral therapy with NDV led to upregulation of a range of activating and inhibitory immune targets in the treated and distant tumors. While NDV therapy shifted the balance from exhausted to effector T cell phenotype in distant tumors, it was not sufficient for complete tumor rejection. We further demonstrate that infection with NDV leads to upregulation of PD-L1, an effect that is mediated early through paracrine action of type I IFN. Consistent with these findings, intratumoral therapy with NDV or IFNa results in upregulation of PD-L1 on both tumor cells and tumor-infiltrating lymphocytes in the treated tumors. In contrast to NDV, intratumoral IFNa therapy had no effect on late PD-L1 expression, immune cell infiltration, or growth of distant tumors, highlighting that late PD-L1 upregulation is likely a reflection of adaptive immune resistance to the increased tumor infiltrating lymphocytes. Systemic therapeutic targeting of PD-1 or PD-L1 in combination with intratumoral NDV resulted in rejection of both treated and distant tumors. Anti-tumor efficacy was fully dependent on CD8 cells and on early presence of NK cells.

Conclusions

These findings provide implications for timing of PD-1/PD-L1 blockade in conjunction with OV therapy and highlight that understanding of adaptive mechanisms of immune resistance to specific OV will be important for rational design of appropriate combinatorial approaches with these agents.

\section{Other}

P416

Anti-tumor effects and immunological response following immune stimulating interstitial laser thermotherapy

Jakob Axelsson, Cristina Pantaleone

Clinical Laserthermia Systems AB, Lund, Sweden

Correspondence: Jakob Axelsson (jakob@clinicallaser.se)

Journal for ImmunoTherapy of Cancer 2017, 5(Suppl 2):P416

\section{Background}

Immune stimulating interstitial laser thermotherapy (imILT) is a local ablation modality designed for local destruction of tumor tissue as well as to optimize systemic immunologically mediated anti-cancer effects. Safety and feasibility in the clinic has been studied and previous rodent data show absopal effects. This study expands on the mechanisms behind these immunological effects.

Methods

An imlLT treatment protocol was developed and a syngenic mouse tumor model was established. Mice were inoculated subcutaneously with tumor cells and tumor growth was monitored. When tumors reached adequate size imILT treatment was performed and subsequently another tumor was inoculated and tumor progression was measured. In another set of experiments, two tumors were inoculated simultaneously (twin tumor model) and one of them was treated at appropriate size. Tumor progression of both tumors were monitored. Immune infiltrate and morphology of all tumors were studied.

Results

Preliminary data show both abscopal effects in the twin tumor model as indicated as reduced growth of the non-treated tumor of the imILT treated mice as compared to the control mice. Memory effects can also be demonstrated in the first set of experiment as seen as reduced growth and take of tumor inoculation in mice which have previously been receiving imILT as compared to control mice.

Conclusions

Taken together these data suggest immunologically mediated effects are evoked following imILT treatment. This new information will be useful for deducing the mechanisms of action involved in these effects.

P417

Post-marketing safety of checkpoint inhibitors: analysis of the FDA adverse event reporting system

Rawad Elias ${ }^{1}$, Jennifer Rider ${ }^{2}$, Osama Rahma

${ }^{1}$ Boston University Medical Center, Boston, MA, USA; ${ }^{2}$ Boston University School of Public Health, Boston, MA, USA; ${ }^{3}$ Dana-Farber Cancer Institute,

Boston, MA, USA

Correspondence: Rawad Elias (rawadelias@hotmail.com)

Journal for ImmunoTherapy of Cancer 2017, 5(Suppl 2):P417

\section{Background}

Information about the toxicity profile of immune checkpoint Inhibitors (ICls) is limited. Available data primarily comes from clinical trials. However, subjects enrolled in clinical trials are usually fitter than patients typically seen in clinical practice and therefore may have a different toxicity profile. 


\section{Methods}

We reviewed data from the FDA Adverse Event Reporting System (FAERS) summarizing adverse events (AEs) associated with the PD-1 inhibitors (nivolumab and pembrolizumab); PD-L1 inhibitor (atezolizumab); and CTLA-4 inhibitor (ipilimumab). We restricted our analysis to reports that included only an $\mathrm{ICl}$ as suspect agent. For each agent, we performed a descriptive analysis of hospitalization (HO) and death (DE) outcomes, as well as AEs of special interest (AESI). We compared the distribution of each outcome within age groups (<65 years; 67-75; >75) using MantelHaenszel chi -quare test for trend.

Results

Our analysis included a total of 21,588 safety reports. 415 for atezolizumab, 10,026 for nivolumab, 4,808 for pembrolizumab, and 6,339 for ipilimumab (Table 1). There was a statistically significant trend of more hospitalization with increasing age for all drugs. Prevalence of any of the AESI was higher as age increased for all drugs $(p<0.0001)$ except for atezolizumab ( $p$ 0.12). However, no statistically significant trend by age was found for any individual AESI (data not shown). Proportion of older patients who experienced death was higher for pembrolizumab $(p<0.001)$ and ipilimumab ( $p$ 0.002)

Conclusions

Our analysis suggests that older patients receiving pembrolizumab, Nivolumab or ipilimumab are more likely to develop immune related AEs, and to be hospitalized. This resulted in higher rate of deaths only in pembrolizumab and ipilimumab. The smaller sample size of the patients receiving atezolizumab may have contributed to the lack of statistical significance. Older patients treated with $\mathrm{ICls}$ should be monitored carefully for treatment-related AEs. Toxicity of ICls should be further evaluated prospectively in the setting of trials in older adults that would take in consideration impact of physiologic and immune aging.

Table 1 (abstract P417). See text for description

\begin{tabular}{|c|c|c|c|c|c|}
\hline & \multicolumn{5}{|c|}{ Atezolizumab } \\
\hline & Overall & $<65 y$ & $65-75 y$ & $>75 y$ & \begin{tabular}{|l|l} 
Pvalue \\
\end{tabular} \\
\hline & $\mathrm{N}=415$ & $\mathrm{~N}=273$ & $\mathrm{~N}=101$ & $\mathrm{~N}=41$ & \\
\hline Hospitalization, N (\%) & $231(56)$ & $124(45)$ & $80(79)$ & $27(66)$ & $<0.0001$ \\
\hline Death, $\mathrm{N}(\%)$ & $85(20)$ & $56(21)$ & $18(18)$ & $111(27)$ & 0.64 \\
\hline \multirow{4}{*}{ Any AE, N (\%) } & $87(21)$ & $52(19)$ & $23(23)$ & $12(29)$ & 0.12 \\
\hline & \multicolumn{5}{|c|}{ Nivolumab } \\
\hline & Overall & $<65 y$ & $65-75 y$ & $>75 y$ & $p$ value \\
\hline & $\mathrm{N}=10026$ & $\mathrm{~N}=7342$ & $\mathrm{~N}=1896$ & $\mathrm{~N}=788$ & \\
\hline Hospitalization, $\mathrm{N}(\%)$ & $3028(30)$ & $1677(23)$ & $970(51)$ & $381(48)$ & $<0.0001$ \\
\hline Death, N (\%) & $3837(38)$ & $2905(40)$ & $670(35)$ & $262(33)$ & $<0.0001$ \\
\hline \multirow{4}{*}{ Any AE, N (\%) } & $1888(19)$ & $1187(16)$ & $456(24)$ & $245(31)$ & $<0.0001$ \\
\hline & \multicolumn{5}{|c|}{ Pembrolizumab } \\
\hline & Overall & $<65 y$ & $65-75 y$ & $>75 y$ & $p$ value \\
\hline & $\mathrm{N}=4808$ & $\mathrm{~N}=3319$ & $\mathrm{~N}=947$ & $\mathrm{~N}=542$ & \\
\hline Hospitalization, N (\%) & $1482(31)$ & $855(26)$ & $386(41)$ & $241(44)$ & $<0.0001$ \\
\hline Death, $N(\%)$ & $1518(32)$ & $970(29)$ & $359(38)$ & $289(35)$ & $<0.0001$ \\
\hline \multirow[t]{4}{*}{ Any AE, N (\%) } & $740(15)$ & $445(13)$ & $174(18)$ & $121(22)$ & $<0.001$ \\
\hline & \multicolumn{5}{|c|}{ Combined PD-1 \& PD-L1 inhibitors } \\
\hline & Overall & $<65 y$ & $65-75 y$ & $>75 y$ & $p$ value \\
\hline & $\mathrm{N}=15249$ & $\mathrm{~N}=11004$ & $\mathrm{~N}=2890$ & $\mathrm{~N}=1355$ & \\
\hline Hospitalization, N (\%) & $4621(30)$ & $2589(24)$ & $1393(48)$ & $639(47)$ & $<0.0001$ \\
\hline Death, N (\%) & 5403 (35) & 3906 (36) & $1039(36)$ & $458(34)$ & 0.44 \\
\hline \multirow[t]{4}{*}{ Any AE, N (\%) } & 2674 (18) & 1656 (15) & $644(22)$ & $374(27)$ & $<0.0001$ \\
\hline & \multicolumn{5}{|c|}{ Ipilimumab } \\
\hline & Overall n (\%) & $<65$ & $65-75$ & $>75$ & $p$ value \\
\hline & $\mathrm{N}=6339$ & $\mathrm{~N}=4697$ & $\mathrm{~N}=1120$ & $\mathrm{~N}=522$ & \\
\hline Hospitalization, $\mathrm{N}(\%)$ & $2401(38)$ & $1477(31)$ & 645 (58) & $279(53)$ & $<0.0001$ \\
\hline Death, $\mathrm{N}(\%)$ & $1421(22)$ & $1021(22)$ & $250(22)$ & $150(29)$ & 0.002 \\
\hline Any AE, N (\%) & $1844(29)$ & $1258(27)$ & $407(36)$ & $179(34)$ & $<0.0001$ \\
\hline
\end{tabular}

P418

Bromodomain and extraterminal region inhibitors slow melanoma tumor growth by altering the tumor microenvironment

Dan A. Erkes ${ }^{1}$, Claudia Capparelli', Shea A. Heilman', Timothy J. Purwin',

Adam C. Berger', Michael A. Davies², Andrew E. Aplin

${ }^{1}$ Thomas Jefferson University, philadephia, PA, USA; ${ }^{2}$ MD Anderson

Cancer Center, houston, TX, USA

Correspondence: Andrew E. Aplin (dan.erkes@jefferson.edu)

Journal for ImmunoTherapy of Cancer 2017, 5(Suppl 2):P418

\section{Background}

Melanoma is the deadliest form of skin cancer, yielding a 2-16\% survival rate for metastatic patients. Despite the onset of new successful therapies, i.e. targeted inhibitors and immunotherapy, there is a large need for drugs that alter the entire tumor microenvironment. To this end, we aim to study drugs that have widespread tumor microenvironment effects, such as epigenetic modifiers, i.e. bromodomain and extra terminal domain inhibitors (BETi), which slow tumor growth in a variety of cancers and effect inflammatory responses. Thus, BETis potentially represent a novel treatment for metastatic melanoma, as they can target tumor cell growth and anti-tumor immune mechanisms.

Methods

Several mouse and human BRAF ${ }^{\mathrm{V} 600 \mathrm{E}}$ melanoma cell lines, immune competent mouse models, and a patient-dreived xenograft model were used to uncover the effects of BETi treatment on melanoma.

Results

Here we show that the BETi PLX51107 was able to differentially impact $\mathrm{BRAF}^{\mathrm{V} 600 \mathrm{E}}$ melanoma tumor growth in a number of mouse and human melanoma cell lines in vitro and in vivo. PLX51107 induced cell cycle arrest and cell death of melanoma cell lines by increasing Bim and decreasing Cyclin B1 in mouse and human lines. Using immune competent mouse models, BETi treatment altered the immunogenicity of the microenvironment of responsive melanoma tumors by lowering the expression of anti-inflammatory PD-L1 and FasL, while increasing pro-inflammatory MHC-I. This was also seen in mouse and human melanoma cell lines and cancer-associated fibroblasts. PLX51107 treatment differentially altered tumor infiltrating lymphocyte (TIL) populations in mouse melanoma models, increasing activated, proliferating, and functional intratumoral CD8+ T cells and leading to a CD8+ T cell mediated tumor growth delay in highly responsive tumors. However, in moderately or poorly responsive tumors, these CD8+ T cell populations were unchanged or decreased after BETi treatment. As BETi treatment was a successful primary therapy, it was prudent to determine its efficacy as a second line therapy. In this vein, an immune compotent mouse model and patient-derived xenograft model were used to demonstrate BETi efficacy on anti-PD-1 resistant tumors. BETi treatment was able to delay the growth of antiPD-1 resistant tumors, by increasing intratumoral CD8+ T cells and pushing tumors to a more responsive IPRES signature.

Conclusions

As hypothesized, BETi treatment had wide-ranging effects on melanoma tumors by altering the tumor cells themselves, the microenvironment, and anti-tumor immunity to induce robust tumor growth delay. From these findings, BETis represent a potential primary or secondary treatment for metastatic melanoma patients.

P419

Tobacco use, awareness and cessation among Malayali tribes, Yelagiri Hills, Tamil nadu, India

Delfin lovelina Francis (delfin_lovelina@yahoo.co.in)

Dr MGR Medical University, Chennai, India

Journal for ImmunoTherapy of Cancer 2017, 5(Suppl 2):P419

Background

Health is a state of complete wellbeing free from any discomfort and pain. Despite remarkable world-wide progress in the field of diagnostic, curative and preventive medicine, still there are large populations of people living in isolation in natural and unpolluted surroundings far away from civilisation, maintaining their traditional 
values, customs, beliefs and myths. India has the second largest tribal population of the world next to the African countries. About half of the world's autochthonous people live in India, thus making India home to many tribes which have an interesting and varied history of origins, customs and social practices. The present study was conducted to assess the tobacco use, awarness and its effect on health among Malayali tribes, Yelagiri Hills, Tamil nadu, India.

Methods

The inhabitants of the 14 villages of the Yelagiri hills, who have completed 18years and residing for more than 15years present on the day of examination and who were willing to participate in the study were included.

Data was collected from a cross-sectional survey, using a Survey Proforma, clinical examinationand a pre-tested questionnaire which included Demographic data, tobacco habits. An intra-oral examination was carried out by a single examiner to assess the Oral Health Status using WHO Oral Health Surveys - Basic Methods Proforma (1997).SPSS version 15 was used for statistical analysis.

Results

Results showed that among 660 study population, 381(57.7\%) had no formal education. Among the study population $75 \%$ ) had the habit of alcohol consumption. Of those who had the habit of smoking, $26 \%$ smoked beedi, $10.9 \%$ smoked cigarette, $65 \%$ chewed raw tobacco, $18 \%$ chewed Hans and $28 \%$ had a combination of smoking and smokeless tobacco usage. The reason for practicing these habits were as a measure to combat the cold, relieving stress and body pain after work, and the lack of awareness of the hazards of the materials used. Prevalence of oral mucosal lesions in the study population was due to tobacco usage and alcohol consumption and lack of awareness regarding the deleterious effects of the products used.

Conclusions

From the results of this study it may be concluded that the Malayali tribes were characterized by a lack of awareness about oral health, deep rooted dental beliefs, high prevalence of tobacco use and limited access to health services.

\section{P420}

Selection of first-in-human starting dose of anti-OX40 agonist monoclonal antibody BMS-986178 using a pharmacokinetic/ pharmacodynamic-based approach

Christine Huang ${ }^{1}$, Yan Feng ${ }^{1}$, Bryan Barnhart ${ }^{2}$, Michael Quigley ${ }^{1}$, John Huber ${ }^{1}$, Akintunde Bello ${ }^{1}$, Punit Marathe', Praveen Aanur ${ }^{1}$

Timothy Reilly', Zheng Yang ${ }^{1}$

${ }^{1}$ Bristol-Myers Squibb, Princeton, NJ, USA; ${ }^{2}$ Bristol-Myers Squibb, Redwood City, CA, USA

Correspondence: Christine Huang (christine.huang@bms.com) Journal for ImmunoTherapy of Cancer 2017, 5(Suppl 2):P420

\section{Background}

Following the clinical success of checkpoint blockade, the field of cancer immunotherapy rapidly expanded. Co-stimulatory molecules from the TNF receptor superfamily, including OX40, may be a promising approach to enhance the benefits of immunotherapy. BMS-986178 is a fully human agonist antibody of the immunoglobulin G1 isotype that binds with high affinity to the human OX40 receptor, currently being developed for the treatment of advanced solid tumors.

\section{Methods}

BMS-986178 does not bind to mouse OX40, necessitating antibody surrogates to assess in vivo activity of OX40 agonism. Two hamster anti-mouse OX40 agonist mAbs (reformatted as mouse antibodies: mlgG1 and mlgG2a) were studied in the mouse MC38 colon adenocarcinoma model. A pharmacokinetic/pharmacodynamic (PK/PD)based approach was employed to integrate the preclinical data and guide the first-in-human $(\mathrm{FIH})$ starting dose selection.

Results

In vitro, binding $\mathrm{EC}_{50}$ of $\mathrm{mlgG} 1$ and $\mathrm{mlgG} 2 \mathrm{a}$ mAbs in activated mouse T-cells was similar to in vitro binding $\mathrm{EC}_{50}$ of BMS-986178 in activated human T-cells. PK/PD analysis of the anti-tumor efficacy data showed maximum efficacy at an AUC of $100 \mu \mathrm{g}^{*} \mathrm{~h} / \mathrm{mL}$ with a $C_{\max }$ of $25 \mu \mathrm{g} / \mathrm{mL}$.
Targeting the same AUC and $\mathrm{C}_{\max }$ in mice into a human PK/PD prediction model, the human efficacious dose was projected to be $1 \mathrm{mg} / \mathrm{kg}$. The proposed FIH starting dose was then selected to be $0.25 \mathrm{mg} / \mathrm{kg}$, 4fold below the projected efficacious dose. First, using preclinical GLP safety assessments, the proposed starting dose was 68- to 80-fold lower than $1 / 6$ th of the highest non-severely toxic dose $(120 \mathrm{mg} / \mathrm{kg} / \mathrm{week})$ in monkeys. Second, the $C_{\max }(6.25 \mu \mathrm{g} / \mathrm{mL})$ at the starting dose was 5 -fold below the highest no-effect concentration $(33 \mu \mathrm{g} / \mathrm{mL})$ evaluated in the in vitro cytokine release assay. Third, there was a $>10$-fold exposure margin between the minimal pharmacologic effect (antigen-specific T-cell or antibody responses) observed in monkeys at doses of $2-4 \mathrm{mg} / \mathrm{kg}$ and the FIH starting dose selected.

The FIH trial was successfully initiated at $20 \mathrm{mg}(0.25 \mathrm{mg} / \mathrm{kg}$ with a BW of $80 \mathrm{~kg}$ ). BMS-986178 was well tolerated without any adverse event at this dose. OX40 receptor occupancy observed in peripheral blood at the starting dose maintained $\sim 80 \%$ in line with the prediction made from in vitro cellular binding affinity and clinical information observed from other anti-OX40 antibodies.

Conclusions

This PK/PD-based approach was successfully applied to select the FIH starting dose of BMS-986178. This strategy appropriately balanced the selection of a safe dose, while minimizing the number of cancer patients receiving sub-therapeutic doses.

\section{P421}

Co-administration of dexamethasone with checkpoint blockade therapy increases survival in brain tumor model

Marsha-Kay Hutchinson, Amber Giles, Heather Sonnemann, Jinkyu Jung, Caitlin Reid, Deric Park, Mark Gilbert

${ }^{1}$ National Institutes of Health, Bethesda, MD, USA

Correspondence: Amber Giles (hutchinsonmn@mail.nih.gov); Deric

Park; Mark Gilbert

Journal for ImmunoTherapy of Cancer 2017, 5(Suppl 2):P421

\section{Background}

The use of corticosteroids for therapeutic benefit must be weighed against risks of adverse consequences associated with these drugs. Brain tumor patients are routinely prescribed dexamethasone to reduce tumor-associated edema. Checkpoint blockade, a type of immune therapy, is currently being investigated as a potential treatment for brain tumors. Although glucocorticoid signaling has been shown to attenuate the immune response, the effect of glucocorticoids on the anti-tumor immune response during checkpoint blockade remains unclear. Here, we propose that dexamethasone's ability to upregulate T-cell checkpoint molecules such as CTLA-4 might be an immunosuppressive mechanism, but can be countered in unison with checkpoint blockade therapy. Methods

Healthy donor T cells were tested for response to dexamethasone. T cell proliferation, cell cycle analysis, apoptosis, glucose uptake, and protein expression were assessed with flow cytometry and Western blots. Transcriptional changes were assessed with qPCR. CTLA-4 was blocked using monoclonal antibodies in vitro on human PBMCs and in vivo with the GL261 syngeneic glioblastoma model.

Results

Checkpoint molecule CTLA-4 was increased by dexamethasone treatment upon stimulation. Unexpectedly, dexamethasone did not elicit a direct lymphotoxic effect on $T$ cells but significantly reduced $T$ cell entry into cell cycle. Dexamethasone also abated CD28 signaling as shown by reduced AKT phosphorylation and reduced glucose uptake. Blockade of CTLA-4 resulted in a substantial reversal of these effects. In addition, we discovered that antigen-experienced memory $\mathrm{T}$ cells were invulnerable to dexamethasone treatment. In vivo, dexamethasone and CTLA-4 blockade provided a survival benefit to tumor bearing mice.

\section{Conclusions}

These results suggest that mature $T$ cells, which predominate in the tumor microenvironment, are resilient to the negative effects of corticosteroids. Thus, corticosteroids are unlikely to destroy anti-tumor $T$ cells. For patients who have not responded to immune therapy, CTLA-4 blockade may provide protection from corticosteroids to the naïve T cell pool. 
P422

Overall survival and treatment patterns among real-world patients with metastatic non-small cell lung cancer not previously treated with systemic therapy for advanced cancer

Jason C. Simeone', Beth Nordstrom ${ }^{1}$, Ketan Patel ${ }^{2}$, Alyssa B. Klein ${ }^{3}$

${ }^{1}$ Evidera, Waltham, MA, USA; ${ }^{2}$ AstraZeneca, Milton, United Kingdom;

${ }^{3}$ AstraZeneca, Gaithersburg, MD, USA

Correspondence: Alyssa B. Klein (caroline.moreau@evidera.com)

Journal for ImmunoTherapy of Cancer 2017, 5(Suppl 2):P422

\section{Background}

The majority of patients with non-small cell lung cancer (NSCLC) are diagnosed at an advanced stage of disease, typically with a poor prognosis. While treatment of metastatic NSCLC is focused on extending survival, real-world data on the effectiveness of existing therapies and patterns of treatment is limited.

\section{Methods}

Patients with metastatic NSCLC, that are EGFR and ALK WT and no prior systemic therapy for advanced disease were identified from the Flatiron Oncology electronic medical record database from 2013-2016. Treatment patterns, including regimens administered and time to treatment, were summarized. The median overall survival (OS) from the initial diagnosis of metastatic NSCLC was calculated from Kaplan-Meier curves, with associated 95\% confidence intervals $(\mathrm{Cl})$.

Results

A total of 10,123 patients were eligible for analysis. The mean age of patients was $68.0 \pm 10.0$ years, and $54.0 \%$ were male. The most commonly observed regimens after the diagnosis of metastatic disease were nivolumab (17.4\%), carboplatin + pemetrexed $(16.0 \%)$, and carboplatin + paclitaxel $(15.0 \%)$, while $25.4 \%$ did not receive treatment. The median time from diagnosis of metastatic disease to start of first-line therapy was 36 days (range: 1-1,770) and the median number of therapy lines was 1 (range: 1-8). The median OS was 11.2 months (95\% Cl: 10.9-11.6) from the initial diagnosis of metastatic disease (Fig. 1).

Conclusions

In this real-world study, overall survival of metastatic patients was less than 12 months, and one quarter of patients did not receive treatment after diagnosis. This evidence indicates a potential unmet need for additional treatment options, as more patient information such as biomarkers become available to physicians at initial diagnosis.

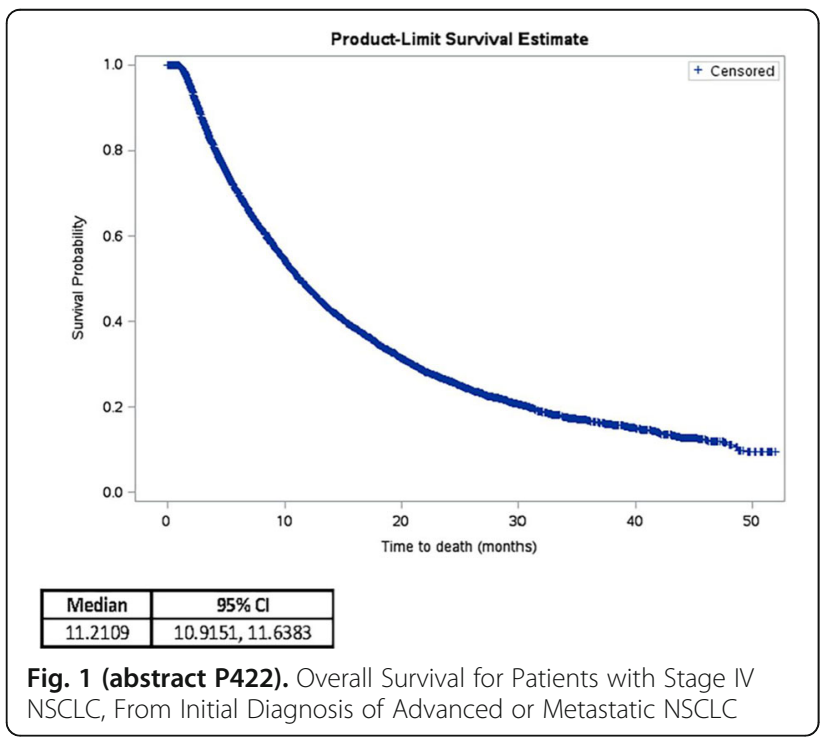

P423

Overall survival and treatment patterns among real-world patients with stage IIIB non-small cell lung cancer treated with platinumbased chemotherapy

Jason C. Simeone', Beth Nordstrom¹, Ketan Patel ${ }^{2}$, Alyssa B. Klein ${ }^{3}$

${ }^{1}$ Evidera, Waltham, MA, USA; ${ }^{2}$ AstraZeneca, Milton, United Kingdom;

${ }^{3}$ AstraZeneca, Gaithersburg, MD, USA

Correspondence: Alyssa B. Klein (caroline.moreau@evidera.com)

Journal for ImmunoTherapy of Cancer 2017, 5(Suppl 2):P423

\section{Background}

Platinum-based chemotherapy has been the standard of care in the management of patients with advanced non-small cell lung cancer (NSCLC), but evidence of effectiveness and patterns of treatment in a real-world setting is needed.

Methods

Electronic medical record data of patients from the Flatiron Oncology database with Stage IIIB NSCLC treated with at least two cycles of platinum-based chemotherapy during 2013-2016 were identified; patients with prior evidence of metastatic disease were excluded. Treatment patterns were examined, including the duration and number of lines received. The median overall survival (OS) from the start date of the third cycle of chemotherapy was calculated from Kaplan-Meier curves, with $95 \%$ confidence intervals $(\mathrm{Cl})$.

Results

A total of 1,226 patients met all criteria for study inclusion. The mean age of patients was $68.5 \pm 9.0$ years, and $51.9 \%$ were male. The most common regimens observed at any time during followup were carboplatin + paclitaxel $(43.9 \%)$, nivolumab $(23.7 \%)$, and carboplatin + pemetrexed (21.0\%); patients received a median of 2 lines of therapy (range: 1-7). The median OS was 19.4 months (95\% Cl: 17.4-21.6) from the start of the third cycle of chemotherapy (Fig. 1).

Conclusions

Overall survival among Stage IIIB NSCLC patients who were treated with platinum-based chemotherapy in a real-world setting was less than 20 months. Previous literature indicates survival of 7-13 months among advanced NSCLC patients treated with chemotherapy. However, the present study excludes patients with metastatic disease. The difference in OS may also be related to the use of newer, more effective treatments that were not available in earlier studies. nt->

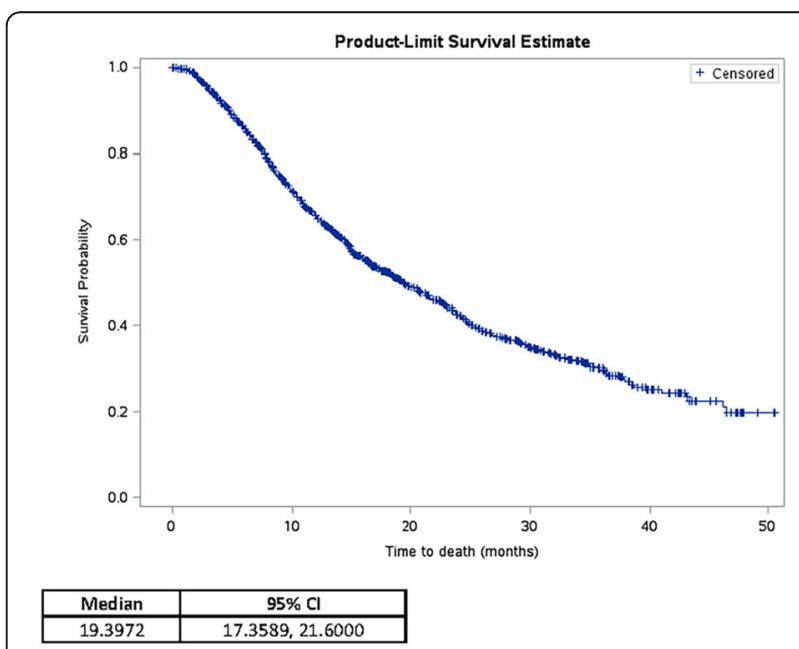

Fig. 1 (abstract P423). Overall Survival of Patients with Stage IIIB NSCLC, From Start of Third Cycle of Chemotherapy 
P424

Efficacy of pembrolizumab (MK-3475) in patients with

adrenocortical carcinoma

Mohammed Amir Habra, Matthew Campbell, Camilo Jimenez,

Daniel Karp, David Hong, Vivek Subbiah, Shubham Pant, Jeane Painter,

Saria Khan, Chantale Bernatchez, Bettzy Stephen, Anas Alshawa,

Coya Tapia, Tito Mendoza, Rivka Colen, Kenneth Hess,

Funda Meric-Bernstam, Aung Naing

MD Anderson Cancer Center, Houston, TX, USA

Correspondence: Aung Naing (anaing@mdanderson.org)

Journal for ImmunoTherapy of Cancer 2017, 5(Suppl 2):P424

\section{Background}

Adrenocortical carcinoma (ACC) is an orphan endocrine malignancy with poor prognosis and limited response to chemotherapy. ACC can express immune markers which could make it susceptible to immunotherapy. So far, there is no published clinical trial about using immunotherapy in ACC.

Methods

This is a phase II study of pembrolizumab in patients with rare tumors including a pre-specified ACC cohort (http://ClinicalTrials. gov identifier, NCT02721732). Patients received pembrolizumab $200 \mathrm{mg}$ intravenously once every 3 weeks. Response was assessed every 9 weeks using RECIST1.1. The primary end point was progression-free survival at 27 weeks (PFS27wk). Mandatory biopsies are taken at baseline, on cycle 1 day $15-21$, and at the time of progression.

Results

A total of 11 patients were enrolled and treated. At the time of analysis, patients received a median of 6 treatment cycles (range 2-17). A PFS of greater than 27 weeks was seen in $3 / 11$ patients $(27 \%)$. The first patient reached $37 \%$ tumor reduction (partial response) while the second patient had a response of $41 \%$ tumor reduction (partial response) and the third patient had stable disease. The remaining 8 patients had evidence of disease progression within 27 weeks of starting the study (median time 18 weeks, range 6-27 weeks). Progression was seen in most patients with hormonally active ACC (6/7) and only in 2/4 patients without evidence of hormonal excess at the time of their initial diagnosis. The safety profile of pembrolizumab was very favorable and none of these patients had severe grade 3 or grade 4 adverse effects.

Conclusions

Single agent pembrolizumab may be an effective option in subset of ACCs without hormonal overproduction while it is associated with high failure rate among patients with hormonally active ACCs. Expanding the study to hormonally silent ACCs is needed to verify the initial observation from limited number of patients treated in this study. Translational data will be presented at this meeting.

Trial Registration

http://ClinicalTrials.gov identifier, NCT02721732.

\section{P425}

Efficacy of pembrolizumab in patients with cutaneous aquamous cell carcinoma

Renata Ferrarotto, Bonnie Glisson, George Blumenschein, David Hong, Sarina Piha-Paul, Dipti Jain, Anas Alshawa, Jeane Painter, Kenneth Hess, Rivka Colen, Chantale Bernatchez, Charles Lu, Bettzy Stephen, Coya Tapia, Tito Mendoza, Funda Meric-Bernstam, Aung Naing The University of Texas MD Anderson Cancer Center, Houston, TX, USA Correspondence: Aung Naing (anaing@mdanderson.org) Journal for ImmunoTherapy of Cancer 2017, 5(Suppl 2):P425

\section{Background}

Cutaneous squamous cell carcinoma ( $\mathrm{CSCC}$ ) is the $2^{\text {nd }}$ most common malignancy in the US. While the majority of patients are cured with surgery, approximately 6,400 die annually as a consequence of disease. There is limited data on the role of chemotherapy in the treatment of metastatic CSCC. The high-mutational burden, the presence of tumor-infiltrating lymphocytes, immunosuppression as a risk factor, and evidence of direct immunosuppressive effects of
UV radiation suggest that $\mathrm{CSCC}$ is appropriate for the clinical study of checkpoint inhibitors.

Methods

This is a phase II study of pembrolizumab in patients with rare tumors including a pre-specified cutaneous squamous cell carcinoma cohort (http://ClinicalTrials.gov identifier, NCT02721732). Patients received pembrolizumab $200 \mathrm{mg}$ intravenously once every three weeks. The response was assessed every nine weeks using RECIST1.1. The primary end point was progression-free survival at 27 weeks (PFS27wk). Mandatory biopsies are taken at baseline, on cycle 1 day $15-21$, and at the time of progression.

Results

A total of 11 patients were enrolled and treated. At the date of analysis, patients received a median of 5.5 treatment cycles (range 1-15). Three out of eleven patients have been treated beyond 27 weeks. Four patients had a partial response and the tumor reduction from the baseline have reached $33 \%, 55 \%, 63 \%$, and $70 \%$. The fifth patient had stable disease while the other five patients had a progressive disease within 27 weeks of starting the study. The last patient has not had his first restaging scans yet. The safety profile of pembrolizumab was very favorable.

Conclusions

Single agent pembrolizumab may be effective in a patient with advanced cutaneous squamous cell carcinoma. Expanding the study is needed to verify the initial observation. Translational data will be presented at this meeting.

Trial Registration

http://ClinicalTrials.gov identifier, NCT02721732.

\section{P426}

Antibody-drug conjugate induced cytotoxicity of tumor cell lines by targeting the SAS1B N-terminus

Arabinda Mandal', Mriganka Mandal ${ }^{2}$, Walter Olson' ${ }^{1}$, Jagathpala Shetty ${ }^{1}$, Kiley Knapp ${ }^{3}$, Eusebio Pires ${ }^{1}$, Todd Bauer', Timothy Bullock ${ }^{4}$, John Herr', Craig Slingluff ${ }^{1}$

${ }^{1}$ University of Virginia, Charlottesville, VA, USA; ${ }^{2}$ Albemarle High School, Charlottesville, VA, USA; ${ }^{3}$ University, Charlottesville, VA, USA; ${ }^{4}$ University of Virginia.edu, Charlottesville, VA, USA

Correspondence: Craig Slingluff (wco3j@virginia.edu)

Journal for ImmunoTherapy of Cancer 2017, 5(Suppl 2):P426

\section{Background}

SAS1B (ASTL, Ovastacin) is a zinc metalloproteinase found normally in growing oocytes that has been detected in uterine cancer cells, and its expression is restricted to oocytes, among adult tissues. Thus, it is classified as a cancer-oocyte neoantigen [1]. Treatment of uterine tumor cells with anti-SAS1B polyclonal antibodies and complement arrested growth, and a saporin-immunotoxin directed to SAS1B caused cell death. The goal of the present study was to develop immunotherapeutic monoclonal antibodies against the human SAS1B target, to reduce risks of off-target effects during therapy.

Methods

Monoclonal antibodies (mAbs) were generated to recombinant $(r)$ human (h) SAS1B immunogen without its signal peptide.

Results

SAS1B transcript in human uterine and lung cancer cell lines was confirmed by PCR and sequencing. SB2 and SB5 mAbs demonstrated Western immunoreactivity to rhSAS1B expressed in E. coli or HEK293T cells, and recognition of SAS1B was confirmed by mass spectrometry on immunoprecipitated antigen. SAS1B deletion constructs mapped the epitopes recognized by the SB2 and SB5 mAbs to the $\mathrm{N}$-terminal domain between residues 32-40. Surface expression of SAS1B in live uterine, lung and pancreatic tumor cell lines was detected by both mAbs. The expression of SAS1B N-terminus on the surface of live tumor cells and its cytoplasmic expression from uterus (SNU539, Fig. 1B), ovary, breast, melanoma (Malme3M, Fig. 1C) lung (H226, Fig. 1D), and were confirmed by flow cytometry and SAS1B peptide blocking. SAS1B expression was not evident on the surface of normal peripheral blood mononuclear cells. Monoclonal antibodies SB2 and SB5, complexed with a Fab-Duocarmycin DM, that incorporated a 
cathepsin-cleavable linker arm, were cytotoxic to SAS1B positive uterine (SNU539, Fig. 1A), pancreatic and lung tumor cells at 10 100 picomolar concentrations.

\section{Conclusions}

The SB2 and SB5 mAbs offer promise as candidate immunotherapeutic entities for targeting SAS1B positive cancer cells with defined epitopes located in the SAS1B N-terminal domain suitable for immunotherapeutic targeting.

\section{References}

1. Pires ES, et al. Membrane Associated Cancer-Oocyte Neoantigen SAS1B/ Ovastacin is a Candidate Immunotherapeutic Target for Uterine Tumors. Oncotarget. 2015; 6:30194-30211.

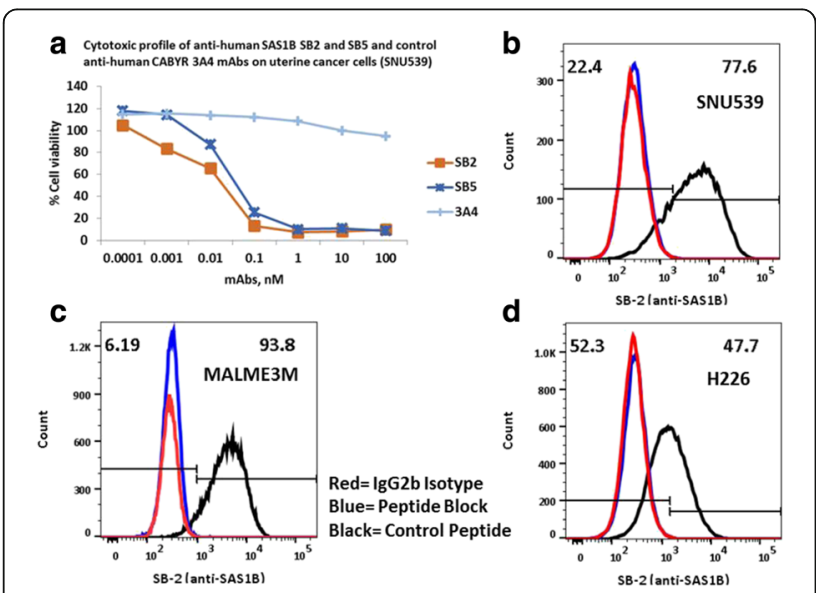

Fig. 1 (abstract P426). Profile of SAS1B expression on tumor cell lines by ADC and flow cytometry

\section{P427}

Democratizing analysis of cancer data using the cancer genomics cloud

Anurag Sethi (anurag.sethi@sbgenomics.com)

Seven Bridges Genomics, Inc, Boston, MA, USA

Journal for ImmunoTherapy of Cancer 2017, 5(Suppl 2):P427

\section{Background}

The advent of next generation sequencing has resulted in the generation of petabytes of multi-dimensional information, but the access and analyses of this data remains challenging. This difficulty is exemplified when we consider data generated by the efforts of The Cancer Genomics Atlas (TCGA) network. Historically, the only means for gaining insight from the TCGA data was to download the complete TCGA repository, which can require several weeks with a highly optimized network connection, followed by analyses in a very expensive high performance computational cluster.

Methods

The Cancer Genomics Cloud Pilots project seeks to democratize cancer data analysis by co-localizing data with the computational resources. Funded by the National Cancer Institute, the Cancer Genomics Cloud Pilot (www.cancergenomicscloud.org) project enables researchers to leverage the power of cloud computing to gain actionable insights on cancer biology and human genetics from massive public datasets including TCGA.

Results

The CGC removes the need to download data, enables easy querying, and much more.

\section{Conclusions}

We will highlight our approach to optimized computation, data mining, and visualization solutions that address the challenges associated with analyses of petabyte-scale datasets and beyond.
P428

PD-1 blockade activates CD4 T cells and the innate immune response for glioblastoma eradication

Sarah R. Klein, Maria C. Speranza, Prafulla C. Gokhale, Margaret K. Wilkens, Kristen L. Jones, Apoorvi Chaudhri, Paul T. Kirschmeier, David A. Reardon, Gordon J. Freeman

Dana-Farber Cancer Institute, Boston, MA, USA

Correspondence: Gordon J. Freeman

(maria_speranza@dfci.harvard.edu)

Journal for ImmunoTherapy of Cancer 2017, 5(Suppl 2):P428

\section{Background}

Cancer immunotherapy has come of age as a result of substantive advances in basic and translational tumor immunology [1]. In fact, multiple advanced clinical trials with human anti-PD-1 antibodies (i.e. nivolumab and pembrolizumab) have convincingly established the ability of anti-PD-1 antibodies to trigger clinically significant tumor destruction in multiple tumor types, reinforced by dramatic anticancer responses in animal models and encouraging durability in early clinical trials [2].

Methods

Through flow cytometry and in in vivo intracranial injection of mouse glioma tumor cells we recently demonstrated that PD-1 blockade elicits an anti-tumor immune response resulting in tumor rejection and long-term survival in approximately $50 \%$ of mice, despite the absence of accumulating CD8+ cytotoxic T cells in the tumor or draining lymph nodes [3].

Results

In this investigation, we provide evidence for the role of conventional CD4+ $T$ cells and the innate immune response in PD-1 mediated antiglioma immunity in this model. In response to anti-PD-1 monotherapy, intratumoral CD4+ T cells, but not CD8+ T cells, expressed significantly elevated levels of IFN- $\gamma$ and TNF- $a$ pro-inflammatory cytokines and the cytotoxic enzyme, granzyme B. Tbet, GATA3, and EOMES, transcription factors required for $T$ cell proliferation, activation, and effector function, were also up-regulated in CD4+, but not CD8+ T cells in the brains of mice treated with PD-1 mAbs when compared to controls. We demonstrated that depletion of CD4+ or CD8+ T cells, but not NK, was sufficient to completely ablate anti-PD-1-mediated tumor eradication and long-term survival. CD4+ T cell activation was accompanied by the classical activation and M1 polarization of resident microglia and tumor-infiltrating macrophages. Moreover, CSF-1 inhibition with PLX3397 to deplete microglia and macrophages did not affect tumor growth, but did lead to a significant survival advantage when combined with anti-PD-1 mAb.

Conclusions

Together, these studies demonstrate for the first time a role for CD4 $+T$ cells and the innate immune response in the eradication of glioblastoma by PD-1 blockade.

\section{References}

1. Mittal D, Gubin MM, Schreiber RD et al. New insights into cancer immunoediting and its three component phases-elimination, equilibrium and escape. Current opinion in immunology. 2014;27:16-25.

2. Freeman GJ, Long AJ, Iwai Y, et al. Engagement of the PD-1 immunoinhibitory receptor by a novel $B 7$ family member leads to negative regulation of lymphocyte activation. J Exp Med. 2000;192:1027-1034.

3. Checkpoint Blockade in an Orthotopic, Immunocompetent Model. Cancer Immunol Res. 2015;4(2): 124-35.

\section{Personalized Vaccines and Technologies/ Personalized Medicine}

P429

Nanoparticle based enrichment and expansion of self and neoepitope specific CD8 ${ }^{+} \mathrm{T}$ cells in murine melanoma

Catherine A Bessell, Joan Glick Bieler, John-William Sidhom, Jonathan P Schneck

Johns Hopkins University, Baltimore, MD, USA

Correspondence: Jonathan P Schneck (cbessel1@jhmi.edu)

Journal for ImmunoTherapy of Cancer 2017, 5(Suppl 2):P429 


\section{Background}

T cell responses against neo-antigens has created a new avenue to target and kill patients' tumors while decreasing autoimmune side effects. Neo-antigen-specific CD8+ T cells have the potential benefit to be a source of T cells that are not limited by peripheral tolerance or deleted from the T cell repertoire by central tolerance. To understand the differences between $T$ cell responses against neo- and self-antigens, these two types of T cell populations must be compared side-by-side for antitumor responses and phenotypic differences.

Methods

Following another group that identified mutated proteins in B16 murine melanoma [1], our current work has identified the short peptide epitopes that can stimulate neo-antigen $\mathrm{T}$ cell responses. Artificial antigen presenting cells (aAPCs) were used to access neo- and self-antigen tumor specific responses in the endogenous T cell population of naïve and B16 tumor bearing animals. In a B16-SIY melanoma model, neo-epitope Kb-SIY serves as a model to collect $\mathrm{T}$ cells for self and neo-epitopes and analyze the T cell receptor (TCR) repertoire of the separate populations.

Results

After exposure to tumor, neo-epitope $\mathrm{T}$ cell responses showed larger fold expansion, while T cell expansions for self-antigen Kb-TRP2 expanded similar in the naïve animals. TCR repertoire analysis of neo- and self-antigen reactive $T$ cells showed a highly conserved $V$ beta gene usage in the naive Kb-SIY TCR repertoire, while the naïve Kb-TRP2 TCR repertoire had a more broad $\mathrm{V}$ beta selection. The tumor did not alter the $\mathrm{V}$ beta usage for Kb-SIY repertoire, however the Kb-TRP2 repertoire changed to different $\mathrm{V}$ beta genes after tumor exposure.

\section{Conclusions}

The increased expansion ability of neo-antigens after tumor exposure demonstrates the potential anti-tumor ability of neo-epitope responses compared to self-epitope T cell responses. TCR repertoire analysis of neo- and self-antigen reactive $T$ cells shows that the tumor shifts the TCR clonal diversity of self-antigen specific populations to but leaves the neo-epitope repertoire intact and similar to the naïve repertoire. From this work, we show proof of concept of a streamlined method to screen candidate neo-epitopes, identify rare endogenous $T$ cell populations, and assess the benefit of $\mathrm{T}$ cell directed immunotherapies on different T cell populations.

\section{References}

1. Castle JC, Kreiter S, Diekmann J, Lower M, van de Roemer N, de Graaf J, Selmi A, Diken M, Boegel S, Paret C, Koslowski M, Kuhn AN, Britten CM, Huber C, Tureci O, Sahin U. Exploiting the Mutanome for Tumor Vaccination. Cancer Res. 2012; 72:1081-1091.

\section{P430}

Neoantigen identification using $\operatorname{ATLAS}^{\mathrm{TM}}$ across multiple tumor types highlights limitations of prediction algorithms

Jason Dobson, Huilei Xu, Kyle Ferber, Johanna Kaufmann, Judy Jacques, Christine McCoy, Michael O'Keeffe, Yana Ostrovsky, Crystal Cabral, Pamela Carroll, Theresa Zhang, Jessica Flechtner, Wendy Broom Genocea Biosciences, Cambridge, MA, USA

Correspondence: Wendy Broom (jason.dobson@genocea.com) Journal for ImmunoTherapy of Cancer 2017, 5(Suppl 2):P430

\section{Background}

Neoantigens arise from tumor-specific, somatic mutations and have the potential to be recognized by T cells that are associated with anti-tumor immune responses. Since they are non-self, they are hypothesized to provide an attractive therapeutic modality since $T$ cells that can respond to those sequences have not undergone thymic selection. The ATLAS ${ }^{T M}$ platform enables identification of biologically relevant $\mathrm{CD} 4^{+}$and $\mathrm{CD} 8^{+} \mathrm{T}$ cell neoantigens in any subject in an unbiased manner.

\section{Methods}

Multiple patients with solid tumors were analyzed. CD14 ${ }^{+}$monocytes and $T$ cell subsets were isolated from patient peripheral blood mononuclear cells. Monocytes were differentiated into dendritic cells (MDDC), and $T$ cells were non-specifically expanded. Whole exome sequencing was performed on tumor biopsies and matched normal genomic DNA and tumor specific changes (single nucleotide variants and insertion/ deletions) were identified and cloned into $E$. coli expression vectors with and without co-expressed listeriolysin $\mathrm{O}$ to enable presentation via MHC class I or class II, respectively. For each patient, their unique clones were co-cultured with autologous MDDCs in an ordered array, then their $\mathrm{CD}^{+}$or $\mathrm{CD}^{+}{ }^{+}$cells were added and incubated overnight. T cell activation was determined by measurement of TNF- $\alpha$ and IFN- $\gamma$ in the supernatants by meso-scale discovery. Cytokine concentrations were normalized against responses to negative control bacteria and neoantigens were defined as clones that elicited responses $>3$ median absolute deviations of the median of negative controls.

Results

ATLAS $^{\text {TM }}$ identified $\mathrm{CD}^{+}$and $\mathrm{CD}^{+}{ }^{+}$cells responses to up to $15 \%$ of mutant polypeptide sequences, across a broad cohort of patients with different tumor types, including tumors with either low or high mutational burden. "Inhibitory" neoantigens, which shut off all cytokine production, were also identified in each subject. Many neoantigens were not predicted by algorithms. When exploring neoantigens by tumor type, no patterns in overall mutational burden, RNA expression level, or DNA mutant allele frequency have yet been identified.

Conclusions

The ATLAS ${ }^{T M}$ platform empirically defines which potential neoantigens created by somatic mutations elicit immune responses in individual patients independently of a patient's HLA type and T cell receptor repertoire. The identification of activating and inhibitory neoantigens for $\mathrm{CD}^{+} \mathrm{T}$ cells, as well as for $\mathrm{CD} 4^{+} \mathrm{T}$ cells for which the algorithmic approaches do not perform nearly as well, provides the opportunity to identify better targets to include in a vaccine formulation. Genocea is currently developing personalized cancer vaccines with neoantigens prioritized by ATLAS $^{\text {TM }}$ which we hypothesize will provide better therapeutic impact than algorithmic approaches.

P431

Identification of immunogenic breast cancer neoantigens exposed by radiation therapy

Claire Lhuillier, Nils Rudqvist, Takahiro Yamazaki, Tuo Zhang, Lorenzo Galluzzi, Sandra Demaria

Weill Cornell Medical College, New-York, NY, USA

Correspondence: Sandra Demaria (cfl2002@med.cornell.edu)

Journal for ImmunoTherapy of Cancer 2017, 5(Suppl 2):P431

\section{Background}

Recent studies have highlighted the key role of mutation-generated neoantigens in tumor response to immunotherapy [1]. We have previously shown in the $4 \mathrm{~T} 1$ mouse model (syngeneic with BALB/C mice) of immune-checkpoint blockade-resistant metastatic breast cancer that local radiation therapy (RT) combined with CTLA-4 blockade induces the $\mathrm{CD} 8^{+}$ $T$ cell-mediated regression of irradiated tumors and limits metastatic lung colonization [2]. Preliminary analysis of the T-cell receptor repertoire indicated that unique clonotypes are expanded in treated tumors, suggesting that tumor rejection involves $T$ cells reactive to a set of tumor antigens that are made available to the immune system by RT. Therefore, we hypothesize that RT, by changing the transcriptional profile of cancer cells, may expose antigenic mutations not transcribed at sufficient levels in untreated tumors and hence promote priming of $T$ cells to these unique mutated antigens. 


\section{Methods}

We performed whole-exome sequencing and RNA sequencing (RNA-seq) of $4 \mathrm{~T} 1$ cells irradiated (8GyX3) or not (0Gy) in vitro to identify tumorspecific mutations. We used several algorithms to predict MHC-I $\left(\mathrm{H}^{\mathrm{d}}\right)^{\mathrm{d}}$ binding epitopes from these mutated genes, and we selected those with a predicted affinity $<500 \mathrm{nM}$. Based on the RNA-seq data, we prioritized variants that were upregulated by radiation. These candidate peptides were synthesized and tested in vitro for binding to $\mathrm{H} 2-\mathrm{L}^{\mathrm{d}}$ or $\mathrm{H} 2-\mathrm{K}^{\mathrm{d}}$ in a MHC stabilization assay using RMA-S cells. Finally, the mutated peptides with the highest affinity were used to vaccinate BALB/c mice, followed by challenge with $4 \mathrm{~T} 1$ cells to test for the induction of protective antitumor immunity.

Results

Out of 309 total mutations initially identified in the 4T1 cancer cells, we selected and tested in vitro 17 candidate neo-epitopes. Two of them bound $\mathrm{H}_{2}-\mathrm{L}^{\mathrm{d}}$ with a high affinity and were used alone or in combination to vaccinate mice. Our preliminary data indicate that the combination of these two neoepitopes is immunogenic and delays the tumor growth after challenge with 4T1 cells.

\section{Conclusions}

In conclusion, these data provide initial proof-of-principle evidence that RT can expose existing neoantigens to the immune system.

\section{References}

1. Schumacher TN, and Schreiber RD. Neoantigens in cancer immunotherapy. Science. 2015; 348: 69-74

2. Demaria S, Kawashima N, Yang AM, Devitt ML, Babb JS, Allison JP, and Formenti SC Immune-mediated inhibition of metastases after treatment with local radiation and CTLA-4 blockade in a mouse model of breast cancer. Clinical cancer research: an official journal of the American Association for Cancer Research. 2005;11:728-734.

\section{P432}

Exploiting large scale HLA peptidomics to generate novel Immunotherapies; A data-driven approach to neoantigen prioritization

Alex S. Powlesland, Geert P.M. Mommen, Ricardo J. Carreira, David Lowne, Michael J. Cundell, Floriana Capuano, Bent K. Jakobsen

Immunocore Ltd, Abingdon, United Kingdom

Correspondence: Alex S. Powlesland (alex.powlesland@gmail.com) Journal for ImmunoTherapy of Cancer 2017, 5(Suppl 2):P432

\section{Background}

Peptides presented to the immune system on HLA complexes are valuable targets for immunotherapeutic treatments. Identifying the full complement of peptides derived from a particular protein that are presented on major class I HLA restrictions will provide a vital step toward increasing the speed and viability of many immunotherapeutic strategies. Advances in next generation sequencing and single cell technologies have enabled the accurate capture of somatic mutations accumulated by a tumour, yet a significant hurdle remains how this information can be utilised for immunotherapeutic benefit. In particular, identifying which somatic mutations produce neoantigens, (peptides that contain a somatic mutation and are presented to the immune system in complex with HLA), is crucial to linking genetic changes with immunological impact.

Methods

Our approach to understanding the targetable human HLA peptidome is based on three key principles; achieving full proteome coverage, maximising individual protein coverage, and focusing on dominant HLA restrictions. By integrating novel cell biology, mass spectrometry, and bioinformatic technologies in over 1000 individual experiments we have dramatically increased the depth of the HLA ligandome captured and achieved near total coverage of the protein-coding genome, with $90 \%$ of the proteome captured for HLA-A*02:01.

Our comprehensive genome coverage has enabled us to probe both directly and indirectly for the presence of neoantigens. Known somatic mutations within immortalised lines were used to generate bespoke reference databases that has led to direct identification of many hundreds of neoantigens.

Results

Proteins that were found to contain neoantigens appeared to follow the processing and presentation behaviours of their unmutated equivalent. We have therefore found our HLA peptide dataset is able to offer significant value in predicting the likelihood of a somatic mutation creating a neoantigen.

To test this, somatic mutations reported in 980 cell lines were probed against the database of HLA peptides. On average we find one peptide containing the mutated amino acid for every 5 somatic mutations reported. By incorporating the HLA background of the cell carrying the mutation we narrow this prediction to one high affinity HLA peptide for every 14 somatic mutations reported. Comparing the peptides predicted in this analysis with those directly identified by mass spectrometry, we are able to show that we can prioritise mutation data by accurately predicting the presence and relative abundance of neoantigens.

Conclusions

An integrative approach to HLA peptidomics has delivered a powerful reference database that can be used as a source for developing novel immunotherapies.

\section{P433}

Computational pipeline for the PGV-001 (personalized genomic vaccine) clinical trial

Alexander Rubinsteyn ${ }^{1}$, Julia Kodysh², Nina Bhardwaj'

Jeffrey Hammerbacher

${ }^{1}$ Icahn School of Medicine at Mount Sinai, New York, NY, USA; ${ }^{2}$ cahn School of Medicine, New York, NY, USA

Correspondence: Alexander Rubinsteyn (alex.rubinsteyn@gmail.com) Journal for ImmunoTherapy of Cancer 2017, 5(Suppl 2):P433

\section{Background}

PGV-001 (NCT02721043) is a phase I clinical trial at Mount Sinai Hospital, studying the safety and immunogenicity of a multipeptide personalized genomic vaccine for treatment of several different malignancies. Patients are treated with a TLR3 agonist (PolyICLC) and ten synthetic long peptides containing tumor mutations that are predicted to be abundantly expressed and to form mutated MHC ligands. The personalized vaccine is administered as an intramuscular injection and is given to each patient 10 times over a span of 6 months. Thus far, two patients (of an eventual twenty) have enrolled in the trial and one has been treated. The vaccine is administered in the adjuvant setting for patients who undergo a complete resection and have no evidence of residual disease.

Methods

Tumor DNA and RNA are extracted from fresh frozen tissue immediately following surgery. Normal DNA is extracted from a patient blood sample. Both DNA samples are enriched for coding regions 
using an exon capture kit. Normal DNA is sequenced to mean ontarget coverage of $150 \mathrm{x}$, whereas tumor DNA is sequenced to $300 \mathrm{x}$ coverage. RNA is enriched for mRNA using poly-A capture and then $>100 \mathrm{M}$ paired-end reads are sequenced. Somatic variants are detected from the tumor and normal DNA sequencing data. The RNA sequencing data is then used to assemble the most abundant coding sequence for each variant, which naturally phases adjacent variants and allows for an allele-specific estimate of expression. Each coding sequence is then translated to a mutated protein fragment, which is ranked according to (1) expression and (2) predicted binding affinity to patient MHC I molecules. The window of wildtype amino acids around a mutation in each peptide is optimized to improve the odds of successful solid phase synthesis.

Results

Though only two patients have enrolled in PGV-001 so far, we have evaluated the pipeline on a variety of patient (and mouse) samples across different kinds of cancers. We demonstrate that even cancers with intermediate mutational burden still yield sufficient predicted neoantigens to potentially benefit from vaccination. Furthermore, even with our existing attempt to optimize peptide sequences for manufacturability, we have still found many peptide sequences cannot be synthesized or purified. Analysis of these peptide sequences hint at additional rules which may improve synthetic yield for peptide-based neoantigen vaccines.

\section{Conclusions}

We hope that discussion of the design and rationale for PGV-001's computational pipeline will help other groups start their own personalized vaccine programs.

Trial Registration

NCT02721043.

\section{P434}

Great Apes Adenoviral vaccine encoding neoantigens synergizes with immunomodulators to cure established tumors in mice

Anna Morena D'Alise', Gabriella Cotugno', Guido Leoni', Francesca Langone', Imma Fichera', Maria De Lucia ${ }^{2}$, Rosa Vitale', Adriano Leuzzi ${ }^{1}$ Elena Di Matteo ${ }^{1}$, Antonella Folgori ${ }^{1}$, Stefano Colloca', Alfredo Nicosia, ${ }^{1}, 2$ Jonathan Zalevsky ${ }^{3}$, Elisa Scarselli ${ }^{1}$

${ }^{1}$ Nouscom srl, Rome, Italy; ${ }^{2}$ University of Naples Federico II, Naples, Italy;

${ }^{3}$ Nektar Therapeutics, San Francisco, CA, USA

Correspondence: Elisa Scarselli (g.napolitano@nouscom.com)

Journal for ImmunoTherapy of Cancer 2017, 5(Suppl 2):P434

\section{Background}

Current immunotherapies based on checkpoint inhibitors (CPI) revealed the importance of neoantigens-specific T cells for tumor control. Here, we developed a novel neoantigens vaccine approach based on the use of a viral vector, Great Apes Adenovirus (GAd), encoding multiple cancer neoantigens in tandem. NKTR-214 is a CD122-biased cytokine agonist currently in clinical trials. NKTR-214 is designed to expand a specific population of cancer-killing $T$ cells, known as tumor-infiltrating lymphocytes. Synergy between the two drugs was investigated.

Methods

Balb/c mice were implanted subcutaneously with CT26 colon carcinoma cells. Once tumors were established, mice were randomized and vaccinated with a single intramuscular injection of GAd encoding up to 20 different CT-26 neoantigens identified by NGS and selected with a NousCom proprietary pipeline. NKTR-214 was administered i.v. on $\mathrm{q} 9 \mathrm{~d} \times 3$ schedule. Tumor growth in treated animals was monitored and neoantigen-specific $T$ cells were measured both in the periphery and in tumors.
Results

Combination treatment of a GAd-CT26 neoantigens vaccine with NKTR-214 results in tumor regression in $90 \%$ of mice. Single agent NKTR-214 treatment led to tumor regression in $<40 \%$ of mice. Interestingly, all animals cured by the combo were resistant to a second tumor challenge. NKTR-214 treatment rescued CD4 T cell reactivity against at least one of the predicted neoantigens, likely induced by the tumor itself. GAd vaccination in the presence of NKTR-214 induced a greater breadth and depth of immune response. When compared to Gad vaccine alone, the Gad + NKTR-214 combination had immune reactivity detected against a larger number of vaccine-encoded neoantigens and there was a greater proportion of IFN-gamma+ CD4 and CD8 T cells against each vaccine-encoded neoantigens. Tumors in regression of mice treated by the combo were highly enriched in T cells reactive to vaccine-encoded neoantigens.

Conclusions

Vaccination with GAd encoding neoantigens is a very effective and safe approach to strengthen and broaden tumor-killing T cells. Therapeutic cancer vaccines have been unsuccessful in the past likely because $T$ cell growing capacity in the tumor is suppressed by the tumor itself. Vigorous growth of vaccine-induced T cells in the tumour can be successfully achieved by the cytokine NKTR-214, overcoming the tumor inhibitory activity. NKTR-214 was shown to be safe and well tolerated in cancer patients and GAd vaccines have been safely used in many healthy volunteers, opening up the opportunity for a fast evaluation of a combo treatment in the clinic.

\section{P435}

Immunogenomics for the development of personalized T cell therapy for ovarian cancer

Muzamil Want, Sebastiano Battaglia, Takemasa Tsuji, Richard Koya Roswell Park Cancer Institute, Buffalo, NY, USA

Correspondence: Sebastiano Battaglia (muzamil.want@roswellpark.org) Journal for ImmunoTherapy of Cancer 2017, 5(Suppl 2):P435

\section{Background}

Ovarian cancer $(\mathrm{OC})$ is the fifth leading cause of cancer death in the United States with approximately 20,000 women being diagnosed every year. Since OC development is mainly asymptomatic, patients are often diagnosed at late stage and present local and distal metastases. This offers a clinical challenge, as roughly $70 \%$ of the patients develop chemoresistant disease. With the advent of cancer immunogenomics it is now possible to identify tumor specific mutations, or neoantigens/neoepitopes, that can be exploited for the development of personalized T cell therapies via DC vaccines or adoptive cell therapy (ACT). We therefore hypothesize that tumor cells isolated from patients with ovarian cancer offer a unique opportunity to identify clinically actionable tumor neoantigens.

Methods

Somatic mutations were identified by whole exome sequencing (WES) and RNASeq analysis in tumor samples, using PBMCs as germline control. In parallel ovarian cancer PDXs were established in NSG mice by injecting $2 \times 106$ patient tumor cells/mouse subcutaneously (SQ, $n=20$ ). Tumor growth was monitored weekly via caliper measurement. Mutational analysis and immunogenicity prediction for the mutated peptides was done using a combination of homedeveloped bioinformatic approaches and NetMHC. Immunogenicity predictions were validated in-vitro by pulsing wild type and mutated peptides into CD8+ depleted PBMCs and co-cultured with CD8+ T cells for up to 20 days. T cell activation was quantitated via ELISA measuring IFN-g release and via flow cytometry by staining for CD137 expression.Lastly, the therapeutic potential of ACT 
targeting neoantiges was tested in-vivo in ovarian cancer PDXs and tumor size is being monitored weekly.

Results

We have established patient-derived xenografts (PDX) mice and interrogated the tumor mutational landscape. Targeted mutations were confirmed in the following PDX passages by NGS and Sanger sequencing. Out of 168 mutated peptides predicted from the patient tumor, 13 were found to have high affinity for different HLAs compared to the wild type peptide. In vitro screening of high affinity peptides showed that few of the mutated peptides can activate the T cells from patient PBMCs and tumor infiltrating lymphocytes thereby confirming the presence of neoantigen reactive T cells.

Conclusions

In this study, we demonstrate that the patient's own T lymphocytes can recognize tumor neoantigens and could be used as personalized therapy in ovarian cancer patients

\section{P436}

In vivo self-assembled albumin/vaccine nanocomplexes for lymphnode-targeted vaccine delivery in combination cancer immunotherapy

Guizhi Zhu, Xiaoyuan Chen

National Institutes of Health, Bethesda, MD, USA

Correspondence: Guizhi Zhu (zhu.julian21@gmail.com)

Journal for ImmunoTherapy of Cancer 2017, 5(Suppl 2):P436

\section{Background}

Cancer immunotherapy aims to normalize the immune system in patients to harness the power of the immune system to systemically treat cancer. Molecular vaccines hold tremendous potential for tumor immunotherapy, but its clinic outcome has yet been optimal, largely due to inefficient co-delivery of heterogeneous peptide antigens and adjuvants to secondary lymphoid organs such as lymph nodes (LNs). Nanovaccines were attempted to improve vaccine delivery, however, clinical translation of most of them have been impeded by complicated large-scale manufacturing and safety concerns.

Methods

We developed an albumin-binding Evans blue derivative as LNhoming moieties and cell uptake mediators for cancer vaccine delivery. The safety of this derivative has been validated in 2 ongoing clinical trials involving more than 200 patients and healthy volunteers. By conjugating this derivative with molecular vaccines (CpG adjuvant, B16F10 melanoma-associated antigen Trp2, and MC38 colon adenocarcinoma-specific neoantigen Adpgk), we developed albumin-binding vaccine (AlbiVax) (Fig. 1A) to efficiently co-deliver molecular adjuvants and antigens to LNs. By PET imaging of radiolabeled AlbiVax in mice, we systematically optimized AlbiVax for LN-targeted delivery, and light-sheet fluorescence imaging and super-resolution confocal microscopy were employed to study vaccine behaviors in transparent LNs and single antigen-presenting cells (APCs). In three tumor models, we studied the antigen-specific immunogenicity and tumor therapy efficacy of AlbiVax, either alone or in combination with anti-PD-1 or Abraxane.

Results

Quantitative PET imaging revealed that AlbiVax was delivered to LNs more efficiently (20-fold for $\mathrm{CpG}$, up to 91 -fold for antigens) than unconjugated vaccines or vaccines emulsified in Incomplete Freud's Adjuvant (IFA) (Fig. 1B, Fig. 1F). Light-sheet fluorescence imaging elucidated the distribution of AlbiVax in transparent draining LNs (Fig. 1, Fig. 1D); and super-resolution imaging revealed efficient intracellular co-delivery of AlbiVax via albumin into endolysosome of APCs (Fig. 1E). AlbiVax elicited $>21$-fold higher frequency of Antigenspecific CD8 ${ }^{+}$cytotoxic T lymphocytes (CTLs) than IFA-emulsified vaccines and induced immune memory for $>5$ months (Fig. g). AlbiVax dramatically regressed or inhibited the progression of established primary or lung metastatic EG7.OVA (Fig. 1G), B16F10 (Fig. 1H), and MC38 tumors (Fig. 11). Remarkably, combining AlbiVax with immune checkpoint inhibitor anti-PD-1 further improved the therapeutic efficacy, and led to the complete regression of $60 \%$ MC38 tumors for at least 5 months, and triple combination of AlbiVax, anti-PD-1, and Abraxane exhibited synergistic therapeutic efficacy (Fig. 1H, Fig. 11). AlbiVax showed excellent safety profile throughout the study.

Conclusions

AlbiVax represents a promising safe, widely applicable, and robust $\mathrm{T}$ cell vaccine for combination cancer immunotherapy.

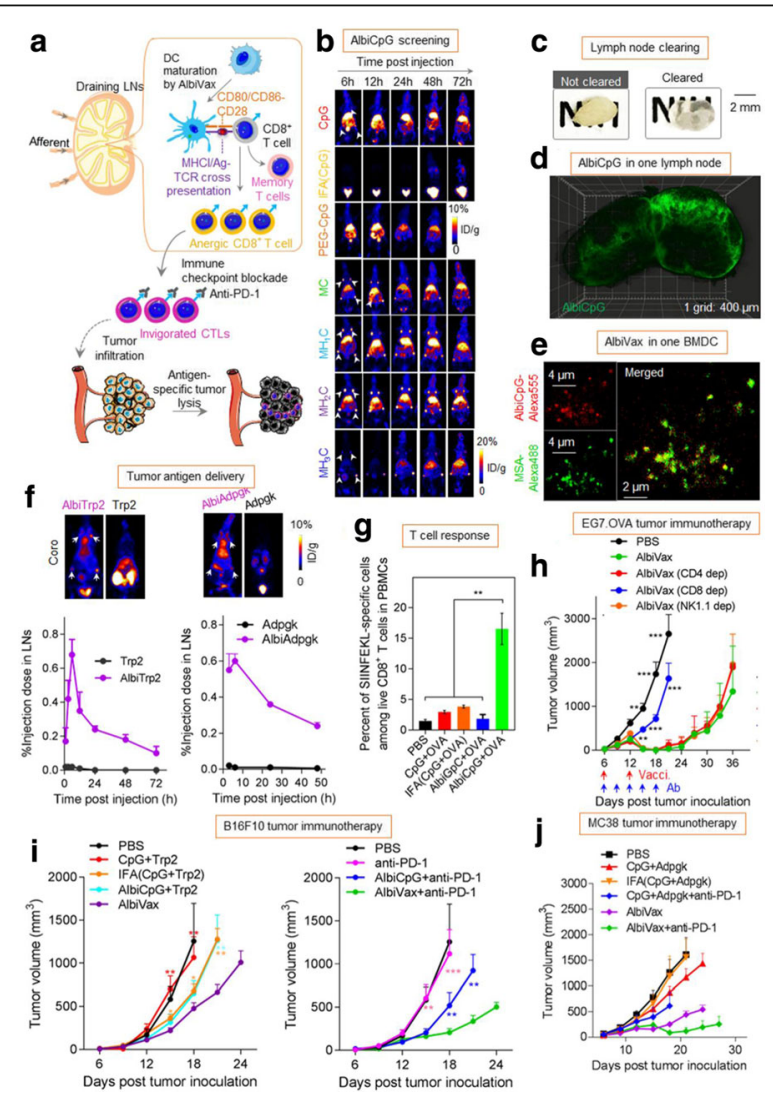

Fig. 1 (abstract P436). In vivo self-assembled albumin/vaccine nanocomplexes for lymph-node-targeted vaccine delivery in combination cancer immunotherapy. (a) Schematics of albuminbinding vaccine (AlbiVax) for LN-targeted vaccine delivery for cancer immunotherapy. $(b, f)$ by PET imaging of radiolabeled AlbiVax in small animals, AlbiVax was systematically optimized for optimal LN-targeted delivery. (c, d) By light-sheet fluorescence imaging, we elucidated the distribution of AlbiVax in draining LNs that were cleared to be transparent. (e) By super-resolution imaging, we discovered efficient intracellular co-delivery of AlbiVax via mouse serum albumin (MSA) into endolysosome of bone marrow derived dendritic cells (BMDCs). (g) AlbiVax elicited 21-fold higher frequency of Antigen-specific CD8+ cytotoxic T lymphocytes (CTLs) than IFAemulsified vaccine. (h-j) AlbiVax dramatically regressed or inhibited the progression of EG7.OVA (g), B16F10 (h), and MC38 tumors (i) 


\section{PHYSICIAN/NURSE/PHARM: Best Practices for Improving Cancer Immunotherapy}

\section{P437}

Mapping the treatment pathway for metastatic uveal melanoma (mUM) patients in England: A qualitative pilot study

Elisabeth Adams ${ }^{1}$, Chih-Yuan Cheng ${ }^{1}$, Joseph Sacco ${ }^{2}$, Sarah Danson ${ }^{3}$, Pippa Corrie ${ }^{4}$, Paul Nathan ${ }^{5}$, Peter Szlosarek ${ }^{6}$, Joanne Upton²,

Abolore Amuludun', Toby Toward ${ }^{7}$

${ }^{1}$ Aquarius Population Health Limited, London, United Kingdom; ${ }^{2}$ The Clatterbridge Cancer Centre, Wirral, United Kingdom; ${ }^{3}$ Weston Park Hospital, Sheffield, United Kingdom; ${ }^{4}$ Addenbrooke's Hospital,

Cambridge, United Kingdom; ${ }^{5}$ Mount Vernon Cancer Centre, London, United Kingdom; ${ }^{6}$ Barts Cancer Institute, London, United Kingdom; ${ }^{7}$ Immunocore Limited, Abingdon, United Kingdom

Correspondence: Toby Toward (chihyuan.cheng@aquariusph.com) Journal for ImmunoTherapy of Cancer 2017, 5(Suppl 2):P437

\section{Background}

mUM is a rare disease (annual UK incidence $<150$ patients), with a reported median overall survival of 7-12 months. In 2015, the UK published their first national uveal melanoma guidelines [1]. However, with few therapeutically effective options available for metastatic patients, it is unclear what patients actually receive as standard of care (SoC). This pilot study aimed to map the real-world pathway for recently diagnosed mUM patients, through qualitatively evaluating their clinical management and treatments received within SoC.

\section{Methods}

Based on treatment recommendations within national guidelines, an outline decision-tree for mUM patients was developed. On presenting the decision-tree to 5 specialist centres across England (January-June, 2017), 6 senior clinicians were interviewed about their typical: clinical management; medical resource use; treatment decision-making and patient flow(s) between local and regional facilities, when treating mUM patients. The interview data were analysed to populate centre-level decision-trees, and consolidated to inform a consensus SoC pathway.

Results

Interviews revealed considerable variation in first-line treatments offered at specialist centres:

- hepatic resection for liver metastases [1-25\%];

- loco-regional therapies for liver metastases (including percutaneous hepatic perfusion and radiofrequency ablation) [0-20\%];

- new treatments in clinical trials (TRAP, SelPac or IMCgp100) [10-80\%];

- immunotherapies (Ipilimumab, Pembrolizumab or Nivolumab) [0-54\%]; and

- chemotherapies (Dacarbazine or Temozolomide) [0-1\%].

There was, however, consensus on treatment priorities - if liver metastases were operable then surgical options were considered first. If non-operable, clinical trials were subsequently considered, before other therapeutic options. Whilst centres agreed that treatment should be initiated at a specialist (supra-regional) mUM centre, there were mixed attitudes on "if" and "how" patients could receive ongoing treatments at more local centres. Conclusions

The main reason suggested for treatment pathway variations amongst centres, was the absence of effective treatments. Introducing an effective therapeutic option at a defined optimum point in the pathway would be considered a "catalyst to transform the management of care". This in turn would lend itself to greater consistency of practice, harmonise care pathways and improve overall outcomes for mUM patients.

\section{References}

1. Nathan P, Cohen V, Coupland S, Curtis K, Damato B, Evans J, Fenwick S, Kirkpatrick L, Li O, Marshall E, McGuirk K, Ottensmeier C, Pearce N, Salvi S, Stedman B, Szlosarek P, Turnbull N. United Kingdom Uveal Melanoma Guideline Development Working Group, Uveal Melanoma UK National Guidelines. European Journal of Cancer. 2015; 51: 2404- 2412.

\section{P438}

Continued challenges and opportunities in oncologists' COMPREHension of clinical immunology and its relationship with cancer immunotherapy

Tara Herrmann', Charlotte Warren', Haleh Kadkhoda², Lianne Wiggins ${ }^{3}$

${ }^{1}$ Medscape Education Oncology, New York, NY, USA; ${ }^{2}$ Medscape

Education, New York, NY, USA; ${ }^{3}$ SITC, Milwaukee, WI, USA

Correspondence: Tara Herrmann (therrmann@medscape.net)

Journal for ImmunoTherapy of Cancer 2017, 5(Suppl 2):P438

\section{Background}

Cancer immunotherapy (CIT) is revolutionizing the treatment of cancer. As part of an ongoing educational curriculum, assessment of oncologists' knowledge, competence, and performance in the use of CIT identified multiple challenges and barriers. This study's objective was to assess and compare practicing oncologists' comprehension of, and confidence in, the interplay between the immune system and cancer tumor development and metastasis.

Methods

An expert panel was convened to identify knowledge, competence, and performance gaps in the area of CIT. Educational curricula consisting of 8 activities that focused on foundational education were developed in 2014-2016, and posted online. Intra-activity questions embedded in each activity allowed learners to self-report their confidence with CIT concepts, while matching participants' responses to knowledge-based questions presented before and after education were collected to measure reinforced learning and gains in comprehension. Confidentiality of respondents was maintained. Responses were collected from 5/2014-10/2016.

Oncologists $(N=4,684)$ who participated in the activities demonstrated on average a $20 \%$ improvement from initial baseline between years 1 and 2 . Additional education in year 3 was provided to help prevent decay in oncologists' comprehension of immune system functions. However, comparison of oncologists' knowledge of the role of immune checkpoints indicates that while gains observed in year 1 were maintained, a drop off was observed between years 2 and 3 . This is likely a result of more data becoming available on alternative CITs while simultaneously less foundational education was available.

Results

Oncologists continued to report a lack of confidence with the concepts of clinical immunology. Only $2 \%-11 \%$ of oncologists were very confident in their comprehension of the relationship between the immune system and tumor development. In addition, fewer than $50 \%$ of participants post-activity could recognize common $\mathrm{T}$ cell coreceptors that could be targeted as part of CIT. One quarter did not realize that tumors interfere with signal 1 by blocking $\mathrm{MHC}$ antigen processing.

\section{Conclusions}

This study demonstrates the value of and continued need for educational interventions on CIT. Retention of CIT concepts and improvements in knowledge of clinical immunology should facilitate competence among oncologists, which has valuable implications for use of CIT and for patient outcomes. 
P439

PDL-1 status and value for Extrapulmonary small cell carcinomas

Mohammed Salhab, Yiqin Xiong, Karen Dresser, Benjamin Chen, James Liebmann

University of Massachusetts, worcester, MA, USA

Correspondence: Mohammed Salhab

(mohammed.salhab@umassmemorial.org)

Journal for ImmunoTherapy of Cancer 2017, 5(Suppl 2):P439

\section{Background}

Extrapulmonary small cell carcinomas (ESCCs) are rare but aggressive tumors with an overall 5 -year survival rate of less than $15 \%$. Relapse is common despite rigorous chemotherapy and radiotherapy. Cancer immunotherapy targeting PD-1/PD-L1 pathway emerges as an increasingly appealing strategy $[1,2,3]$.

\section{Methods}

We investigated PD-L1 expression by immunochemistry (IHC) in ESCCs diagnosed at our institution from 1999-2016. 34 cases with sufficient material were selected for PD-L1 IHC analysis using clone E1L3N. Sites of origin include bladder (9), cervix (3), prostate (2), ovary (2), gallbladder (2), duodenum (1), esophagus (1), biliary tract (1), vagina (1), palate (1), ureter (1), and unknown primary (10).

Results

Two cases showed diffuse PD-L1 expression in both tumor and stromal cells, one from ureter and one from a gallbladder in origin. 10 cases $(29 \%)$ showed at least patchy PD-L1 expression in stromal cells. 22 cases (65\%) were completely negative for PD-L1 expression. Patients of both groups who was treated, did received chemotherapy +/- surgery or radiation. The overall response rate for PDL positive group was $80 \%$ while it was $67 \%$ for PDL-1 negative group (Table 1 ). The median overall survival for PD-L1 positive staining group, regardless of stage was 11.5 months' vs 7 months for PDL-1 negative group (Fig. 1). Patients with limited stage disease with positive PDL-1 showed an impressive 53 months' survival compared to PDL-1 negative limited disease with 15 months.

\section{Conclusions}

This study suggests that PD-L1 staining in extrapulmonary small cell carcinoma may be of prognostic relevance and further studies are required to understand the implications of immune dysregulation in these aggressive tumors. PD-1 inhibitors should be investigated in this group of patients.

\section{References}

1. Annemiek ME, Walenkamp A, Sonke G, Sleijfer D. Clinical and therapeutic aspects of extrapulmonary small cell carcinoma. Cancer Treatment Reviews. 2009; 35 :228-236.

2. Wong YN, Jack R, Mak V, Henrik M, Davies E. The epidemiology and survival of extrapulmonary small cell carcinoma in South East England, 1970-2004. BMC Cancer. 2009; 9:209.

3. Brennan S, Gregory D, Stillie A, Herschtal A, Manus M, Ball D. Should Extrapulmonary Small Cell Cancer BeManaged Like Small Cell Lung Cancer? Cancer.2010

Table 1 (abstract P439). See text for description

\begin{tabular}{l|l|l}
\hline Table 1 & $\begin{array}{l}\text { Positive PDL-1 } \\
(\mathbf{n = 1 2 )}\end{array}$ & $\begin{array}{l}\text { Negative PDL-1 } \\
(\mathbf{n = 2 2})\end{array}$ \\
\hline $\begin{array}{l}\text { Received } \\
\text { Treatment }\end{array}$ & $10(83 \%)$ & $16(73 \%)$ \\
\hline $\begin{array}{l}\text { Response rate to } \\
\text { treatments }\end{array}$ & $8(80 \%)$ & $11(67 \%)$ \\
\hline $\begin{array}{l}\text { Median Overall } \\
\text { Survival }\end{array}$ & 11.5 months & 7 months \\
\hline
\end{tabular}

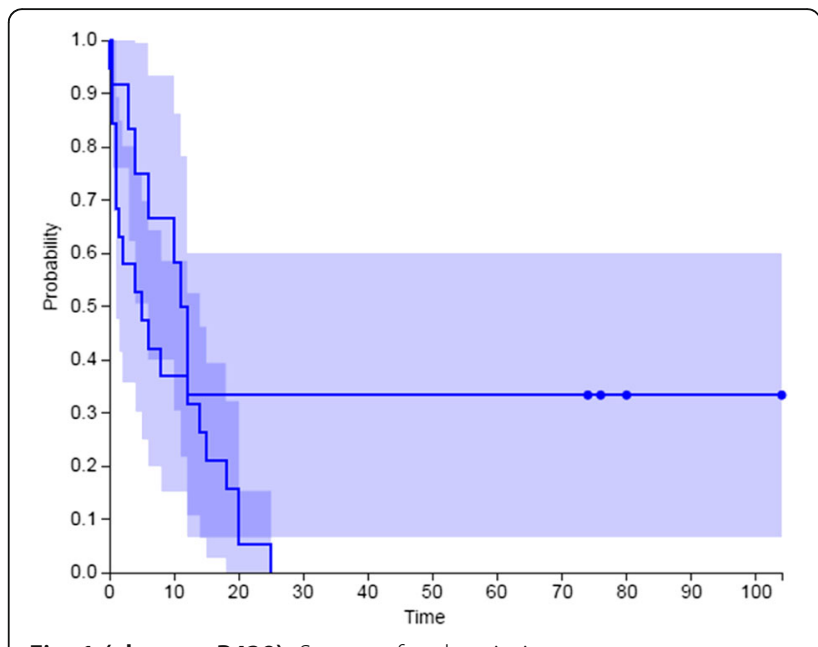

Fig. 1 (abstract P439). See text for description

\section{PHYSICIAN/NURSE/PHARM: Case Studies demonstrating exceptional responses}

P440

Durable complete response with PANVAC and Trastuzumab in metastatic triple positive breast cancer (mTPBC)

Margaret Gatti-Mays', Mahsa Mohebtash², Ravi Madan', Julius Strauss', Marijo Bilusic ${ }^{1}$, Caroline Jochems ${ }^{1}$, Myrna Rauckhorst ${ }^{1}$, Elizabeth Jones ${ }^{3}$, William Dahut ${ }^{1}$, Jeffrey Schlom, James Gulley ${ }^{1}$

${ }^{1}$ National Cancer Institute, NIH, Bethesda, MD, USA; ${ }^{2}$ Medstar Union Memorial Hospital, Baltimore, MD, USA; ${ }^{3}$ National Institutes of Health, Bethesda, MD, USA

Correspondence: Margaret Gatti-Mays (margaret.gatti-mays@nih.gov) Journal for ImmunoTherapy of Cancer 2017, 5(Suppl 2):P440

\section{Background}

First line treatment of metastatic HER2+ breast cancer with Docetaxel+Trastuzumab(TH) carries a median PFS of 12 months and OS of 40months. ${ }^{1}$ The addition of Trastuzumab+/-Pertuzumab to chemotherapy prolongs survival in the metastatic setting ${ }^{1}$ but no therapies are curative. Trastuzumab has immune-related effects including antibody-dependent cell-mediated cytotoxicity and cross-presentation of tumor antigens. PANVAC is a recombinant poxviral vaccine that contains transgenes for MUC-1, CEA and Tcell costimulatory molecules (B7.1, ICAM-1 and LFA-3). For optimal response, PANVAC employs a prime-boost regimen, using vaccinia (PANVAC-V) as the primer and fowlpox (PANVAC-F) as the boost. We report an exceptional response of mTPBC to PANVAC+Trastuzumab after progression on Letrozole+Trastuzumab.

Methods

A pilot study of PANVAC+Sargramostim(NCT00088413) demonstrated minimal side effects and suggested clinical benefit in low disease burden. $^{2}$ On Day 1(D1), patients received PANVAC-V $2 \times 10$ e8pfu SC followed by PANVAC-F $1 \times 10$ e9pfu SC every 2 weeks $\times 3$, then monthly x 12 followed by maintenance every 3 months until progression. Sar-

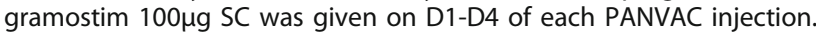
Scans were performed every 2-3months.

Results

A 32-year-old female, BRCA wildtype, with poorly differentiated, invasive ductal carcinoma, ER60\%, PR70\%, HER2+(IHC3+) had biopsyconfirmed metastasis to an axillary node and liver shortly after diagnosis. After bilateral oophorectomy, she received THx6cycles followed by a partial hepatectomy, which demonstrated a pathologic complete 
response. She received radiation to supraclavicular lymph nodes with a tumor bed boost. After 12 months on Letrozole+Trastuzumab she recurred. While on Letrozole she completed 6injections for a HER2/neu peptide vaccine trial(NCT00194714). Due to progression in bilateral hilar and subcarinal $(2.8 \mathrm{~cm})$ lymph nodes determined by PET, she enrolled on trial at $\mathrm{NCl}$ in January 2008. Per protocol above, she received PANVAC-V followed by PANVAC-F. Restaging at 10months showed a partial response $(0.8 \mathrm{~cm})$ and at 18 months showed a complete response(CR). She has remained on-study and receives PANVAC-F+Sargramostim every 90days and Trastuzumab every 3weeks. Restaging in May 2017 shows a continued $C R$ at 113 months.

\section{Conclusions}

This case report highlights the potential of immunotherapy and possibly multiple immunotherapies in a patient with breast cancer. Further study is required to understand the mechanism of response in this patient and if it can be applied on a broader scale.

Trial Registration

NCT00088413

\section{References}

1. Swain SM, Baselga J, Kim SB, et al. Pertuzumab, trastuzumab, and docetaxel in HER2-positive metastatic breast cancer. N Engl J Med. 2015;372(8):724-734

2. Mohebtash M, Tsang KY, Madan RA, et al. A pilot study of MUC-1/CEA TRICOM poxviral-based vaccine in patients with metastatic breast and ovarian cancer. Clin Cancer Res. 2011;17(22):7164-7173.

\section{P441}

Activity of Nivolumab (Opdivo ${ }^{\circledR}$ in relapsed progressive peripheral T-cell Lymphoma-NOS (PTCL-NOS)

Philip A Haddad', David Sommerhalder ${ }^{2}$

'Overton Brooks VAMC, Shreveport, LA, USA; ${ }^{2}$ LSUHSC-S, Shreveport, LA, USA

Correspondence: Philip A Haddad (haddad8838@msn.com)

Journal for ImmunoTherapy of Cancer 2017, 5(Suppl 2):P441

\section{Background}

Nivolumab is a member of the family of check-point inhibitors. It is a PD-L1 inhibitor that has been approved by the FDA as treatment for several solid tumors including non-small cell lung cancer. It's also approved to treat Hodgkin's Lymphoma after failure of several lines of therapy. PTCL is often aggressive and incurable. Compared to B-cell lymphomas, they tend to present at more advanced stages and respond less frequently and with shorter duration to the same chemotherapy regimens. Nevertheless, PCTL is still approached with suboptimal B-cell therapeutic strategies. As to the recently approved PTCL specific therapies, they are palliative in nature. An urgent need for better therapeutic agents in this desperate lymphoma continues to exist.

Methods

We report on the activity of Nivolumab as a $4^{\text {th }}$ line treatment in a case of relapsed and rapidly progressive PTCL-NOS.

Results

Our patient was a 62 y.o. white man who was diagnosed with poor risk stage IVA PTCL and Stage IA lung squamous cell carcinoma in 2014. Due to his borderline functional performance status and decreased left ventricular ejection fraction, he was treated with Pralatrexate that is approved for PTCL and has activity in lung cancer. He received 2 cycles of Pralatrexate which stabilized both malignancies for 3 months after which they progressed. Subsequently, he was given Carboplatin and Gemcitabine combination that has activity in both malignancies. This combination seemed to stabilize his lung cancer and PTCL for 4 months. After the $5^{\text {th }}$ cycle, the patient's lymphoma progressed. At this time, the patient was started on Belinostat to address his PTCL. Initially, PTCL responded to Belinostat and then stabilized for 5 months. After the fifth cycle, his lung cancer started progressing requiring lung cancer directed therapy. He was started on Nivolumab which stabilized his lung cancer. After the $4^{\text {th }}$ dose of Nivolumab further unexpected PTCL response was noted clinically and on follow up imaging. This response was maintained for a period of 15 months spanning 27 cycles of Nivolumab without any significant toxicity. Unfortunately, by the $27^{\text {th }}$ cycles the patient's lung cancer progressed and he decided to stop all treatment and enroll in hospice where he eventually expired due to his progressive lung cancer.

Conclusions

This case demonstrates for the first time the notable activity and clinical benefit of Nivolumab as a single agent in PTCL-NOS. Further evaluation of Nivolumab's activity in PTCL as well its incorporation into PTCL treatment approaches need additional study and validation.

P442

Pseudoprogression manifesting as recurrent ascities with anti-PD-1 therapy

Randy Sweis, Yuanyuan Zha, Russell Szmulewitz, Lomax Pass,

Tara Chongsuwat, Jason Luke, Thomas Gajewski

University of Chicago, Chicago, IL, USA

Correspondence: Randy Sweis (rsweis@uchicago.edu)

Journal for ImmunoTherapy of Cancer 2017, 5(Suppl 2):P442

\section{Background}

Pseudoprogression occurs when patients treated with immunotherapy have an initial increase in apparent tumor size prior to regression. This phenomenon results from an influx of activated immune cells, occurring in up to $7 \%$ of patients [1]. Ascites is also a common manifestation of progressive malignancy from peritoneal metastases. However, ascites has not been previously reported as a manifestation of pseudoprogression. We describe a metastatic urothelial cancer patient with progressive ascites and concurrent reduction in tumor burden on anti-PD-1 therapy.

Methods

Pembrolizumab was given 200 mg IV every 3 weeks. Computed tomography scans were obtained and tumor burden assessed by RECIST v1.1. Peritoneal fluid was analyzed for cytology and fluorescence-activated cell sorting (FACS) using FlowJo10.1r5. Multiplex cytokine assay was performed on peritoneal fluid. Normal donor PBMCs stimulated with phorbol myristate acetate/ionomycin was used as a positive control.

Results

A 62-year-old female with urothelial bladder cancer previously treated with chemotherapy and radiation presented with recurrent metastatic disease including two peritoneal nodules and an enlarged para-aortic lymph node. Pembrolizumab was started and she had a significant partial response with a $49 \%$ reduction in target lesions. During therapy, she developed large-volume ascites. Initial paracentesis showed a serum-ascites albumin gradient $>1.5$, total protein of $1.2 \mathrm{~g} / \mathrm{dL}$, and leukocyte count of $231 / \mu \mathrm{L}$ including $45 \%$ lymphocytes. Cytology from four separate paracenteses over 8 weeks showed reactive cellular changes/inflammation, but no malignant cells were identified.

FACS analysis on peritoneal fluid showed $\mathrm{CD}^{+}$lymphocytes comprised $53 \%$ of $\mathrm{CD}^{2} 5^{+} \mathrm{CD}^{+}$cells. The majority of $\mathrm{CD} 8^{+}$lymphocytes were PD-1 ${ }^{+}$(78\%). Class II-MHC (HLA-DR) and CD40 ligand (CD154) were expressed on $43 \%$ and $20 \%$ of $C D 8^{+}$lymphocytes, respectively. All three markers were increased relative to normal donor PBMC controls, suggesting a locally high number of antigen-experienced, activated T cells. FoxP3 ${ }^{+} \mathrm{CD} 25^{+}$Tregs were also detected in $17 \%$ of the $\mathrm{CD}_{4} 5^{+} \mathrm{CD}^{+} \mathrm{CD}^{+}$compartment. Multiplex cytokine assay for 38 analytes was performed. The most significantly elevated analytes were IL-6, IL-15, G-GSF and FGF-2 (each >2-fold above control).

Conclusions

Pseudoprogression in cancer patients treated with immunotherapy can manifest as recurrent ascites due to a rapid influx of activated T lymphocytes into the peritoneum. Since ascites can also be caused by disease progression, it is important to clinically distinguish the two, so that treatment may be appropriately continued or stopped.

\section{References}

1.Blumenthal,

et al. Treatment beyond progression with immune checkpoint

inhibitors-known unknowns. JAMA Oncology. 2017. 
P443

Malignant melanoma metastatic to the heart: exploring patient outcomes and cardiovascular complications in the era of immunotherapy

Laura Talamo, William Grosh

University of Virginia, Charlottesville, VA, USA

Correspondence: William Grosh (It3j@virginia.edu)

Journal for ImmunoTherapy of Cancer 2017, 5(Suppl 2):P443

\section{Background}

Cardiac metastasis (CM) from melanoma is being recognized more frequently, but often presents without signal symptoms. A rate of CM of $64 \%$ has been quoted in one autopsy series [1], though the clinical diagnosis rate is considerably lower [2]. We evaluated patients with known CM for cardiac complications and impact of therapy, and defined survival among these patients.

\section{Methods}

This retrospective review identified patients with CM seen in the University of Virginia Oncology clinic between 2010 and 2017. Eligible patients were at least 18 years old with metastatic melanoma (MM) and CM.

Our primary outcome metrics were 2 year survival from time of diagnosis of melanoma (M) and MM. We recorded cardiac complications, diagnostic procedures, patient outcomes, and treatment modality. Ten patients were identified with the demographics outlined below. Longest follow up was 21 months from diagnosis of CM (DCM).

Results

Two year survival from $\mathrm{M}$ diagnosis was $70 \%$. Two year survival from DCM could not be calculated (two patients are still living at 9 and 21 months from DCM). Figure 1 shows Kaplan-Meier survival analysis. Of the eight patients who died, all died within one year of DCM. Responses to immunotherapy (IO) are shown in Table 2.

\section{Conclusions}

The rate of cardiac complications was low. Only one death was a direct result of CM (patient 10).

$90 \%$ of patients received IO. At the time of data collection, two patients remain ( 2 and 3 ) alive nine and 21 months since DCM; both received $>20$ cycles of P. Patients $1,2,3,8,9$, and 10 received $I O$ after DCM, but only patient 3's CM responded to IO (IN induction, $\mathrm{P}$ maintenance). Patient 1 did not respond to I; patient 2 was lost to follow up on IL-2; patient 8's response cannot be determined due to death from septic shock; patient 9 progressed through $\mathrm{P}$; and patient 10 did not respond after one cycle of $\mathrm{I}$. This study is limited by small patient numbers, but supports a poor prognosis for patients with $\mathrm{CM}$. Responses can be seen with $\mathrm{IO}$, specifically PD-L1 inhibitors, which may improve survival. Other treatments cannot be assessed. The only patient who survived one year after DCM has had an ongoing response to $\mathrm{P}$.

\section{References}

1. Glancy DL, Roberts WC. The heart in malignant melanoma. A study of 70 autopsy cases. Am J Cardiol. 1968:21:555-71.

2. Villa et al. Cardiac metastases of melanoma as first manifestation of the disease. J Radiol Case Rep. 2014;1;8(4):8-15.

\section{PHYSICIAN/NURSE/PHARM: irAE Management: Clinical Care and Best Practices}

\section{P444}

Inflammatory arthritis induced by the use of checkpoint inhibitors for immunotherapy of cancer

Noha Abdel-Wahab ${ }^{1,2}$, Jean H. Tayar ${ }^{1}$, Adi Diab ${ }^{1}$, Sang T. Kim ${ }^{1}$

Huifang Lu', Maria E. Suarez-Almazor ${ }^{1}$

'The University of Texas MD Anderson Cancer Center, Houston, TX, USA; ${ }^{2}$ Assiut University Hospitals, Faculty of Medicine, Assiut, Egypt

Correspondence: Maria E. Suarez-Almazor (nahassan@mdanderson.org) Journal for ImmunoTherapy of Cancer 2017, 5(Suppl 2):P444

\section{Background}

Checkpoint inhibitors (CPI) have revolutionized cancer treatment with remarkable survival benefits in multiple cancer patients. Yet, their use can be hampered by frequent immune-related adverse events
(irAEs), affecting any organ, with signs and symptoms usually seen in inflammatory and autoimmune diseases. There is paucity of data on CPI induced arthritis, primarily from case reports or small series. Methods

Patients developed arthritis while using CPI were identified from the Rheumatology Clinic at UT MD Anderson Cancer Center. We diagnosed arthritis if patients developed joint swelling after receiving any FDA approved $\mathrm{CPI}$, after ruling out other potential underlying causes or pathologies. Here we report 13 pts. Cancer types included melanoma $(n=5)$, non-small cell lung cancer $(n=4)$, renal cell carcinoma $(n=2)$, acute myeloid leukemia, and cancer colon (1 each). CPI included nivolumab ( $n=8)$, pembrolizumab ( $n=3)$, and ipilimumab and atezolizumab (1 each).

Results

Patients age ranged from $47-74$ years and $62 \%$ were male. CPI induced arthritis was the sole irAE in eight pts (62\%), and was associated with other irAEs including sicca symptoms (xerostomia, dry eyes), colitis, pancreatitis, or dermatitis in the remaining five (38\%). Overall, median time to onset of arthritis after the initiation of CPI was 6 (range 1 to 15) months. When occurred with other irAEs, arthritis always manifested up to 4 months after the first occurring irAE despite sufficient immunosuppressant treatment (infliximab and/or high dose steroid) and symptoms control, with the exception of one patient with sicca symptoms. Initial symptoms varied widely between small and large joints of upper and lower extremities. Over time, symmetrical polyarthritis resembling rheumatoid arthritis, predominantly involving small joints of the hands and wrists, along with larger joints was reported in six patients. Two had oligoarthritis resembling seronegative spondyloarthropathy with axial pain and enthesopathy. Four had asymmetrical inflammatory polyarthritis, and one had oligoarthritis. Rheumatoid factor and/or anticitrullinated peptide antibodies were positive in two patients, and antinuclear antibodies in two others. Eleven patients were treated with steroids, six were controlled, while five needed additional biological agents (4 tocilizumab, 1 infliximab) to achieve appropriate steroid taper (<7.5 mg). One patient improved with intra-articular steroid injection, and one received tocilizumab as first line.

Conclusions

Our data suggest that arthritis is a late occurring irAE that may be due to a different immunopathology. A multi-institutional collaborative work including tissue analysis is needed to enhance our understanding of the pathogenesis and design optiomal therapy without dampening antitumor immunity.

P445

Clinical features of immune checkpoint inhibitor-induced inflammatory arthritis differ in those treated with combination versus single agent therapy

Laura Cappelli, Clifton Bingham, Julie Brahmer, Patrick Forde, Dung Le,

Evan Lipson, Jarushka Naidoo, Lei Zhang, Ami Shah

Johns Hopkins, Baltimore, MD, USA

Correspondence: Laura Cappelli (Icappel1@jhmi.edu)

Journal for ImmunoTherapy of Cancer 2017, 5(Suppl 2):P445

\section{Background}

Multi-system immune-related adverse events (iRAE) can occur as a result of immune checkpoint inhibitors (ICls) targeting PD-1, PD-L1, and CTLA-4, with variations depending on tumor type and agent used. Inflammatory arthritis (IA) has been increasingly recognized as an iRAE that may occur as a result of anti-PD-1/PD-L1/CTLA-4 therapy used in the treatment of malignancy [1,2]. We evaluated associations between $\mathrm{ICl}$ regimen and clinical presentation of $\mathrm{IA}$ in a single institution.

Methods

Patients referred to rheumatology for suspected ICl-induced IA, were identified January 2013- July 2017. Patients were included if they developed de novo IA during or after ICl therapy and had no history of pre-existing autoimmune disease. Patients were included for the single agent group if they were on a PD-1 or PD-L1 inhibitor without CTLA-4 inhibition. Those in the combination group were treated with PD-1 and CTLA-4 inhibition. Clinical, demographic and laboratory data were obtained from the medical records. 


\section{Results}

Tumor types for included patients were melanoma, non-small cell lung cancer, small cell lung cancer, colon cancer, Hodgkin or cutaneous lymphoma, renal cell carcinoma, endometrial carcinoma, duodenal carcinoma, merkel cell carcinoma, basal cell carcinoma, or squamous cell carcinoma. The group treated with combination CTLA-4/PD-1 inhibition was significantly younger than the monotherapy group; those in the combination group were also more likely to be male and to have melanoma (Table 1). Combination therapy was associated with higher levels of C-reactive protein $(4 \mathrm{mg} / \mathrm{dl}$ vs. $0.7 \mathrm{mg} / \mathrm{dL})$ and a higher likelihood of having a large joint affected first in the arthritis course (table 1). All instances of reactive arthritis-like presentation (arthritis + sterile urethritis, conjunctivitis) were in the combination therapy group. Patients who received PD-1/PD-L1 monotherapy were more likely to have IA as their first IRAE. No patients had CCP antibodies; two patients were positive for rheumatoid factor.

\section{Conclusions}

This study illustrates the diversity in clinical phenotypes of patients with ICl-induced IA. Differences in presentation may be explained by the regimen used to treat a patient's malignancy. Understanding the diversity of clinical presentations will help oncologists identify and treat this increasingly important iRAE.

\section{References}

1. Cappelli LC, Gutierrez AK, Baer AN, et al. Inflammatory arthritis and sicca syndrome induced by nivolumab and ipilimumab. Ann Rheum Dis 2017; 761):43-50.

2. Calabrese C, Kirchner E, Kontzias K, et al. Rheumatic immune-related adverse events of checkpoint therapy for cancer: case series of a new nosological entity. RMD open 2017;3(1):e000412.

Table 1 (abstract P445). See text for description

\begin{tabular}{|c|c|c|c|c|}
\hline & All patients $(n=32)$ & \begin{tabular}{|l|} 
PD-1/PD-L1 \\
monotherapy (n=18) \\
\end{tabular} & $\begin{array}{l}\text { Combination CTLA-4/ } \\
\text { PD-1 therapy ( } n=14)\end{array}$ & \begin{tabular}{|l|}
$\begin{array}{l}\text { p- } \\
\text { value }\end{array}$ \\
\end{tabular} \\
\hline Age: median (IOR) & \begin{tabular}{|l|}
$59(54-67.5)$ \\
\end{tabular} & $\begin{array}{l}62(55-73) \\
\end{array}$ & $57.5(45-59)$ & \begin{tabular}{|l|l|}
0.02 \\
\end{tabular} \\
\hline Female sex: $N(\%)$ & $13(40.6 \%)$ & $10(55.6 \%)$ & $3(21.4 \%)$ & 0.08 \\
\hline Tumor Type: $N(\%)$ & $\begin{array}{l}\text { Melanoma: } 8(25 \%) \\
\text { NSCLC: } 12(37.5 \%) \\
\text { Other. } 12(37.5 \%)\end{array}$ & $\begin{array}{l}\text { Melanoma: } 2(11.1 \%) \\
\text { NsCLC: } 9(50 \%) \\
\text { Other. } 7(38.9 \%)\end{array}$ & $\begin{array}{l}\text { Melanoma: } 6(42.9 \%) \\
\text { Nsac: } 3(21.4 \%) \\
\text { Other: } 5(35.7 \%)\end{array}$ & 0.09 \\
\hline $\begin{array}{l}\text { Partial/complete } \\
\text { tumor response: } N(\%) \\
N=31\end{array}$ & Yes: $20(64.5 \%)$ & Yes: $11(64.7 \%)$ & Yes: $9(64.3 \%)$ & 0.98 \\
\hline $\begin{array}{l}\text { Additional IRAE } \\
\text { present: } \mathrm{N}(\%)\end{array}$ & \begin{tabular}{|l|} 
None: $10(31.3 \%)$ \\
1 IRAE: $13(40.6 \%)$ \\
>1 IIRAE: $9(28.1 \%)$
\end{tabular} & $\begin{array}{l}\text { None: } 7(38.9 \%) \\
\text { 1 IRAE: } 8(44.4 \%) \\
\text { >1 IRAE: } 2(16.7 \%)\end{array}$ & $\begin{array}{l}\text { None: } 3(21.4 \%) \\
1 \text { IRAE: } 5(35.7 \%) \\
\text { >1 IRAE: } 6(42.9 \%)\end{array}$ & 0.32 \\
\hline $\begin{array}{l}\text { Co-occurrence of } \\
\text { other IRAEs"* }\end{array}$ & \begin{tabular}{|l|} 
Colitis: 10 \\
Thyroid disease: 5 \\
Pneumonit is: 4 \\
Hypophysitis: 3 \\
Rash: 4 \\
Sicca: 3 \\
Other: 4 \\
\end{tabular} & \begin{tabular}{|l|} 
Colitis: 2 \\
Thyroid dise ase: 2 \\
Pneumonit is: 3 \\
Hypophysitis: 1 \\
Rash: 2 \\
Sicca: 1 \\
Other: 2 \\
\end{tabular} & $\begin{array}{l}\text { Colitis: } 8 \\
\text { Thyroid dise ase: } 3 \\
\text { Pneumonit is: } 1 \\
\text { Hypophysitis: } 2 \\
\text { Rash: } 2 \\
\text { Sicca: } 2 \\
\text { Other: } 2\end{array}$ & \\
\hline $\begin{array}{l}\text { Time to first IRAE Dx } \\
\text { (months): median } \\
\text { (IQR) } \\
\text { Total }=22\end{array}$ & $3(15-10)$ & $7.5(2-16)$ & $2(1-4.25)$ & 0.06 \\
\hline $\begin{array}{l}\text { Time to lA Sx } \\
\text { (months): median } \\
\text { (IQR) }\end{array}$ & $5(2-8)$ & $4(2-7)$ & $5.75(5-8)$ & 0.27 \\
\hline $\begin{array}{l}\text { Time to LA DX } \\
\text { (months): median } \\
\text { (IQR) }\end{array}$ & $9(4.5-17.5)$ & $7(4-21)$ & $9.5(6-16)$ & 0.79 \\
\hline $\begin{array}{l}\text { IA as } 1^{n} \text { IRAE: } n(\%) \\
\text { First joint/s affected: } N \\
\text { (\%) }\end{array}$ & \begin{tabular}{|l|}
$17(53.1 \%)$ \\
Knee: $17(53.1 \%)$ \\
Other large: $7(21.9 \%)$ \\
Small joint/s: $8(25 \%)$
\end{tabular} & \begin{tabular}{|l|}
$13(72.2 \%)$ \\
Knee: $:(38.9 \%)$ \\
Other large: $3(16.7 \%)$ \\
Small joint/s: $8(44.4 \%)$
\end{tabular} & $\begin{array}{l}4(28.6 \%) \\
\text { Knee: } 10(71.4 \%) \\
\text { Other large: } 4(28.6 \%) \\
\text { Small joint/s: } 0(0 \%)\end{array}$ & $\begin{array}{l}0.03 \\
0.01\end{array}$ \\
\hline $\begin{array}{l}\text { \# Swollen joints: } \\
\text { median (IQR) } \\
\text { Reactive arthritis triad: } \\
\text { N (\%) }\end{array}$ & $\begin{array}{l}7(4-10.5) \\
\text { Yes: } 3(9.7 \%)\end{array}$ & $\begin{array}{l}8.5(5-11) \\
\text { Yes: } 0(0 \%)\end{array}$ & $\begin{array}{l}6(4-10) \\
\text { Yes: } 3(23.0 \%)\end{array}$ & $\begin{array}{l}0.60 \\
0.06\end{array}$ \\
\hline $\begin{array}{l}\text { CRP (mg/dL) median } \\
\text { (IQR) }\end{array}$ & $2(0.3-6.3)$ & $0.7(0.2 \cdot 3.3)$ & $4(0.5 \cdot 9.2)$ & 0.02 \\
\hline
\end{tabular}

Demographic and clinical variables at initial evaluation for inflammatory arthritis
P446

Immunosuppression use and clinical outcomes in patients with immune checkpoint inhibitor -induced inflammatory arthritis Laura Cappelli, Clifton Bingham, Julie Brahmer, Patrick Forde, Dung Le, Evan Lipson, Jarushka Naidoo, Lei Zheng, Ami Shah Johns Hopkins, Baltimore, MD, USA

Correspondence: Laura Cappelli (Icappel1@jhmi.edu)

Journal for ImmunoTherapy of Cancer 2017, 5(Suppl 2):P446

\section{Background}

Inflammatory arthritis (IA) resulting from immune checkpoint inhibitors (ICls) is an increasingly recognized immune-related adverse event. The requirement for immunosuppression to treat $I A$ including TNF-inhibitors (TNF-I), has been documented in previous case series [1, 2]. How often immunosuppression is required to treat ICl-induced IA is unclear. The consequences of longterm immunosuppression on tumor response from $\mathrm{ICls}$ is also uncertain.

Methods

Patients treated at Johns Hopkins Rheumatology for IA due to ICls were included if they received anti-CTLA-4, anti-PD-1, and/or anti-PDL1 therapy and developed IA during or after therapy. Patients with pre-existing autoimmune disease were excluded. Patients were managed by rheumatology and oncology based on preliminary recommendations for ICl-induced IA treatment [3].

Results

Included patients $(n=32)$ had melanoma, non-small cell lung cancer (NSCLC), small cell lung cancer, colon cancer, Hodgkin or cutaneous lymphoma, renal cell carcinoma, endometrial carcinoma, duodenal carcinoma, merkel cell carcinoma, basal cell carcinoma, or squamous cell carcinoma. Of these patients, 20 (63\%) had a partial or complete tumor response to ICls clinically or by RECIST criteria. Twenty six (81.3\%) required corticosteroids for management of their IA. Eleven patients (34.3\%) required additional immunosuppression. TNF-Is were used in 7 patients (tumor types: melanoma $n=6, \operatorname{RCC} n=1$ ). Methotrexate or leflunomide was used in 4 patients (tumor types: NSCLC, small cell lung cancer, duodenal carcinoma, endometrial carcinoma). Duration of TNF-I use ranged from 2-16 months. Four patients treated with TNF-I, all with melanoma, had complete response by RECIST criteria or no evidence of disease at ICl start. None of these patients had tumor progression while on TNF-I (duration: 3-16 months). Of the patients treated with methotrexate or leflunomide, 3 had a complete or partial response to $\mathrm{ICls}$, and none had tumor progression during 2-12 months of IA management.

Conclusions

In a cohort of patients with ICl-induced IA, the majority of patients required corticosteroids to control IA symptoms. In this series, TNFinhibitors and other forms of non-corticosteroid immunosuppression did not affect tumor response in those who had an initial response to ICls for malignancy.

\section{References}

1. Cappelli LC, Gutierrez AK, Baer AN, et al. Inflammatory arthritis and sicca syndrome induced by nivolumab and ipilimumab. Ann Rheum Dis 2017;76(1):43-50

2. Calabrese C, Kirchner E, Kontzias K, et al. Rheumatic immune-related adverse events of checkpoint therapy for cancer: case series of a new nosological entity. RMD open 2017;3(1):e000412.

3. Naidoo J, Cappelli LC, Forde PM, et al. Inflammatory Arthritis: A Newly Recognized Adverse Event of Immune Checkpoint Blockade. Oncologist 2017;22(6):627-30 
P447

Infliximab associated with faster symptom resolution compared to corticosteroids alone for management of immune related enterocolitis

Daniel H Johnson ${ }^{1}$, Chrystia Zobniw², Van A Trinh'², Roland Bassett ${ }^{2}$, Junsheng $\mathrm{Ma}^{2}$, Jamie Anderson ${ }^{2}$, Jennifer Davis' ${ }^{2}$, Jocelyn Joseph², Marc Uemura ${ }^{2}$, Cassian Yee ${ }^{2}$, Rodabe Amaria², Sapna Patel², Hussein Tawbi ${ }^{2}$, Isabella Glitza², Michael A Davies's, Michael Wong², Scott Woodman'2, Patrick Hwu', Wen-Jen Hwu'2, Yinghong Wang', Adi Diab ${ }^{2}$

${ }^{1}$ University of Texas MD Anderson Cancer Center, Houston, TX, USA; ${ }^{2}$ UT-MD Anderson Cancer Center, Houston, TX, USA

Correspondence: Daniel H Johnson (dhjohnson@mdanderson.org); Adi Diab Journal for ImmunoTherapy of Cancer 2017, 5(Suppl 2):P447

\section{Background}

Immune-related enterocolitis (irEC) is the most common serious complication from checkpoint inhibitors (CPIs) that can lead to bowel perforation, sepsis, and death. The current front-line treatment for irEC is high-dose corticosteroids (CS). However, prolonged high-dose CS therapy has significant toxicities and side effects, and prolonged therapy may reduce CPI anti-tumor activity. Early addition of TNF-alpha inhibitors such as infliximab may expedite symptom resolution and shorten CS duration. The only prospective trial evaluating infliximab with CS versus CS alone for irEC (NCT02763761) has recently been terminated. Thus we conducted a retrospective study to evaluate symptom resolution in patients with irEC treated with infliximab + CS versus CS alone. Methods

The medical records of patients with irEC between January 1, 2012 and June 30, 2017 were reviewed under an institution review boardapproved protocol. Patient demographics, CPI regimen (aCTLA-4, aPD$1 / L-1$, alone or in combination), colitis grade, colitis treatment, treatment-related toxicities, time to symptom resolution, and CS duration were collected. The primary-endpoint was time to symptom resolution for irEC (times to diarrhea resolution and to initiation of steroid titration) for patients managed with versus without infliximab. Duration of CS and rate of CS-associated toxicities were secondary-endpoints. Categorical and continuous variables were assessed between groups by Fisher's exact test and Kruskal-Wallis test (or two-sample t-test).

Results

Among 75 patients with irEC, $39(52 \%)$ received CS alone and 36 $(48 \%)$ received infliximab within a median of 8 days after CS initiation. Patient characteristics (age, comorbidities, cancer type, immunotherapy regimen, and initial steroid dose), were similar between the two arms. The incidence of grade $3+$ colitis was higher in the infliximab + CS arm $(86.1 \%$ vs. $34.2 \%, p<0.001)$. Despite this, times to diarrhea resolution (median 3 vs. 9 days, $p<0.001$ ) and to steroid titration (median 4 vs. 13 days, $\mathrm{p}<0.001$ ) were shorter in patients treated with infliximab + CS versus CS alone. Rates of CSassociated toxicities ( $39 \%$ vs. $54 \% ; p=0.249)$ and total steroid duration (median 35 days vs. 51 days; $p=0.241$ ) were numerically lower in the infliximab-treated patients (Table 1).

\section{Conclusions}

To our knowledge this is the first study to compare time to symptom resolution for irEC with infliximab + CS versus CS alone. Despite higher incidence of grade 3+ colitis, infliximab treatment was associated with a statistically significant shorter time to symptom resolution without additional toxicities. The data suggest that early introduction of infliximab should be considered for patients with irEC until definitive prospective clinical trials.

Table 1 (abstract P447). See text for description

\begin{tabular}{|c|c|c|c|c|c|}
\hline Variable & $\mathbf{N}$ & Overall & Infliximab & Steroid & $\mathbf{P}$ \\
\hline Grade of colitis, $\mathbf{n}(\%)$ & 74 & & & & $<0.001$ \\
\hline Grade 1 & & $8(10.8)$ & $0(0.0)$ & $8(21.1)$ & \\
\hline Grade 2 & & $22(29.7)$ & $5(13.9)$ & $17(44.7)$ & \\
\hline Grade 3+ & & $44(59.5)$ & $31(86.1)$ & $13(34.2)$ & \\
\hline \multicolumn{6}{|l|}{ Symptom Resolution } \\
\hline $\begin{array}{l}\text { Time to steroid titration, } \\
\text { Median }\end{array}$ & 74 & 6 & 4 & 13 & $<0.001$ \\
\hline $\begin{array}{l}\text { Time to diarrhea resolution, } \\
\text { Median }\end{array}$ & 68 & 4 & 3 & 9 & $<0.001$ \\
\hline Total duration of steroid, Median & 67 & 42 & 35 & 51 & 0.241 \\
\hline
\end{tabular}

P448

Incidence of thyroid test function abnormalities in patients receiving immune-checkpoint inhibitors for cancer treatment Nisha Patel, Anais Oury, Gregory Daniels, Lyudmila Bazhenova,

Sandip Patel

University of California San Diego, La Jolla, CA, USA

Correspondence: Nisha Patel (nip011@ucsd.edu)

Journal for ImmunoTherapy of Cancer 2017, 5(Suppl 2):P448

\section{Background}

With the advent of immune-checkpoint inhibitor (ICl) therapy (antiCTLA-4, anti-PD-1), immune related adverse events (irAE) such as thyroid function test abnormalities (TFTA) are common with a reported incidence range of $2-15 \%$ depending upon the ICI utilized $[1,2]$. The aim of this study was to describe the incidence of TFTAs retrospectively in patients who received ICI therapy.

Methods

A total of 285 patients were reviewed (178 male, 107 female; ages 16-94) of which 218 had no baseline TFTA, 61 had baseline TFTAs, and 6 had history of thyroidectomy and were excluded. Patients received at least one dose of ipilimumab and/or nivolumab or pembrolizumab. Post-ICl therapy TFTA was classified according to definitions of thyroid abnormalities when possible [3].

Results

A total of 35\% (76/218) patients had new onset TFTAs on ICI therapy. Of note, $70.5 \%$ (43/61) had baseline TFTA that were exacerbated by ICl therapy. Median time to new onset or exacerbated baseline TFTA were 46 \& 33 days respectively. Of note, $65 \%$ (20/31) of patients on both ipilimumab and nivolumab had new onset TFTA, compared to $31.3 \%$ (15/48) with ipilimumab, 31.5\% (28/89) nivolumab, 26\% (13/50) pembrolizumab (Table 1).

Conclusions

The incidence of TFTAs with ICl therapy was higher than previously reported. Patients with baseline TFTA and/or receiving ipilimumab and nivolumab combination therapy had a higher incidence of TFTA than one agent ICl therapy. In conclusion, we recommend more frequent evaluation of TFT in the first two months, especially in those with baseline TFTA.

\section{References}

1. Corsello S, Barnabei A, Marchetti P, et al. Endocrine Side Effects Induced by Immune Checkpoint Inhibitors.J Clin Endocrinol Metab. 2013:98(4):1361-1375.

2. Morganstein D.L, Lai Z, Spain L, et al. Thyroid abnormalities following the use of cytotoxic T-lymphocyte antigen-4 and programmed death receptor protein -1 inhibitors in the treatment of melanoma. Clinical Endocrinology. 2017;(0) 1-7

3. Rossi E, Sgambato A, De Chiara G, et al. Thyroid-Induced Toxicity of Check-Point Inhibitors Immunotherapy in the Treatment of Advanced Non-Small Cell Lung Cancer. J Endocrinol Diabetes. 2016; 3 (1):1-10.

Table 1 (abstract P448). See text for description

\begin{tabular}{|c|c|c|c|c|c|}
\hline $\begin{array}{l}\text { Thyroid function test } \\
\text { abnormality }\end{array}$ & $\begin{array}{l}\mid \& N \\
n=31\end{array}$ & $n=48$ & $\begin{array}{l}N \\
n=89\end{array}$ & $\begin{array}{l}P \\
n=50\end{array}$ & $\begin{array}{l}\text { Total } \\
n=218\end{array}$ \\
\hline Primary hypothyroidism & $\begin{array}{l}6.5 \% \\
(2)\end{array}$ & $\begin{array}{l}4.2 \%( \\
2)\end{array}$ & $\begin{array}{l}10.1 \%( \\
9)\end{array}$ & $\begin{array}{l}14 \% \\
(7)\end{array}$ & 20 \\
\hline Subclinical hypothyroidism & $\begin{array}{l}6.5 \% \\
(2)\end{array}$ & $\begin{array}{l}8.3 \% \\
(4)\end{array}$ & $\begin{array}{l}11.2 \\
(10)\end{array}$ & $8 \%(4)$ & 20 \\
\hline Central hypothyroidism & $\begin{array}{l}16.1 \% \\
(5)\end{array}$ & $\begin{array}{l}8.3 \% \\
(4)\end{array}$ & $\begin{array}{l}1.1 \% \\
(1)\end{array}$ & $2 \%(1)$ & 11 \\
\hline Primary overt hyperthyroidism & $\begin{array}{l}25.8 \% \\
(8)\end{array}$ & $\begin{array}{l}2.1 \% \\
(1)\end{array}$ & $0 \%(0)$ & $2 \%(1)$ & 10 \\
\hline Subclinical hyperthyroidism & $0 \%(0)$ & $\begin{array}{l}4.2 \% \\
(2)\end{array}$ & $\begin{array}{l}5.6 \% \\
(5)\end{array}$ & $0 \%(0)$ & 7 \\
\hline $\begin{array}{l}\text { Thyroiditis } \\
\text { (Hyperthyroidism } \square \text { Hypothyroidi } \\
\text { sm) }\end{array}$ & $\begin{array}{l}9.7 \% \\
\text { (3) }\end{array}$ & $\begin{array}{l}4.2 \% \\
\text { (2) }\end{array}$ & $\begin{array}{l}3.4 \% \\
\text { (3) }\end{array}$ & $0 \%(0)$ & 8 \\
\hline Total \% $(x / x)$ & $\begin{array}{l}64.5 \% \\
(20 / 31\end{array}$ & $\begin{array}{l}31.3 \% \\
(15 / 48 \\
)\end{array}$ & $\begin{array}{l}31.5 \% \\
(28 / 89)\end{array}$ & $\begin{array}{l}26 \% \\
(13 / 50\end{array}$ & $\begin{array}{l}35 \% \\
(76 / 218 \\
)^{2}\end{array}$ \\
\hline $\begin{array}{l}\text { Required thyroid replacement } \\
\text { therapy }\end{array}$ & $\begin{array}{l}32.3 \% \\
(10 / 31\end{array}$ & $\begin{array}{l}14.6 \% \\
(7 / 48)\end{array}$ & $\begin{array}{l}13.5 \% \\
(12 / 89)\end{array}$ & $\begin{array}{l}12 \% \\
(6 / 50)\end{array}$ & $\begin{array}{l}17.4 \% \\
(35 / 218\end{array}$ \\
\hline $\begin{array}{l}\text { Required thionamide treatment } \\
\text { for overt hyperthyroidism }\end{array}$ & $\begin{array}{l}22.6 \% \\
(7 / 31)\end{array}$ & $0 \%(0)$ & $0 \%(0)$ & $\begin{array}{l}2 \% \\
(1 / 50)\end{array}$ & $\begin{array}{l}3.7 \% \\
(8 / 218)\end{array}$ \\
\hline
\end{tabular}

Summary of new onset TFTA 
P449

Neurotoxicity in patients with metastatic solid tumors treated with immune checkpoint inhibitors: a single institution retrospective analysis

Bianca Santomasso, Rachna Malani, Aya Haggiagi, Jenessa Holder, Yelena Shames, Samuel Briggs, Margaret Callahan

Memorial Sloan Kettering Cancer Center, New York, NY, USA

Correspondence: Bianca Santomasso (Santomab@mskcc.org); Margaret Callahan

Journal for ImmunoTherapy of Cancer 2017, 5(Suppl 2):P449

\section{Background}

Immune checkpoint inhibitors (ICls) have dramatically improved survival for patients with melanoma and are playing an increasingly important role in the management of other tumor types. Anti-CTLA4, anti-PD1, and anti-PDL1 antibodies have been approved for multiple tumor types based on their clinical benefit, however they can be associated with a unique set of toxicities termed immune related adverse events (irAEs). We aimed to identify the incidence and clinical manifestations of patients who developed neurologic irAEs with ICls at our institution in order to inform investigation and management guidelines.

Methods

An IRB approved retrospective study involved review of charts and institutional databases at MSKCC. We identified patients who developed neurotoxicity while on at least one of the following ICls: antiCTLA4, anti-PD1 or anti-PD-L1 over a 6 year period (1/1/2010-11/16/ 2016).

Results

We identified a total of 3,804 patients who were treated with one or more $\mathrm{ICl}$ during the period of review. Neurotoxicity was observed in 81 patients $(2.1 \%)$ and affected both central and peripheral nervous systems. 31 patients $(38.2 \%)$ received more than one ICl. Median number of cycles prior to developing toxicity was 3 (1-29). Ten patients had more than one neurotoxicity. The various neurologic phenotypes observed in patients included: sensory neuropathy, encephalopathy, headache and aseptic meningitis, myasthenia gravislike syndrome, myopathy, autonomic neuropathy, radiculopathy, brachial plexitis, mononeuritis multiplex, Guillain-Barre syndrome or chronic inflammatory demyelinating polyneuropathy, paraneoplastic neurologic disorder, and PRES. 43 patients required hospital admission and 2 required ICU level of care. A diagnosis of neurotoxicity was based upon the temporal association with $\mathrm{ICls}$ and appropriate workup including but not limited to neurologic consultation, lumbar puncture, and neuroimaging. Patients were treated with drug holiday, observation, corticosteroids, plasma exchange and/or IVIG.

\section{Conclusions}

As ICls are being used with increased frequency, oncologists and other health care providers should be aware of the varied manifestations of neurotoxicity in order to ensure appropriate diagnosis, work up and treatment. Prompt treatment may relay a benefit in regards to outcome.

\section{PHYSICIAN/NURSE/PHARM: Patient Experience: Patient Education Regarding I}

\section{P450}

Educating cancer patients about immunotherapy: gains from attending a national educational immunotherapy workshop Claire Saxton, Heather Hollen, Marni Amsellem

Cancer Support Community, Washington, DC, USA

Correspondence: Claire Saxton

(hhollen@cancersupportcommunity.org)

Journal for ImmunoTherapy of Cancer 2017, 5(Suppl 2):P450

\section{Background}

Immunotherapy continues to offer promising treatments for many tumor types. Unfortunately, patients potentially eligible for immunotherapy are often not aware of options. According to a 2014 Cancer Support Community (CSC) online survey, only $34.8 \%$ of cancer survivors knew the term "immuno-oncology" and $64.9 \%$ had heard of "immunotherapy." Despite low awareness, most (84\%) wanted to know more about these topics. Recognizing this need, the CSC developed a national psychoeducational immunotherapy workshop. The current analyses investigate the program's informational and empowerment outcomes among attendees with cancer.

Methods

From 2014-16, 706 program attendees of the CSC's Frankly Speaking About Cancer: Your Immune System \& Cancer Treatment workshop completed a post-program evaluation assessing knowledge (on 5-point scale) and program outcomes, including program satisfaction. Individuals with cancer $(n=478)$ comprised $68.0 \%$ of participants in the workshop. These attendees were White $(81.7 \%)$, female $(79.6 \%)$, and averaged 62.6 years old [1]. Workshop outcomes were ascertained by descriptive analyses and ANOVAs.

Results

Over half of workshop attendees with cancer (53.0\%) reported generally being very involved in their overall treatment decision-making, yet $47.2 \%$ had been unsure whether immunotherapy was a treatment option for them[m1]. [AKZ2] About one-third (36.8\%) had spoken with their doctor about immunotherapy and $26.2 \%$ inquired about potential side effects of immunotherapy; $44.1 \%$ had searched for information about immunotherapy on their own before the workshop. The majority (74.6\%) reported low knowledge about immunotherapy before the workshop.

After the workshop, most attendees with cancer reported a high level of knowledge of immunotherapy $(57.8 \%)$, representing a significant gain from attending the program $(F(4,416)=39.2, p<.01)$. Post-workshop, most (86.5\%) felt confident speaking about immunotherapy with their doctors. Furthermore, a majority felt "more confident" discussing potential side effects (86.3\%), asking about treatment options generally (90.3\%), and making decisions with their doctors $(84.8 \%)$ post-workshop. Nearly all $(92.5 \%)$ strongly or moderately recommend attending the workshop.

Conclusions

Results from the attendee evaluations demonstrate significant informational and confidence gains from attending a two-hour psychoeducational workshop. Attendees reported increased awareness of options, knowledge, and efficacy in discussing potential options for them. Results suggest that improving access to comprehensive information about immunotherapy and promoting communication between the patient and the healthcare team about treatment options and decision-making via an educational workshop is viable and can be implemented nationally to reach many.

\section{References}

1. Cancer Support Community. CSC Immuno-Oncology online survey. 2014.

\section{Tumor Microenvironment (Mechanisms and Therapies)}

P451

Alpha-TEA demonstrates antitumor activity against trastuzumab sensitive and resistant breast cancer

Emmanuel Akporiaye ${ }^{1}$, Mary Disis², Ekram Gad², Tobias Hahn', Brook Cole ${ }^{3}$, Michael Gough', Talicia Savage ${ }^{4}$, Hailing Lu ${ }^{5}$

${ }^{1}$ Providence Portland Medical Center, Portland, OR, USA; ${ }^{2}$ University of Washington School of Medicine, Seattle, WA, USA; ${ }^{3}$ University of

Minnesota, Minneapolis, MN, USA; ${ }^{4}$ Albert Einstein College of Medicine, Bronx, NY, USA; ${ }^{5}$ Immune Design, Seattle, WA, USA

Correspondence: Emmanuel Akporiaye (etakporiaye@gmail.com) Journal for ImmunoTherapy of Cancer 2017, 5(Suppl 2):P451 


\section{Background}

Alpha-tocopheryloxyacetic acid (a-TEA) is an orally administered, small molecule, multi-pathway cancer therapeutic agent that drives tumor cell apoptosis and autophagy to stimulate antigen cross-presentation. Anti-tumor activity of a-TEA in combination with trastuzumab was evaluated in vivo against established Her2/neu-expressing mouse tumors (MMC) and in vitro against HER2 positive, human trastuzumab-sensitive (BT-474) and trastuzumab-resistant (HR6) tumor cells.

Methods

In vivo studies: Alpha-TEA was formulated in mouse chow and administered to mice ad libitum. FVB/N-TgN (MMTVneu) on nutrient-matched control chow mice received s.c. implant of 1 million Her2/neu positive murine mammary cancer (MMC) cells. On day 25, after tumors were established, mice were transferred to a-TEA diet, and treatment with anti-Her2/neu monoclonal antibody (7.16.4) was initiated. Mice received 3 injections of 7.16.4 antibody 3 times a week for 1 month and tumor growth was monitored until day 60 . Mice demonstrating complete tumor regression were challenged with viable tumor cells to assess the establishment of a memory response. Spleen cells were removed from treated mice and assessed for cytokine production by ELISPOT assay. In vitro studies: Human trastuzumab-sensitive (BT-474) and a resistant sub-clone (HR-6) were treated continuously with a-TEA alone or in combination with trastuzumab, both at various doses and evaluated for clonogenic potential and activation of ERK and AKT signaling pathways.

Results

Treatment of mice harboring established MMC tumors with a-TEA plus trastuzumab caused complete tumor regression, and increased cytokine production by splenic lymphocytes. Individually, a-TEA and trastuzumab caused cell death and reduced colony formation of BT474 tumor cells in vitro; however only a-TEA was able to kill and reduce the clonogenicity of the trastuzumab-resistant HR6 cell line. Furthermore, $a$-TEA-mediated cell death of HR6 tumor cells involved inhibition of AKT and ERK phosphorylation. Treatment with a-TEA did not affect the expression of HER2/neu by both cell lines.

Conclusions

- Alpha-TEA synergizes with trastuzumab to cause regression of established Her2/neu-expressing tumors.

- Anti tumor activity of a-TEA is due to apoptosis induction and suppression of AKT and ERK signaling pathways.

- Alpha-TEA represents a potential opportunity for combination treatment with Herceptin in HER2 positive breast cancer.

\section{P452}

Defining IL-15 expressing myeloid cells in the tumor microenvironment

Figen Beceren-Braun, Rosa M. Santana Carrero, Sarai C. Rivas, Kimberly S. Schluns

University of Texas MD Anderson Cancer Center, Houston, TX, USA

Correspondence: Figen Beceren-Braun (fbeceren@mdanderson.org)

Journal for ImmunoTherapy of Cancer 2017, 5(Suppl 2):P452

\section{Background}

Loss of IL-15 within colorectal tumors correlates with higher risk of relapse, decreased survival, lower $\mathrm{T}$ cell density and decreased $\mathrm{T}$ cell proliferation [1] suggesting IL-15 expression within the tumor is important for anti-tumor responses. Furthermore, we have found that soluble IL-15 (sIL-15) complexes are produced in early tumors of mice implanted with tumor cell lines. However, the cell types expressing IL-15 and the mechanisms regulating IL-15 responses in the tumor microenvironment are not known. The goal of this study was to identify myeloid cell populations expressing IL-15 in the tumor microenvironment and investigate the mechanisms of expression.

Methods

Both IL-15 transcriptional and translational EGFP (enhanced green fluorescent protein) reporter mice were injected subcutaneously with $3 \times 10^{5}$ B16 melanoma, MCA-205 fibrosarcoma or MC-38 murine adenocarcinoma cell line. After two weeks tumors were stained for myeloid cell surface markers and analyzed via flow cytometry. Distribution of myeloid cell populations in tumors were also monitored via confocal laser-scanning microscopy of IL-15 reporter mice. CCR2 ${ }^{+} \mathrm{IL}-15^{+}$ double reporter mice were used to further characterize myeloid cells. To investigate the cellular source of slL-15 complexes, we used mice with a conditional deletion of IL-15Ra from DCs (CD11c-Cre x IL-15Rafl/ $\mathrm{fl}$ ) or macrophages (LysM-Cre $\mathrm{x} \mathrm{IL-15Rafl/fl);} \mathrm{or} \mathrm{mice} \mathrm{were} \mathrm{treated} \mathrm{with}$ anti-Ly6G depleting antibodies.

Results

In both IL-15 reporter lines, we found that the EGFP expressing cells in the tumor microenvironment are composed of three main populations:

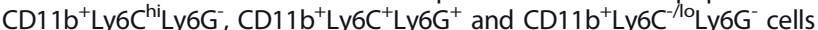
with the CD11 $\mathrm{b}^{+} \mathrm{L} y 6 \mathrm{C}^{\text {hi }} \mathrm{CCR} 2^{+}$cells being the most abundant. Similar composition of EGFP ${ }^{+}$myeloid populations was also seen in MCA-205 and MC-38 tumors. Imaging studies of MCA-205 tumors showed that the majority of the $\mathrm{F} 4 / 80^{+}$or $\mathrm{CCR2}^{+}$cells were also EGFP ${ }^{+}$. In MCA-205

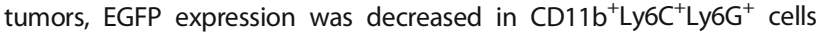
compared to the spleen. Analyses with conditional knockout mice or depleting antibodies showed that the monocytic cells and macrophages are major sources of sIL-15 complexes in early B16 tumors. Conclusions

In the tumor microenvironment, IL-15 is primarily produced by inflammatory monocytes and macrophages in early tumors and is down regulated with tumor growth.

\section{References}

1. Mlecnik B, et al. Functional Network Pipeline Reveals Genetic Determinants Associated with in Situ Lymphocyte Proliferation and Survival of Cancer Patients. Sci Transl Med. 2014; 6228:228ra37.

\section{P453}

Targeted tumor stroma depletion in combination with a whole cell tumor vaccine enhances effector $\mathrm{T}$ cell infiltration in a mouse model of pancreatic cancer

Alex Blair ${ }^{1}$, Victoria Kim", Stephen Muth', Todd Armstrong ${ }^{1}$,

Sanna Rosengren ${ }^{2}$, Lei Zheng ${ }^{1}$

'Johns Hopkins Medical Institution, Baltimore, MD, USA; ${ }^{2}$ Halozyme

Therapeutics, San Diego, CA, USA

Correspondence: Lei Zheng (ablair9@jhmi.edu)

Journal for ImmunoTherapy of Cancer 2017, 5(Suppl 2):P453

\section{Background}

Pancreatic ductal adenocarcinoma (PDAC) is an aggressive disease with dismal prognosis due to conventional therapy resistance. The tumor microenvironment (TME) of PDAC is characterized by a desmoplastic reaction that excludes effector immune cell infiltration, thus preventing optimal efficacy of immunotherapy. A human recombinant hyaluronidase (PEGPH20) has been clinically formulated to enzymatically deplete hyaluronan $(\mathrm{HA})$ in the tumor extracellular matrix. We tested the hypothesis that combining the stromal targeting agent PEGPH2O with a GMCSF secreting allogenic pancreatic tumor vaccine (GVAX) would increase immune cell infiltration and improve survival of PDAC bearing mice. Methods

C57BL/6 mice were orthotopically transplanted with $2 \times 10^{6}$ murine KPC pancreatic tumor cells to form liver metastases by a hemisplenectomy technique on day 0 . Following tumor transplantation, mice were treated subcutaneously with GVAX in combination with intravenous PEGPH20 or the appropriate control. PEGPH20 was given on day 6 and GVAX on post-operative day 7. An immune modulating dose of Cyclophosphamide (Cy) was given the day before GVAX in all groups. Tumor immune lymphocyte infiltration was assessed by immunohistochemistry and flow cytometry. RT-PCR was performed on tumor associated macrophages isolated with CD11b beads. Mice were sacrificed on day 16 for immune and gene expression analysis. Additional survival analysis followed mice until death.

Results

Combination therapy with PEGPH20 and GVAX yielded higher infiltration of effector CD8+ and CD4+ tumor infiltrating lymphocytes in the 
TME compared to untreated and monotherapy groups (all $p<0.05$ ) (Fig. 1). Murine survival was improved in the combination therapy group as compared to GVAX therapy alone (Hazard Ratio: 0.286 [0.10-0.82] $\mathrm{p}=0.02$ ). Additionally, a larger percent of tumor-infiltrating CD8+ cells were noted to express the lymphocyte activation surface marker CD137 in combination therapy compared to monotherapy controls. A trend towards lower CXCR4 expression was noted in the combination therapy group, suggesting a possible mechanism to CD8+CD137+ infiltration beyond decreased vascular impedance.

\section{Conclusions}

Our preclinical murine model of PDAC demonstrates enhanced efficacy of GVAX in combination with the stromal targeting agent PEGPH2O with increased immune cell infiltration and a survival advantage. This study provides rationale for further study of this combination for PDAC treatment.

Total number of live (A) CD8+ and (B) CD4+ tumor infiltrating lymphocytes after KPC hemispleen surgery and indicated therapy. Data represent mean \pm SEM from one representative experiment with three mice per group that was repeated twice. (C) Kaplan-Meier survival curves of mice that were implanted with PDAC cells and treated with Cy/GVAX with and without combination of PEGPH20. ${ }^{*}=p$.

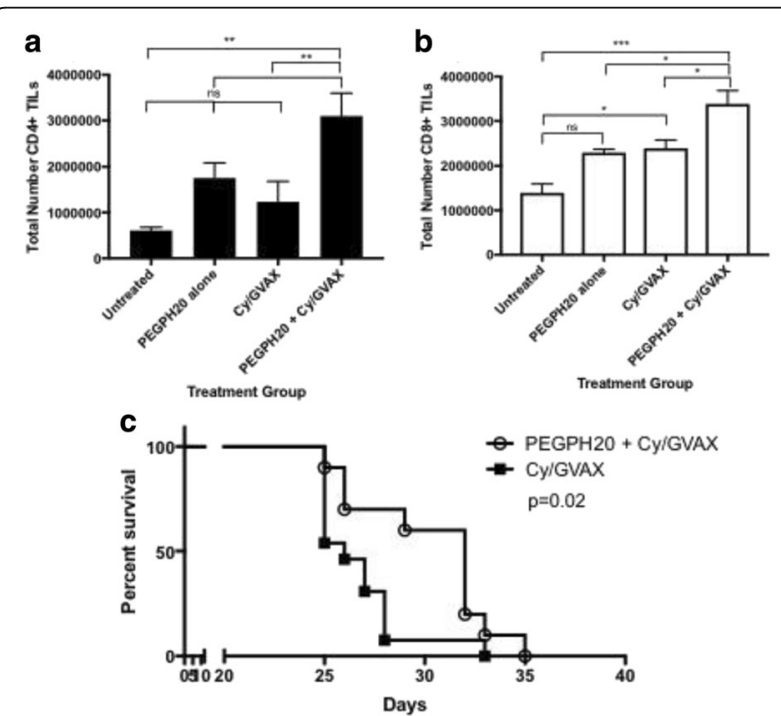

Fig. 1 (abstract P453). Combination therapy of PEGPH20 with Cy/GVAX improves immune cell infiltration and survival in a PDAC mouse model

\section{P454}

Molecular targeted radiotherapy facilitates in situ vaccination in a syngeneic murine melanoma model

Peter M Carlson' ', Clinton Heinze', Joseph Grudzinski', Reinier Hernandez ${ }^{1}$ Stephen D Gillies², Hans Loibner", Alexander L Rakhmilevich', Mario Otto', Bryan Bednarz', Jamey Weichert', Paul M Sondel', Zachary S Morris ${ }^{1}$ 'University of Wisconsin School of Medicine and Public Health, Madison, WI, USA; ${ }^{2}$ Provenance Biopharmaceuticals, Carlisle, MA, USA; ${ }^{3}$ Apeiron Biologics, Vienna, Austria

Correspondence: Zachary S Morris (pcarlson2@wisc.edu) Journal for ImmunoTherapy of Cancer 2017, 5(Suppl 2):P454

\section{Background}

We reported that $12 \mathrm{~Gy}$ external beam radiation (EBRT) and intratumoral (IT) administration of the hu14.18-IL2 immunocytokine (IC) [an anti-disialoganglioside (GD2) mAb fused to IL2] five days later elicits an in situ vaccination effect in $\mathrm{GD2}^{+}$murine tumors. An established distant, untreated secondary tumor exerts an antagonistic effect, "concomitant immune tolerance" (CIT), which drastically reduces the efficacy of in situ vaccination. We have shown that CIT is mediated, in part, by tumor-infiltrating regulatory $\mathrm{T}$ cells (Tregs) and that CIT can be overcome by delivering 12Gy EBRT to both tumors. Clinically, it is not feasible to deliver this dose to all sites of metastatic disease. Systemically delivered molecular targeted radiotherapy (MTRT) with the tumor-selective alkyl phosphocholine analog ${ }^{131}$ I-NM404 could overcome this challenge, and potentially deplete immunosuppressive cells responsible for CIT in practically all sites of metastasis. We evaluated doses of EBRT to distant tumor sites needed to eliminate CIT performed dosimetry to determine the administered activity of ${ }^{131}$ INM404 that approximates this EBRT absorbed dose, and tested whether systemic administration of MTRT may overcome CIT in a syngeneic murine melanoma model.

Methods

We monitored tumor volume, survival, and infiltrating lymphocyte populations in primary and secondary tumors treated with EBRT+IT$\mathrm{IC}+$ /-intravenous MTRT in C57BL/6 mice bearing one or two $\sim 250 \mathrm{~mm}^{3}$ syngeneic GD2+ B78 melanoma flank tumors.

Results

We demonstrate that CIT can be substantially attenuated using lowdose (2Gy) EBRT to the secondary tumor. This lower dose is not adequate to generate an in situ vaccine response but appeared comparable to $12 \mathrm{~Gy}$ in its capacity to overcome CIT. Using longitudinal ${ }^{124}$ I-NM404 PET/CT imaging with Monte Carlo dosimetry, we estimated that $60 \mathrm{MCi}$ of ${ }^{131} \mathrm{I}$-NM404, a dose which does not destroy B78 tumors, given IV provides approximately $2 \mathrm{~Gy}$ to both tumors in animals bearing two B78 tumors. Following $60 \mu \mathrm{Ci}$ of ${ }^{131} \mathrm{I}$-NM404 to mice with two B78 tumors, we observed depletion of tumor-infiltrating Tregs by IHC staining over a 4-week period, without bone marrow suppression (thrombocytopenia/leukopenia/anemia). We observed that pretreatment with systemic ${ }^{131}$ I-NM404 one week prior to $12 \mathrm{~Gy}$ EBRT+IT-IC to a single tumor site may substantially attenuate CIT and rescue a systemic response to in situ tumor vaccination.

Conclusions

We demonstrate that 2Gy EBRT is sufficient to attenuate CIT, and that this dose can be approximated using systemic MTRT. This work suggests that MTRT may enable conditioning of the tumor immune microenvironment to overcome intrinsic immune evasion mechanisms and restore efficacy of in situ tumor vaccination.

P455

Investigating the specificity and response of a native leukemiareactive $\mathrm{CD}^{+} \mathrm{T}$ cell clone

Xiufen Chen ${ }^{1}$, Brendan MacNabb ${ }^{1}$, Emily Curran ${ }^{1}$, Bruce Blazar ${ }^{2}$, Justin

Kline

'University of Chicago, Chicago, IL, USA; ${ }^{2}$ University of Minnesota,

Minneapolis, MN, USA

Correspondence: Justin Kline (xchen82@bsd.uchicago.edu)

Journal for ImmunoTherapy of Cancer 2017, 5(Suppl 2):P455

\section{Background}

We previously reported that high-affinity, antigen-specific $\mathrm{CD}^{+} \mathrm{T}$ cells were induced to undergo abortive proliferation and deletion in leukemia-bearing hosts, and have subsequently found that this deletional $\mathrm{CD}^{+} \mathrm{T}$ cell tolerance requires cross-presentation of the leukemia antigen by splenic Batf3-lineage dendritic cells.

Methods

Recently, we generated a $T$ cell receptor (TCR) transgenic mouse strain (referred to as Tg101) that expresses the TCR- $\alpha$ and $-\beta$ chains of a $C D 8^{+} T$ cell clone specific for a native leukemia antigen.

Results

Having confirmed that $\mathrm{Tg} 101 \mathrm{CD}^{+} \mathrm{T}$ cells specifically recognize C1498 acute myeloid leukemia (AML) cells in the context of the MHC class I molecule, $\mathrm{H}-2 \mathrm{D}^{\mathrm{b}}$, we investigated the behavior and fate these cells in naïve and C1498 leukemia-bearing hosts. In naïve mice, Tg101 thymocytes predominantly developed along the $\mathrm{CD} 8^{+}$lineage, and existed as naïve $\mathrm{CD} 8^{+} \mathrm{T}$ cells in peripheral lymphoid organs, suggesting that the Tg101 antigen is likely leukemia-specific. In leukemia-bearing mice, antigen encounter by $\operatorname{Tg} 101 \mathrm{~T}$ cells was delayed (day 10-12), and 
primarily occurred in the liver - a prominent site of leukemia progression. Subsequently, $\mathrm{Tg} 101$ cells expanded and upregulated expression of co-inhibitory receptors, including PD-1, LAG-3, TIM-3 and TIGIT, which correlated with a progressively dysfunctional phenotype. Surprisingly, antigen encounter by Tg101 T cells in leukemia-bearing mice occurred independently of Batf3-lineage DCs, suggesting that the Tg101 antigen was poorly cross-presented in vivo. Given this finding, we deleted $\mathrm{H}-2 \mathrm{D}^{\mathrm{b}}$ in $\mathrm{C} 1498$ cells to determine the extent to which direct antigen presentation by leukemia cells is responsible for the acquisition of dysfunctional properties in Tg101 T cells in vivo. In vitro expansion was 2 -fold decreased in Tg101 T cells compared to high-affinity $2 \mathrm{C} \mathrm{CD8}{ }^{+} \mathrm{T}$ cells (which recognize the SIY peptide in the context of $\mathrm{H}-2 \mathrm{~K}^{\mathrm{b}}$ ) upon culture with SIY-expressing C1498 cells (C1498.SIY), indicative of a lower avidity of Tg101 vs the 2C T cells for antigen. In contrast to Tg101 T cells, 2C T cells encountered leukemia antigen rapidly (day 3-4) in the context of splenic CD8a ${ }^{+}$DCs, proliferated briefly, and were effectively deleted from the host by day 12 .

Conclusions

Collectively, our data reveal that $\mathrm{CD}^{+} \mathrm{T}$ cell tolerance is effectively generated in the context of AML. However, the nature of the leukemia antigen, as well as the context in which it is presented, are critical determinants in the underlying mechanism of tolerance that ensues.

\section{P456}

\section{CDK4 \& 6 inhibitor Abemaciclib exerts intrinsic}

\section{immunomodulatory effects on human $\mathrm{T}$ cells in vitro}

Darin Chin ${ }^{1}$, Carmine Carpenito', Nelusha Amaladas', Linda Chung ${ }^{2}$, Kirk

Staschke ${ }^{2}$, David Schaer ${ }^{3}$, Richard Beckmann², Ruslan Novosiadly?',

Michael Kalos ${ }^{1}$

${ }^{1}$ Eli Lilly \& Co, New York, NY, USA; ${ }^{2}$ Eli Lilly \& Co, Indianapolis, IN, USA;

${ }^{3}$ Eli Lilly \& Co, Brooklyn, NY, USA

Correspondence: Carmine Carpenito (chin_darin@lilly.com); Kirk Staschke, David Schaer, Richard Beckmann, Ruslan Novosiadly, Michael Kalos

Journal for ImmunoTherapy of Cancer 2017, 5(Suppl 2):P456

\section{Background}

Abemaciclib is an inhibitor of cyclin-dependent kinases 4 and 6 (CDK4 \& 6) that suppresses DNA synthesis and cell proliferation as result of cell cycle arrest in G1 phase. Significant clinical activity of abemaciclib has been observed in hormone receptorpositive breast cancer patients highlighting the therapeutic potential of this compound in oncology, including combinations with other therapeutic modalities. In preclinical studies, pretreatment with abemaciclib followed by PD-L1 checkpoint blockade has demonstrated synergistic immune activation and antitumor efficacy. This has generated interest in combination studies of abemaciclib with immunotherapeutic agents. However, the mechanisms underlying immunomodulatory activity of abemaciclib have not been fully elucidated. Consequently, the objective of this study was to understand the direct effects of abemaciclib on primary human T cells.

Methods

Jurkat and primary human T cells were stimulated in vitro with antiCD3/CD28 and treated with increasing concentrations of abemaciclib ranging between $0 \mathrm{uM}$ to $1 \mathrm{uM}$ for up to ten days. Cells were harvested at the end of the experiments and nuclear factor of activated T cells (NFAT) activation, cell surface immune markers (costimulatory and coinhibitory receptors and ligands) and expression of immunerelated genes were analyzed by signal reporter assay, flow cytometry and Quantigene Plex, respectively.

Results

Treatment of Jurkat $T$ cells showed a dose dependent increase in NFAT reporter activation during abemaciclib treatment which was replicated in primary human T cells. Additionally, treatment of primary $\mathrm{T}$ cells with abemaciclib resulted in a distinct phenotype exemplified by up-regulation of CD137, GITR, TIM3, PD-1, PD-L1, and TIGIT on the surface of both CD4+ and CD8+ T cells correlating with gene expression changes associated with $T$ cell activation phenotype (upregulated Granzyme B, CD137, OX40, TNF-alpha, IL2R, IP10, ICAM1) with only a modest concentration-dependent decrease in T cell expansion. Furthermore, intermittent exposure of $\mathrm{T}$ cells to abemaciclib during various stages of expansion revealed that $T$ cells were capable of recovering from abemaciclib-induced growth inhibition whereas expression of costimulatory/coinhibitory molecules was maintained once the drug was removed.

Conclusions

Taken together, these results demonstrate that abemaciclib can exert T cell-intrinsic effects at concentrations comparable to clinical exposure. This is exemplified by increased T cell activation phenotype with only a modest and reversible decrease in $T$ cell expansion, providing further rationale for clinical exploration of combination studies with immunomodulatory agents such as PD-1 pathway inhibitors.

\section{P457}

Pharmacodynamic activity of MEDI9197, a TLR7/8 agonist, administered intratumorally in subjects with solid subcutaneous or cutaneous tumors

Zachary A. Cooper ${ }^{1}$, Christopher A. Morehouse', Yuling Wu' ${ }^{1}$ Keith Steele', David Brucker', Leo Tseng', Maria Jure-Kunkel', Nancy Mueller',

Patricia Ryan', Juneko Grilley-Olson ${ }^{3}$, David Hong ${ }^{4}$, Aurélien Marabelle ${ }^{5}$

Pamela Munster ${ }^{6}$, Rahul Aggarwal ${ }^{6}$, Sandrine Aspeslagh ${ }^{5}$, Robert G. Dixon ${ }^{3}$ Manish Patel ${ }^{7}$, Vivek Subbiah', Joshua Brody ${ }^{8}$, Shilpa Gupta ${ }^{7}$

${ }^{1}$ Medlmmune, Gaithersburg, MD, USA; ${ }^{2}$ Definiens, Cambridge, MA, USA; ${ }^{3}$ University of North Carolina Lineberger Comprehensive Cancer Center, Chapel Hill, NC, USA; ${ }^{4}$ MD Anderson Cancer Center, Houston, TX, USA; ${ }^{5}$ Institut Gustave Roussy, 94800 Villejuif, France; ${ }^{6}$ UCSF Helen Diller

Family Comprehensive Cancer Center, San Francisco, CA, USA; ${ }^{7}$ Masonic Cancer Center, Minneapolis, MN, USA; ${ }^{8}$ Mount Sinai (Hess Center for

Science and Medicine), New York, NY, USA

Correspondence: Zachary A. Cooper

(robert.mulvey@ashfieldhealthcare.com)

Journal for ImmunoTherapy of Cancer 2017, 5(Suppl 2):P457

\section{Background}

MEDI9197 (formerly 3M-052), is a novel TLR7/8 dual agonist formulated for intratumoral (IT) injection and optimized for retention within the tumor after IT injection. IT administration aims to focus antigen presentation in the tumor by activation of both plasmacytoid (TLR7 expressing) and conventional dendritic (TLR8 expressing) cells, thus minimizing systemic inflammatory toxicities. We report the pharmacodynamic results of MEDI9197 from a phase I, first-in-human, dose-escalation study in patients with solid tumors (NCT02556463).

Methods

The dose-escalation trial of MEDI9197 (0.005 to $0.055 \mathrm{mg}$ ) has enrolled 24 patients with subcutaneous/cutaneous tumors. As reported at AACR 2017, the maximum tolerated dose is $0.037 \mathrm{mg}$ and IT administration is feasible and safe. Here we present updated tumoral and peripheral pharmacodynamic effects in patients treated with MEDI9197 0.005, 0.012, and $0.037 \mathrm{mg}$.

Results

Local pharmacodynamic effects assessed by immunohistochemistry $(\mathrm{IHC})$ in longitudinal biopsies demonstrated the majority of patients treated with $0.037 \mathrm{mg}$ had an increase ( $\geq 2$ fold) in CD8, CD40, CD56, or PD-L1 (tumor and immune cells) markers 3 weeks after a single dose of MEDI9197. RNAseq analysis of paired tumor biopsies showed an increase in TLR7/8-downstream regulated genes and innate and adaptive immune activation signatures such as Type 1 IFN, IFN $\gamma$ and T effector signatures ( $\geq 1.5$ fold) consistent with IHC.

Increases in peripheral levels of IFN $\gamma$, CXCL10, and CXCL11 were observed across all 3 doses $(0.037,0.012$, and $0.005 \mathrm{mg})$. Within 
24 hours of IT administration of MEDI9197 $0.037 \mathrm{mg}$, median peak values of IFN $\gamma, C X C L 10$, and CXCL11 observed were 236, 9286, and $558 \mathrm{pg} / \mathrm{mL}$ respectively. The fold change increase ranged from 4.5-43,30-132, and 3.7-52 vs. baseline for IFNY, CXCL10, and CXCL11 respectively. Peripheral levels of IFNY showed a significant trend for a dose response with median peak values for those treated with MEDI9197 $0.005 \mathrm{mg} \mathrm{4.1-fold} \mathrm{less}$ than those treated with $0.037 \mathrm{mg}$. Analysis of whole blood microarray data across all doses demonstrated increases ( $\geq 2$ fold) in $\mathrm{T}_{\mathrm{H}} 1$ and Type 1 IFN gene expression signatures with a transient decrease ( $\geq 1.5$ fold) in CD8A transcript and NK cell signature expression, suggesting trafficking of $T$ and NK cells.

\section{Conclusions}

IT administration of MEDI9197 induces Type 1 and 2 IFN as well as $\mathrm{T}_{\mathrm{H}} 1$ responses, suggesting activation of both plasmacytoid and conventional dendritic cells. A combination trial of MEDI9197 with durvalumab (anti-PD-L1) and radiation in patients with advanced solid tumors is currently ongoing.

Trial Registration

NCT02556463.

\section{P458}

Nitric oxide synthase inhibition results in PDL-1 upregulation and ADAR1 inhibition, triggering immunogenic cell death: an in-vitro and in-vivo analysis

Daniel Davila-Gonzalez, Roberto Rosato, Wei Qian, Anthony Kozielski,

Wen Chen, Dong Soon Choi, Patrick Tucker, Jenny Chang

Houston Methodist, Houston, TX, USA

Correspondence: Jenny Chang

(ddavilagonzalez@houstonmethodist.org)

Journal for ImmunoTherapy of Cancer 2017, 5(Suppl 2):P458

\section{Background}

Response failure in checkpoint inhibitors, such as death ligand 1 (PD-L1) and its receptor programmed death 1 (PD-1), is associated with cancer-related immunosuppression. Inducible nitric oxide synthase (iNOS) product, nitric oxide, has been implicated in immunosuppressive environments. In triple negative breast cancer (TNBC), iNOS expression is associated with poor survival and increased tumor aggressiveness. We've shown ADAR1 (adenosine deaminase acting on RNA 1) is upregulated by iNOS activation in TNBC cell lines. ADAR1 RNA editing suppresses inflammation, thereby damping the immune response. This study aims to evaluate whether NOS inhibition plus anti-PD-1 is a feasible therapeutic combination in TNBC.

Methods

TNBC cell lines BT-549, SUM-159, SUM-157, HCC-70, MDA-MB-231 and MDA-MD-468 were treated with NOS inhibition therapy (LNMMA $4 \mathrm{mM})+$ amlodipine $(5 \mu \mathrm{M})$. PDL-1, HMGB1, and ADAR1 expression was assessed via western blot. BALBC mice growing orthotopically injected $4 \mathrm{t} 1$ cells were treated weekly with: 1 ) vehicle (saline, oral/Rat-lgG2 i.p.); 2) NOS inhibition [L-NMMA $(200 \mathrm{mg} / \mathrm{kg}$ oral) + amlodipine (10mg/kg, i.p.), days 1-5]; 3) anti-PD-1 antibody (10 mg/ kg i.p.; Bio X Cell Clone: RMP1-14, days 1, 3, 5); or 4) anti-PD-1 + NOS inhibition. Humanized mice were developed by injecting hematopoietic stem cells (HSC, CD34+) into the tail vein of irradiated NOD-scid IL2Rynull mice. Mice were sorted and TNBC patient-derived xenografts (PDXs), representing 12 different patients, were implanted into the mammary fat pad ( 3 mice/PDX). Mice were sorted and

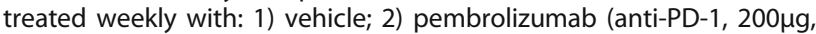
day 1, IV); or 3) pembrolizumab + NOS inhibitor.

Results

NOS inhibition increased PD-L1 in most cell lines. 4T1 syngeneic model helped assess the potential benefit of combined therapy. There were no significant differences in tumor growth between monotherapy and control group. However, anti-PD1 + NOS-inhibition resulted in a significant reduction in tumor growth. In immunehumanized TNBC PDXs, 40\% responded to anti-PD1 monotherapy, $60 \%$ had improved response following combination therapy, and $33 \%$ responded only to combination therapy. In total, $66 \%$ of PDXs analyzed benefited from combination therapy. Furthermore, both in vitro and in-vivo samples showed downregulation of ADAR1 levels when treated with NOS inhibition, with a subsequent increase in HMGB1, a marker of immunogenic cell death.

\section{Conclusions}

NOS inhibition upregulates PDL-1 expression in TNBC cell lines. Furthermore, combining NOS inhibition with anti-PD1 therapy improved antibody response by activating the immunogenic cell death pathway. This combination treatment may benefit cancer patients who do not respond to anti-PD-1 monotherapy.

P459

Class lla HDAC inhibition promotes an anti-tumor macrophage phenotype that induces breast tumor regression and inhibits metastasis

Jennifer Guerriero', Alaba Sotayo', Holly Ponichtera', Jessica Castrillon', Alexandra Pourzia', Sara Schad', Shawn Johnson', Ruben Carrasco' Suzan Lazo', Roderick Bronson², Scott Davis's, Mercedes Lobera ${ }^{3}$,

Michael Nolan ${ }^{3}$, Anthony Letai

${ }^{1}$ Dana-Farber Cancer Institute, Boston, MA, USA; ${ }^{2}$ Harvard Medical

School, Boston, MA, USA; ${ }^{3}$ GlaxoSmithKline, Cambridge, MA, USA

Correspondence: Jennifer Guerriero

(Jennifer_Guerriero@dfci.harvard.edu)

Journal for ImmunoTherapy of Cancer 2017, 5(Suppl 2):P459

\section{Background}

The breast tumor environment is complex and includes both neoplastic and immune cells. Among the most prevalent immune cell population within breast tumors is tumor associated macrophages (TAMs). Macrophages have the ability to polarize into either $\mathrm{M} 1$ cells, which have potent anti-tumor capabilities, or M2 macrophages which promote tumor progression by stimulating tumor vasculature, invasion, metastasis, and can enhance tumor resistance to chemotherapy. Generally TAMs in breast tumors are considered M2 macrophages and a high tumor density of TAMs clinically correlates to both overall worse prognosis and increased metastasis. Several therapeutic strategies exist to modulate TAMs clinically, focusing on depleting or inhibiting TAMs. However, macrophage are required for optimal tumor regression in response to both chemotherapy and immunotherapy. Their embedded location and their untapped potential provide impetus to the discovery of strategies to turn them against tumors and to harness for cancer therapy. Therefore here, we describe a novel method to polarize pro-tumor macrophages to an anti-tumor phenotype.

Methods

We recently reported that a first in class selective class Ila HDAC inhibitor (TMP195) influenced human monocyte responses to colony stimulating factors CSF-1 and CSF-2 in vitro. Here, we utilize a macrophage-dependent autochthonous mouse model of breast cancer to demonstrate that in vivo TMP195 treatment alters the tumor microenvironment and reduces tumor burden and pulmonary metastases through macrophage modulation.

Results

Here, we demonstrate that a first in class, class lla histone deacetylase (HDAC) inhibitor, TMP195, can activate tumor macrophages in vivo to induce tumor regression and inhibit pulmonary metastases in a mouse model of breast cancer. We find that TMP195 induces macrophage recruitment and differentiation of highly phagocytic cells within the tumor, which increases tumor cell death while decreasing angiogenesis. Strikingly, we find that TMP195 enhances chemotherapy and immunotherapy to induce durable tumor reduction. 


\section{Conclusions}

Our findings reveal an immunostimulatory effect of class Ila HDAC inhibition that contrasts with strategies of depleting or inhibiting TAMs for cancer therapy. Class Ila HDAC inhibition leverages the effector functions of macrophages, opening the door to clinically-relevant cooperation of checkpoint blockade, agonistic aCD40 or inhibitory aCD47 therapy, peptide vaccine therapy or the antibody-dependent cellular phagocytosis (ADCP) associated with mAb therapy. Indeed, the innate immune system is the natural complement to the adaptive immune system that surveys and fights tumors, and these studies demonstrate a novel approach to harness innate immune cells to cooperate with agents that stimulate an adaptive anti-tumor immune response in an otherwise resistant cancer.

\section{P460}

Selenium, the element of the moon, improves immunotherapies on earth

Claudia Lennicke', Shamim Ahmad', Rajeev Shrimali', Barbara Seliger², Youcef Rustum³, John Janik', Mikayel Mkrtichyan', Seema Gupta',

Samir Khleif ${ }^{1}$

${ }^{1}$ Augusta University, Augsuta, GA, USA: ${ }^{2}$ Institute of Medical

Immunology, Halle, Germany; ${ }^{3}$ University of Buffalo, Buffalo, NY, USA

Correspondence: Samir Khleif (segupta@augusta.edu)

Journal for ImmunoTherapy of Cancer 2017, 5(Suppl 2):P460

\section{Background}

Recent advances in immunotherapy have shown amazing results in cancer patients, albeit in small fraction. Attempts in developing immunotherapy to generate effective response against tumors are still faced with immune suppressive environment. The abundance of tumor-infiltrated T regulatory cells (Tregs) highly is one of the main mechanism by which tumors overcome immune responsiveness. Tregs also correlates with poor patient prognosis.

Selenium (Se), an essential trace element named after the goddess of the moon, is described to exhibit anti-carcinogenic potentials [1]. However, the underlying mechanisms of Se action are insufficiently understood. Since Se is also known to exhibit immune-modulatory properties [2] we hypothesized that Se in combination with vaccinebased immunotherapy might enhance anti-tumor responses.

Methods

TC-1 tumor-bearing C57BL/6 mice were treated either with Vaccine (E7, $100 \mu \mathrm{g} /$ mouse; PADRE, $20 \mu \mathrm{g} / \mathrm{mouse}$; every $7^{\text {th }}$ day), Methylselenocysteine, as source of Se (MSC, $100 \mu \mathrm{g} / \mathrm{mouse}$; daily) or with a combination of both. In addition, an untreated group served as the control. Spleens and tumor tissues were analyzed for T cell subsets via flow cytometry. ELISpot assays were performed to determine IFNg production in E7/ PADRE restimulated splenocytes. In vitro, CD4+ T cells isolated from spleens of C57BL/6 mice were cultured under iTreg conditions, treated with Se and analyzed via Flow Cytometry and Immunoblotting.

Results

We found that combining Selenium with specific antigen vaccine led to significant enhancement of immune response leading to delay in tumor growth thereby prolonging the survival rate of mice. Importantly, Se significantly reduced the infiltration of Tregs into the tumor environment thereby enhancing the CD8+/Treg ratio leading to an increased vaccine-induced immune response. In vitro analysis of iTregs revealed that Se reduces Foxp3 expression and destabilizes Tregs.

\section{Conclusions}

Here we show for the first time that Se an essential trace element improves vaccine-based immunotherapies thereby opening a new window for the development of novel strategies to fight against cancer.

\section{References}

1. Misra S, Boylan M, Selvam A. Redox-active selenium compounds-from toxicity and cell death to cancer treatment. Nutrients. 2015;7:3536-56.

2. Huang $Z$, Rose $A H$, Hoffmann PR. The role of selenium in inflammation and immunity: from molecular mechanisms to therapeutic opportunities. Antioxid Redox Signal. 2012;16:705-43.
P461

Listeria monocytogenes-based immunotherapies alter the suppressive phenotype of intratumoral regulatory $\mathrm{T}$ cells Rachelle E. Kosoff, Nithya Thambi, Lauren K. Pettit, Daniel O. Villarreal, Kimberly Ramos, Michael F. Princiotta, Robert G. Petit, Sandra M. Hayes Advaxis, Inc., Princeton, NJ, USA

Correspondence: Sandra M. Hayes (hayes@advaxis.com)

Journal for ImmunoTherapy of Cancer 2017, 5(Suppl 2):P461

\section{Background}

Advaxis' Listeria monocytogenes $(\mathrm{Lm})$-based immunotherapies are live attenuated bacterial vectors that elicit tumor-reactive T cells through the delivery of tumor-associated antigens directly to dendritic cells. In addition, our $L m$-based immunotherapies reduce the frequency and function of intratumoral regulatory T cells (Tregs), thereby counteracting the immunosuppressive tumor microenvironment (TME) and sustaining the newly generated antitumor immune response. The purpose of this study is to investigate further the mechanism by which Advaxis' $L m$-based immunotherapies alter Treg function.

Methods

C57BL/6 mice were implanted with TC-1 tumor cells, which express the human papillomavirus (HPV)16 E6 and E7 proteins. On day 8, when tumors were palpable, mice were treated with either one dose of ADXSHPV-Quad, an $\mathrm{Lm}$-based vector targeting the E6 and E7 proteins from both HPV16 and HPV18, or PBS. Five days post-treatment, tumor infiltrating lymphocytes were phenotyped by flow cytometry and intact tumors were analyzed by immunofluorescence microscopy.

Results

We reasoned that $L m$-based immunotherapies, to be effective at tumor control, must reprogram the immunosuppressive TME early during the course of treatment in order to induce and sustain infiltration of tumorspecific T cell effectors. Accordingly, we analyzed the TME 5 days after a single dose of ADXS-HPV-Quad. By flow cytometry, we compared the proliferative status and viability of Tregs in tumors of mice treated with either PBS or ADXS-HPV-Quad. The percentage of proliferating $\left(\mathrm{Ki}-67^{+}\right)$ Tregs was comparable between the two treatment groups, but the percentage of Tregs undergoing apoptosis was 2-fold higher in ADXSHPV-Quad-treated mice than in PBS-treated mice. Further phenotypic analysis revealed that the percentage of Tregs expressing CCR8, a chemokine receptor whose expression is essential for Treg survival and function, was almost 2-fold lower in ADXS-HPV-Quad-treated mice than in PBS-treated mice. Expression levels of other Treg phenotypic markers, namely Foxp3 and CD39, were also reduced in the CCR8 negative Tregs. The phenotypic changes in intratumoral Tregs were associated with a loss in in vivo suppressive activity, as there was a dramatic increase in the infiltration of activated cytokine-producing $T$ cell effectors into the tumor core of ADXS-HPV-Quad-treated mice but not into the tumor core of PBS-treated mice.

Conclusions

Within 5 days of administration, a single dose of ADXS-HPV-Quad alters the tumor microenvironment by impairing Treg survival and function and by promoting effector $\mathrm{T}$ cell recruitment and function. Changes in the suppressive Treg phenotype, specifically the downregulation of CCR8 expression, may be a key mechanism by which Advaxis' $L m$-based immunotherapies impair Treg function.

\section{P462}

Ubiquitin-specific protease 6 (USP6) oncogene promotes immune cell recruitment in sarcoma

lan Henrich', Laura Quick', Rob Young ${ }^{2}$, Andre Oliveira ${ }^{3}$, Margaret Chou ${ }^{1}$ ${ }^{1}$ University of Pennsylvania, Philadelphia, PA, USA; ${ }^{2}$ Children's Hospital of Pennsylvania, Philadelphia, PA, USA; ${ }^{3}$ Mayo Clinic, Rochester, MN, USA Correspondence: lan Henrich (ihenrich@mail.med.upenn.edu) Journal for ImmunoTherapy of Cancer 2017, 5(Suppl 2):P462

\section{Background}

The de-ubiquitylating enzyme USP6 is a target of chromosomal translocation and the key etiologic agent in several benign bone and soft tissue tumors (BSTTs) [1]. We have shown that USP6 drives tumorigenesis by directly de-ubiquitylating the Jak1 kinase, leading to its 
stabilization and activation of STAT transcription factors [2]. We recently found that USP6 is also upregulated in sarcomas, malignancies of mesenchymal origin. Activation of the Jak-STAT pathway plays a key role in modulating the tumor microenvironment, however it is not known if or how USP6 affects immune cell infiltration and/or composition. We sought to determine USP6 effects on sarcoma tumor growth and immune cell migration/infiltration.

Methods

USP6 was expressed in a doxycycline-inducible manner in the patientderived sarcoma cell lines A673 and RD-ES. USP6 expression levels were confirmed to approximate those in primary patient tumor samples.

Results

Overexpression of USP6 results in the production of several immunomodulatory cytokines, most notably CXCL10, CCL5, and CCL20. USP6 expression led to the hallmarks of a heightened immune response, such as enhanced migration, polarization, and activation of several classes of immune cells, such as $\mathrm{CD} 4^{+}$and $\mathrm{CD} 8^{+} \mathrm{T}$ cells, monocytes, and macrophages. In murine xenografts, sarcoma cells expressing USP6 had attenuated tumor growth and enhanced immune cell infiltration compared to parental lines. Furthermore, analysis of sarcoma patient datasets revealed that elevated USP6 expression correlated with an immune cell gene signature, and immunohistochemistry of USP6-translocated tumors revealed a high degree of $\mathrm{CD}^{+}, \mathrm{CD}^{+}$, and $\mathrm{CD} 163^{+}$infiltration.

\section{Conclusions}

USP6 expression results in the production of several immunomodulatory cytokines that are known to promote the activation and migration of several classes of immune cells such as T cells, monocytes, and natural killer cells. High USP6 expression correlated with increased immune cell infiltration in mouse xenografts and patient samples. The ability of USP6 to provoke an enhanced immune response could potentially serve as a biomarker for susceptibility to immunotherapy.

\section{References}

1. Oliveira A, Chou M. The TRE17/USP6 oncogene: a riddle wrapped in a mystery inside an enigma. Front Biosci (Schol Ed).2012:4:321-340.2.

2. Quick L, Young R, Henrich I, Wang X, Asmann Y, Oliveira A, Chou M.Jak1STAT3 signals are essential effectors of the USP6/TRE17 oncogene in tumorigenesis. Cancer Res. 2016;76(18):5337-47.

\section{P463}

TAM RTKs facilitate MDSC-mediated tumor immunosuppression

Alisha Holtzhausen ${ }^{1}$, William Harris ${ }^{1}$, Debra Hunter ${ }^{1}$, Eric Ubil ${ }^{1}$,

Xiaodong Wang ${ }^{1}$, Douglas Graham ${ }^{2}$, Stephen Frye ${ }^{1}$, H. Shelton Earp ${ }^{1}$

${ }^{1}$ University of North Carolina at Chapel Hill, Chapel Hill, NC, USA; ${ }^{2}$ Emory University, Atlanta, GA, USA

Correspondence: $\mathrm{H}$. Shelton Earp (alisha_holtzhausen@unc.edu)

Journal for ImmunoTherapy of Cancer 2017, 5(Suppl 2):P463

\section{Background}

The innate immune-regulatory receptor tyrosine kinases Tyro3, Axl and MerTK (TAM RTKs) and their ligands, Gas 6 and Protein S, suppress the immune response through various mechanisms, including down regulating $\mathrm{M} 1$ cytokines, suppressing antigen presenting cell function and decreasing $\mathrm{CD}^{+}$tumor infiltration. Myeloid-derived suppressor cells (MDSCs) are potently immunosuppressive and inhibit $\mathrm{T}$ cell activity via various mechanisms, including increased expression of arginase, inducible nitric oxide synthase (iNOS), and the production of reactive oxygen species (ROS).

Methods

We studied the expression of TAM RTKs in MDSCs and their respective roles in MDSC-mediated immune suppression in the setting of cancer.
Using syngeneic murine tumor models implanted into WT and TAM RTK $^{-1-}$ mice, we FACS isolated MDSCs and analyzed the TAM RTKs' effect on MDSC suppressive mechanisms. To complement our genetic models, we employed a pan-TAM RTK inhibitor, UNC4241, and evaluated MDSC function and tumor growth.

Results

TAM RTKs and their ligands are significantly (M-MDSCs>20 fold, GMDSCs $>15$ fold) upregulated in MDSCs from tumor-bearing mice compared to non-tumor bearing controls. MDSCs isolated from tumor bearing TAM RTK ${ }^{-/}$mice have significantly diminished arginase, iNOS and ROS activity compared to WT counterparts. Consistent with these findings, M-MDSC functional suppression assays showed that loss of either mertk or tyro3 yielded tumor-elicited MMDSCs exhibiting reduced suppressive capabilities in $\mathrm{T}$ cell proliferation and IFN-g ELISPOT assays. We performed tumor and MDSC co-implantation studies and demonstrated that WT and $a x^{\%}$ MDSCs migrate readily to the TDLN while mertk $k^{-1}$ and tyro $^{-1-}$ MDSCs exhibit a migration defect and remain within the tumor, suggesting that the TAM RTKs play several roles in MDSC-mediated inhibition of T cell priming. Adoptive transfer of TAM RTK ${ }^{-1-}$ MDSCs results in diminished B16 melanoma development in syngeneic hosts relative to those receiving WT MDSCs. To evaluate whether inhibition of TAM RTKs has clinical potential, we employed a panTAM inhibitor, UNC4241, and found this agent to suppress MDSC arginase, iNOS and ROS activity, all while increasing $T$ cell proliferation and diminishing the growth of a BRAF ${ }^{\mathrm{V} 600 \mathrm{E}} \mathrm{PTEN}^{-1-}$ melanoma model. In vivo MDSC ablation reversed the effect of UNC4241 indicating that the mechanism of this agent is MDSC-dependent.

Conclusions

These results indicate that coordinate TAM RTK action mediates MDSC suppressive capabilities and that their pharmacologic inhibition reverses MDSC-dependent $T$ cell suppression and diminishes tumor growth. These findings suggest that inhibition of TAM RTKs represents a novel approach for modulating MDSC function and augmenting the efficacy of checkpoint inhibitor therapy.

\section{P464}

Use of evofosfamide for targeting immune-suppressive hypoxia in head and neck squamous cell carcinoma

Francis Hunter', Avik Shome', Way Wong', Pratha Budhani', Neil Senzer ${ }^{3}$, E. Gabriela Chiorean ${ }^{4}$, Dan Li', Peter Tsai', Nooriyah Poonawala',

Purvi Kakadiya', Maria Kondratyev ${ }^{5}$, Courtney Lynch', Cho Rong Hong',

Tet-Woo Lee', Dennis Kee ${ }^{6}$, Andrew Macann", Nicholas Mclvor ${ }^{6}$,

Kevin Hicks ${ }^{1}$, Stefan Bohlander ${ }^{1}$, Cristin Print ${ }^{1}$, Stew Kroll ${ }^{7}$, Charles Hart' ${ }^{7}$,

Bradly Wouters ${ }^{5}$, William Wilson ${ }^{1}$, Michael Curran ${ }^{2}$, Stephen Jamieson ${ }^{1}$

${ }^{1}$ University of Auckland, Auckland, New Zealand; ${ }^{2} \mathrm{MD}$ Anderson Cancer

Center, Houston, TX, USA; ${ }^{3}$ Mary Crowley Cancer Research Center, Dallas,

TX, USA; Indiana University Melvin and Bren Simon Cancer Center,

Indianapolis, IN, USA; 5 University Health Network, Toronto, ON, Canada;

${ }^{6}$ Auckland City Hospital, Auckland, New Zealand; ${ }^{7}$ Threshold

Pharmaceuticals, San Francisco, CA, USA

Correspondence: Francis Hunter (f.hunter@auckland.ac.nz)

Journal for ImmunoTherapy of Cancer 2017, 5(Suppl 2):P464

\section{Background}

Hypoxia is prevalent in head and neck squamous cell carcinoma (HNSCC), where it limits radiotherapy outcomes and may create an immune-suppressive microenvironment. The hypoxia-activated prodrug (HAP) evofosfamide (TH-302) targets hypoxia by undergoing oxygensensitive reductive activation (Figure 1). Evofosfamide is being clinically evaluated in combination with ipilimumab in solid tumours, including HNSCC (NCT03098160). The present study investigated the efficacy and sensitivity determinants of evofosfamide in HNSCC. 


\section{Methods}

Case reports were retrieved from an unpublished phase 2 evofosfamide monotherapy expansion cohort in solid tumours (480 or $575 \mathrm{mg} \cdot \mathrm{m}^{2}$ on days 1,8 and 15 of 28-day cycles). A collection of 22 human papillomavirus-negative HNSCC cell lines derived from lesions of varying TNM stages was assessed for sensitivity to $3 \mathrm{HAPs}$ - evofosfamide, PR-104A and SN30000 - in addition to cisplatin, 5-fluorouracil and the active metabolite of evofosfamide, bromo-iso-phosphoramide mustard (Br-IPM). Reductive activation of evofosfamide in cultured cells was measured by LC-MS/MS. The lines were molecularly characterised by RNAseq and whole-exome sequencing and their genomic and transcriptomic features compared to public domain HNSCC clinical samples. Molecular predictors of evofosfamide sensitivity were investigated using hierarchical clustering, differential expression and correlation analyses. The antitumour activity of evofosfamide $\left(50 \mathrm{mg} \cdot \mathrm{kg}^{-1} \mathrm{qdx} 5\right.$ for 2-3 cycles with/without 10 Gy radiation on day 5 of cycle 1) was evaluated in two HNSCC xenografts and two HNSCC PDX models derived in house. Evofosfamide was evaluated in combination with CTLA-4 blockade in the murine SCC-VII model. Hypoxic fraction was assessed using pimonidazole.

Results

Of 5 metastatic or locally-advanced HNSCC patients who received evofosfamide after failing standard-of-care, two showed partial responses and three showed stable disease. Evofosfamide was highly selective for hypoxic HNSCC cells and more potent and selective than PR-104A or SN30000. Cell line sensitivity to evofosfamide was correlated with $\mathrm{Br}$ IPM and cisplatin but not with PR-104A, SN30000 or 5-FU, indicating distinct sensitivity determinants. Evofosfamide sensitivity was associated with the expression of genes relating to proliferation. Accordingly, a proliferation metagene identified subtypes within the cell lines that were differentially sensitive to evofosfamide. Xenografts chosen on the basis of putative predictive biomarkers (tumour hypoxia, proliferation subtype) showed the expected patterns of response. Two PDX models were also highly responsive to evofosfamide. SCC-VII was refractory to evofosfamide monotherapy but showed increased growth delay when evofosfamide was combined with CTLA-4 inhibition.

\section{Conclusions}

This study provides a rationale for the clinical evaluation of evofosfamide with immunotherapy and/or radiotherapy in genetically defined subsets of HNSCC.

\section{P465}

Characterization of immune cells in the pre-metastatic niche in a murine model of rhabdomyosarcoma

Sabina Kaczanowska, Meera Murgai, Daniel Beury, Rosandra Kaplan National Cancer Institute, Bethesda, MD, USA

Correspondence: Rosandra Kaplan (sabina.kaczanowska@nih.gov) Journal for ImmunoTherapy of Cancer 2017, 5(Suppl 2):P465

\section{Background}

Tumor metastasis is a critical step in the progression of cancer that is associated with patient mortality. Prior to the arrival of tumor cells at the metastatic site, hematopoietic stem and progenitor cells expand in the bone marrow and mobilize to the pre-metastatic niche, where they differentiate into immunosuppressive myeloid cells [1,2]. Furthermore, recent data has demonstrated that perivascular cells undergo phenotypic changes in the lung that are dependent on the transcription factor KLF4 and enhance metastasis in response to tumor-derived factors [3]. Targeting the pre-metastatic niche by either antibody-mediated depletion of myeloid cells or inhibition of perivascular cell phenotypic switching significantly decreases metastasis $[2,3]$. However, changes in other immune cell populations in the pre-metastatic niche are not well defined. We hypothesize that primary tumor growth will alter immune cell infiltration and/or function in the pre-metastatic niche.

\section{Methods}

Lungs and bone marrow from mice bearing M3-9-M, a metastatic embryonal rhabdomyosarcoma cell line, were harvested at various time points and analyzed by flow cytometry for lymphoid and myeloid populations.

Results

Primary tumor progression was correlated with infiltration of immune cells into the lung before detectable metastasis. There was a dramatic increase of $\mathrm{CD} 11 \mathrm{~b}^{+} \mathrm{Ly} 6 \mathrm{G}^{+}$and $\mathrm{CD} 11 \mathrm{~b}^{+} \mathrm{Ly} 6 \mathrm{C}^{+}$myeloid cells in the lung, and a shift in macrophage and dendritic cell populations and phenotypes in the lung and bone marrow. Furthermore, the proportion of both $\mathrm{CD}^{+}$and $\mathrm{CD} 8^{+} \mathrm{T}$ cells that express PD- 1 is significantly enhanced in the lung, as is the number of leukocytes expressing PD-L1.

\section{Conclusions}

We demonstrate that the immune compartment in the bone marrow and in pre-metastatic lungs changes significantly in response to primary tumor growth. The differences in immune populations that were observed in the lung and bone marrow were distinct, indicating that the immune changes occurring at these sites in response to tumor progression are specific to their unique microenvironment. We postulate that disrupting the formation of the pre-metastatic niche by targeting stromal cell populations combined with immunotherapy could have anti-metastatic potential. These studies will provide insight into the immune signature of metastatic disease and identify new targets for therapeutic intervention with the potential to reduce metastasis.

\section{References}

1. Rosandra Natasha Kaplan et al. VEGFR1-positive haematopoietic bone marrow progenitors initiate the pre-metastatic niche. Nature. 2005; 438(7069):820-7.

2. Amber Jin Giles et al: Activation of Hematopoietic Stem/Progenitor Cells Promotes Immunosuppression Within the Pre-metastatic Niche. Cancer. Res. 2016;76(6):1335-47.

3. Meera Murgai et al. KLF4-dependent perivascular plasticity mediates premetastatic niche formation and metastasis. Nat. Med. In Press.

\section{P466}

Rational design of immuno-oncology biologics with improved safety and efficacy

Margaret Karow ${ }^{1}$, Kurt Jenkins ${ }^{2}$, Miso Park ${ }^{2}$, Parker Johnson ${ }^{2}$

Asaul Gonzalez², Veronica Flesch², Tim Miles², Ulrich Rodeck ${ }^{3}$, John Williams ${ }^{2}$

${ }^{1}$ Akriveia Therapeutics, Thousand Oaks, CA, USA; ${ }^{2}$ City of Hope, Duarte, CA, USA; ${ }^{3}$ Thomas Jefferson University, Philadelphia, PA, USA

Correspondence: Margaret Karow (karow@akriveiatx.com) Journal for ImmunoTherapy of Cancer 2017, 5(Suppl 2):P466

\section{Background}

The benefit of enhancing anti-tumor immune response has been demonstrated by the unprecedented clinical responses of immune checkpoint antagonists and cell-based therapies ${ }^{1}$. However, the challenge with these therapeutic approaches is that they necessarily involve a systemic activation of the immune system ${ }^{2}$. This leads to treatmentlimiting and often debilitating immune related adverse events (irAEs). Attempts to control irAEs include reducing dosing frequency and dosing levels, co-dosing immune suppressants, and drug withdrawal; all of which may limit the utility of immune therapies ${ }^{3}$. Focusing the activity of immuno-oncology agents to the tumor microenvironment is expected to overcome the limitations posed by irAEs. 


\section{Methods}

Using anti-mouse CTLA4 antibody 9D9 and anti-mouse PD1 antibody J43 as a proof of concept, we have applied a rational approach to design molecules that are activated in the tumor microenvironment by tumor-associated proteases. In vitro testing using antigen binding by Surface Plasmon Resonance (SPR), antigen-capture ELISA and kinetic assays provided important information to support the design process. Candidates were further characterized in vivo in C57BI/6 mice implanted with syngeneic MC38 tumors using tumor regression as a measure of efficacy. The potential for improved safety resulting from inhibition of systemic activity was evaluated using a Fluorescent Activated Cell (FAC) analysis of the T cell population in the spleen for the CTLA4 targeting agents. Induction of diabetes in NOD mice was used to evaluate safety potential for the anti-PD1 agents.

Results

The tumor-activated antibodies exhibit significantly reduced affinity for their targets without activation, and full binding affinity is restored with proteolytic activation. In vivo testing of the tumoractivated antibodies led to tumor reduction similar to the parental antibody. Critically, the tumor-activated antibodies exhibit reduced levels of the systemic activity compared to their parental antibody. Conclusions

We have developed and demonstrated a rational approach to efficiently design immunotherapeutics that are locally activated in the tumor microenvironment and exhibit reduced systemic activities. Our approach, which we have termed Aklusion ${ }^{\mathrm{TM}}$, has the potential to improve the safety and efficacy of immuno-oncology therapeutics.

\section{References}

1. Hoos, A. Development of immuno-oncology drugs - from CTLA4 to PD1 to the next generations. Nat Rev Drug Discov. 2016;4:235-47.

2. June $\mathrm{CH}$, Warshauer, JT, Bluestone JA. Is autoimmunity the Achilles' heel of cancer immunotherapy? Nat Med. 2017;23(5):540-547.

3. Abdel-Wahab N, Shah M, Suarez-Almazor ME. Adverse Events Associated with Immune Checkpoint Blockade in Patients with Cancer: A Systematic Review of Case Reports. PLoS ONE. 2016;11 (7): e0160221.

\section{P467}

CCR4 antagonists inhibit regulatory $T$ cell $\left(T_{\text {reg }}\right)$ recruitment and increase effector $T$ cell $\left(T_{\text {eff }}\right)$ numbers in the tumor microenvironment potentiating an anti-tumor response

Oezcan Talay ${ }^{1}$, Lisa Marshall', Cesar Meleza', Maureen Reilly', Omar Robles ${ }^{1}$, Mikhail Zibinsky ${ }^{1}$, Abood Okal' ${ }^{1}$, Jenny McKinnell', Scott Jacobson', Erin Riegler', Sashie Marubayashi', Emily Karbarz', David Chian', Angela Wadsworth', David Wustrow', Jordan Fridman², Paul Kassner

${ }^{1} \mathrm{FLX}$ Bio Inc., South San Francisco, CA, USA; ${ }^{2}$ Formerly at FLX Bio Inc., South San Francisco, CA, USA

Correspondence: Paul Kassner (pkassner@flxbio.com)

Journal for ImmunoTherapy of Cancer 2017, 5(Suppl 2):P467

\section{Background}

Regulatory $T$ cells $\left(T_{\text {reg }}\right)$ are essential for immune tolerance and $T_{\text {reg }}$-mediated suppression of effector $T$ cells ( $\left.T_{\text {eff }}\right)$ is important to control inflammation and prevent autoimmune diseases. However, the presence of $\mathrm{T}_{\text {reg }}$ in the tumor microenvironment (TME) has been shown to dampen anti-tumor immune responses. Human $\mathrm{T}_{\text {reg }}$ express CCR4, the receptor for the chemokines CCL17 and CCL22. Preclinical and clinical data supports a role for CCR4-mediated recruitment and accumulation of $T_{\text {reg }}$ in the TME which can be associated with poor prognosis. Furthermore, patients receiving immunomodulatory agents demonstrate an influx of $\mathrm{T}_{\text {reg }}$ in responding patients which may dampen optimal anti-tumor responses. Therefore, CCR4 is an ideal target to selectively block $T_{\text {reg }}$ recruitment into the TME.

\section{Methods}

We have developed a structurally unique series of small molecule antagonists of CCR4 with cellular potencies in multiple assays (e.g. chemotaxis of primary human $\mathrm{T}_{\text {reg }}$ in $100 \%$ serum) in the low double-digit $\mathrm{nM}$ range. Moreover, these compounds have excellent in vitro and in vivo ADME properties, consistent with convenient oral dosing. These CCR4 antagonists were tested in murine syngeneic tumor models alone and in combination with immunomodulatory agents. During and following treatment, CCR4 ligand levels, tumor infiltrating lymphocytes, and tumor volumes were evaluated.

Results

Preclinically, these CCR4 antagonists block $\mathrm{T}_{\text {reg }}$ migration and support expansion of activated $T_{\text {eff }}$ in the tumor. Our antagonists reduce $T_{\text {reg }}$ in the tumor, but not in peripheral tissues such as blood, spleen or skin; which presents a potential safety advantage to the non-selective approach of depleting anti-CCR4 antibodies. In preclinical efficacy studies, treatment with various checkpoint inhibitors and immune stimulators (e.g., anti-CTLA-4 or anti-CD137) induce the upregulation of CCR4 ligand expression. Combination therapy with CCR4 antagonist and immunomodulatory agents reduced intratumoral $\mathrm{T}_{\text {reg }}$ number and increased number of activated and total $\mathrm{T}_{\text {eff, }}$ resulting in an increase in the intratumoral ratios of both $\mathrm{CD}^{+}$and $\mathrm{CD}^{+} \mathrm{T}_{\text {eff }}$ to $\mathrm{T}_{\text {reg. }}$. The change in these $T_{\text {eff }}$ to $T_{\text {reg }}$ ratios is greater for our CCR4 antagonist in combination than with the immunomodulatory agent alone and correlates with enhanced tumor growth inhibition and increased tumor regression.

Conclusions

Combination therapy with CCR4 antagonist and immunomodulatory

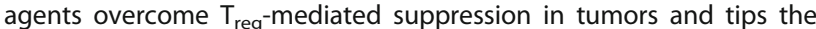
balance toward tumor rejection.

\section{P468}

A novel 3D ex vivo platform of fresh patient tumor tissue with intact tumor microenvironment for immuno-oncology drug development and biomarker discovery

Melanie Mediavilla-Varela, Melba Marie Page, Jenny Kreahling, Scott Antonia, Soner Altiok

Nilogen Oncosystems, Tampa, FL, USA

Correspondence: Soner Altiok (jenny@nilogen.com)

Journal for ImmunoTherapy of Cancer 2017, 5(Suppl 2):P468

\section{Background}

Cancer immunotherapy delivers treatment of high specificity, low toxicity and prolonged activity in subsets of patients. There is an unmet need for clinically relevant preclinical models and translational strategies that recapitulate the complexity of tumor immune microenvironment to test the therapeutic potential of immuno-oncology (IO) drugs, identify rational combination therapies and to develop novel predictive biomarkers of clinical response. Here we describe a novel 3D ex vivo drug assay utilizing fresh patient tumor samples with intact immune microenvironment to facilitate oncology and IO drug development and biomarker discovery.

Methods

$3 \mathrm{D}$ ex vivo studies were performed with fresh tumor tissue obtained from consented patients with non-small cell lung, renal, urothelial, head \& neck and breast carcinoma. 3D tumor microspheroids (150 $\mu \mathrm{m}$ in size) prepared according to a proprietary protocol were treated in their intact immune microenvironment with PD1 inhibitors pembrolizumab or nivolumab at $10 \mu \mathrm{g} / \mathrm{ml}$ for 36 hours. Culture media was collected over the course of the experiments to simultaneously analyze the differential release of cyto-and chemokines. Treatment-mediated changes in T-cell activation and immune cell populations were monitored by flow cytometry while NanoString PanCancer Immune Profiling platform containing probes to quantitate 770 immune function genes was 
used to determine positive and negative associations between expression of immune function genes and TIL activation by ex vivo treatment. Immunohistochemical studies were performed to identify PD-L1 expression and immune cell composition in tumor samples.

Results

$3 \mathrm{D}$ ex vivo samples treated with pembrolizumab or nivolumab demonstrated PD1 occupation in all tumors, while approximately $30 \%$ of tumors showed increased CD8 T-cell activation that closely correlated with proinflammatory cytokine release in the conditioned media. Immune gene expression profiling studies revealed increased expression of IFNg inducible genes in tumors showing activated CD8 population by flow cytometry. No significant correlation was found between tumor PD-L1 expression and ex vivo response to treatment. Furthermore, we showed pembrolizumab or nivolumab treatment ex vivo led to changes in tumor immune cell composition in specifically including changes in monocyte populations in subset of tumors used in the ex vivo experiments.

\section{Conclusions}

Our studies showed that this approach can be used to identify rationale drug combinations and to develop potential companion diagnostics to facilitate biomarker-driven drug development efforts and personalized medicine in immuno-oncology.

\section{P469}

Tumor oxygenator OMX reverses multiple modes of

immunosuppression, activates anti-tumor immunity and cures as a single agent, and in combination with anti-PD-1 in resistant orthotopic tumors

Ana Krtolica, Natacha Le Moan, Philberta Leung, Sarah Ng, Tina Davis, Carol Liang, Jonathan Winger, Stephen P. L. Cary

Omniox, Inc San Carlos, CA, USA

Correspondence: Ana Krtolica (akrtolica@omnioxinc.com) Journal for ImmunoTherapy of Cancer 2017, 5(Suppl 2):P469

\section{Background}

Hypoxia, a common feature of solid tumors that correlates with poor patient outcomes, is a central mediator of escape from innate and adaptive anti-tumor immune responses. Numerous classes of immunotherapy target specific pathways of hypoxia-driven immunosuppression. Reversing tumor hypoxia, however, would work upstream at one of the primary nodes of immunosuppression to stimulate effective responses. The hypoxic tumor microenvironment arises due to dysfunctional tumor vasculature, and oxygen diffusion is restricted to within $\sim 80 \mathrm{~mm}$ of active capillaries. To reverse tumor hypoxia and create an immunopermissive microenvironment, we engineered OMX, a stable, non-vasoactive, high affinity oxygen carrier that preferentially accumulates in solid tumors and specifically re-oxygenates hypoxic microenvironments without affecting normoxic tissues. OMX improves antitumor immune responses.

Methods

We used immunohistochemical, flow cytometric, and multiplex cytokine analyses to evaluate OMX's ability to weaken immunosuppression in the tumor microenvironment of orthotopic GL261 brain tumors and promote tumor cures.

Results

A single dose of OMX in GL261 tumor-bearing mice reduces tumor hypoxia, enhances $T$ cell accumulation into the tumor, decreases Tregs and increases activation and proliferation of cytotoxic $T$ lymphocytes (CTLs). Specifically, OMX increases the Teff/Treg ratio by $\sim 3$-fold, indicating a switch from an immunosuppressive to an immunopermissive microenvironment. In addition, OMX stimulates secretion of a major chemoattractant for Th1 cells, and increases the accumulation of CD8+ tumor-specific T cells in the tumor tissue. When combined with antiPD-1, OMX increases CTL infiltration, proliferation and cytotoxic activity, and modulates IFNY and IFNy-inducible cytokines that may polarize T cells towards a Th1 phenotype. OMX alone resulted in $\sim 50 \%$ tumor cures when treatment is initiated at day 7 in GL261 tumor-bearing mice. Furthermore, the combination of OMX with anti-PD-1 in latestage GL261 tumor-bearing mice increases mouse survival by $~ 80 \%$ and in large PD-1 resistant GL261 tumors, OMX significantly increases the median survival by $\sim 50 \%$. The survival benefit observed with OMX could be predicted with an identified circulating chemokine signature (post-hoc test).

Conclusions

By delivering oxygen specifically to the hypoxic tumor microenvironment, OMX may restore anti-cancer immune responses in cancer patients through activation of inflammatory cytokine cascades and mobilization and activation of tumor-specific T cells. As a unique, pleiotropic immunotherapy, OMX may enhance immune control of tumors to improve patient outcomes.

\section{P470}

Tertiary lymphoid organs as a good prognostic indicator following neoadjuvant chemo (radio) therapy for pancreatic cancer

Shota Kuwabara, Takahiro Tsuchikawa, Yoshitsugu Nakanishi, Toshimichi Asano, Takehiro Noji, Yo Kurashima, Yuma Ebihara, Soichi Murakami, Toru Nakamura, Keisuke Okamura, Toshiaki Shichinohe, Satoshi Hirano Hokkaido University Graduate School of Medicine, Sapporo Hokkaido, Japan Correspondence: Takahiro Tsuchikawa (medicineman424@yahoo.co.jp) Journal for ImmunoTherapy of Cancer 2017, 5(Suppl 2):P470

\section{Background}

Pancreatic cancer (pancreatic ductal adenocarcinoma; PDAC) is a highly malignant tumor that frequently develops local recurrence and distant metastasis even after a curative resection. Prognosis of PDAC is very poor, and the overall 5-year survival rate for patients with PDAC treated by curative resection is $15-25 \%$. Recently, neoadjuvant chemo (radio) therapy had been reported to improve treatment outcome. Therefore, it is an urgent need to explore surrogate markers predicting patients' outcome. We herein focused on the tumor microenvironment as one of the candidates for monitoring local pathological and immunological reactions, and put a spotlight on tertiary lymphoid organs (TLO, alternatively, ectopic lymphoid tissue) as a biomarker reflecting the effect of preoperative neoadjuvant chemotherapy (NAC).

Methods

In this study, we retrospectively analyzed the area of TLO, dividing 140 patients diagnosed with PDAC from January 2009 to December 2015 into two groups, those who performed upfront surgery (SF group; $n=93$ ) and those who underwent NAC (NAC group; $n=47$ ). Then we measured the area $\left(\mathrm{mm}^{2}\right)$ of TLO, the total area $\left(\mathrm{mm}^{2}\right)$ of the tumor, and calculated the area ratio of total tumor to the total TLO (TLO/ Tumor ratio) by microscopic observation, and statistically analyzed the association between the level of TLO formation and prognosis in the tumor microenvironment. All the microscopic images were imported as digital photo files using a NanoZoomer Digital Pathology system (Hamamatsu Photonics, Hamamatsu, Japan), and imaging analysis were performed using Image J software $(\mathrm{NIH}$, Bethesda, MD, USA).

Results

The TLO formation was recognized in 128 patients (91.4\%; 128/140). There were no significant difference in terms of TLO formation nor TLO/ Tumor ratio between the two groups $(94.6 \% ; 88 / 93$ v.s. $85.1 \%$; $40 / 47, p=0.0575$ and $0.66 \pm 1.22$ v.s. $0.64 \pm 8.62, p=0.2342$, respectively). The 5-year-overall survival rate of NAC group had been significantly better than SF group ( $41 \%$ v.s. $16 \%, p=0.0203)$. On multivariate analysis, lymphnode metastaisis (HR 0.047, 95\% Cl: 0.005-0.265, $p<0.0001)$ and high TLO/ Tumor ratio (HR 0.108, 95\% Cl: $0.010-0.593$, $p=0.008$ ) were revealed as independent favorable prognostic factors. Conclusions

Our present results indicate that the level of TLO serves as one of the valuable independent prognostic markers following neoadjuvant chemo (radio) therapy for PDAC. 
P471

Patterns of immune cell infiltration in murine models of melanoma: roles of antigen and tissue site in creating inflamed tumors

Katie Leick', Joel Pinczewski ${ }^{2}$, Donna Deacon' ${ }^{1}$, Amber Woods ${ }^{1}$, Marcus Bosenberg $^{3}$, Victor Engelhard ${ }^{1}$, Craig Slingluff ${ }^{\prime}$

'University of Virginia, Charlottesville, VA, USA; ${ }^{2}$ Dorevitch Pathology, North Melbourne, Australia; ${ }^{3}$ Yale University, New Haven, CT, USA

Correspondence: Victor Engelhard, Craig Slingluff (kml2h@virginia.edu) Journal for ImmunoTherapy of Cancer 2017, 5(Suppl 2):P471

\section{Background}

Immune cell infiltration is associated with improved survival in melanoma and other cancers. Melanoma metastases in humans may be grouped into three Immunotypes representing patterns of immune cell infiltration: A (sparse), B (perivascular cuffing), and C (diffuse) ${ }^{1}$. Prior work suggests that anatomical site and intratumoral vascularity can have dramatic effects on $T$ cell responses and immune infiltration. Murine models provide opportunities to understand factors that control immune infiltrates, but Immunotypes have not been defined for common murine models. We hypothesize that patterns of immune cell infiltration can be defined in Braf/Pten and B16 murine models that are similar to Immunotypes observed in human melanoma metastases ${ }^{1}$, and that AAD and OVA transfectants and intraperitoneal (i.p.) location will facilitate greater immune infiltration in the B16 model.

Methods

We performed immunohistochemistry for S100, CD31, and CD45, with immune cell enumeration, Immunotyping, and scoring of vascular density in spontaneous melanoma models and in transplantable melanoma models (B16-F1, B16-OVA, and B16-AAD). The transplantable tumors were grown either in subcutaneous (s.c.) or i.p. locations.

Results

The Braf/Pten and Braf/Pten/ $\beta$-catenin tumors had low immune cell counts in $6 / 6$ tumors that were consistent with Immunotype A (Fig. 1A, Fig. 1B), which was also seen in 11/12 B16-F1 tumors (Fig. 1C). In comparison, 9/10 tumors in B16-OVA s.c. and i.p. and 5/6 B16-AAD s.c. tumors were characterized as Immunotype B. Only the B16-AAD i.p. tumors were Immunotype $C$ in $4 / 6$ tumors. The i.p. location was characterized by significantly higher immune cell counts in B16-OVA tumors $(P=0.0008$, Fig. $1 D)$ and higher tumor vascularity. Interestingly, mutated cancer antigens in mutant BRAF models and in B16 melanoma were insufficient to induce significant immune infiltrates, suggesting the existence of barriers to immune infiltration. Addition of model neoantigens (OVA or AAD) overcame existing barriers in a manner that was dependent in part on tumor site.

\section{Conclusions}

These murine models may be useful for preclinical studies of combination immune therapy, and suggest that both tumor antigen expression and tumor location contribute to the extent of immune cell infiltration and Immunotype in murine melanoma.

Spontaneous Braf/Pten and Braf/Pten/ $\beta$-catenin dermal melanomas lacked significant intratumoral immune cell infiltrates characteristic of Immunotype A (A), and the mean CD45+ cell counts for 3 mice of each tumor type were all less than 10 per hpf in murine melanoma (B). Associations of B16 tumor cell type and tumor location with immune infiltrate. B16-F1 melanomas (B16-F1), or those transfected with OVA or AAD, were grown in from s.c. or i.p. locations and evaluated for Immunotype (C) and overall CD45+ cell counts (D). Significance is noted by asterisks: ${ }^{*}<0.05,{ }^{* *}<0.001,{ }^{* * *}<0.0001$.

\section{References}

1. Erdag G, Schaefer JT, Smolkin ME, et al. Immunotype and immunohistologic characteristics of tumor-infiltrating immune cells are associated with clinical outcome in metastatic melanoma. Cancer Res. 2012;72(5):1070.
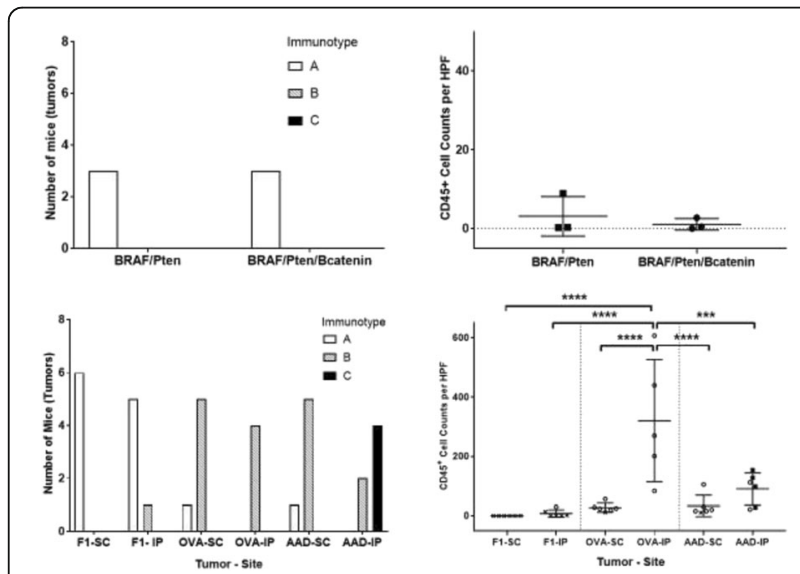

Fig. 1 (abstract P471). Immunotype and immune cell density of Braf/Pten and B16 murine melanomas

P472

DARPin ${ }^{\circledR}$-based fibroblast activation protein-targeted agonists of 41BB and OX40 co-stimulate T-cells in a Fc-receptor-independent, tumor-restricted manner

Alexander Link, Julia Hepp, Ulrike Fiedler, Christian Reichen,

Clara Metz, Alexan der Titz, Ivana Tosevski, Joanna Taylor,

Zita Arany, Laurent Juglair, Patricia Schildknecht, Yvonne Kaufmann,

Denis Villemagne, Simon Fontane, Ralph Bessey, Christof Zitt,

Keith Dawson, Dan Snell, Daniel Steiner, Michael Stumpp, Victor Levitsky

Molecular Partners AG, Schlieren-Zürich, Switzerland

Correspondence: Victor Levitsky

(victor.levitsky@molecularpartners.com)

Journal for ImmunoTherapy of Cancer 2017, 5(Suppl 2):P472

\section{Background}

In addition to check point blockade, immunosuppressive tumor microenvironment can be counteracted by enhanced triggering of T-cell co-stimulatory receptors. In vivo agonistic activity of antibodies against such receptors usually requires $\mathrm{Fc}$-dependent crosslinking and comes at a cost of various systemic, immune related toxicities. We developed a new class of biologics based on the DARPin ${ }^{\oplus}$ technology platform which allow tumorrestricted engagement of co-stimulatory molecules independently of Fc-receptors. Our preclinical in vitro and in vivo results indicate that DARPin ${ }^{\oplus}$-based co-stimulatory receptor agonists are comparable to or exceed conventional antibodies in agonistic potency but restrict their activity to the tumor site effectively widening the therapeutic window for compounds exploiting this mode of action. 


\section{Methods}

We generated multi-specific DARPin ${ }^{\circledast}$ therapeutics, which bind to human fibroblast activation protein (FAP) and either to 4-1BB or OX40 co-stimulatory receptor, preferentially expressed on CD8+ and CD4+ lymphocytes, respectively. The agonistic activity of both drug candidates was analyzed using NFkB-pathway activation reporter cells lines and several types of T-cell activation assays. The 4-1BBagonist was tested in a xenotransplantation tumor model established in NOG mice humanized by PBMCs.

Results

Both 4-1BB and OX40-specific FAP-targeted DARPin ${ }^{\circledR}$ drug candidates efficiently induced activation of the NFkB pathway in 4-1BB or OX40 expressing reporter cells but only after crosslinking on FAP, either surface immobilized or expressed on cells. The capacity of the molecules to enhance lymphokine secretion and upregulate expression of activation markers on T-cells stimulated by polyclonal T-cell receptor crosslinking or specific antigen recognition also strictly required FAPdependent oligomerization.

Urelumab, a clinically tested 4-1BB agonistic antibody associated with liver toxicity, led to signs of severe graft-versus-host disease (GVHD) and weight loss greater than 20\% in the humanized NOG mice transplanted with HT-29 tumor cells at termination of the experiment. In contrast, mice treated with several doses of the 41BB-specific DARPin ${ }^{\circledast}$ drug candidate showed only minor signs of GVHD and marginal weight loss. Further analysis indicated that urelumab induced CD8+ T-cell expansion both in the peripheral blood and tumor tissue of animals while the 4-1BB-specific DAR$\mathrm{Pin}^{\circledast}$ drug candidate triggered a similar change only in the tumor sites.

Conclusions

Thus, tumor targeted DARPin ${ }^{\oplus}$ agonists of co-stimulatory molecules can enhance T-cell activity in a manner independent of Fccrosslinking and limited to the tumor site, thereby avoiding systemic toxicity caused by antibodies against the same immunologic targets. This approach with DARPin ${ }^{\circledR}$ therapeutics can be broadly applied to a range of tumor targets and immune receptors.

\section{P473}

Immuno-oncological efficacy of RXDX-106, a novel TAM (TYRO3, AXL, MER) family small molecule kinase inhibitor

Erin Lew ${ }^{1}$, Yumi Yokoyama', Colin Walsh', Jack Lee', Joanne Oh'

Elizabeth Tindall', Robin Navarez', Amy Dilberto ${ }^{1}$, Heather Ely',

Ruth Seelige ${ }^{2}$, Amanda Albert ${ }^{1}$, Jack Bui ${ }^{2}$, Gary Li ${ }^{1}$

${ }^{1}$ Ignyta, Inc, San Diego, CA, USA; ${ }^{2}$ University of California, San Diego, San Diego, CA, USA

Correspondence: Gary Li (elew@ignyta.com)

Journal for ImmunoTherapy of Cancer 2017, 5(Suppl 2):P473

\section{Background}

The receptor tyrosine kinases (RTKs), TYRO3, AXL, and MER (TAM), are emerging targets for cancer therapy. Particularly, TAM RTKs play a key homeostatic role as negative regulators of immune responses. RXDX-106 is a potent and selective TAM family inhibitor in preclinical development. Here, we sought (1) to evaluate the efficacy of RXDX-106 as a single agent and in combination with immune checkpoint inhibitors; (2) to identify the immunomodulatory mechanisms of action; and (3) to decipher the reciprocal regulation of TAM expression on cancer and immune cells, and how pharmacological inhibition of TAM signaling would be beneficial, given their complex interaction and regulation in the tumor microenvironment.

Methods

The anti-tumor efficacy of RXDX-106, as single agent or in combination with checkpoint inhibitors, was studied in tumors grown either in syngeneic models or in immunocompromised mice. Immuno-phenotyping was performed in tissue and tumor samples collected during or at the end of studies by flow cytometry, gene expression and protein analyses, and Luminex bead-based multiplex assays.

Results

Here, we demonstrated that tumor growth inhibition in an MC38 model was associated with increase in intra-tumoral M1 macrophages, CD169 ${ }^{\text {hi }}$ antigen presenting macrophages, and PD-L1 expression. Also observed were a higher ratio of CD8 $+/ C D 4+$ T cells and an increased expression of CD69 and PD-1 on CD8+ $T$ cells, indicative of activation of cytotoxic $T$ cells. Additionally, an increase in Granzyme B and IFNY with a concomitant decrease in VEGF in tumor cell lysates indicted $T$ cell activation and M1 polarization of macrophages. Furthermore, in a CT26 syngeneic model, we demonstrated that RXDX-106 inhibited tumor growth as a single agent, and the effect was further potentiated by combination therapy with immune checkpoint inhibitors, as evidenced by upregulation of anti-tumor gene expression patterns, upregulation of anti-tumor cytokines in the tumor cell lysates, and an increase in T cell function. Finally, in an AXL-driven tumor model, we demonstrated that AXL expressing tumors induced a pro-tumorigenic immune environment, and treatment with RXDX-106 resulted in complete tumor regression and re-polarization of macrophages towards an $\mathrm{M} 1$, anti-tumor phenotype.

Conclusions

RXDX-106 has the potential to restore and enhance immune function in macrophages and T cells, resulting in repolarization of the immune response towards an anti-tumor microenvironment. The unique mechanism of activating both innate and adaptive immunity, plus regulating cross-talk between immune cells and tumor cells by RXDX-106 supports clinical development of RXDX-106 to potentially treat a wide variety of cancers.

\section{P474}

Gene expression in MHC II pathway may predict triple negative breast cancer prognosis

Amy Zhao', Gavin Bond', Katherine Varley², Andres Forero-Torres ${ }^{3}$,

Albert LoBuglio ${ }^{3}$, Dongquan Chen ${ }^{3}$

${ }^{1}$ University of Pennsylvania, Philadelphia, PA, USA; ${ }^{2}$ University of Utah,

Salt Lake City, UT, USA; ${ }^{3}$ University of Alabama at Birmingham,

Birmingham, AL, USA

Correspondence: Amy Zhao (yli@uabmc.edu)

Journal for ImmunoTherapy of Cancer 2017, 5(Suppl 2):P474

\section{Background}

Triple negative breast cancer (TNBC) is a subtype with heterogeneous patient outcomes. It behaves aggressively, and patients are not a candidate for ER or HER2/Neu targeted therapy. Although there have been major research efforts directed at establishing genomic signatures to assess prognosis of breast cancer, prognostic gene expression signatures are not well developed for TNBC. In this study, we investigated twenty-four genes [1] related to the MHC II pathway in 47 TNBC patients and explored gene(s) that can be used to distinguish who will relapse. This work may lead to TNBC management and treatment and could also provide mechanism for the generation of the anti-tumor immune response.

Methods

Forty-seven snap frozen primary TNBC tumor specimens were analyzed using RNA-Seq. Individual gene expressions were transformed to fit a normal distribution. The Logistic regression analysis was used to identify genes that can predict no relapse. The optimal cutpoint was determined from the receiver operating characteristic analysis. This study has the approval of UAB's Institutional Review Board.

Results

CIITA had significantly high expression in TNBC patients who had not relapsed $(\mathrm{P}<0.0001)$. With high CIITA expression, TNBC were 10 times more likely to have not relapsed than those with low expression (OR $=10.8,95 \% \mathrm{Cl} 2.8-41.9, \mathrm{p}=0.006)$. The sensitivity and specificity are 
$75 \%$ and $78.3 \%$, respectively. Using multivariable approach with all 24-gene panel, we identified three genes, CIITA, CTSH and KRT14, which together can predict no relapse with $87.5 \%$ sensitivity, and $78.3 \%$ specificity. Further, at the optimal cut point, we found CIITA alone can predict TNBC no relapse with $84 \%$ sensitivity and $77 \%$ specificity and accuracy of $81 \%$. It predicted $17 / 22$ relapse, and 21/25 no relapse correctly. These biomarkers were independent predictors of TNBC prognosis regardless of age, race, tumor grade and stage. The findings were confirmed in public microarray data from 199 patients with TNBC confirmed.

\section{Conclusions}

High expression of genes in the MHC II pathway, e.g. CIITA, may predict TNBC prognosis with high sensitivity and specificity regardless of age, race, and tumor grade and tumor stage. This work may lead TNBC management and treatment. Although we explored the optimal point to distinguish patients' outcome, it is important to further establish the threshold in a larger TNBC population.

\section{References}

1. Forero A, et al. Expression of the MHC Class II Pathway in Triple-Negative Breast Cancer Tumor Cells is Associated with a Good Prognosis and Infiltrating Lymphocytes. Cancer Immunol Res. 2016; 4(5): 390-399.

\section{P475}

Profiling Immune Infiltration of Glioblastoma

Camilo Fadul, Craig Slingluff, lleana Mauldin

University of Virginia, Charlottesville, VA, USA

Correspondence: Craig Slingluff (ileanasoto@virginia.edu)

Journal for ImmunoTherapy of Cancer 2017, 5(Suppl 2):P475

\section{Background}

The median survival for patients with glioblastoma (GBM) treated with standard of care therapies is 15-20 months. The efficacy of immunotherapy for many types of cancer make this an attractive strategy to improve outcomes for patients with GBM; unfortunately, thus far these have not had demonstrated efficacy in GBM. In other cancers, the presence of immune cell infiltration, the pattern of infiltration, and the expression of immune markers have been associated with survival and response to some types of immunotherapy. However, the immune infiltrate in GBM has not been well-characterized. Methods

Multispectral immunohistochemistry images were obtained from 6 GBM specimens stained with CD8, CD20, FoxP3, PNAD, CD83 and Ki67, and analyzed for tumor infiltrating lymphocytes using digital software. Results

In preliminary studies, we have observed that GBM can have dense B cell infiltrates located near blood vessels; however, the functional role of $B$ cells in GBM, and the prevalence of these cells has been unstudied. Few studies have been performed evaluating B cells in GBM and suggest contradictory roles for B cells in GBM. Candolfi et al. studied the role of $B$ cells in a GBM murine model and concluded that B cells act as antigen presenting cells, lending a critical role in clonal expansion of tumor antigen specific T cells, and brain tumor regression in mice. Conversely, Saraswathula et al. showed that patients with GBM have Bregs in peripheral blood. Bregs downregulate effector T cell responses through the secretion of immunosuppressive cytokines such as IL-10 and through cell-cell contact. Thus, these studies present conflicting roles for B cells in the GBM setting. As a preliminary assessment, we examined the association between survival and overexpression of the MS4A1 gene which encodes for CD20, using RNA-seq gene expression data obtained from TCGA. The expression data showed that patients with overexpression of the gene encoding for CD20 had decreased overall survival $(p=0.0111)$. While these data provide interesting preliminary findings, they need to be further investigated, by evaluating markers of $B$ cell effector functions in GBM and the impact of $B$ cells on T cells infiltrating tumor. However, considering these gene expression data, we hypothesize that $\mathrm{CD}^{2} \mathrm{O}^{+} \mathrm{B}$ cells in GBM may negatively impact patient survival, and that these cells may constitute Bregs.

Conclusions

To optimize immunotherapy, there is a necessity to understand the prevalence and the functional role that B cells play in GBM and their impact on patient survival.

P476

Targeting the IDO/TDO pathway through degradation of the immunosuppressive metabolite kynurenine and inhibition of the downstream AHR pathway

Karen McGovern' ${ }^{1}$ X. Michelle Zhang ${ }^{1}$, Everett Stone ${ }^{3}$, Todd Triplett ${ }^{3}$, Kendra Garrison ${ }^{3}$, Silvia Coma', Luis Felipe Campesato ${ }^{2}$, Meghan Walsh', Jeremy Tchaicha', Candice Lamb ${ }^{3}$, Christos Karamitros ${ }^{3}$, John Blazek ${ }^{3}$, Taha Merghoub ${ }^{2}$, Jedd Wolchok ${ }^{2}$, George Georgiou ${ }^{3}$, Mark Manfredi ${ }^{\prime}$ ${ }^{1}$ Kyn Therapeutics, Cambridge, MA, USA; ${ }^{2}$ Memorial Sloan Kettering Cancer Center, New York, NY, USA; ${ }^{3}$ University of Texas, Austin, TX, USA

Correspondence: Karen McGovern (karen@kyntherapeutics.com) Journal for ImmunoTherapy of Cancer 2017, 5(Suppl 2):P476

\section{Background}

Checkpoint inhibitors have become the cornerstone of immunebased oncology therapy. Several orthogonal immune pathways are currently being investigated to relieve suppression or boost activity of the innate and adaptive immune system, including the study of the immune-modulating role of metabolites. Both Indoleaminepyrrole 2,3-dioxygenase 1 (IDO1) and tryptophan 2,3-dioxygenase 2 (TDO2) enzymes metabolize tryptophan forming kynurenine, which binds the aryl hydrocarbon receptor (AHR) in multiple innate and adaptive immune cell types, causing a net immunosuppressive environment. Both enzymes are upregulated across many tumor types, and the IDO1 enzyme has been clinically validated with small molecule inhibitors in combination with checkpoint inhibition, lending credence to the tumor microenvironment containing small molecule metabolites that induce immune cell tolerance, and leaving the opportunity for broader efficacy if both pathways can be targeted. Kynureninase (Kynase), an enzyme degrading kynurenine generated from IDO1 and TDO2 pathway, as well as AHR antagonism through small molecule inhibition, can potentially relieve the immunosuppression on multiple cells types and provides novel approaches to inhibiting this pathway in multiple tumor types. Methods

For Kynase, a pegylated enzyme was used for in vitro and in vivo studies. Pharmacodynamic (kynurenine depletion) and efficacy studies were performed in syngeneic tumor models as single agent or in combination with checkpoint inhibitors (anti-PD1 or anti-CTLA4). Exvivo studies were done to analyze the immune cell composition of tumors post-treatment and the effect on AHR signaling. For AHR antagonists, in vitro and in vivo studies were performed to assess kynurenine-dependent signaling through AHR.

Results

Kynase depleted kynurenine produced by IDO1+, TDO2+ and dual positive human cancer cells whereas, the IDO1 inhibitor epacadostat or TDO2 inhibitor 680C91 selectively inhibited Kyn production in IDO1+ or $\mathrm{TDO} 2+$ cells, respectively. Kynase ameliorated the suppressive effects of kynurenine on CD4+ and CD8+ T-cells. In B16F10 tumor-bearing mice, a single subcutaneous dose of Kynase depleted kynurenine in the plasma and tumors, leading to an increase in tumor infiltrating CD8+ and CD4+ lymphocytes. Kynurenine activation of AHR in cells, measured by gene expression, is inhibited with AHR antagonists. Kynase demonstrated significant tumor growth inhibition and survival benefit either as a single agent or in combination with checkpoint inhibitors in B16F10, CT26 and 4T1 models. Interestingly, Kynase combined with anti-PD1, showed greater efficacy than epacadostat / anti-PD1 combination.

Conclusions

Our data support clinical development of human Kynase for the treatment of cancers where both IDO1/TDO2 pathways play a significant immunosuppressive role through kynurenine production. 
P477

Novel effector phenotype of Tim3 + regulatory T cells leads to enhanced suppressive function in head and neck cancer patients Zhuqing Liu1, Elizabeth McMichael ${ }^{2}$, Gulidanna Shayan ${ }^{3}$, Jing Li ${ }^{3}$,

Rghvendra Srivastava ${ }^{2}$, Lawrence Kane ${ }^{2}$, Robert Ferris ${ }^{2}$

${ }^{1}$ Tongji University, Shanghai, China; ${ }^{2}$ University of Pittsburgh, Pittsburgh, PA, USA; ${ }^{3}$ Tsinghua University, Beijing, China

Correspondence: Robert Ferris (cmichaele@upmc.edu)

Journal for ImmunoTherapy of Cancer 2017, 5(Suppl 2):P477

\section{Background}

Regulatory T (Treg) cells are important suppressive cells among tumor infiltrating lymphocytes. Treg express the well-known immune checkpoint receptor PD-1, which is reported to mark "exhausted" Treg with lower suppressive function. T cell immuglobulin mucin (Tim)-3, a negative regulator of Th1 immunity, is expressed by a sizable fraction of TIL Tregs, but the functional status of Tim $-3^{+}$Tregs remains unclear.

Methods

CD4 $4^{+}$CTLA- 4 CD25 ${ }^{\text {high }}$ Treg were sorted from freshly excised head and neck squamous cell carcinoma (HNSCC) TIL based on Tim-3 expression. Functional and phenotypic features of these $\mathrm{Tim}^{-3^{+}}$and $\mathrm{Tim}-3^{-} \mathrm{TIL}$ Tregs were tested by in vitro suppression assays and multi-color flow cytometry. Gene expression profiling and NanoString analysis of Tim $-3^{+}$ TIL Treg was performed. A murine HNSCC model was used to test the effect of anti-PD-1 immunotherapy o $\mathrm{nTim}-3^{+}$Treg.

Results

Despite high PD-1 expression, Tim- $3^{+}$TIL Treg displayed a greater capacity to inhibit naïve T cell proliferatin than Tim-3- Treg. Tim $-3^{+}$Treg from human HNSCC TIL also displayed an effector-like phenotype, with more robust expression of CTLA-4, PD-1, CD39, and IFN- $\gamma$ receptor. Exogenous IFN- $\gamma$ treatment could partially reverse the suppressive function of Tim-3+ TIL Treg. Anti-PD-1 immunotherapy downregulated Tim3 expression on Tregs isolated from murine HNSCC tumors, and this treatment reversed the suppressive function of HNSCC TIL Tregs.

Conclusions

Tim-3+ Treg are functionally and phenotypically distinct in HNSCC $\mathrm{TIL}$, and are highly effective at inhibiting T cell proliferation despite high PD-1 expression. IFN- $\gamma$ induced by anti-PD-1 immunotherapy may be beneficial by reversing Tim $-3^{+}$Treg suppression.

\section{P478}

Formation and functional associations of CD49a-, CD49b- and CD103-expressing CD8 T cell populations in human metastatic melanoma

Marit Melssen', Walter Olson', Nolan Wages', Ciara Hutchison', lleana Mauldin ${ }^{1}$, Cornelis Melief ${ }^{2}$, Timothy Bullock', Victor Engelhard', Craig Slingluff, J $\mathrm{r}^{1}$

'University of Virginia, Charlottesville, VA, USA; ${ }^{2}$ ISA Pharmaceuticals, Leiden, Netherlands

Correspondence: Marit Melssen (mm2xz@virginia.edu)

Journal for ImmunoTherapy of Cancer 2017, 5(Suppl 2):P478

\section{Background}

Integrins $a 1 \beta 1$ (CD49a), a2 $\beta 1$ (CD49b) and aE $\beta 7$ (CD103) mediate retention of lymphocytes in peripheral tissues, and their expression is upregulated on $\mathrm{CD}^{+}$tumor infiltrating lymphocytes (TIL) compared to circulating lymphocytes. Little is known about what induces expression of these retention integrins (RI). We hypothesized that RI expression marks functionally distinct $T$ cell subsets in the tumor microenvironment (TME) and that these subsets can be induced by selected cytokines and T cell receptor (TCR) stimulation.

Methods

$\mathrm{CD}^{+}$TIL from 19 human melanoma metastatic lesions were stained for CD49a, CD49b, CD103, and cytokines either directly or after PMA/ lonomycin stimulation. For in vitro studies, circulating $\mathrm{CD}^{+}{ }^{+} \mathrm{T}$ cells from normal donors $(n=4-7)$ were cultured with cytokines and/or $\mathrm{CD} 3 / \mathrm{CD} 28$ stimulation and evaluated by flow cytometry.
Results

Among $\mathrm{CD}^{+}$TIL from melanoma metastases, $\mathrm{RI}^{\text {neg }}, \mathrm{CD} 49 \mathrm{a}$ ${ }^{+} \mathrm{CD} 49 \mathrm{~b}^{\text {neg }} \mathrm{CD} 103^{\text {neg }}$ and $\mathrm{CD} 49 \mathrm{a}^{+} \mathrm{CD} 49 \mathrm{~b}^{+} \mathrm{CD} 103^{\text {neg }}$ subpopulations were found in virtually all patients. However, only a subset of patients had $\mathrm{CD}_{103}{ }^{+}$subpopulations $\left(\mathrm{CD} 49 \mathrm{a}^{+} \mathrm{CD} 49 \mathrm{~b}^{\text {neg }} \mathrm{CD} 103^{+}\right.$and $\mathrm{CD} 49 \mathrm{a}^{+} \mathrm{CD} 49 \mathrm{~b}$ $\left.{ }^{+} \mathrm{CD} 103^{+}\right)$. Each subset was assessed for cytokine production. A large fraction of $\mathrm{CD} 49 \mathrm{a}^{+} \mathrm{CD} 49 \mathrm{~b}^{\text {neg }} \mathrm{CD} 103^{\text {neg }} \mathrm{TIL}$ was multifunctional, producing IFNY, TNFa and IL-2. In contrast, CD49a ${ }^{+} C D 49 b^{\text {neg }} C D 103^{+}$TIL only expressed IFN $\gamma$, and both $\mathrm{CD} 49 \mathrm{a}^{+} \mathrm{CD} 49 \mathrm{~b}^{+} \mathrm{CD} 103^{\text {neg }}$ and $\mathrm{CD} 49 \mathrm{a}^{+} \mathrm{CD} 49 \mathrm{~b}$ ${ }^{+} \mathrm{CD} 103^{+}$subsets were incapable of inducing IFN $\gamma$, TNFa or IL-2 at comparable levels (Fig.1). Next, we evaluated Rl expression of naïve T cells in response to different stimuli in vitro. TCR or IL-2 stimulation alone induced two $\mathrm{Rl}^{+}$cell populations: one that co-expressed CD49a and CD49b and another that expressed CD49a alone. Adding TNFa to TCR stimulation further induced these populations, whereas TGF $\beta+$ TCR stimulation generated two additional populations; CD49a ${ }^{+} \mathrm{CD} 49 \mathrm{~b}^{\text {neg }} \mathrm{CD}_{103^{+}}$and $\mathrm{CD} 49 \mathrm{a}^{+} \mathrm{CD} 49 \mathrm{~b}^{+} \mathrm{CD} 103^{+}$. Each of these subsets was found among melanoma TIL, suggesting that TCR stimulation, IL-2, TNFa and/or TGF $\beta$ may be responsible for the generation of $\mathrm{RI}^{+}$subsets in the TME.

Conclusions

These observations suggest that CD49b and/or CD103 expression is upregulated as effector TIL lose the capability of producing cytokines and become more exhausted. Generation of $\mathrm{CD}_{103}{ }^{+}$subsets in the TME may be driven by TCR stimulation and TGF $\beta$ and given their absence in a fraction of tumors, may only be present in tumors producing sufficient TGF $\beta$. The other $\mathrm{RI}^{+}$subsets can be induced by IL-2, TCR engagement and/or TNFa, which we hypothesize will be available in all tumors. Together, our findings identify opportunities to modulate RI expression in the TME by cytokine therapies and to augment subsets with specific RI expression in the interest of improving immune therapies for cancer or other immune-related diseases.

P479

MPL-5821, an ESM ${ }^{\mathrm{TM}}$-p38 MAPK Inhibitor, modulates macrophage plasticity leading to enhanced IL-12p70 and IFN gamma, reduced IL-10 and the reversal of macrophage induced T-cell suppression Martin Perry ${ }^{1}$, David Moffat ${ }^{1}$, Justyna Rzepecka ${ }^{2}$, Lucia Janicova ${ }^{2}$, Anastasia Nika ${ }^{2}$, Darryl Turner', Clare Doris ${ }^{2}$, Stephen Anderton ${ }^{2}$

${ }^{1}$ Macrophage Pharma Limited, Windsor, United Kingdom; ${ }^{2}$ Aquila BioMedical Limited, Edinburgh, United Kingdom

Correspondence: David Moffat (david@macrophagepharma.com) Journal for ImmunoTherapy of Cancer 2017, 5(Suppl 2):P479

\section{Background}

Myelomonocytic cells orchestrate both innate and adaptive immune response to tumours but frequently adopt an immunosuppressive, tumor supportive phenotype (M2-like). However, their plasticity offers the opportunity for therapeutic repolarization to one that is non-immunosuppressive and supportive of an anti-tumor immune response (M1-like). A challenge in identifying such pharmaceuticals is their counter-active functionality in other important immune cell types such as lymphocytes. Macrophage Pharma Limited's Esterase Sensitive Motif $\left(E M^{T M}\right)$ technology predominantly targets myelomonocytic cells [1]. We describe the preliminary in vitro characterisation of MPL-5821, an ESM-p38 MAPK inhibitor, contrasting it with conventional inhibitors, with reference to its cell type specificity, sparing of myelomonocytic - lymphocyte communication and ability to reverse macrophage driven lymphocyte immunosuppression.

Methods

Human Macrophage Assays: M2 polarised macrophages (M-CSF, +/IL-4, IL-10, TGF $\beta$ ) were stimulated with LPS/IFN $+/$ - compounds overnight. Cytokine production and macrophage markers were measured by ELISA and flow cytometry respectively.

Human PBMC Assay: PBMCs were stimulated with either LPS or anti-CD3 +/- compounds for 72 hours and cytokine production measured by ELISA. CD4 and CD8 T-cell proliferation was measured by flow cytometry. 
Macrophage suppression of Human T-cells: M2-polarised macrophages were incubated with autologous PBMCs $+/$ - compound. T cell stimulation was provided by anti-CD3/anti-CD28. Cytokine production was analysed by ELISA and expression of CD4, CD8 and Ki67 by flow cytometry.

Results

Human Macrophage Assays: MPL-5821 and reference p38 MAPK inhibitors exhibited a similar profile in inhibiting IL-10 production and enhancing IL-12p70 production.

Human PBMC Assay: In comparison with reference p38 MAPK inhibitors, MPL-5821 was several orders of magnitude more potent in enhancing IL-12p70 production and was the only inhibitor to enhance IFNy production under LPS stimulation. Similarly, MPL-5821 was the only inhibitor to enhance IFNY production in response to anti-CD3 stimulation.

Macrophage suppression of Human T-cells: MPL-5821 reversed the suppressive effects of the $\mathrm{M} 2$ macrophages as evidenced by a return of CD4 and CD8 proliferation to control levels accompanied by a concomitant increase in IFNY and reduction in IL-10.

Conclusions

MPL-5821 modulates the macrophage phenotype to IL-12p70 $10^{\mathrm{LO}}$ and reverses the $\mathrm{M} 2$ macrophage immune suppression of T-cell functionality. The ESM ${ }^{\mathrm{TM}}$ cell selectivity differentiates MPL-5821 from other p38 MAPK inhibitors by its sparing of other immune cells such as lymphocytes. Thus MPL-5821 enables the maintenance of the myelomonocytic/lymphocyte IL-12p70/IFNy axis, key to an effective anti-tumour immune response.

\section{References}

1. Needham LA et al, J Pharmacol Exp Ther. (2011), $339: 132-42$.

\section{P480}

Tumor infiltrating $T$ regulatory cells in head and neck cancer patients treated with cetuximab demonstrate increased inhibitory receptors and survival

Jessica Moskovitz, Tullia Bruno, Robert Ferris, Dario Vignali

University of Pittsburgh, Pittsburgh, PA, USA

Correspondence: Jessica Moskovitz (jessiemoskovitz@gmail.com)

Journal for ImmunoTherapy of Cancer 2017, 5(Suppl 2):P480

\section{Background}

Patients with locally advanced head and neck squamous cell carcinoma (HNSCC) incur significant treatment morbidity and have a poor 5 year overall [1]. The idea of treating HNSCC by targeting epidermal growth factor receptor (EGFR) developed from the demonstration of EGFR overexpression correlating with advanced tumor size and decreased survival [2]. Clinical anti-tumor activity of cetuximab, a monoclonal antibody that prevents ligand binding of EGFR, did not correlate with expected response rates despite high HNSCC tumor cell EGFR expression. Further studies to clarify the mechanism of action of cetuximab in HNSCC revealed an increase in CTLA4+ T regulatory cells (Tregs) that suppressed NK cell mediated cytotoxicity, which correlated with poor response to neoadjuvant cetuximab treatment [3].

Methods

We aimed to further measure inhibitory receptor [e.g. PD1, TIM3, TIGIT, CTLA4, neuropilin-1(NRP1)] expression on T cells in tumor and peripheral blood lymphocytes (TIL/PBL) of cetuximab treated patients. Patients with locally advance HNSCC were treated with neoadjuvant cetuximab for four weeks prior to definitive surgery with $\mathrm{CT}$ scans performed prior to each intervention. Nine samples (TIL and PBL) pre and post cetuximab were analyzed using flow cytometry, and tumor area change was measured using delta CT.

Results

Tumor infiltrating CTLA4+ Tregs had increased expression of NRP1 and TIGIT after cetuximab therapy, specifically in patients that had a poor response to cetuximab. Further, tumor infiltrating CTLA4+TIGIT+ Tregs correlated with patients having larger baseline tumors and minimal change in tumor area post cetuximab. Finally, Tregs from these patients displayed an increase in mean flouresecence intensity of pro-survival markers such as $\mathrm{Bcl} 2$.

\section{Conclusions}

Increased inhibitory receptors such as TIGIT and NRP1 on CTLA4+ Tregs combined with increased pro-survival markers in patients that had a minimal response to cetuximab suggests that these Tregs maintain suppressive function and survival despite cetuximab treatment. Further characterization of the immune resistance mechanism to cetuximab will provide the basis for designing knowledgeable combinatorial trials with other immunotherapy agents i.e. CTLA-4.

Trial Registration

clinical trials.gov:NCT01218048,NCT0193592.

\section{References}

1. Vokes EE, Weichselbaum RR, et al. Head and neck cancer. N Engl J Med 1993;328:184-194.

2. Ang KK, Berkey BA,et al. Impact of epidermal growth factor receptor expression on survival and pattern of relapse in patients with advanced head and neck carcinoma. Cancer Res. 2002;62(24):7350-6.

3. Jie HB, Ferris RL, et al. CTLA4+ Regulatory $T$ cells are increased in head and neck cancer patients, supress NK cell cytotoxicity and correlate with poor prognosis. Cancer Res. 2015;75 (11):2200-2210.

\section{P481}

Non-oncogenic acute viral infection modulates the innate immune response and reduces tumor growth in hosts with established cancer

Jenna Newman, Charles Brent Chesson, Andrew Zloza

Rutgers Cancer Institute of New Jersey, New Brunswick, NJ, USA

Correspondence: Jenna Newman jhn33@gsbs.rutgers.edu

Journal for ImmunoTherapy of Cancer 2017, 5(Suppl 2):P481

\section{Background}

The impact of infection on the development and progression of cancer has been a source of debate throughout the past century. Several pathogens have been linked to the development of cancer, while conversely, pathogen components (including Coley's toxins) and oncolytic viruses have demonstrated tumor regression. Since infection and cancer are two often-concomitant challenges to the immune system, we sought to understand the impact of acute, non-oncogenic, nononcolytic infections - which constitute the majority of infections affecting humans - on tumor growth and host immunity.

Methods

C57BL/6 (B6) or NOD-scid-gamma (NSG) mice were challenged with $1.2 \times 10^{5}$ cells B16-F10 melanoma intradermally (right flank) on day 0 and influenza A/PR8/H1N1 (10,000 PFU) administered intranasally (prior to tumor challenge, after tumor challenge before clinical appearance of the tumor, or once the tumor was established). Tumor growth and influenza infection were monitored via caliper measurement and host weight, respectively, every 2-3 days. Immune cell populations within tumors, lungs, and spleens were analyzed by flow cytometry at day 20. Statistical comparisons between groups were determined using the student's t test with $p<0.05$ considered statistically significant.

Results

Mice infected with influenza prior to or during the subclinical phase of melanoma development displayed accelerated tumor growth relative to influenza-naïve mice $(p<0.01)$. Contrarily, mice with established $(\sim 3 x$ $3 \mathrm{~mm}$ ) tumors displayed slower tumor growth after infection compared to uninfected controls $(p<0.01)$. For the latter, infected mice were found to have a 9-fold reduction of systemic MHC-II Ly6-G ${ }^{+}$Ly6- $\mathrm{C}^{+}$myeloidderived suppressor cells (MDSCs) in the spleen, relative to that observed in influenza-naïve counterparts. Furthermore, the result of curtailed tumor growth in concomitantly challenged mice was observed in immunocompromised NSG mice as well.

\section{Conclusions}

Previously we reported that influenza infection in the lung accelerates tumor growth of subclinical melanoma in the flank. However, our recent data demonstrate that the reverse effect is observed if influenza is administered to hosts with an established tumor in the flank. The latter effect is observed also in NSG mice, which lack adaptive immunity and maintain limited innate immunity. These findings, 
in conjunction with a decrease in MDSCs observed in immunocompetent infected B6 mice exhibiting reduced tumor growth, suggest that the innate immune system may promote anti-tumor immunity in the context of infection. Further research into the mechanisms by which influenza alters tumor growth are being investigated in preparation for clinical trials that will focus on harnessing microbial immunity for the treatment of cancer.

\section{P482}

Somatic TP53 mutations alter the immune micro-environment after chemotherapy in breast cancer

Mellissa Nixon ${ }^{1}$, M. Valeria Estrada², Susan Opalenik', Donna Hicks³

Michael Korrer ${ }^{1}$, Mark Pilkinton', Melinda Sanders', Roberto Salgado ${ }^{4}$,

Simon Mallal', Young Kim', Rebecca Cook ${ }^{3}$, Carlos Arteaga',

Justin Balko

${ }^{1}$ Vanderbilt University Medical Center, Nashville, TN, USA; ${ }^{2}$ UC San Diego, La Jolla, CA, USA; ${ }^{3}$ Vanderbilt University, Nashville, TN, USA; ${ }^{4}$ nstitut Jules Bordet, Brussels, Belgium

Correspondence: Justin Balko (mellissa.nixon@vanderbilt.edu) Journal for ImmunoTherapy of Cancer 2017, 5(Suppl 2):P482

\section{Background}

Neoadjuvant chemotherapy (NAC), followed by surgery, is the standard of care for triple negative breast cancer (TNBC) patients. Unfortunately, only $30 \%$ achieve a pathological complete response (pathCR). In patients who do not achieve a pathCR, tumor infiltrating lymphocytes (TILS) after NAC correlate with improved survival. This suggests there is an immune response after NAC which may be augmented by immune checkpoint blockade (ICB). Clinical trials are underway to examine the efficacy of ICB, therefore it is vital to identify biomarkers of response to identify patients who may benefit from these modalities. As more than $80 \%$ of TNBC patients harbor somatic TP53 mutations, we investigated the role of TP53 mutations on the immune microenvironment of breast cancer after NAC.

Methods

We examined matched pre and post NAC primary breast cancer. TILs were scored by a pathologist. RNA and DNA were extracted and analyzed using Nanostring Pan-Cancer Immune panel (>700 genes) and ImmunoSeq T-cell Receptor (TCR) sequencing respectively. CRISPR technology was applied to mouse tumor cell lines to develop Trp53 mutations commonly found in TNBC. Cells were treated with chemotherapy in vitro to determine tumor specific changes in cytokines and PD-L1 expression. To determine alterations in the immune microenvironment, cell lines were injected into syngeneic mice or genetically engineered mouse models (GEMM) were utilized Expression of antigen presentation by the tumor as well as immune phenotyping of TIL after NAC was performed.

Results

TILs in residual disease of patients correlated with improved survival. A third of patients had increased immune gene signatures after NAC which correlated with increased TCR clonality and increased overall survival. CRISPR induced Trp53 mutant cells were used to elucidate the role of p53. Cells in vitro had higher basal levels of immunerecruiting chemokines which were further induced with chemotherapy treatment. Trp53 mutant cells also exhibited increased antigen presentation (MHC-I/II) and PD-L1 expression after NAC. In a GEMM of breast cancer that had recurred after chemotherapy, TILs had increased expression of CXCR3, LAG3 and PD-1 by flow cytometry.

\section{Conclusions}

Our work suggests that NAC induces immune gene signatures in a subset of TNBC which is correlated with a better prognosis. TP53 mutations in the tumor may contribute to an increased immune infiltrate through upregulating chemokines, antigen presentation, and increased cytokine receptor CXCR3 + T cells that display increased exhaustion marks PD-1 and LAG3. Ongoing studies with ICB will indicate if p53-mutant tumors have a better response to ICB after NAC.

\section{P483}

A novel non-competitive and non-brain penetrant adenosine $A_{2 A}$ receptor antagonist designed to reverse adenosine-mediated suppression of anti-tumor immunity

Erica Houthuys, Reece Marillier, Théo Deregnaucourt, Margreet Brouwer, Romain Pirson, Joao Marchante, Paola Basilico, Shruthi Prasad,

Julie Preillon, Anne-Catherine Michaux, Kim Frederix, Annelise Hermant, Florence Nyawouame, Charlotte Moulin, Vanesa Bol, Bruno Gomes,

Florence Lambolez, Véronique Bodo, Jakub Swiercz, Gregory Driessens, Noémie Wald, Chiara Martinoli, Michel Detheux, Christophe Quéva, Stefano Crosignani

iTeos Therapeutics, Gosselies, Belgium

Correspondence: Christophe Quéva

(christophe.queva@iteostherapeutics.com)

Journal for ImmunoTherapy of Cancer 2017, 5(Suppl 2):P483

\section{Background}

High levels of extracellular adenosine in the tumor microenvironment promote tumor immune evasion by suppressing Th1 cytokine production and the activation and cytolytic activity of $\mathrm{T}$ cells and NK cells.

Methods

We measured extracellular adenosine concentration using intratumoral microdialysis of 11 patient-derived xenografts from various cancer indications. In parallel, we defined the expression of the four adenosine receptors in primary human immune cells using nanostring.

Results

Intratumoral adenosine concentrations reached a range of 1 to 37 $\mu \mathrm{M}$ (mean, $11 \mu \mathrm{M}$ ), significantly higher than the $0.1-0.2 \mu \mathrm{M}$ reported in normal brain tissue.

$A_{2 A}$ was the main adenosine receptor expressed by CD4 and CD8 T lymphocytes and monocytes, and the only one in mature monocytederived dendritic cells and NK cells. $A_{2 B}$ mRNA were not abundant in $T$ cells and monocytes, while $A_{1}$ and $A_{3}$ mRNA were not detected in any of the cell types evaluated. We further studied $A_{2 A}$ functions in primary human $\mathrm{T}$ lymphocytes and monocytes and demonstrated that selective $A_{2 A}$ agonists such as CGS-21680 suppressed cytokine production by activated $T$ lymphocytes and monocytes, highlighting the role of $A_{2 A}$ as the main receptor mediating adenosine signaling in these cells.

We showed that $A_{2 A}$ antagonists initially designed for Parkinson's disease but recently repurposed for immuno-oncology dramatically lost potency in a high adenosine environment. We therefore developped a novel non-brain penetrant and non-competitive inhibitor of $A_{2 A}$ with sub-nanomolar $\mathrm{Ki}$ and selectivity versus $A_{1}, A_{2 B}$, and $A_{3}$ receptors. Our compound potently inhibited $A_{2 A}$ signaling in human $T$ lymphocytes independently of adenosine concentrations $\left(\mathrm{IC}_{50}\right.$ in $\mathrm{T}$ cell CAMP assay $=2.8$ and $5.5 \mathrm{nM}$ in 1 and $100 \mu \mathrm{M}$ adenosine, respectively), and rescued cytokine production in the presence of high concentrations of $A_{2 A}$ agonists. iTeos $A_{2 A}$ antagonist potently rescued $T_{H 1}$ cytokine production in human whole blood treated by $A_{2 A}$ agonists, and increased CD8 $\mathrm{T}$ cell cytotoxicity in a co-culture assay of effector CD8 T cells and target cancer cells.

Conclusions

iTeos Therapeutics non-competitive $A_{2 A}$ receptor antagonist is uniquely designed to address the challenge of counteracting elevated adenosine concentrations in tumors in order to restore antitumor immunity.

\section{P484}

A novel metabolic therapeutic that harnesses the anti-tumor immune response

Dayana Rivadeneira, Nicole Scharping, Greg Delgoffe

University of Pittsburgh, Pittsburgh, PA, USA

Correspondence: Greg Delgoffe (rivadeneirad@upmc.edu)

Journal for ImmunoTherapy of Cancer 2017, 5(Suppl 2):P484 


\section{Background}

In the past decade immunotherapy has shown impressive clinical responses, quickly becoming the first line treatment for cancers such as melanoma. Despite these promising results, all patients do not have the same level of response, and recent efforts have focused on delineating avenues to understand and avoid therapy resistance. Our lab and others have recently proposed that tumor cells may compromise $\mathrm{T}$ cell function by generating a metabolically inhospitable microenvironment. Most importantly immune or tumor metabolism can be modified to modulate the T cell response to immunotherapy. Methods

We hypothesize that leptin, a hormone that affects a wide range of metabolic processes, might be utilized as a means to remodel the metabolic state of the tumor microenvironment, consequently modulating tumor growth and immunotherapy response. Previous studies have shown that leptin can enhance $T$ cell proliferation and activation, but the effects of leptin on tumor-infiltrating $T$ cells have not been thoroughly investigated. In order to assess the effects of leptin in the tumor microenvironment, we obtained tumor infiltrating $T$ cells (TIL) from tumor-bearing mice treated with recombinant leptin. Additionally, we generated PTEN/BRAF melanoma cell lines stably overexpressing leptin.

Results

As we have previously shown, glucose uptake in TIL is decreased compared to ndLN. When mice were treated with recombinant leptin, we observed an increase in TIL glucose uptake, suggesting metabolic remodeling. Furthermore, PTEN/BRAF melanoma cells which overexpress leptin have significantly slower growth kinetics compared to control tumors and an increase in overall survival. Leptin overexpressing tumors have increased $\mathrm{T}$ cell infiltration compared to control tumors, and these TIL are metabolically and functionally superior.

\section{Conclusions}

Taken together, these data suggest leptin can have a direct effect on tumor infiltrating $T$ cell function and tumor growth. Our goal is to further characterize how leptin can metabolically remodel the tumor microenvironment and modulate immunotherapeutic response.

\section{P485}

Use of the NanoString gene expression profiling platform to capture the immunological status of the leukemia microenvironment

Jayakumar Vadakekolathu' ${ }^{1}$ Tasleema Patel ${ }^{2}$, Stephen Reeder ${ }^{1}$,

Heidi Schaarschmidt ${ }^{3}$, Marc Schmitz ${ }^{4}$, Martin Bornhäuser ${ }^{3}$, Sarah Warren 5 , Tressa Hood ${ }^{5}$, Patrick Danaher ${ }^{5}$, Alessandra Cesano ${ }^{5}$, Joseph M.

Beechem ${ }^{5}$, A. Graham Pockley', Sarah Tasian², Sergio Rutella'

${ }^{1}$ Nottingham Trent University, Nottingham, United Kingdom; ${ }^{2}$ Children's

Hospital of Philadelphia, Philadelphia, PA, USA; ${ }^{3}$ University Hospital Carl

Gustav Carus, Dresden, Germany; ${ }^{4}$ Medical Faculty Carl Gustav Carus,

Dresden, Germany; ${ }^{5}$ NanoString Technologies, Inc., Seattle, WA, USA

Correspondence: Sergio Rutella (sergio.rutella@ntu.ac.uk)

Journal for ImmunoTherapy of Cancer 2017, 5(Suppl 2):P485

\section{Background}

Acute myeloid leukemia (AML) is characterized by clonal expansion of poorly differentiated myeloid precursors and results in impaired hematopoiesis and often bone marrow failure. Immune responses are defective in patients with AML due to powerful immune suppressive circuits that are activated by soluble factors and immune checkpoint molecules, including PD-L1 and IDO1. The development and delivery of new therapeutic strategies for high-risk $A M L$, including immunotherapy, therefore remains a priority.

\section{Methods}

This multi-institutional study was undertaken to collect comprehensive immune profiles across the biological heterogeneity of childhood and adult $\mathrm{AML}$, with the aim to implement new molecularly targeted agents for patients with specific immunologic subtypes of AML. We employed the nCounter ${ }^{\mathrm{TM}}$ system (NanoString Technologies, Seattle, USA) to characterize bone marrow (BM) specimens from 70 patients with nonpromyelocytic AML (42 children and 28 adults). Ninety BM samples (63 de novo AMLs, 18 AMLs in complete remission [CR] and 9 relapsed AMLs) were analyzed on the nCounter ${ }^{\oplus}$ FLEX platform, using the RNA Pan-Cancer Immune Profiling Panel. Transcriptomic data were normalized using the $\mathrm{nSolver}{ }^{\mathrm{TM}}$ software package.

Results

Hierarchical clustering identified patient subgroups with heightened expression of CD8 T-cell, Th1-cell, B-cell and cytotoxicity-related genes, including genes related to NK-cell function, in the leukemia microenvironment ("immune enriched" patients). Interestingly, a select group of patients over-expressed genes associated with monocyte/macrophage, neutrophil and mast cell functions, with low expression of T-cell and B-cell genes. AML patients with 'immune enriched' gene profiles also expressed CD8A, IFNG, FOXP3, and inhibitory molecules including IDO1 and the immune checkpoints $L A G 3$, CTLA4 and PD-L1.

Gene set analyses identified differences in immune gene expression levels between childhood and adult AMLs, as well as patients with newly diagnosed $A M L$, relapsed $A M L$ or $A M L$ in $C R$. In particular, genes highly expressed in adult AMLs compared with childhood disease included chemokine genes, dendritic cell and macrophage genes and NF-kB inhibitor-a. In contrast, immune genes downregulated in adult compared with childhood AMLs included integrin family members, cytokine receptors, TCR complex components and lipocalin-2, a neutrophil gelatinase-associated molecule implicated in suppression of tumor invasiveness.

Conclusions

Our analysis has captured the immunological status of the leukemia tumor microenvironment in children and adults with AML at different disease stages. From a clinical standpoint, 'immune enriched' AMLs might be amenable to immunotherapy approaches tailored to the BM microenvironment.

P486

Utilization of dual IHC and quantitative image analysis techniques to evaluate LAG-3-positive T cells in the tumor microenvironment of NSCLC tissue

Malik Khenkhar ${ }^{1}$, Philipp Uhlig' ${ }^{1}$ Nickels Winkler ${ }^{1}$, Hartmut Juhl ${ }^{1}$,

Alison Bigley², Lorcan Sherry ${ }^{2}$

${ }^{1}$ Indivumed GmbH, Hamburg, Germany; ${ }^{2}$ OracleBio Limited, Glasgow,

United Kingdom

Correspondence: Lorcan Sherry lorcan.sherry@oraclebio.com

Journal for ImmunoTherapy of Cancer 2017, 5(Suppl 2):P486

\section{Background}

Non-small cell lung cancer (NSCLC) patients have impaired immune responses, where the expression of inhibitory checkpoint molecules on T cells may lead to reduced immune cell activity. The presence of lymphocyte activation gene-3(LAG-3)-positive $T$ cells in the tumor microenvironment and their potential impact on prognosis have been investigated over many years. One implication has been that the engagement of LAG-3 inhibits T cell proliferation resulting in T cell suppression and poor prognosis [1]. We aimed to quantify CD3 and LAG-3 immune cell relationships in terms of cell infiltrations and proportions within the tumor microenvironment of NSCLC tissue. 


\section{Methods}

Ten individual NSCLC clinical tissue samples were immunohistochemically $(\mathrm{IHC})$ stained by Indivumed using the Ventana DISCOVERY XT automated staining platform. The first section was dual-labeled for LAG-3 and CD3 and the second for pan-Cytokeratin (CK). Image analysis was performed by OracleBio using Indica Labs Halo software. Tumor and stroma regions of interest (ROI) were classified using the CK section. ROI classifications were automatically transferred, during analysis, from the sample-associated CK section to the co-registered dual IHC section. Cellular analysis was performed on the ROI using threshold established to identify and count CD3, LAG-3 and duallabeled cells.

Results

Evaluation of tumor samples demonstrated a mean number of LAG3/CD3-dual positive cells within the stroma and tumor of $132 \pm 59$ and $54 \pm 20$ per $\mathrm{mm}^{2} \mathrm{ROI}$ area, respectively. Interestingly however, when the proportion of LAG-3/CD3-dual positive cells was normalized to the total population of CD3-positive cells within these regions, an opposite relationship was observed where the mean \% LAG-3/CD3-positive cells relative to the total CD3 immune cell population was $5 \pm 2 \%$ within the stroma and $12 \pm 3 \%$ within the tumor. Conclusions

These example data highlight the benefits of utilizing dual IHC staining with whole slide image analysis to characterize immune cell relationships within the tumor microenvironment. These techniques can assist in the assessment of changing relationships between proportions of immune cells between tissue compartments and evaluation of LAG-3 as a potential target for modulating $T$ cell responses in NSCLC.

\section{References}

1. He Y, Yu H, Rozeboom L. LAG-3 Protein Expression in Non-Small Cell Lung Cancer and Its Relationship with PD-1/PD-L1 and Tumor-Infiltrating Lymphocytes. Journal of Thoracic Oncology. 2017;12(5):814-823.

\section{P487}

Multiplex immunofluorescence evaluation of an immune cell marker panel in the tumor microenvironment of NSCLC tissue using tailored analysis of multi-spectral image component data Richard Bystry ${ }^{1}$, Edward Durley ${ }^{1}$, Lee Dawson', Rebecca Houliston?', Alison Bigley ${ }^{2}$, Lorcan Sherry

${ }^{1}$ Asterand Bioscience, Royston, United Kingdom; ${ }^{2}$ OracleBio Limited, Glasgow, United Kingdom

Correspondence: Lorcan Sherry lorcan.sherry@oraclebio.com Journal for ImmunoTherapy of Cancer 2017, 5(Suppl 2):P487

\section{Background}

Multiplex fluorescence immunohistochemistry (MIF), multispectral imaging and image analysis of immune cells within the tumor microenvironment has been increasingly applied to profile and correlate cell interactions in relation to cancer treatment and predicting patient outcome. Tumor-infiltrating $\mathrm{T}$ lymphocytes are known to play an important role in anti-cancer mechanisms where coexisting infiltrations of CD8 and CD4 T cells affords a good prognosis in nonsmall-cell lung carcinoma (NSCLC) [1]. Here we use CD8/CD4 evaluation to exemplify the use of MIF combined with multispectral imaging and tailored spectral and spatial quantification to examine relationships between immune cell populations within tumor and stroma.

Methods

An exemplar NSCLC lung tumor section was MIF labelled by Asterand for 5 immune cell markers, including CD4 and CD8, a pan cytokeratin tumor marker and DAPI nuclear marker. The stained slide was digitised using the Vectra Polaris multispectral scanner (Perkin Elmer) and exemplar region of interest $(\mathrm{ROI})$ images exported in component data format. The component data images were analysed by OracleBio using a 3-tiered sequence of tailored applications developed in Visiopharm Oncotopix Software. The first tier enabled the classification of tumor and stroma ROI, the second tier facilitated cell detection, classification and analysis and the third tier refined cell relationships and measured immune cell distances to the nearest tumor cell or between defined immune cells.

Results

Selected ROI displayed a range of tumor and stroma content with varying populations of CD4 and CD8 positive immune cell infiltrations. The mean number of CD4 and CD8 positive cells in tumor was 30 and 250 per $\mathrm{mm}^{2}$ respectively and in stroma was 474 and 915 per $\mathrm{mm}^{2}$ respectively. Evaluation of tumor-immune cell spatial relationships within the stroma, at a $20 \mu \mathrm{m}$ distance to the tumor border, highlighted that the percentage of CD4 and CD8 positive cells within this stromal border was $33 \%$ and $54 \%$ respectively and the average distance between the 2 immune cell types was $13 \mu \mathrm{m}$.

Conclusions

The exemplar data generated provides information relating to the potential influences of immune cell recruitment and activation within the tumor microenvironment, correlation of spatial distributions and the inter-dependencies between immune cells and cancer cells, which may be indicative of response to treatment or predicting patient outcome.

References

1. Hiraoka K, Miyamoto M, Cho Y: British Journal of Cancer. 2006;94:275-280.

\section{P488}

Single-cell RNA sequencing of CD8+ T cells to prioritize immunotherapy combinations

Yashaswi Shrestha, Michael Kuziora, Lydia Greenlees, Brandon Higgs, Katie Streicher, Rajiv Raja, Koustubh Ranade

MedImmune, Gaithersburg, MD, USA

Correspondence: Yashaswi Shrestha

(robert.mulvey@ashfieldhealthcare.com)

Journal for ImmunoTherapy of Cancer 2017, 5(Suppl 2):P488

\section{Background}

Immunotherapy has transformed cancer treatment. To address remaining unmet needs, combinations of checkpoint inhibitors and agonists are under investigation, although prioritization strategies are unclear. We hypothesized that molecular understanding of T-cell phenotypes under chronic stimulation that mimics the tumor microenvironment could aid in optimizing combinations. Additionally, target co-expression is important for designing bispecific monoclonal antibodies (mAbs). Although aggregate gene expression analysis of CD8+ T cells is valuable, it does not show target co-expression at the single-cell level. Hence, we explored single-cell RNAseq to identify targetable CD8 + T-cell subpopulations at various dysfunctional states.

Methods

CD8+ T cells were negatively selected from PBMCs and treated with IL2 (NonStim) or IL2+anti-CD3,antiCD28 antibodies (Stim) for 13 days, including two cycles of treatment separated by a two-day rest in IL2 [1]. Over 1500 Stim and NonStim cells were profiled on 10X Genomics platform and analyzed with corresponding pipeline. Gene expression corresponded to 2 unique molecular indices. Published consensus gene-sets for functional states of CD8+ T cells in cancer [2] were used for GeneSet Variant Analysis (GSVA) to identify populations with significant differences in the four states - dysfunctional, senescent, terminal-effector, and stem-like memory - between Stim and NonStim.

Results

GSVA scores indicated significant upregulation of a dysfunction signature and downregulation of terminal-effector function in Stim versus NonStim cells $(P<0.001)$. PD- 1 was most differentially expressed between top and bottom tertiles of Stim cells with dysfunctional signature. Similarly, cells with high expression of $\mathrm{TIM} 3$, another dysfunction marker, showed downregulation of cytotoxicity and antigen-presentation associated genes, compared to those with low TIM3 expression.

The percentage of cells expressing dysfunction markers increased under chronic stimulation, while those producing effector cytokines decreased. Cells expressing PD-1, CTLA-4 and GATA3 increased from 
$5 \%, 3 \%$ and $16 \%$ to $17 \%, 80 \%$ and $21 \%$, respectively, whereas cells expressing IFNy decreased from $16 \%$ to $7 \%$. Of the Stim cells, $11 \%$, $15 \%$ and $71 \%$ co-expressed PD-1 and TIM3, PD-1 and CTLA-4, and CTLA-4 and LAG3, respectively. CD137 and TIM3 were co-expressed in $>30 \%$ of Stim cells, but only $1 \%$ of NonStim.

\section{Conclusions}

Our results provide new insights into the heterogeneity of activated/ exhausted/dysfunctional CD8+ T cells at the single-cell level, which could aid in prioritizing immunotherapy combinations and designing new bispecific mAbs.

\section{References}

1. McKinney E, et al. T-cell exhaustion, co-stimulation and clinical outcome in autoimmunity and infection. Nature. 2015;523:612-616.

2. Apetoh $L$, et al.Consensus nomenclature for CD8+ T cell phenotypes in cancer. Oncoimmunology. 2015;4:e998538.

\section{P489}

Studying the cellular heterogeneity and spatial arrangement of immune cells in FFPE sections of Hodgkin Lymphoma and normal lymph node using a highly multiplexed Imaging Mass Cytometry Mohan Singh, Parvesh Chaudhry, Erik Gerdtsson, Lisa Harton, James Hicks, Peter Kuhn, Wendy Cozen, Imran Siddiqi, Akil Merchant

University of Southern California, Los Angeles, Los Angeles, CA, USA Correspondence: Akil Merchant (mohansin@usc.edu)

Journal for ImmunoTherapy of Cancer 2017, 5(Suppl 2):P489

\section{Background}

Clinical successes with immuno-oncology agents have demonstrated the potency of the immune system in controlling cancers, most strikingly in Hodgkin lymphoma (HL), where response rates to PD1/PDL1 inhibitors approach $90 \%$. The majority of tumor bulk in $\mathrm{HL}$ is made up of non-malignant immune cells. High parameter methods such as gene expression profiling or flow cytometry have been applied to the study of the tumor immune microenvironment (TME) in Hodgkin disease, however due to the inherent limitations of these techniques, several questions remain unanswered. Data on cellular heterogeneity and rare cells is lost with gene expression studies while the spatial relationship between tumor and immune cells is lost with flow cytometry. The Fluidigm Hyperion imaging mass cytometry (IMC) system combines a CyTOF mass cytometer with a laser ablation system allowing for $40+$ parameter simultaneous immunophenotyping on a single slide of FFPE tissue, with sub-cellular resolution. Here we describe our efforts to develop and optimize a 26 parameter antibody panel for the characterization of the lymphoma TME on the IMC platform.

\section{Methods}

Literature was reviewed to identify markers relevant to HL TME. Antigen retrieval, blocking, and antibody staining concentrations were optimized to identify universal staining conditions. Slides were imaged using the Fluidigm Hyperion IMC system and image analysis was performed with MCD viewer (Fluidigm) or CellProfiler/miCAT (https://doi.org/10.1101/109207).

Results

We successfully performed IMC imaging of Hodgkin lymphoma, NK lymphoma and normal lymph nodes (LN) using a 26 parameter panel. We were able to clearly identify tissue architecture (Fig. 1, normal LN, germinal center(GC), mantle zone(MZ), cortex/paracortex(PC), Fig. 2A) and characterize specific cellular populations such as CD8+Tcells, $\mathrm{Th}_{1} /$ $\mathrm{Th}_{2}$ subsets, FoxP3+ $\mathrm{T}_{\text {reg, }}$ tissue associated macrophages, CD56+NK cells and tumor vasculature. Important immuno-oncology targets such as LAG3, PDL1/PDL2 and PD1 are included. Cellular resolution is similar to $\mathrm{IHC}$, allowing for imaging of tumor, effector and regulatory cell interactions at a single cell level (Fig. 2B, Reed Sternberg(RS)cells (red). CD8+(green) adjacent to RS cell flanked by $\mathrm{T}_{\mathrm{REG}}$ (FoxP3-teal) and $\mathrm{Th}_{2}$ (CCR4-yellow) CD4+(not shown) cells. Abundant CD68+(magenta) cells which co-express PDL1/PDL2 (not shown) in the background.)

\section{Conclusions}

IMC allows for successful multiplex imagining of the TME in FFPE tissues. The method does not require special processing and can be applied to archival specimens and tumor microarrays. Methods developed here should be applicable for the study of the TME in other tumor types and could be used to identify additional biomarkers of response to immunooncology agents.
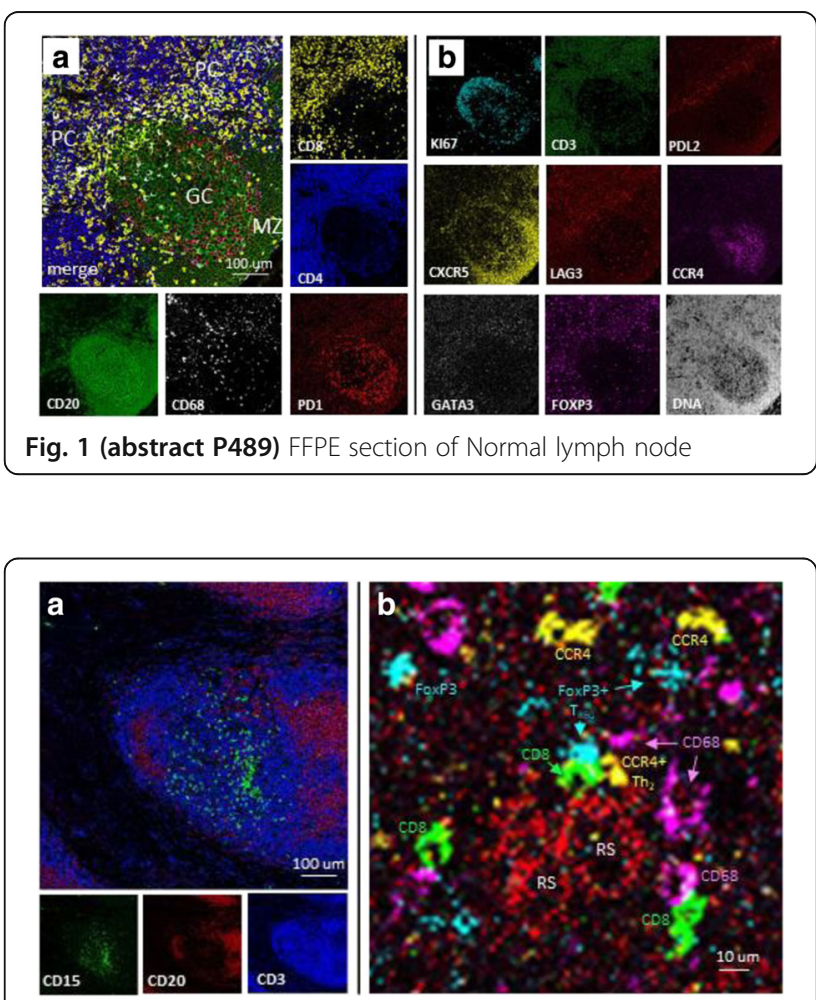

Fig. 2 (abstract P489) FFPE section of Hodgkin lymphoma

\section{P490}

PSMA-specific CARTyrin T-stem cell memory therapy eliminates solid tumor in subcutaneous prostate cancer model Jenessa B. Smith, Rebecca Codde, Yening Tan, Burton E. Barnett, David Hermanson, Srinivas Rengarajan, Eric M. Ostertag, Devon J. Shedlock Poseida Therapeutics, San Diego, CA, USA

Correspondence: Devon J. Shedlock (jbsmith0711@gmail.com) Journal for ImmunoTherapy of Cancer 2017, 5(Suppl 2):P490

\section{Background}

Prostate-specific membrane antigen (PSMA) is a transmembrane glycoprotein overexpressed in $>80 \%$ of metastatic prostate cancer. Although PSMA is a promising target for peptide and antibody drug conjugate therapies, early chimeric antigen receptor (CAR) T-cell therapies lacked clinical efficacy. Here we developed a novel CAR T-cell product (P-PSMA-101) via piggyBac ${ }^{T M}$ transposition of a tri-cistronic transgene encoding a safety switch, a PSMA-specific Centyrin-based CAR (CARTyrin), and a selection gene, features that may improve safety and efficacy compared with previous anti-PSMA CAR T-cell therapies.

Methods

We developed and identified a lead anti-PSMA CARTyrin from over 250 available Centryin binders. We also tested the previously clinically- 
tested anti-PSMA J591 scFv-based CAR for comparability. Initial assessment utilized mRNA delivery of candidate CARTyrins to confirm CAR surface expression and specific T-cell degranulation against PSMA+ prostate tumor cells or PSMA-engineered cells. We then used piggyBac to deliver our tri-cistronic vector system encoding the lead PSMA CARTyrin (P-PSMA-101), J591 scFv CAR, or a BCMA-specific CARTyrin to Tcells, resulting in $>95 \%$ CAR+ T-cells after selection and expansion. Importantly, our unique production methodology leads to $>60 \%$ Tstem cell memory (Tscm) cells, an early memory population that correlates with complete responses in CD19 CAR T-cell clinical trials. In vitro, P-PSMA-101 and J591 CAR T-cells specifically proliferated, lysed, and secreted IFN- $\gamma$ against PSMA+ LNCaP or PSMA-engineered K562s. No evidence of tonic signaling or exhaustion was detected.

Results

P-PSMA-101 demonstrated significantly enhanced anti-tumor efficacy and survival in comparison to J591 CAR T-cell-treated mice in a low "stress test" dose of T-cells against established subcutaneous LNCaP(fLuc+) solid tumors in NSG mice. The $>60 \%$ Tscm P-PSMA-101 expanded in vivo and gave rise to differentiated effector CAR+ T-cells that were detected in the peripheral blood concomitant with a decrease in tumor burden below detectable caliper and bioluminescent imaging limits. P-PSMA-101 then contracted, yet persisted in the peripheral blood with $>70 \%$ of T-cells exhibiting a Tscm phenotype. On the contrary, J591 CAR T-cells did not significantly control tumor burden. In a dose titration study, P-PSMA-101 eliminated established LNCaP tumor in $100 \%$ of animals for the duration of the studies ( 42 days posttreatment), while $2 / 3$ low-dose animals remained tumor-free.

\section{Conclusions}

P-PSMA-101 is a first-in-class Centyrin-based CAR T-cell therapeutic that exhibits a persistently high frequency of $\mathrm{Ts} \mathrm{cm}$ and mediates durable anti-solid tumor efficacy that surpasses previously established anti-PSMA CAR T-cell therapy in our in vivo model. Future efforts will continue towards clinical application of P-PSMA-101 in patients with metastatic castrate resistance prostate cancer.

\section{P491}

Alerting the immune system by removing epigenetic silencing of $\mathrm{T}_{\mathrm{H}} 1$ chemokines

Heather Sonnemann, Amber Giles, Jinkyu Jung, Caitlin Reid, Marsha-Kay Hutchinson, Deric Park, Mark Gilbert

$\mathrm{NIH}, \mathrm{NCl}$, Bethesda, MD, USA

Correspondence: Amber Giles (heather.sonnemann@nih.gov)

Journal for ImmunoTherapy of Cancer 2017, 5(Suppl 2):P491

\section{Background}

BACKGROUND: Solid tumors employ multiple mechanisms to evade immunologic detection and destruction. However, the potential to enhance the immune response to cancer has been proven in several malignancies and is under investigation in many others, including primary CNS tumors. Brain tumors, in particular, lack robust T-cell infiltration, suggesting poor immune surveillance. Recent studies have found that tumor cells express T-cell attracting chemokines, CXCL9 and CXCL10, in response to interferon gamma (IFNY). Chemokine expression can be further amplified by drugs that prevent epigenetic silencing, such as histone methyltransferase inhibitors or histone deacetylase (HDAC) inhibitors. This approach increases T-cell trafficking to tumors in vivo and enhances the anti-tumor efficacy of checkpoint blockade. We hypothesized that T-cell trafficking to brain tumors could be increased by epigenetically enhancing CXCL9 and CXCL10 expression.

Methods

Assays were performed on human glioma brain tumor cell lines. CXCL9 and CXCL10 expression were measured by real-time PCR and Western blot. GSK126, a commercially available methyltransferase inhibitor, was utilized to demethylate histone H3 (K9 and K27). LB201 (Lixte Biotechnology, Setatuket, NY) and SAHA were used to inhibit class 1 and class 2 HDACs and Entinostat was used to inhibit class I and class III HDACs. Histone methylation and acetylation status were examined using Western blot analysis. T-cell migration was measured using transwell migration assays. RESULTS: Expression of CXCL9 and CXCL10 was induced by IFNY in brain tumor lines. Chemokine expression was further increased when cells were treated with GSK126 and HDAC inhibitors compared to IFNy alone. Migration assays confirmed that tumor cells treated with GSK126 and HDAC inhibitors released chemotactic factors that increased T-cell migration.

Results

Expression of CXCL9 and CXCL10 was induced by IFNY in brain tumor lines. Chemokine expression was further increased when cells were treated with GSK126 and HDAC inhibitors compared to IFNy alone. Migration assays confirmed that tumor cells treated with GSK126 and HDAC inhibitors released T-cell chemotactic factors that increased T-cell migration. Conclusions

These studies demonstrate that brain tumors express T-cell attracting chemokines CXCL9 and 10 in response to IFNy. Further, single agent and the combination of GSK126 and HDAC inhibitors enhanced expression of CXCL9 and CXCL10, resulting in increased T-cell migration toward tumor cells. Together, these data provide a potential means to enhance the efficacy of immune therapies for brain tumors by promoting T-cell trafficking toward tumor cells.

\section{P492}

Galectin-3 inhibition with GR-MD-02 synergizes with agonist antiOX40 mAb therapy leading to reduced immune suppression and improved overall survival

Elizabeth Sturgill, Stephanie Linch, Courtney Mick, Melissa Kasiewicz, William Redmond

Providence Portland Medical Center, Portland, OR, USA

Correspondence: William Redmond (elizabeth.sturgill@providence.org) Journal for ImmunoTherapy of Cancer 2017, 5(Suppl 2):P492

\section{Background}

Cancer immunotherapy aims to harness the immune system to destroy tumor cells as well as reverse tumor-induced immune suppression. One such molecule reported to contribute to immune suppression is galectin-3 (Gal3). Indeed, Gal3 is upregulated on a wide variety of tumors and is associated with poor patient prognosis. In addition, increased amounts of Gal3 are found in patients with metastatic disease. Gal3 has been shown to recruit myeloid derived suppressor cells (MDSCs) into the tumor microenvironment, thus aiding in tumor escape. Therefore, we hypothesized that the addition of a galectin-3 inhibitor (GR-MD-02) in conjunction with an agonist anti-OX40 antibody (aOX40) would synergize to promote tumor regression and increased survival via a reduction in tumor-induced immune suppression.

Methods

MCA-205 sarcoma cells or TRAMP-C1 prostate adenocarcinoma cells were implanted into the flank of $\mathrm{C} 57 \mathrm{BI} / 6$ mice; $4 \mathrm{~T}-1$ mammary carcinoma cells were implanted orthotopically into the mammary fat pad. GR-MD-02 (2.4 mg/dose) and aOX40 ( $250 \mu \mathrm{g} /$ dose) were administered to mice intraperitoneally (ip) either $3 x /$ week for 2 weeks or $2 x /$ week for 1 week, respectively. Tumor growth (area) was assessed $2 x /$ week and mice were sacrificed when tumors exceeded $150 \mathrm{~mm} 2$. Tumors were either digested and immune cells were analyzed by flow cytometry or homogenized into tumor lysate was assayed by cytokine bead array.

Results

We observed that combined GR-MD-02/aOX40 therapy significantly improved survival of MCA-205, 4T-1, and TRAMP-C1 tumor-bearing mice, and additionally reduced lung metastases in the 4T-1 model. Further analysis revealed that GR-MD-02/aOX40 therapy significantly reduced the amount of both monocytic (Mo-) and granulocytic (Gr-) MDSCs within the tumor compared to aOX40 alone $(p<0.05)$. In addition, we observed a significant decrease in PD-L1, iNOS, arginase $1(p<0.05)$, and CD206 $(p<0.001)$ expression in both Mo- and Gr-MSDCs. Moreover, GRMD-02/aOX40 therapy led to increased $\mathrm{CD}^{+} \mathrm{T}$ cell proliferation in the lymph node and increased intratumoral concentrations of IL-17a and IL-15 $(p<0.05)$, suggesting a potential role in modulating immune infiltrate and/or prolonged T cell survival.

\section{Conclusions}

In summary, our data suggests that Gal3 inhibition plus agonist aOX40 therapy may reduce myeloid recruitment to the tumor microenvironment, thus reducing immune suppression and subsequently mediating tumor regression and increased survival. 
P493

LTX-315: A first-in-class oncolytic peptide that reshapes the tumor microenvironment

Baldur Sveinbjørnsson ${ }^{1}$, Ketil Camilio², Meng-Yui Wang², Janne Nestvold ${ }^{3}$,

Gunhild Mælandsmo², Gunnar Kvalheim², Øystein Rekdal ${ }^{1}$

${ }^{1}$ Lytix Biopharma, Oslo, Norway; ${ }^{2}$ Radiumhospitalet, Oslo, Norway;

${ }^{3}$ Rikshospitalet, Oslo, Norway

Correspondence: Øystein Rekdal

(baldur.sveinbjornsson@lytixbiopharma.com)

Journal for ImmunoTherapy of Cancer 2017, 5(Suppl 2):P493

\section{Background}

The oncolytic peptide LTX-315, which has been de novo designed based on structure-activity relationship studies of host-defense peptides, has the ability to kill human cancer cells and induce long-lasting anticancer immune response when injected locally into tumors established in immunocompetent murine models. The oncolytic effect of LTX-315 involves perturbation of the plasma membrane and the mitochondria with subsequent release of danger-associated molecular pattern molecules (DAMPs) such as ATP, Cytochrome C and HMGB1. Furthermore, LTX-315 effectively disintegrates the cellular compartments with subsequent release of tumor antigens as demonstrated by a greater T-cell infiltration (TILs), TILs clonality and the number of clones with greater abundance in the tumor microenvironment. In experimental tumor models, LTX-315 exerts abscopal effects and reshapes the tumor microenvironment by decreasing the local abundance of immunosuppressive cells and by increasing the frequency of effector Tcells. LTX-315's ability to convert immunogenically "cold" tumors to "hot" makes it ideal combination partner with other immunotherapies or immunochemotherapy. Indeed, in preclinical tumor models, a combination of LTX-315 and immune checkpoint inhibitor (anti-CTLA4) demonstrates significant synergy.

Methods

In the present study the antitumor efficacy and potential synergy of LTX-315 in combination with low-dose chemotherapy was investigated in experimental mouse models. Subcutaneously established murine A20 lymphoma was treated with LTX-315 alone, cyclophosphamide alone or LTX-315 in combination with cyclophosphamide. Similarily, orthotopically established $4 \mathrm{~T} 1$ murine mammary carcinoma was treated with either LTX-315 alone, liposomal doxorubicin (CAELYX) alone or in combination.

Results

LTX-315 showed significantly enhanced anticancer efficacy against A20 lymphomas and 4T1 breast carcinomas when combined with cyclophosphamide and doxorubicin, respectively.

\section{Conclusions}

The LTX-315 unique "release and reshape" properties make it a promising candidate for combination with several types of anticancer therapies. Phase $1 \mathrm{~b}$ study combining LTX-315 with ipilimumab (antiCTLA-4) in malignant melanoma patients, as well as LTX-315 with Pembrolizumab (anti-PD1) in metastatic breast cancer is ongoing.

\section{P494}

Reversal of adenosine-mediated immune suppression by AB421, a potent and selective small-molecule CD73 inhibitor

Joanne BL Tan, Annette Becker, Daniel DiRenzo, Kristen Zhang, Ada

Chen, Rhiannon Thomas-Tran, Joel Beatty, Debashis Mandal, Guifen Xu,

Steve Young, Matthew Walters, Ulrike Schindler, Jay Powers

Arcus Biosciences, Hayward, CA, USA

Correspondence: Joanne BL Tan jtan@arcusbio.com

Journal for ImmunoTherapy of Cancer 2017, 5(Suppl 2):P494

\section{Background}

Extracellular adenosine-triphosphate (ATP) is efficiently hydrolyzed to adenosine by ecto-nucleotidases CD39 and CD73, which converts adenosine-monophosphate (AMP) into adenosine (ADO). ADO suppresses immune responses including those of $T$ cells, natural killer (NK) cells, and dendritic cells (DCs) through activation of $A_{2 a} R$ and $A_{2 b} R$ receptors. $\mathrm{CD} 73$ inhibition is a promising therapeutic approach for preventing ADO-mediated immunosuppression in the tumor microenvironment.

\section{Methods}

Human $\mathrm{CD} 8^{+} \mathrm{T}$ cells, $\mathrm{CD} 4^{+} \mathrm{T}$ cells, and $\mathrm{CD} 14^{+}$monocytes were isolated from buffy coats using various RosetteSep and EasySep Enrichment cocktails. The ability of AB421 to rescue AMP-mediated inhibition of T cell activation was evaluated using CD3/CD28/CD2 stimulation. Mixed lymphocyte reactions (MLRs) were established by mixing GM-CSF and IL-4 differentiated monocytic DCs with allogeneic $C D 4^{+} T$ cells. AMP and ADO levels in C57BL/6J mice were measured by mass spectrometry in plasma samples isolated at various time points after administration of AB421.

Results

We have designed a highly potent and selective small-molecule CD73 inhibitor, AB421, which inhibits endogenous CD73 activity in human $\mathrm{CD}^{+}$and $\mathrm{CD}^{+} \mathrm{T}$ cells with $\mathrm{IC}_{50}$ values of $4.5 \mathrm{pM}$ and $6.2 \mathrm{pM}$, respectively. $A B 421$ prevents $A M P-$ mediated inhibition of $T$ cell activation in CD3/CD28/CD2 activated human $\mathrm{CD}^{+}$and $\mathrm{CD} 8^{+} \mathrm{T}$ cells, as well as allogeneic $\mathrm{CD} 4^{+} \mathrm{T}$ cell activation in MLRs. Addition of exogenous AMP abrogated the enhanced allogeneic $\mathrm{CD}^{+} \mathrm{T}$ cell activation and IFN- $\gamma$ production mediated by a-PD-L1 monoclonal antibody (mAb), an effect that was blocked by AB421. Mechanistically, addition of AMP repressed expression of activation markers (CD25) and immune checkpoint proteins (CTLA-4, PD-1, TIM-3, LAG-3, ICOS, CD28) in the MLR assays. This suggests that activation of the adenosinergic pathway is dominant and may limit the utility of most antibodies targeting immune checkpoint proteins by curtailing their expression and/or upregulation (as was seen with an a-PD-L1 mAb and AMP coculture systems). Analysis of TCGA databases and tumor microarrays showed differential expression of CD73 across tumor types, with high expression detected in non-small cell lung carcinoma (adenocarcinoma), colorectal, head and neck, esophageal, and stomach cancers. Analysis of dissociated tumor cells by flow cytometry showed CD73 expression in both hematopoietic and non-hematopoietic cells. Finally, we show that $A B 421$ can elevate AMP-to-ADO ratios in vivo in a dose-dependent manner, reflecting systemic inhibition of CD73.

Conclusions

AB421 represents a class of potent, reversible and selective CD73 inhibitors that exhibit picomolar potency in primary human immune cells and is currently undergoing preclinical evaluation as a candidate for clinical evaluation.

\section{P495}

Separate molecular pathways mediate anti-tumor versus tumorpromoting Aspects of dsRNA signaling in cancer microenvironments

Marie-Nicole Theodoraki', Saumendra Sarkar', Ravi Muthuswamy', Jamie Voyten ${ }^{1}$, Robert Edwards' ${ }^{2}$, David Bartlett ${ }^{1}$, Kalinski Pawel ${ }^{1}$

${ }^{1}$ University of Pittsburgh, Pittsburgh, PA, USA; ${ }^{2}$ Magee-Womens Hospital of UPMC, Pittsburgh, PA, USA

Correspondence: Marie-Nicole Theodoraki

(marie.nicole.theodorakis@gmail.com)

Journal for ImmunoTherapy of Cancer 2017, 5(Suppl 2):P495

\section{Background}

Infiltration of tumors with cytotoxic T cells (CTLs) predicts improved prognosis and has been shown critical for antitumor effectiveness of checkpoint blockers. In contrast, tumor infiltration with regulatory $\mathrm{T}$ cells (Tregs) and myeloid-derived suppressor cells (MDSCs) typically predicts rapid progression and poor outcomes. Poly-l:C, a frequently used adjuvant which induces not only the CTL-attracting chemokines but also Treg attractants. Here we evaluated the molecular pathways, which lead to the induction of chemokines by dsRNA in TME and different types of tumor-associated cells, in order to develop improved adjuvants which selectively attract the desirable effector cells rather than suppressive cells. Methods

Isolated cells or human cancer biopsies were cultured in the absence or presence of one of two synthetic TLR3 ligands Poly-l:C (non-selective activator of TLR3 and helicases) or rintatolimod (selective TLR3 ligand) and in the absence or presence of a COX-1/2 inhibitor, NF-kBor TNFa inhibitors. mRNA assays. ELISA, chemotaxis assays and molecular biology assays were used to analyze the chemokine production and tumor-associated suppressive factors. Confocal microscopy 
of macrophage cultures treated with the TLR3 ligands was performed to evaluate the activation of the different signaling pathways by the TLR3 receptor and cytoplasmic helicases.

Results

We observed that poly-l:C induced activation of NF-kB- and COX-2 pathways leading to induction of COX2-dependent suppressive factors and Treg- and MDSC-attracting chemokines. These undesirable effects were blocked with inhibitors of both NF-kB- or COX-2 pathways. In contrast rintatolimod selectively induced the desirable chemokines, which were associated with lack of direct activation of NF-kB- or COX-2 pathways, and strongly suppressed attraction of Tregs and MDSCs, with elevated CTL attraction in ex vivo migration assays. Looking at the upstream signaling pathways, both TLR3 ligands induced IRF3 (Type-1 interferon pathway). However, only Poly-l:C induced activation of TRAF3 and RIP-1 (TLR3 dependent NF-kB pathway) as well as MAVS (cytoplasmic helicases).

\section{Conclusions}

Our data implicate an important new role of helicases by the induction of tumor-promoting factors by dsRNA and points out to new targets to enhance the immunogenic and antitumor activities of adjuvants.

\section{P496}

Degradation of hyaluronan (HA) by PEGPH20 promotes anti-tumor immunity and enhances the effect of checkpoint blockade in an HA-accumulating mouse syngeneic tumor model

Benjamin J Thompson, Renee Clift, Trevor B Kimbler, Jesse D Bahn, Chunmei Zhao, Barbara Blouw, Curtis B Thompson, Sanna Rosengren Halozyme, San Diego, CA, USA

Correspondence: Benjamin J Thompson (bthompson@halozyme.com) Journal for ImmunoTherapy of Cancer 2017, 5(Suppl 2):P496

\section{Background}

The glycosaminoglycan hyaluronan $(\mathrm{HA})$ is abundant in many solid tumors, and its accumulation is often associated with poor patient outcomes. Its degradation by intravenously administered PEGylated recombinant human hyaluronidase $\mathrm{PH} 20$ ( $\mathrm{PEGPH} 20)$ remodels the tumor stroma, reduces intratumoral pressure, decompresses tumor blood vessels, and facilitates drug delivery. Recently, PEGPH20 was shown to enhance tumor growth inhibition induced by the immune checkpoint inhibitor anti-PD-L1 in HA-accumulating pancreatic and orthotopic breast syngeneic tumor models, and to enhance tumor T cell infiltration. Here, the HA content of commonly used syngeneic models was systematically studied, and MC38 (Charles River Laboratories) was identified to further evaluate the combinatorial effect of PEGPH20 and anti-PD-L1 in HA-accumulating tumors.

\section{Methods}

Eight syngeneic tumor models were screened for HA content by ELISA, and immune activity by qPCR for a panel of transcripts that included surface markers, cytokines, chemokines, immune checkpoints, and immunomodulatory enzymes. For tumor growth and immunophenotyping studies, MC38 tumors were implanted subcutaneously, and PEGPH20 $(37.5 \mu \mathrm{g} / \mathrm{kg})$ was administered intravenously $24 \mathrm{~h}$ prior to anti-PD-L1 (10F.9G2, $5 \mathrm{mg} / \mathrm{kg}$ ) using a biweekly dosing regimen. Tumor HA was visualized by immunohistochemistry followed by positive pixel count. Tumor-infiltrating immune cells were evaluated by flow cytometry following tumor dissociation.

Results

Of the eight models tested, MC38 tumors had the highest HA content, and displayed evidence of robust immune activity in the qPCR screen. PEGPH20 reduced MC38 tumor HA by nearly $90 \% 24 \mathrm{~h}$ after either a single dose, or the last of three biweekly doses. In two out of three blinded studies, PEGPH2O significantly enhanced inhibition of MC38 tumor growth induced by anti-PD-L1, and combination treatment also significantly prolonged the survival of tumor-bearing mice compared to either treatment alone. PEGPH20-treated tumors contained significantly higher numbers of CD8+ and CD4+ (both Th and Tregs) T cells, as well as natural killer cells, compared to controls. Among CD45+ cells, tumor-associated macrophages (TAMs) were the most abundant cell type; PEGPH20 significantly reduced the frequency of TAMs, while increasing the frequency of dendritic cells.

Conclusions

Collectively, these results suggest that degradation of tumor HA by PEGPH20 can facilitate an anti-tumor immune response by promoting effector cell infiltration and skewing the immune microenvironment toward a more anti-tumor composition, thereby enhancing the effect of anti-PD-L1. These findings support the ongoing clinical evaluation of PEGPH2O in combination with checkpoint inhibitors in HA-accumulating solid tumors, and provide rationale for investigating PEGPH2O in combination with additional immune modulating cancer therapies.

P497

Tumor microenvironment immune gene signature asscoiated with axicabtagene ciloleucel (axi-cel, KTE-C19), an anti-CD19 chimeric antigen receptor (CAR) T cell, in a multicenter trial (ZUMA-1) John Rossi ${ }^{1}$, Jérôme Galon², Sarah Turcan², Corinne Danan², Frederick Locke³, Sattva Neelapu ${ }^{4}$, David Miklos ${ }^{5}$, Nancy Bartlett ${ }^{6}$, Caron Jacobson? Ira Braunschweig ${ }^{8}$, Olalekan Oluwole, Tanya Siddiqi ${ }^{10}$, Yi Lin $^{11}$, John Timmerman ${ }^{12}$, Patrick Reagan ${ }^{13}$, Lazaros Lekakis ${ }^{14}$, Sherryonne Unabia', William Go', Jeff Wiezorek', Adrian Bot ${ }^{1}$

${ }^{1}$ Kite Pharma, Santa Monica, CA, USA; ${ }^{2}$ HalioDx, Marseille, France; ${ }^{3}$ Moffitt Cancer Center, Tampa, FL, USA; ${ }^{4}$ MD Anderson Cancer Center, Houston, TX, USA; ${ }^{5}$ Stanford University School of Medicine, Stanford, CA, USA; ${ }^{6}$ Washington University Medical School, Saint Louis, MO, USA; ' DanaFarber Cacner Institute, Boston, MA, USA; ${ }^{8}$ Montefiore Medical Center, Albert Einstein College of Medicine, Bronx, NY, USA; ${ }^{9}$ Vanderbilt-Ingram Cancer Center, Nashville, TN, USA; ${ }^{10}$ City of Hope National Medical Center, Duarte, CA, USA; ${ }^{11}$ Mayo Clinic, Rochester, MN, USA; ${ }^{12}$ UCLA David Geffen School of Medicine, Santa Monica, CA, USA; ${ }^{13}$ University of Rochester Medical Center, Rochester, NY, USA; ${ }^{14}$ University of Miami Health System, Sylvester Comprehensive Care Center, Miami, FL, USA Correspondence: Adrian Bot (kate.trueblood@nexusggmed.com) Journal for ImmunoTherapy of Cancer 2017, 5(Suppl 2):P497

\section{Background}

The efficacy of axicabtagene ciloleucel (axi-cel), an autologous anti-CD19 CAR T cell therapy, was evaluated in the multicenter, pivotal ZUMA-1 study in patients with refractory, aggressive non-Hodgkin lymphoma (NHL). In a pre-specified interim analysis, ZUMA-1 met its primary endpoint with an objective response rate of $82 \%$ and a complete response rate of 54\% [1]. Based on an analysis of patients enrolled in ZUMA-1, we describe for the first time a tumor microenvironment immune gene signature associated with CAR T cell treatment of patients with aggressive NHL. Methods

In this post-hoc analysis, paired biopsies were taken before and within 3 weeks of axi-cel treatment from 14 patients enrolled in ZUMA-1. These biopsies were analyzed by digital gene expression (Nanostring ${ }^{\mathrm{TM}}$ ) and a pre-specified bioinformatics algorithm was then applied to IGES15 and IGES21 genes, which are hypothesized to be involved in immune-mediated tumor regression (Immunosign ${ }^{\circledR}$ ) [2]. The Immunosign ${ }^{\circledR}$ profiles evaluated a pre-defined set of genes for effector T cells, Th1 cells, chemokines, and cytokines. Expression analysis and hierarchical clustering were used to define an axi-cel-related tumor immune gene signature. Wilcoxon signed rank test with multiple test correction by false discovery rate (FDR; Benjamini-Hochberg) was used.

Results

A comparison of gene expression profiles of biopsies from 14 patients on ZUMA-1 taken before and after axi-cel treatment showed profound changes in gene expression within the tumor environment after infusion. The most upregulated genes post-axi-cel treatment were CCL5 (RANTES), CTLA4, and GZMA (log2 fold change $>2, P<0.05$, FDR <0.050). Immune checkpoints $P D-L 1$ and $L A G 3$ were also upregulated post-axi-cel treatment 
(log2 fold change $>1.6, P<0.05$, FDR $<0.055)$. Other genes associated with $T$ cell proliferation, homing, and effector function that were upregulated included IL-15, GZMK, CXC3CL1 (Fractalkine), CD8A, and STAT4 (log2 fold change $>1.6 ; P<0.05$, FDR $<0.074$ ). Additional baseline tumor characteristics and associative analysis will be presented.

\section{Conclusions}

We define a mechanistic tumor immune gene signature in NHL patients associated with axi-cel treatment. This signature comprises upregulation of T cell activation, effector, chemokine, and immune checkpoint genes. These data will potentially lead to rational optimization of T cell interventions in cancer.

Trial Registration

NCT02348216.

\section{References}

1. Locke FL, Neelapu SS, Bartlett, NL, et al. AACR 2017. 9986.

2. Galon J, Angell HK, Bedognetti D, Farincola FM. Immunity. 2013;39: 11-26.

\section{P498}

$A B 928$, a dual antagonist of the $A_{2 a} R$ and $A_{2 b} R$ adenosine receptors for the treatment of cancer

Matthew Walters, Daniel DiRenzo, Joanne Tan, Ulrike Schindler, Dana Piovesan, Devika Ashok, Tim Park, Nell NarasappaFangfang Yin, Wan Hsin Lim, Stefan Garrido-Shaqfeh, Jenna Jeffrey, Laurent Debien, Brandon Rosen, Ehesan Sharif, Dillon Miles, Manmohan Leleti, Juan Jaen, Jay Powers

Arcus Biosciences, Hayward, CA, USA

Correspondence: Matthew Walters (mwalters@arcusbio.com)

Journal for ImmunoTherapy of Cancer 2017, 5(Suppl 2):P498

\section{Background}

In the tumor micro-environment (TME), extracellular ATP is sequentially hydrolyzed to adenosine by the ecto-nucleotidases CD39 $($ ATP $\rightarrow$ AMP) and CD73 (AMP $\rightarrow$ adenosine), where it acts to suppress immune cell function. $A_{2 a} R$ is expressed by a variety of lymphocytes, while myeloid cells express both $A_{2 a} R \& A_{2 b} R . A_{2 a} R$ activation results in decreased $T$ cell activation, while binding of adenosine to $A_{2 b} R$ on myeloid cells is thought to promote a tolerogenic phenotype. To alleviate immune suppression within the TME, we have designed $A B 928$, a potent and selective dual antagonist of $A_{2 a} R$ and $A_{2 b} R$ Methods

Human immune cells were isolated from healthy donor PBMC and cultured as appropriate for the cell type. PCREB assays were performed using freshly collected whole mouse blood.

Results

AB928 inhibits both $A_{2 a} R$ and $A_{2 b} R$ with similar potencies $\left(K_{B}: 1.4 n M\right.$ and $2 \mathrm{nM}$, respectively). In PBMC sorted from healthy donors, similar levels of $A_{2 a} R$ mRNA were observed in naïve and central memory CD4 and CD8 T cells, as well as NK and $B$ cells; $A_{2 a} R$ was also expressed in both dendritic cells and monocytes. In contrast to this, $A_{2 b} R$ was absent from lymphocytes but was present in both dendritic cell and monocyte populations. Adenosine-mediated suppression of CD8 $\mathrm{T}$ cell activation was reversed by $A B 928$, which restored IFN-g and granzyme B production from these cells. Adenosine-mediated suppression of NK lytic activity was inhibited by $A B 928$, resulting in a significant increase in cell killing. Monocytederived dendritic cells ( $m o D C$ ) express high levels of $A_{2 b} R$ and those cells, when cultured in the presence of adenosine, had lower levels of costimulatory molecules upon activation and a reduced capacity to stimulate CD4 $T$ cells in a mixed-lymphocyte reaction (MLR). Inhibition of adenosine-mediated signaling by AB928 resulted in a significant increase in the frequency of activated T cells in the MLR assay. Adenosine receptor activation leads to phosphorylation of CREB, which can be detected in circulating leukocytes, allowing for the establishment of PK/PD relationships in mice. AB928 exhibited a dose dependent inhibition of pCREB in whole blood.

\section{Conclusions}

In conclusion, $A B 928$ is a potent dual inhibitor of $A_{2 a} R$ and $A_{2 b} R$ receptors with the potential to block all immunosuppressive effects of extracellular adenosine in the TME. This molecule is anticipated to enter clinical development later in 2017.
P499

Expression pattern and prognostic value of IL-36g in colorectal cancer

Aliyah M Weinstein ${ }^{1}$, Nicolas A Giraldo², Catherine Julie ${ }^{3}$, Laetitia

Lacroix ${ }^{2}$, Florent Petitprez ${ }^{2}$, Jean-François Emile ${ }^{3}$, Wolf H Fridman²,

Walter J Storkus ${ }^{1}$, Catherine Sautès-Fridman ${ }^{2}$

${ }^{1}$ University of Pittsburgh, Pittsburgh, PA, USA; ${ }^{2}$ UMR_S1138, Centre de

Recherche des Cordeliers, Paris, France; ${ }^{3}$ Hopital Ambroise Paré,

Boulogne, France

Correspondence: Walter J Storkus (alw151@pitt.edu); Catherine SautèsFridman

Journal for ImmunoTherapy of Cancer 2017, 5(Suppl 2):P499

\section{Background}

IL-36g/LL-1F9 is a member of the IL-1 cytokine superfamily and a mediator of Type 1 immunity. It was recently reported to be a necessary mediator of tertiary lymphoid organ (TLO) formation in a murine model of colon carcinoma [1]. Specifically, when IL-36g was introduced therapeutically into the tumor microenvironment of MC38bearing mice, tumors exhibited delayed progression in correlation with TLO formation; this was not observed in control-treated mice. Co-treatment with the antagonist to IL-36g, IL-1F5, blocked the efficacy of the therapy. CD11c+ dendritic cells (DC) were observed to be a major source of IL-36g in the tumor microenvironment (TME), and signaling through IL-36R+ host cells (including those of the vasculature) was necessary for the efficacy of IL-36g-based treatment. In the present study, we sought to identify a role for IL-36g in the de novo formation of TLO.

Methods

In a cohort of 33 primary colorectal cancer patients, the histologic localization and magnitude of expression of IL-36g, IL-1F5/L-36RA, CD20, CD138, CD8, CD31, PNAd, DC-LAMP, CD68, and Thet were determined using immunohistochemical and immunofluorescent imaging. Images were analyzed using CaloPix and Visiopharm software packages.

Results

Both IL-36g and IL-1F5 can be expressed by vasculature-associated smooth muscle cells/myofibroblasts as well as immune cells. Expression of IL-36g by the tumor vasculature positively correlates with CD20+ B cell density in TLO, but not the number of TLO observed in the tumor; neither observation holds for IL-1F5. Some IL-36g+ vessels appear to be high endothelial venules, specialized vessels that aid in recruiting lymphocytes to TLO. Interestingly, while B cells are the primary cellular component of TLO in this model, they do not produce IL-36g. Within the immune compartment, CD8+ T cells (including those expressing Tbet) and CD68+ macrophages are major sources of IL-36g in the TME, while DC-LAMP+ DC are less so.

Conclusions

These results support that the pro-inflammatory conditions of colorectal cancer can promote a Type 1-polarized immune microenvironment; that IL-36g is secreted by diverse cell types within the TME; and that IL-36g expression may play a role in the maintenance of TLO and in B cell recruitment to these structures, within the immune contexture of colorectal cancer.

\section{References}

1. Weinstein $A M$, Chen L, Brzana EA, Patil PR, Taylor JL, Fabian KL, Wallace $C T$, Jones SD, Watkins SC, Lu B, Stroncek DF, Denning TL, Fu YX, Cohen PA, Storkus WJ. Tbet and IL-36g cooperate in therapeutic DC-mediated promotion of ectopic lymphoid organogenesis in the tumor microenvironment. Oncolmmunology. 2017; 6(6).

P500

CD73 blockade restores the abscopal response to radiation therapy and anti-CTLA-4 in cGAS-deficient tumors

Erik Wennerberg, Claire Vanpouille-Box, Silvia Chiara Formenti, Sandra Demaria

Weill Cornell Medicine, New York, NY, USA

Correspondence: Erik Wennerberg (erik.ag.wennerberg@gmail.com) Journal for ImmunoTherapy of Cancer 2017, 5(Suppl 2):P500 


\section{Background}

We have recently shown that in poorly immunogenic tumors resistant to immune checkpoint blockers (ICB), radiation therapy (RT) used at 8GyX3 induces cancer cell-intrinsic interferon type I (IFN-I) through cGAS-activation. This process is required for recruitment of BATF3dependent $\mathrm{CD}_{103^{+}}$dendritic cells (DCs) and for priming of tumorspecific $C D 8^{+} \mathrm{T}$ cells that mediated abscopal responses in the presence of ICBs [1]. Importantly, knockdown of cGAS in the cancer cells at the irradiated tumor site abrogates the induction of abscopal responses by RT+ICB. Extracellular ATP, which is released from irradiated cancer cells, provides an alternative signal for recruitment and activation of DCs. However, rapid conversion of ATP into immunosuppressive adenosine effectively abrogates this process. Therefore, we hypothesized that antibody blockade of the adenosine-converting ectoenzyme CD73 could restore abscopal responses by RT+ICB in cGAS-deficient tumors by augmenting ATP-mediated DC recruitment.

\section{Methods}

Mice bearing subcutaneously inoculated flank tumors (CGAS-silenced TSA $^{\text {shcGAS }}$ or mock-silenced TSA ${ }^{\text {shNS }}$ as primary tumors and TSA ${ }^{\text {shNS }}$ as abscopal tumors) were treated with (1) control antibody; (2) RT; (3) RT tanti-CD73; (4) RT+anti-CTLA-4 or (5) RT+anti-CD73+anti-CTLA-4. AntiCD73 was administered on day 11, 14, 17 and 20 and anti-CTLA-4 on day 14,17 and 20. RT was administered to the primary tumor in doses of $8 \mathrm{~Gy}$ on day 12,13 and 14 . Tumors were analyzed for DC infiltration on day 18. Results

In mice bearing primary TSA ${ }^{\text {shNS }}$ tumors, RT+anti-CTLA-4 elicited abscopal responses. In mice bearing primary TSA $A^{\text {shcGAS }}$ tumors, RT+anti-CTLA4 did not induce abscopal responses, while RT+anti-CTLA-4+anti-CD73 did (Tumor size day $37: 873 \pm 533 \mathrm{~mm}^{3}$ in control; $827 \pm 732 \mathrm{~mm}^{3}$ in RT tanti-CTLA-4; $131 \pm 169 \mathrm{~mm}^{3}$ in RT+anti-CTLA-4+anti-CD73, $p<0.01$ ). Treatment of mice bearing TSA $A^{\text {shcGAS }}$ tumors with RT+anti-CD73 resulted in significantly increased tumor infiltration of $\mathrm{CD} 103^{+} \mathrm{DCs}$ among intratumoral leukocytes compared to mice receiving RT alone $(1.2 \pm 0.8 \%$ in RT v. $2.5 \pm 0.7 \%$ in RT+anti-CD73, $p<0.05)$.

\section{Conclusions}

Our results demonstrate that blocking CD73 recovers the ability of RT to induce recruitment of $\mathrm{CD}_{103^{+}} \mathrm{DCs}$ to CGAS-deficient tumors and restores the abscopal anti-tumor responses elicited by RT+ICB.

\section{References}

1. Vanpouille-Box C, et al. DNA exonuclease Trex1 regulates radiotherapyinduced tumour immunogenicity. Nat Commun. 2017;8:15618.

\section{P501}

Involvement of local renin-angiotensin system in immunosuppression of tumor microenvironment and its modulation for augmentation of cancer immunotherapy

Tomonori Yaguchi ${ }^{1}$, Kenta Nakamura², Kenji Morii ${ }^{1}$, Yutaka Kawakami

${ }^{1}$ Keio University School of Medicine, Shinjuku-ku, Tokyo, Japan; ${ }^{2}$ Shinshu University School of medicine, Matsumoto, Nagano, Japan

Correspondence: Yutaka Kawakami beatless@rr.iij4u.or.jp Journal for ImmunoTherapy of Cancer 2017, 5(Suppl 2):P501

\section{Background}

To improve the current cancer immunotherapies, strategies to modulate various immunosuppressive stromal cells need to be developed. Local renin-angiotensin system (RAS) in cancer tissues has been reported to be involved in cellular migration, proliferation, inflammation, and angiogenesis in the tumor and supporting stromal cells, few studies have evaluated the effect of local RAS on anti-tumor immune responses. In this study we have evaluated the role of local RAS in the tumor immune-microenvironment.

Methods

We have evaluated the effect of administration of angiotensin receptor blockers (ARBs) on phenotypes of CD11+ myeloid cells and cancer associated fibroblasts (CAF) in cancer microenvironments, induction of tumor antigen specific T cells, and therapeutic effects of PD-1/PD-L1 immune checkpoint blockade therapies, using murine models bearing tumor cell lines in which RAS was not involved in their proliferation and angiogenetic ability.

Results

Administration of ARBs to tumor-bearing mice resulted in significant enhancement of tumor antigen specific T cells. The ARB administration did not change the numbers of CD11b+ myeloid cells in tumors, but significantly reduced their T-cell inhibitory ability along with decreased production of various immunosuppressive factors including IL-6, IL-10, VEGF, and arginase by CD11b+ cells in tumors. ARB also decreased expression of immunosuppressive factors such as chemokine ligand 12 and nitric oxide synthase 2 in CAFs. Lastly, combination of ARB and anti-programmed death-ligand 1 (PD-L1) antibodies resulted in significant augmentation of anti-tumor effects in a CD8positive $T$ cell-dependent manner.

Conclusions

These results demonstrated that RAS is involved in generation of immunosuppressive tumor microenvironments caused by myeloid cells and fibroblasts, other than the previously shown proliferative and angiogenetic properties of cancer cells and macrophages, and that ARB can transform the immunosuppressive properties of MDSCs and CAFs and could be used in combination with PD-1/PD-L1 immune checkpoint blockade therapies.

\section{P502}

ID01, an immune checkpoint with distinct mechanisms and prognostic significance in glioblastoma

Lijie Zhai ${ }^{1}$, Derek Wainwright ${ }^{1}$, Erik Ladomersky', Carlos Dostal ${ }^{2}$, Kristen Lauing ${ }^{1}$, Robert McCusker ${ }^{2}$, David Binder ${ }^{3}$, Meijing Wu', Matthew Genet ${ }^{1}$, Galina Gritsina ${ }^{1}$, Balaz Gyorffy ${ }^{4}$, Priscilla Brastinanos ${ }^{5}$, Jeffrey Sosman?

Craig Horbinski ${ }^{1}$, David James ${ }^{1}$, Roger Stupp ${ }^{1}$, Francis Giles ${ }^{1}$

${ }^{1}$ Northwestern University Feinberg School of Medicine, Chicago, IL, USA; ${ }^{2}$ University of Illinois at Urbana-Champaign, Urbana, IL, USA; ${ }^{3}$ University of Colorado School of Medicine, Aurora, CO, USA; ${ }^{4}$ Semmelweis University,

Budapest, Hungary; ${ }^{5}$ Massachusetts General Hospital, Boston, MA, USA

Correspondence: Derek Wainwright (zhailijie12@gmail.com)

Journal for ImmunoTherapy of Cancer 2017, 5(Suppl 2):P502

\section{Background}

It is commonly accepted that indoleamine 2, 3 dioxygenase 1 (IDO1), a pivotal immune checkpoint, mediates potent immunosuppression in cancers through its tryptophan (Trp)-to-kynurenine (Kyn) catabolizing activity. However, this enzymatic-dependent mechanism of IDO1 has not been established in glioblastoma (GBM), the most common malignant brain tumor in adults. Additionally, the basis for elevated IDO1 expression in GBM is also poorly understood. The major objective of this study is to address this gap in our understanding of how IDO1 contributes to the biology of GBM and immunotherapy of this deadly malignance, as well as whether its level of expression is a determinant of GBM patients' outcome.

Methods

Freshly resected human GBM, human T cell:GBM co-cultures, HPLC analysis, CTLA-4 and PD-L1 immune checkpoint blockade, IDO1 $1^{-1-}$ and $\mathrm{IDO}^{+/+} \mathrm{C} 57 \mathrm{BL} / 6$ mice, spontaneous GBM [GFAP(ERT2) $\rightarrow \mathrm{Cre}^{+/-}$; $\mathrm{pTEN}^{\mathrm{fl} /}$ $\left.{ }_{\mathrm{fl}} ; \mathrm{Rb}^{\mathrm{fl} / \mathrm{fl}} ; \mathrm{p} 53^{\mathrm{fl} / \mathrm{fl}} ;\left(+/ / \mathrm{IDO1}^{\mathrm{fl} / \mathrm{fl}}\right) ;\left(+/-\mathrm{IDOO} 1^{-/}\right)\right]$mice, nu/nu mice, NOD-scid and humanized (NSG-SGM3-BLT) mice engrafted human GBM as well as the cancer genome atlas form the basis of our investigation. Results

(1) Depletion of GBM-derived IDO1 did not affect brain tumor Trp catabolism, while systemic knockout of IDO1 resulted in decreased Trp$>$ Kyn conversion in both naïve and tumor-bearing mice; (2)CTLA-4 and PD-L1-based dual immunotherapy did not alter Trp/Kyn level in IDO1 ${ }^{-1}$ and $\mathrm{IDO} 1^{+/+}$mice, but only reached to maximal treatment effect in IDO1 
${ }^{+/+}$mice; (3) In situ hybridization for IDO1 revealed consistent transcript expression in all human GBM samples $(n=172)$, whereas immunohistochemical IDO1 expression was highly variable; (4) Multivariate analysis revealed that higher levels of IDO1 transcript predicted/correlated with shorter survival in the TCGA dataset $(P=0.0076)$ and IDO1 mRNA levels positively correlate with increased gene expression for markers of cytolytic and regulatory T cells, but no correlation with IFNs gene expression; (5) Humanized mice intracranially-engrafted with human GBM revealed an IFNY-dependent and T cell-mediated increase of intratumoral IDO1 expression.

\section{Conclusions}

Our data indicate that tumor cell-derived IDO1 mediates its immunosuppression in GBM via a non-enzymatic mechanism, while the nontumor IDO1 dominantly contributes to its Trp catabolic function and is essential for the immune checkpoint blockade therapy. TCGA analysis also revealed high intratumoral IDO1 mRNA levels correlate with inferior GBM patient outcome and infiltrating $T$ cells. Future immunotherapeutic efforts aiming to increase $T$ cell-mediated immune response should consider combinatorial approaches that inhibit potential T cellmediated IDO1 upregulation during therapy.

\section{P503}

Comprehensive characterization of immune microenvironment of cholangiocarcinoma

Tao Xia', Dwayne Thomas ${ }^{1}$, Linda Chen ${ }^{1}$, Qingfeng Zhu' ${ }^{1}$ Jin He', Robert Anders $^{1}$, Shafat Quadri ${ }^{2}$, Elaine Pinherio ${ }^{2}$, Adrian Murphy ${ }^{1}$, Lei Zheng ${ }^{1}$

${ }^{1}$ Johns Hopkins University School of Medicine, Baltimore, MD, USA;

${ }^{2}$ Merck, Merck, NJ, USA

Correspondence: Lei Zheng Izheng6@jhmi.edu

Journal for ImmunoTherapy of Cancer 2017, 5(Suppl 2):P503

\section{Background}

Background: Biliary tract cancers (BTC) are groups of tumors that include both intrahepatic cholangiocarcinoma (ICC), extrahepatic cholangiocarcinoma (ECC) and gallbladder carcinoma (GC). BTC comprise an uncommon cancer type that is currently on the rise with a high mortality rate. New modalities of treatment for BTC are highly demanded. Immunotherapy has emerged as a promising treatment for many malignant diseases. Although many risk factors of BTC are associated with chronic inflammatory conditions, the immune microenvironment of BTC has been examined to a very limited extent. Therefore, we perfored a comprehensive analysis of tumor microenvironment of BTCs to identify immune mechanistic targets for immunotherapy and identify optimal strategies of combinational immunotherapy.

Methods

Materials and methods: Surgically resected tumors from 31 cases of ECC and 24 cases of ICC were stained with multiplex immunohistochemistry of 14 immune cell markers including CD3, CD4, CD8, CD20, CD45, CD68, Cd163, CSF1R, DC-LAMP, DC-Sign, Foxp3, Ki-67, PD-1, PD-L1 on single slide section and analyzed by the Halo software. Nanostring of immune panels and cytokine arrays PCR were also performed by RNA purified from FFPE slides.

Results

Results: Higher density of CD8 T cells is significantly associated with longer overall survival $(p=0.024)$. Higher densities of PD- $1+$ T cells and PD-L1+ myeloid cells are significantly associated with longer overall survival in ECC (PD-L1, $p=0.019 ; \mathrm{PD}-1, p=0.018$ ) (Fig. 1, Fig. 2), but higher density of PD-L1+ cells are significantly associated with shorter overall survival in ICC ( $p=0.003$ ) (Fig. 3, Fig. 4). CD68 is not prognostic. However, higher density of CD163+CD68+ M2 macrophages is associated with poorer survival in ECC (Fig. 5). Nanostring and cytokine array PCR results are being analyzed. More cases are being stained by multiplex immunothistochemistry.

\section{Conclusions}

Conclusion: Our study represents so far the most comprehensive characterization of immune cell subtypes in the tumor microenvironment of cholangiocarcinoma. The results suggest that PD-1/PD-L1 is not a good target in ECC, but a potentially good target in ICC. An ideal macrophage-targeting agent would be the one that depletes the M2 macrophages more specifically.

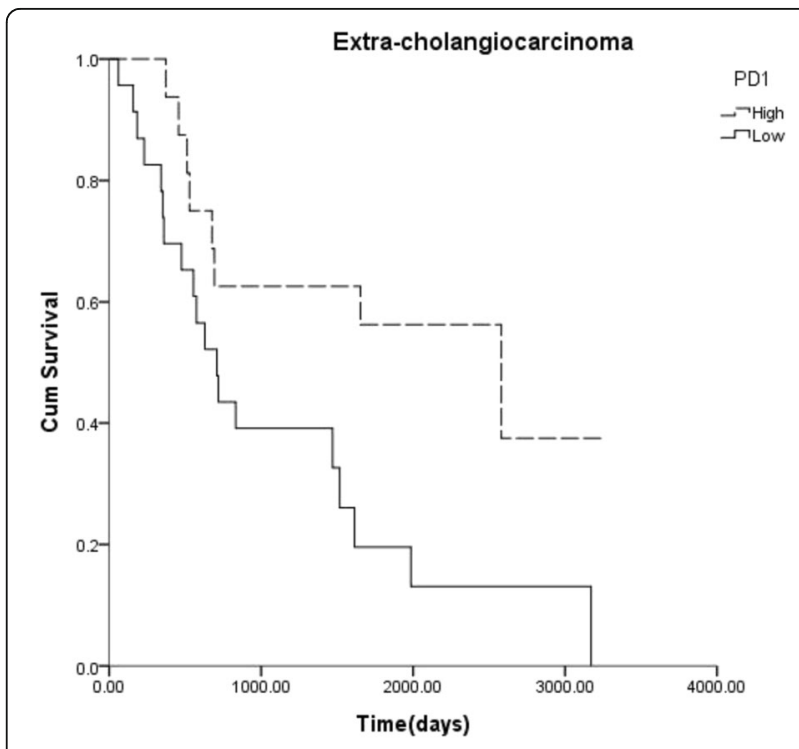

Fig. 1 (abstract P503) See text for description

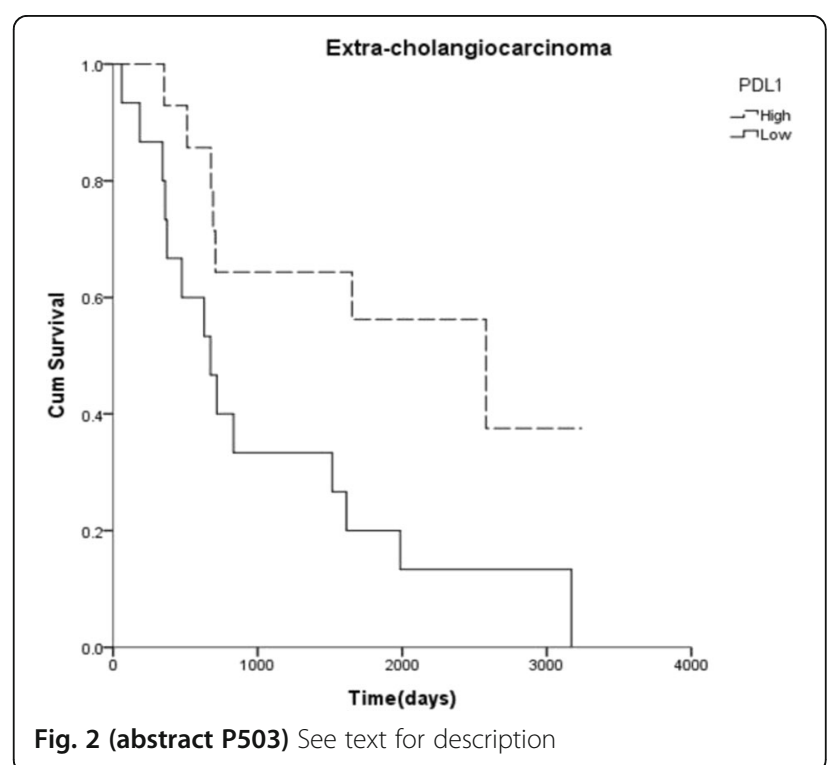




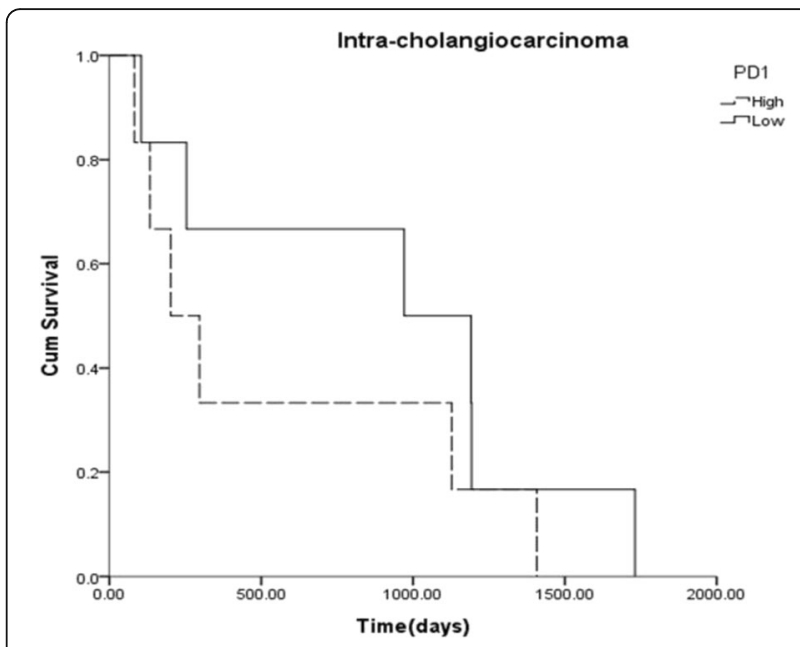

Fig. 3 (abstract P503) See text for description

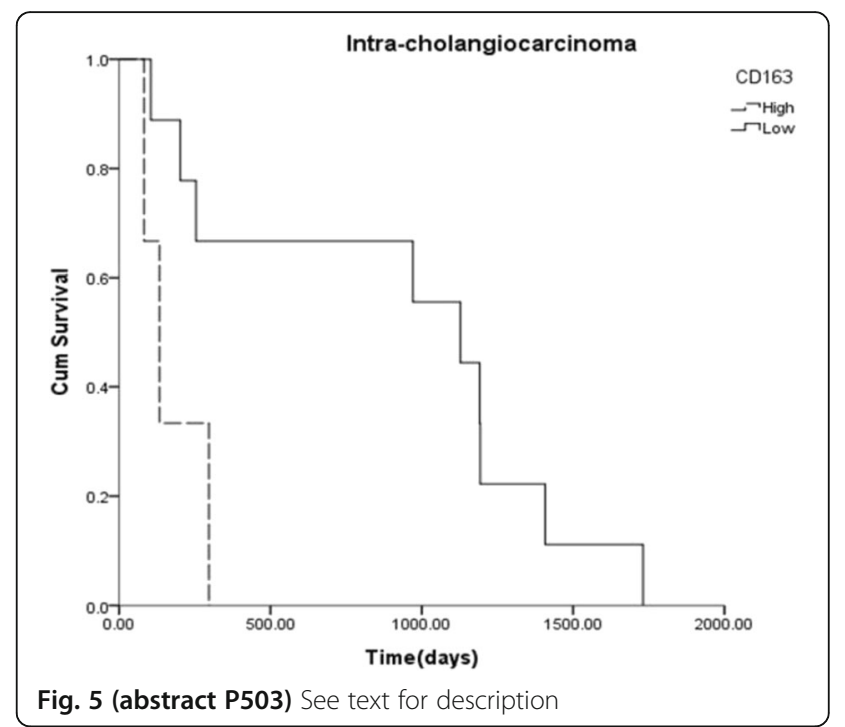

P504

Enhancement of CAR-T cell tumor tropism through expression of CXCR2 chemokine receptors

Ismahene Benzaid', Daniel Santiago', Cecilia Ramello', Mibel Pabon'1 Wendy Kandell', Theresa Boyle', Anders Berglund', Scott Antonia', Daniel Abate-Daga'

${ }^{1}$ Moffitt Cancer Center, Tampa, FL, USA

Correspondence: Daniel Santiago Daniel.AbateDaga@moffitt.org Journal for ImmunoTherapy of Cancer 2017, 5(Suppl 2):P504

\section{Background}

Chimeric Antigen Receptor (CAR)-T cell-based immunotherapies are novel approaches to cancer therapy. With the expected FDA approval of an anti-CD19 CAR for B-cell malignancies, adoptive immunotherapies will become the standard of care for a large number of patients. However, the clinical success of CAR-T cells beyond hematological malignancies has been scarce. We hypothesize that this is in part due to suboptimal bioavailability of CAR-T cells in solid tumors, and we propose to manipulate the expression of chemokine receptors to enhance the CAR-T cells homing and increase their therapeutic efficacy against the tumor.

Methods

We first analyzed the expression of chemokines by human lung tumors, using publicly available databases of RNA expression (TCGA). In addition, we measured their expression in the supernatants of shortterm cultures of human lung tumors. Retroviral vectors were generated for the expression of chemokine receptors, in combination with an anti-mesothelin (MSLN) second generation CAR. T cell migration and function were assessed using migration transwell and cytotoxic assays. Anti-tumor efficacy was assessed using a xenograft model of lung cancer. 
Results

IL-8 (CXCL8) was consistently expressed by lung tumors, and secreted by short-term cultures of surgically-removed lesions. Co-expression of an anti-MSLN CAR and CXCR2 or CXCR1 increased the migration of human $T$ cells towards medium containing recombinant IL-8, or conditioned media from lung cancer cell cultures, in transwell assays. Importantly, co-expression of chemokine receptors did not compromise CAR expression or function. Furthermore, expression of CXCR2 (but not CXCR1) in CAR T cells increased the number of intratumoral $T$ cells, and significantly enhanced antitumor efficacy in a xenograft tumor model based on $\mathrm{H} 2110$ cells.

Conclusions

Tumor homing and therapeutic efficacy of CAR-T cells can be improved through co-expression of chemokine receptors. This approach may apply to other types of $\mathrm{T}$ cell-based immunotherapies, for the treatment of lung cancer, and other solid tumors.

\section{P505}

First in human, single ascending dose study in healthy volunteers of SNDX-6352, a humanized IgG4 monoclonal antibody targeting colony stimulating factor-1 receptor (CSF-1R)

Renger Tiessen', Anniek Visser ${ }^{2}$, Henko Tadema ${ }^{2}$, Caryn Peterson ${ }^{2}$, Helen Pentikis ${ }^{3}$, Lei Wang ${ }^{3}$, Michael L. Meyers ${ }^{4}$, Peter Ordentlich ${ }^{3}$

${ }^{1}$ PRA Health Sciences, Groningen, Netherlands;

${ }^{2}$ PRA Bioanalytical Laboratory, Assen, Netherlands;

${ }^{3}$ Syndax Pharmaceuticals, Inc., Waltham, Massachussets, USA;

${ }^{4}$ Syndax Pharmaceuticals, Inc., New York, New York, USA

Correspondence: Renger Tiessen tiessenrenger@prahs.com

Journal for ImmunoTherapy of Cancer 2017, 5(Suppl 2):P505

\section{Background}

CSF-1R is expressed on immunosuppressive tumor associated macrophages (TAMs). High numbers of TAMs in tumors are associated with poor prognosis. SNDX-6352 is a humanized lgG4 monoclonal antibody with high affinity against CSF-1R and has demonstrated antitumor activity in preclinical tumor models by enhancing the immune response.

Methods

Study SNDX-6352-0001 is a first-in-human, double-blind, randomized, placebo-controlled, single ascending dose, phase I trial in healthy volunteers. The planned dose levels were $0.15,1.0,3.0,6.0$, and 10.0 $\mathrm{mg} / \mathrm{kg}$. All treated subjects were observed for 72 hours post-infusion and underwent follow-up evaluations for 12 weeks. Safety assessments included adverse event (AE) monitoring, clinical laboratories, vital signs, 12-lead electrocardiogram (ECG), eye examination, and physical examination. Blood samples were collected to characterize the pharmacokinetics (PK) and pharmacodynamics (PD; change in plasma colony stimulating factor-1 [CSF-1] and interleukin-34 [IL-34]), as well as to evaluate changes in circulating classical and non-classical CD16+ monocytes in blood and change in CSF-1 receptor occupancy (RO).

Results

The first three placebo-controlled cohorts were completed with 14 subjects receiving SNDX-6352 (2 at $0.15 \mathrm{mg} / \mathrm{kg}, 6$ at $1 \mathrm{mg} / \mathrm{kg}$ and 6 at $3 \mathrm{mg} / \mathrm{kg}$ ) and 6 subjects receiving placebo ( 2 in each cohort). Dosing up to $1.0 \mathrm{mg} / \mathrm{kg}$ was tolerated well with the most common complaint being mild to moderate itching (lasting 2 or 3 days). At the 3 $\mathrm{mg} / \mathrm{kg}$ dose, similar itching complaints were observed. At this dose, mild to moderate eyelid swelling was observed in all subjects (median duration of 40 days), with no impact on vision. Dose escalation was then terminated. In blood, a transient reduction in non-classical CD16+ monocytes was observed, and an upward trend in LDH and ASAT. Cmax increases corresponded with dose, while AUC increases were greater than dose proportional, indicating non-linear PK. Incr.

Publisher's Note

Springer Nature remains neutral with regard to jurisdictional claims in published maps and institutional affiliations.

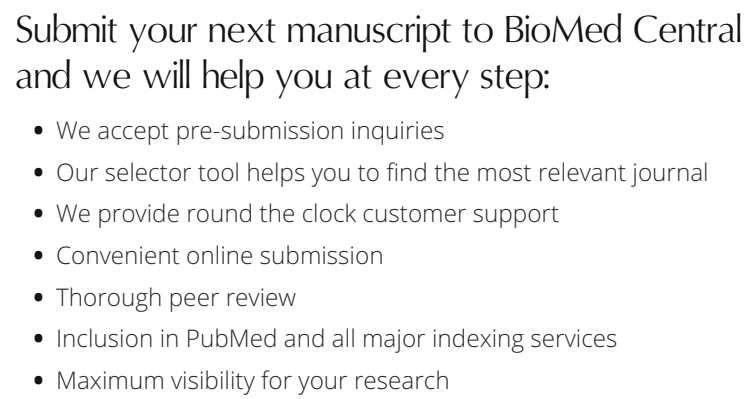

Biomed Central 